\title{
Radical $\alpha$-Trifluoromethoxylation of Ketones by Means of Organic Photoredox Catalysis
}

\author{
Thibaut Duhail, ${ }^{[a] \dagger}$ Tommaso Bortolato,,${ }^{[b] \dagger}$ Javier Mateos, ${ }^{[b]}$ Elsa Anselmi, ${ }^{[a, c]}$ Benson Jelier, ${ }^{[d]}$ Antonio \\ Togni, ${ }^{[\mathrm{d}]}$ Emmanuel Magnier, ${ }^{[\mathrm{a}] *}$ Guillaume Dagousset ${ }^{[\mathrm{a}] *}$ and Luca Dell'Amico ${ }^{[\mathrm{b}] *}$
}

\begin{abstract}
The first light-driven method for the $\alpha$-trifluoromethoxylation of ketones is reported. Enol carbonates, in particular Boc derivatives, react with $N$-trifluoromethoxy-4-cyano-pyridinium triflimide (2a) using the photoredox-catalyst 4-CzIPN (5 mol-\%) under irradiation at 456 $\mathrm{nm}$ affording the corresponding $\alpha$-trifluoromethoxy ketones in up to $50 \%$ isolated yield and virtually complete chemoselectivity. As shown by 35 examples, representing a great variety of substrates, the reaction is general and proceeds rapidly under batch (1h) and flow conditions (2 min). Mechanistic investigations reveal that a radicalchain propagation is operative, as efficiently orchestrated by the activity of the organic photoredox catalyst. Diverse products manipulations, including ketone reduction and reductive amination, demonstrate the synthetic potential of the disclosed method to accessing elusive trifluoromethoxylated potentially bioactive ingredients.
\end{abstract}

Among the rapidly emerging perfluorinated groups whose introduction into organic structures is of great interest, the $\mathrm{OCF}_{3}$ moiety occupies a very special place. ${ }^{[1]}$ Electronic and steric properties are among the main reasons for the popularity of this group. It brings indeed a high lipophilicity (Hansch parameter: $\pi=$ $+1.04)^{[2]}$ to the molecules and possesses a high electronegativity (Pauling's electronegativity scale: $\chi=3.7$ ) which earned it the nickname of super-halogen. ${ }^{[3]}$ These remarkable physicochemical properties associated to good metabolic stability and unique conformational properties make this group particularly attractive for the life sciences. ${ }^{[4]}$

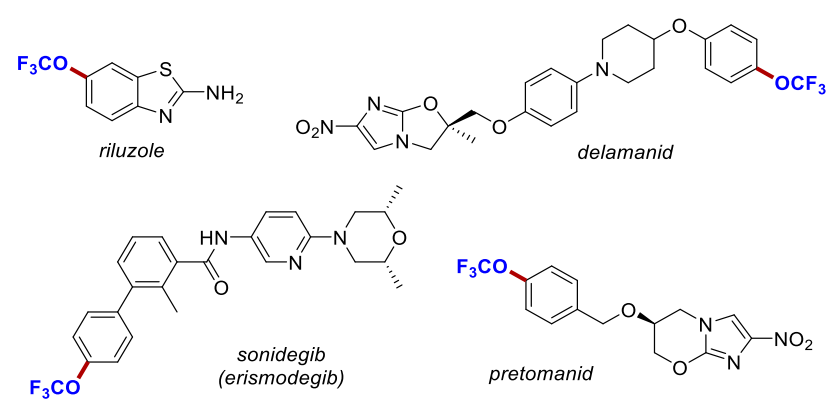

Figure 1. The four drugs containing a $\mathrm{OCF}_{3}$ moiety in their skeleton.

[a] T. Duhail, Dr. E. Anselmi, Dr. E. Magnier, Dr. G. Dagousset Université Paris-Saclay, UVSQ, CNRS, UMR 8180

Institut Lavoisier de Versailles, 78035 Versailles Cedex (France)

E-mail: emmanuel.magnier@uvsq.fr, guillaume.dagousset@uvsq.fr

[b] T. Bortolato, J. Mateos, Dr. L. Dell'Amico. Department of Chemical Sciences, University of Padova, Via Marzolo 1, 35131, Padova (Italy) E-mail: luca.dellamico@unipd.it

[c] Dr. E. Anselmi. Université de Tours, Faculté des Sciences et Techniques, 37200 Tours (France)

[d] Dr. B. Jelier, Prof. Dr. A Togni

Department of Chemistry and Applied Biosciences, Swiss Federal Institute of Technology, ETH Zurich, Vladimir-Prelog-Weg 2, 8093 Zurich (Switzerland)

† These authors contributed equally.
Despite this, the number of marketed pharmaceutical and agrochemical products containing $\mathrm{OCF}_{3}$ remains low. To date, only 4 of the 340 identified drugs containing at least one fluorine atom bear a $\mathrm{OCF}_{3}$ group (Figure 1). ${ }^{[5]}$ Among the 424 fluoroagrochemicals, 10 with $\mathrm{OCF}_{3}$ are listed. ${ }^{[6]}$ It should also be pointed out that for these 14 commercial molecules the $\mathrm{OCF}_{3}$ is always attached to an aromatic ring. This contrasting situation is mainly due to the small number of existing methods and/or the lack of reagents capable of delivering

this functional target under selective conditions at intermediate or late stage of a synthetic route. Pioneering works have focused on the construction of the $\mathrm{O}-\mathrm{CF}_{3}$ bond from already installed $\mathrm{OH}$ group, via i) multistep processes, requiring harsh conditions/toxic reagents (e.g. $\left.\mathrm{HF}, \mathrm{SF}_{4}\right),{ }^{[7]}$ or ii) direct electrophilic trifluoromethylation of alcohols, either with hypervalent iodine reagents which require large excesses of alcohol (5-75 equiv) to achieve reasonable yields, or with an unstable oxonium salt. ${ }^{[8]}$ Despite recent improvements, ${ }^{[9]}$ such approaches are still limited in practicality and scope. An elegant alternative to access trifluoromethoxylated compounds is the direct introduction of the $\mathrm{OCF}_{3}$ functionality (Scheme 1). To this end, nucleophilic routes have been proposed, with recent leading advances involving the description of new sources of the trifluoromethoxide anion or new methods for its in-situ formation (Scheme 1a). ${ }^{[10]}$ However, the use of this approach is hampered by several issues: 1) decomposition of the $\mathrm{OCF}_{3}$ entity to fluorophosgene, 2) reagent synthesis from gaseous or expensive chemicals, 3) the requirement of several additives and inert-atmosphere techniques, and 4) the need for pre-functionalized starting materials. Previously unknown, the radical approach emerged in 2018 and has seen rapid and dramatic developments, ${ }^{[11]}$ in particular with the invention of three new reagents (Scheme $1 b$ ).

(a) Nucleophilic trifluoromethoxylation

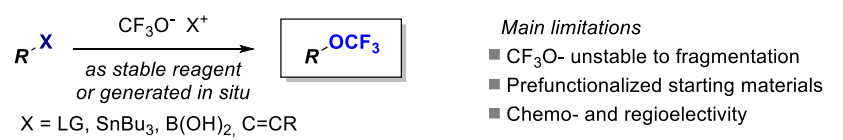

(b) Radical trifluoromethoxylation

$A r^{-}-\stackrel{[R u]}{\underset{\text { blue LEDs }}{\longrightarrow}} A r^{-\mathrm{OCF}_{3}}$
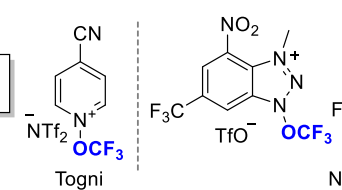
$\mathrm{NO}_{2}$

- Limited to aromatic substrates - Selectivity issues

Togni

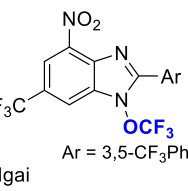

(c) This work Light-mediated trifluoromethoxylation of ketones

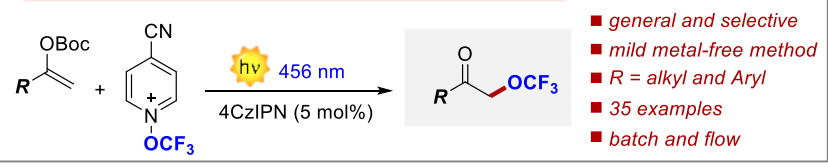

Scheme 1. Direct trifluoromethoxylation processes.

One of us designed a pyridine $\mathrm{N}$-oxide reagent ${ }^{[12]}$ and the group of Ngai reported the use of azole-based compounds. ${ }^{[13]}$ Under 
photoredox conditions, these three reagents proved efficient for the catalytic $\mathrm{C}-\mathrm{H}$ trifluoromethoxylation of arenes and heteroarenes. ${ }^{[14]}$ To date, their scope has not been extended beyond (hetero)aromatic substrates. This represents an unprecedented challenge, the success of which would give access to new or hitherto poorly described molecules due to their cumbersome synthesis.

We herein report a mild metal-free visible-light-driven strategy to tackle this unsolved synthetic issue. We identified enol carbonates as substrates for their peculiar stereoelectronic properties, their ease of preparation and for the molecular diversity they offer in view of the trifluoromethoxylation of structually diverse ketones (Scheme 1c). ${ }^{[15]}$

Table 1. Selected optimisation results for the light-driven $\alpha$ trifluoromethoxylation of ketones.
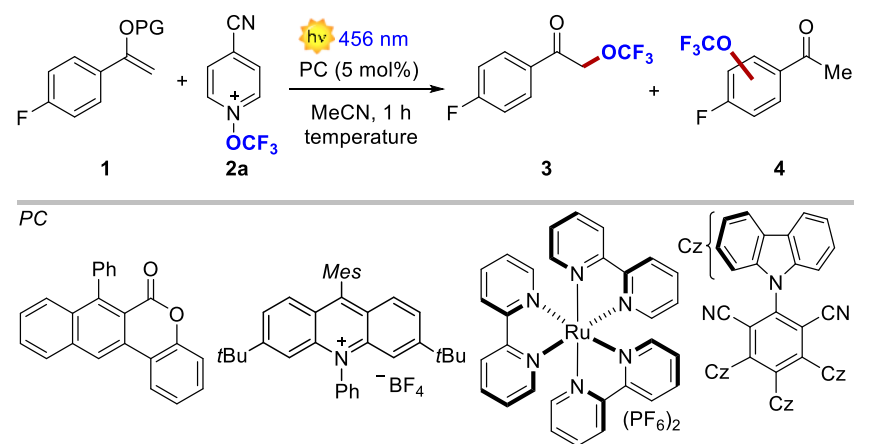

\begin{tabular}{|c|c|c|c|c|c|c|}
\hline \multicolumn{2}{|c|}{$\begin{array}{c}\text { NTC1 } \\
E_{\text {ox }}^{*}=-1.27 \mathrm{~V} \\
E_{\text {red }}^{*}=1.28 \mathrm{~V}\end{array}$} & \multicolumn{2}{|c|}{$\begin{array}{c}\text { Mes-Acr } \\
-\end{array}$} & \multicolumn{2}{|c|}{ 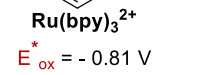 } & \multirow{2}{*}{$\begin{array}{c}\text { 4-CzIPN } \\
E_{o x}^{*}=-1.18 \mathrm{~V} \\
\mathrm{E}_{\text {red }}^{*}=1.43 \mathrm{~V} \\
\mathbf{3}, \\
\text { Yield }\end{array}$} \\
\hline $\begin{array}{l}E_{\text {red }}= \\
\text { Entry }\end{array}$ & $1, P G$ & $\begin{array}{l}1, \\
\text { eq. }\end{array}$ & $\begin{array}{r}2.10 \mathrm{~V} \\
\mathrm{PC}\end{array}$ & $\begin{array}{l}\text { E red }= \\
\text { Light } \\
\text { source }\end{array}$ & $\begin{array}{c}3: 4 \\
\text { ratio }\end{array}$ & \\
\hline 1 & $1 \mathrm{a}, \mathrm{Ac}$ & 10 & - & $400 \mathrm{~nm}$ & $8: 1$ & 17 \\
\hline 2 & 1a, Ac & 10 & NTC1 & $420 \mathrm{~nm}$ & 13:1 & 20 \\
\hline 3 & $1 \mathrm{a}, \mathrm{Ac}$ & 10 & Mes-Acr ${ }^{+}$ & $456 \mathrm{~nm}$ & $9: 1$ & 7 \\
\hline 4 & 1a, Ac & 10 & $\mathrm{Ru}(\mathrm{bpy})_{3}$ & $456 \mathrm{~nm}$ & $12: 1$ & 33 \\
\hline 5 & 1a, Ac & 10 & 4-CzIPN & $456 \mathrm{~nm}$ & $>20: 1$ & 37 \\
\hline $6^{[c]}$ & $1 \mathrm{a}, \mathrm{Ac}$ & 10 & 4-CzIPN & $456 \mathrm{~nm}$ & $>20: 1$ & 41 \\
\hline $7^{[\mathrm{c}, \mathrm{d}]}$ & 1a, Ac & 10 & 4-CzIPN & $456 \mathrm{~nm}$ & $>20: 1$ & 44 \\
\hline $8^{[c, d]}$ & 1b, Boc & 10 & 4-CzIPN & $456 \mathrm{~nm}$ & $>20: 1$ & 52 \\
\hline $9^{[c, d]}$ & 1b, Boc & 5 & 4-CzIPN & $456 \mathrm{~nm}$ & $>20: 1$ & 50 \\
\hline $10^{[\mathrm{c}, \mathrm{d}]}$ & 1b, Boc & 5 & 4-CzIPN & dark & - & 0 \\
\hline
\end{tabular}

[a] Unless otherwise stated reaction conditions: $1.5 \mathrm{~mL}$ of MeCN, [2a $]_{0}=0.033$ $\mathrm{M}$, at $\mathrm{rt}$ for $1 \mathrm{~h}$ irradiation time (see $\mathrm{SI}$ for details). ${ }^{[b]}{ }^{19} \mathrm{~F}-\mathrm{NMR}$ yield using $\mathrm{CF}_{3}-\mathrm{Ph}$ as the internal standard. ${ }^{[c]}$ The reaction was performed at $[\mathbf{2} \mathbf{a}]_{0}=0.01 \mathrm{M}$. ${ }^{[\mathrm{d}]}$ The reaction was conducted at $50^{\circ} \mathrm{C}$.

Our optimization began by studying the reaction between enol acetate $\mathbf{1 a}$ (10 equiv.) and the $\mathrm{N}$-trifluoromethoxypyridinium 2a, commercially available as $\mathrm{NTf}_{2}{ }^{-}$salt (Table 1 , entry 1 ). We initially evaluated the possibility of exploiting an electron-donor-acceptor (EDA) complex between the two reagents. ${ }^{[16]}$ Indeed, by mixing 1a and 2a a clear charge-transfer (CT) band was observed in the absorption spectra. Irradiation of the CT band at $400 \mathrm{~nm}$ delivered the trifluoromethoxylated target $\mathbf{3}$ in $17 \%$ yield. Quite unexpectedly, product 3 was accompanied by the undesired sideproduct 4 , where the $\mathrm{OCF}_{3}$ group was introduced onto the aromatic ring. ${ }^{[2]}$ We reasoned that the low chemoselectivity of the process could be overcome by channeling the process towards a purely photoredox manifold, possibly resulting in a chainpropagation process (vide infra). We thus screened various photocatalysts (PCs) characterized by diverse redox and photochemical properties. ${ }^{[17]}$ Naphthochromenone (NTC1, Table 1 , entry 2$)$ resulted in only slight improvements $(20 \%$ yield and 12:1 ratio). ${ }^{[18]}$ This fact was attributed to the EDA-based pathway being concomitantly operating at $420 \mathrm{~nm}$. We thus selected a more red-shifted light source $(456 \mathrm{~nm})$ and evaluated the performance of Mes-Acr ${ }^{+}, \mathrm{Ru}(\mathrm{bpy})_{3}{ }^{2+}$ and $4-\mathrm{CzIPN}$. The low yield $(7 \%)$ and chemoselectivity (9:1) obtained with the highly oxidizing Mes-Acr ${ }^{+}$is attributed to a possibly operating unproductive reductive quenching cycle involving the initial oxidation of $\mathbf{1 a} .^{[19]}$ On the other hand, PCs, characterized by a balanced distribution of the reductive and oxidative power (entry 4 and 5), delivered the product 3 in promising yield and selectivity, up to $37 \%$ and $>20: 1$, respectively. Remarkably, when using the 4-CzIPN PC, 4 was not detected. Interestingly, we observed additional improvements by increasing the temperature in a more diluted medium (entry 6 and 7). Finally, replacing the acetyl (Ac) for a tertbutyloxycarbonyl group (Boc) led to $52 \%$ yield in only $1 \mathrm{~h}$ reaction time (entry 8). Under these conditions we were able to halve the substrate loading with minimal yield erosion (entry 9). Longer reaction times $(3 \mathrm{~h})$ resulted in the appearance of the side-product 4. Furthermore, the reaction did not proceed in the dark, confirming the light-driven nature of the process (entry 10).

Before exploring the generality of the optimised conditions, we decided to decipher the operative mechanism to understand the impact of alternative reaction manifolds on the reaction outcome. As mentioned, the EDA-based pathway resulted inefficient and poorly chemoselective. The poor chemoselectivity was ascribed to the rapid in-situ deprotection of $1 \mathrm{a}$, promoted by its singleelectron oxidation and the following trifluoromethoxylation of the resulting acetophenone. ${ }^{[12]}$ This observation was supported by the fact that more diluted conditions disfavour the EDA complex formation supporting a chain-propagation mechanism (Table 1 entry 5 vs entry 6$)$.
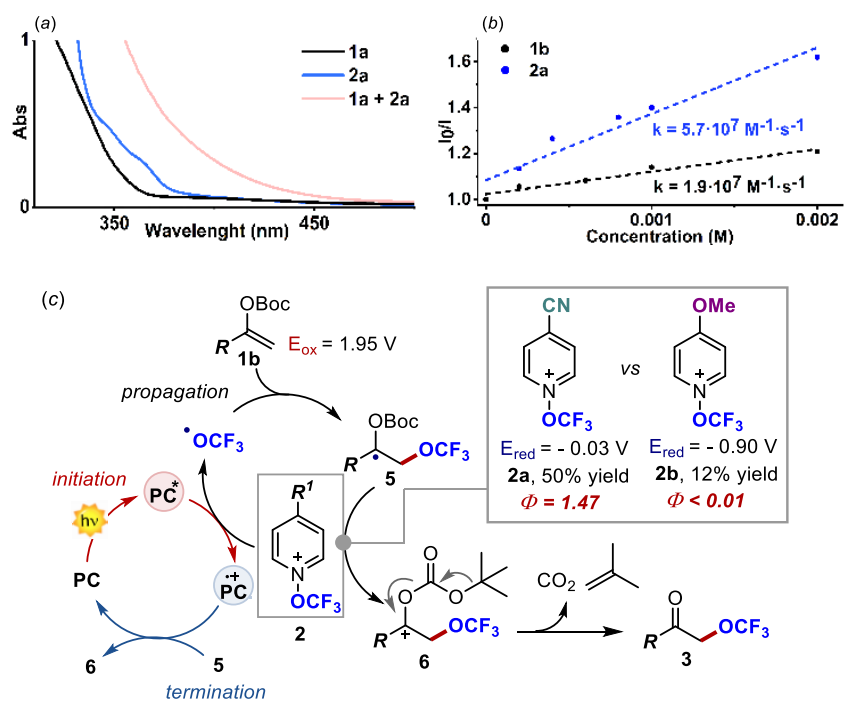

Figure 2 a) UV absorption spectra of 1a, 2a and their mixture. b) Stern-Volme quenching experiment of $4-\mathrm{CzIPN}$ with $\mathbf{1 a}$ versus $\mathbf{2 b}$. c) Proposed reaction manifold under the optimised reaction conditions. 
The reaction catalysed by Mes-Acr further corroborated this hypothesis, indicating that the initial oxidation of $\mathbf{1 a}$ is detrimental to the reaction outcome. Hence, the available concentration of $\mathbf{1 a}$ in the reaction mixture is a key parameter to channel the reactivity towards the intended $\alpha$-trifluoromethoxylation. Interestingly, the identification of enol carbonate $\mathbf{1 b}$ was key to increase the overall reactivity of the system. Specifically, we speculated that the use of the Boc group facilitates the formation of the final product by exploiting the driving force for $\mathrm{CO}_{2}$ and isobutylene formation. The higher reactivity observed under the optimised reaction conditions, together with the Stern-Volmer analysis (Figure 2b) led us to depict the mechanistic scenario shown in Figure 2c. Upon excitation, the PC (4-CzIPN) reaches an electronically excited state that reduces 2 by SET, with the generation of the $\mathrm{OCF}_{3}$ radical, and the formation of the $\mathrm{PC}^{\cdot+}$ radical cation. The $\mathrm{OCF}_{3}$ radical is readily intercepted by 1 , with the formation of the $\mathrm{O}-\mathrm{C}$ bond within the radical intermediate $\mathbf{5}$. At this juncture, $\mathbf{5}$ can reduce a second molecule of 2 , in a radical-chain process which delivers carbocation $\mathbf{6}$. This rapidly evolves to the final product 3 with the formation of $\mathrm{CO}_{2}$ and isobutylene. Finally, the chain process is terminated by the oxidation of 5 by $\mathrm{PC}^{\cdot+}$. This mechanistic hypothesis was also supported by quantum yield measurements in the presence of different trifluoromethoxylating agents $\mathbf{2 a}$ and $\mathbf{2} \mathbf{b}$. When using the easily reducible $\mathbf{2 a}\left(\mathrm{E}_{\mathrm{red}}=-\right.$ $0.03 \mathrm{~V}$ ) we measured a quantum yield of 1.47 , indicating that a Table 2. Scope of the developed visible-light-driven $\alpha$-trifluoromethoxylation of ketones.

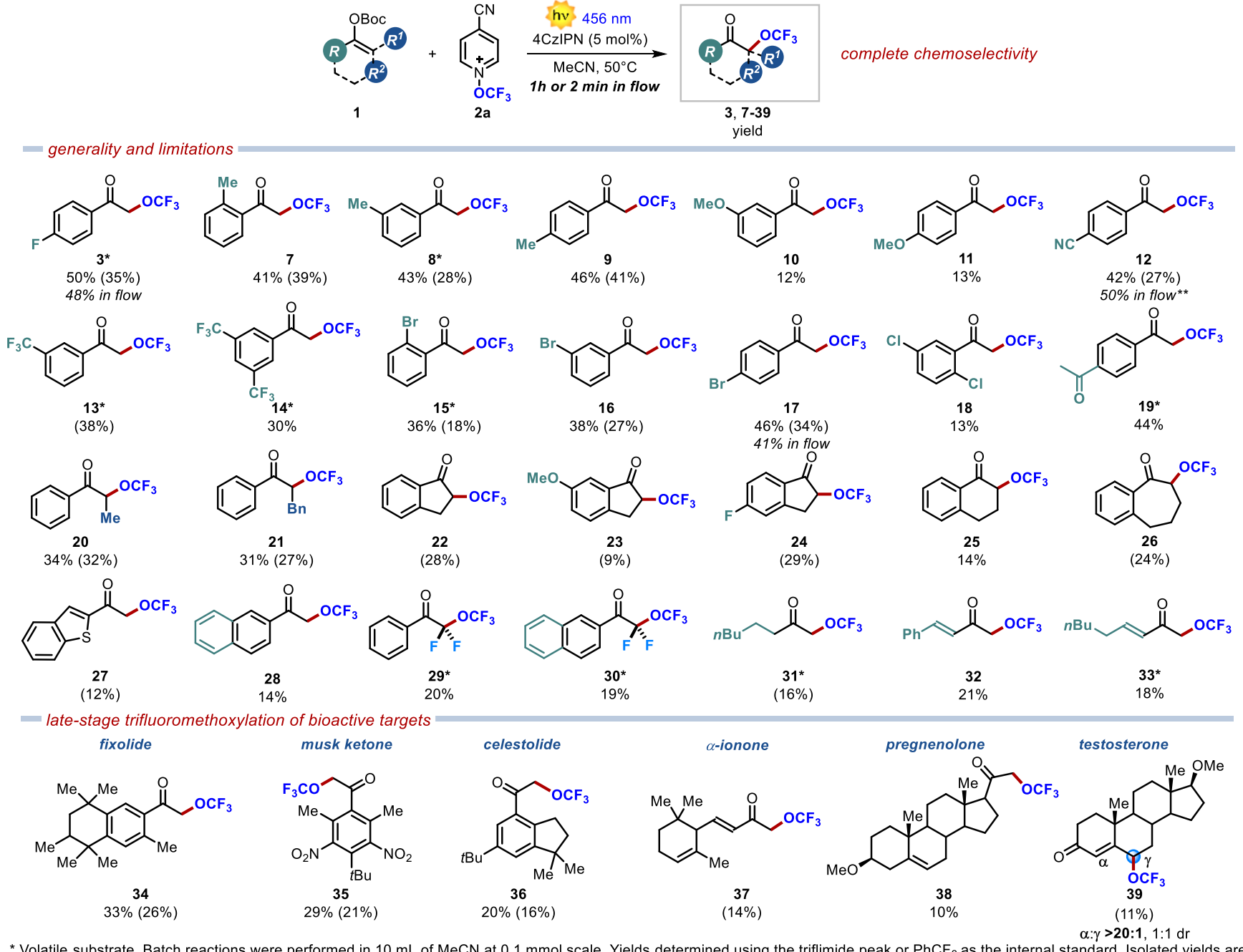

chain propagation is operating. ${ }^{[20]}$ On the contrary, when using the more electron-rich $\mathbf{2 b}\left(\mathrm{E}_{\mathrm{red}}=-0.90 \mathrm{~V}\right)$ the quantum yield dropped drastically to less than 0.01 , indicating that intermediate $\mathbf{5}$ is not able to reduce this pyridinium reagent and the mechanism switches to a classic photoredox cycle. Additionally, the reaction appeared much slower affording in $1 \mathrm{~h}$ product 3 in $12 \%$ yield instead of $50 \%$.

Having deciphered the operative reaction manifold, we moved to test the generality of the developed trifluoromethoxylation method. We first focused our attention on acetophenone derivatives. We were pleased to see that substitutions on all the positions of the aromatic ring were tolerated. Alkyl substituents (79) gave comparable results with yields up to $46 \%$. Remarkably, the reaction was easily transferred into a flow photoreactor without any significant erosion of the yield (48\%), while allowing a shorter reaction time of only $2 \mathrm{~min}$. On the other hand, electrondonating groups led to a considerable yield decrease (10-11). As investigated, this was due to a competing undesired reductive quenching mechanism hampering the chain-propagation process (Figure 2c). Interestingly, electron-withdrawing functionalities such as cyano and mono- and disubstituted trifluoromethyl enol carbonates are competent substrates under the developed trifluoromethoxylation method (12-14), affording the products in up to $42 \%$ yield in $1 \mathrm{~h}$.

Volatile substrate. Batch reactions were performed in $10 \mathrm{~mL}$ of $\mathrm{MeCN}$ at $0.1 \mathrm{mmol}$ scale. Yields determined using the triflimide peak or $\mathrm{PhCF}_{3}$ as the internal 
The $\mathrm{OCF}_{3}$ group was readily installed also onto mono- and dihalogenated derivatives (15-18) in yields up to $46 \%$, as well as on the diketo derivative (19), in $44 \%$ yield. It is worth noting that this mild photoredox-catalyzed protocol is not limited to terminal enol carbonates, and that trifluoromethoxylated ketones (20-21) bearing a methyl, or benzyl group at the $\alpha$ position can also be prepared.

We then turned our attention to cyclic ketones (22-26). 1Indanone- and benzosuberone-derived enol carbonates $(22,26)$ were found to react more efficiently than the corresponding tetralone (25). Regarding the substitution pattern on the indanone scaffold, we observed a trend similar to that of acyclic derivatives. Electron-withdrawing groups such as a fluorine atom led a better yield $(29 \%)$ than the electron-donating OMe group (9\%). The developed method was amenable to diverse aromatic systems. Thiophene and 2-naphtyl derivatives delivered the corresponding products 27 and $\mathbf{2 8}$ in $12 \%$ and $14 \%$ yield, respectively. Synthetically appealing difluorinated enol carbonates were also investigated. Remarkably, the unprecedented perfluoroalkylated ketones $\mathbf{2 9}$ and $\mathbf{3 0}$ were easily obtained. We then attempted the trifluoromethoxylation of challenging enol carbonates derived from aliphatic ketones and enones. We were pleased to see that the intended trifluoromethoxylated ketones (31-33) were still successfully produced, in spite of a less important stabilization of the corresponding radical intermediate. Noteworthy, $\mathbf{3 2}$ and $\mathbf{3 3}$ were formed as single regioisomers despite the presence of two conjugated double bonds in the starting materials. We next tested the versatility of the developed method for the mild late-stage trifluoromethoxylation of biorelevant targets. To our delight, $\alpha$ $\mathrm{OCF}_{3}$ ketones 34-36 derived from Fixolide, Musk ketone and Celestolide were readily obtained. Remarkably, even the structurally complex bioactive natural products $\alpha$-ionone, and pregnenolone participated in the developed trifluoromethoxylation process although with inferior results (37-39). Despite the presence of several double bonds, full chemoselectivity was observed in all these reactions, while preserving the fragile nature of these complex natural scaffolds. Encouraged by these results, we attempted the installation of the $\mathrm{OCF}_{3}$ fragment into the testosterone scaffold. In this case however, two conjugated double bonds are present in the starting material, possibly leading to the formation of two diverse regioisomers $\left(\alpha-\mathrm{vs} \gamma-\mathrm{OCF}_{3}\right)$. We were pleased to see that under the developed method the trifluoromethoxylation occurred selectively at the vinylogous $\gamma$ position, furnishing 39 exclusively, in $11 \%$ isolated yield and 1:1 dr.

To further demonstrate the synthetic potential of the developed visible-light-driven method, we performed a large-scale flowsynthesis of the trifluoromethoxylated ketones 12 and 17. ${ }^{[21]}$ By applying a flow rate of $5 \mathrm{~mL} \cdot \mathrm{min}^{-1}$ and a residence time of $2 \mathrm{~min}$, we were able to perform a 20 -fold scale up of the process, without yield erosion. A routine reduction with $\mathrm{NaBH}_{4}$ furnished the synthetically appealing mono-trifluoromethylated vicinal diol $\mathbf{4 0}$. This molecule was subsequently subjected to a BuchwaldHartwig amination with morpholine, resulting in the formation of derivative 41. Simple treatment with ammonium acetate under reductive conditions of $\mathbf{1 7}$ delivered the trifluoromethylated amino alcohol 42, which is an essential ingredient for the synthesis of allosteric modulators of muscarinic receptors. ${ }^{[22]}$ Additionally, ketone 12 was reacted with dimethylformamide-dimethylacetal exploiting its pronucleophilic nature giving the trifluoromethoxylated enaminone 43. These experiments demonstrate the synthetic potential of the developed method and the versatility of the products as synthetic building blocks.

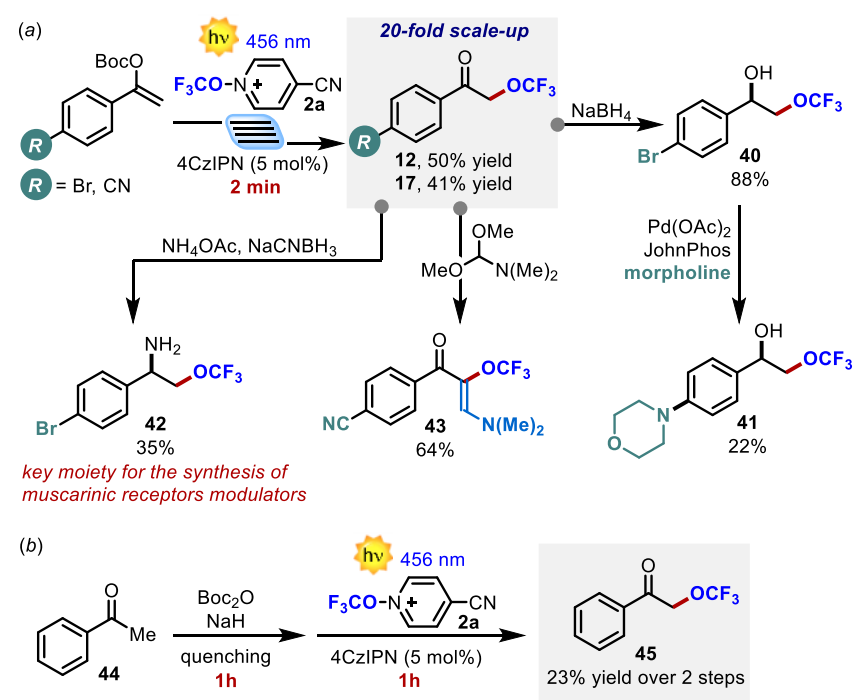

Scheme 2. a) In-flow implementation and products manipulations. b) One-pot two-steps sequence to access biorelevant synthetic target $\mathbf{4 5}$

Finally, to test the robustness of the described process we set up a one-pot two-step sequence starting from acetophenone $\mathbf{4 4}$. The crude enol carbonate was directly subjected to the optimized reaction conditions without any purification step. Noteworthy, also under these conditions the reaction proceeded with the formation of the biorelevant target $\mathbf{4 5}$ in $23 \%$ yield within an overall reaction time of $2 \mathrm{~h}$, while previous approaches required longer times (up to $92 \mathrm{~h}$ ) and prefunctionalised substrates. ${ }^{[23]}$

In conclusion, a mild and selective visible-light method for the $\alpha$ trifluoromethoxylation of ketones has been developed. The process uses a commercially available trifluoromethoxylating reagent, an organic photocatalyst and a wide range of structurally diverse enol carbonates. Mechanistic investigations revealed that a radical chain mechanism is essential to access the desired trifluoromethoxylated products in useful synthetic yields. The easy in-flow upscaling and the straightforward manipulations of the products make of this methodology an unprecedented general tool for the incorporation of the $\mathrm{OCF}_{3}$ fragment into synthetically relevant targets, including bioactive ones.

\section{Acknowledgements}

This work was supported by a grant from the Agence Nationale de la Recherche (ANR) with PRCI funding (ANR-17-CE07-004801) and by the University of Padova P-DiSC\#11BIRD2020UNIPD, CariParo Foundation, Synergy - Progetti di Eccellenza 2018 (L.D). Giovanni Salvagno is acknowledged for preliminary experiments. Prof. Tommaso Carofiglio and Dr Paolo Zardi are acknowledged for technical assistance.

Keywords: Organofluorine compounds - Trifluoromethoxylation - Photoredox catalysis - Trifluoromethylethers • Flow chemistry 
[1] a) P. Tang, $X$. Jiang in Emerging Fluorinated Motifs Synthesis, Properties, and Applications, edited by D. Cahard and J.-A. Ma, 2020 Wiley - VCH Weinheim, Germany; b) F. Toulgouat, F. Liger, T. Billard in Organofluorine Chemistry, Synthesis, Modeling, and Applications, edited by K. J. Szabó and N. Selande, 2020, Wiley - VCH Weinheim, Germany.

[2] C. Hansch, A. Leo, Substituent Constants for Correlation Analysis in Chemistry and Biology, Wiley, New York, 1979.

[3] M. A. McClinton, D. A. McClinton, Tetrahedron, 1992, 48, 6555-6666.

[4] See, e.g.: a) J. Klocker, A. Karpfen, P. Wolschann, Chem. Phys. Lett. 2003, 367, 566-575; b) J. Klocker, A. Karpfen, P. Wolschann, J. Chem. Phys. A, 2003, 107, 23652-2368; c) B. Manteau, P. Genix, L. Brelot, J.P. Vors, S. Pazenok, F. Giornal, C. Leuenberger, F. R. Leroux, Eur. J. Org. Chem. 2010, 6043-6066.

[5] M. Inoue, Y. Sumii, N; Shibata, ACS Omega, 2020, 19, 10633-10640.

[6] Y. Ogawa, E. Tokunaga, O. Kobayashi, K. Hirai, N. Shibata, iScience, 2020, 23, 101467.

[7] For reviews, see a) A. Tlili, F. Toulgoat, T. Billard, Angew. Chem. Int. Ed. 2016, 55, 11726-11735; b) X. Zhang, P. Tang, Sci. China Chem. 2019, $62,525-532$.

[8] a) T. Umemoto, K. Adachi, S. Ishihara, J. Org. Chem. 2007, 72, 69056917 ; b) R. Koller, K. Stanek, D. Stolz, R. Aardoom, K. Niedermann, A. Togni, Angew. Chem. Int. Ed. 2009, 48, 4332-4336.

[9] a) M. Yoritate, A. T. Londregan, Y. Lian, J. F. Hartwig, J. Org. Chem. 2019, 84, 15767-15776; b) J.-B. Liu, X.-H. Xu, F.-L. Qing, Org. Lett. 2015 17, 5048-5051; c) J. Kalim, T. Duhail, E. Pietrasiak, E. Anselmi, E. Magnier, A. Togni Chem. Eur. J. 2021, 67, 2638-2642.

[10] Selected recent examples: a) C. Huang, T. Liang, S. Harada, E. Lee, T. Ritter, J. Am. Chem. Soc. 2011, 133, 13308-13310; b) S. Guo, F. Cong R. Guo, L. Wang, P. Tang, Nat. Chem. 2017, 9, 546-551; c) M. Zhou, C $\mathrm{Ni}$, Y. Zeng, J. Hu, J. Am. Chem. Soc. 2018, 140, 6801-6805; d) A Turksoy, T. Scattolin, S. Bouayad-Gervais, F. Schoenebeck, Chem. Eur J. 2020, 26, 2183-2186; e) Y. Li, Y. Yang, P. Tang, Nat. Chem. 2020, 11, 755-762.

[11] Reviews: a) C. Ghiazza, T. Billard, A. Tlili, Chem. Eur. J. 2019, 25, 64826495; b) J. W. Lee, K. N. Lee, M. Ngai, Angew. Chem. Int. Ed. 2019, 58 11171-11181.
[12] B. J. Jelier, P. F. Tripet, E. Pietrasiak, I. Franzoni, G. Jeschke, A. Togni, Angew. Chem. Int. Ed. 2018, 57, 13784-13789.

[13] a) W. Zheng, C. A. Morales-Rivera, J. W. Lee, P. Liu, M.-Y. Ngai, Angew. Chem. Int. Ed. 2018, 57, 9645-9649; b) W. Zheng, J. W. Lee, C. A Morales-Rivera, P. Liu, M.-Y. Ngai, Angew. Chem. Int. Ed. 2018, 57, 13795-13799.

[14] For a radical trifluoromethoxylation of arenes and azoarenes see: a) $\mathrm{S}$ Yang, M. Chen, P. Tang, Angew. Chem. Int. Ed. 2019, 58, 7840-7844. b) S. Dix, P. Golz, J. R. Schmid, S. Riedel, M. N. Hopkinson, Chem. Eur. J. 2021, doi: 10.1002/chem.202101621.

[15] R. Zriba, E. Magnier, J.-C. Blazejewski, Synlett, 2009, 7, 1131-1135;

[16] a) G. E. M. Crisenza, D. Mazzarella, P. Melchiorre, J. Am. Chem. Soc. 2020, 142, 5461-5476; b) E. Arceo, I. D. Jurberg, A. Alvarez-Fernàndez, P. Melchiorre Nat. Chem. 2013, 5, 750-756.

[17] a) C. K. Prier, D. A. Rankic, D. W. C. MacMillan, Chem. Rev. 2013, 113, 5322-5363; b) A. Vega-Peñaloza, J. Mateos, X. Companyó, M. Escudero-Casao, L. Dell'Amico, Angew. Chem. Int. Ed. 2020, 133, 1096-1111.

[18] J. Mateos, F. Rigodanza, A. Vega-Peñaloza, A. Sartorel, M. Natali, T. Bortolato, G. Pelosi, X. Companyó, M. Bonchio, L. Dell'Amico, Angew. Chem. Int. Ed. 2020, 59, 1302-1312.

[19] N. A. Romero, D. A. Nicewicz, Chem. Rev. 2016, 116, 10075-100166.

[20] M. A. Cismesia, T. P. Yoon, Chem. Sci. 2015, 6, 5426-5434.

[21] a) D. Cambié, C. Bottecchia, N. J. W. Straathof, V. Hessel, T. Noël, Chem. Rev. 2016, 116, 10276-10341; b) A. V. Nyuchev, T. Wan, B. Cendón, C. Sambiagio, J. J. C. Struijs, M. Ho, M. Gulías, Y. Wang, T Noël, Beilstein J. Org. Chem. 2020, 16, 1305-1312; c) P. Franceschi, J. Mateos, A. Vega-Peñaloza, L. Dell'Amico, Eur. J. Org. Chem. 2020 43, 6718-6722

[22] H. Teller, A. Straub, M. Brechmann, T. Müller, M. Meininghaus, K. Nowak-Reppel, H. Tinel, K. Münter, D. Fliegner, T. Mondritzki, M Boutadakis Arapinis, T. Marquardt, A. Vakalopolous, A.-S. Rebstock, M. B. Wittwer, (Bayer PA), US20190367516, 2019

[23] J. Barbion, T. Billard, B. Langlois, O. Marrec, S. Pazenok, J.-P. Vors, (Bayer CropScience AG), EP2628722, 2013. 


\section{Supporting Information}

\section{Table of Contents}

A. GENERAL INFORMATION

B. LIGHT SOURCES EMISSION SPECTRA

C. EXPERIMENTAL SETUP $\quad 10$

456 NM REACTION SETUP - ROOM TEMPERATURE $\quad 10$

$\begin{array}{lr}456 \text { NM REACTION SETUP }-50^{\circ} \mathrm{C} & 10\end{array}$

D. REACTION OPTIMIZATION 11

E. SYNTHETIC PROCEDURES AND CHARACTERIZATION 13

GENERAL PROCEDURES FOR THE SYNTHESIS OF STARTING MATERIALS $\quad 13$

GENERAL PROCEDURES FOR THE TRIFLUOROMETHOXYLATION OF ENOL CARBONATES 224

$\begin{array}{lr}\text { SCALE-UP EXPERIMENTS } & 35\end{array}$

$\begin{array}{ll}\text { POST-MODIFICATIONS } & 36\end{array}$

ONE-POT PROCEDURE FOR THE TRIFLUOROMETHOXYLATION OF ACETOPHENONE 37

\begin{tabular}{lr} 
F. & MECHANISTIC STUDIES \\
\hline
\end{tabular}

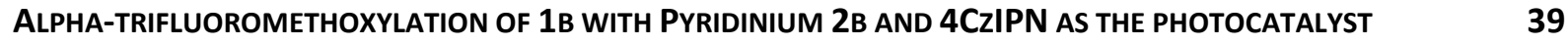
LUMINESCENCE QUENCHING EXPERIMENTS $\quad 39$

$\begin{array}{lr}\text { QUANTUM YIELD MEASUREMENTS } & 40\end{array}$

G. CYCLIC VOLTAMMETRIES $\quad 46$

\begin{tabular}{lr} 
H. UV-VIS SPECTRA & 47 \\
\hline
\end{tabular}

\begin{tabular}{lr} 
I. REFERENCES & 49 \\
\hline
\end{tabular}

\begin{tabular}{lr} 
J. & NMR SPECTRA \\
\hline
\end{tabular} 


\section{A. General Information}

All reactions were carried out in anhydrous solvents purchased from commercial suppliers over molecular sieves in a sealed bottle which were used without further purification. Chemicals were purchased from commercial sources (Sigma-Aldrich, Alfa Aesar, Fluorochem, TCl or ABCR) and used without further purification. Organic solvents were purchased from Sigma-Aldrich and Carlo Erba companies. Reactions were monitored by thin-layer chromatography on silica gel 60F254, and/or by ${ }^{19} \mathrm{~F}$ NMR spectroscopy.

NMR spectra were collected on a Bruker AC-300 spectrometer fitted with a Bruker PABBO BB/19F$1 \mathrm{H} / \mathrm{D}$ probe head, Bruker 200 equipped with a QNP probehead, Bruker 400 Avance III HD spectrometer equipped with a BBI-z grad probehead, Bruker 500 Avance III equipped with a BBI-ATM-z grad probehead and Bruker Neo 600 equipped with a Prodigy probehead operating at the denoted spectrometer frequency given in $\mathrm{MHz}$ for the specified nucleus. Reported coupling constants and chemicals shifts were based on a first order analysis. The internal reference for ${ }^{1} \mathrm{H}$ NMR was the

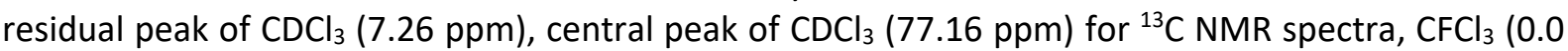
$\mathrm{ppm}$ ) as internal reference for ${ }^{19} \mathrm{~F}$ NMR spectra. All coupling constants $(\mathrm{J})$ are reported in $\mathrm{Hz}$ with the following abbreviations: $s=$ singlet, $d=$ doublet, $d d=$ double doublet, $t=$ triplet, $d t=$ double triplet, $q$ = quadruplet, $\mathrm{m}=$ multiplet, $\mathrm{br}=$ broad. High resolution mass spectrometry (HRMS) was recorded on a Mass Spectrometer XEVO-QTOF in the Institute Lavoisier of Versailles - University of Versailles Saint Quentin or on a Xevo G2-XS QToF in the Department of Pharmaceutical Sciences - University of Padua.

Thin-layer chromatography (TLC) analysis was performed on pre-coated Merck TLC plates (silica gel $60 \mathrm{G}$ F254, $0.25 \mathrm{~mm}$ ). Visualization of the developed purification was performed by checking UV absorbance $(254 \mathrm{~nm})$ as well as with aqueous ceric ammonium molybdate and potassium permanganate solutions. Chromatographic purification of the products was accomplished using flash chromatography on silica gel $\left(\mathrm{SiO}_{2}, 0.04-0.0063 \mathrm{~mm}\right)$ purchased from Sigma-Aldrich, with the indicated solvent system according to the standard techniques, or with pre-coated Merck preparative TLC plates (silica gel 60G F254, 20×20 cm). Organic solutions were concentrated under reduced pressure on a Büchi rotary evaporator.

Steady-state absorption spectroscopy studies were performed at room temperature on a Varian Cary 50 UV-vis; $10 \mathrm{~mm}$ path length Hellma Analytics 100 QS quartz cuvettes were used.

The electrochemical characterizations were carried out in acetonitrile (MeCN)/0.1 M tetrabutylammonium hexafluorophosphate $\left(\mathrm{TBAPF}_{6}\right)$ at room temperature, on an BASi EC Epsilon potentiostat-galvanostat in a glass cell. All the cyclic voltammograms were recorded with a scan rate of $0.1 \mathrm{~V} / \mathrm{s}$. A typical three-electrode cell was employed, which was composed of a glassy carbon (GC) working electrode ( $3 \mathrm{~mm}$ diameter), a platinum wire as counter electrode and a saturated aqueous calomel electrode (SCE) as reference electrode. The glass electrochemical cell was kept closed with a stopper annexed to the potentiostat. Oxygen was removed by purging the solvent with high-purity Nitrogen $\left(\mathrm{N}_{2}\right)$, introduced from a line into the cell by means of a glass pipe. The potential of ferrocenium/ferrocene $(\mathrm{Fc}+\mathrm{Fc})$ couple was used as internal reference system to calibrate the potentiostat. All the results are subsequently converted in $\mathrm{V}$ vS SCE, in agreement with the value reported in literature $\left[\mathrm{E}_{1 / 2}(\mathrm{Fc}+/ \mathrm{Fc})=+0.395 \mathrm{~V}\right.$ vs SCE]. ${ }^{[1]}$ The IR compensation implemented within the potentiostat was used, and every effort was made throughout the experiments to minimize the resistance of the solution. The full electrochemical reversibility of the voltammetric wave of ferrocene was taken as an indicator of the absence of uncompensated resistance effects. The GC electrode was polished before any measurement with diamond paste, carefully rinsed with de-ionized water, ethanol, acetone and ultrasonically rinsed with a methanol/ethanol/acetone 1:1:1 $(\mathrm{v} / \mathrm{v})$ mixture for 5 minutes. After each series of CV experiments, the electrochemical cell was carefully rinsed with 
ethanol, acetone and de-ionized water; afterwards, the cell and the magnetic stirrer were sonicated for 5-10 min with acetone.

$456 \mathrm{~nm}$ LEDs were purchased on Amazon (https://www.amazon.it/Striscia-Decorazioni-ConnettoriConnessione-Alimentatore/dp/B0OHSF64E6), as well as the waterproof silicone tubing used for submerging the LEDs under water (https://www.amazon.it/Silicone-Guaine-Flessibile-BobinaStriscia/dp/B00TOM6NWO/ref=sr 14 ? $\mathrm{mk}$ it $\mathrm{IT}=\% \mathrm{C3} \% 85 \mathrm{M} \% \mathrm{C3} \% 85 \% \mathrm{C5} \% \mathrm{BD} \% \mathrm{C} 3 \% 95 \% \mathrm{C3} \% 91 \& \mathrm{~d}$ child=1\&keywords=tubo+silicone+led\&qid=1622812658\&s=lighting\&sr=1-4).

Flow reactions were performed on a Vapourtec apparatus equipped with a UV-150 $10 \mathrm{~mL}$ photochemical reactor. 


\section{B. Light sources emission spectra}

The following spectrum was recorded by an AvaSpec ULS3648 high-resolution fiber-optic spectrometer which was placed at a fixed distance of $0.5 \mathrm{~cm}$ from the light source.

(more info at: https://www.avantes.com/products/spectrometers/starline/item/209-avaspeculs3648-high-resolution-spectrometer ).

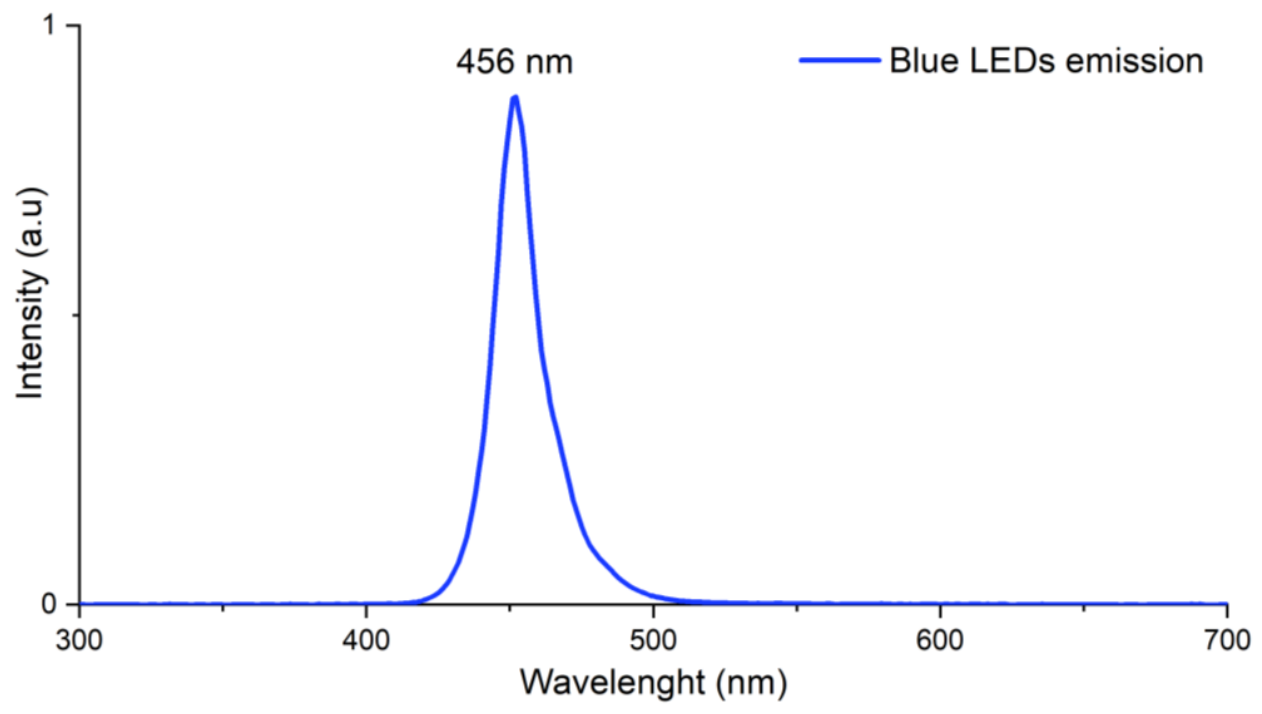

Figure S1 Emission spectrum of the $456 \mathrm{~nm}$ LEDs used in this work 


\section{Experimental setup}

\section{$456 \mathrm{~nm}$ reaction setup - room temperature}

Figure S2 shows the general setup of a batch reaction under $456 \mathrm{~nm}$ irradiation. The reaction mixture was placed in the middle of the photoreactor (at about $1.5 \mathrm{~cm}$ distance from the light source. To maintain a stable reaction temperature one fan was placed close to the vials $\left(25 \pm 2{ }^{\circ} \mathrm{C}\right)$ and the temperature was controlled by a thermometer.

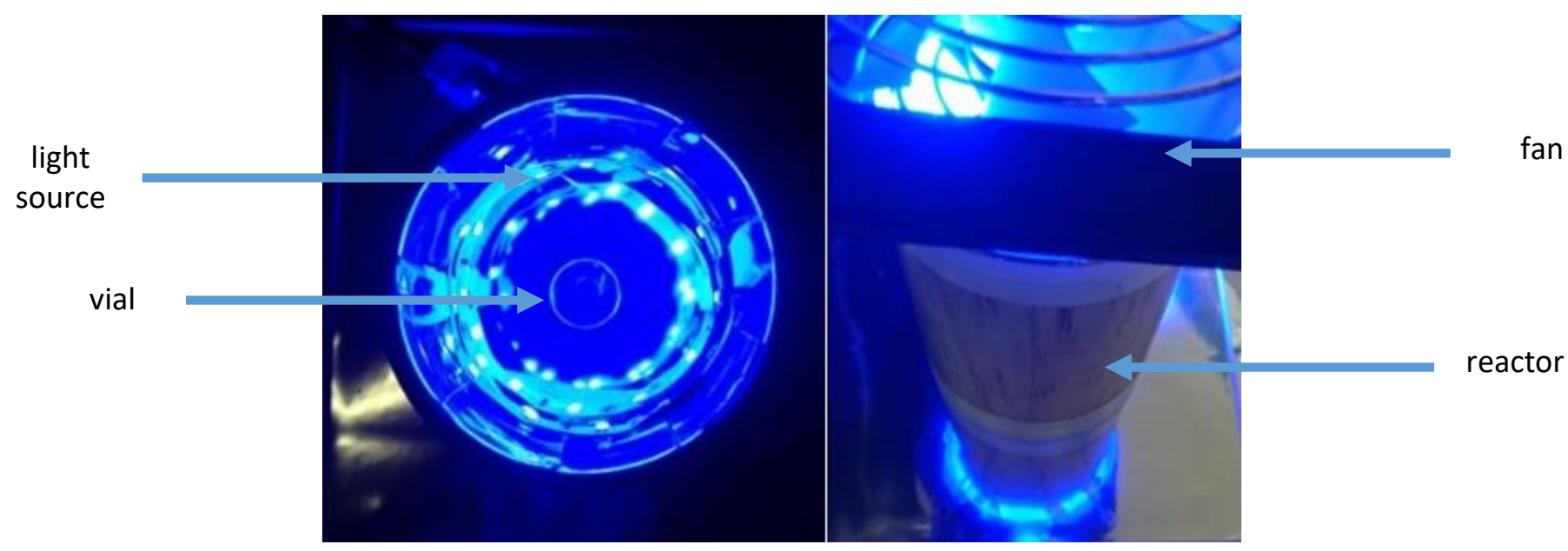

Figure S2 Left: top view of the photoreactor used in this work. Right: Front view of the photoreactor used in this work.

\section{$456 \mathrm{~nm}$ reaction setup $-50^{\circ} \mathrm{C}$}

Figure S3 shows the general setup of a batch reaction under $456 \mathrm{~nm}$ irradiation at $50^{\circ} \mathrm{C}$. The reaction mixture was wrapped by $456 \mathrm{~nm}$ LEDs inserted in the silicone tubing to avoid contact with water and placed in a water bath at a stable temperature of $50^{\circ} \mathrm{C}$.

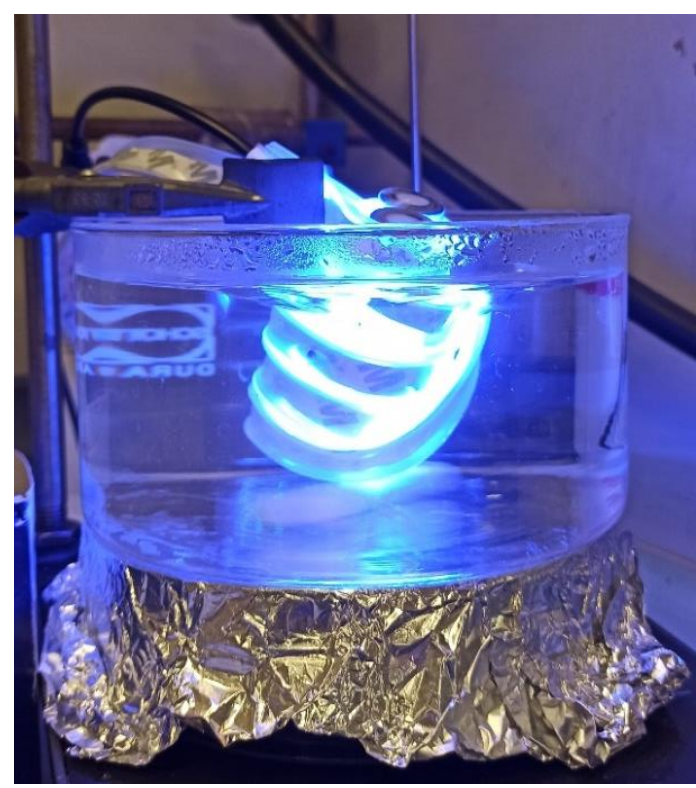

Figure S3 Front view of the reaction setup under $456 \mathrm{~nm}$ irradiation at $50^{\circ} \mathrm{C}$ 


\section{Reaction optimization}

For the trifluoromethoxylation reaction between enol derivatives $\mathbf{1}$ and the pyridinium reagent $\mathbf{2 a}$ investigated in the present work, different reaction conditions were tested in function of the used enol derivative, reagents ratio, concentration, solvent, photocatalyst, additives, temperature and reaction time (Table S1). Photocatalysts NTC1, 4CzIPN and 3DPAFIPN were synthesised according to reported procedures. ${ }^{[2,3]}$
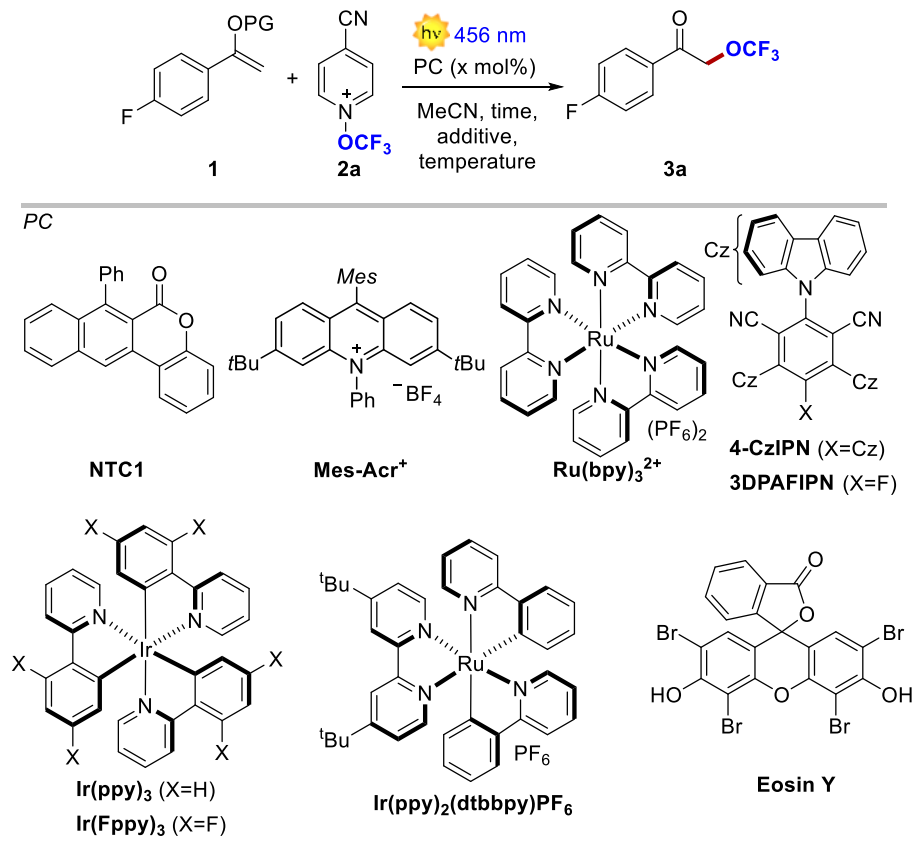

Table S1 Exploratory studies of the reaction between enol derivative 1 and pyridinium $2 a$

\begin{tabular}{|c|c|c|c|c|c|c|}
\hline Entry & PG & $\begin{array}{c}\text { Ratio } \\
(1 \mathrm{a} / 2 \mathrm{a})\end{array}$ & Concentration & PC (mol\%) & Additive & $\begin{array}{l}\text { Yield of } \\
\text { 3a (\%) }\end{array}$ \\
\hline 1 & TIPS & 1:3 & $0.1 \mathrm{M}$ & $\mathrm{Ru}(\mathrm{bpy})_{3}\left(\mathrm{PF}_{6}\right)_{2}(1)$ & - & 0 \\
\hline 2 & $A c$ & 1:3 & $0.1 \mathrm{M}$ & $\mathrm{Ru}(\mathrm{bpy})_{3}\left(\mathrm{PF}_{6}\right)_{2}(1)$ & - & 8 \\
\hline 3 & $A c$ & 1:1.5 & $0.1 \mathrm{M}$ & $\mathrm{Ru}(\mathrm{bpy})_{3}\left(\mathrm{PF}_{6}\right)_{2}(1)$ & - & 5 \\
\hline 4 & $A c$ & 3:1 & $0.1 \mathrm{M}$ & $\mathrm{Ru}(\mathrm{bpy})_{3}\left(\mathrm{PF}_{6}\right)_{2}(1)$ & - & 6 \\
\hline 7 & Ac & 1:3 & $0.1 \mathrm{M}$ & $\mathrm{Ru}(\mathrm{bpy})_{3}\left(\mathrm{PF}_{6}\right)_{2}(1)$ & $\mathrm{K}_{3} \mathrm{PO}_{4}(2 \mathrm{eq})$ & 0 \\
\hline 8 & $A c$ & 1:3 & $0.1 \mathrm{M}$ & $\mathrm{Ru}(\mathrm{bpy})_{3}\left(\mathrm{PF}_{6}\right)_{2}(1)$ & $\mathrm{K}_{2} \mathrm{CO}_{3}(2 \mathrm{eq})$ & 0 \\
\hline 9 & Ac & $1: 3$ & $0.1 \mathrm{M}$ & $\mathrm{Ru}(\mathrm{bpy})_{3}\left(\mathrm{PF}_{6}\right)_{2}(1)$ & $\mathrm{K}_{2} \mathrm{HPO}_{4}(2 \mathrm{eq})$ & 17 \\
\hline 10 & $A c$ & $10: 1$ & $0.1 \mathrm{M}$ & $\mathrm{Ru}(\mathrm{bpy})_{3}\left(\mathrm{PF}_{6}\right)_{2}(5)$ & $\mathrm{K}_{2} \mathrm{HPO}_{4}(2 \mathrm{eq})$ & 23 \\
\hline 11 & $A c$ & $10: 1$ & $0.1 \mathrm{M}$ & $\mathrm{Ru}(\mathrm{bpy})_{3}\left(\mathrm{PF}_{6}\right)_{2}(5)$ & - & 30 \\
\hline 12 & Ac & $10: 1$ & $0.033 \mathrm{M}$ & $\mathrm{Ru}(\mathrm{bpy})_{3}\left(\mathrm{PF}_{6}\right)_{2}(5)$ & - & 33 \\
\hline $13^{\mathrm{b})}$ & $A c$ & $10: 1$ & $0.033 \mathrm{M}$ & $\mathrm{Ru}(\mathrm{bpy})_{3}\left(\mathrm{PF}_{6}\right)_{2}(5)$ & - & 28 \\
\hline $14^{c)}$ & $A c$ & $10: 1$ & $0.033 \mathrm{M}$ & $\mathrm{Ru}(\mathrm{bpy})_{3}\left(\mathrm{PF}_{6}\right)_{2}(5)$ & - & 30 \\
\hline $15^{d)}$ & $A c$ & $10: 1$ & $0.033 \mathrm{M}$ & $\mathrm{Ru}(\mathrm{bpy})_{3}\left(\mathrm{PF}_{6}\right)_{2}(5)$ & - & 26 \\
\hline
\end{tabular}




\begin{tabular}{|c|c|c|c|c|c|c|}
\hline Entry & PG & $\begin{array}{c}\text { Ratio } \\
\text { (1a/2a) }\end{array}$ & Concentration & PC (mol\%) & Additive & $\begin{array}{l}\text { Yield of } \\
\text { 3a (\%) }\end{array}$ \\
\hline $16^{\mathrm{e})}$ & $A c$ & $10: 1$ & $0.033 \mathrm{M}$ & $\mathrm{Ru}(\mathrm{bpy})_{3}\left(\mathrm{PF}_{6}\right)_{2}(5)$ & - & 25 \\
\hline $17^{f)}$ & $A c$ & $10: 1$ & $0.033 \mathrm{M}$ & - & - & 17 \\
\hline 18 & $A c$ & $10: 1$ & $0.033 \mathrm{M}$ & $\operatorname{Ir}(\mathrm{ppy})_{3}(5)$ & - & 0 \\
\hline 19 & $A c$ & $10: 1$ & $0.033 \mathrm{M}$ & $\operatorname{Ir}(\mathrm{Fppy})_{3}(5)$ & - & 15 \\
\hline 20 & $A c$ & $10: 1$ & $0.033 \mathrm{M}$ & $\operatorname{Ir}(\mathrm{ppy})_{2}(\mathrm{dtbbpy}) \mathrm{PF}_{6}(5)$ & - & 3 \\
\hline 21 & $A c$ & $10: 1$ & $0.033 \mathrm{M}$ & Eosin Y (5) & - & 14 \\
\hline 22 & $A c$ & $10: 1$ & $0.033 \mathrm{M}$ & Mes-Acr-BF 4 (5) & - & 7 \\
\hline 23 & $A c$ & $10: 1$ & $0.033 \mathrm{M}$ & 3-DPAFIPN (5) & - & 27 \\
\hline $24^{\mathrm{g})}$ & $A c$ & $10: 1$ & $0.033 \mathrm{M}$ & NTC1 (5) & - & 20 \\
\hline 25 & $A c$ & $10: 1$ & $0.033 \mathrm{M}$ & 4CzIPN (5) & - & 37 \\
\hline 26 & $A c$ & $10: 1$ & $0.033 \mathrm{M}$ & 4CZIPN (10) & - & 30 \\
\hline 27 & $A c$ & $1: 3$ & $0.033 \mathrm{M}$ & 4CZIPN (5) & - & 2 \\
\hline 28 & $A c$ & $10: 1$ & $0.01 \mathrm{M}$ & 4CZIPN (5) & - & 41 \\
\hline 29 & $A c$ & $10: 1$ & $0.01 \mathrm{M}$ & 4CZIPN (2) & - & 33 \\
\hline $30^{\text {h) }}$ & $A c$ & $10: 1$ & $0.01 \mathrm{M}$ & 4CZIPN (5) & - & 44 \\
\hline $31^{h)}$ & Boc & $10: 1$ & $0.01 \mathrm{M}$ & 4CZIPN (5) & - & 52 \\
\hline $32^{h)}$ & Boc & $5: 1$ & $0.01 \mathrm{M}$ & 4CZIPN (5) & - & 50 \\
\hline $33^{h)}$ & Boc & $1.5: 1$ & $0.01 \mathrm{M}$ & 4CZIPN (5) & - & 30 \\
\hline $34^{h)}$ & Boc & $5: 1$ & $0.01 \mathrm{M}$ & 4CzIPN (5) & $\mathrm{BF}_{3} \mathrm{OEt}_{2}(1 \mathrm{eq})$ & 8 \\
\hline $35^{\text {h) }}$ & Boc & $5: 1$ & $0.01 \mathrm{M}$ & 4CZIPN (5) & $\mathrm{Bu}_{4} \mathrm{NOAc}(1 \mathrm{eq})$ & 0 \\
\hline $36^{\mathrm{h}, \mathrm{i})}$ & Boc & $5: 1$ & $0.01 \mathrm{M}$ & 4CZIPN (5) & - & 0 \\
\hline
\end{tabular}

a) ${ }^{19} \mathrm{~F}-\mathrm{NMR}$ yield using $\mathrm{PhCF}_{3}$ or the peak of triflimide as the internal standard b) The reaction was performed with a total reaction time of $6 \mathrm{~h} \mathrm{c}$ ) The reaction was performed in acetone as solvent d) The reaction was performed in ethyl acetate as solvent e) 2a was added with a driver syringe at a flow of $0.2 \mathrm{~mL}$ per hour in $0.6 \mathrm{~mL}$ of acetonitrile f) The reaction was performed under $400 \mathrm{~nm}$ light irradiation g) The reaction was performed under $420 \mathrm{~nm}$ light irradiation $\mathrm{h}$ ) The reaction was performed at $50^{\circ} \mathrm{C}$ i) The reaction was performed in the dark 


\section{E. Synthetic procedures and characterization}

\section{General procedures for the synthesis of starting materials}

\section{General procedure A, for enol carbonates synthesis}

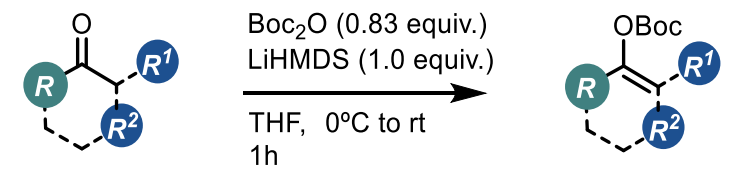

A round-bottomed flask was charged with the required ketone $(3.2 \mathrm{mmol}, 1.2$ equiv.) and suspended in THF ( $5 \mathrm{~mL}, 0.6 \mathrm{M})$ at $0^{\circ} \mathrm{C}$ under argon atmosphere. LiHMDS (1.06 M in THF, $3.0 \mathrm{~mL}, 1.2$ equiv.) was added at once to this suspension, which was then stirred for 5 mins at $0^{\circ} \mathrm{C}$. Boc ${ }_{2} \mathrm{O}(600 \mu \mathrm{L}, 2.6 \mathrm{mmol}$, 1 equiv.) was then added and the reaction medium was stirred for $1 \mathrm{~h}$ at room temperature. The resulting mixture was then quenched with water $(20 \mathrm{~mL})$ and extracted with $\mathrm{CH}_{2} \mathrm{Cl}_{2}(3 \times 20 \mathrm{~mL})$. The organic phases were then dried over $\mathrm{MgSO}_{4}$ and concentrated under vacuum. Corresponding enol carbonates were isolated by column chromatography on silica gel (Pentane/EtOAc 95/5).

\section{General procedure B, for enol carbonates synthesis}

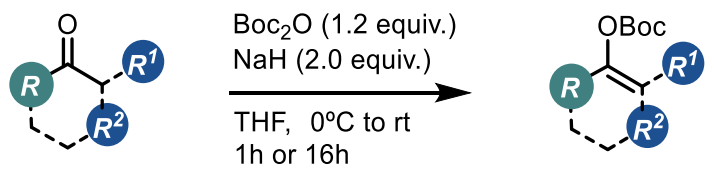

This procedure is the modification of a literature one. ${ }^{[4]} \mathrm{A}$ round-bottomed flask was charged with the required ketone ( $5 \mathrm{mmol}, 1$ equiv.) and dissolved in dry THF $(5 \mathrm{~mL}, 1 \mathrm{M})$ at $0^{\circ} \mathrm{C}$ under nitrogen atmosphere. $\mathrm{NaH}$ ( $60 \%$ dispersion in mineral oil, $400 \mathrm{mg}, 10 \mathrm{mmol}, 2$ equiv.) was added portionwise to the reaction mixture, which was then stirred for $15 \mathrm{mins}$ at $0^{\circ} \mathrm{C}$. Boc ${ }_{2} \mathrm{O}(1.38 \mathrm{~mL}, 6 \mathrm{mmol}, 1.2$ equiv.) was then added dropwise and the reaction mixture was stirred for $16 \mathrm{~h}$ at room temperature. The resulting mixture was then quenched with water $(20 \mathrm{~mL})$ and DMAP ( $61 \mathrm{mg}, 0.5 \mathrm{mmol}, 0.1$ equiv.) was added and the reaction mixture stirred for 2 hours. The crude was then diluted with $\mathrm{CH}_{2} \mathrm{Cl}_{2}(50 \mathrm{~mL})$ and washed with brine $(3 \times 20 \mathrm{~mL})$. The organic phase was then dried over $\mathrm{MgSO}_{4}$ and concentrated under vacuum. Corresponding enol carbonates were isolated by column chromatography on silica gel with the indicated eluent system.

General procedure C, for enol carbonates synthesis

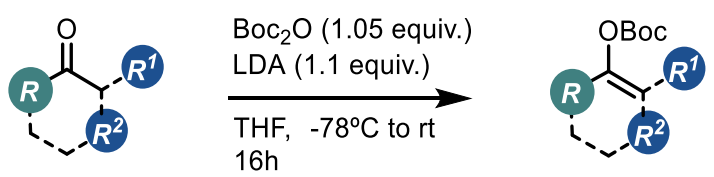

A flame-dried, nitrogen flushed round-bottomed flask was charged with the required ketone $(5 \mathrm{mmol}$, 1 equiv.) and dissolved in dry THF $(5 \mathrm{~mL}, 1 \mathrm{M})$ under nitrogen atmosphere. A freshly prepared solution of LDA ( $0.5 \mathrm{M}$ in THF, $11 \mathrm{~mL}, 5.5 \mathrm{mmol}, 1.1$ equiv.) was dropwise at $-78^{\circ} \mathrm{C}$ to the reaction mixture, which was then stirred for 90 minutes at $-78^{\circ} \mathrm{C}$. Boc ${ }_{2} \mathrm{O}(1.21 \mathrm{~mL}, 5.25 \mathrm{mmol}, 1.05$ equiv.) was then added dropwise and the reaction medium was stirred for 30 minutes at $-78^{\circ} \mathrm{C}$ and at room temperature for 16 hours. The resulting mixture was then quenched with water $(20 \mathrm{~mL})$, DMAP (61 mg, $0.5 \mathrm{mmol}, 0.1$ equiv.) was added and the resulting mixture stirred for 2 hours. The crude was then diluted with $\mathrm{CH}_{2} \mathrm{Cl}_{2}$ $(50 \mathrm{~mL})$ and washed with brine $(3 \times 20 \mathrm{~mL})$. The organic phase was then dried over $\mathrm{MgSO}_{4}$ and 
concentrated under vacuum. Corresponding enol carbonates were isolated by column chromatography on silica gel with the indicated eluent system.

\section{1-(4-fluorophenyl)ethenyl acetate 1a}<smiles>C=C(OC)c1ccc(F)cc1</smiles>

$1 a$

1-(4-fluorophenyl)ethenyl acetate 1a was synthesized according to a procedure reported in the literature..$^{[5]}$ The spectral data matched with those reported in the literature.

tert-butyl (1-(4-fluorophenyl)vinyl) carbonate 1b<smiles>C=C(OC)c1ccc(F)cc1</smiles>

$1 \mathrm{~b}$

According to general procedure B, 1-(4-fluorophenyl)vinyl tert-butyl carbonate $\mathbf{1 b}$ was obtained from 1-(4-fluorophenyl)ethan-1-one as a yellow oil in $41 \%$ yield $(494 \mathrm{mg}, 2.07 \mathrm{mmol}$ ) after purification by column chromatography on silica gel (hexane/EtOAc 95/5). ${ }^{1} \mathbf{H ~ N M R ~}\left(500 \mathbf{~ M H z}, \mathrm{CDCl}_{3}\right) \delta 7.50-7.44(\mathrm{~m}$, 2H), $7.06-7.00(\mathrm{~m}, 2 \mathrm{H}), 5.32(\mathrm{~d}, J=2.4 \mathrm{~Hz}, 1 \mathrm{H}), 5.07(\mathrm{~d}, J=2.4 \mathrm{~Hz}, 1 \mathrm{H}), 1.49(\mathrm{~s}, 9 \mathrm{H}) \mathrm{ppm} .{ }^{13} \mathrm{C}$ NMR (126 $\left.\mathrm{MHz} \mathrm{CDCl}_{3}\right) \delta 163.2(\mathrm{~d}, J=248.7 \mathrm{~Hz}, \mathrm{C}-\mathrm{F}), 152.5,151.4,130.8(\mathrm{~d}, J=3.4 \mathrm{~Hz}), 126.9(\mathrm{~d}, J=8.2 \mathrm{~Hz}), 115.6$ $(\mathrm{d}, J=21.9 \mathrm{~Hz}), 101.4\left(\mathrm{~d}, J=1.7 \mathrm{~Hz}, \mathrm{C}=\mathrm{CH}_{2}\right), 83.4\left(\mathrm{O}-\mathrm{C}-\left(\mathrm{CH}_{3}\right)_{3}\right), 27.6\left(\mathrm{C}-\left(\mathrm{CH}_{3}\right)_{3}\right) \mathrm{ppm} .{ }^{19} \mathrm{~F} \mathrm{NMR}(\mathbf{1 8 8 ~ M H z}$, ${ }^{1} \mathrm{H}$ decoupled, $\left.\mathrm{CDCl}_{3}\right) \delta-112.84$ ppm. HRMS (ESI+) Calcd. for $\mathrm{C}_{8} \mathrm{H}_{6} \mathrm{~F}^{+}[\mathrm{M}-\mathrm{OBoc}]^{+}:$121.0448. Found: 121.0409.

\section{tert-butyl (1-phenylvinyl) carbonate 1c}<smiles>C=C(OC)c1ccccc1</smiles>

$1 \mathrm{c}$

According to general procedure B, tert-butyl (1-phenylvinyl) carbonate 1c was obtained from acetophenone as a yellow oil in $21 \%$ yield $(233 \mathrm{mg}, 1.06 \mathrm{mmol})$ after purification by column chromatography on silica gel (hexane/DCM 75/25). The spectral data matched with those reported in the literature. ${ }^{[4]}$

\section{tert-butyl (1-(o-tolyl)vinyl) carbonate 1d}

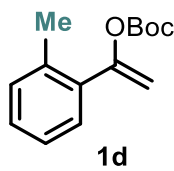

According to general procedure A, tert-butyl (1-(o-tolyl)vinyl) carbonate $1 \mathbf{d}$ was obtained from 2'methylacetophenone as a yellow oil in $96 \%$ yield $(600 \mathrm{mg}, 2.5 \mathrm{mmol}) .{ }^{1} \mathrm{H} \mathrm{NMR}\left(300 \mathrm{MHz}, \mathrm{CDCl}_{3}\right) \delta 7.40$ $(\mathrm{d}, J=7.0 \mathrm{~Hz}, 1 \mathrm{H}), 7.29-7.14(\mathrm{~m}, 3 \mathrm{H}), 5.25(\mathrm{~s}, 1 \mathrm{H}), 4.97(\mathrm{~s}, 1 \mathrm{H}), 2.44(\mathrm{~s}, 3 \mathrm{H}), 1.44(\mathrm{~s}, 9 \mathrm{H}) \mathrm{ppm} .{ }^{13} \mathrm{C}$ NMR (75 MHz, CDCl $)$ ) $154.1,151.1,136.0,135.2,130.6,129.0,128.8,125.7,104.9\left(\mathrm{~s}, \mathrm{C}=\mathrm{CH}_{2}\right), 83.1(\mathrm{~s}, 1 \mathrm{C}$, O-C- $\left.\left(\mathrm{CH}_{3}\right)_{3}\right), 27.7\left(\mathrm{~s}, 3 \mathrm{C}, \mathrm{C}-\left(\mathrm{CH}_{3}\right)_{3}\right), 20.5$ ppm. HRMS (ASAP +) Calcd for $\mathrm{C}_{14} \mathrm{H}_{18} \mathrm{O}_{3} \mathrm{Na}^{+}[\mathrm{M}+\mathrm{Na}]^{+}: 257.1148$. Found: 257.1156. 
<smiles>C=C(OC)c1cccc(C)c1</smiles>

According to general procedure A, tert-butyl (1-(m-tolyl)vinyl) carbonate 1e was obtained from 3'methylacetophenone as a yellow oil in $90 \%$ yield $(550 \mathrm{mg}, 2.3 \mathrm{mmol}) .{ }^{1} \mathbf{H ~ N M R}\left(\mathbf{3 0 0} \mathbf{~ M H z}, \mathrm{CDCl}_{3}\right) \delta 7.35$ (d, $J=7.6 \mathrm{~Hz}, 2 \mathrm{H}), 7.28(\mathrm{t}, J=7.6 \mathrm{~Hz}, 1 \mathrm{H}), 7.18(\mathrm{~d}, J=7.5 \mathrm{~Hz}, 1 \mathrm{H}), 5.43(\mathrm{~d}, J=2.2 \mathrm{~Hz}, 1 \mathrm{H}), 5.12$ (d, $J=2.3$ $\mathrm{Hz}, 1 \mathrm{H}), 2.40$ (s, 3H), 1.55 (s, 9H) ppm. ${ }^{13} \mathrm{C}$ NMR (75 MHz, CDCl $\left.{ }_{3}\right) \delta 153.6,151.5,138.2,134.4,129.8$, 128.5, 125.6, 122.0, $101.4\left(\mathrm{~s}, \mathrm{C}=\mathrm{CH}_{2}\right), 83.2\left(\mathrm{~s}, 1 \mathrm{C}, \mathrm{O}-\mathrm{C}-\left(\mathrm{CH}_{3}\right)_{3}\right), 27.7$ (s, 3C, C- $\left.\left(\mathrm{CH}_{3}\right)_{3}\right), 21.5$ ppm. HRMS $\left(\right.$ ASAP +) Calcd for $\mathrm{C}_{14} \mathrm{H}_{18} \mathrm{O}_{3} \mathrm{Na}^{+}[\mathrm{M}+\mathrm{Na}]^{+}: 257.1148$. Found: 257.1154 .

tert-butyl (1-(p-tolyl)vinyl) carbonate 1f<smiles>C=C(OC)c1ccc(C)cc1</smiles>

According to general procedure A, tert-butyl (1-(p-tolyl)vinyl) carbonate 1f was obtained from 4'methylacetophenone as a yellow oil in $90 \%$ yield $(550 \mathrm{mg}, 2.3 \mathrm{mmol}) .{ }^{1} \mathrm{H} \mathrm{NMR}\left(300 \mathrm{MHz}, \mathrm{CDCl}_{3}\right) \delta 7.40$ (d, $J=8.5 \mathrm{~Hz}, 2 \mathrm{H}), 7.17(\mathrm{~d}, J=8.1 \mathrm{~Hz}, 2 \mathrm{H}), 5.36(\mathrm{~d}, J=2.4 \mathrm{~Hz}, 1 \mathrm{H}), 5.04(\mathrm{~d}, J=2.5 \mathrm{~Hz}, 1 \mathrm{H}), 2.35(\mathrm{~s}, 3 \mathrm{H})$, 1.50 (s, 9H) ppm. ${ }^{13} \mathrm{C} \mathrm{NMR}\left(75 \mathrm{MHz}, \mathrm{CDCl}_{3}\right) \delta$ 153.5, 151.6, 139.1, 131.7, 129.4, 124.9, 100.9 (s, C= $\left.\mathrm{CH}_{2}\right)$, $83.3\left(\mathrm{~s}, 1 \mathrm{C}, \mathrm{O}-\mathrm{C}-\left(\mathrm{CH}_{3}\right)_{3}\right), 27.8\left(\mathrm{~s}, 3 \mathrm{C}, \mathrm{C}-\left(\mathrm{CH}_{3}\right)_{3}\right), 21.4$ ppm. HRMS (ASAP +) Calcd for $\mathrm{C}_{14} \mathrm{H}_{18} \mathrm{O}_{3} \mathrm{Na}^{+}[\mathrm{M}+$ $\mathrm{Na}]^{+}:$257.1148. Found: 257.1153.

\section{tert-butyl 1-(3-methoxyphenyl)vinyl carbonate $1 \mathrm{~g}$}<smiles>C=C(O[Na])c1cccc(OC)c1</smiles>

According to general procedure B, tert-butyl 1-(3-methoxyphenyl)vinyl tert-butyl carbonate $\mathbf{1 g}$ was obtained from 1-(3-methoxyphenyl)ethan-1-one as a yellow solid in $38 \%$ yield $(472 \mathrm{mg}, 1.90 \mathrm{mmol}$ ) after purification by column chromatography on silica gel (hexane/EtOAc 9/1). ${ }^{1} \mathbf{H} \mathbf{~ N M R}(\mathbf{5 0 0} \mathbf{~ M H z}$, $\left.\mathrm{CDCl}_{3}\right) \delta 7.27(\mathrm{t}, J=8.0 \mathrm{~Hz}, 1 \mathrm{H}), 7.10(\mathrm{ddd}, J=7.7,1.7,0.9 \mathrm{~Hz}, 1 \mathrm{H}), 7.03(\mathrm{dd}, J=2.6,1.7 \mathrm{~Hz}, 1 \mathrm{H}), 6.88$ (ddd, $J=8.3,2.6,0.9 \mathrm{~Hz}, 1 \mathrm{H}$ ), $5.40(\mathrm{~d}, J=2.2 \mathrm{~Hz}, 1 \mathrm{H}), 5.10(\mathrm{~d}, J=2.2 \mathrm{~Hz}, 1 \mathrm{H}), 3.81(\mathrm{~s}, 3 \mathrm{H}), 1.50(\mathrm{~s}, 9 \mathrm{H})$ ppm. ${ }^{13} \mathrm{C}$ NMR $\left(126 \mathrm{MHz}, \mathrm{CDCl}_{3}\right) \delta 159.8,153.3,151.5,136.0,129.7,117.5,114.8,110.6,102.1\left(\mathrm{C}=\mathrm{CH}_{2}\right)$, $83.4\left(\mathrm{O}-\mathrm{C}-\left(\mathrm{CH}_{3}\right)_{3}\right), 55.4,27.8\left(\mathrm{C}-\left(\mathrm{CH}_{3}\right)_{3}\right)$ ppm. HRMS (ESI+) Calcd. for $\mathrm{C}_{14} \mathrm{H}_{18} \mathrm{O}_{4} \mathrm{Na}^{+}[\mathrm{M}+\mathrm{Na}]^{+}: 273.1097$. Found: 273.1083.

tert-butyl 1-(4-methoxyphenyl)vinyl carbonate $1 \mathrm{~h}$<smiles>C=C(OC)c1ccc(OC)cc1</smiles>

According to general procedure B, tert-butyl 1-(4-methoxyphenyl)vinyl tert-butyl carbonate $\mathbf{1 h}$ was obtained from 1-(4-methoxyphenyl)ethan-1-one as a yellow solid in $10 \%$ yield (130 mg, $0.52 \mathrm{mmol}$ ) after purification by column chromatography on silica gel (hexane/EtOAc 9/1). ${ }^{1} \mathbf{H} \mathbf{~ N M R}(\mathbf{5 0 0} \mathbf{~ M H z}$, $\left.\mathrm{CDCl}_{3}\right) \delta 7.47-7.40(\mathrm{~m}, 2 \mathrm{H}), 6.92-6.84(\mathrm{~m}, 2 \mathrm{H}), 5.28(\mathrm{~d}, J=2.2 \mathrm{~Hz}, 1 \mathrm{H}), 4.99(\mathrm{~d}, J=2.2 \mathrm{~Hz}, 1 \mathrm{H}), 3.82$ $(\mathrm{s}, 3 \mathrm{H}), 1.50(\mathrm{~s}, 9 \mathrm{H}) \mathrm{ppm} .{ }^{13} \mathrm{C}$ NMR (126 MHz, $\left.\mathrm{CDCl}_{3}\right) \delta 160.3,153.3,151.6,127.2,126.4,114.1,100.0$ 
$\left(\mathrm{C}=\mathrm{CH}_{2}\right), 83.3\left(\mathrm{O}-\mathrm{C}-\left(\mathrm{CH}_{3}\right)_{3}\right), 55.4,27.8\left(\mathrm{C}-\left(\mathrm{CH}_{3}\right)_{3}\right)$ ppm. HRMS (ESI+) Calcd. for $\mathrm{C}_{9} \mathrm{H}_{11} \mathrm{O}_{2}{ }^{+}[\mathrm{M}-\mathrm{Boc}+\mathrm{H}]^{+}$: 151.0754. Found: 151.0729.

tert-butyl (1-(4-cyanophenyl)vinyl) carbonate 1i<smiles>C=C(OC)c1ccc(C#N)cc1</smiles>

A round-bottomed flask was charged with 4-acetylbenzonitrile ( $23 \mathrm{mmol}, 1.2$ equiv.) and suspended in THF ( $50 \mathrm{~mL}, 0.5 \mathrm{M})$ at $0^{\circ} \mathrm{C}$ under argon atmosphere. LiHMDS (1.06 M in THF, $21.7 \mathrm{~mL}, 1.2$ equiv.) was added at once to this suspension, which was then stirred for 5 mins at $0^{\circ} \mathrm{C}$. Boc ${ }_{2} \mathrm{O}(4.4 \mathrm{ml}, 19 \mathrm{mmol}, 1$ equiv.) was then added and the reaction medium was stirred for $1 \mathrm{~h}$ at room temperature. The resulting mixture was then quenched with water $(50 \mathrm{~mL})$ and extracted with $\mathrm{CH}_{2} \mathrm{Cl}_{2}(3 \times 100 \mathrm{~mL})$. The organic phases were then dried over $\mathrm{MgSO}_{4}$ and concentrated under vacuum. Tert-butyl (1-(4cyanophenyl)vinyl) carbonate 1i was isolated by column chromatography on silica gel (Pentane/EtOAc 95/5) as a white solid in $87 \%$ yield $(4.1 \mathrm{~g}, 16.7 \mathrm{mmol}) .{ }^{1} \mathbf{H} \mathbf{~ N M R ~}\left(300 \mathrm{MHz}, \mathrm{CDCl}_{3}\right) \delta 7.66(\mathrm{~d}, J=8.1 \mathrm{~Hz}$, $2 \mathrm{H}), 7.59(\mathrm{~d}, J=8.3 \mathrm{~Hz}, 2 \mathrm{H}), 5.53(\mathrm{~s}, 1 \mathrm{H}), 5.28(\mathrm{~s}, 1 \mathrm{H}), 1.50(\mathrm{~s}, 9 \mathrm{H}) \mathrm{ppm} .{ }^{13} \mathrm{C} \mathbf{N M R}\left(75 \mathrm{MHz}, \mathrm{CDCl}_{3}\right) \delta$ 151.8, 151.2, 139.0, 132.6, 125.6, 118.6, 112.6, $105.0\left(\mathrm{C}=\mathrm{CH}_{2}\right), 84.2\left(\mathrm{O}-\mathrm{C}-\left(\mathrm{CH}_{3}\right)_{3}\right), 27.8\left(\mathrm{C}-\left(\mathrm{CH}_{3}\right)_{3}\right) \mathrm{ppm}$. MP 59-61 ${ }^{\circ} \mathrm{C}$. HRMS (ASAP +) Calcd for $\mathrm{C}_{14} \mathrm{H}_{16} \mathrm{NO}_{3}{ }^{+}[\mathrm{M}+\mathrm{H}]^{+}: 246.1125$. Found: 246.1119 .

tert-butyl (1-(3-(trifluoromethyl)phenyl)vinyl) carbonate $1 \mathbf{j}$<smiles>C=C(OC)c1cccc(C(F)(F)F)c1</smiles>

According to general procedure A, tert-butyl (1-(3-(trifluoromethyl)phenyl)vinyl) carbonate $\mathbf{1 j}$ was obtained from 1-(3-(trifluoromethyl)phenyl)ethanone as a yellow oil in 96\% yield ( $725 \mathrm{mg}, 2.5 \mathrm{mmol}$ ). ${ }^{1} \mathrm{H}$ NMR $\left(300 \mathrm{MHz}, \mathrm{CDCl}_{3}\right) \delta 7.74(\mathrm{~s}, 1 \mathrm{H}), 7.67(\mathrm{~d}, J=8.0 \mathrm{~Hz}, 1 \mathrm{H}), 7.57(\mathrm{~d}, J=7.9 \mathrm{~Hz}, 1 \mathrm{H}), 7.45(\mathrm{t}, J=7.8$ $\mathrm{Hz}, 1 \mathrm{H}), 5.47(\mathrm{~s}, 1 \mathrm{H}), 5.20(\mathrm{~s}, 1 \mathrm{H}), 1.49(\mathrm{~s}, 5 \mathrm{H}) \mathrm{ppm} .{ }^{13} \mathrm{C} \mathrm{NMR}\left(75 \mathrm{MHz}, \mathrm{CDCl}_{3}\right) \delta$ 152.1, 151.3, 135.5, $131.2\left(\mathrm{q}, J=32.5 \mathrm{~Hz}\right.$ ), 129.2, 128.2, 125.6 (q, $J=3.8 \mathrm{~Hz}$ ), 124.0 (q, $\left.J=272.3 \mathrm{~Hz}, \mathrm{CF}_{3}\right), 121.7$ (q, $J=3.9$ $\mathrm{Hz}), 103.1\left(\mathrm{~s}, \mathrm{C}=\mathrm{CH}_{2}\right), 83.8\left(\mathrm{~s}, 1 \mathrm{C}, \mathrm{O}-\mathrm{C}-\left(\mathrm{CH}_{3}\right)_{3}\right), 27.5\left(\mathrm{~s}, 3 \mathrm{C}, \mathrm{C}-\left(\mathrm{CH}_{3}\right)_{3}\right) \mathrm{ppm} .{ }^{19} \mathrm{~F} \mathrm{NMR}\left(\mathbf{1 8 8} \mathrm{MHz}, \mathrm{CDCl}_{3}\right) \delta-$ 63.4 ppm. HRMS (ASAP +) Calcd for $\mathrm{C}_{14} \mathrm{H}_{16} \mathrm{~F}_{3} \mathrm{O}_{3}{ }^{+}[\mathrm{M}+\mathrm{H}]^{+}:$: 289.1046. Found: 289.1060.

\section{1-(3,5-bis(trifluoromethyl)phenyl)vinyl tert-butyl carbonate $1 \mathrm{k}$}<smiles>C=C(OCCOCCCCCC(F)(F)F)c1cc(C(F)(F)F)cc(C(F)(F)F)c1</smiles>

According to general procedure B, 1-(3,5-bis(trifluoromethyl)phenyl)vinyl tert-butyl carbonate $\mathbf{1} \mathbf{k}$ was obtained from 1-(3,5-bis(trifluoromethyl)phenyl)ethan-1-one as a brown solid in 14\% yield (241 mg, $0.68 \mathrm{mmol}$ ) after purification by column chromatography on silica gel (hexane/DCM 9/1). ${ }^{1} \mathrm{H}$ NMR (400 $\left.\mathrm{MHz}, \mathrm{CDCl}_{3}\right) \delta 7.92(\mathrm{~s}, 2 \mathrm{H}), 7.84(\mathrm{~s}, 1 \mathrm{H}), 5.58(\mathrm{~d}, J=2.9 \mathrm{~Hz}, 1 \mathrm{H}), 5.34(\mathrm{~d}, J=2.9 \mathrm{~Hz}, 1 \mathrm{H}), 1.51(\mathrm{~s}, 9 \mathrm{H}) \mathrm{ppm}$. ${ }^{13} \mathrm{C}$ NMR (101 MHz, CDCl $)_{3} \delta 151.1,150.8,136.9,132.3$ (q, J = 33.5 Hz, C-CF $), 125.1$ (d, J = $\left.2.7 \mathrm{~Hz}\right)$, $123.2\left(\mathrm{q}, J=272.9 \mathrm{~Hz}, \mathrm{C}-\mathrm{CF}_{3}\right), 122.6(\mathrm{p}, J=7.1,3.7,3.4 \mathrm{~Hz}), 104.9\left(\mathrm{C}=\mathrm{CH}_{2}\right), 84.5\left(\mathrm{O}-\mathrm{C}-\left(\mathrm{CH}_{3}\right)_{3}\right), 27.7(\mathrm{C}-$ $\left.\left(\mathrm{CH}_{3}\right)_{3}\right)$ ppm. ${ }^{19} \mathrm{~F}$ NMR (188 MHz, ${ }^{1} \mathrm{H}$ decoupled, $\left.\mathrm{CDCl}_{3}\right) \delta-63.6 \mathrm{ppm}$. HRMS (ASAP +) Calcd for $\mathrm{C}_{10} \mathrm{H}_{7} \mathrm{~F}_{6} \mathrm{O}^{+}$ $[\mathrm{M}-\mathrm{Boc}+\mathrm{H}]^{+}:$257.0396. Found: 257.0390 . 
<smiles>C=C(OC)c1ccccc1Br</smiles>

According to general procedure B, 1-(2-bromophenyl)vinyl tert-butyl carbonate 1 l was obtained from 1-(2-bromophenyl)ethan-1-one as a yellow oil in $34 \%$ yield $(508 \mathrm{mg}, 1.7 \mathrm{mmol}$ ) after purification by column chromatography on silica gel (hexane/EtOAc 95/5). ${ }^{1} \mathbf{H} \mathbf{~ N M R ~}\left(\mathbf{5 0 0} \mathbf{~ M H z}, \mathbf{C D C l}_{3}\right) \delta 7.57$ (dd, $J=$ 8.0, 1.2 Hz, 1H), 7.44 (dd, $J=7.7,1.7 \mathrm{~Hz}, 1 \mathrm{H}$ ), 7.29 (td, $J=7.5,1.2 \mathrm{~Hz}, 1 \mathrm{H}$ ), 7.17 (ddd, $J=8.0,7.4,1.7$ $\mathrm{Hz}, 1 \mathrm{H}), 5.32(\mathrm{~d}, J=2.0 \mathrm{~Hz}, 1 \mathrm{H}), 5.16(\mathrm{~d}, J=2.0 \mathrm{~Hz}, 1 \mathrm{H}), 1.44(\mathrm{~s}, 9 \mathrm{H}) \mathrm{ppm} .{ }^{13} \mathrm{C} \mathrm{NMR}\left(126 \mathrm{MHz}, \mathrm{CDCl}_{3}\right) \delta$ $152.4,150.8,136.4,133.4,130.8,130.1,127.3,121.5,106.3\left(\mathrm{C}=\mathrm{CH}_{2}\right), 83.3\left(\mathrm{O}-\mathrm{C}-\left(\mathrm{CH}_{3}\right)_{3}\right), 27.7\left(\mathrm{C}-\left(\mathrm{CH}_{3}\right)_{3}\right)$ ppm. HRMS (ESI+) Calcd. for $\mathrm{C}_{8} \mathrm{H}_{6} \mathrm{Br}^{+}[\mathrm{M}-\mathrm{OBoc}]^{+}: 180.9647$. Found: 180.9623.

\section{1-(3-bromophenyl)vinyl tert-butyl carbonate 1m}<smiles>C=C(OC)c1cccc(Br)c1</smiles>

According to general procedure B, 1-(2-bromophenyl)vinyl tert-butyl carbonate $1 \mathrm{~m}$ was obtained from 1-(3-bromophenyl)ethan-1-one as a yellow oil in $67 \%$ yield $(772 \mathrm{mg}, 3.35 \mathrm{mmol}$ ) after purification by column chromatography on silica gel (hexane/EtOAc 95/5). ${ }^{1} \mathbf{H} \mathbf{~ N M R ~}\left(\mathbf{5 0 0} \mathbf{~ M H z}, \mathrm{CDCl}_{3}\right) \delta 7.64$ (t, $J=1.9$ $\mathrm{Hz}, 1 \mathrm{H}$ ), 7.45 (ddd, $J=7.9,2.0,1.0 \mathrm{~Hz}, 1 \mathrm{H}), 7.42$ (ddd, $J=7.9,1.8,1.0 \mathrm{~Hz}, 1 \mathrm{H}), 7.21(\mathrm{t}, J=7.9 \mathrm{~Hz}, 1 \mathrm{H}$ ), $5.41(\mathrm{~d}, J=2.5 \mathrm{~Hz}, 1 \mathrm{H}), 5.14(\mathrm{~d}, J=2.5 \mathrm{~Hz}, 1 \mathrm{H}), 1.50(\mathrm{~s}, 9 \mathrm{H}) \mathrm{ppm} .{ }^{13} \mathrm{C} \mathrm{NMR}\left(126 \mathrm{MHz}, \mathrm{CDCl}_{3}\right) \delta 152.0$, 151.3, 136.6, 132.0, 130.1, 128.1, 123.5, 122.8, $102.9\left(\mathrm{C}=\mathrm{CH}_{2}\right), 83.7\left(\mathrm{O}-\mathrm{C}-\left(\mathrm{CH}_{3}\right)_{3}\right), 27.7\left(\mathrm{C}-\left(\mathrm{CH}_{3}\right)_{3}\right) \mathrm{ppm}$. HRMS (ASAP +) Calcd for $\mathrm{C}_{8} \mathrm{H}_{8} \mathrm{BrO}^{+}[\mathrm{M}-\mathrm{BoC}+\mathrm{H}]^{+}:$198.9753. Found: 198.9759 .

\section{1-(4-bromophenyl)vinyl tert-butyl carbonate 1n}<smiles>C=C(OC)c1ccc(Br)cc1</smiles>

According to general procedure B, 1-(4-bromophenyl)vinyl tert-butyl carbonate $\mathbf{1 n}$ was obtained from 1-(4-bromophenyl)ethan-1-one as a yellow solid in $39 \%$ yield $(586 \mathrm{mg}, 1.95 \mathrm{mmol}$ ) after purification by column chromatography on silica gel (hexane/EtOAc 95/5). ${ }^{1} \mathrm{H}$ NMR $\left(500 \mathbf{~ M H z}, \mathbf{C D C l}_{3}\right) \delta 7.48(\mathrm{~d}, J=8.7$ $\mathrm{Hz}, 2 \mathrm{H}), 7.36(\mathrm{~d}, J=8.7 \mathrm{~Hz}, 2 \mathrm{H}), 5.40(\mathrm{~d}, J=2.4 \mathrm{~Hz}, 1 \mathrm{H}), 5.12(\mathrm{~d}, J=2.4 \mathrm{~Hz}, 1 \mathrm{H}), 1.50(\mathrm{~s}, 9 \mathrm{H}) \mathrm{ppm} .{ }^{13} \mathrm{C}$ NMR $\left(126 \mathrm{MHz}, \mathrm{CDCl}_{3}\right) \delta 152.5,151.4,133.6,131.8,126.6,123.2,102.4\left(\mathrm{C}=\mathrm{CH}_{2}\right), 83.7\left(\mathrm{O}-\mathrm{C}-\left(\mathrm{CH}_{3}\right)_{3}\right)$, $27.8\left(\mathrm{C}-\left(\mathrm{CH}_{3}\right)_{3}\right)$ ppm. HRMS (ESI+) Calcd. for $\mathrm{C}_{13} \mathrm{H}_{15} \mathrm{BrO}_{3} \mathrm{Na}^{+}[\mathrm{M}+\mathrm{Na}]^{+}: 321.0102$. Found: 321.0110.

tert-butyl 1-(2,5-dichlorophenyl)vinyl carbonate 10<smiles>C=C(OC)c1cc(Cl)ccc1Cl</smiles>

According to general procedure B, tert-butyl 1-(2,5-dichlorophenyl)vinyl carbonate 10 was obtained from 1-(2,5-dichlorophenyl)ethan-1-one as a yellow oil in $10 \%$ yield $(150 \mathrm{mg}, 0.52 \mathrm{mmol}$ ) after purification by column chromatography on silica gel (hexane/DCM 7/3). $\left.{ }^{1} \mathbf{H} \mathbf{~ N M R ~ ( 4 0 0 ~} \mathbf{~ M H z}, \mathbf{C D C l}_{3}\right) \delta$ $7.45(\mathrm{~d}, J=2.5 \mathrm{~Hz}, 1 \mathrm{H}), 7.32(\mathrm{~d}, J=8.5 \mathrm{~Hz}, 1 \mathrm{H}), 7.24(\mathrm{dd}, J=8.5,2.5 \mathrm{~Hz}, 1 \mathrm{H}), 5.37(\mathrm{~d}, J=2.2 \mathrm{~Hz}, 1 \mathrm{H}), 5.24$ (d, $J=2.2 \mathrm{~Hz}, 1 \mathrm{H}), 1.46(\mathrm{~s}, 9 \mathrm{H}) \mathrm{ppm} .{ }^{13} \mathrm{C}$ NMR $\left(101 \mathrm{MHz}, \mathrm{CDCl}_{3}\right) \delta 150.8,150.1,135.8,132.7,131.4$, 
130.6, 130.1, 129.9, $107.6\left(\mathrm{C}=\mathrm{CH}_{2}\right), 83.8\left(\mathrm{O}-\mathrm{C}-\left(\mathrm{CH}_{3}\right)_{3}\right), 27.7\left(\mathrm{C}-\left(\mathrm{CH}_{3}\right)_{3}\right)$ ppm. HRMS (ESI+) Calcd. for $\mathrm{C}_{13} \mathrm{H}_{14} \mathrm{Cl}_{2} \mathrm{O}_{3} \mathrm{Na}^{+}[\mathrm{M}+\mathrm{Na}]^{+}:$311.0212. Found: 311.0193 .

\section{1-(4-acetylphenyl)vinyl tert-butyl carbonate 1p}

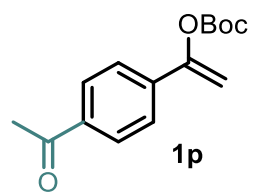

According to general procedure C, 1-(4-acetylphenyl)vinyl tert-butyl carbonate $1 \mathrm{p}$ was obtained from 1,4-diacetylbenzene as a colourless oil in $26 \%$ yield ( $344 \mathrm{mg}, 1.3 \mathrm{mmol}$ ) after purification by column chromatography on silica gel (hexane/EtOAc 8/2). ${ }^{1} \mathrm{H} \mathrm{NMR}\left(400 \mathrm{MHz}, \mathrm{CDCl}_{3}\right) \delta 7.98-7.90(\mathrm{~m}, 2 \mathrm{H}), 7.61$ - $7.53(\mathrm{~m}, 2 \mathrm{H}), 5.52(\mathrm{~d}, J=2.5 \mathrm{~Hz}, 1 \mathrm{H}), 5.22(\mathrm{~d}, J=2.5 \mathrm{~Hz}, 1 \mathrm{H}), 2.59(\mathrm{~s}, 3 \mathrm{H}), 1.49(\mathrm{~s}, 9 \mathrm{H}) \mathrm{ppm}{ }^{13} \mathrm{C}$ NMR (101 MHz, CDCl $\left.)_{3}\right) \delta 197.4,152.5,151.3,138.9,137.2,128.8,125.1,104.1\left(\mathrm{C}=\mathrm{CH}_{2}\right), 83.8\left(\mathrm{O}-\mathrm{C}-\left(\mathrm{CH}_{3}\right)_{3}\right)$, $27.7\left(\mathrm{C}-\left(\mathrm{CH}_{3}\right)_{3}\right), 26.7$ ppm. HRMS (ESI+) Calcd. for $\mathrm{C}_{15} \mathrm{H}_{19} \mathrm{O}_{4} \mathrm{H}^{+}[\mathrm{M}+\mathrm{H}]^{+}:$263.1278. Found: 263.1286.

tert-butyl (1-phenylprop-1-en-1-yl) carbonate 1q<smiles>[14CH3]OC(=O)c1ccccc1</smiles>

According to general procedure B, tert-butyl (1-phenylprop-1-en-1-yl) carbonate 1q was obtained from propiophenone as a colourless oil in $24 \%$ yield $(276 \mathrm{mg}, 1.2 \mathrm{mmol}$ ) after purification by column chromatography on silica gel (hexane/DCM 7/3). $\left.{ }^{1} \mathbf{H ~ N M R ~ ( 5 0 0 ~ M H z , ~} \mathrm{CDCl}_{3}\right) \delta 7.37-7.32(\mathrm{~m}, 1 \mathrm{H}), 7.27$ $-7.21(\mathrm{~m}, 2 \mathrm{H}), 7.20-7.15(\mathrm{~m}, 1 \mathrm{H}), 5.75(\mathrm{q}, J=7.0 \mathrm{~Hz}, 1 \mathrm{H}), 1.69(\mathrm{~d}, J=7.0 \mathrm{~Hz}, 3 \mathrm{H}), 1.42(\mathrm{~s}, 9 \mathrm{H}) \mathrm{ppm} .{ }^{13} \mathrm{C}$ NMR (126 MHz, CDCl $\left.)_{3}\right) \delta 151.2,147.4,135.4,128.6,128.1,124.4,112.7$ (C=CHR), 83.1 (O-C-(CH $\left.)_{3}\right)$, $27.8\left(\mathrm{C}-\left(\mathrm{CH}_{3}\right)_{3}\right), 11.4$ ppm. HRMS (ESI+) Calcd. for $\mathrm{C}_{9} \mathrm{H}_{9}{ }^{+}[\mathrm{M}-\mathrm{OBoc}]^{+}:$117.0699. Found: 117.0701.

tert-butyl (1,3-diphenylprop-1-en-1-yl) carbonate $1 r$<smiles></smiles>

According to general procedure C, tert-butyl (1,3-diphenylprop-1-en-1-yl) carbonate $1 \mathbf{r}$ was obtained from dihydrochalcone as a white solid in $79 \%$ yield $(1.23 \mathrm{~g}, 3.9 \mathrm{mmol})$ after purification by column chromatography on silica gel (hexane/DCM 7/3). ${ }^{1} \mathbf{H}$ NMR ( $\left.500 \mathbf{~ M H z}, \mathrm{CDCl}_{3}\right) \delta 7.41-7.35(\mathrm{~m}, 2 \mathrm{H}), 7.28$ - $7.15(\mathrm{~m}, 7 \mathrm{H}), 7.16-7.09(\mathrm{~m}, 1 \mathrm{H}), 5.82(\mathrm{t}, J=7.5 \mathrm{~Hz}, 1 \mathrm{H}), 3.48(\mathrm{~d}, J=7.5 \mathrm{~Hz}, 2 \mathrm{H}), 1.41(\mathrm{~s}, 9 \mathrm{H}) \mathrm{ppm} .{ }^{13} \mathrm{C}$ NMR (126 MHz, $\left.\mathrm{CDCl}_{3}\right) \delta 151.3,147.0,139.8,135.2,128.7,128.6,128.6,128.4,126.4,124.6,116.8$ (C=CHR), $83.4\left(\mathrm{O}-\mathrm{C}-\left(\mathrm{CH}_{3}\right)_{3}\right), 32.3,27.7\left(\mathrm{C}-\left(\mathrm{CH}_{3}\right)_{3}\right)$ ppm. HRMS (ESI+) Calcd. for $\mathrm{C}_{15} \mathrm{H}_{13}{ }^{+}[\mathrm{M}-\mathrm{OBoc}]^{+}$: 193.1012. Found: 193.1002.

\section{tert-butyl 1H-inden-3-yl carbonate 1s}<smiles>CCOc1ccccc1-c1ccccc1</smiles>

According to general procedure $\mathrm{A}$, tert-butyl $1 \mathrm{H}$-inden-3-yl carbonate $1 \mathrm{~s}$ was obtained from 1 Indanone as a yellow oil in $99 \%$ yield $(600 \mathrm{mg}, 2.6 \mathrm{mmol}) .{ }^{1} \mathbf{H} \mathbf{~ N M R}\left(300 \mathrm{MHz}, \mathbf{C D C l}_{3}\right) \delta 7.45$ (dd, $J=$ 
15.5, 7.3 Hz, 2H), $7.39-7.27(\mathrm{~m}, 2 \mathrm{H}), 6.34(\mathrm{~s}, 1 \mathrm{H}), 3.44(\mathrm{~s}, 2 \mathrm{H}), 1.62(\mathrm{~s}, 9 \mathrm{H}) \mathrm{ppm} .{ }^{13} \mathrm{C}$ NMR $(75 \mathrm{MHz}$, $\left.\mathrm{CDCl}_{3}\right) \delta 150.9,149.6,142.0,139.0,126.4,125.8,124.2,118.3,114.6,83.7\left(\mathrm{~s}, 1 \mathrm{C}, \mathrm{O}-\mathrm{C}-\left(\mathrm{CH}_{3}\right)_{3}\right), 34.9$, $27.8\left(\mathrm{~s}, 3 \mathrm{C}, \mathrm{C}-\left(\mathrm{CH}_{3}\right)_{3}\right) \mathrm{ppm}$.

tert-butyl (5-methoxy-1H-inden-3-yl) carbonate 1t<smiles>COC1=CC([Te])c2ccc(OC)cc21</smiles>

According to general procedure A, tert-butyl (5-methoxy-1H-inden-3-yl) carbonate 1 t was obtained from 6-methoxy-2,3-dihydro-1H-inden-1-one as a yellow oil in $88 \%$ yield ( $600 \mathrm{mg}, 2.3 \mathrm{mmol}$ ). ${ }^{1} \mathrm{H}$ NMR (300 MHz, $\left.\mathrm{CDCl}_{3}\right) \delta 7.30(\mathrm{~d}, J=8.2 \mathrm{~Hz}, 1 \mathrm{H}), 6.91(\mathrm{~s}, 1 \mathrm{H}), 6.80(\mathrm{~d}, J=8.2 \mathrm{~Hz}, 1 \mathrm{H}), 6.33(\mathrm{~s}, 1 \mathrm{H}), 3.84(\mathrm{~s}$, 3H), 3.34 (s, 2H), 1.59 (s, 9H) ppm. ${ }^{13} \mathrm{C} \mathrm{NMR} \mathrm{(75} \mathrm{MHz,} \mathrm{CDCl} 3$ ) $\delta$ 159.0, 150.9, 149.3, 140.3, 134.0, 124.7, $115.8,112.6,103.4,83.8\left(\mathrm{~s}, 1 \mathrm{C}, \mathrm{O}-\mathrm{C}-\left(\mathrm{CH}_{3}\right)_{3}\right), 55.7,34.2,27.8\left(\mathrm{~s}, 3 \mathrm{C}, \mathrm{C}-\left(\mathrm{CH}_{3}\right)_{3}\right) \mathrm{ppm}$.

tert-butyl (6-fluoro-1H-inden-3-yl) carbonate 1u<smiles>O=C(OCc1ccccc1)C1=CCCC1</smiles>

According to general procedure A, tert-butyl (6-fluoro-1H-inden-3-yl) carbonate $1 \mathrm{u}$ was obtained from 5-fluoro-2,3-dihydro-1H-inden-1-one as a yellow oil in $92 \%$ yield $(600 \mathrm{mg}, 2.4 \mathrm{mmol}) .{ }^{1} \mathrm{H}$ NMR (300 $\left.\mathrm{MHz}, \mathrm{CDCl}_{3}\right) \delta 7.34-7.25(\mathrm{~m}, 3 \mathrm{H}), 7.15(\mathrm{~d}, J=8.8 \mathrm{~Hz}, 1 \mathrm{H}), 7.03(\mathrm{t}, J=8.9 \mathrm{~Hz}, 1 \mathrm{H}), 6.28(\mathrm{~s}, 1 \mathrm{H}), 3.39(\mathrm{~s}$, 2H), 1.59 (s, 9H) ppm. $\left.{ }^{13} \mathrm{C} \mathrm{NMR} \mathrm{(75} \mathrm{MHz,} \mathrm{CDCl}_{3}\right) \delta 163.8,160.5,150.8,148.9,144.0$ (d, $\left.J=8.8 \mathrm{~Hz}, 1 \mathrm{C}\right)$, $135.0(\mathrm{~d}, J=2.2 \mathrm{~Hz}, 1 \mathrm{C}), 119.1(\mathrm{~d}, J=8.9 \mathrm{~Hz}, 1 \mathrm{C}), 114.0(\mathrm{~d}, J=3.8 \mathrm{~Hz}, 1 \mathrm{C}), 112.70$ (dd, $J=121.1,23.3$ $\mathrm{Hz}), 83.8\left(\mathrm{~s}, 1 \mathrm{C}, \mathrm{O}-\mathrm{C}-\left(\mathrm{CH}_{3}\right)_{3}\right), 34.9(\mathrm{~d}, J=2.4 \mathrm{~Hz}, 1 \mathrm{C}), 27.8\left(\mathrm{~s}, 3 \mathrm{C}, \mathrm{C}-\left(\mathrm{CH}_{3}\right)_{3}\right)$ ppm. ${ }^{19} \mathrm{~F}$ NMR (188 MHz, $\left.\mathrm{CDCl}_{3}\right) \delta-117.84(\mathrm{td}, J=9.0,5.0 \mathrm{~Hz}, 1 \mathrm{~F}) \mathrm{ppm}$.

tert-butyl 3,4-dihydronaphthalen-1-yl carbonate 1v<smiles>CC1C=C(c2ccccc2O)c2ccccc2C1</smiles>

According to general procedure B, tert-butyl 3,4-dihydronaphthalen-1-yl carbonate $1 \mathbf{v}$ was obtained from $\alpha$-tetralone as a colourless oil in $20 \%$ yield $(244 \mathrm{mg}, 0.99 \mathrm{mmol})$ after purification by column chromatography on silica gel (hexane/EtOAc 9/1). $\left.{ }^{1} \mathbf{H ~ N M R ~ ( 5 0 0 ~ M H z , ~} \mathrm{CDCl}_{3}\right) \delta 7.21-7.12(\mathrm{~m}, 4 \mathrm{H}), 5.78$ (t, $J=4.7 \mathrm{~Hz}, 1 \mathrm{H}$ ), 2.86 (ddd, $J=8.3,7.6,0.9 \mathrm{~Hz}, 2 \mathrm{H}$ ), 2.44 (ddd, $J=8.9,7.6,4.7 \mathrm{~Hz}, 2 \mathrm{H}$ ), $1.54(\mathrm{~s}, 9 \mathrm{H})$ ppm. ${ }^{13} \mathrm{C} \mathrm{NMR}\left(126 \mathrm{MHz}, \mathrm{CDCl}_{3}\right) \delta 152.0,146.2,136.5,130.7,128.0,127.7,126.6,120.7,115.3$ (C=CHR), $83.3\left(\mathrm{O}-\mathrm{C}-\left(\mathrm{CH}_{3}\right)_{3}\right), 27.8\left(\mathrm{C}-\left(\mathrm{CH}_{3}\right)_{3}\right), 27.6,22.1 \mathrm{ppm}$. HRMS (ESI+) Calcd. for $\mathrm{C}_{15} \mathrm{H}_{18} \mathrm{O}_{3} \mathrm{Na}^{+}[\mathrm{M}+\mathrm{Na}]^{+}$: 269.1148. Found: 269.1136. 
<smiles>C[C@H]1C=Cc2c(O)cccc2C1</smiles>

According to general procedure A, tert-butyl (6,7-dihydro-5H-benzo[7]annulen-9-yl) carbonate $\mathbf{1 w}$ was obtained from 1-Benzosuberone as a colorless oil in $92 \%$ yield $(620 \mathrm{mg}, 2.4 \mathrm{mmol}) .{ }^{1} \mathrm{H} \mathrm{NMR}$ (300 MHz, $\left.\mathrm{CDCl}_{3}\right) \delta 7.47-7.34(\mathrm{~m}, 1 \mathrm{H}), 7.27-7.16(\mathrm{~m}, 3 \mathrm{H}), 5.93(\mathrm{t}, J=6.1 \mathrm{~Hz}, 1 \mathrm{H}), 2.83(\mathrm{t}, J=6.0 \mathrm{~Hz}, 2 \mathrm{H}), 2.18(\mathrm{q}$, $J=6.5 \mathrm{~Hz}, 2 \mathrm{H}), 2.13-7.02(\mathrm{~m}, 2 \mathrm{H}), 1.47(\mathrm{~s}, 9 \mathrm{H}) \mathrm{ppm} .{ }^{13} \mathrm{C} \mathrm{NMR}\left(75 \mathrm{MHz}, \mathrm{CDCl}_{3}\right) \delta 152.3,146.2,141.8$, 134.5, 129.2, 128.2, 126.2, 125.3, 119.3, $82.9\left(\mathrm{~s}, 1 \mathrm{C}, \mathrm{O}-\mathrm{C}-\left(\mathrm{CH}_{3}\right)_{3}\right), 33.8,31.1,27.8\left(\mathrm{~s}, 3 \mathrm{C}, \mathrm{C}-\left(\mathrm{CH}_{3}\right)_{3}\right), 25.3$ ppm.

\section{1-(benzo[b]thiophen-2-yl)vinyl tert-butyl carbonate $1 x$}

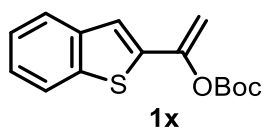

According to general procedure A, 1-(benzo[b]thiophen-2-yl)vinyl tert-butyl carbonate $\mathbf{1 x}$ was obtained from 1-(benzo[b]thiophen-2-yl)ethanone as a yellow oil in $95 \%$ yield $(680 \mathrm{mg}, 2.5 \mathrm{mmol}) .{ }^{1} \mathbf{H}$ NMR (300 MHz, CDCl$) \delta 7.82-7.70(\mathrm{~m}, 2 \mathrm{H}), 7.38(\mathrm{~s}, 1 \mathrm{H}), 7.37-7.28(\mathrm{~m}, 2 \mathrm{H}), 5.47(\mathrm{~m}, 1 \mathrm{H}), 5.20(\mathrm{~m}$, 1H), 1.57 (s, 9H) ppm. ${ }^{13} \mathrm{C}$ NMR $\left(75 \mathrm{MHz}, \mathrm{CDCl}_{3}\right) \delta 151.2,148.3,139.7,139.5,137.9,125.3,124.7,124.1$, 122.2, 121.5, $103.3\left(\mathrm{~s}, \mathrm{C}=\mathrm{CH}_{2}\right), 83.8\left(\mathrm{~s}, 1 \mathrm{C}, \mathrm{O}-\mathrm{C}-\left(\mathrm{CH}_{3}\right)_{3}\right), 27.7\left(\mathrm{~s}, 3 \mathrm{C}, \mathrm{C}-\left(\mathrm{CH}_{3}\right)_{3}\right)$ ppm. HRMS (ASAP +) Calcd for $\mathrm{C}_{15} \mathrm{H}_{16} \mathrm{NaO}_{3} \mathrm{~S}^{+}[\mathrm{M}+\mathrm{Na}]^{+}: 299,0712$. Found: 299,0718 .

\section{tert-butyl 1-(naphthalen-2-yl)vinyl carbonate 1y}<smiles>C=C(OC)c1ccc2ccccc2c1</smiles>

According to general procedure B, tert-butyl 1-(naphthalen-2-yl)vinyl carbonate $1 \mathbf{y}$ was obtained from 1-(naphthalen-2-yl)ethan-1-one as a white solid in $12 \%$ yield $(165 \mathrm{mg}, 0.61 \mathrm{mmol}$ ) after purification by column chromatography on silica gel (hexane/EtOAc 95/5). ${ }^{1} \mathbf{H} \mathbf{~ N M R}\left(\mathbf{4 0 0} \mathbf{~ M H z}, \mathbf{C D C l}_{3}\right) \delta 7.95$ (d, $J=0.9$ $\mathrm{Hz}, 1 \mathrm{H}), 7.90-7.77(\mathrm{~m}, 3 \mathrm{H}), 7.63(\mathrm{dd}, J=8.7,1.9 \mathrm{~Hz}, 1 \mathrm{H}), 7.52-7.45(\mathrm{~m}, 2 \mathrm{H}), 5.56(\mathrm{~d}, J=2.4 \mathrm{~Hz}, 1 \mathrm{H})$, $5.21(\mathrm{~d}, J=2.4 \mathrm{~Hz}, 1 \mathrm{H}), 1.53(\mathrm{~s}, 9 \mathrm{H}) \mathrm{ppm} .{ }^{13} \mathrm{C} \mathrm{NMR}\left(101 \mathrm{MHz}, \mathrm{CDCl}_{3}\right) \delta 153.5,151.6,133.6,133.2,131.8$, $128.6,128.5,127.8,126.7,126.6,124.2,122.8,102.3\left(\mathrm{C}=\mathrm{CH}_{2}\right), 83.6\left(\mathrm{O}-\mathrm{C}-\left(\mathrm{CH}_{3}\right)_{3}\right), 27.8\left(\mathrm{C}-\left(\mathrm{CH}_{3}\right)_{3}\right) \mathrm{ppm}$. HRMS (ESI+) Calcd. for $\mathrm{C}_{17} \mathrm{H}_{18} \mathrm{O}_{3} \mathrm{Na}^{+}[\mathrm{M}+\mathrm{Na}]^{+}:$293.1148. Found: 293.1138.

\section{tert-butyl 2,2-difluoro-1-phenylvinyl carbonate $1 z$}<smiles>FC(F)=C(OCc1ccccc1)c1ccccc1</smiles>

tert-butyl 2,2-difluoro-1-phenylvinyl carbonate $\mathbf{1 z}$ was synthesized according to a procedure reported in the literature. ${ }^{[6]}$ The spectral data matched with those reported in the literature. 
<smiles>COC(=O)C(F)=C(F)c1ccc2ccccc2c1</smiles>

2,2,2-trifluoro-1-(naphthalen-2-yl)ethan-1-one was synthesized according to a reported literature procedure. ${ }^{[7]}$ The spectral data matched with those reported in the literature.

2,2,2-trifluoro-1-(naphthalen-2-yl)ethan-1-one (433 mg, $1.93 \mathrm{mmol} 1$ equiv.) was dissolved in $\mathrm{MeOH}$ $(5 \mathrm{~mL}, 0.4 \mathrm{M})$ at $0^{\circ} \mathrm{C} . \mathrm{NaBH}_{4}(80 \mathrm{mg}, 2.12 \mathrm{mmol}, 1.1$ equiv.) was then added portionwise and the reaction stirred for $1 \mathrm{~h}$ at room temperature. The reaction was then cooled to $0^{\circ} \mathrm{C}$, quenched with sat. $\mathrm{NH}_{4} \mathrm{Cl}$ until gas evolution stopped and evaporated to dryness. The crude residue was diluted with DCM (20 $\mathrm{mL}$ ) and washed with brine ( $3 \times 10 \mathrm{~mL})$. The organic phase was then dried over $\mathrm{MgSO}_{4}$ and concentrated under reduced pressure. The crude 2,2,2-trifluoro-1-(naphthalen-2-yl)ethan-1-ol product was used in the subsequent step without further purification. The spectral data matched with those reported in the literature. ${ }^{[8]}$

Crude 2,2,2-trifluoro-1-(naphthalen-2-yl)ethan-1-ol (340 mg) was dissolved in MeCN (7 mL) and DMAP $(18.3 \mathrm{mg}, 0.15 \mathrm{mmol})$ was added. $\mathrm{Boc}_{2} \mathrm{O}(517 \mu \mathrm{L}, 2.25 \mathrm{mmol})$ was then added dropwise and the reaction mixture stirred for $20 \mathrm{~h}$, after which time the mixture was concentrated under reduced pressure. The residue was filtered on silica using DCM as eluent and the eluted solution evaporated under reduced pressure. The crude tert-butyl (2,2,2-trifluoro-1-(naphthalen-2-yl)ethyl) carbonate product was used in the subsequent step without further purification.

To a solution of crude tert-butyl (2,2,2-trifluoro-1-(naphthalen-2-yl)ethyl) carbonate (485 mg) in dry THF $(7 \mathrm{~mL})$ at $-78^{\circ} \mathrm{C}$ was added dropwise a freshly prepared solution of LDA (0.7 M, 3.21 mL). After stirring for $1 \mathrm{~h}$ at $-78{ }^{\circ} \mathrm{C}$, the reaction mixture was stirred for an additional $1 \mathrm{~h}$ at room temperature. The reaction mixture was then diluted with water $(10 \mathrm{~mL})$ and extracted with EtOAc $(3 \times 10 \mathrm{~mL})$, dried over anhydrous $\mathrm{MgSO}_{4}$, and the solvent evaporated to give a residue. Finally, the residue was purified by silica gel chromatography (hexane/EtOAc 97/3) to give tert-butyl 2,2-difluoro-1-(naphthalen-2yl)vinyl carbonate 1 aa in $45 \%$ yield over 3 steps $(266 \mathrm{mg}, 0.87 \mathrm{mmol}) .{ }^{1} \mathbf{H} \mathbf{~ N M R}\left(\mathbf{5 0 0} \mathbf{~ M H z}, \mathbf{C D C l}_{3}\right) \delta 7.92$ $-7.88(\mathrm{~m}, 1 \mathrm{H}), 7.89-7.79(\mathrm{~m}, 3 \mathrm{H}), 7.56(\mathrm{dt}, J=8.7,1.8 \mathrm{~Hz}, 1 \mathrm{H}), 7.54-7.47(\mathrm{~m}, 2 \mathrm{H}), 1.53(\mathrm{~s}, 9 \mathrm{H}) \mathrm{ppm}$. ${ }^{13} \mathrm{C}$ NMR $\left(126 \mathrm{MHz}, \mathrm{CDCl}_{3}\right) \delta 155.2\left(\mathrm{dd}, J=292.4,290.5 \mathrm{~Hz},\left(\mathrm{C}=\mathrm{CF}_{2}\right), 151.0(\mathrm{dd}, J=3.5,2.3 \mathrm{~Hz}), 133.2\right.$ (d, $J=10.5 \mathrm{~Hz}$ ), 128.6, 128.4, 127.8, 126.9, 126.9, 126.8, 126.8, 125.1 (t, $J=5.3 \mathrm{~Hz}), 122.9$ (dd, $J=7.0$, $2.3 \mathrm{~Hz}), 113.4\left(\mathrm{dd}, J=38.8,19.5 \mathrm{~Hz}, \mathrm{C}=\mathrm{CF}_{2}\right), 84.7\left(\mathrm{O}-\mathrm{C}-\left(\mathrm{CH}_{3}\right)_{3}\right), 27.7\left(\mathrm{C}-\left(\mathrm{CH}_{3}\right)_{3}\right)$ ppm. ${ }^{19} \mathrm{~F} \mathrm{NMR}(188 \mathrm{MHz}$, $\left.\mathrm{CDCl}_{3}\right) \delta-92.4(\mathrm{~d}, J=46.6 \mathrm{~Hz}),-103.9(\mathrm{~d}, J=46.6 \mathrm{~Hz})$ ppm. HRMS (ASAP +) Calcd for $\mathrm{C}_{12} \mathrm{H}_{9} \mathrm{~F}_{2} \mathrm{O}^{+}[\mathrm{M}-\mathrm{Boc}$ $+\mathrm{H}]^{+}:$207.0616. Found: 207.0621 .

tert-butyl oct-1-en-2-yl carbonate 1ab<smiles>C=C(C)CCCCCCC</smiles>

According to general procedure $C$, tert-butyl oct-1-en-2-yl carbonate 1zb was obtained from octan-2one as a colourless oil in $33 \%$ yield ( $380 \mathrm{mg}, 1.66 \mathrm{mmol}$ ) after purification by column chromatography on silica gel (hexane/EtOAc 98/2). ${ }^{1} \mathrm{H}$ NMR $\left(400 \mathrm{MHz}, \mathrm{CDCl}_{3}\right) \delta 4.75-4.67(\mathrm{~m}, 1 \mathrm{H}), 4.62-4.56(\mathrm{~m}, 1 \mathrm{H})$, $2.21-2.10(\mathrm{~m}, 2 \mathrm{H}), 1.43(\mathrm{~s}, 9 \mathrm{H}), 1.41-1.38(\mathrm{~m}, 4 \mathrm{H}), 1.24-1.21(\mathrm{~m}, 4 \mathrm{H}), 0.83-0.80(\mathrm{~m}, 3 \mathrm{H}) \mathrm{ppm} .{ }^{13} \mathrm{C}$ NMR (101 MHz, CDCl $)_{3} \delta 156.6,151.46,100.4\left(\mathrm{C}=\mathrm{CH}_{2}\right), 82.4\left(\mathrm{O}-\mathrm{C}-\left(\mathrm{CH}_{3}\right)_{3}\right), 33.1,31.6,28.6,27.6$ (C$\left.\left(\mathrm{CH}_{3}\right)_{3}\right), 26.4,22.5,14.0$ ppm. HRMS (ESI+) Calcd. for $\mathrm{C}_{13} \mathrm{H}_{24} \mathrm{O}_{3} \mathrm{Na}^{+}[\mathrm{M}+\mathrm{Na}]^{+}:$251.1618. Found: 251.1596. 
(E)-tert-butyl (4-phenylbuta-1,3-dien-2-yl) carbonate 1ac<smiles>C=C(C)OCC=Cc1ccccc1</smiles>

According to general procedure A, (E)-tert-butyl (4-phenylbuta-1,3-dien-2-yl) carbonate 1ac was obtained from 4- (E)-4-phenylbut-3-en-2-one as a colorless oil in $63 \%$ yield ( $400 \mathrm{mg}, 1.6 \mathrm{mmol}$ ). This product had to be stored at $-20^{\circ} \mathrm{C}$, decomposition was noticed after few days in the fridge. ${ }^{1} \mathbf{H}$ NMR (300 MHz, CDCl 3 ) $\delta 7.44(\mathrm{~d}, J=7.5 \mathrm{~Hz}, 2 \mathrm{H}), 7.40-7.27(\mathrm{~m}, 3 \mathrm{H}), 6.70$ (dd, $J=16.1,11.3 \mathrm{~Hz}, 2 \mathrm{H}), 5.10$ (d, $J=8.7 \mathrm{~Hz}, 2 \mathrm{H}), 1.58(\mathrm{~s}, 9 \mathrm{H}) \mathrm{ppm} .{ }^{13} \mathrm{C}$ NMR $\left(75 \mathrm{MHz}, \mathrm{CDCl}_{3}\right) \delta 152.3,136.2,130.1,128.8,128.4,127.0$, 122.6, $105.7\left(\mathrm{~s}, \mathrm{C}=\mathrm{CH}_{2}\right), 83.4\left(\mathrm{~s}, 1 \mathrm{C}, \mathrm{O}-\mathrm{C}-\left(\mathrm{CH}_{3}\right)_{3}\right), 27.8\left(\mathrm{~s}, 3 \mathrm{C}, \mathrm{C}-\left(\mathrm{CH}_{3}\right)_{3}\right) \mathrm{ppm}$.

(E)-tert-butyl nona-1,3-dien-2-yl carbonate 1ad<smiles>C=C(C)OC(=O)OC=CCCCCC</smiles>

According to general procedure A, (E)-tert-butyl nona-1,3-dien-2-yl carbonate 1ad was obtained from (E)-non-3-en-2-one as a colorless oil in $99 \%$ yield $(620 \mathrm{mg}, 2.6 \mathrm{mmol}) .{ }^{1} \mathbf{H ~ N M R}\left(300 \mathrm{MHz}, \mathrm{CDCl}_{3}\right) \delta 5.99$ $-5.78(\mathrm{~m}, 2 \mathrm{H}), 4.83(\mathrm{~s}, 2 \mathrm{H}), 2.10(\mathrm{q}, J=7.0 \mathrm{~Hz}, 2 \mathrm{H}), 1.52(\mathrm{~s}, 9 \mathrm{H}), 1.40(\mathrm{t}, J=7.0 \mathrm{~Hz}, 2 \mathrm{H}), 1.29(\mathrm{~s}, 4 \mathrm{H})$, $0.92-0.82(\mathrm{t}, J=6.7 \mathrm{~Hz}, 3 \mathrm{H}) \mathrm{ppm} .{ }^{13} \mathrm{C}$ NMR $\left(75 \mathrm{MHz}, \mathrm{CDCl}_{3}\right) \delta 152.2,151.4,132.9,124.0,102.8(\mathrm{~s}$, $\left.\mathrm{C}=\mathrm{CH}_{2}\right), 82.8\left(\mathrm{~s}, 1 \mathrm{C}, \mathrm{O}-\mathrm{C}-\left(\mathrm{CH}_{3}\right)_{3}\right), 32.2,31.3,28.6,27.6\left(\mathrm{~s}, 3 \mathrm{C}, \mathrm{C}-\left(\mathrm{CH}_{3}\right)_{3}\right), 22.5,14.0 \mathrm{ppm}$. HRMS (ASAP +) Calcd for $\mathrm{C}_{14} \mathrm{H}_{24} \mathrm{NaO}_{3}{ }^{+}[\mathrm{M}+\mathrm{Na}]^{+}: 263,1613$. Found: 263,1618 .

tert-butyl 1-(3,5,5,6,8,8-hexamethyl-5,6,7,8-tetrahydronaphthalen-2-yl)vinyl carbonate 1ae<smiles>C=C(O[Na])c1cc2c(cc1N=O)C(C)(C)C(C)CC2(C)C</smiles>

According to general procedure C, tert-butyl 1-(3,5,5,6,8,8-hexamethyl-5,6,7,8-tetrahydronaphthalen2-yl)vinyl) carbonate 1ae was obtained from 6-Acetyl-1,1,2,4,4,7-hexamethyltetralin as a white solid in $42 \%$ yield $(752 \mathrm{mg}, 2.1 \mathrm{mmol})$ after purification by column chromatography on silica gel (hexane/DCM 7/3). ${ }^{1} \mathrm{H}$ NMR (500 MHz, CDCl $) \delta 7.29(\mathrm{~s}, 1 \mathrm{H}), 7.15(\mathrm{~s}, 1 \mathrm{H}), 5.21(\mathrm{~d}, J=1.6 \mathrm{~Hz}, 1 \mathrm{H}), 4.96$ (d, $J=1.6 \mathrm{~Hz}, 1 \mathrm{H}), 2.39(\mathrm{~s}, 3 \mathrm{H}), 1.85(\mathrm{dqd}, J=13.5,6.8,2.6 \mathrm{~Hz}, 1 \mathrm{H}), 1.61(\mathrm{t}, J=13.2 \mathrm{~Hz}, 1 \mathrm{H}), 1.43(\mathrm{~s}, 9 \mathrm{H})$ $1.36(\mathrm{dd}, J=13.5,2.6 \mathrm{~Hz}, 1 \mathrm{H}), 1.31(\mathrm{~s}, 3 \mathrm{H}), 1.27(\mathrm{~s}, 3 \mathrm{H}), 1.23(\mathrm{~s}, 3 \mathrm{H}), 1.06(\mathrm{~s}, 3 \mathrm{H}), 0.98(\mathrm{~d}, J=6.8 \mathrm{~Hz}, 3 \mathrm{H})$ ppm. ${ }^{13} \mathrm{C}$ NMR $\left(126 \mathrm{MHz}, \mathrm{CDCl}_{3}\right) \delta 153.9,151.2,146.8,142.3,132.8,132.4,129.3,126.5,104.3\left(\mathrm{C}=\mathrm{CH}_{2}\right)$, $83.0\left(\mathrm{O}-\mathrm{C}-\left(\mathrm{CH}_{3}\right)_{3}\right), 43.8,37.7,34.7,34.1,32.5,32.1,28.7,27.8\left(\mathrm{C}-\left(\mathrm{CH}_{3}\right)_{3}\right), 25.0,20.5,17.0$ ppm. HRMS (ESI+) Calcd. for $\mathrm{C}_{18} \mathrm{H}_{25}{ }^{+}[\mathrm{M}-\mathrm{OBoc}]^{+}:$241.1951. Found: 241.1967.

tert-butyl (1-(4-(tert-butyl)-2,6-dimethyl-3,5-dinitrophenyl)vinyl) carbonate 1af<smiles>C=C(OC)c1c([N+](=O)[O-])c([N+](=O)[O-])c([18O])c([N+](=O)[O-])c1[N+](=O)[O-]</smiles>

According to general procedure A, tert-butyl (1-(4-(tert-butyl)-2,6-dimethyl-3,5-dinitrophenyl)vinyl) carbonate 1af was obtained from musk ketone as a colorless oil in $99 \%$ yield $(620 \mathrm{mg}, 2.6 \mathrm{mmol}) .{ }^{1} \mathbf{H}$ NMR $\left(300 \mathrm{MHz}, \mathrm{CDCl}_{3}\right) \delta 5.56(\mathrm{~s}, 1 \mathrm{H}), 4.94(\mathrm{~s}, 1 \mathrm{H}), 2.28(\mathrm{~s}, 6 \mathrm{H}), 1.48(\mathrm{~s}, 9 \mathrm{H}), 1.44(\mathrm{~s}, 9 \mathrm{H}) \mathrm{ppm} .{ }^{13} \mathrm{C} \mathrm{NMR}$ (75 MHz, $\left.\mathrm{CDCl}_{3}\right) \delta 150.4,148.6,136.8,131.9,131.8,108.2\left(\mathrm{~s}, \mathrm{C}=\mathrm{CH}_{2}\right), 84.2$ (s, 1C, O-C- $\left.\left(\mathrm{CH}_{3}\right)_{3}\right), 37.5$, 30.5, $27.7\left(\mathrm{~s}, 3 \mathrm{C}, \mathrm{C}-\left(\mathrm{CH}_{3}\right)_{3}\right), 16.1 \mathrm{ppm}$. HRMS (ASAP +) Calcd for $\mathrm{C}_{19} \mathrm{H}_{27} \mathrm{~N}_{2} \mathrm{O}_{7}{ }^{+}[\mathrm{M}+\mathrm{H}]^{+}:$395.1813. Found: 395.1813. 
<smiles>C=C(OC(C)C)c1cc(Br)cc2c1CCC2(C)C</smiles>

According to general procedure A, tert-butyl (1-(6-(tert-butyl)-1,1-dimethyl-2,3-dihydro-1H-inden-4$\mathrm{yl}$ )vinyl) carbonate $1 \mathrm{ag}$ was obtained from celestolide as a white solid in $70 \%$ yield $(630 \mathrm{mg}, 1.8 \mathrm{mmol})$. ${ }^{1} \mathrm{H}$ NMR (300 MHz, CDCl $) \delta 7.28(\mathrm{~s}, 1 \mathrm{H}), 7.14(\mathrm{~s}, 1 \mathrm{H}), 5.16(\mathrm{~d}, J=9.7 \mathrm{~Hz}, 2 \mathrm{H}), 2.96(\mathrm{t}, J=7.1 \mathrm{~Hz}, 2 \mathrm{H})$, $1.93(\mathrm{t}, J=7.2 \mathrm{~Hz}, 2 \mathrm{H}), 1.45(\mathrm{~s}, 9 \mathrm{H}), 1.32(\mathrm{~s}, 9 \mathrm{H}), 1.26(\mathrm{~s}, 6 \mathrm{H}) \mathrm{ppm} .{ }^{13} \mathrm{C} \mathrm{NMR}\left(75 \mathrm{MHz}, \mathrm{CDCl}_{3}\right) \delta 153.9$, 153.5, 151.4, 150.0, 137.3, 130.7, 121.5, 119.6, 103.8 (s, C=CH $)_{2}, 83.1\left(\mathrm{~s}, 1 \mathrm{C}, \mathrm{O}-\mathrm{C}-\left(\mathrm{CH}_{3}\right)_{3}\right), 43.9,41.6$, 34.8, 31.7, 30.1, 28.8, $27.8\left(\mathrm{~s}, 3 \mathrm{C}, \mathrm{C}-\left(\mathrm{CH}_{3}\right)_{3}\right)$ ppm. HRMS (ASAP +) Calcd for $\mathrm{C}_{34} \mathrm{H}_{50} \mathrm{O}_{2}{ }^{+}[2(\mathrm{M}-\mathrm{Boc}+\mathrm{H})+$ $\mathrm{H}]^{+}:$489.3727. Found: 489.3726 .

(E)-tert-butyl (4-(2,6,6-trimethylcyclohex-2-en-1-yl)buta-1,3-dien-2-yl) carbonate 1ah<smiles>C=C(/C=C/C1=CC=CCC1(C)C)OCC</smiles>

According to general procedure A, (E)-tert-butyl (4-(2,6,6-trimethylcyclohex-2-en-1-yl)buta-1,3-dien2 -yl) carbonate 1 ah was obtained from $\alpha$-ionone as an orange oil in $72 \%$ yield ( $550 \mathrm{mg}, 1.9 \mathrm{mmol}) .{ }^{1} \mathbf{H}$ NMR $\left(300 \mathrm{MHz}, \mathrm{CDCl}_{3}\right) \delta 5.91(\mathrm{~d}, J=15.6 \mathrm{~Hz}, 1 \mathrm{H}), 5.64(\mathrm{dd}, J=15.4,9.4 \mathrm{~Hz}, 1 \mathrm{H}), 5.34(\mathrm{~d}, J=36.8 \mathrm{~Hz}$, $1 \mathrm{H}), 4.84(\mathrm{~s}, 2 \mathrm{H}), 2.16(\mathrm{~d}, J=9.5 \mathrm{~Hz}, 1 \mathrm{H}), 1.98(\mathrm{~s}, 2 \mathrm{H}), 1.55(\mathrm{~s}, 3 \mathrm{H}), 1.49(\mathrm{~s}, 9 \mathrm{H}), 1.42-1.32(\mathrm{~m}, 1 \mathrm{H}), 1.23$ - $1.09(\mathrm{~m}, 1 \mathrm{H}), 0.88(\mathrm{~s}, 3 \mathrm{H}), 0.80(\mathrm{~s}, 3 \mathrm{H}) \mathrm{ppm} .{ }^{13} \mathrm{C}$ NMR $\left(75 \mathrm{MHz}, \mathrm{CDCl}_{3}\right) \delta 152.2,151.4,133.8,133.6$, 125.2, 121.5, $103.3\left(\mathrm{~s}, \mathrm{C}=\mathrm{CH}_{2}\right), 82.9\left(\mathrm{~s}, 1 \mathrm{C}, \mathrm{O}-\mathrm{C}-\left(\mathrm{CH}_{3}\right)_{3}\right), 54.1,32.6,31.7,27.7\left(\mathrm{~s}, 3 \mathrm{C}, \mathrm{C}-\left(\mathrm{CH}_{3}\right)_{3}\right), 27.5$, 27.1, 23.1, 23.0 ppm. HRMS (ASAP +) Calcd for $\mathrm{C}_{13} \mathrm{H}_{21} \mathrm{O}^{+}[\mathrm{M}-\mathrm{Boc}+\mathrm{H}]^{+}:$193.1587. Found: 193.1586.

tert-butyl

(1-((3S,10R,13S,17S)-3-methoxy-10,13-dimethyl-tetradecahydro-1Hcyclopenta[a]phenanthren-17-yl)vinyl) carbonate 1ai<smiles>C=C(OC(=O)C1CCC2C3CC=C4CC(OC)CCC4(C)C3CCC12C(=O)OCc1ccccc1)C(=O)O</smiles>

Pregnenolone methyl ether was synthesised according to a literature procedure. ${ }^{[9]}$ Spectral data matched with those reported in the literature.

According to general procedure $C$, tert-butyl (1-((3S,10R,13S,17S)-3-methoxy-10,13-dimethyltetradecahydro-1H-cyclopenta[a]phenanthren-17-yl)vinyl) carbonate 1 ai was obtained from pregnenolone methyl ether as a white solid in $57 \%$ yield $(1.23 \mathrm{~g}, 2.85 \mathrm{mmol})$ after purification by column chromatography on silica gel (hexane/EtOAc 9/1). ${ }^{1} \mathrm{H} \mathrm{NMR}\left(500 \mathrm{MHz}, \mathrm{CDCl}_{3}\right) \delta 5.34(\mathrm{dt}, J=5.4$, $2.1 \mathrm{~Hz}, 1 \mathrm{H}), 4.85(\mathrm{dd}, J=1.9,1.0 \mathrm{~Hz}, 1 \mathrm{H}), 4.74(\mathrm{t}, J=1.7 \mathrm{~Hz}, 1 \mathrm{H}), 3.34(\mathrm{~s}, 3 \mathrm{H}), 3.04(\mathrm{tt}, J=11.4,4.6 \mathrm{~Hz}$, $1 \mathrm{H}), 2.43-2.31(\mathrm{~m}, 2 \mathrm{H}), 2.19-2.10(\mathrm{~m}, 1 \mathrm{H}), 2.02-1.94(\mathrm{~m}, 2 \mathrm{H}), 1.93-1.81(\mathrm{~m}, 2 \mathrm{H}), 1.74-1.64(\mathrm{~m}$, 2H), $1.58-1.50(\mathrm{~m}, 2 \mathrm{H}), 1.49(\mathrm{~s}, 9 \mathrm{H}), 1.45-1.35(\mathrm{~m}, 2 \mathrm{H}), 1.25$ (ddd, $J=18.7,9.0,4.6 \mathrm{~Hz}, 2 \mathrm{H}), 1.21-$ $1.14(\mathrm{~m}, 2 \mathrm{H}), 1.14-1.02(\mathrm{~m}, 2 \mathrm{H}), 0.99(\mathrm{~s}, 3 \mathrm{H}), 0.65(\mathrm{~s}, 3 \mathrm{H}) .{ }^{13} \mathrm{C}$ NMR $\left(126 \mathrm{MHz}, \mathrm{CDCl}_{3}\right) \delta$ 157.6, 151.8, 141.1, 121.4, $101.8\left(\mathrm{C}=\mathrm{CH}_{2}\right), 82.6\left(\mathrm{O}-\mathrm{C}-\left(\mathrm{CH}_{3}\right)_{3}\right), 80.4,56.1,55.7,53.3,50.3,43.1,38.8,38.0,37.3,37.1$, 32.3, 31.9, 28.1, $27.9\left(\mathrm{C}-\left(\mathrm{CH}_{3}\right)_{3}\right), 24.9,24.3,21.1,19.5,12.7$ ppm. HRMS (ESI+) Calcd. for $\mathrm{C}_{27} \mathrm{H}_{42} \mathrm{O}_{4} \mathrm{Na}^{+}$ $[\mathrm{M}+\mathrm{Na}]^{+}:$453.2975. Found: 453.2980 . 
tert-butyl ((10R,13S,17S)-17-methoxy-10,13-dimethyl-dodecahydro-1H-cyclopenta[a]phenanthren3-yl) carbonate 1aj<smiles>COC1=CC2=CCC3C(CCC4(C)C(OC(C)(C)C)CCC34)C2CC1</smiles>

$17 \beta$-methoxy-4-androsten-3-one was synthesised according to a literature procedure. ${ }^{[10]}$ Spectral data matched with those reported in the literature.

According to general procedure $A$, tert-butyl ((10R,13S,17S)-17-methoxy-10,13-dimethyldodecahydro-1H-cyclopenta[a]phenanthren-3-yl) carbonate 1aj was obtained from 17 $\beta$-methoxy-4androsten-3-one as a white solid in $76 \%$ yield $\left.(800 \mathrm{mg}, 2.0 \mathrm{mmol}) .{ }^{1} \mathrm{H} \mathrm{NMR} \mathrm{(300} \mathrm{MHz}, \mathrm{CDCl}_{3}\right) \delta 5.38$ (s, $1 \mathrm{H}), 5.25(\mathrm{~s}, 1 \mathrm{H}), 3.33(\mathrm{~s}, 3 \mathrm{H}), 3.20(\mathrm{t}, J=8.3,1 \mathrm{H}), 2.23(\mathrm{t}, J=7.7 \mathrm{~Hz}, 4 \mathrm{H}), 2.05-1.85(\mathrm{~m}, 2 \mathrm{H}), 1.74-$ $1.55(\mathrm{~m}, 2 \mathrm{H}), 1.49(\mathrm{~d}, 9 \mathrm{H}), 1.46-1.35(\mathrm{~m}, 4 \mathrm{H}), 1.33-1.07(\mathrm{~m}, 3 \mathrm{H}), 1.00-0.90(\mathrm{~m}, 5 \mathrm{H}), 0.77(\mathrm{~s}, 3 \mathrm{H})$ ppm. ${ }^{13} \mathrm{C}$ NMR $\left(75 \mathrm{MHz}, \mathrm{CDCl}_{3}\right) \delta 152.0,149.5,145.1,115.6,107.2,90.8,82.9$ (s, 1C, O-C- $\left.\left(\mathrm{CH}_{3}\right)_{3}\right), 58.0$, 54.7, 50.9, 43.0, 38.1, 38.0, 37.2, 36.2, 31.2, 30.8, 27.9, 27.8 (s, 3C, $\left.\mathrm{C}-\left(\mathrm{CH}_{3}\right)_{3}\right), 23.6,21.4,17.5,11.6 \mathrm{ppm}$. HRMS (ASAP +) Calcd for $\mathrm{C}_{25} \mathrm{H}_{38} \mathrm{O}_{4} \mathrm{Na}^{+}[\mathrm{M}+\mathrm{Na}]^{+}:$425.2662. Found: 425.2677 .

\section{General procedures for the trifluoromethoxylation of enol carbonates}

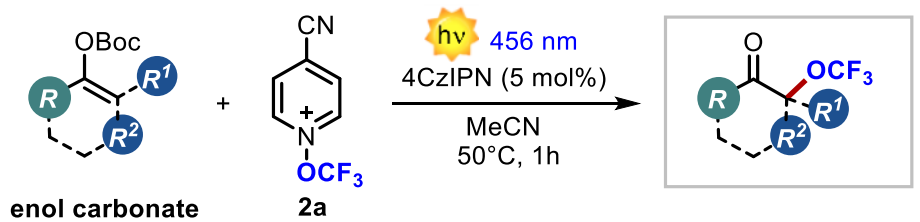

\section{General procedure D, for trifluoromethoxylation of enol carbonates}

A sealable tube surrounded by blue LEDs with a watertight protection, purged with argon and equipped with a cap was charged with 4-cyano-1-(trifluoromethoxy)pyridin-1-ium bis((trifluoromethyl)sulfonyl)amide $2 \mathrm{a}(46.9 \mathrm{mg}, 0.1 \mathrm{mmol}, 1$ equiv.), the desired enol carbonate $(0.5$ mmol, 5 equiv.) and 4CzIPN ( $3.9 \mathrm{mg}, 0.005 \mathrm{mmol}, 0.05$ equiv.). Dry acetonitrile $(10 \mathrm{~mL}, 0.01 \mathrm{M}$ ) was finally added and the reaction medium purged with argon again. The resulting mixture in the sealable tube was placed in a water bath and allowed to stir vigorously for $1 \mathrm{~h}$ at $50^{\circ} \mathrm{C}$ under irradiation of the blue LEDs. The crude was then concentrated under vacuum. Alpha-trifluoromethoxylated ketones were isolated by preparative thin layer chromatography (PTLC).

\section{General procedure E, for trifluoromethoxylation of enol carbonates}

Two 8-mL vials surrounded by blue LEDs with a watertight protection, equipped with a screwcap and a septum were purged with argon and each one charged with 4-cyano-1-(trifluoromethoxy)pyridin-1ium bis((trifluoromethyl)sulfonyl)amide $2 \mathrm{a}(23.5 \mathrm{mg}, 0.05 \mathrm{mmol}, 1$ equiv.), the desired enol carbonate ( $0.25 \mathrm{mmol}, 5$ equiv.) and 4CzIPN (1.9 mg, $0.0025 \mathrm{mmol}, 0.05$ equiv.). Dry acetonitrile $(5 \mathrm{~mL}, 0.01 \mathrm{M})$ was finally added and the reaction medium purged with argon again. The resulting mixtures in the vials were placed in a water bath and allowed to stir vigorously for $1 \mathrm{~h}$ at $50^{\circ} \mathrm{C}$ under irradiation of the blue LEDs. The crude reaction mixture was then transferred to a round-bottom flask, $1 \mathrm{~mL}$ of $6 \mathrm{M} \mathrm{HCl}$ was added and the reaction mixture stirred until complete hydrolysis of the unreacted starting material (controlled by $\mathrm{TLC}$ analysis). The crude reaction mixture was then quenched with sat. $\mathrm{NaHCO}_{3}$ until gas evolution stopped, diluted with DCM $(30 \mathrm{~mL})$ and washed with brine $(3 \times 10 \mathrm{~mL})$. The organic phase was 
dried over $\mathrm{MgSO}_{4}$ and concentrated under vacuum. Corresponding alpha-trifluoromethoxylated ketones were isolated by column chromatography on silica gel.

\section{1-(4-fluorophenyl)-2-(trifluoromethoxy)ethanone 3}<smiles>O=C(COC(F)(F)F)c1ccc(F)cc1</smiles>

According to general procedure D, 1-(4-fluorophenyl)-2-(trifluoromethoxy)ethanone $\mathbf{3}$ was obtained from the corresponding enol carbonate $\mathbf{1 b}$; the yield of the desired product was determined by comparing the integration of the ${ }^{19} \mathrm{~F}$ NMR resonance of the product with that of bis((trifluoromethyl)sulfonyl)amide. ${ }^{19} \mathrm{~F}$ NMR yield of $50 \%$. The product was isolated by PTLC (Pentane/Et ${ }_{2} \mathrm{O}\left(85 / 15,2\right.$ elutions)) in $32 \%$ yield $(7.0 \mathrm{mg}, 0.031 \mathrm{mmol}) .{ }^{1} \mathrm{H}$ NMR (300 MHz, $\left.\mathrm{CDCl}_{3}\right) \delta 8.02$ $-7.89(\mathrm{~m}, 1 \mathrm{H}), 7.19(\mathrm{t}, J=8.4 \mathrm{~Hz}, 1 \mathrm{H}), 5.13(\mathrm{~s}, 1 \mathrm{H}) \mathrm{ppm} .{ }^{13} \mathrm{C} \mathrm{NMR}\left(75 \mathrm{MHz}, \mathrm{CDCl}_{3}\right) \delta 188.9(\mathrm{C}=0), 166.5$ (d, J = 257.2 Hz, C1), 130.9 (d, J = 9.5 Hz, C2), 130.4 (d, J = 3.1 Hz, C4), 121.8 (q, J = 256.6 Hz, ROCF 3 ), 116.48 (d, $J=22.2 \mathrm{~Hz}, \mathrm{C} 3), 68.3\left(\mathrm{q}, J=2.9 \mathrm{~Hz}, \mathrm{RCH}_{2}-\mathrm{OCF}_{3}\right)$ ppm. ${ }^{19} \mathrm{~F} \mathrm{NMR}\left(\mathbf{1 8 8 ~ M H z}, \mathrm{CDCl}_{3}\right) \delta-61.56$ (s, 3F), -102.88 (m, 1F) ppm. HRMS (ASAP +) Calcd for $\mathrm{C}_{9} \mathrm{H}_{7} \mathrm{~F}_{4} \mathrm{O}_{2}{ }^{+}[\mathrm{M}+\mathrm{H}]^{+}: 223.0377$. Found: 223.0386.

\section{1-(o-tolyl)-2-(trifluoromethoxy)ethanone 7}<smiles>CCOC(=O)COc1ccccc1C(=O)OCc1ccccc1</smiles>

According to general procedure $\mathrm{D}, 1$-(o-tolyl)-2-(trifluoromethoxy)ethanone $\mathbf{7}$ was obtained from the corresponding enol carbonate $\mathbf{1 d}$; the yield of the desired product was determined by comparing the integration of the ${ }^{19} \mathrm{~F} N M R$ resonance of the product with that of bis((trifluoromethyl)sulfonyl)amide. ${ }^{19} \mathrm{~F} \mathrm{NMR}$ yield of $41 \%$. The product was isolated by PTLC (Pentane/Et $2 \mathrm{O}(95 / 5,2$ elutions)) in $36 \%$ yield (8.5 mg, $0.036 \mathrm{mmol}) .{ }^{1} \mathrm{H}$ NMR (300 MHz, $\left.\mathrm{CDCl}_{3}\right) \delta 7.54(\mathrm{~d}, J=7.7 \mathrm{~Hz}, 1 \mathrm{H}), 7.46(\mathrm{t}, J=7.6 \mathrm{~Hz}, 1 \mathrm{H}), 7.31$ $(\mathrm{d}, J=8.0 \mathrm{~Hz}, 2 \mathrm{H}), 5.04(\mathrm{~s}, 2 \mathrm{H}), 2.54(\mathrm{~s}, 3 \mathrm{H}) \mathrm{ppm} .{ }^{13} \mathrm{C} \mathrm{NMR}\left(75 \mathrm{MHz}, \mathrm{CDCl}_{3}\right) \delta 193.6,139.7,133.8,132.8$, 132.7, 128.3, 126.0, 121.8 (q, $J=256 \mathrm{~Hz}, \mathrm{ROCF}_{3}$ ), 69.3 (q, $J=2.9 \mathrm{~Hz}, \mathrm{RCH}_{2}-\mathrm{OCF}_{3}$ ), 21.4 ppm. ${ }^{19} \mathrm{~F} \mathrm{NMR}$ (188 MHz, $\mathrm{CDCl}_{3}$ ) $\delta-61.40$ ppm. HRMS (ASAP +) Calcd for $\mathrm{C}_{10} \mathrm{H}_{10} \mathrm{~F}_{3} \mathrm{O}_{2}{ }^{+}[\mathrm{M}+\mathrm{H}]^{+}:$219.0627. Found: 219.0634 .

\section{1-(m-tolyl)-2-(trifluoromethoxy)ethanone 8}<smiles>CCOC(=O)COC(F)(F)F</smiles>

According to general procedure D, 1-( $m$-tolyl)-2-(trifluoromethoxy)ethanone $\mathbf{8}$ was obtained from the corresponding enol carbonate $\mathbf{1} \mathbf{e}$; the yield of the desired product was determined by comparing the integration of the ${ }^{19} \mathrm{~F} N M R$ resonance of the product with that of bis((trifluoromethyl)sulfonyl)amide. ${ }^{19} \mathrm{~F}$ NMR yield of $43 \%$. The product was isolated by PTLC (Pentane/Et ${ }_{2} \mathrm{O}(95 / 5,2$ elutions)) in $28 \%$ yield (6.1 mg, $0.028 \mathrm{mmol}) .{ }^{1} \mathrm{H}$ NMR $\left(300 \mathrm{MHz}, \mathrm{CDCl}_{3}\right) \delta 7.74-7.65(\mathrm{~m}, 2 \mathrm{H}), 7.49-7.34(\mathrm{~m}, 2 \mathrm{H}), 5.17(\mathrm{~s}$, $2 \mathrm{H}), 2.43(\mathrm{~s}, 3 \mathrm{H}) \mathrm{ppm} .{ }^{13} \mathrm{C} \mathrm{NMR}\left(75 \mathrm{MHz}, \mathrm{CDCl}_{3}\right) \delta 190.4,139.2,135.2,133.9,129.0,128.5,125.2,121.8$ (q, $\left.J=256.5 \mathrm{~Hz}, \mathrm{ROCF}_{3}\right), 68.4\left(\mathrm{q}, J=2.9 \mathrm{~Hz}, \mathrm{RCH}_{2}-\mathrm{OCF}_{3}\right), 21.5 \mathrm{ppm} .{ }^{19} \mathrm{~F} \mathrm{NMR}\left(188 \mathrm{MHz}, \mathrm{CDCl}_{3}\right) \delta-61.41$ ppm. HRMS (ASAP +) Calcd for $\mathrm{C}_{10} \mathrm{H}_{10} \mathrm{~F}_{3} \mathrm{O}_{2}{ }^{+}[\mathrm{M}+\mathrm{H}]^{+}: 219.0627$. Found: 219.0625 . 
<smiles>CCCCC(C)(C)OCC(=O)c1ccc(C)cc1</smiles>

According to general procedure $D, 1$-( $p$-tolyl)-2-(trifluoromethoxy)ethanone 9 was obtained from the corresponding enol carbonate 1f; the yield of the desired product was determined by comparing the integration of the ${ }^{19} \mathrm{~F}$ NMR resonance of the product with that of bis((trifluoromethyl)sulfonyl)amide. ${ }^{19} \mathrm{~F}$ NMR yield of $46 \%$. The product was isolated by PTLC (Pentane/Et ${ }_{2} \mathrm{O}(95 / 5,2$ elutions)) in $41 \%$ yield (9.0 mg, $0.041 \mathrm{mmol}) .{ }^{1} \mathrm{H}$ NMR (300 MHz, $\left.\mathrm{CDCl}_{3}\right) \delta 7.80(\mathrm{~d}, J=7.8 \mathrm{~Hz}, 2 \mathrm{H}), 7.30(\mathrm{~d}, J=7.8 \mathrm{~Hz}, 2 \mathrm{H}), 5.15$ $(\mathrm{s}, 2 \mathrm{H}), 2.43(\mathrm{~s}, 3 \mathrm{H}) \mathrm{ppm} .{ }^{13} \mathrm{C} \mathrm{NMR}\left(75 \mathrm{MHz}, \mathrm{CDCl}_{3}\right) \delta 189.9,145.6,131.4,129.8,128.2,121.8$ (q, $J=257$ $\left.\mathrm{Hz}, \mathrm{ROCF}_{3}\right) .68 .4\left(\mathrm{q}, J=2.8 \mathrm{~Hz}, \mathrm{RCH}_{2}-\mathrm{OCF}_{3}\right), 21.9 \mathrm{ppm} .{ }^{19} \mathrm{~F} \mathrm{NMR}\left(188 \mathrm{MHz}, \mathrm{CDCl}_{3}\right) \delta-61.46 \mathrm{ppm} . \mathrm{HRMS}$ (ASAP +) Calcd for $\mathrm{C}_{10} \mathrm{H}_{10} \mathrm{~F}_{3} \mathrm{O}_{2}^{+}[\mathrm{M}+\mathrm{H}]^{+}:$219.0627. Found: 219.0627 .

\section{1-(3-methoxyphenyl)-2-(trifluoromethoxy)ethan-1-one 10}<smiles>CCOC(=O)c1cccc(OC)c1</smiles>

According to general procedure E, 1-(3-methoxyphenyl)-2-(trifluoromethoxy)ethan-1-one $\mathbf{1 0}$ was obtained from the corresponding enol carbonate $1 \mathrm{~g}$. The yield of the desired product was determined by comparing the integration of the ${ }^{19} \mathrm{~F} N M R$ resonance of the product with that of bis((trifluoromethyl)sulfonyl)amide. ${ }^{19} \mathrm{~F}$ NMR yield of $12 \% .{ }^{19} \mathrm{~F} \mathrm{NMR}\left(188 \mathrm{MHz}, \mathrm{CDCl}_{\mathbf{3}}\right) \delta-61.05 \mathrm{ppm}$.

\section{1-(4-methoxyphenyl)-2-(trifluoromethoxy)ethan-1-one 11}<smiles>COCC(=O)c1ccc(OC)cc1</smiles>

According to general procedure E, 1-(4-methoxyphenyl)-2-(trifluoromethoxy)ethan-1-one $\mathbf{1 1}$ was obtained from the corresponding enol carbonate $\mathbf{1 h}$. The yield of the desired product was determined by comparing the integration of the ${ }^{19} \mathrm{~F} N M R$ resonance of the product with that of bis((trifluoromethyl)sulfonyl)amide. ${ }^{19} \mathrm{~F} \mathrm{NMR}$ yield of $\left.13 \% .{ }^{19} \mathrm{~F} \mathrm{NMR} \mathrm{(188} \mathbf{M H z}, \mathrm{CDCl}_{3}\right) \delta-60.98 \mathrm{ppm}$.

\section{4-(2-(trifluoromethoxy)acetyl)benzonitrile 12}<smiles>N#[N+]c1ccc(C(=O)COC(F)(F)F)cc1</smiles>

12

According to general procedure E, 4-(2-(trifluoromethoxy)acetyl)benzonitrile $\mathbf{1 2}$ was obtained from the corresponding enol carbonate $\mathbf{1} \mathbf{i}$; the yield of the desired product was determined by comparing the integration of the ${ }^{19} \mathrm{~F}$ NMR resonance of the product with that of bis((trifluoromethyl)sulfonyl)amide. ${ }^{19} \mathrm{~F}$ NMR yield of $42 \%$. The product was isolated by flash column chromatography on silica gel hexane/EtOAc $8 / 2)$ in $27 \%$ yield $(6.2 \mathrm{mg}, 0.027 \mathrm{mmol}) .{ }^{1} \mathrm{H} \mathrm{NMR}(300 \mathrm{MHz}$, $\left.\mathrm{CDCl}_{3}\right) \delta 8.01(\mathrm{~d}, J=7.8 \mathrm{~Hz}, 2 \mathrm{H}), 7.82(\mathrm{~d}, J=8.0 \mathrm{~Hz}, 2 \mathrm{H}), 5.15(\mathrm{~s}, 2 \mathrm{H})$ ppm. ${ }^{13} \mathrm{C} \mathrm{NMR}\left(75 \mathrm{MHz}, \mathrm{CDCl}_{3}\right) \delta$ 189.6, 136.8, 133.0, 128.7, 121.7 (q, $J=257.4 \mathrm{~Hz}, \mathrm{ROCF}_{3}$ ), 117.8, 117.6, 68.5 (q, J = 3.0 Hz, RCH $\mathrm{RCCF}_{3}$ ) ppm. ${ }^{19} \mathrm{~F}$ NMR (188 MHz, CDCl $\left.{ }_{3}\right) \delta-61.63$ ppm. HRMS (ASAP +) Calcd for $\mathrm{C}_{10} \mathrm{H}_{7} \mathrm{~F}_{3} \mathrm{NO}_{2}{ }^{+}[\mathrm{M}+\mathrm{H}]^{+}$: 230.0423. Found: 230.0422. 
<smiles>O=C(COC(F)(F)F)c1cccc(C(F)(F)F)c1</smiles>

13

According to general procedure D, 2-(trifluoromethoxy)-1-(3-(trifluoromethyl)phenyl)ethanone $\mathbf{1 3}$ was obtained from the corresponding enol carbonate $\mathbf{1 j}$; the yield of the desired product was determined by comparing the integration of the ${ }^{19} \mathrm{~F} N M R$ resonance of the product with that of bis((trifluoromethyl)sulfonyl)amide. ${ }^{19} \mathrm{~F}$ NMR yield of $38 \%$. PTLC conditions: 2 successive purifications with Pentane/ $\mathrm{Et}_{2} \mathrm{O}(9 / 1)$ and then $100 \% \mathrm{DCM}$. The product was isolated in $15 \%$ yield $(4.0 \mathrm{mg}, 0.015$ mmol). ${ }^{1} \mathrm{H}$ NMR $\left(300 \mathrm{MHz}, \mathrm{CDCl}_{3}\right) \delta 8.16(\mathrm{~s}, 1 \mathrm{H}), 8.10(\mathrm{~d}, J=7.8 \mathrm{~Hz}, 1 \mathrm{H}), 7.90(\mathrm{~d}, J=7.7 \mathrm{~Hz}, 1 \mathrm{H}), 7.68$ $(\mathrm{t}, J=7.8 \mathrm{~Hz}, 1 \mathrm{H}), 5.18(\mathrm{~s}, 2 \mathrm{H}) \mathrm{ppm} .{ }^{13} \mathrm{C} \mathrm{NMR}\left(75 \mathrm{MHz}, \mathrm{CDCl}_{3}\right) \delta 189.4,134.4,131.9(\mathrm{~d}, J=33 \mathrm{~Hz}), 131.3$, 130.8 (q, $J=3.5 \mathrm{~Hz}$ ), 129.9, 125.1 (q, $J=3.9 \mathrm{~Hz}), 123.6(\mathrm{~d}, J=272 \mathrm{~Hz}) 121.7$ (q, J = 256 Hz, ROCF 3 ), 68.4 $\left(q, J=3.0 \mathrm{~Hz}, \mathrm{RCH}_{2}-\mathrm{OCF}_{3}\right) \mathrm{ppm} .{ }^{19} \mathrm{~F}$ NMR $\left(188 \mathrm{MHz} \mathrm{CDCl}_{3}\right) \delta-61.59\left(\mathrm{OCF}_{3}\right),-63.44\left(\mathrm{CF}_{3}\right)$ ppm. HRMS $\left(\right.$ ASAP +) Calcd for $\mathrm{C}_{10} \mathrm{H}_{7} \mathrm{~F}_{6} \mathrm{O}_{2}{ }^{+}[\mathrm{M}+\mathrm{H}]^{+}:$273.0345. Found: 273.0341 .

\section{1-(3,5-bis(trifluoromethyl)phenyl)-2-(trifluoromethoxy)ethan-1-one 14}<smiles>CCOC(=O)c1cc(C(F)(F)F)cc(C(F)(F)F)c1</smiles>

According to general procedure E, 1-(3,5-bis(trifluoromethyl)phenyl)-2-(trifluoromethoxy)ethan-1-one 14 was obtained from the corresponding enol carbonate $\mathbf{1 k}$. The yield of the desired product was determined by comparing the integration of the ${ }^{19} \mathrm{~F} N M R$ resonance of the product with that of bis((trifluoromethyl)sulfonyl)amide. ${ }^{19} \mathrm{~F}$ NMR yield of $\left.13 \% .{ }^{19} \mathrm{~F} \mathrm{NMR} \mathrm{(188} \mathrm{MHz,} \mathrm{CDCl}_{3}\right) \delta-61.23(\mathrm{~s}, 3 \mathrm{~F})$, $-63.36(s, 6 F)$ ppm.

\section{1-(2-bromophenyl)-2-(trifluoromethoxy)ethan-1-one 15}<smiles>O=C(COC(F)(F)F)c1ccccc1Br</smiles>

15

According to general procedure E, 1-(2-bromophenyl)-2-(trifluoromethoxy)ethan-1-one $\mathbf{1 5}$ was obtained from the corresponding enol carbonate 1 . The yield of the desired product was determined by comparing the integration of the ${ }^{19} \mathrm{~F} N M R$ resonance of the product with that of bis((trifluoromethyl)sulfonyl)amide. ${ }^{19} \mathrm{~F}$ NMR yield of $36 \%$. The product was isolated by flash column chromatography on silica gel (hexane/EtOAc 8/2) in $18 \%$ yield $(5.1 \mathrm{mg}, 0.018 \mathrm{mmol}$ ). Particular care was taken during solvent evaporation steps due to the observed volatility of the product. ${ }^{1} \mathbf{H}$ NMR (500 $\left.\mathbf{M H z}, \mathrm{CDCl}_{3}\right) \delta 7.66(\mathrm{~d}, J=7.8 \mathrm{~Hz}, 1 \mathrm{H}), 7.51-7.33(\mathrm{~m}, 3 \mathrm{H}), 5.05(\mathrm{~s}, 2 \mathrm{H})$ ppm. ${ }^{13} \mathrm{C}$ NMR $\left(\mathbf{1 2 6} \mathbf{~ M H z}, \mathbf{C D C l}_{3}\right)$ $\delta$ 194.8, 137.8, 134.0, 133.1, 129.6, 127.8, 121.6 (q, $\left.J=256.8 \mathrm{~Hz}, \mathrm{ROCF}_{3}\right), 119.4,69.8$ (q, $J=3.0 \mathrm{~Hz}$, $\mathrm{RCH}_{2}-\mathrm{OCF}_{3}$ ) ppm. ${ }^{19} \mathrm{~F}$ NMR (188 MHz, $\mathrm{CDCl}_{3}$ ) $\delta-61.53$ ppm. HRMS (ASAP +) Calcd for $\mathrm{C}_{8} \mathrm{H}_{5} \mathrm{BrF}_{3} \mathrm{O}_{2}{ }^{+}[\mathrm{M}+$ $H]^{+}:$282.9576. Found: 282.9580 . 
<smiles>O=C(COc1ccccc1)c1cccc(Br)c1</smiles>

16

According to general procedure E, 1-(3-bromophenyl)-2-(trifluoromethoxy)ethan-1-one $\mathbf{1 6}$ was obtained from the corresponding enol carbonate $1 \mathrm{~m}$. The yield of the desired product was determined by comparing the integration of the ${ }^{19} \mathrm{~F}$ NMR resonance of the product with that of bis((trifluoromethyl)sulfonyl)amide. ${ }^{19} \mathrm{~F}$ NMR yield of $38 \%$. The product was isolated by flash column chromatography on silica gel (hexane/DCM 7/3) in $27 \%$ yield $(7.6 \mathrm{mg}, 0.027 \mathrm{mmol}) .{ }^{1} \mathbf{H ~ N M R ~ ( 5 0 0 ~} \mathbf{~ M H z}$, $\left.\mathrm{CDCl}_{3}\right) \delta 8.04(\mathrm{t}, J=1.8 \mathrm{~Hz}, 1 \mathrm{H}), 7.83(\mathrm{dt}, J=7.8,1.3 \mathrm{~Hz}, 1 \mathrm{H}), 7.77$ (ddd, $\left.J=8.0,2.0,1.0 \mathrm{~Hz}, 1 \mathrm{H}\right), 7.40(\mathrm{t}$, $J=7.9 \mathrm{~Hz}, 1 \mathrm{H}), 5.13(\mathrm{~s}, 2 \mathrm{H}) \mathrm{ppm} .{ }^{13} \mathrm{C} \mathrm{NMR}\left(126 \mathrm{MHz}, \mathrm{CDCl}_{3}\right) \delta 189.2,137.4,135.6,131.2,130.7,126.6$, 123.6, 121.8 (q, $\left.J=256.8 \mathrm{~Hz}, \mathrm{ROCF}_{3}\right), 68.3$ (q, $\left.J=3.0 \mathrm{~Hz}, \mathrm{RCH}_{2}-\mathrm{OCF}_{3}\right)$ ppm. ${ }^{19} \mathrm{~F}$ NMR $\left(188 \mathrm{MHz}, \mathrm{CDCl}_{3}\right) \delta$ -61.59 ppm. HRMS (ASAP +) Calcd for $\mathrm{C}_{8} \mathrm{H}_{5} \mathrm{BrF}_{3} \mathrm{O}_{2}{ }^{+}[\mathrm{M}+\mathrm{H}]^{+}:$: 282.9576. Found: 282.9576.

\section{1-(4-bromophenyl)-2-(trifluoromethoxy)ethan-1-one 17}<smiles>CCOCC(=O)c1ccc(Br)cc1</smiles>

17

According to general procedure E, 1-(4-bromophenyl)-2-(trifluoromethoxy)ethan-1-one $\mathbf{1 7}$ was obtained from the corresponding enol carbonate $1 \mathrm{n}$. The yield of the desired product was determined by comparing the integration of the ${ }^{19} \mathrm{~F}$ NMR resonance of the product with that of bis((trifluoromethyl)sulfonyl)amide. ${ }^{19} \mathrm{~F}$ NMR yield of $46 \%$. The product was isolated by flash column chromatography on silica gel (hexane/EtOAc $95 / 5)$ in $34 \%$ yield $(9.6 \mathrm{mg}, 0.034 \mathrm{mmol}) .{ }^{1} \mathbf{H}$ NMR (500 $\mathrm{MHz}^{\mathrm{CDCl}} \mathrm{CD}_{3} \delta 7.78(\mathrm{~d}, J=8.6 \mathrm{~Hz}, 2 \mathrm{H}), 7.66(\mathrm{~d}, J=8.6 \mathrm{~Hz}, 2 \mathrm{H}), 5.12(\mathrm{~s}, 2 \mathrm{H}) \mathrm{ppm} .{ }^{13} \mathrm{C}$ NMR $(126 \mathrm{MHz}$, $\left.\mathrm{CDCl}_{3}\right) \delta 189.6,132.6,132.6,129.8,129.6, \delta 121.8$ (q, $\left.J=256.8 \mathrm{~Hz}, \mathrm{ROCF}_{3}\right), 68.3\left(\mathrm{q}, J=3.0 \mathrm{~Hz}, \mathrm{RCH}_{2^{-}}\right.$ $\mathrm{OCF}_{3}$ ) ppm. ${ }^{19} \mathrm{~F}$ NMR (188 MHz, $\left.\mathrm{CDCl}_{3}\right) \delta-61.56$ ppm. HRMS (ASAP +) Calcd for $\mathrm{C}_{8} \mathrm{H}_{5} \mathrm{BrF}_{3} \mathrm{O}_{2}{ }^{+}[\mathrm{M}+\mathrm{H}]^{+}$: 282.9576. Found: 282.9572.

\section{1-(2,5-dichlorophenyl)-2-(trifluoromethoxy)ethan-1-one 18}<smiles>CCOCC(=O)c1cc(Cl)ccc1Cl</smiles>

18

According to general procedure E, 1-(2,5-dichlorophenyl)-2-(trifluoromethoxy)ethan-1-one $\mathbf{1 8}$ was obtained from the corresponding enol carbonate 10 . The yield of the desired product was determined by comparing the integration of the ${ }^{19} \mathrm{~F} N M R$ resonance of the product with that of bis((trifluoromethyl)sulfonyl)amide. ${ }^{19} \mathrm{~F}$ NMR yield of $13 \% .{ }^{19} \mathbf{F ~ N M R ~}\left(188 \mathbf{M H z}, \mathrm{CDCl}_{3}\right) \delta-61.20 \mathrm{ppm}$.

\section{1-(4-acetylphenyl)-2-(trifluoromethoxy)ethan-1-one 19}<smiles>CC(=O)OCC(=O)c1ccc(C(F)(F)F)cc1</smiles>

According to general procedure E, 1-(4-acetylphenyl)-2-(trifluoromethoxy)ethan-1-one 19 was obtained from the corresponding enol carbonate $1 \mathbf{p}$. The yield of the desired product was determined 
by comparing the integration of the ${ }^{19} \mathrm{~F}$ NMR resonance of the product with that of bis((trifluoromethyl)sulfonyl)amide. ${ }^{19} \mathrm{~F} \mathrm{NMR}$ yield of $\left.44 \% .{ }^{19} \mathrm{~F} \mathrm{NMR} \mathrm{(188} \mathbf{M H z}, \mathrm{CDCl}_{3}\right) \delta-61.32 \mathrm{ppm}$.

1-phenyl-2-(trifluoromethoxy)propan-1-one 20<smiles>CCOC(=O)C(=O)c1ccccc1</smiles>

According to general procedure E, 1-phenyl-2-(trifluoromethoxy)propan-1-one $\mathbf{2 0}$ was obtained from the corresponding enol carbonate 1q. The yield of the desired product was determined by comparing the integration of the ${ }^{19} \mathrm{~F}$ NMR resonance of the product with that of bis((trifluoromethyl)sulfonyl)amide. ${ }^{19} \mathrm{~F}$ NMR yield of $34 \%$. The product was isolated by flash column chromatography on silica gel (hexane/DCM 8/2) in $32 \%$ yield $(7.0 \mathrm{mg}, 0.032 \mathrm{mmol}) .{ }^{1} \mathbf{H}$ NMR (500 MHz, $\left.\mathrm{CDCl}_{3}\right) \delta 7.97(\mathrm{~d}, J=8.0 \mathrm{~Hz}, 2 \mathrm{H}), 7.63(\mathrm{t}, J=7.4 \mathrm{~Hz}, 1 \mathrm{H}), 7.51(\mathrm{t}, J=7.7 \mathrm{~Hz}, 2 \mathrm{H}), 5.48(\mathrm{q}, J=6.9 \mathrm{~Hz}, 1 \mathrm{H})$, $1.66(\mathrm{~d}, J=6.9 \mathrm{~Hz}, 3 \mathrm{H}) \mathrm{ppm} .{ }^{13} \mathrm{C}$ NMR $\left(126 \mathrm{MHz}, \mathrm{CDCl}_{3}\right) \delta 195.1,134.2,133.7,129.1,128.9,121.6$ (q, $J$ $\left.=256.2 \mathrm{~Hz}, \mathrm{ROCF}_{3}\right), \delta 75.5$ (q, $\left.J=2.5 \mathrm{~Hz}, \mathrm{RCH}_{2}-\mathrm{OCF}_{3}\right), 18.8 \mathrm{ppm} .{ }^{19} \mathrm{~F} \mathbf{N M R}\left(188 \mathrm{MHz}, \mathrm{CDCl}_{3}\right) \delta-59.29$ ppm. HRMS (ASAP +) Calcd for $\mathrm{C}_{10} \mathrm{H}_{10} \mathrm{~F}_{3} \mathrm{O}_{2}^{+}[\mathrm{M}+\mathrm{H}]^{+}:$219.0627. Found: 219.0629.

\section{1,3-diphenyl-2-(trifluoromethoxy)propan-1-one 21}<smiles>O=C(c1ccccc1)C(F)(Br)OC(F)(F)F</smiles>

21

According to general procedure E, 1,3-diphenyl-2-(trifluoromethoxy)propan-1-one $\mathbf{2 1}$ was obtained from the corresponding enol carbonate $1 r$. The yield of the desired product was determined by comparing the integration of the ${ }^{19} \mathrm{~F}$ NMR resonance of the product with that of bis((trifluoromethyl)sulfonyl)amide. ${ }^{19} \mathrm{~F}$ NMR yield of $31 \%$. The product was isolated by flash column chromatography on silica gel (hexane/DCM 8/2) in $27 \%$ yield $(7.9 \mathrm{mg}, 0.027 \mathrm{mmol}) .{ }^{1} \mathbf{H} \mathbf{~ N M R}(\mathbf{5 0 0} \mathbf{~ M H z}$, $\left.\mathrm{CDCl}_{3}\right) \delta 7.98-7.83(\mathrm{~m}, 2 \mathrm{H}), 7.69-7.55(\mathrm{~m}, 1 \mathrm{H}), 7.55-7.42(\mathrm{~m}, 2 \mathrm{H}), 7.32-7.28(\mathrm{~m}, 2 \mathrm{H}), 7.27-7.24$ $(\mathrm{m}, 1 \mathrm{H}), 7.24-7.20(\mathrm{~m}, 2 \mathrm{H}), 5.50(\mathrm{dd}, J=7.8,5.3 \mathrm{~Hz}, 1 \mathrm{H}), 3.31-3.14(\mathrm{~m}, 2 \mathrm{H}) \mathrm{ppm} .{ }^{13} \mathrm{C}$ NMR $(126 \mathrm{MHz}$, $\mathrm{CDCl}_{3}$ ) $\delta 194.7,135.0,134.2,134.2,129.5,129.1,128.9,127.6,121.4$ (q, J = 256.8 Hz, ROCF $), 79.5$ (q, $\left.J=2.0 \mathrm{~Hz}, \mathrm{RCH}_{2}-\mathrm{OCF}_{3}\right), 39.0 \mathrm{ppm} .{ }^{19} \mathrm{~F} \mathrm{NMR}\left(188 \mathrm{MHz}, \mathrm{CDCl}_{3}\right) \delta-59.46 \mathrm{ppm}$. HRMS (ASAP +) Calcd for $\mathrm{C}_{10} \mathrm{H}_{10} \mathrm{~F}_{3} \mathrm{O}_{2}^{+}[\mathrm{M}+\mathrm{H}]^{+}:$295.0940. Found: 295.0941.

\section{2-(trifluoromethoxy)-2,3-dihydro-1H-inden-1-one 22}

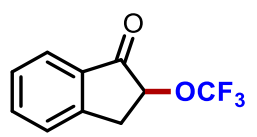

22

According to general procedure D, 2-(trifluoromethoxy)-2,3-dihydro-1H-inden-1-one $\mathbf{2 2}$ was obtained from the corresponding enol carbonate 1s; the yield of the desired product was determined by comparing the integration of the ${ }^{19} \mathrm{~F}$ NMR resonance of the product with that of bis((trifluoromethyl)sulfonyl)amide. ${ }^{19} \mathrm{~F}$ NMR yield of $28 \%$. PTLC conditions: Pentane/Et ${ }_{2} \mathrm{O}(85 / 15,2$ elutions). ${ }^{1} \mathrm{H}$ NMR (300 MHz, CDCl$) \delta 7.82(\mathrm{~d}, J=7.7 \mathrm{~Hz}, 1 \mathrm{H}), 7.68(\mathrm{t}, J=7.1 \mathrm{~Hz}, 1 \mathrm{H}), 7.50-7.40(\mathrm{~m}$, $2 \mathrm{H}), 4.98-4.89(\mathrm{~m}, 1 \mathrm{H}), 3.68(\mathrm{dd}, J=17.1,8.0 \mathrm{~Hz}, 1 \mathrm{H}), 3.26(\mathrm{dd}, J=16.7,4.2 \mathrm{~Hz}, 1 \mathrm{H})$ ppm. ${ }^{13} \mathrm{C}$ NMR $(75$ $\mathrm{MHz}_{2} \mathrm{CDCl}_{3}$ ) $\delta 197.6,149.7,136.6,133.8,128.7,126.8,125.0,122.1$ (q, $J=257 \mathrm{~Hz}, \mathrm{ROCF}_{3}$ ), $33.9 \mathrm{ppm}$. ${ }^{19} \mathrm{~F}$ NMR (188 MHz, CDCl $) \delta$ - 59.73 ppm. HRMS (ASAP +) Calcd for $\mathrm{C}_{20} \mathrm{H}_{15} \mathrm{~F}_{6} \mathrm{O}_{4}{ }^{+}[2 \mathrm{M}+\mathrm{H}]^{+}: 433.0869$. Found: 433.0864 . 
<smiles>COc1ccc2c(c1)C(=O)C(OC(F)(F)F)C2</smiles>

23

According to general procedure D, 6-methoxy-2-(trifluoromethoxy)-2,3-dihydro-1H-inden-1-one 23 was obtained from the corresponding enol carbonate $\mathbf{1 t}$; the yield of the desired product was determined by comparing the integration of the ${ }^{19} \mathrm{~F}$ NMR resonance of the product with that of bis((trifluoromethyl)sulfonyl)amide. ${ }^{19} \mathrm{~F}$ NMR yield of $9 \%$. PTLC conditions: Pentane/Et $2 \mathrm{O}(8 / 2) .{ }^{1} \mathrm{H}$ NMR (300 MHz, CDCl $) \delta 7.36(\mathrm{~d}, J=8.3 \mathrm{~Hz}, 1 \mathrm{H}), 7.30-7.21(\mathrm{~m}, 3 \mathrm{H}), 4.97-4.89(\mathrm{~m}, 1 \mathrm{H}), 3.85(\mathrm{~s}, 3 \mathrm{H}), 3.60$ (dd, $J=16.6,7.9 \mathrm{~Hz}, 1 \mathrm{H}), 3.17$ (dd, $J=16.9,4.6 \mathrm{~Hz}, 1 \mathrm{H}) \mathrm{ppm} .{ }^{13} \mathrm{C} \mathrm{NMR}\left(75 \mathrm{MHz}, \mathrm{CDCl}_{3}\right) \delta 197.7,160.3$, 142.5, 134.9, 127.6, 126.0, 122.07 ( $\left.\mathrm{q}, J=256.8 \mathrm{~Hz}, \mathrm{ROCF}_{3}\right), 106.0,55.9,33.3 \mathrm{ppm} .{ }^{19} \mathrm{~F} \mathrm{NMR}(188 \mathrm{MHz}$, $\mathrm{CDCl}_{3}$ ) $\delta$-59.72 ppm. HRMS (ASAP +) Calcd for $\mathrm{C}_{11} \mathrm{H}_{10} \mathrm{~F}_{3} \mathrm{O}_{3}{ }^{+}[\mathrm{M}+\mathrm{H}]^{+}: 247.0577$. Found: 247.0572.

\section{5-fluoro-2-(trifluoromethoxy)-2,3-dihydro-1H-inden-1-one 24}<smiles>O=C1c2ccc(F)cc2CC1OC(F)(F)F</smiles>

24

According to general procedure D, 5-fluoro-2-(trifluoromethoxy)-2,3-dihydro-1H-inden-1-one $\mathbf{2 4}$ was obtained from the corresponding enol carbonate $1 \mathbf{u}$; the yield of the desired product was determined by comparing the integration of the ${ }^{19} \mathrm{~F} N M R$ resonance of the product with that of bis((trifluoromethyl)sulfonyl)amide. ${ }^{19} \mathrm{~F}$ NMR yield of $29 \%$. The product was isolated by PTLC (Pentane/Et ${ }_{2} \mathrm{O}\left(85 / 15,2\right.$ elutions)) in $22 \%$ yield $(5.2 \mathrm{mg}, 0.022 \mathrm{mmol}) .{ }^{1} \mathrm{H} \mathrm{NMR}\left(300 \mathrm{MHz}, \mathrm{CDCl}_{3}\right) \delta 7.88$ $-7.80(\mathrm{~m}, 1 \mathrm{H}), 7.20-7.09(\mathrm{~m}, 2 \mathrm{H}), 4.98-4.89(\mathrm{~m}, 1 \mathrm{H}), 3.67(\mathrm{dd}, J=17.3,7.9 \mathrm{~Hz}, 1 \mathrm{H}), 3.25(\mathrm{dd}, J=17.1$, $4.8 \mathrm{~Hz}, 1 \mathrm{H}$ ) ppm. ${ }^{13} \mathrm{C}$ NMR $\left(75 \mathrm{MHz}, \mathrm{CDCl}_{3}\right) \delta 195.6,168.19$ (d, J $\left.=259 \mathrm{~Hz}, \mathrm{~F}-\mathrm{C}_{\mathrm{Ar}}\right), 152.66$ (d, J = $10.5 \mathrm{~Hz}$ ), $130.3,127.61\left(\mathrm{~d}, J=10.6 \mathrm{~Hz}\right.$ ), $122.03\left(\mathrm{q}, J=257 \mathrm{~Hz}, \mathrm{ROCF}_{3}\right), 117.20$ (d, $J=23.8 \mathrm{~Hz}$ ), $113.62(\mathrm{~d}, J=22.8$ $\mathrm{Hz}$ ), 76.38 (q, $J=2.4 \mathrm{~Hz}, \mathrm{RCH}_{2}-\mathrm{OCF}_{3}$ ), $33.8 \mathrm{ppm} .{ }^{19} \mathrm{~F} \mathrm{NMR} \mathrm{(188} \mathrm{MHz}, \mathrm{CDCl}_{3}$ ) $\delta-59.80$ (s, $\left.\mathrm{CF}_{3}\right),-99.41$ (q, J $=7.7 \mathrm{~Hz}$ ) ppm. HRMS (ASAP +) Calcd for $\mathrm{C}_{10} \mathrm{H}_{7} \mathrm{~F}_{4} \mathrm{O}_{2}{ }^{+}[\mathrm{M}+\mathrm{H}]^{+}:$235.0377. Found: 235.0380 .

\section{2-(trifluoromethoxy)-3,4-dihydronaphthalen-1(2H)-one 25}<smiles>O=C1c2ccccc2CCC1OC(F)(F)F</smiles>

25

According to general procedure E, 2-(trifluoromethoxy)-3,4-dihydronaphthalen-1(2H)-one 25 was obtained from the corresponding enol carbonate $1 \mathbf{v}$. The yield of the desired product was determined by comparing the integration of the ${ }^{19} \mathrm{~F} N M R$ resonance of the product with that of bis((trifluoromethyl)sulfonyl)amide. ${ }^{19} \mathrm{~F}$ NMR yield of $14 \% .{ }^{19} \mathrm{~F} \mathrm{NMR} \mathrm{(188} \mathrm{MHz,} \mathrm{CDCl3)} \delta-58.76 \mathrm{ppm}$.

\section{6-(trifluoromethoxy)-6,7,8,9-tetrahydro-5H-benzo[7]annulen-5-one 26}<smiles>O=C1c2ccccc2CCC1OC(F)(F)F</smiles>

According to general procedure D, 6-(trifluoromethoxy)-6,7,8,9-tetrahydro-5H-benzo[7]annulen-5one $\mathbf{2 6}$ was obtained from the corresponding enol carbonate $\mathbf{1} \mathbf{w}$; the yield of the desired product was 
determined by comparing the integration of the ${ }^{19} \mathrm{~F} N M R$ resonance of the product with that of bis((trifluoromethyl)sulfonyl)amide. ${ }^{19} \mathrm{~F} N M R$ yield of $24 \%$. PTLC conditions: Pentane/Et ${ }_{2} \mathrm{O}(85 / 15) .{ }^{1} \mathbf{H}$ NMR $\left(300 \mathrm{MHz}, \mathrm{CDCl}_{3}\right) \delta 7.71(\mathrm{~d}, J=7.6 \mathrm{~Hz}, 1 \mathrm{H}), 7.44(\mathrm{t}, J=7.4 \mathrm{~Hz}, 1 \mathrm{H}), 7.33(\mathrm{t}, J=7.6 \mathrm{~Hz}, 1 \mathrm{H}), 7.23(\mathrm{~s}$, $1 \mathrm{H}), 4.99(\mathrm{t}, J=7.0 \mathrm{~Hz}, 1 \mathrm{H}), 3.14-2.91(\mathrm{~m}, 2 \mathrm{H}), 2.40-2.24(\mathrm{~m}, 1 \mathrm{H}), 2.22-2.0(\mathrm{~m}, 2 \mathrm{H}), 1.99-1.81(\mathrm{~m}$, 1H) ppm. ${ }^{13} \mathrm{C} \mathrm{NMR}\left(75 \mathrm{MHz}, \mathrm{CDCl}_{3}\right.$ ) $\delta 198.9,141.7,136.5,132.4,130.2,129.4,127.0,121.50$ (q, J = 257 $\mathrm{Hz}, \mathrm{ROCF}_{3}$ ), 81.0 (q, $\left.J=2.6 \mathrm{~Hz}, \mathrm{RCH}_{2}-\mathrm{OCF}_{3}\right) .34 .2,30.8,23.2 \mathrm{ppm} .{ }^{19} \mathrm{~F} \mathrm{NMR}\left(188 \mathrm{MHz}, \mathrm{CDCl}_{3}\right) \delta-59.52$ ppm. HRMS (ASAP +) Calcd for $\mathrm{C}_{12} \mathrm{H}_{12} \mathrm{~F}_{3} \mathrm{O}_{2}^{+}[\mathrm{M}+\mathrm{H}]^{+}: 245.0784$. Found: 245.0781 .

\section{1-(benzo[b]thiophen-2-yl)-2-(trifluoromethoxy)ethanone 27}<smiles>CCOC(=O)COC(F)(F)F</smiles>

According to general procedure D, 1-(benzo[b]thiophen-2-yl)-2-(trifluoromethoxy)ethanone $\mathbf{2 7}$ was obtained from the corresponding enol carbonate $\mathbf{1 x}$; the yield of the desired product was determined by comparing the integration of the ${ }^{19} \mathrm{~F} N M R$ resonance of the product with that of bis((trifluoromethyl)sulfonyl)amide. ${ }^{19} \mathrm{~F}$ NMR yield of $12 \%$. PTLC conditions: Pentane/Et $\mathrm{E}_{2} \mathrm{O}(9 / 1) .{ }^{1} \mathrm{H}$ NMR (300 MHz, CDCl 3$) \delta 8.05(\mathrm{~s}, 1 \mathrm{H}), 7.92(\mathrm{t}, J=8.2 \mathrm{~Hz}, 2 \mathrm{H}), 7.49(\mathrm{dt}, J=22.0,7.4 \mathrm{~Hz}, 2 \mathrm{H}), 5.13(\mathrm{~s}, 1 \mathrm{H})$ ppm. ${ }^{13} \mathrm{C} \mathrm{NMR} \mathrm{(75} \mathrm{MHz,} \mathrm{CDCl} 3$ ) $\delta 185.2,142.7,139.5,139.0,130.4,128.4,126.5,125.6,123.1,121.7$ (d, $\left.J=257.2 \mathrm{~Hz}, \mathrm{ROCF}_{3}\right), 68.5$ (q, $\left.J=3.7,3.0 \mathrm{~Hz}, \mathrm{RCH}_{2}-\mathrm{OCF}_{3}\right)$ ppm. ${ }^{19} \mathrm{~F} \mathrm{NMR}\left(\mathbf{1 8 8} \mathbf{~ M H z}, \mathrm{CDCl}_{3}\right) \delta-61.41$ ppm. HRMS (ASAP +) Calcd for $\mathrm{C}_{11} \mathrm{H}_{8} \mathrm{~F}_{3} \mathrm{O}_{2} \mathrm{~S}^{+}[\mathrm{M}+\mathrm{H}]^{+}:$261.0192. Found: 261.0204 .

\section{1-(naphthalen-2-yl)-2-(trifluoromethoxy)ethan-1-one 28}<smiles>O=C(COC(F)(F)F)c1ccc2ccccc2c1</smiles>

28

According to general procedure E, 1-(naphthalen-2-yl)-2-(trifluoromethoxy)ethan-1-one 28 was obtained from the corresponding enol carbonate $\mathbf{1 y}$. The yield of the desired product was determined by comparing the integration of the ${ }^{19} \mathrm{~F} N M R$ resonance of the product with that of bis((trifluoromethyl)sulfonyl)amide. ${ }^{19} \mathrm{~F}$ NMR yield of $14 \% .{ }^{19} \mathrm{~F} \mathrm{NMR}\left(188 \mathbf{~ M H z}, \mathrm{CDCl}_{3}\right) \delta-60.95 \mathrm{ppm}$.

\section{2,2-difluoro-1-phenyl-2-(trifluoromethoxy)ethan-1-one 29}<smiles>O=C(OC(F)(F)F)C(F)(F)c1ccccc1</smiles>

29

According to general procedure E, 2,2-difluoro-1-phenyl-2-(trifluoromethoxy)ethan-1-one 29 was obtained from the corresponding enol carbonate $1 \mathbf{z}$. The yield of the desired product was determined by comparing the integration of the ${ }^{19} \mathrm{~F} N M R$ resonance of the product with that of bis((trifluoromethyl)sulfonyl)amide. ${ }^{19} \mathrm{~F}$ NMR yield of $20 \% .{ }^{19} \mathrm{~F} \mathrm{NMR}\left(188 \mathrm{MHz}, \mathrm{CDCl}_{3}\right) \delta-54.91(\mathrm{t}, \mathrm{J}=$ 8.7 Hz, 3F), -76.80 (q, J = 9.1 Hz, 2F) ppm.

\section{2,2-difluoro-1-(naphthalen-2-yl)-2-(trifluoromethoxy)ethan-1-one 30}<smiles>O=C(c1ccc2ccccc2c1)C(F)(F)OC(F)(F)F</smiles> 
According to general procedure E, 2,2-difluoro-1-(naphthalen-2-yl)-2-(trifluoromethoxy)ethan-1-one 30 was obtained from the corresponding enol carbonate 1aa. The yield of the desired product was determined by comparing the integration of the ${ }^{19} \mathrm{~F}$ NMR resonance of the product with that of bis((trifluoromethyl)sulfonyl)amide. ${ }^{19} \mathrm{~F}$ NMR yield of $\left.19 \% .{ }^{19} \mathrm{~F} \mathrm{NMR} \mathrm{(188} \mathbf{~ M H z}, \mathrm{CDCl}_{3}\right) \delta-54.92(\mathrm{t}, \mathrm{J}=$ 8.7 Hz, 3F), -76.23 (q, J = 8.5 Hz, 2F) ppm.

\section{1-(trifluoromethoxy)octan-2-one 31}<smiles>CCCCC(=O)CO[Na]</smiles>

31

According to general procedure E, 1-(trifluoromethoxy)octan-2-one $\mathbf{3 1}$ was obtained from the corresponding enol carbonate $1 \mathbf{a b}$. The yield of the desired product was determined by comparing the integration of the ${ }^{19} \mathrm{~F}$ NMR resonance of the product with that of bis((trifluoromethyl)sulfonyl)amide. ${ }^{19} \mathrm{~F}$ NMR yield of $16 \% .{ }^{19} \mathrm{~F}$ NMR (188 MHz, $\left.\mathrm{CDCl}_{3}\right) \delta-61.30 \mathrm{ppm}$.

\section{(E)-4-phenyl-1-(trifluoromethoxy)but-3-en-2-one 32}<smiles>O=C(C=Cc1ccccc1)COC(F)(F)F</smiles>

32

According to general procedure D, (E)-4-phenyl-1-(trifluoromethoxy)but-3-en-2-one 32 was obtained from the corresponding enol carbonate 1ac; the yield of the desired product was determined by comparing the integration of the ${ }^{19} \mathrm{~F}$ NMR resonance of the product with that of bis((trifluoromethyl)sulfonyl)amide. ${ }^{19} \mathrm{~F}$ NMR yield of $21 \%$. PTLC conditions: Pentane/Et ${ }_{2} \mathrm{O}(9 / 1,2$ elutions). ${ }^{1} \mathrm{H}$ NMR (300 MHz, $\left.\mathrm{CDCl}_{3}\right) \delta 7.76(\mathrm{~d}, J=16.1 \mathrm{~Hz}, 1 \mathrm{H}), 7.65-7.56(\mathrm{~m}, 2 \mathrm{H}), 7.48-7.36(\mathrm{~m}, 3 \mathrm{H})$, $6.97(\mathrm{~d}, J=16.1 \mathrm{~Hz}, 1 \mathrm{H}), 4.69(\mathrm{~s}, 2 \mathrm{H}) \mathrm{ppm} .{ }^{13} \mathrm{C} \mathrm{NMR}\left(75 \mathrm{MHz}, \mathrm{CDCl}_{3}\right) \delta 191.4,145.7,134.0,131.5,129.2$, 128.9, 128.6, 121.7 (q, $\left.J=256 \mathrm{~Hz}, \mathrm{ROCF}_{3}\right), 69.9$ (q, $\left.J=2.5 \mathrm{~Hz}, \mathrm{RCH}_{2}-\mathrm{OCF}_{3}\right)$ ppm. ${ }^{19} \mathrm{~F}$ NMR (188 MHz, $\mathrm{CDCl}_{3}$ ) $\delta$-61.63 ppm. HRMS (ASAP +) Calcd for $\mathrm{C}_{11} \mathrm{H}_{10} \mathrm{~F}_{3} \mathrm{O}_{2}{ }^{+}[\mathrm{M}+\mathrm{H}]^{+}:$231.0627. Found: 231.0634.

\section{(E)-1-(trifluoromethoxy)non-3-en-2-one 33}<smiles>CCCCCCC(=O)COC(C)(F)F</smiles>

33

According to general procedure D, (E)-1-(trifluoromethoxy)non-3-en-2-one $\mathbf{3 3}$ was obtained from the corresponding enol carbonate 1ad; the yield of the desired product was determined by comparing the integration of the ${ }^{19} \mathrm{~F} \mathrm{NMR}$ resonance of the product with that of bis((trifluoromethyl)sulfonyl)amide. ${ }^{19} \mathrm{~F}$ NMR yield of $18 \% .{ }^{19} \mathrm{~F}$ NMR (188 MHz, CDCl $) \delta-61.67$ ppm. HRMS (ASAP +) Calcd for $\mathrm{C}_{10} \mathrm{H}_{16} \mathrm{~F}_{3} \mathrm{O}_{2}{ }^{+}$ $[\mathrm{M}+\mathrm{H}]^{+}:$225.1097. Found: 225.1093 . 


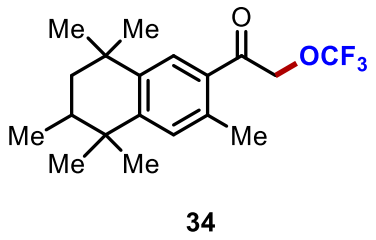

According to general procedure E, 1-(3,5,5,6,8,8-hexamethyl-5,6,7,8-tetrahydronaphthalen-2-yl)-2(trifluoromethoxy)ethan-1-one $\mathbf{3 4}$ was obtained from the corresponding enol carbonate 1ae. The yield of the desired product was determined by comparing the integration of the ${ }^{19} \mathrm{~F} N \mathrm{NR}$ resonance of the product with that of bis((trifluoromethyl)sulfonyl)amide. ${ }^{19} \mathrm{~F}$ NMR yield of $33 \%$. The product was isolated by flash column chromatography on silica gel (hexane/DCM 7/3) in $26 \%$ yield ( $8.9 \mathrm{mg}, 0.026$ mmol). ${ }^{1} \mathrm{H}$ NMR (500 MHz, $\left.\mathrm{CDCl}_{3}\right) \delta 7.48(\mathrm{~s}, 1 \mathrm{H}), 7.26(\mathrm{~s}, 1 \mathrm{H}), 5.02(\mathrm{~s}, 2 \mathrm{H}), 2.50(\mathrm{~s}, 3 \mathrm{H}), 1.88(\mathrm{dqd}, J=$ $13.5,6.8,2.6 \mathrm{~Hz}, 1 \mathrm{H}), 1.64(\mathrm{t}, J=13.3 \mathrm{~Hz}, 1 \mathrm{H}), 1.41(\mathrm{dd}, J=13.6,2.6 \mathrm{~Hz}, 1 \mathrm{H}), 1.33(\mathrm{~s}, 3 \mathrm{H}), 1.32(\mathrm{~s}, 3 \mathrm{H})$, $1.27(\mathrm{~s}, 3 \mathrm{H}), 1.08(\mathrm{~s}, 3 \mathrm{H}), 1.00(\mathrm{~d}, J=6.8 \mathrm{~Hz}, 3 \mathrm{H}) \mathrm{ppm} .{ }^{13} \mathrm{C} \mathrm{NMR}\left(126 \mathrm{MHz}, \mathrm{CDCl}_{3}\right) \delta$ 193.0, 151.7, 142.7, $136.5,131.3$ (x2), 127.2, 121.8, (q, J = 256.2 Hz, ROCF 3 ), 69.3 (q, $\left.J=2.9 \mathrm{~Hz}, \mathrm{RCH}_{2}-\mathrm{OCF}_{3}\right), 43.4,38.2,34.5$, 34.2, 32.6, 32.0, 28.4, 24.8, 21.3, 16.9 ppm. ${ }^{19} \mathrm{~F}$ NMR (188 $\left.\mathrm{MHz}, \mathrm{CDCl}_{3}\right) \delta-61.29 \mathrm{ppm}$. HRMS (ESI+) Calcd. for $\mathrm{C}_{19} \mathrm{H}_{26} \mathrm{~F}_{3} \mathrm{O}_{2}{ }^{+}\left[\mathrm{M}+\mathrm{H}^{+}\right]^{+}:$343.1879. Found: 343.1870 .

\section{1-(4-(tert-butyl)-2,6-dimethyl-3,5-dinitrophenyl)-2-(trifluoromethoxy)ethanone 35}<smiles>Cc1c(C(=O)COC(F)(F)F)c([N+](=O)[O-])c([N+](=O)[O-])c([N+](=O)[O-])c1[N+](=O)[O-]</smiles>

35

According to general procedure D, 1-(4-(tert-butyl)-2,6-dimethyl-3,5-dinitrophenyl)-2(trifluoromethoxy)ethanone $\mathbf{3 5}$ was obtained from the corresponding enol carbonate 1af; the yield of the desired product was determined by comparing the integration of the ${ }^{19} \mathrm{~F} N \mathrm{NR}$ resonance of the product with that of bis((trifluoromethyl)sulfonyl)amide. ${ }^{19} \mathrm{~F}$ NMR yield of $29 \%$. The product was isolated by PTLC (Pentane/Et ${ }_{2} \mathrm{O} 9 / 1$ ) in $21 \%$ yield $(7.9 \mathrm{mg}, 0.021 \mathrm{mmol})$ as a white solid. ${ }^{1} \mathrm{H}$ NMR (300 $\left.\mathbf{M H z}, \mathrm{CDCl}_{3}\right) \delta 4.74(\mathrm{~s}, 2 \mathrm{H}), 2.12(\mathrm{~s}, 6 \mathrm{H}), 1.45(\mathrm{~s}, 9 \mathrm{H}) \mathrm{ppm} .{ }^{13} \mathrm{C} \mathbf{N M R}\left(75 \mathrm{MHz}, \mathrm{CDCl}_{3}\right) \delta$ 197.6, 150.7, $138.5,133.8,128.5,121.4$ (q, $J=258 \mathrm{~Hz}, \mathrm{ROCF}_{3}$ ), 70.7 (q, $J=2.9 \mathrm{~Hz}, \mathrm{RCH}_{2}-\mathrm{OCF}_{3}$ ), 37.8, 30.4, $15.2 \mathrm{ppm}$. ${ }^{19}$ F NMR (188 MHz, CDCl $\left.{ }_{3}\right) \delta-61.74$ ppm. HRMS (ASAP +) Calcd for $\mathrm{C}_{15} \mathrm{H}_{18} \mathrm{~F}_{3} \mathrm{~N}_{2} \mathrm{O}_{6}{ }^{+}[\mathrm{M}+\mathrm{H}]^{+}: 379.1111$. Found: 379.1126.

1-(6-(tert-butyl)-1,1-dimethyl-2,3-dihydro-1H-inden-4-yl)-2-(trifluoromethoxy)ethanone 36<smiles>CC(C)(C)OCC(=O)c1cc(Br)cc2c1CCC2(C)C</smiles>

According to general procedure D, 1-(6-(tert-butyl)-1,1-dimethyl-2,3-dihydro-1H-inden-4-yl)-2(trifluoromethoxy)ethanone $\mathbf{3 6}$ was obtained from the corresponding enol carbonate $\mathbf{1 a g}$; the yield of the desired product was determined by comparing the integration of the ${ }^{19} \mathrm{~F} N \mathrm{NR}$ resonance of the product with that of bis((trifluoromethyl)sulfonyl)amide. ${ }^{19} \mathrm{~F} \mathrm{NMR}$ yield of $20 \%$. The product was isolated by PTLC (Pentane/EtOAc (98/2, 2 elutions)) in $16 \%$ yield $(5.2 \mathrm{mg}, 0.016 \mathrm{mmol}) .{ }^{1} \mathrm{H}$ NMR (300 $\left.\mathrm{MHz}, \mathrm{CDCl}_{3}\right) \delta 7.52(\mathrm{~s}, 1 \mathrm{H}), 7.40(\mathrm{~s}, 1 \mathrm{H}), 5.12(\mathrm{~s}, 2 \mathrm{H}), 3.17(\mathrm{t}, J=7.3 \mathrm{~Hz}, 2 \mathrm{H}), 1.96(\mathrm{t}, J=7.3 \mathrm{~Hz}, 2 \mathrm{H}), 1.36$ $(\mathrm{s}, 9 \mathrm{H}), 1.27$ (s, 6H) ppm. ${ }^{13} \mathrm{C}$ NMR $\left(75 \mathrm{MHz}, \mathrm{CDCl}_{3}\right) \delta 191.8,155.2,150.5,142.2,130.2,124.7,123.2$, 117.5 (q, $J=336.7 \mathrm{~Hz}, \mathrm{ROCF}_{3}$ ), 69.2 (q, $J=2.6 \mathrm{~Hz}, \mathrm{RCH}_{2}-\mathrm{OCF}_{3}$ ). 43.7, 41.5, 34.9, 31.6, 30.9, $28.9 \mathrm{ppm} .{ }^{19} \mathrm{~F}$ 
NMR (188 MHz, CDCl $) \delta-61.24$ ppm. HRMS (ASAP +) Calcd for $\mathrm{C}_{18} \mathrm{H}_{24} \mathrm{~F}_{3} \mathrm{O}_{2}{ }^{+}[\mathrm{M}+\mathrm{H}]^{+}:$329.1723. Found: 329.1679 .

(E)-1-(trifluoromethoxy)-4-(2,6,6-trimethylcyclohex-2-en-1-yl)but-3-en-2-one 37<smiles>CCOC(=O)C=CC1=CCCCC1(C)O</smiles>

37

According to general procedure $D$, (E)-1-(trifluoromethoxy)-4-(2,6,6-trimethylcyclohex-2-en-1-yl)but3-en-2-one $\mathbf{3 8}$ was obtained from the corresponding enol carbonate 1ah; the yield of the desired product was determined by comparing the integration of the ${ }^{19} \mathrm{~F} N \mathrm{NR}$ resonance of the product with that of bis((trifluoromethyl)sulfonyl)amide. ${ }^{19} \mathrm{~F}$ NMR yield of $14 \%$. The product was isolated by PTLC (Pentane/EtOAc 98/2) in 11\% yield (3.2 mg, $0.011 \mathrm{mmol}) .{ }^{1} \mathbf{H}$ NMR (200 MHz, $\left.\mathbf{C D C l}_{3}\right) \delta 6.87$ (dd, $J=$ 15.7, $9.8 \mathrm{~Hz}, 1 \mathrm{H}), 6.28(\mathrm{~d}, J=15.6 \mathrm{~Hz}, 1 \mathrm{H}), 5.53(\mathrm{bs}, 1 \mathrm{H}), 4.61(\mathrm{~s}, 2 \mathrm{H}), 2.32(\mathrm{~d}, J=9.6 \mathrm{~Hz}, 1 \mathrm{H}), 1.66(\mathrm{~s}$, $1 \mathrm{H}), 2.14-1.98(\mathrm{~m}, 3 \mathrm{H}), 1.52(\mathrm{~s}, 3 \mathrm{H}), 0.93(\mathrm{~s}, 3 \mathrm{H}), 0.85(\mathrm{~s}, 3 \mathrm{H}) \mathrm{ppm} .{ }^{13} \mathrm{C} \mathrm{NMR}\left(151 \mathrm{MHz}, \mathrm{CDCl}_{3}\right) \delta 191.04$, $151.82,131.35,125.79,123.39,69.54$ (q $J=2.9 \mathrm{~Hz}, \mathrm{CH}_{2} \mathrm{OCF}_{3}$ ), 54.77, 28.00, 27.81, 26.89, 23.14, 22.87 ppm. $\left.{ }^{19} \mathrm{~F} \mathrm{NMR} \mathrm{(188} \mathrm{MHz,} \mathrm{CDCl} 3\right) \delta-61.62 \mathrm{ppm}$. HRMS (ASAP +) Calcd for $\mathrm{C}_{14} \mathrm{H}_{20} \mathrm{~F}_{3} \mathrm{O}_{2}{ }^{+}[\mathrm{M}+\mathrm{H}]^{+}: 277.1410$. Found: 277.1407.

1-((3S,8S,9S,10R,13S,14S)-3-methoxy-10,13-dimethyl-tetradecahydro-1Hcyclopenta[a]phenanthren-17-yl)-2-(trifluoromethoxy)ethan-1-one 38<smiles>COC(=O)CC1CCC2C3CC=C4CC(OC)CCC4(C)C3CCC12N</smiles>

38

According to general procedure E, 1-((3S,8S,9S,10R,13S,14S)-3-methoxy-10,13-dimethyltetradecahydro-1H-cyclopenta[a]phenanthren-17-yl)-2-(trifluoromethoxy)ethan-1-one 38 was obtained from the corresponding enol carbonate 1ai. The yield of the desired product was determined by comparing the integration of the ${ }^{19} \mathrm{~F} N M R$ resonance of the product with that of bis((trifluoromethyl)sulfonyl)amide. ${ }^{19} \mathrm{~F}$ NMR yield of $10 \% .{ }^{19} \mathrm{~F} \mathrm{NMR} \mathrm{(188} \mathrm{MHz,} \mathrm{CDCl3)} \delta-61.10 \mathrm{ppm}$.

(8R,9S,10R,13S,14S,17S)-17-methoxy-10,13-dimethyl-6-(trifluoromethoxy)-dodecahydro-1Hcyclopenta[a]phenanthren-3(2H)-one 39

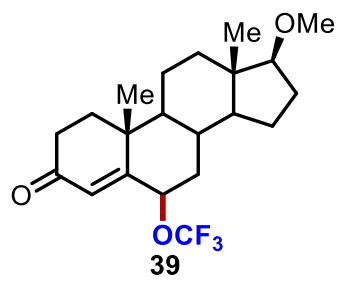

According to general procedure D, (8R,9S,10R,13S,14S,17S)-17-methoxy-10,13-dimethyl-6(trifluoromethoxy)-dodecahydro-1H-cyclopenta[a]phenanthren-3(2H)-one 39 was obtained from the corresponding enol carbonate $\mathbf{1} \mathbf{a j}$; the yield of the desired product was determined by comparing the integration of the ${ }^{19} \mathrm{~F}$ NMR resonance of the product with that of bis((trifluoromethyl)sulfonyl)amide. ${ }^{19} \mathrm{~F}$ NMR yield of $8 \%$. PTLC conditions: Pentane/ $\mathrm{Et}_{2} \mathrm{O}$ (9/1). The compound was isolated as an 
inseparable 1:1 mixture of diastereoisomers. $\ln { }^{1} \mathrm{H}$ NMR, only characteristic peaks are listed. ${ }^{[10]}{ }^{1} \mathbf{H}$ NMR $\left(600 \mathrm{MHz}, \mathrm{CDCl}_{3}\right) \delta 5.82(\mathrm{~d}, J=1.0 \mathrm{~Hz}, 1 \mathrm{H}, \mathrm{C}=\mathrm{CHC}=0), 5.77$ (d, J=1.4 Hz, 1H, C=CHC=O), 4.72 (dd, $J=$ 14.0, $\left.5.3 \mathrm{~Hz}, 1 \mathrm{H}, \mathrm{CHOCF}_{3}\right), 4.57\left(\mathrm{dd}, J=9.2,4.5 \mathrm{~Hz}, 1 \mathrm{H}, \mathrm{CHOCF}_{3}\right), 3.35\left(\mathrm{~s}, 3 \mathrm{H} \mathrm{x} 2, \mathrm{OCH}_{3}\right), 3.25-3.20(\mathrm{~m}$, $2 \mathrm{H} \times 2, \mathrm{CHOCH} 3), 2.54-2.45\left(\mathrm{~m}, 1 \mathrm{H}, \mathrm{CH}_{2} \mathrm{C}=0\right), 2.34$ (dt, $\left.J=4.9,2.3 \mathrm{~Hz}, 1 \mathrm{H}, \mathrm{CH}_{2} \mathrm{C}=0\right), 2.29$ (dd, $J=4.2$, $\left.2.6 \mathrm{~Hz}, 1 \mathrm{H}, \mathrm{CH}_{2} \mathrm{C}=\mathrm{O}\right), 2.27\left(\mathrm{dt}, J=5.2,2.7 \mathrm{~Hz}, 1 \mathrm{H}, \mathrm{CH}_{2} \mathrm{C}=\mathrm{O}\right), 1.32\left(\mathrm{~s}, 3 \mathrm{H}, \mathrm{C}-19 \mathrm{CH}_{3}\right), 1.27\left(\mathrm{~s}, 3 \mathrm{H}, \mathrm{C}-19 \mathrm{CH}_{3}\right)$, 0.80 (s, 3H, C-18 CH $\mathrm{CH}_{3}, 0.80$ (s, 3H, C-18 CH CH$_{3}{ }^{13} \mathrm{C} \mathrm{NMR}\left(151 \mathrm{MHz}, \mathrm{CDCl}_{3}\right) \delta 191.5,191.1,173.6,171.3$, 123.5 (d, $\left.J=239.1 \mathrm{~Hz}, \mathrm{ROCF}_{3}\right), 121.8,120.8,117.1,90.8,90.5,75.2-75.1\left(\mathrm{~m}, \mathrm{CHOCF}_{3}\right), 74.5-74.4(\mathrm{~m}$, $\mathrm{CHOCF}_{3}$ ), 58.0 (x2), 54.4, 54.2, 52.7, 51.0, 50.7 (x2), 43.2, 43.0, 42.9, 42.7, 40.9, 40.4, 39.6, 38.0, 37.8, $37.7,37.6,35.7,35.5,35.0,33.7,33.6,33.1,32.7,32.5,32.1,31.6,29.8,27.8,27.7,27.6,23.4(x 3)$, 21.9, 21.8, 21.2, 20.8, 18.3, 17.6, 11.8, 11.7 (x2). $\left.{ }^{19} \mathrm{~F} \mathrm{NMR} \mathrm{(188} \mathrm{MHz,} \mathrm{CDCl}_{3}\right) \delta$-59.05 (s, $\left.\mathrm{CF}_{3}\right),-59.11$ (s, $\mathrm{CF}_{3}$ ) ppm. HRMS (ASAP +) Calcd for $\mathrm{C}_{21} \mathrm{H}_{30} \mathrm{~F}_{3} \mathrm{O}_{3}{ }^{+}[\mathrm{M}+\mathrm{H}]^{+}: 387.2142$. Found: 387.2152 .

\section{Scale-up experiments}

\section{4-(2-(trifluoromethoxy)acetyl)benzonitrile 12}

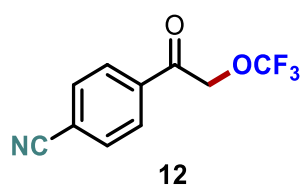

A round-bottomed flask purged with argon was charged with 4-cyano-1-(trifluoromethoxy)pyridin-1ium bis((trifluoromethyl)sulfonyl)amide 2a $(939 \mathrm{mg}, 2.1 \mathrm{mmol}, 1$ equiv.), tert-butyl (1-(4cyanophenyl)vinyl) carbonate $1 \mathrm{i}(2.58 \mathrm{~g}, 10.5 \mathrm{mmol}, 5$ equiv.) and 4CzIPN (83 mg, $0.11 \mathrm{mmol}, 0.05$ equiv.). Dry acetonitrile $(210 \mathrm{~mL}, 0.01 \mathrm{M})$ was finally added and the reaction medium purged with argon again. $200 \mathrm{~mL}$ of the resulting mixture were taken at a flow rate of $5 \mathrm{~mL} / \mathrm{min}$ ( 2 minutes of residence time in a $10 \mathrm{~mL}$ coil UV-150 reactor) by the VapourTec apparatus, equipped with a blue lamp irradiating at $450 \mathrm{~nm}$ inside the reactor, at $50^{\circ} \mathrm{C}$. The resulting mixture exiting the apparatus was concentrated under vacuum. ${ }^{19} \mathrm{~F}$ NMR yield of $37 \%$. 4-(2-(trifluoromethoxy)acetyl)benzonitrile 12 was isolated as a white solid by column chromatography on silica gel (Pentane/EtOAc 9/1) in 36\% yield (165 $\mathrm{mg}, 0.72 \mathrm{mmol})$.

The scaled-up synthesis of 12 was then repeated with 4-cyano-1-(trifluoromethoxy)pyridin-1-ium bis((trifluoromethyl)sulfonyl)amide 2a (1.06 g, $2.26 \mathrm{mmol}, 1$ equiv.) and tert-butyl (1-(4cyanophenyl)vinyl) carbonate $\quad \mathbf{1 i} \quad(3.0 \quad \mathrm{~g}, \quad 13.6 \mathrm{mmol}, 6$ equiv.). 4-(2(trifluoromethoxy)acetyl)benzonitrile $\mathbf{1 2}$ was isolated as a white solid by column chromatography on silica gel (Pentane/EtOAc 9/1) in 50\% yield ( $260 \mathrm{mg}, 1.13 \mathrm{mmol}$ ).

\section{1-(4-bromophenyl)-2-(trifluoromethoxy)ethan-1-one 17}<smiles>CCOCC(=O)c1ccc(Br)cc1</smiles>

17

A $250 \mathrm{~mL}$ round-bottomed flask purged with argon was charged with 4-cyano-1(trifluoromethoxy)pyridin-1-ium bis((trifluoromethyl)sulfonyl)amide 2 a (469 mg, 1 mmol, 1 equiv.), 1(4-bromophenyl)vinyl tert-butyl carbonate $1 \mathrm{n}(1.50 \mathrm{~g}, 5 \mathrm{mmol}, 5$ equiv.) and 4CzIPN ( $39 \mathrm{mg}, 0.05 \mathrm{mmol}$, 0.05 equiv.). Dry acetonitrile $(100 \mathrm{~mL}, 0.01 \mathrm{M})$ was finally added and the reaction medium purged with argon again. The resulting mixture was taken at a flow rate of $5 \mathrm{~mL} / \mathrm{min}(2$ minutes of residence time in a $10 \mathrm{~mL}$ coil UV-150 reactor) by the VapourTec apparatus, equipped with a medium pressure mercury lamp set at $75 \%$ of its maximum power output and a band-pass filter $\mathrm{N}^{\circ} 4$ irradiating at 370 $\mathrm{nm}$ inside the reactor, at $50^{\circ} \mathrm{C}$. The resulting mixture exiting the apparatus was treated with $10 \mathrm{~mL}$ of 
$6 \mathrm{M} \mathrm{HCl}$ and the reaction mixture stirred until complete hydrolysis of the unreacted starting material (controlled by TLC analysis). The crude reaction mixture was then quenched with sat. $\mathrm{NaHCO}_{3}$ until gas evolution stopped, diluted with DCM $(200 \mathrm{~mL})$ and washed with brine $(3 \times 50 \mathrm{~mL})$. The organic phase was dried over $\mathrm{MgSO}_{4}$ and concentrated under vacuum. ${ }^{19} \mathrm{~F} \mathrm{NMR} \mathrm{yield} \mathrm{of} \mathrm{37 \% .} \mathrm{1-(4-bromophenyl)-2-}$ (trifluoromethoxy)ethan-1-one $\mathbf{1 7}$ was isolated as a yellow solid by column chromatography on silica gel (hexane/EtOAc 95/5) in 33\% yield (94 mg, $0.33 \mathrm{mmol}$ ).

\section{Post-modifications}

\section{1-(4-bromophenyl)-2-(trifluoromethoxy)ethan-1-ol 40}<smiles>OC(COC(F)(F)F)c1ccc(Br)cc1</smiles>

A $4 \mathrm{~mL}$ vial was charged with 1-(4-bromophenyl)-2-(trifluoromethoxy)ethan-1-one $\mathbf{1 7}$ (30 mg, 0.106 mmol, 1 equiv.) and $\mathrm{MeOH}$ was added $(300 \mu \mathrm{L})$. The solution was cooled to $0^{\circ} \mathrm{C}$ and $\mathrm{NaBH}_{4}(4.8 \mathrm{mg}$, $0.127 \mathrm{mmol}, 1.2$ equiv.) was added. The reaction mixture was stirred at $0^{\circ} \mathrm{C}$ for $1 \mathrm{~h}$, after which time it was quenched with $1 \mathrm{M} \mathrm{HCl}(1 \mathrm{~mL})$, extracted with EtOAc $(3 \times 10 \mathrm{~mL})$ and washed with brine $(10 \mathrm{~mL})$. The organic phase was dried over $\mathrm{MgSO}_{4}$ and concentrated under vacuum. 1-(4-bromophenyl)-2(trifluoromethoxy)ethan-1-ol $\mathbf{4 0}$ was isolated without the need of further purification as a yellow oil, which solidified upon standing, in $88 \%$ yield $(26.6 \mathrm{mg}, 0.093 \mathrm{mmol}) .{ }^{1} \mathbf{H} \mathbf{~ N M R}\left(500 \mathbf{~ M H z}, \mathrm{CDCl}_{3}\right) \delta 7.52$ (d, $J=8.4 \mathrm{~Hz}, 2 \mathrm{H}$ ), 7.28 (d, $J=8.4 \mathrm{~Hz}, 2 \mathrm{H}$ ), 4.97 (dd, $J=8.5,3.4 \mathrm{~Hz}, 1 \mathrm{H}$ ), 4.05 (dd, $J=10.2,3.5 \mathrm{~Hz}, 1 \mathrm{H}$ ), 3.98 (dd, $J=10.2,8.5 \mathrm{~Hz}, 1 \mathrm{H}), 2.44$ (br s, $1 \mathrm{H})$ ppm. $\left.{ }^{13} \mathrm{C} \mathrm{NMR} \mathrm{(126} \mathrm{MHz}, \mathrm{CDCl}_{3}\right) \delta$ 137.5, 132.1, 128.0, 122.8, 119.7 (q, $\left.J=238.3 \mathrm{~Hz}, \mathrm{ROCF}_{3}\right), 71.6$ (q, $\left.J=2.6 \mathrm{~Hz}, \mathrm{RCH}_{2}-\mathrm{OCF}_{3}\right), 71.4 \mathrm{ppm} .{ }^{19} \mathrm{~F}$ NMR (188 MHz, $\mathrm{CDCl}_{3}$ ) $\delta$-61.24 ppm. HRMS (ESI-) Calcd. for $\mathrm{C}_{18} \mathrm{H}_{14} \mathrm{O}_{4} \mathrm{Br}_{2} \mathrm{~F}_{6} \mathrm{~K}^{-}[2 \mathrm{M}-2 \mathrm{H}+\mathrm{K}]^{-}: 604.8806$. Found: 604.8813.

\section{1-(4-morpholinophenyl)-2-(trifluoromethoxy)ethan-1-ol 41}<smiles>OC(COC(F)(F)F)c1ccc(N2CCOCC2)cc1</smiles>

The procedure was adapted from the literature. ${ }^{[11]} \mathrm{A} 4 \mathrm{~mL}$ vial purged with nitrogen was charged with 1-(4-bromophenyl)-2-(trifluoromethoxy)ethan-1-ol 40 (14 mg, $0.05 \mathrm{mmol}, 1$ equiv.), $\mathrm{Pd}(\mathrm{OAc})_{2}$ (0.22 $\mathrm{mg}, 0.001 \mathrm{mmol}, 0.02$ equiv.), 2-(di-tert-butylphosphino)biphenyl (JohnPhos, $0.44 \mathrm{mg}, 0.0015 \mathrm{mmol}$, 0.03 equiv.) and anhydrous $\mathrm{Cs}_{2} \mathrm{CO}_{3}(24 \mathrm{mg}, 0.075 \mathrm{mmol}, 1.5$ equiv.). The vial was closed with a cap equipped with a septum and $400 \mu \mathrm{L}$ of degassed anhydrous 1,4-dioxane were added. Morpholine (5 $\mu \mathrm{l}, 0.06 \mathrm{mmol}, 1.2$ equiv.) was added and the reaction was stirred at $80^{\circ} \mathrm{C}$ for 16 hours, after which time the reaction mixture was concentrated under reduced pressure. ${ }^{19} \mathrm{~F}$ NMR yield of $19 \%$. 1-(4morpholinophenyl)-2-(trifluoromethoxy)ethan-1-ol $\mathbf{4 1}$ was isolated as a yellow oil by column chromatography on silica gel (hexane/EtOAc $1 / 1$ ) in $22 \%$ yield $\left(3.2 \mathrm{mg}, 0.011 \mathrm{mmol}\right.$ ). ${ }^{1} \mathbf{H}$ NMR (500 $\left.\mathrm{MHz}, \mathrm{CDCl}_{3}\right) \delta 7.32-7.27(\mathrm{~m}, 2 \mathrm{H}), 6.94-6.89(\mathrm{~m}, 2 \mathrm{H}), 4.93(\mathrm{t}, J=6.2 \mathrm{~Hz}, 2 \mathrm{H}), 4.07-3.98(\mathrm{~m}, 2 \mathrm{H}), 3.91$ $-3.78(\mathrm{~m}, 4 \mathrm{H}), 3.20-3.13(\mathrm{~m}, 4 \mathrm{H}), 2.34(\mathrm{~d}, J=2.9 \mathrm{~Hz}, 1 \mathrm{H}) \mathrm{ppm} .{ }^{13} \mathrm{C} \mathrm{NMR}\left(126 \mathrm{MHz}, \mathrm{CDCl}_{3}\right) \delta 151.71$, 129.65, 127.39, 121.78 (q, $J=255.0 \mathrm{~Hz}, \mathrm{ROCF}_{3}$ ), 115.76, 71.90 (q, $J=2.3 \mathrm{~Hz}, \mathrm{RCH}_{2}-\mathrm{OCF}_{3}$ ), 71.63, 66.98, 49.23 ppm. ${ }^{19} \mathrm{~F}$ NMR (188 MHz, $\left.\mathrm{CDCl}_{3}\right) \delta-61.10$ ppm. HRMS (ASAP +) Calcd for $\mathrm{C}_{13} \mathrm{H}_{17} \mathrm{~F}_{3} \mathrm{NO}_{3}{ }^{+}[\mathrm{M}+\mathrm{H}]^{+}$: 292.1155. Found: 292.1161. 
<smiles>COCC(N)c1ccc(Br)cc1</smiles>

The procedure was adapted from the literature. ${ }^{[12]} \mathrm{A}$ flame-dried schlenk tube purged with nitrogen was charged with 1-(4-bromophenyl)-2-(trifluoromethoxy)ethan-1-one 17 (14 mg, 0.05 mmol), anhydrous $\mathrm{NH}_{4} \mathrm{OAc}$ ( $77 \mathrm{mg}, 1 \mathrm{mmol}, 20$ equiv.) and $\mathrm{NaBH}_{3} \mathrm{CN}(8.5 \mathrm{mg}, 0.135 \mathrm{mmol}, 2.7$ equiv.). Finally, $200 \mu \mathrm{L}$ of anhydrous $\mathrm{MeOH}$ were added and the schlenk tube sealed with a screw cap. The reaction was stirred at $70^{\circ} \mathrm{C}$ for 16 hours, after which time the reaction was concentrated under reduced pressure. ${ }^{19} \mathrm{~F}$ NMR yield of 34\%. 1-(4-bromophenyl)-2-(trifluoromethoxy)ethan-1-aminium acetate 42 was isolated as a white solid by column chromatography on silica gel (DCM/MeOH 95/5) in $31 \%$ yield (5.3 mg, $0.016 \mathrm{mmol}) .{ }^{1} \mathrm{H}$ NMR $\left(500 \mathrm{MHz}, \mathrm{CDCl}_{3}\right) \delta 7.50(\mathrm{~d}, J=8.4 \mathrm{~Hz}, 2 \mathrm{H}), 7.28(\mathrm{~d}, J=8.4 \mathrm{~Hz}, 2 \mathrm{H}), 4.66$ $(\mathrm{s}, 3 \mathrm{H}), 4.31(\mathrm{dd}, J=9.1,4.2 \mathrm{~Hz}, 1 \mathrm{H}), 4.05(\mathrm{dd}, J=9.7,4.0 \mathrm{~Hz}, 1 \mathrm{H}), 3.94(\mathrm{t}, J=9.1 \mathrm{~Hz}, 1 \mathrm{H}), 2.09(\mathrm{~s}, 3 \mathrm{H})$ ppm. ${ }^{13} \mathrm{C}$ NMR (126 MHz, CDCl $\left.{ }_{3}\right) \delta 175.8,132.3,132.1,128.8,122.4,121.7$ (q, $J=255.3 \mathrm{~Hz}, \mathrm{ROCF}_{3}$ ), 71.9, 54.1, 20.7 ppm. $\left.{ }^{19} \mathrm{~F} \mathrm{NMR} \mathrm{(188} \mathrm{MHz,} \mathrm{CDCl}_{3}\right) \delta-61.24$ ppm. HRMS (ESI+) Calcd. for $\mathrm{C}_{9} \mathrm{H}_{7} \mathrm{OBrF}_{3}{ }^{+}[\mathrm{M}$ $\left.+\mathrm{H}-\mathrm{NH}_{3}\right]^{+}:$266.9627. Found: 266.9619 .

\section{4-(3-(dimethylamino)-2-(trifluoromethoxy)acryloyl)benzonitrile 43}<smiles>CN(C)/C=C(\OC(F)(F)F)C(=O)c1ccc(C#N)cc1</smiles>

The procedure was adapted from the literature. ${ }^{[13]}$ A $4 \mathrm{~mL}$ vial was charged with 4-(2(trifluoromethoxy)acetyl)benzonitrile $12(20 \mathrm{mg}, 0.09 \mathrm{mmol}, 1$ equiv.) and $\mathrm{N}, \mathrm{N}$-dimethylformamide dimethyl acetal $\left(25 \mu \mathrm{L}, 0.18 \mathrm{mmol}, 2\right.$ equiv.). The vial was closed with a septum and stirred at $90^{\circ} \mathrm{C}$ for $3 \mathrm{~h}$. The resulting mixture was then diluted in $\mathrm{CH}_{2} \mathrm{Cl}_{2}(10 \mathrm{~mL})$ and washed with water $(10 \mathrm{~mL})$, dried over $\mathrm{MgSO}_{4}$ and concentrated under vacuum. The product was isolated by PTLC (Pentane/EtOAc 6/4) in 64\% yield (16.5 mg, $0.06 \mathrm{mmol}$ ) as a yellow solid. ${ }^{1} \mathrm{H}$ NMR (300 MHz, $\left.\mathrm{CDCl}_{3}\right) \delta 7.69(\mathrm{~s}, 4 \mathrm{H}), 7.11$ (bs, $1 \mathrm{H}), 3.16$ (s, 6H) ppm. ${ }^{13} \mathrm{C} \mathrm{NMR}\left(75 \mathrm{MHz}, \mathrm{CDCl}_{3}\right)$ 186.5, 145.2 (bs, $\left.\mathrm{RC}=\mathrm{C}-\mathrm{N}\left(\mathrm{CH}_{3}\right)_{2}\right), 143.3,132.1,129.0$, 122.1 (bs, RC=C-N(CH$\left.)_{2}\right), 121.2$ (q, $\left.J=257.1 \mathrm{~Hz}, \mathrm{ROCF}_{3}\right), 118.5,114.1 \mathrm{ppm} .{ }^{19} \mathrm{~F} \mathrm{NMR}\left(188 \mathrm{MHz}, \mathrm{CDCl}_{3}\right)$ $\delta$-59.29 (s, 3F) ppm. HRMS (ASAP +) Calcd for $\mathrm{C}_{13} \mathrm{H}_{11} \mathrm{~F}_{3} \mathrm{~N}_{2} \mathrm{NaO}_{2}{ }^{+}[2 \mathrm{M}+\mathrm{Na}]^{+}: 591.1438$. Found: 591.1440 .

\section{One-pot procedure for the trifluoromethoxylation of acetophenone}<smiles>O=C(COC(F)(F)F)c1ccccc1</smiles>

A round-bottomed flask was charged with acetophenone ( $5 \mathrm{mmol}, 1$ equiv.) and dissolved in dry THF $(5 \mathrm{~mL}, 1 \mathrm{M})$ at $0^{\circ} \mathrm{C}$ under nitrogen atmosphere. $\mathrm{NaH}$ (60\% dispersion in mineral oil, $400 \mathrm{mg}, 10 \mathrm{mmol}$, 2 equiv.) was added portionwise to the reaction mixture, which was then stirred for 15 mins at $0^{\circ} \mathrm{C}$. $\mathrm{Boc}_{2} \mathrm{O}(1.38 \mathrm{~mL}, 6 \mathrm{mmol}, 1.2$ equiv.) was then added dropwise and the reaction mixture was stirred for $1 \mathrm{~h}$ at room temperature. The resulting mixture was then quenched with water $(20 \mathrm{~mL})$, diluted with $\mathrm{CH}_{2} \mathrm{Cl}_{2}(50 \mathrm{~mL})$ and washed with brine $(3 \times 20 \mathrm{~mL})$. The organic phase was then dried over $\mathrm{MgSO}_{4}$ and concentrated under vacuum. The resulting crude compound was diluted with $1 \mathrm{~mL}$ of $\mathrm{CDCl}_{3}$ and trichloroethylene ( $450 \mu \mathrm{L}, 5 \mathrm{mmol}$ ) was added as the internal standard to determine the yield by ${ }^{1} \mathrm{H}$ NMR. ${ }^{1} \mathrm{H}$ NMR yield: $43 \%$. The crude was then evaporated and redissolved in $25 \mathrm{~mL}$ of DCM in a volumetric flask. $2.9 \mathrm{~mL}$ of the resulting solution (containing $0.25 \mathrm{mmol}$ of enol carbonate according to the previously measured ${ }^{1} \mathrm{H}$ NMR yield) were placed in an $8 \mathrm{~mL}$ vial and the solvent evaporated. The 
vial was then purged with argon and charged with 4-cyano-1-(trifluoromethoxy)pyridin-1-ium bis((trifluoromethyl)sulfonyl)amide 2 a $(23.5 \mathrm{mg}, 0.05 \mathrm{mmol}, 1$ equiv.) and 4CzIPN (1.9 mg, 0.0025 mmol, 0.05 equiv.) and subjected to the trifluoromethoxylation reaction according to General procedure $\mathrm{E}$. The yield of product $\mathbf{4 5}$ was determined by comparing the integration of the ${ }^{19} \mathrm{~F} \mathrm{NMR}$ resonance of the product with that of bis((trifluoromethyl)sulfonyl)amide. ${ }^{19} \mathrm{~F} \mathrm{NMR}$ yield of $23 \%$. The compound is reported in the literature. ${ }^{[14]}$ 


\section{F. Mechanistic studies}

\section{Alpha-trifluoromethoxylation of $1 \mathrm{~b}$ with Pyridinium $2 \mathrm{~b}$ and $4 \mathrm{CzIPN}$ as the photocatalyst}<smiles>C=C(OC(C)(C)C)c1ccc(F)cc1</smiles>

$1 b$

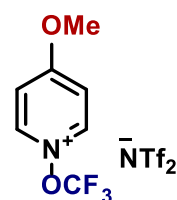

2b

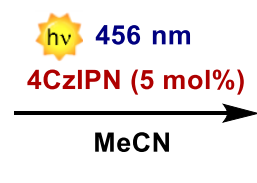$$
\mathbf{F}
$$<smiles>O=C(COC(F)(F)F)c1ccc(F)cc1</smiles>

3

An 8-mL vial surrounded by blue LEDs with a watertight protection, equipped with a screwcap and a septum was purged with argon and charged with 4-methoxy-1-(trifluoromethoxy)pyridin-1-ium bis((trifluoromethyl)sulfonyl)amide $\mathbf{2 b}(23.7 \mathrm{mg}, 0.05 \mathrm{mmol}, 1$ equiv.), 1-(4-fluorophenyl)vinyl tertbutyl carbonate 1 b $(0.25 \mathrm{mmol}, 5$ equiv.) and 4CzIPN (1.9 mg, $0.0025 \mathrm{mmol}, 0.05$ equiv.). Dry acetonitrile $(5 \mathrm{~mL}, 0.01 \mathrm{M})$ was finally added and the reaction medium purged with argon again. The resulting mixture in the vial was placed in a water bath and allowed to stir vigorously for $1 \mathrm{~h}$ at $50^{\circ} \mathrm{C}$ under irradiation of the blue LEDs. The yield of product $\mathbf{3}$ was determined by comparing the integration of the ${ }^{19} \mathrm{~F}$ NMR resonance of the product with that of bis((trifluoromethyl)sulfonyl)amide. ${ }^{19} \mathrm{~F} \mathrm{NMR}$ yield of $12 \%$.

\section{Luminescence quenching experiments}

Rates of quenching $\left(\mathrm{k}_{\mathrm{q}}\right)$ were determined using Stern-Volmer kinetics:

$$
\mathrm{I}_{0} / \mathrm{I}=\mathrm{k}_{\mathrm{q}} \tau_{0}[\text { quencher }]+1
$$

Where $I_{0}$ is the luminescence intensity without the quencher, $I$ is the intensity with the quencher, and $\tau_{0}$ is the excited state lifetime of the photocatalyst.

The following stock solutions were prepared in distilled acetonitrile and degassed by three freezepump-thaw cycles.

\section{General procedure:}

A stock solution of 4CzIPN was prepared by dissolving 4CzIPN ( $25 \mu \mathrm{mol})$ in $10 \mathrm{~mL}$ of acetonitrile. Of this solution, $0.1 \mathrm{~mL}$ were further diluted with acetonitrile to give a total volume of $10 \mathrm{~mL}$. Concentration of [4CzIPN] $=2.5 \times 10^{-5} \mathrm{M}$. A stock solution of 4-cyano-1-(trifluoromethoxy)pyridin-1ium bis((trifluoromethyl)sulfonyl)amide $2 \mathrm{a}$ was prepared by dissolving $2 \mathrm{a}(30 \mu \mathrm{mol})$ in $10 \mathrm{~mL}$ of acetonitrile. Concentration of [2a] = $3 \times 10-3 \mathrm{M}$. A stock solution of tert-butyl (1-(4-fluorophenyl)vinyl) carbonate $\mathbf{1 b}$ was prepared by dissolving $\mathbf{1 b}(30 \mu \mathrm{mol})$ in $10 \mathrm{~mL}$ of acetonitrile. Concentration of [1 $\mathbf{b}$ ] $=3 \times 10-3 \mathrm{M}$. For each experiment, 6 samples were prepared in the dark. Quartz cuvettes $(3.5 \mathrm{~mL})$ were filled with photocatalyst stock solution $(0.3 \mathrm{~mL})$, reagent stock solution $(0 \mathrm{~mL}, 0.2 \mathrm{~mL}, 0.4 \mathrm{~mL}, 0.6$ $\mathrm{mL}, 0.8 \mathrm{~mL}, 1.0 \mathrm{~mL}$ ) and acetonitrile $(2.7 \mathrm{~mL}, 2.5 \mathrm{~mL}, 2.3 \mathrm{~mL}, 2.1 \mathrm{~mL}, 1.9 \mathrm{~mL}, 1.7 \mathrm{~mL})$ to obtain a total volume of $3 \mathrm{~mL}$. The final concentrations were $[4 \mathrm{CzIPN}]=2.510^{-6} \mathrm{M}$ and [quencher] $=2 \times 10^{-4} \mathrm{M}, 4 \mathrm{x}$ $10^{-4} \mathrm{M}, 6 \times 10^{-4} \mathrm{M}, 8 \times 10^{-4} \mathrm{M}, 1 \times 10^{-3} \mathrm{M}$. For each sample, emission spectra were acquired between $470 \mathrm{~nm}$ and $700 \mathrm{~nm}$ (excitation at $450 \mathrm{~nm}$ ). 


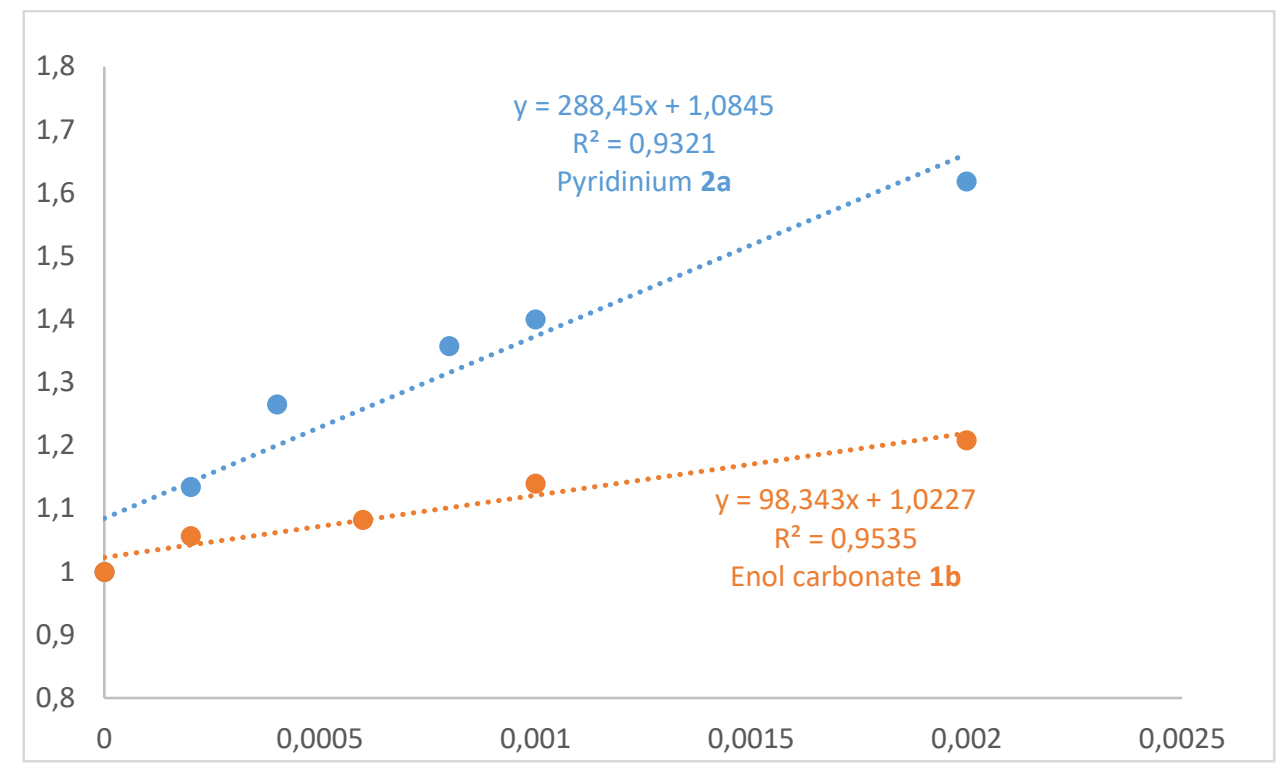

Figure S4 Stern-Volmer plots for pyridinium $2 a$ and enol carbonate $1 b$ as quenchers

$k_{2}=5.7^{*} 10^{7} \mathrm{~L} \cdot \mathrm{mol}^{-1} \cdot \mathrm{s}^{-1} \quad \mathrm{k}_{1}=1.9 * 10^{7} \mathrm{~L} \cdot \mathrm{mol}^{-1} \cdot \mathrm{s}^{-1} \quad\left(\tau_{0=5.1} \mu \mathrm{s} \text { for 4CzIPN }\right)^{[15]}$

\section{Quantum yield measurements}

A ferrioxalate actinometry solution was prepared by following the Hammond variation of the Hatchard and Parker procedure outlined in Handbook of Photochemistry. ${ }^{[16]}$ Ferrioxalate actinometer solution measures the decomposition of ferric ions to ferrous ions, which are complexed by 1,10 phenanthroline and monitored by UV/Vis absorbance at $510 \mathrm{~nm}$. The moles of iron-phenanthroline complex formed are related to moles of photons absorbed. The solutions were prepared and stored in the dark:

1. Potassium ferrioxalate solution: $589.5 \mathrm{mg}$ of potassium ferrioxalate (commercially available from Sigma Aldrich) and $278 \mu \mathrm{L}$ of sulfuric acid (96\%) were added to a $100 \mathrm{~mL}$ volumetric flask and filled to the mark with water (MilliQ grade).

2. Phenantroline solution: $0.2 \%$ by weight of 1,10 -phenanthroline in water $(200 \mathrm{mg}$ in $100 \mathrm{~mL}$ volumetric flask).

3. Buffer solution: to a $100 \mathrm{~mL}$ volumetric flask $4.94 \mathrm{~g}$ of $\mathrm{NaOAc}$ and $1 \mathrm{~mL}$ of sulfuric acid (96\%) were added and filled to the mark with water (MilliQ grade).

The actinometry measurements were done as follows:

1a. $456 \mathrm{~nm}$ LED: $1 \mathrm{~mL}$ of the actinometer solution was added to a quartz cuvette $(I=10 \mathrm{~mm})$. The actinometry solutions (placed $1 \mathrm{~cm}$ away from a $456 \mathrm{~nm}$ LED) were irradiated for specified time intervals $(0,15,30,45,60)$ seconds.

1b. $400 \mathrm{~nm}$ LED: $1 \mathrm{~mL}$ of the actinometer solution was added to a quartz cuvette $(I=10 \mathrm{~mm})$. The actinometry solutions (placed $2 \mathrm{~cm}$ away from a $3 W 400 \mathrm{~nm}$ LED) were irradiated for specified time intervals $(0,15,20,30,40)$ seconds.

2. After irradiation all the actinometer solution was removed and placed in a $10 \mathrm{~mL}$ volumetric flask. $0.5 \mathrm{~mL}$ of 1,10 -phenanthroline solution and $2 \mathrm{~mL}$ of buffer solution was added to this flask and filled to the mark with water (MilliQ grade).

3. The UV-Vis spectra of actinometry samples were recorded for each time interval (Figure S5 and Figure S6). The absorbance of the actinometry solution was monitored at $510 \mathrm{~nm}$. 


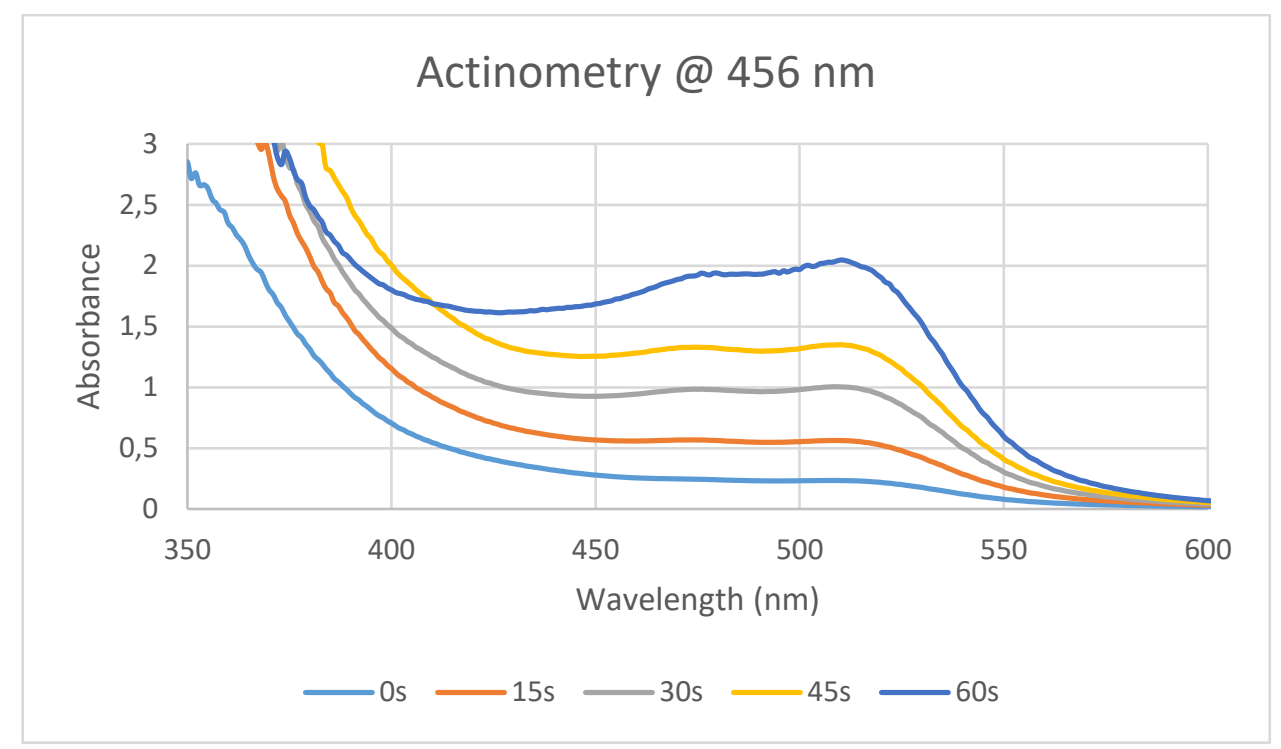

Figure S5 UV-Vis spectra of actinometry samples irradiated with $456 \mathrm{~nm}$ light for the indicated time intervals

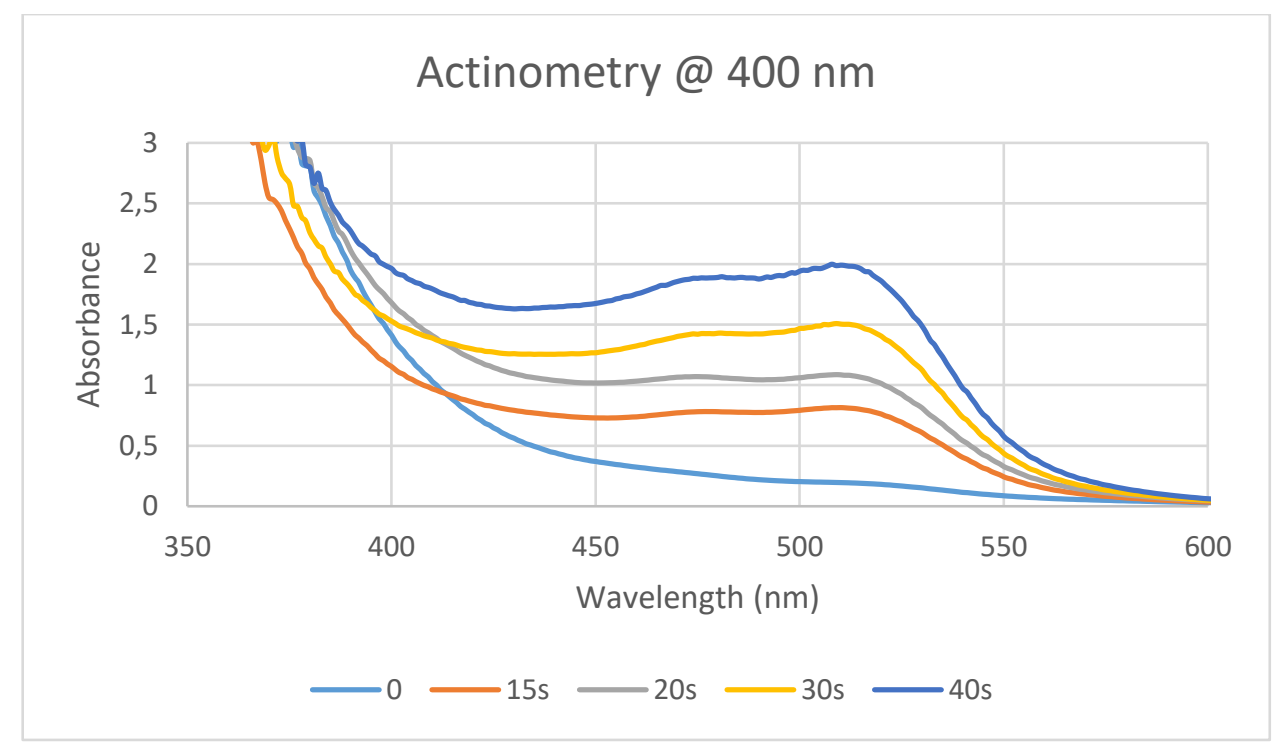

Figure S6 UV-Vis spectra of actinometry samples irradiated with $400 \mathrm{~nm}$ light for the indicated time intervals

4. The moles of $\mathrm{Fe}^{2+}$ formed for each sample are determined using Beers' Law:

$$
\text { Moles } \mathrm{Fe}^{2+}=\frac{V_{1} V_{3} \Delta A_{(510 \mathrm{~nm})}}{10^{3} V_{2} l \varepsilon_{(510 \mathrm{~nm})}}
$$

where $V_{1}$ is the irradiated volume $(1 \mathrm{~mL}), V_{2}$ is the aliquot of the irradiated solution taken for the determination of the ferrous ions $(1 \mathrm{~mL}), \mathrm{V}_{3}$ is the final volume after complexation with phenanthroline $(10 \mathrm{~mL}), \mathrm{I}$ is the optical path-length of the irradiation cell $(1 \mathrm{~cm}), \Delta \mathrm{A}_{(510 \mathrm{~nm})}$ the optical difference in absorbance between the irradiated solution and that taken in the dark, $\varepsilon_{(510 \mathrm{~nm})}$ is the molar extinction coefficient of the complex Fe(phen) $)_{3}{ }^{2+}\left(11100 \mathrm{~L} \mathrm{~mol}^{-1} \mathrm{~cm}^{-1}\right)$.

5. The moles of $\mathrm{Fe}^{2+}$ formed $(\mathrm{N})$ are plotted as a function of time (t) (Figure $\mathrm{S} 7$ and Figure S8). The slope is a product of the photon flux (F) and the quantum yield for $\mathrm{Fe}^{2+}\left(\phi\left(\mathrm{Fe}^{2+}\right)=1.13\right)$, since $\mathrm{F}=\mathrm{N} / \phi\left(\mathrm{Fe}^{2+}\right) \mathrm{t}$.

$456 \mathrm{~nm}$ : The $\mathrm{F}_{456 \mathrm{~nm}}$ was determined to be $2.20 \cdot 10^{-8}$ einstein $\mathrm{s}^{-1}$.

$400 \mathrm{~nm}$ : The $\mathrm{F}_{400 \mathrm{~nm}}$ was determined to be $3.52 \cdot 10^{-8}$ einstein $\mathrm{s}^{-1}$. 


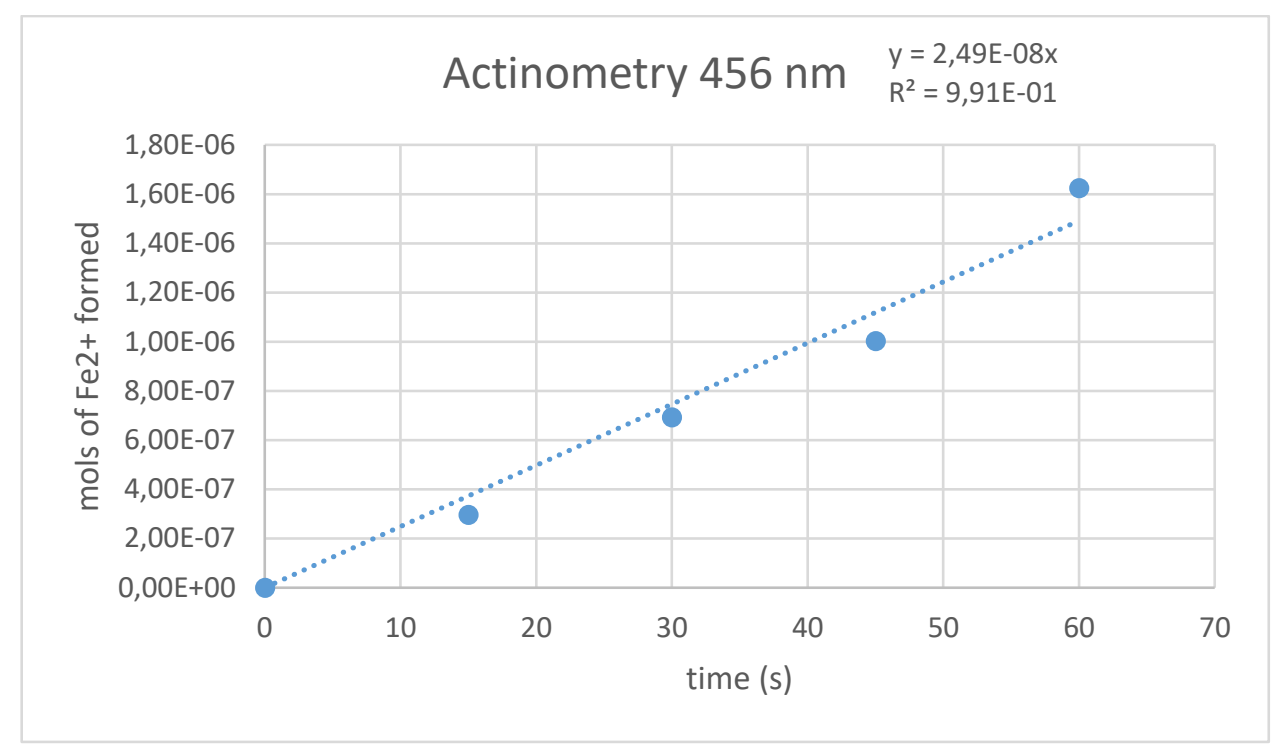

Figure S7 Moles of $\mathrm{Fe}^{2+}$ formed after irradiation with $456 \mathrm{~nm}$ light as a function of time

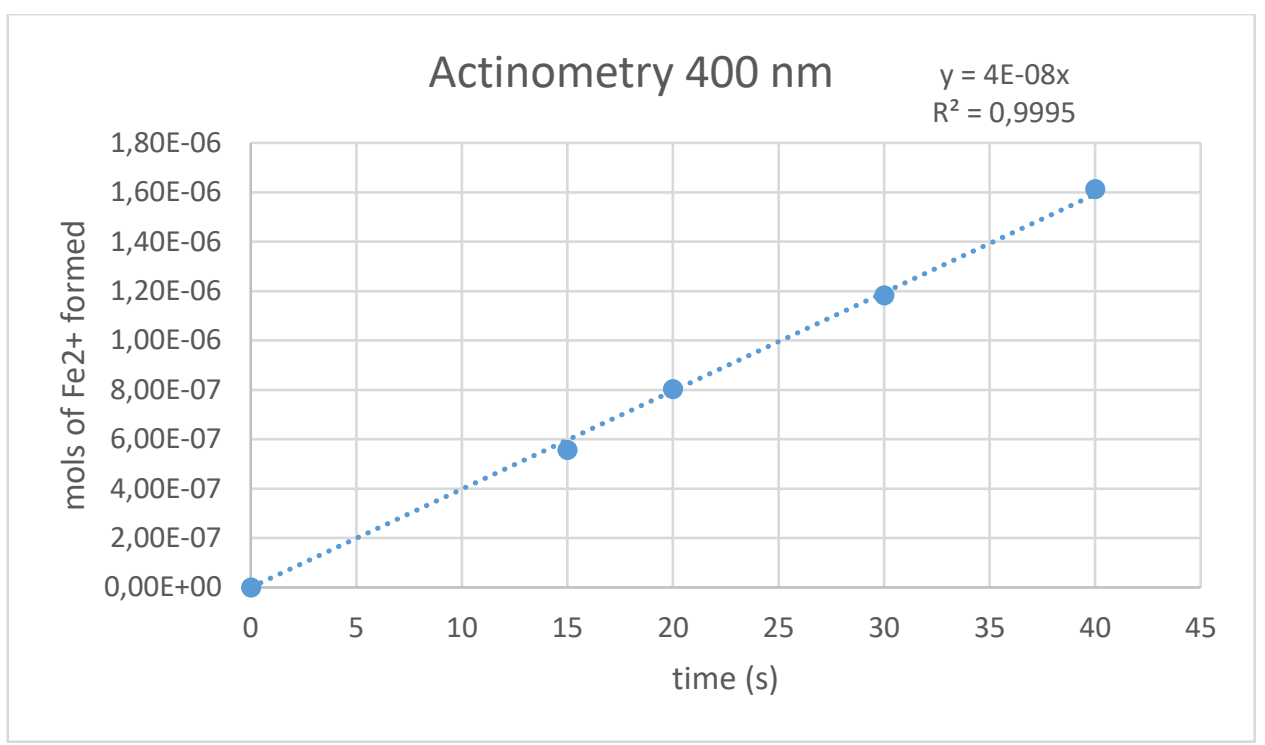

Figure S8 Moles of $\mathrm{Fe}^{2+}$ formed after irradiation with $400 \mathrm{~nm}$ light as a function of time

Since the ferrioxalate actinometer absorbance at $456 \mathrm{~nm}$ is 0.12 and at $400 \mathrm{~nm}$ is 0.47 and inferior to 2 in both cases (if is major than 2 at the wavelength used, it can be assumed that the entire incident light is absorbed), a correction factor (C) based on the fraction of light absorbed by the actinometer has to be considered to calculate the photon flux and the final quantum yield of the reaction (note that this is also needed if the absorbance of the reaction under study at the optimized concentration is inferior to 2 at the wavelength used). Thus, according to the definition of quantum yield: ${ }^{[17]}$

$$
\Phi_{\mathrm{Fe} 2+}=\frac{\left(\frac{\partial_{\text {moles } F e 2+}}{\partial_{\text {time }}}\right)}{F C_{F e 2+}} \rightarrow F=\frac{\left(\frac{\partial_{\text {moles } F e 2+}}{\partial_{\text {time }}}\right)}{\Phi_{F e 2+} C_{F e 2+}} \quad C=1-10^{-A(\lambda)}
$$

The photon flux (F) previously found must be divided by the appropriate correction factor $\mathrm{C}$.

$456 \mathrm{~nm}: \mathrm{C}=0.24$, calculated with $\mathrm{A}_{456 \mathrm{~nm}}=0.12$. Corrected photon flux: $5.33 \cdot 10^{-9}$ einstein s${ }^{-1}$ $400 \mathrm{~nm}: \mathrm{C}=0.66$, calculated with $\mathrm{A}_{400 \mathrm{~nm}}=0.47$. Corrected photon flux: $2.33 \cdot 10^{-8}$ einstein $\mathrm{s}^{-1}$ 
6. $1 \mathrm{~mL}$ of the model reaction solutions were irradiated using the same systems and the moles of product formed for the reaction of interest are described below. The moles of product formed were determined by ${ }^{19} \mathrm{~F}$ NMR measurements using the peak of triflimide as the internal standard. The number of moles of product per unit of time is related to the number of photons absorbed.

\section{Alpha-trifluoromethoxylation of $1 \mathrm{~b}$ with Pyridinium 2a and 4CzIPN as the photocatalyst}<smiles>C=C(OC(=O)OCc1ccc(F)cc1)c1ccc(OC)cc1</smiles>

$1 b$<smiles>N#Cc1cc[n+](OC(F)(F)F)cc1</smiles>

$2 \mathrm{a}$

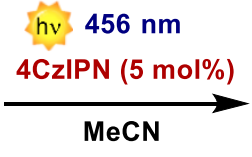

$\mathrm{MeCN}$<smiles>O=C(COC(F)(F)F)c1ccc(F)cc1</smiles>

3

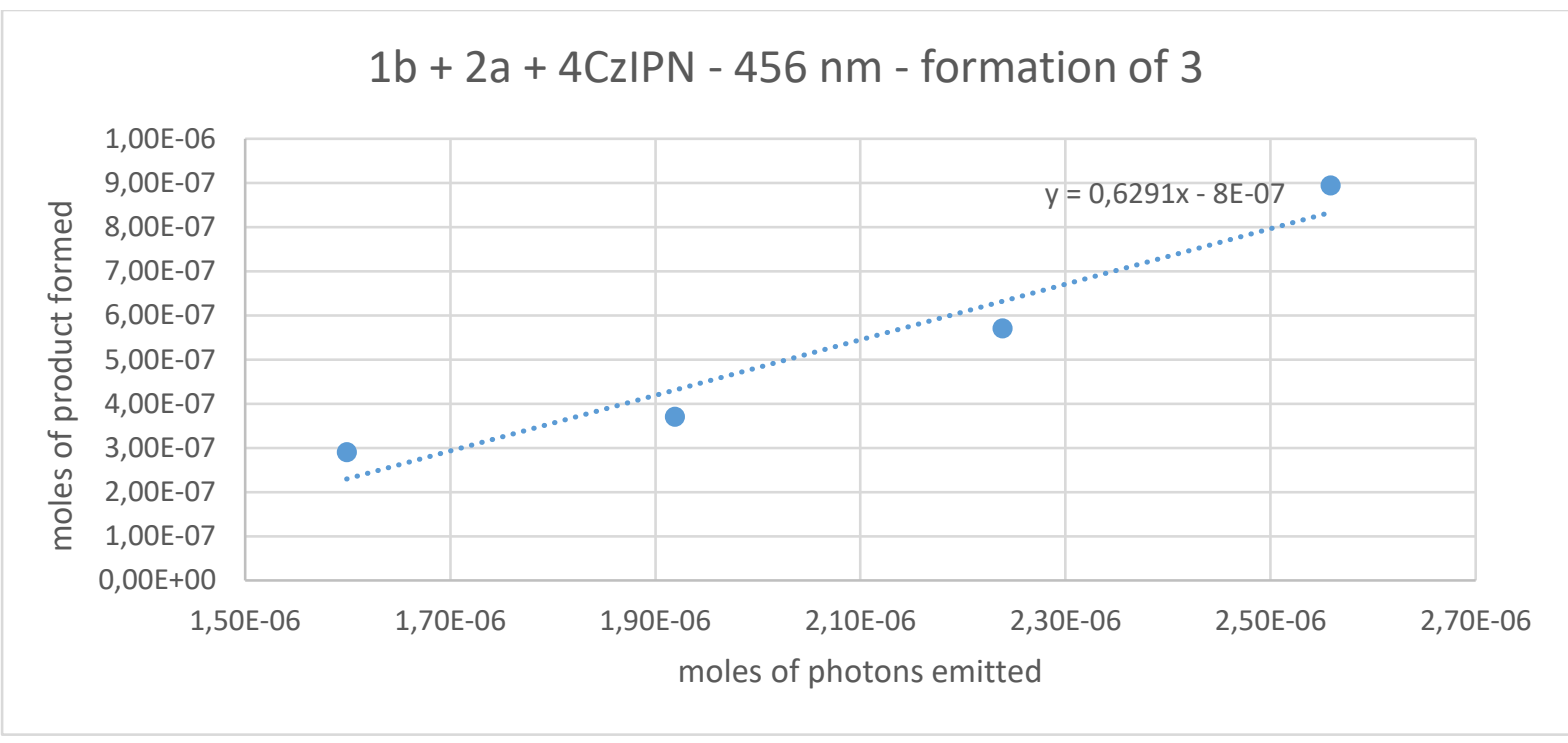

Figure S9 Moles of product formed after irradiation with $456 \mathrm{~nm}$ light as a function of the moles of emitted photons

The absorbance of the reaction at the irradiation wavelength $(456 \mathrm{~nm})$ is $A_{(456 \mathrm{~nm})}>2$, hence no correction factor must be applied since it can be assumed that the entire incident light is absorbed. The quantum yield $(\phi)$ corresponding to the registered slope is 0.63 (Figure 59 ).

According to the IUPAC definition of the quantum yield, ${ }^{[17]}$ we also calculated it considering the overall consumption of $\mathbf{2 a}$ :

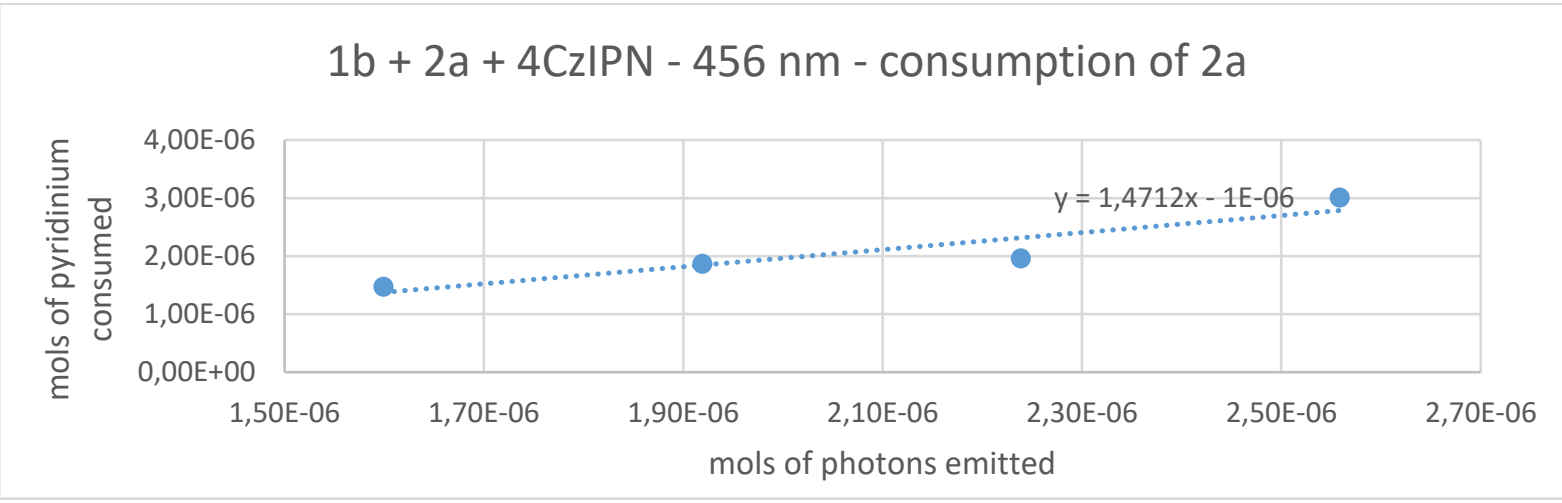

Figure S10 Moles of pyridinium consumed after irradiation with $456 \mathrm{~nm}$ light as a function of the moles of emitted photons

The quantum yield $(\phi)$ corresponding to the registered slope is 1.47 (Figure S10). 
Alpha-trifluoromethoxylation of $1 \mathrm{~b}$ with pyridinium $2 \mathrm{a}$ in the absence of a photocatalyst

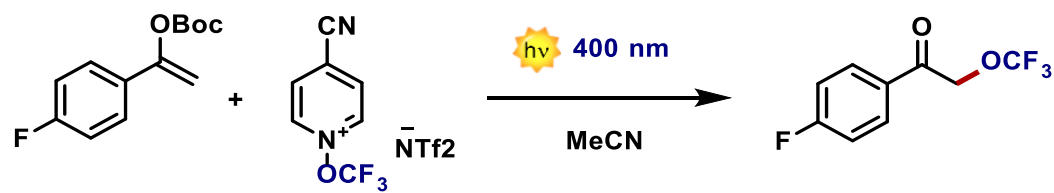

1b

2a

3

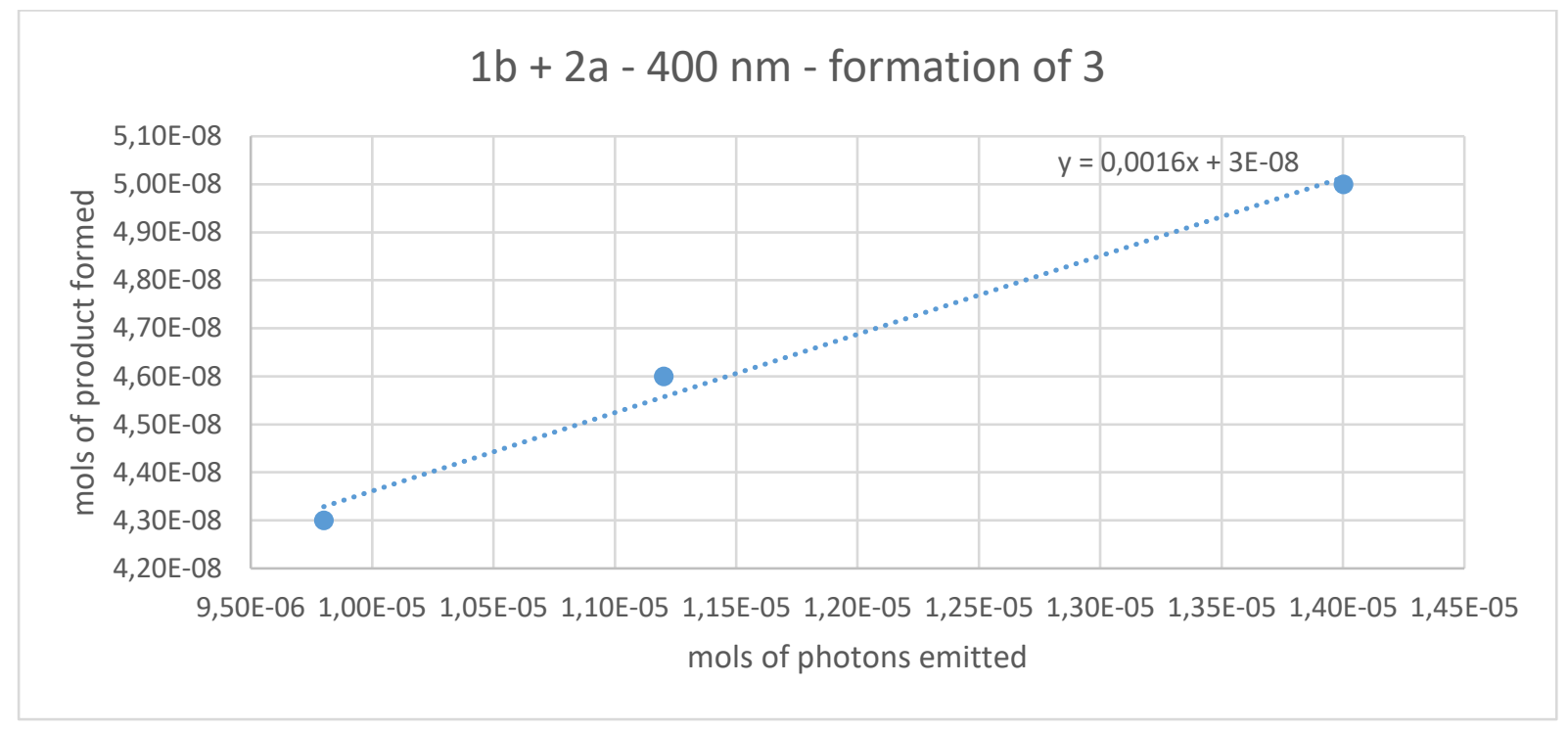

Figure S11 Moles of product formed after irradiation with $400 \mathrm{~nm}$ light as a function of the moles of emitted photons

The registered slope is 0.0016 . The absorbance of the reaction at the irradiation wavelength $(400 \mathrm{~nm})$ is $\mathrm{A}_{(400 \mathrm{~nm})}=0.086$ (Figure S11). The registered slope must be divided by the correction factor $C=1-$ $10^{-A(400 \mathrm{~nm})}=0.18$ to obtain the quantum yield $\phi=0.0089$.

Alpha-trifluoromethoxylation of $1 \mathrm{~b}$ with pyridinium $2 \mathrm{~b}$ and $4 \mathrm{CzIPN}$ as the photocatalyst<smiles>C=C(OC(=O)OCc1ccc(F)cc1)c1ccc(O)cc1</smiles>

$1 b$<smiles>COc1cc[n+](OC(F)(F)F)cc1</smiles>

$2 b$

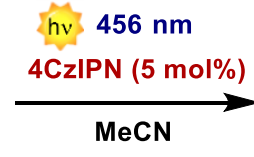

3

After 30 minutes of irradiation, no product 3 could be detected by ${ }^{19} \mathrm{~F}$ NMR. Hence, the quantum yield can be estimated to be $\phi<0.01$.

\section{Chain length calculation}

The length $L$ of the radical chain propagation can be calculated according to the following equation: ${ }^{[18]}$

$L=\frac{\Phi}{Q}$

with

$$
Q=\frac{k_{q}[\text { quencher }]}{\tau_{0}^{-1}+k_{q}[\text { quencher }]+\text { other processes }}
$$

Where $\tau_{0}$ is the lifetime of the photocatalyst and $k_{q}$ is the quenching rate constant obtained from SternVolmer analysis and "other processes" include quenching of the photocatalyst through non-productive pathways. The quenching fraction $Q$ expresses the ratio of the rate at which the excited photocatalyst is productively quenched by $2 a$ to the sum of the rates of all the relaxation processes available to the excited state. Based on our mechanistic hypothesis (see main text Figure 2), assuming that i) the 
quenching of 4 CzIPN by $\mathbf{2 a}$ is the only productive pathway for the reaction, ii) the quenching of $4 \mathrm{CzIPN}$ by $\mathbf{1 b}$ is not productive (falling under "other processes"), and iii) considering $\tau_{0}=5.1 \mu \mathrm{s}$ for 4 CzIPN: ${ }^{[15]}$

$L=\frac{\Phi}{Q}=\frac{0.63}{0.33}=1.9 \quad$ with $\quad Q=\frac{5.7 \cdot 10^{7} \mathrm{M}^{-1} \mathrm{~S}^{-1} \cdot 0.01 \mathrm{M}}{\left(5.1 \cdot 10^{-6}\right)^{-1}+5.7 \cdot 10^{7} \mathrm{M}^{-1} \mathrm{~S}^{-1} \cdot 0.01 \mathrm{M}+1.9 \cdot 10^{7} \mathrm{M}^{-1} \mathrm{~S}^{-1} \cdot 0.05 \mathrm{M}}=0.33$

The calculated length of the radical chain propagation $L=1.9$, suggests that about 2 cycles of radical propagation are carried out between $\mathbf{2 a}$ and intermediate $\mathbf{5}$ termination. 


\section{G. Cyclic Voltammetries}

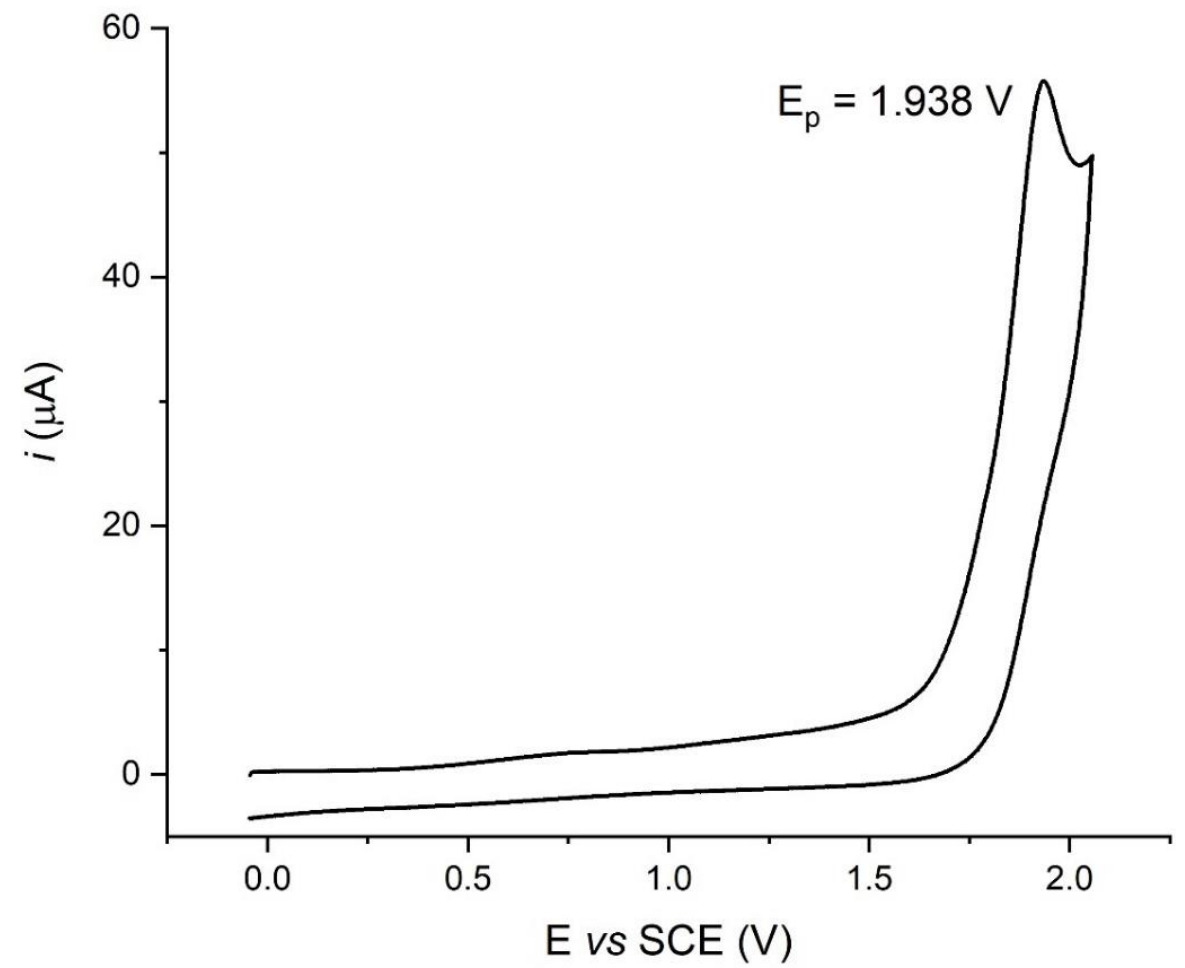

Figure S12 Cyclic Voltammogram of $1 a$ in a supporting electrolyte $0.1 \mathrm{M}\left[\mathrm{Bu}_{4} \mathrm{~N}\right]\left[\mathrm{PF} \mathrm{F}_{6}\right]$ in MeCN referenced to SCE at $0.1 \mathrm{Vs}^{-1}$ sweep rate.

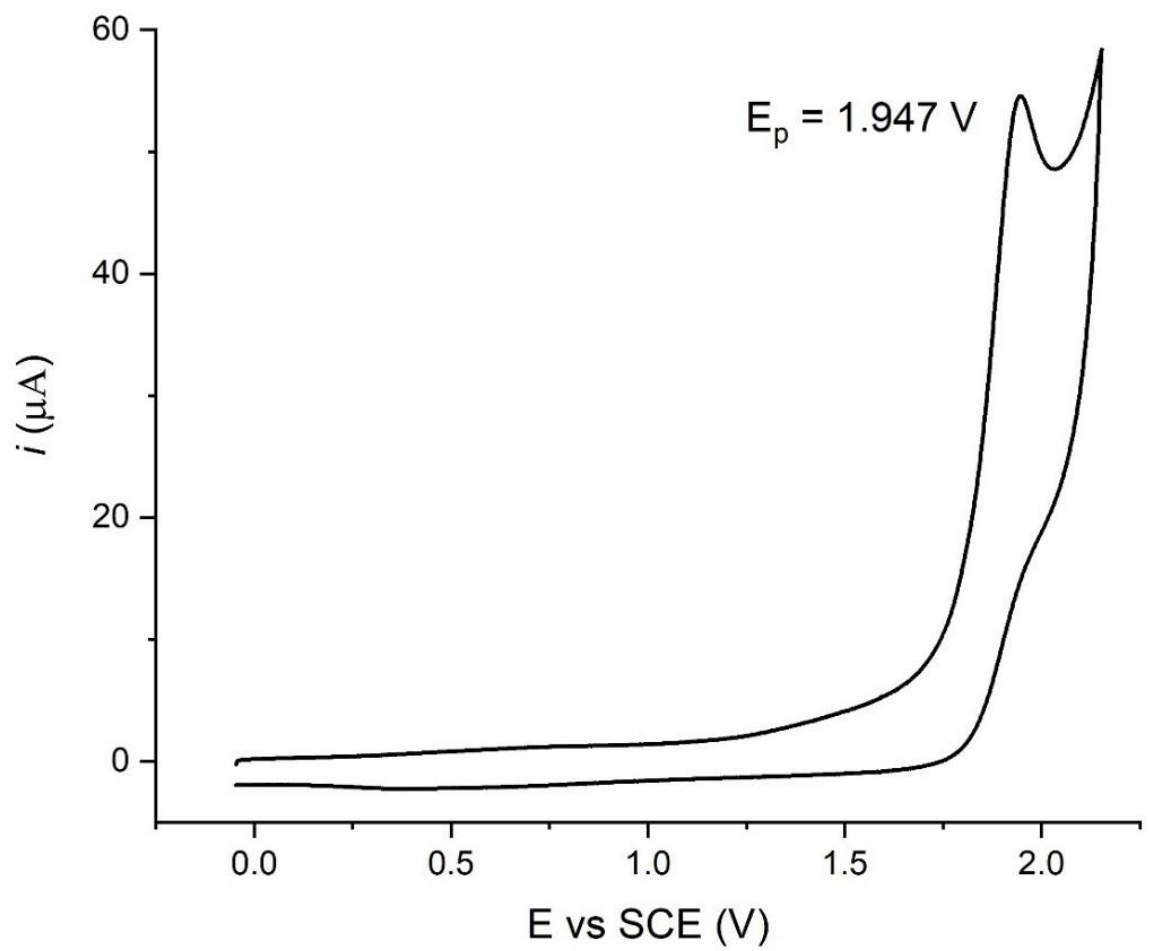

Figure S13 Cyclic Voltammogram of $1 \mathrm{~b}$ in a supporting electrolyte $0.1 \mathrm{M}\left[\mathrm{Bu}_{4} \mathrm{~N}\right]\left[\mathrm{PF} \mathrm{F}_{6}\right]$ in MeCN referenced to SCE at $0.1 \mathrm{Vs}^{-1}$ sweep rate. 


\section{H. UV-Vis spectra}

Figure S14 shows the UV-Vis spectra of $1 \mathrm{a}$ and $2 \mathrm{a}$ and the mixture of both components. The redshift of the absorbance in the orange trace with respect to the individual components suggests the formation of an EDA complex.

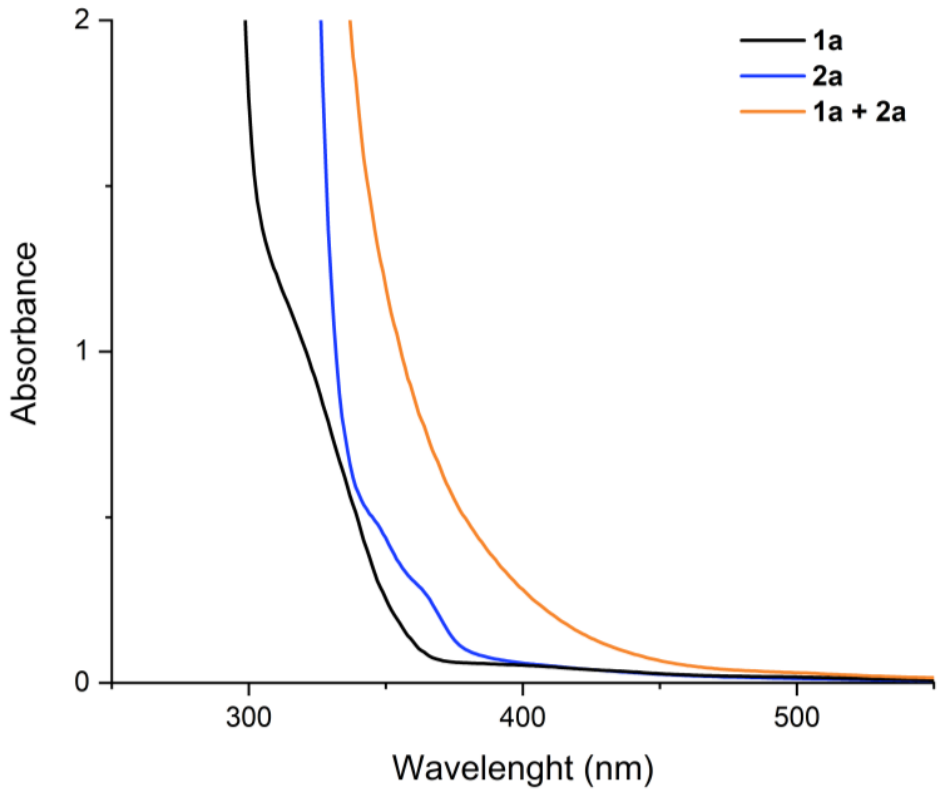

Figure S14 UV-Vis traces of enol acetate $1 a$, pyridinium $2 a$ and the mixture of $1 a$ and $2 a .[1 a]=0.1 \mathrm{M}$ (black trace); [2a]=0.1 M (blue trace); $[1 a]=0.1 \mathrm{M}$ and $[2 a]=0.1 \mathrm{M}$ (orange trace).

Figure S15 shows the UV-Vis traces of $1 \mathrm{a}, 2 \mathrm{a}$, mixture of $1 \mathrm{a}+2 \mathrm{a}(5: 1$ equiv.) and the reaction mixture in the optimized reaction conditions ([2a] $=0.01 \mathrm{M}, 5$ equiv. of enol derivative and $5 \mathrm{~mol} \%$ of $4 \mathrm{CzIPN}$ ).

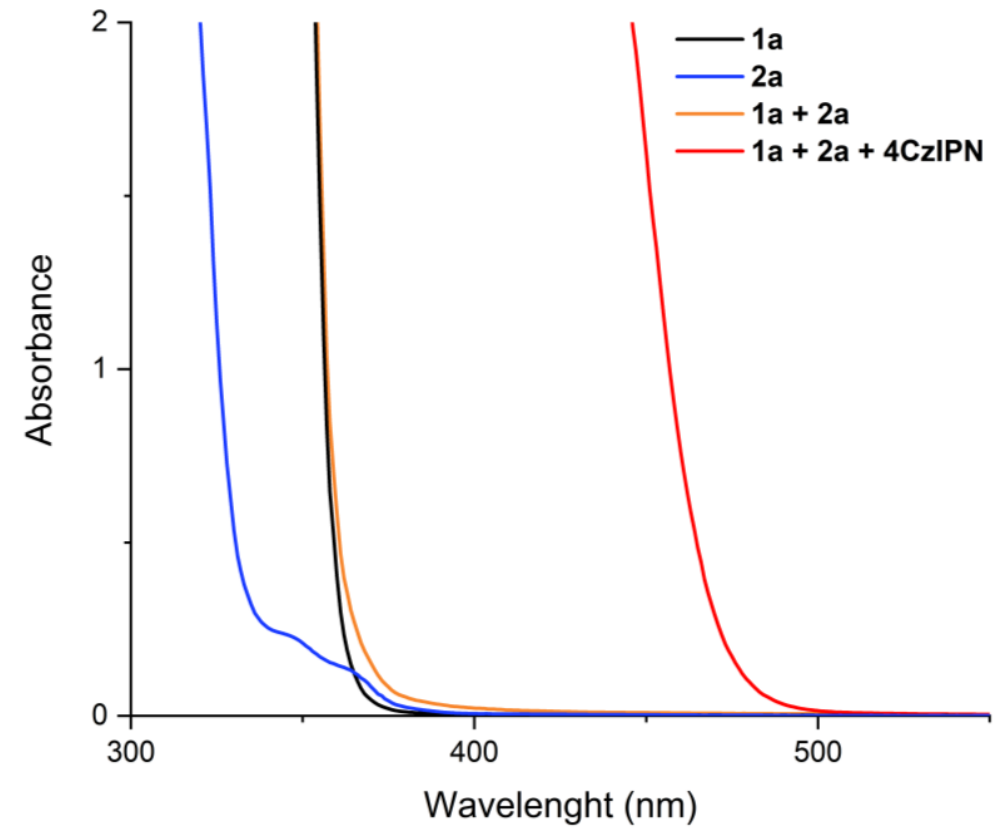

Figure S15 UV-Vis traces of enol acetate $1 a$, pyridinium 2a, mixture of $1 a$ and $2 a$ and the mixture of $1 a$ and $2 a$ with 5 mol\% of $4 C z I P N$. [1a]=0.05 M (black trace); [2a]=0.01 M (blue trace); [1a]=0.05 $\mathrm{M}$ and [2a]=0.01 M (orange trace); [1a]=0.05 M, $[2 a]=0.01 \mathrm{M}$ and $[4 C z I P N]=0.0005 \mathrm{M}$ (red trace). 
Figure S16 shows the UV-Vis traces of $1 b, 2 a$, mixture of $1 b+2 a(5: 1$ equiv.) and the reaction mixture in the optimized reaction conditions ([2a] $=0.01 \mathrm{M}, 5$ equiv. of enol derivative and $5 \mathrm{~mol} \%$ of $4 \mathrm{CzIPN})$.

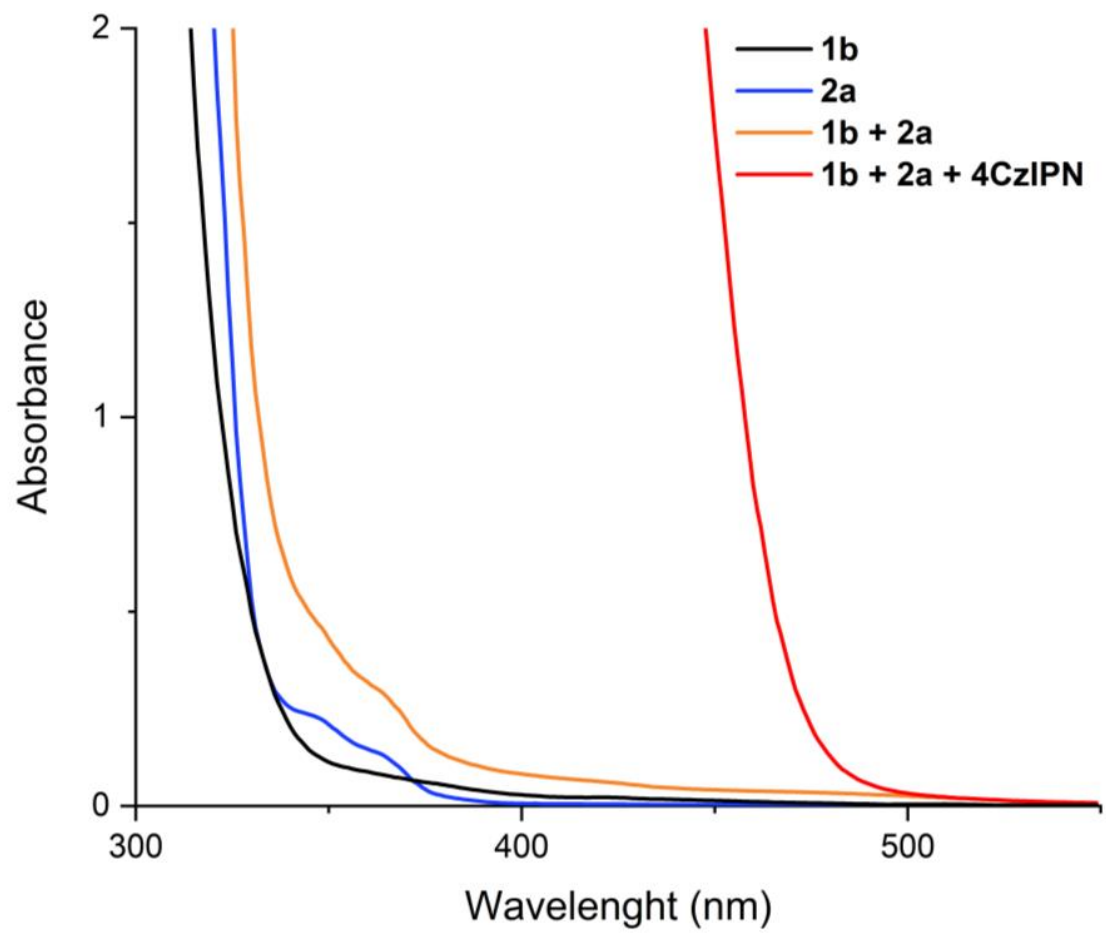

Figure S16 UV-Vis traces of enol carbonate $1 b$, pyridinium $2 a$, mixture of $1 b$ and $2 a$ and the mixture of $1 b$ and $2 a$ with 5 mol\% of 4CzIPN. [1b]=0.05 M (black trace); [2a]=0.01 M (blue trace); [1b]=0.05 M and [2a]=0.01 M (orange trace); [1b]=0.05 M, $[2 a]=0.01 \mathrm{M}$ and $[4 \mathrm{CZIPN}]=0.0005 \mathrm{M}$ (red trace). 


\section{References}

[1] G. Ragazzon, C. Schäfer, P. Franchi, S. Silvi, B. Colasson, M. Lucarini, A. Credi, Proc. Natl. Acad. Sci. 2018, 115, 9385 LP -9390.

[2] J. Mateos, F. Rigodanza, A. Vega-Peñaloza, A. Sartorel, M. Natali, T. Bortolato, G. Pelosi, X. Companyó, M. Bonchio, L. Dell'Amico, Angew. Chemie Int. Ed. 2020, 59, 1302-1312.

[3] E. Speckmeier, T. G. Fischer, K. Zeitler, J. Am. Chem. Soc. 2018, 140, 15353-15365.

[4] F. Houlihan, F. Bouchard, J. M. J. Fréchet, C. G. Willson, Can. J. Chem. 1985, 63, 153-162.

[5] W. Li, Y. Zhu, Y. Zhou, H. Yang, C. Zhu, Tetrahedron 2019, 75, 1647-1651.

[6] S. Sasaki, T. Suzuki, T. Uchiya, S. Toyota, A. Hirano, M. Tanemura, H. Teramoto, T. Yamauchi, K. Higashiyama, J. Fluor. Chem. 2016, 192, 78-85.

[7] A. Parrilla, I. Villuendas, A. Guerrero, Bioorg. Med. Chem. 1994, 2, 243-252.

[8] Q. Xu, H. Zhou, X. Geng, P. Chen, Tetrahedron 2009, 65, 2232-2238.

[9] Y. Hirayama, K. Okuzumi, H. Masubuti, H. Uekusa, J.-P. Girault, Y. Fujimoto, J. Org. Chem. 2014, $79,5471-5477$.

[10] R. Floresca, M. Kurihara, D. S. Watt, A. Demir, J. Org. Chem. 1993, 58, 2196-2200.

[11] L. Da Costa, E. Scheers, A. Coluccia, A. Rosetti, M. Roche, J. Neyts, T. Terme, R. Cirilli, C. Mirabelli, R. Silvestri, et al., Eur. J. Med. Chem. 2017, 140, 528-541.

[12] C. Yates, Metalloenzyme Inhibitor Compounds, 2018, WO2018/165520Al.

[13] Y. A. Davydova, T. M. Sokolenko, Y. L. Yagupolskii, J. Fluor. Chem. 2014, 157, 58-62.

[14] R. Zriba, E. Magnier, J.-C. Blazejewski, Synlett 2009, 2009, 1131-1135.

[15] H. Uoyama, K. Goushi, K. Shizu, H. Nomura, C. Adachi, Nature 2012, 492, 234-238.

[16] J. für Prakt. Chemie 1976, 318, 702-703.

[17] S. E. Braslavsky, Pure Appl. Chem. 2007, 79, 293.

[18] M. A. Cismesia, T. P. Yoon, Chem. Sci. 2015, 6, DOI 10.1039/c5sc02185e. 


\section{J. NMR spectra}

tert-butyl (1-(4-fluorophenyl)vinyl) carbonate 1b

${ }^{1} \mathrm{H}$ NMR (500 MHz, $\mathrm{CDCl}_{3}$ )

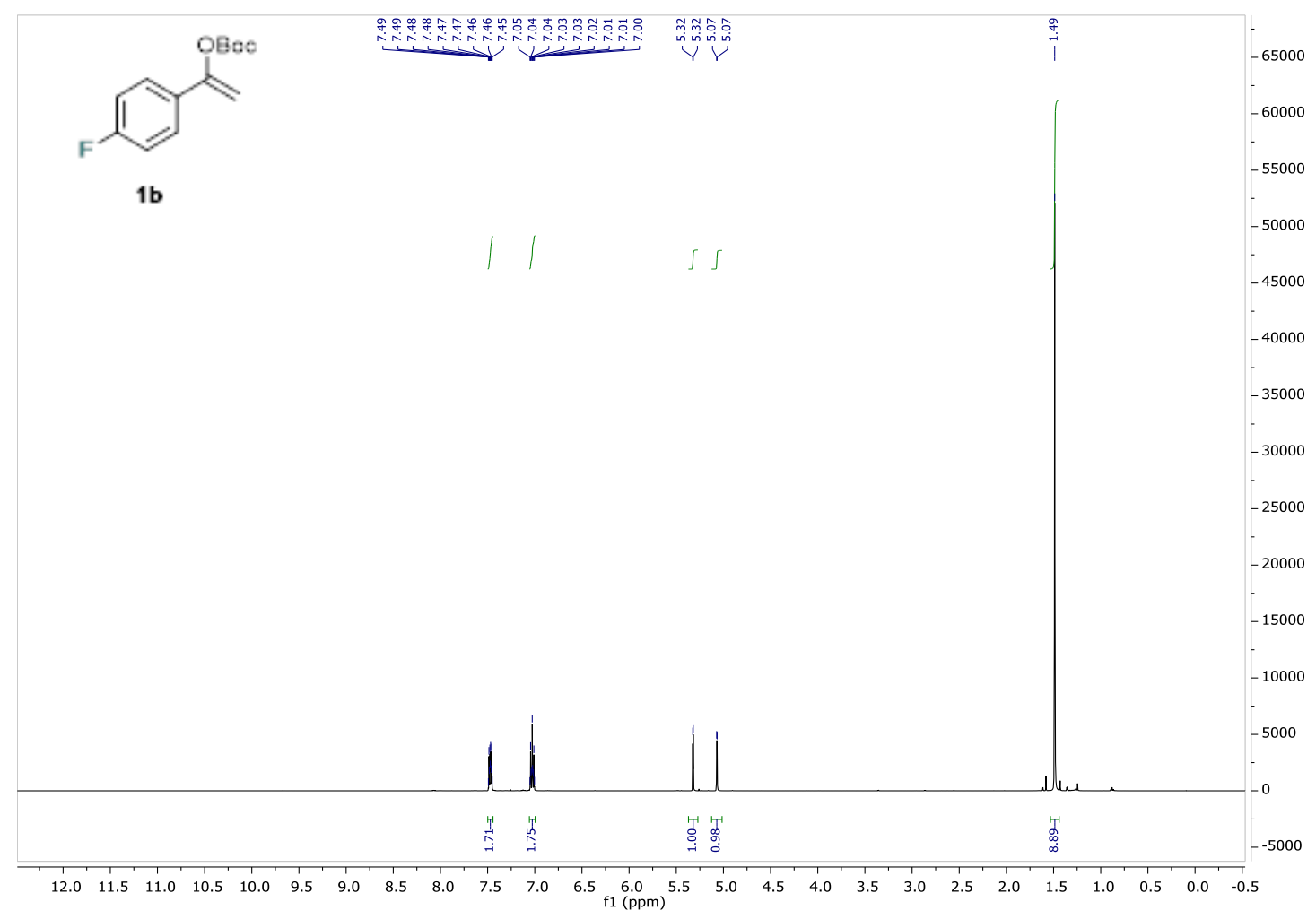

${ }^{13} \mathrm{C}$ NMR (126 MHz, $\left.\mathrm{CDCl}_{3}\right)$

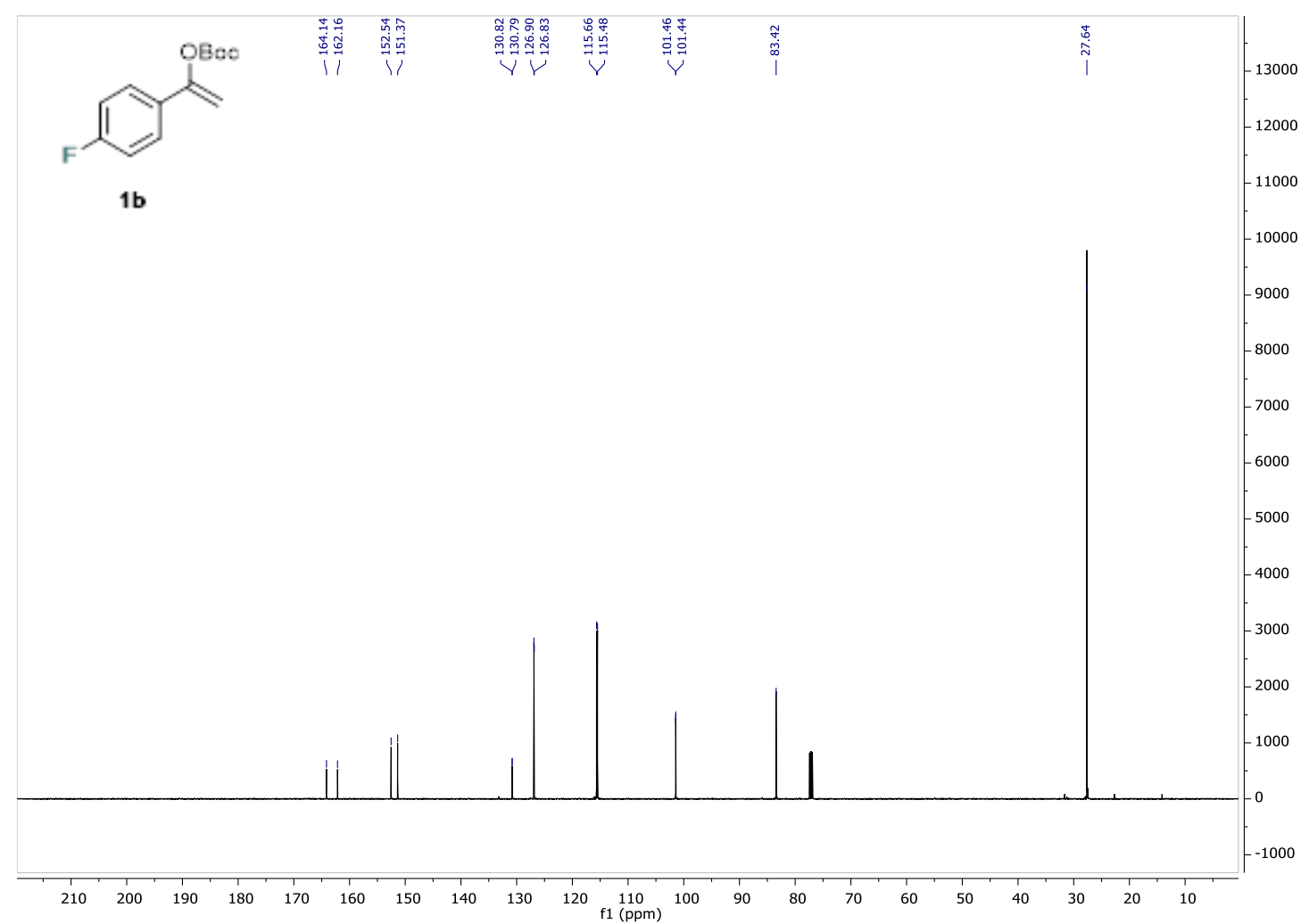


${ }^{19} \mathrm{~F}$ NMR (188 MHz, ${ }^{1} \mathrm{H}$ decoupled, $\mathrm{CDCl}_{3}$ )

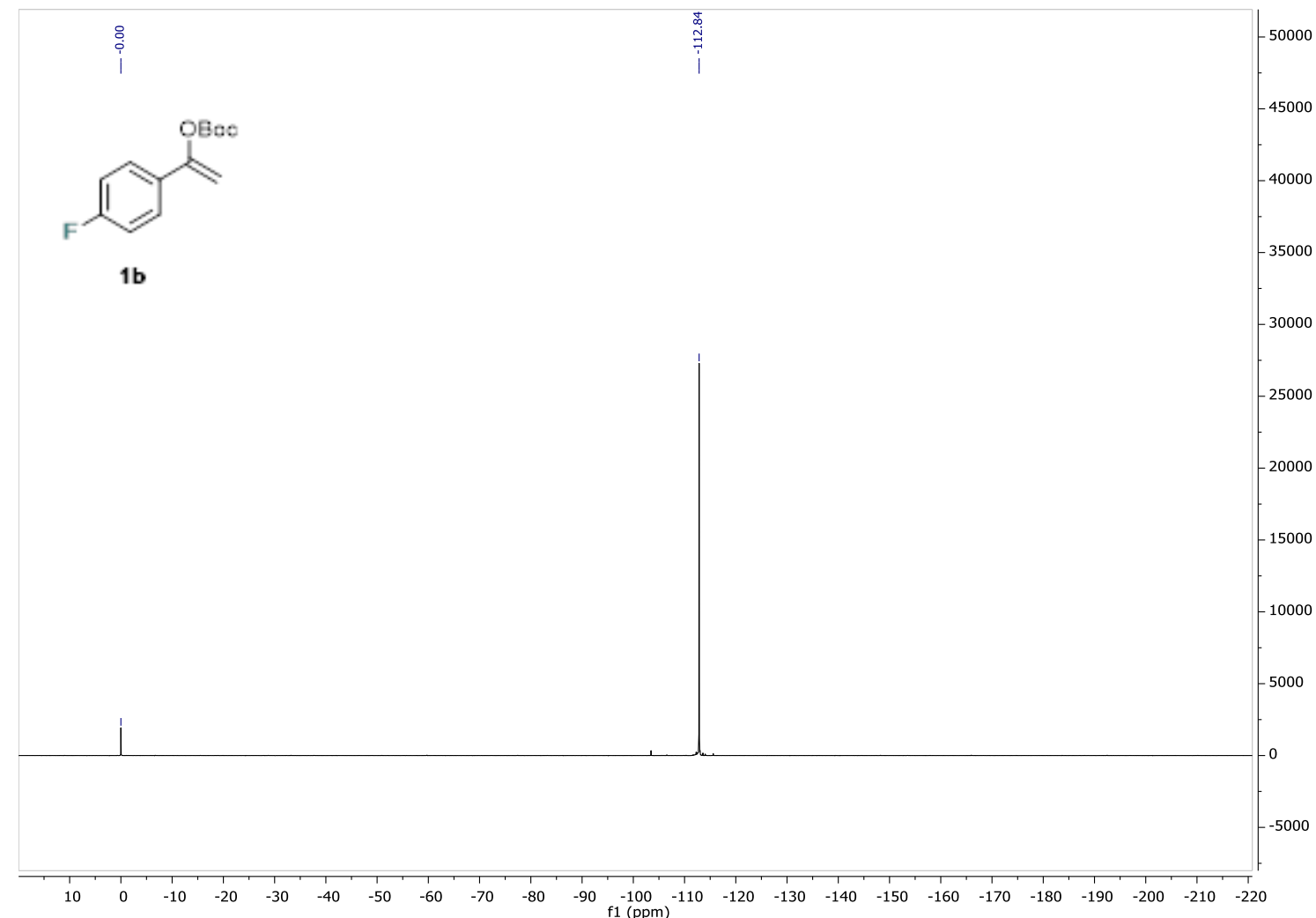

tert-butyl (1-(o-tolyl)vinyl) carbonate 1d

${ }^{1} \mathrm{H}$ NMR $\left(300 \mathrm{MHz}, \mathrm{CDCl}_{3}\right)$

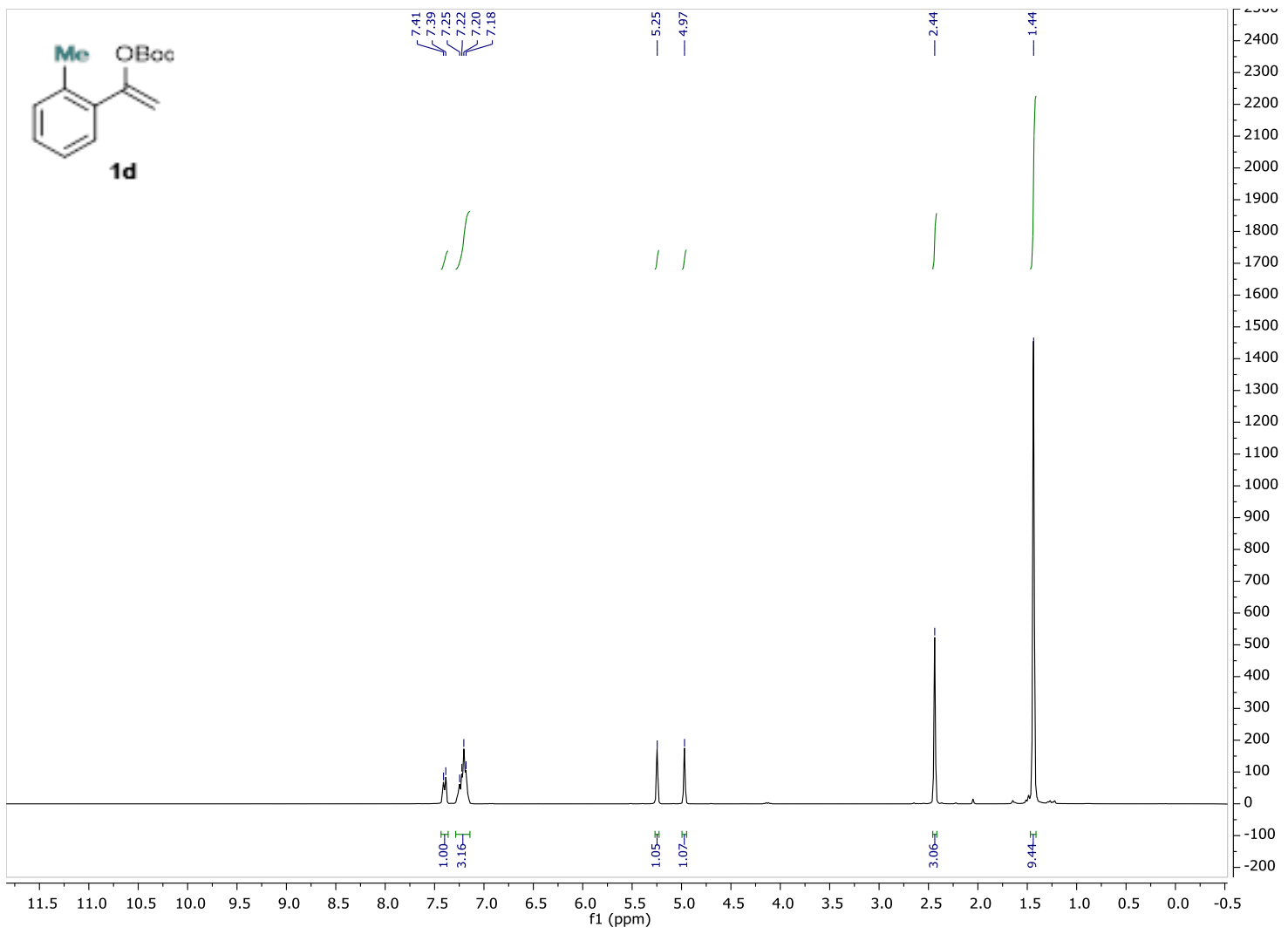


${ }^{13} \mathrm{C}$ NMR (75 MHz, $\mathrm{CDCl}_{3}$ )

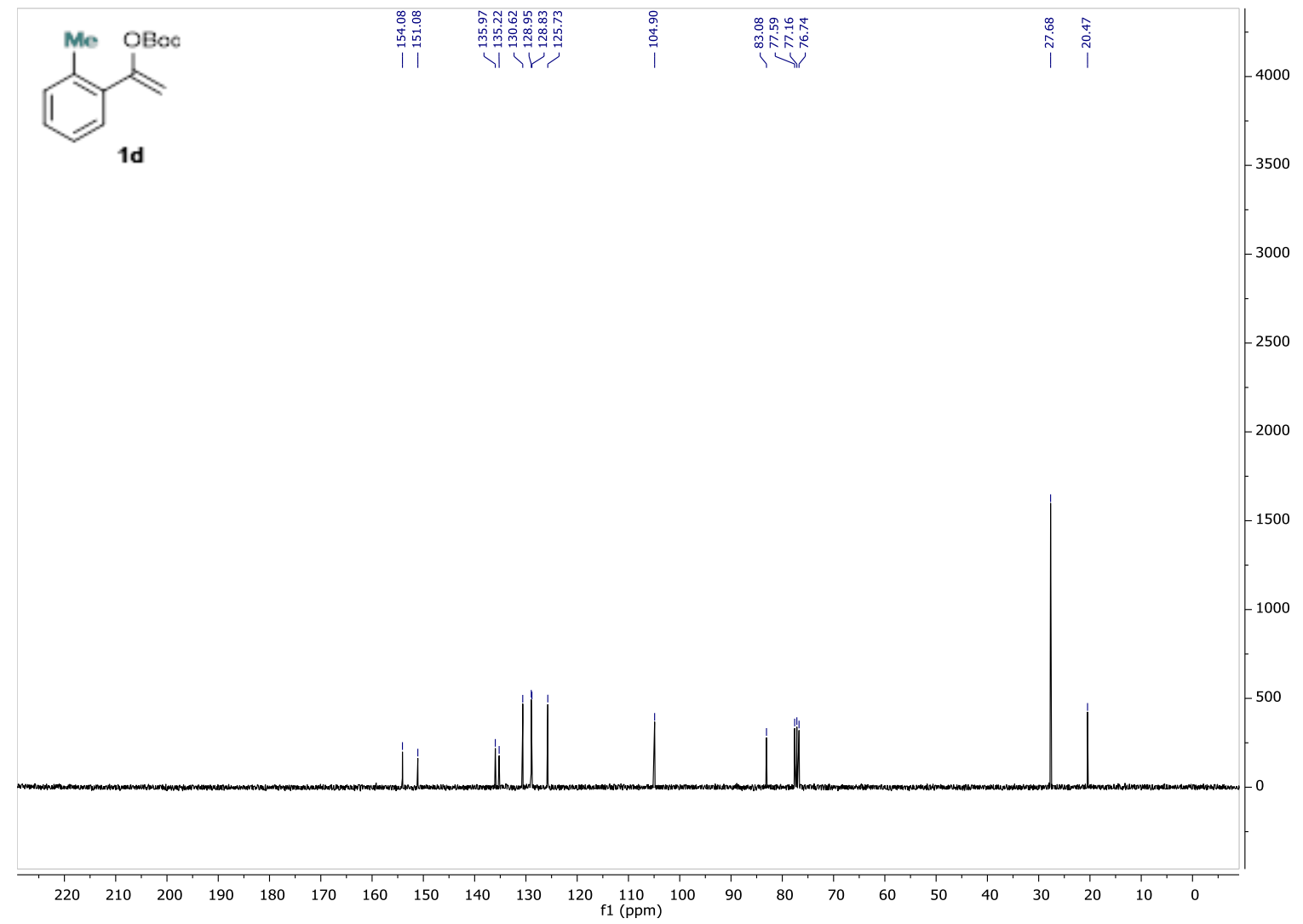

tert-butyl (1-(m-tolyl)vinyl) carbonate 1e

${ }^{1} \mathrm{H}$ NMR (300 MHz, $\mathrm{CDCl}_{3}$ )

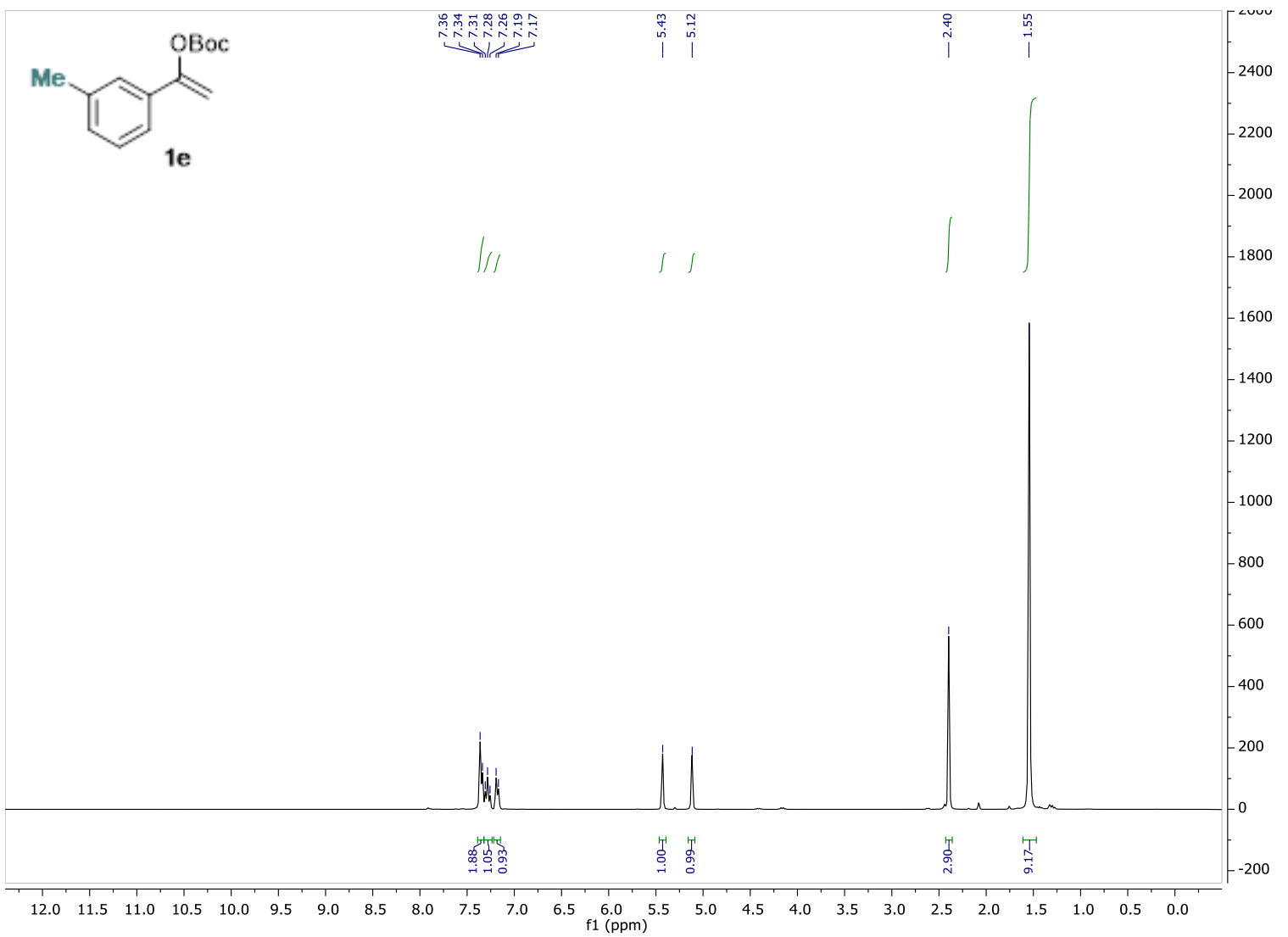


${ }^{13} \mathrm{C}$ NMR $\left(75 \mathrm{MHz}, \mathrm{CDCl}_{3}\right)$

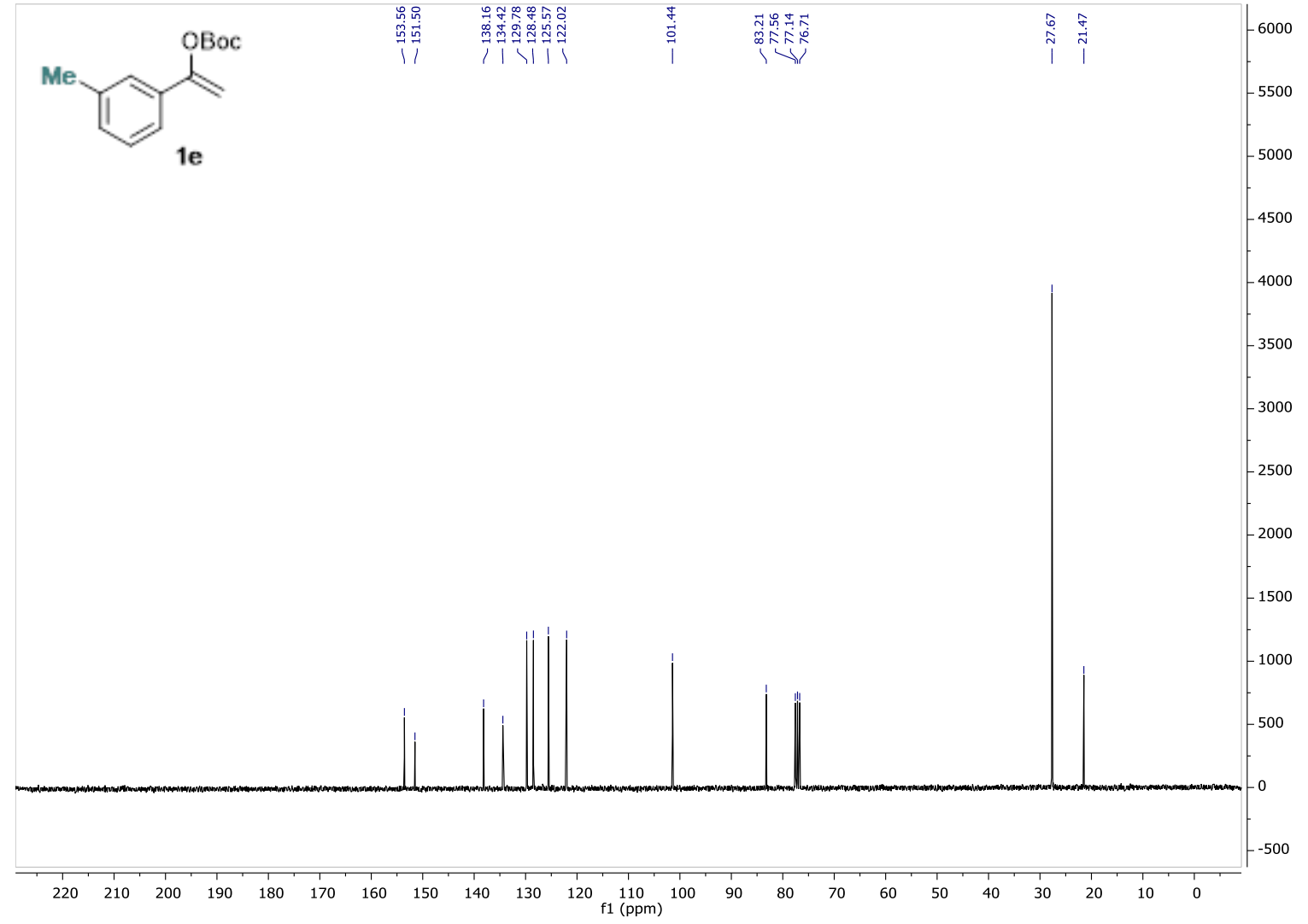

tert-butyl (1-(p-tolyl)vinyl) carbonate 1 f

${ }^{1} \mathrm{H}$ NMR (300 MHz, $\left.\mathrm{CDCl}_{3}\right)$

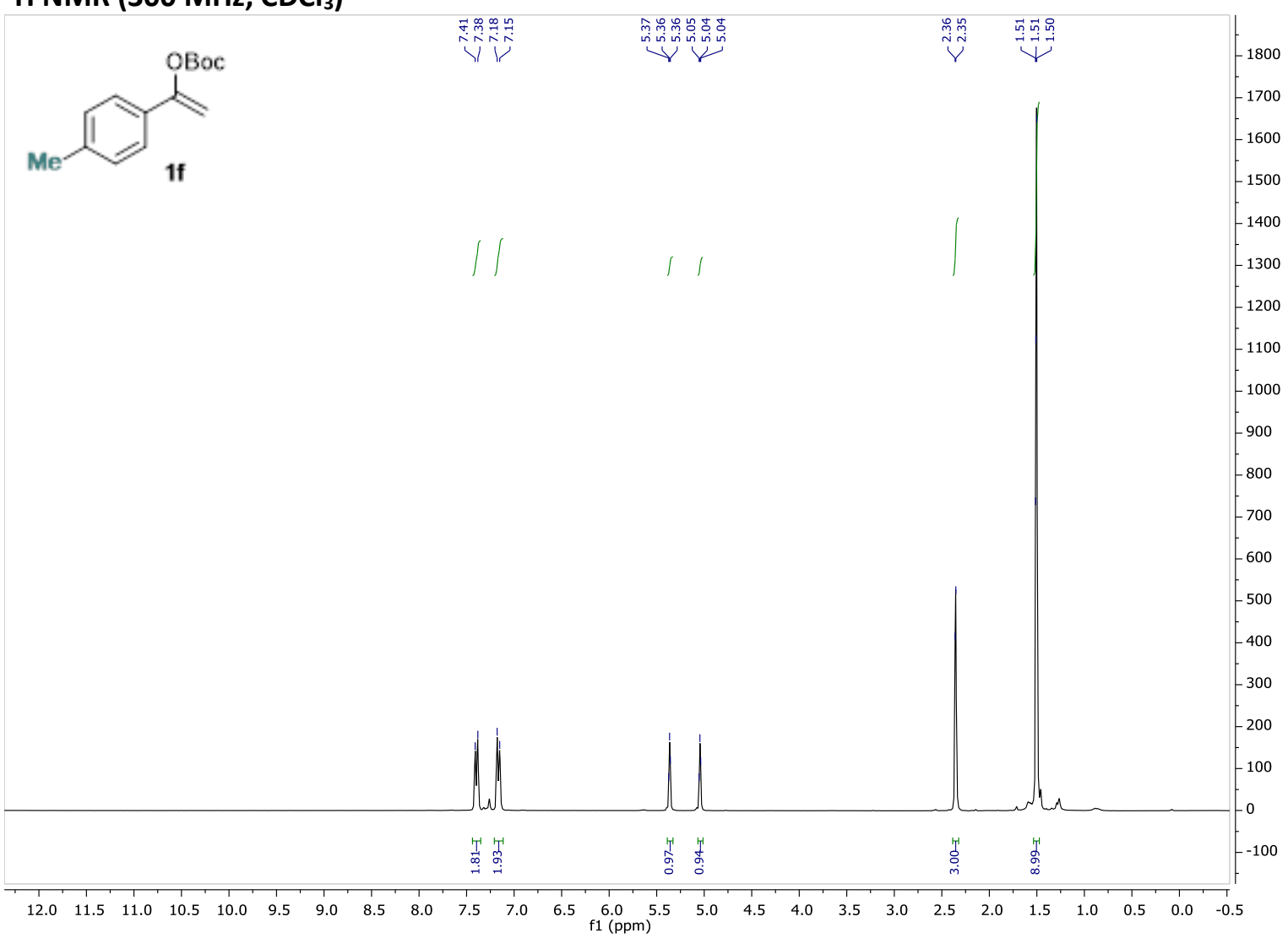


${ }^{13} \mathrm{C}$ NMR $\left(75 \mathrm{MHz}, \mathrm{CDCl}_{3}\right)$

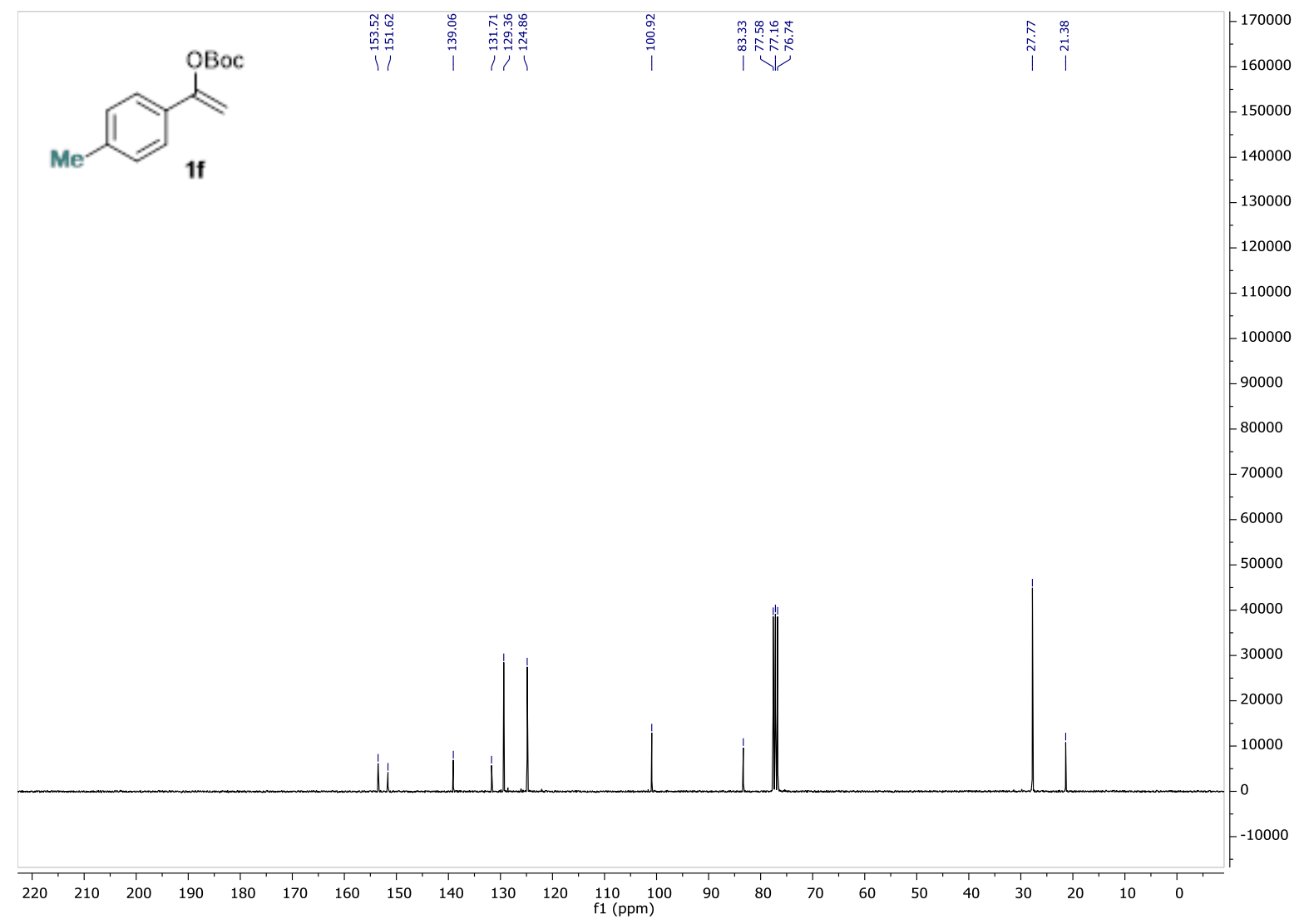

tert-butyl 1-(3-methoxyphenyl)vinyl carbonate $1 \mathrm{~g}$

${ }^{1} \mathrm{H}$ NMR (500 MHz, $\left.\mathrm{CDCl}_{3}\right)$

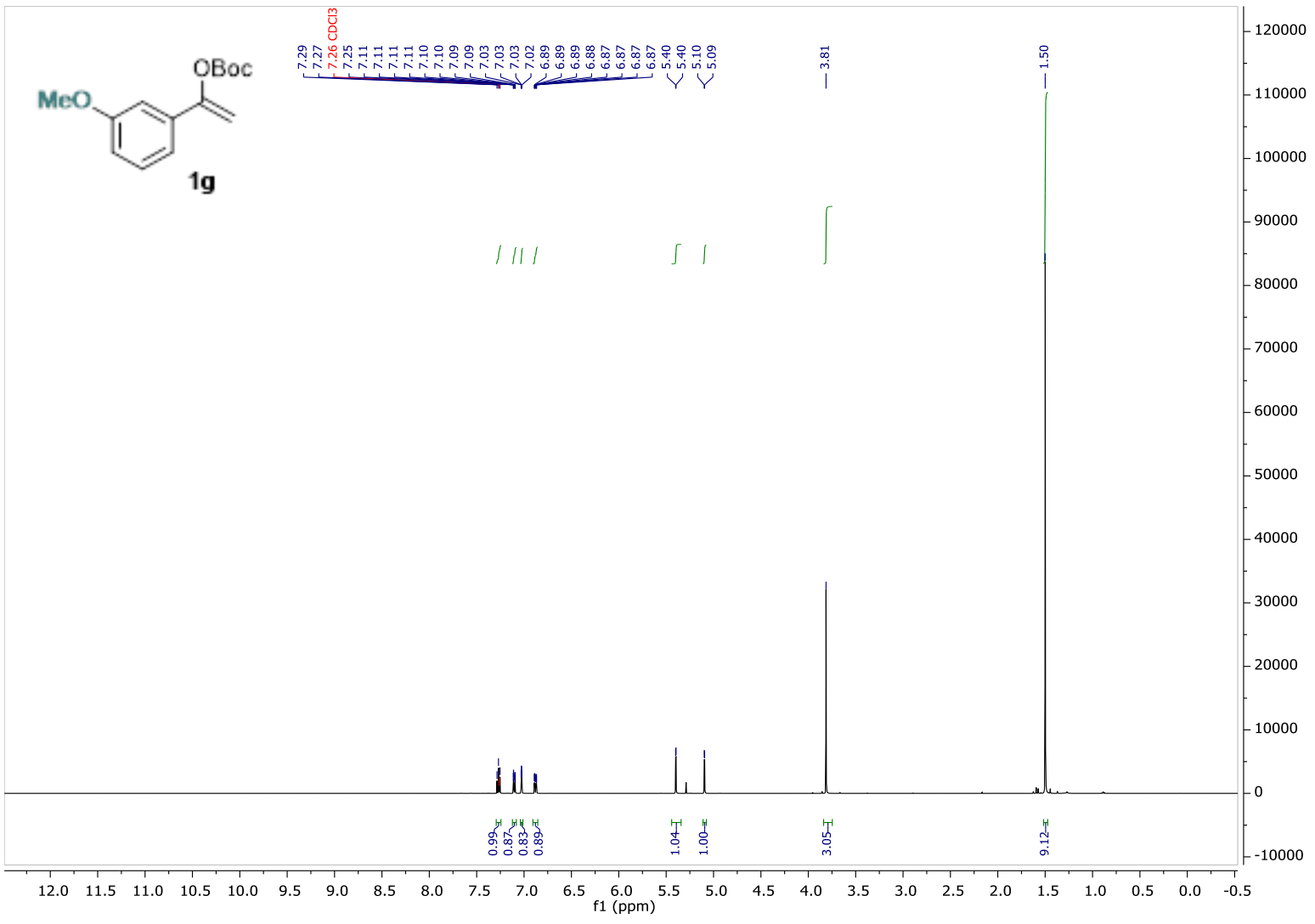


${ }^{13} \mathrm{C}$ NMR (126 MHz, $\left.\mathrm{CDCl}_{3}\right)$

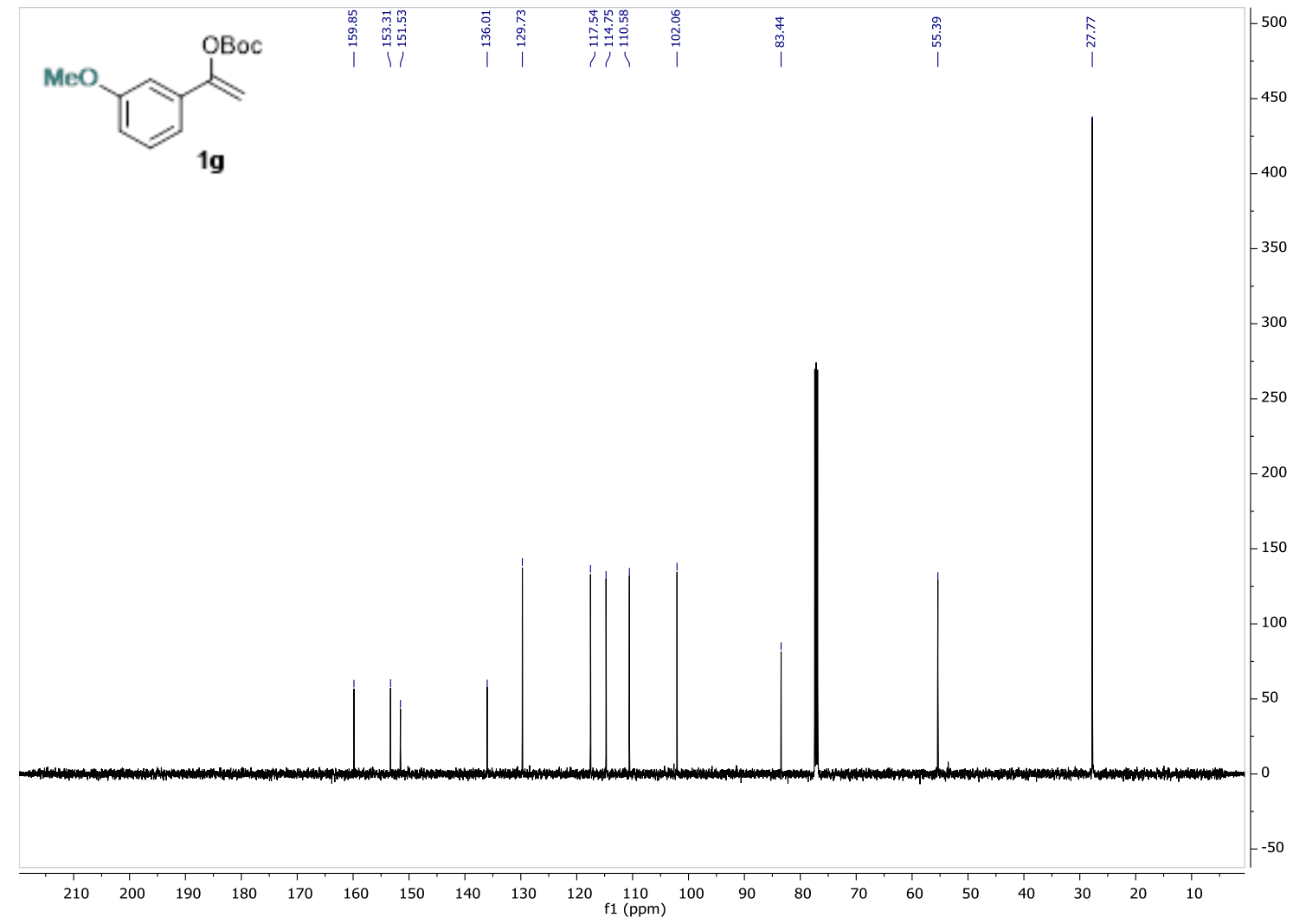

tert-butyl 1-(4-methoxyphenyl)vinyl carbonate $\mathbf{1 h}$

${ }^{1} \mathrm{H}$ NMR $\left(500 \mathrm{MHz}, \mathrm{CDCl}_{3}\right)$

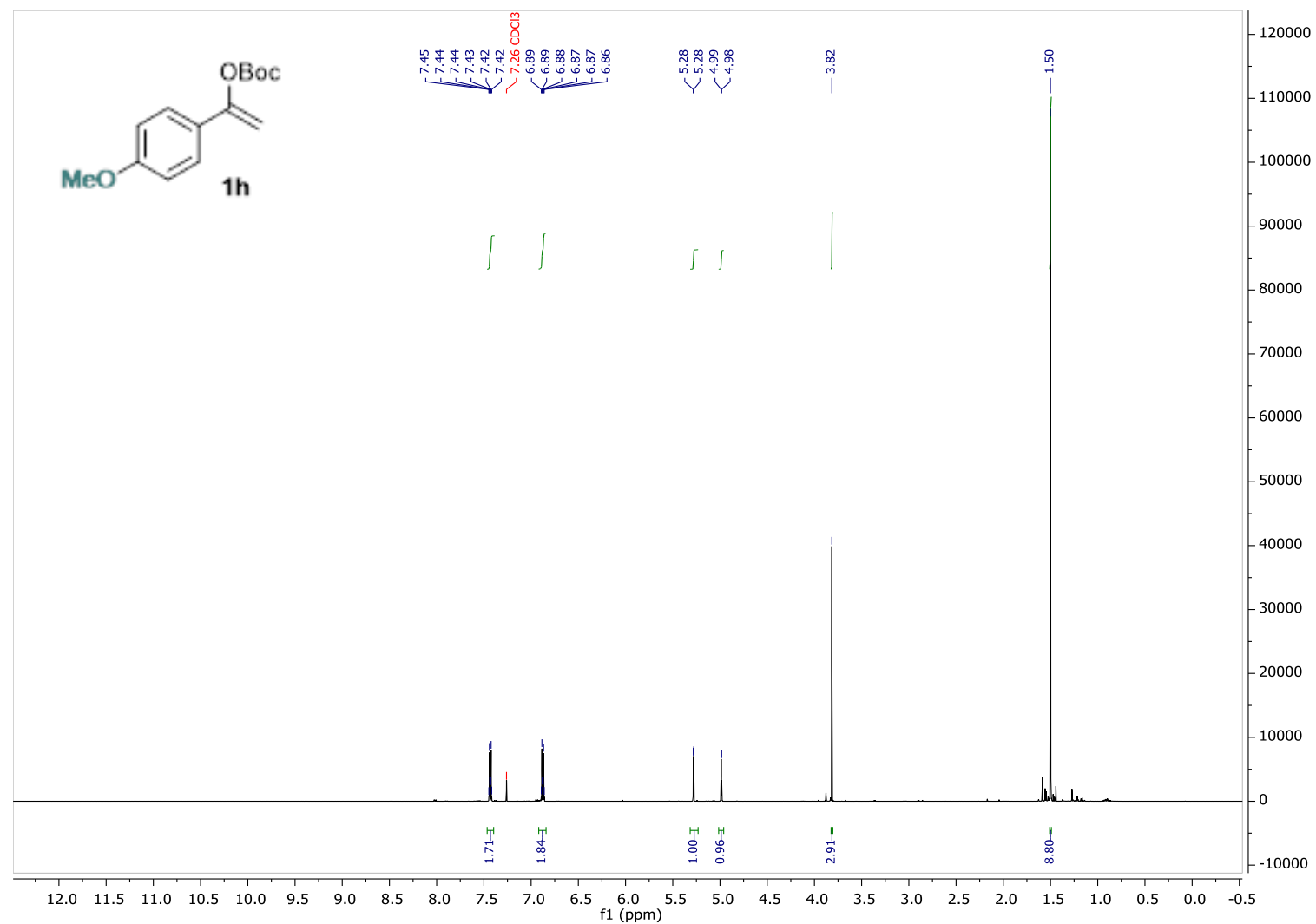


${ }^{13} \mathrm{C}$ NMR (126 MHz, $\left.\mathrm{CDCl}_{3}\right)$

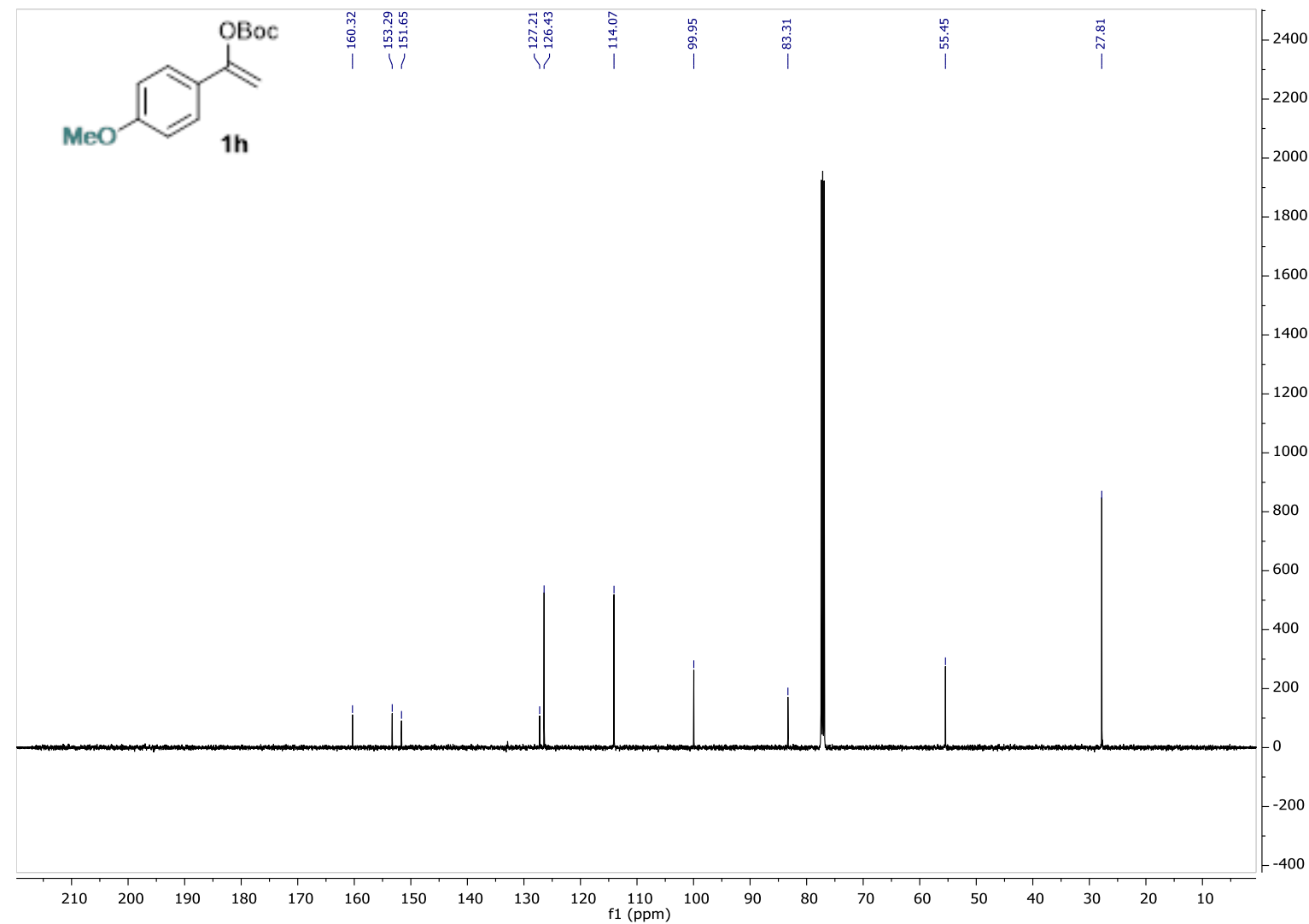

tert-butyl (1-(4-cyanophenyl)vinyl) carbonate $1 \mathrm{i}$

${ }^{1} \mathrm{H}$ NMR $\left(300 \mathrm{MHz}, \mathrm{CDCl}_{3}\right)$

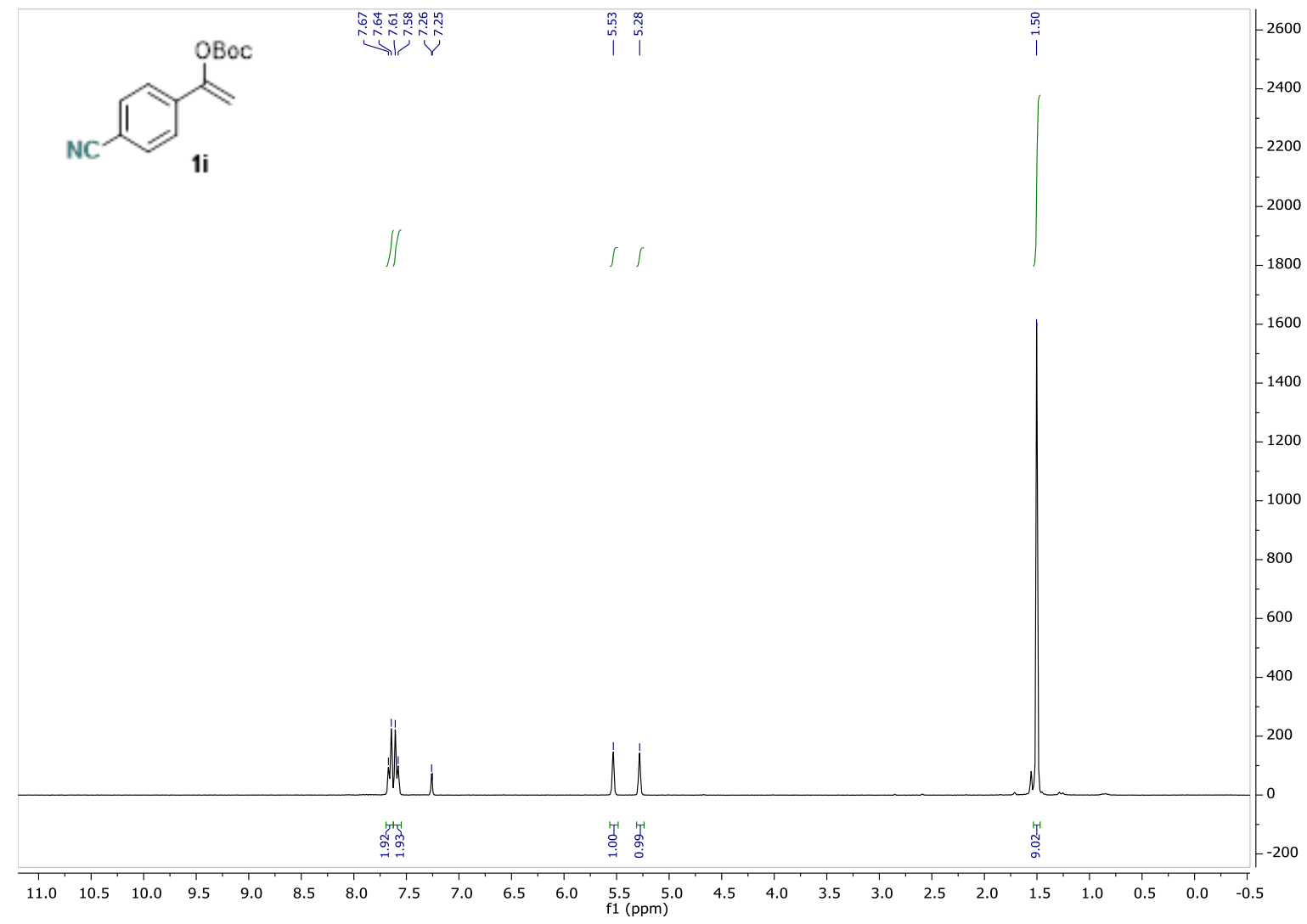


${ }^{13} \mathrm{C}$ NMR $\left(75 \mathrm{MHz}, \mathrm{CDCl}_{3}\right)$

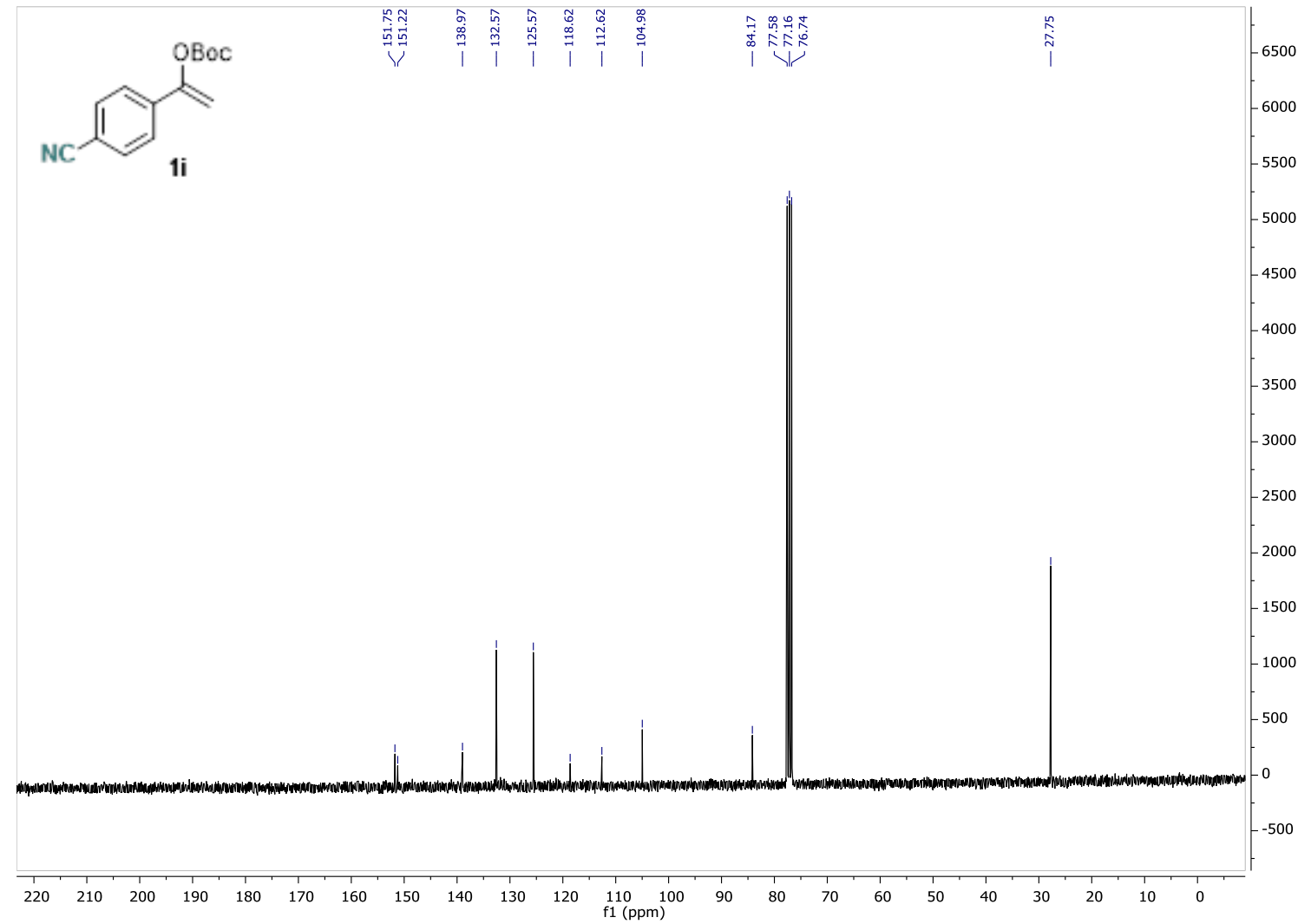

tert-butyl (1-(3-(trifluoromethyl)phenyl)vinyl) carbonate $\mathbf{1 j}$

${ }^{1} \mathrm{H}$ NMR $\left(300 \mathrm{MHz}, \mathrm{CDCl}_{3}\right)$

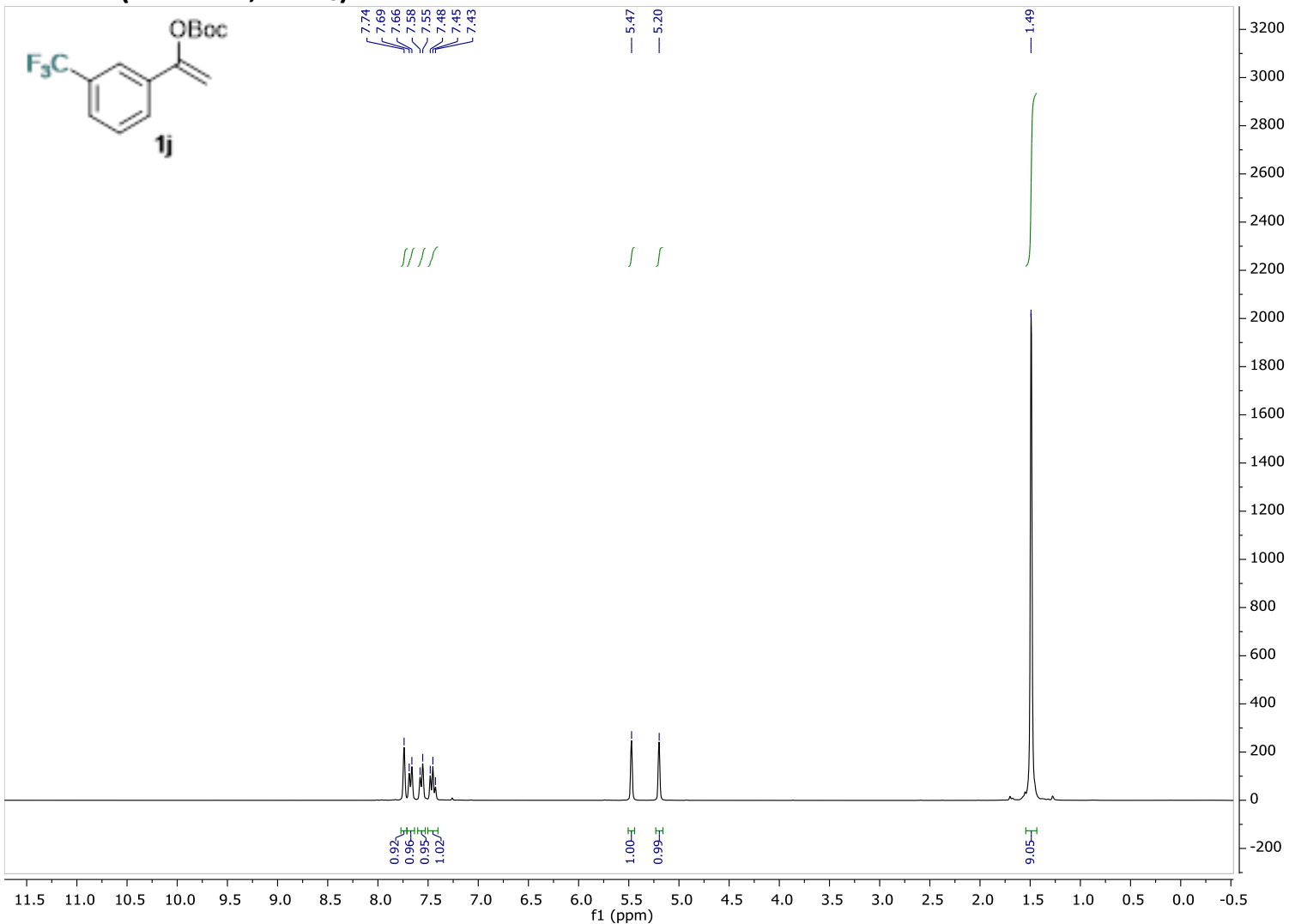


$\left.{ }^{13} \mathrm{C} \mathrm{NMR} \mathrm{(75} \mathrm{MHz,} \mathrm{CDCl}_{3}\right)$

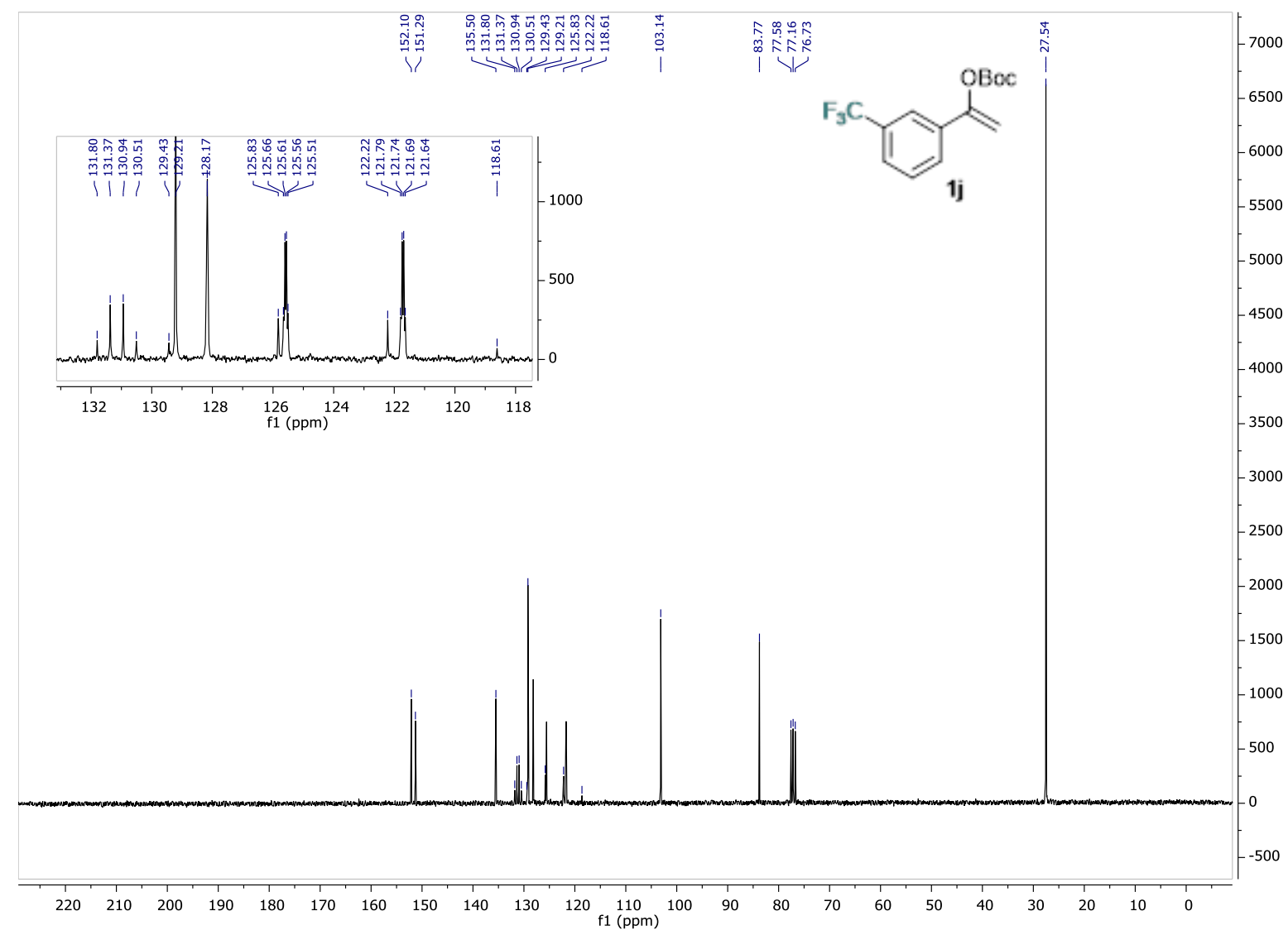

${ }^{19} \mathrm{~F}$ NMR (188 MHz, $\mathrm{CDCl}_{3}$ )

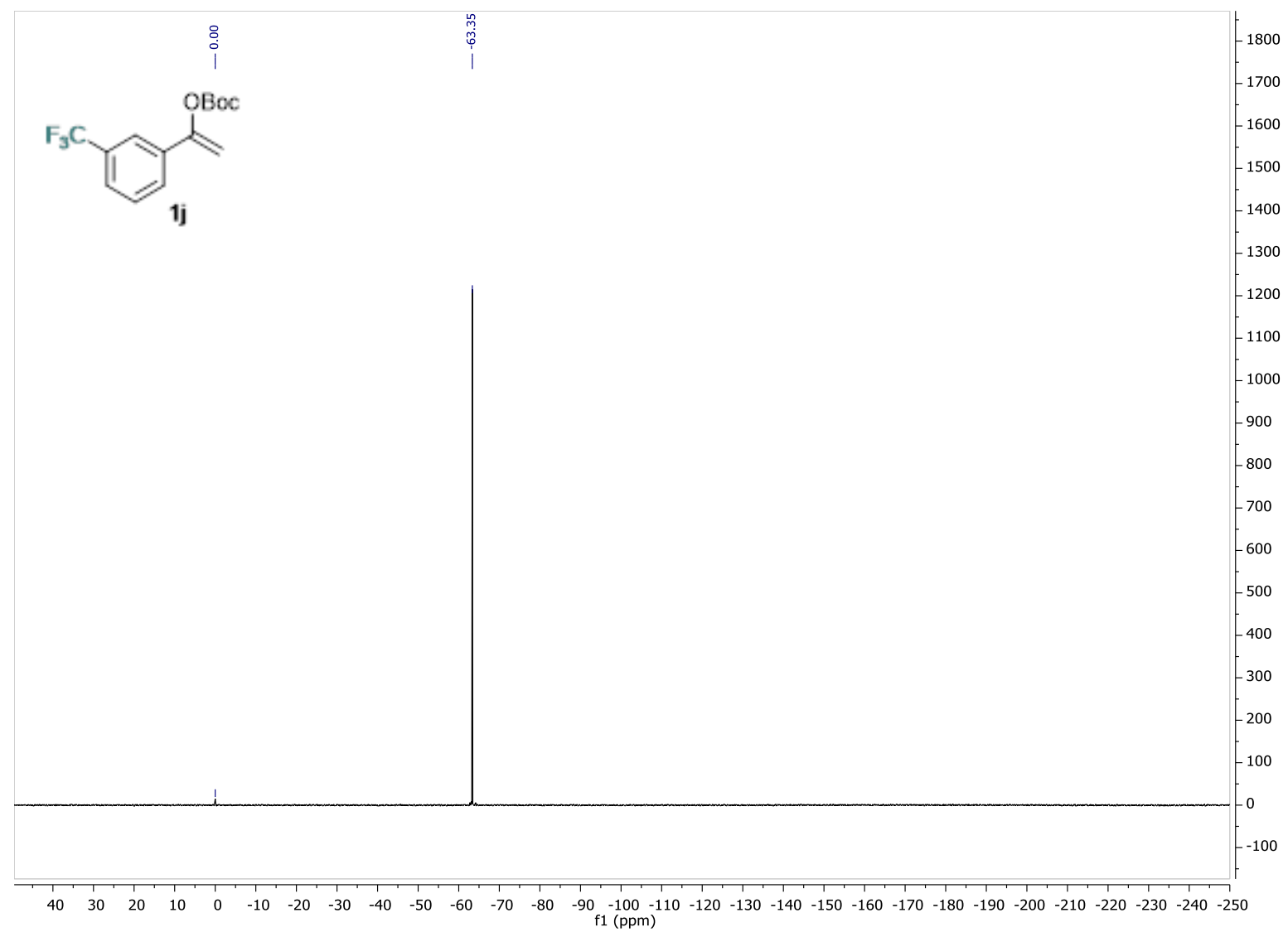




\section{1-(3,5-bis(trifluoromethyl)phenyl)vinyl tert-butyl carbonate $1 \mathrm{k}$}

${ }^{1} \mathrm{H}$ NMR $\left(400 \mathrm{MHz}, \mathrm{CDCl}_{3}\right)$

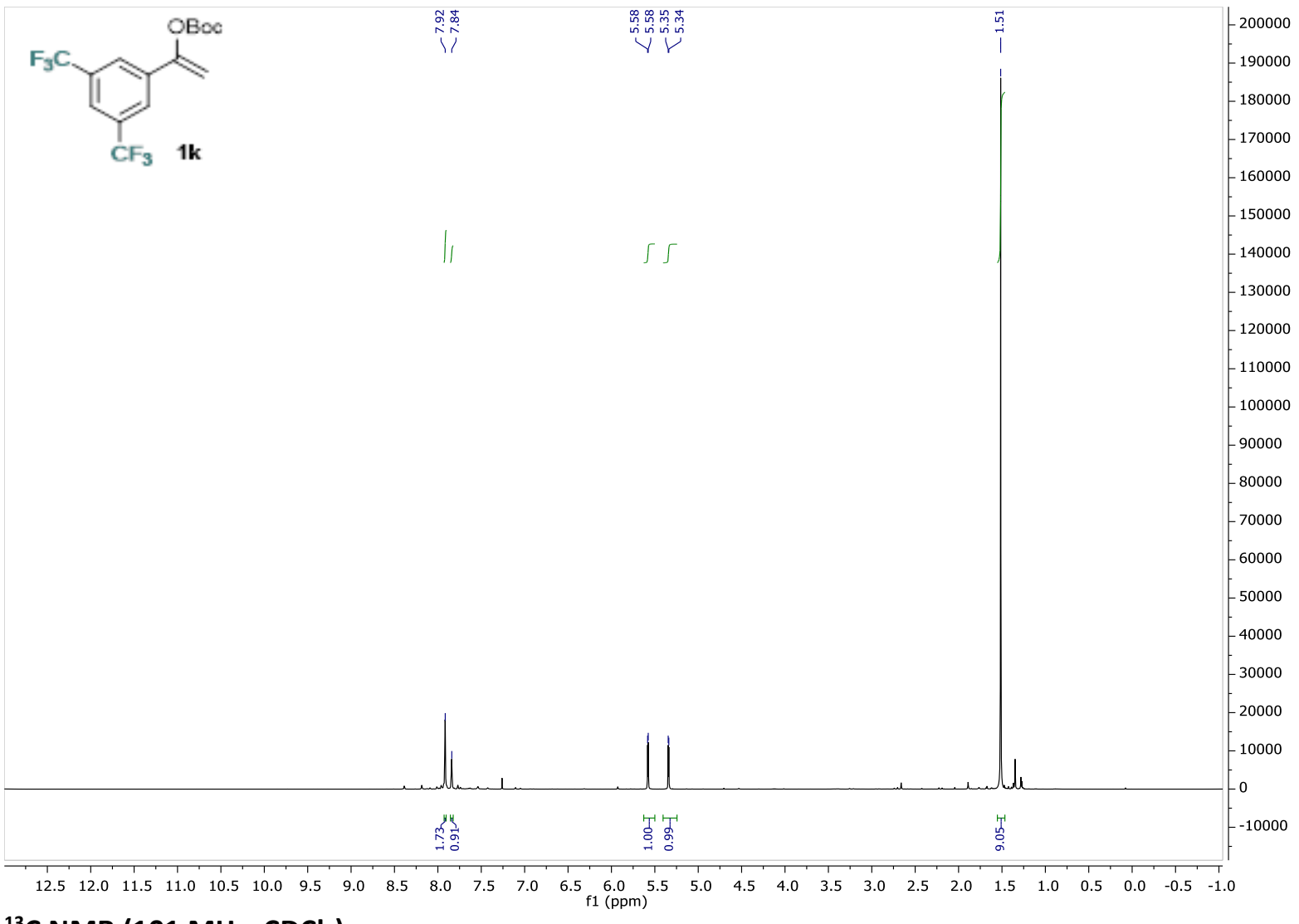

${ }^{13} \mathrm{C}$ NMR (101 MHz, $\mathrm{CDCl}_{3}$ )

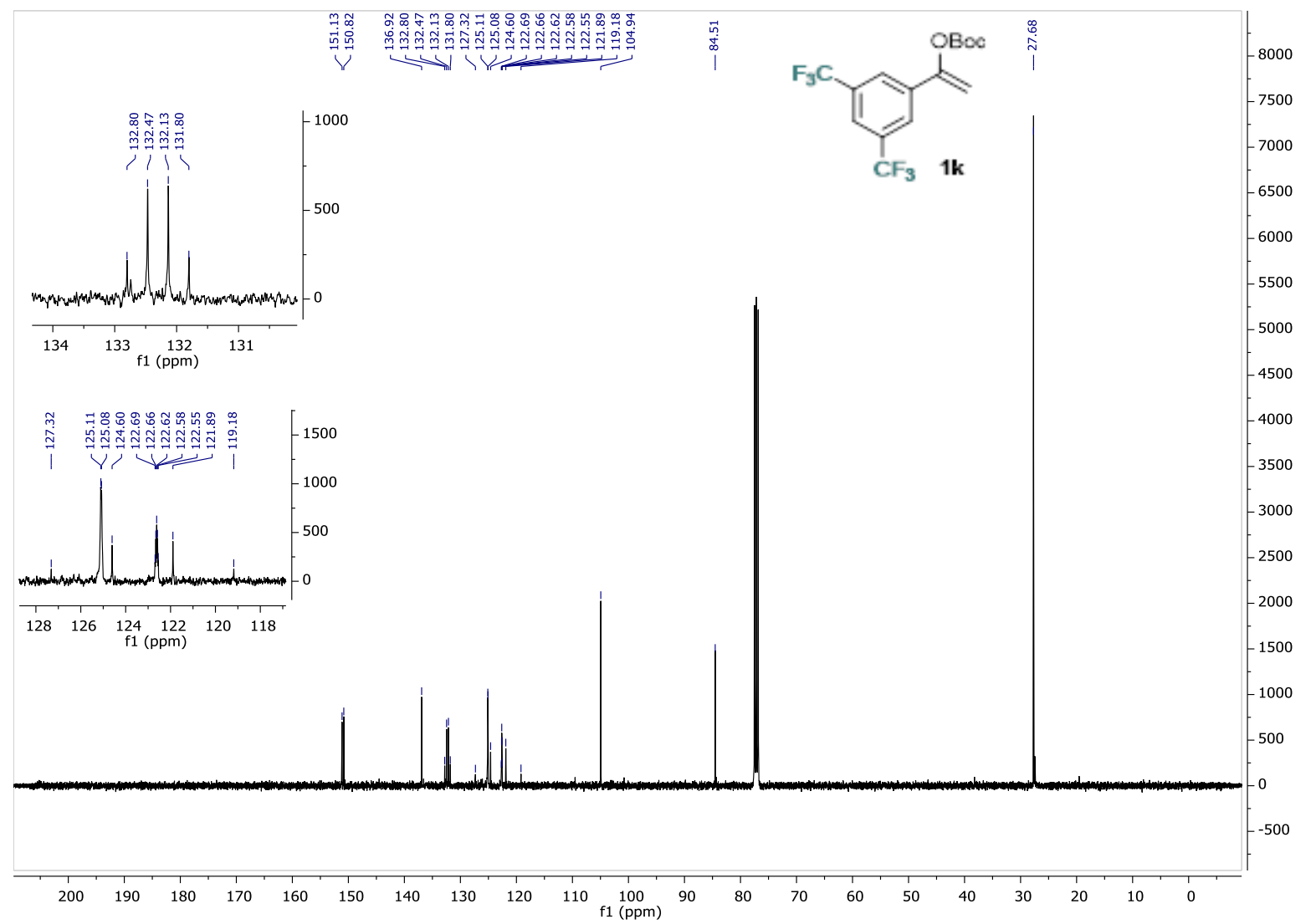


${ }^{19} \mathrm{~F}$ NMR (188 MHz, ${ }^{1} \mathrm{H}$ decoupled, $\mathrm{CDCl}_{3}$ )

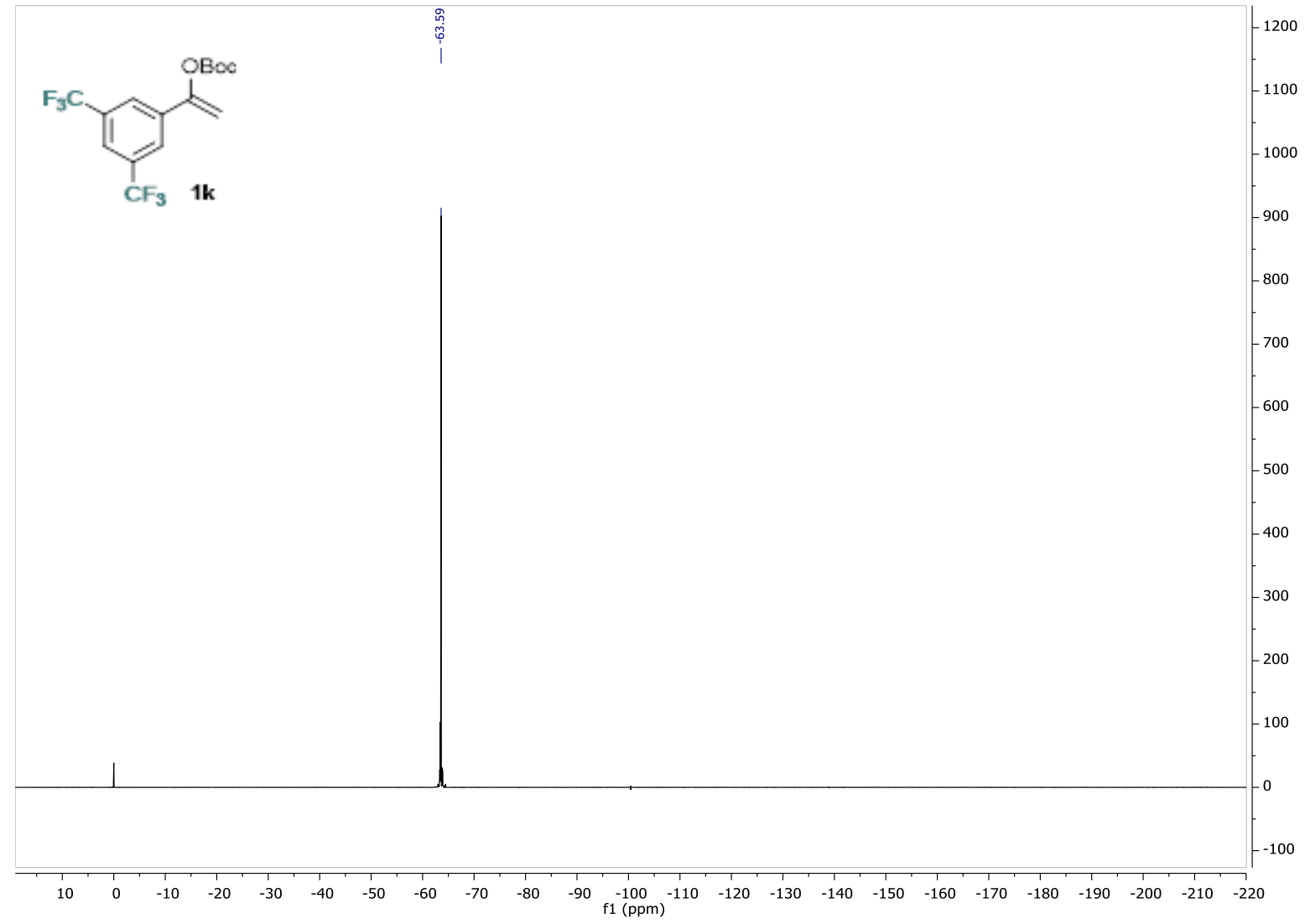

1-(2-bromophenyl)vinyl tert-butyl carbonate $\mathbf{1 l}$ ${ }^{1} \mathrm{H}$ NMR $\left(500 \mathrm{MHz}, \mathrm{CDCl}_{3}\right)$

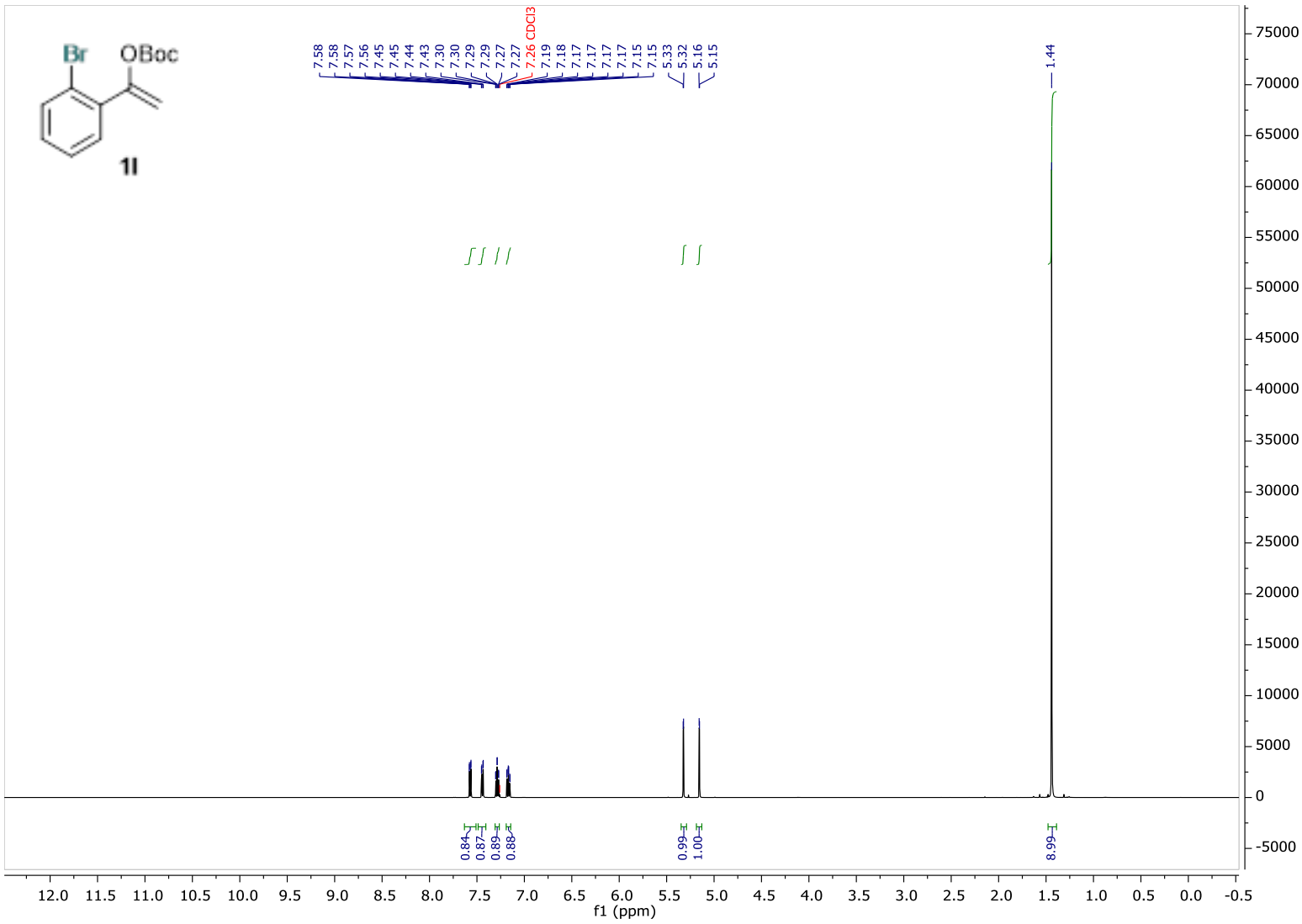


${ }^{13} \mathrm{C}$ NMR (126 MHz, $\left.\mathrm{CDCl}_{3}\right)$

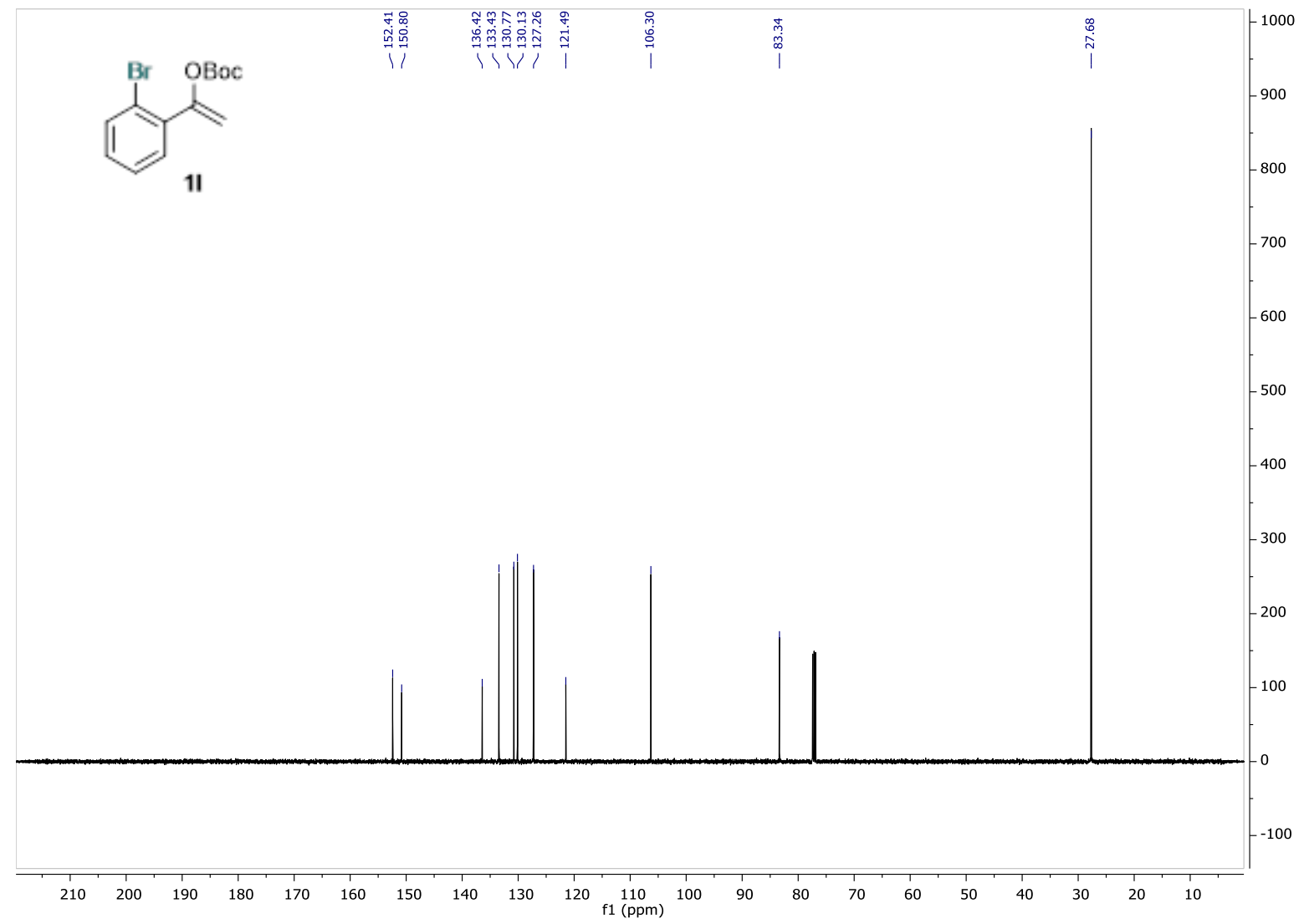

1-(3-bromophenyl)vinyl tert-butyl carbonate 1m

${ }^{1} \mathrm{H}$ NMR (500 MHz, $\mathrm{CDCl}_{3}$ )

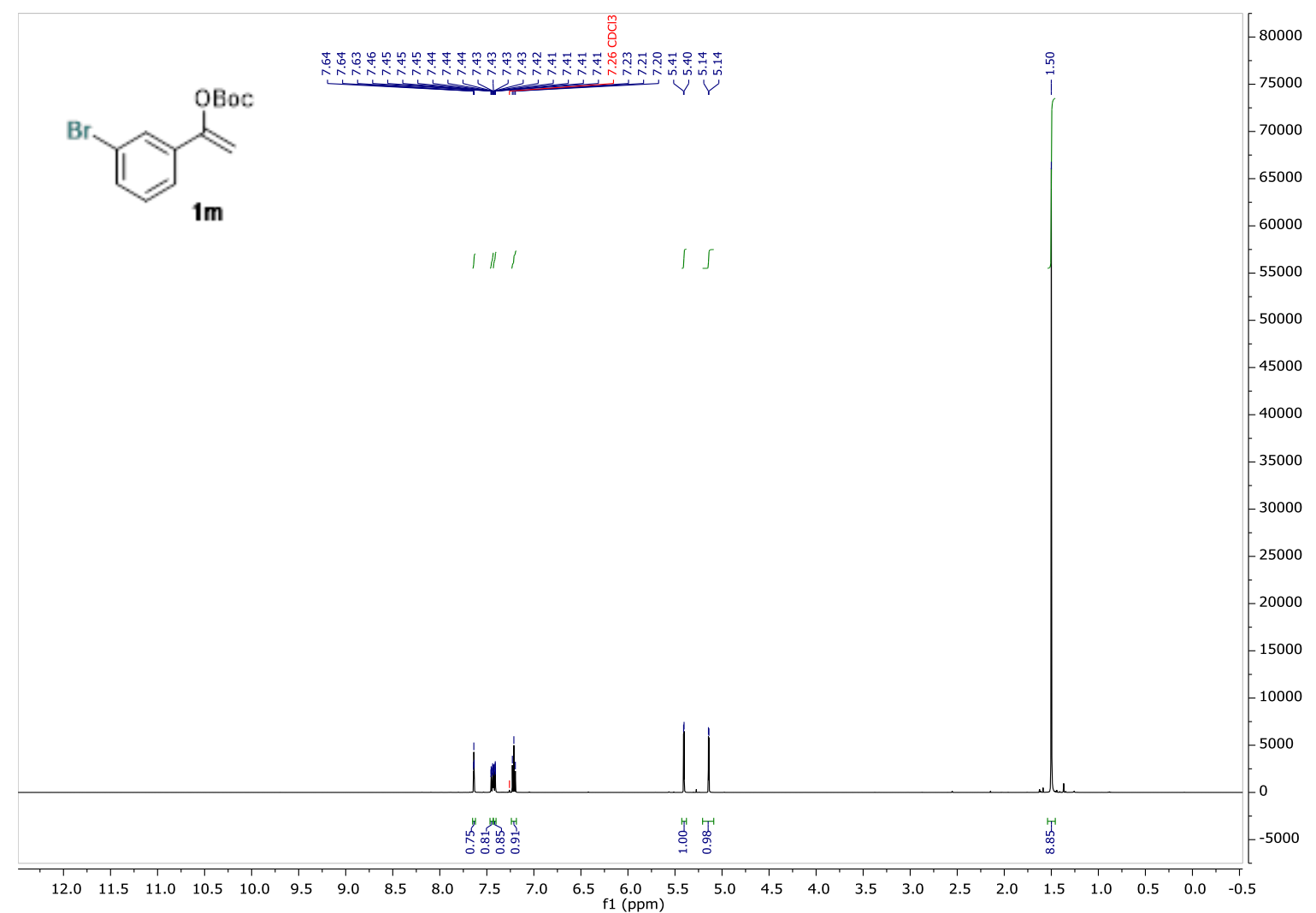


.${ }^{13} \mathrm{C}$ NMR (126 MHz, $\left.\mathrm{CDCl}_{3}\right)$

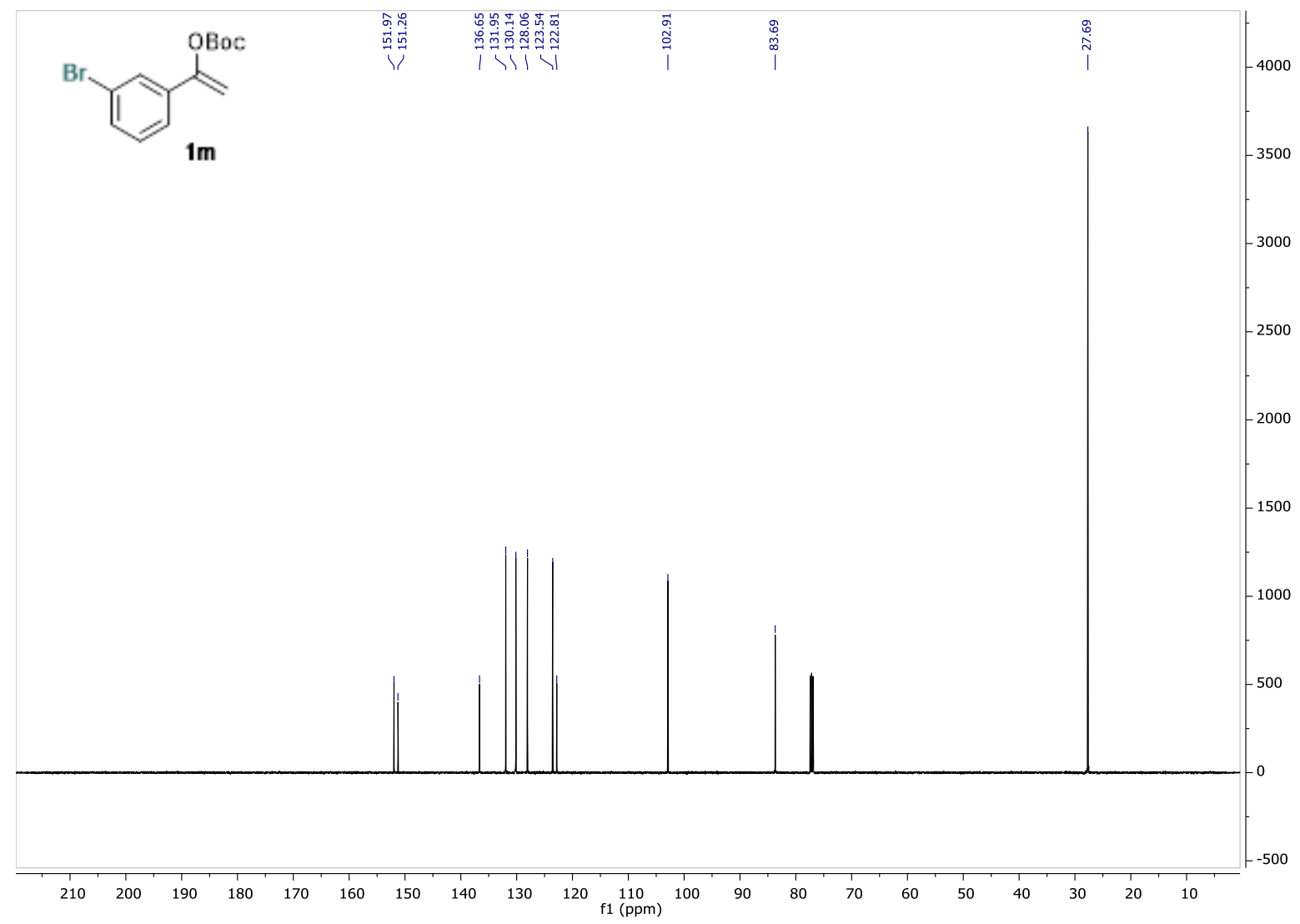

1-(4-bromophenyl)vinyl tert-butyl carbonate 1n

${ }^{1} \mathrm{H}$ NMR (500 MHz, $\mathrm{CDCl}_{3}$ )

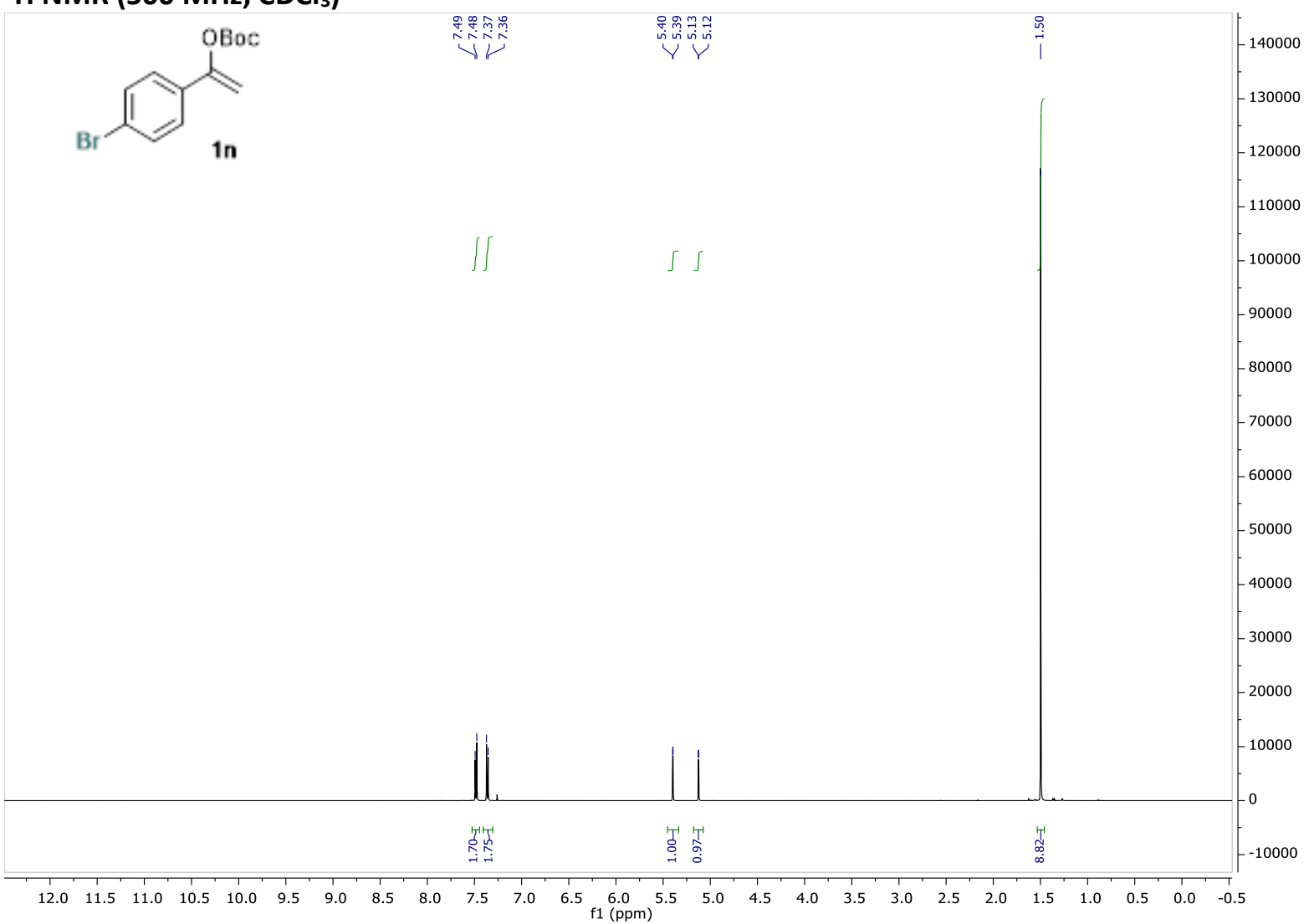


${ }^{13} \mathrm{C}$ NMR (126 MHz, $\left.\mathrm{CDCl}_{3}\right)$

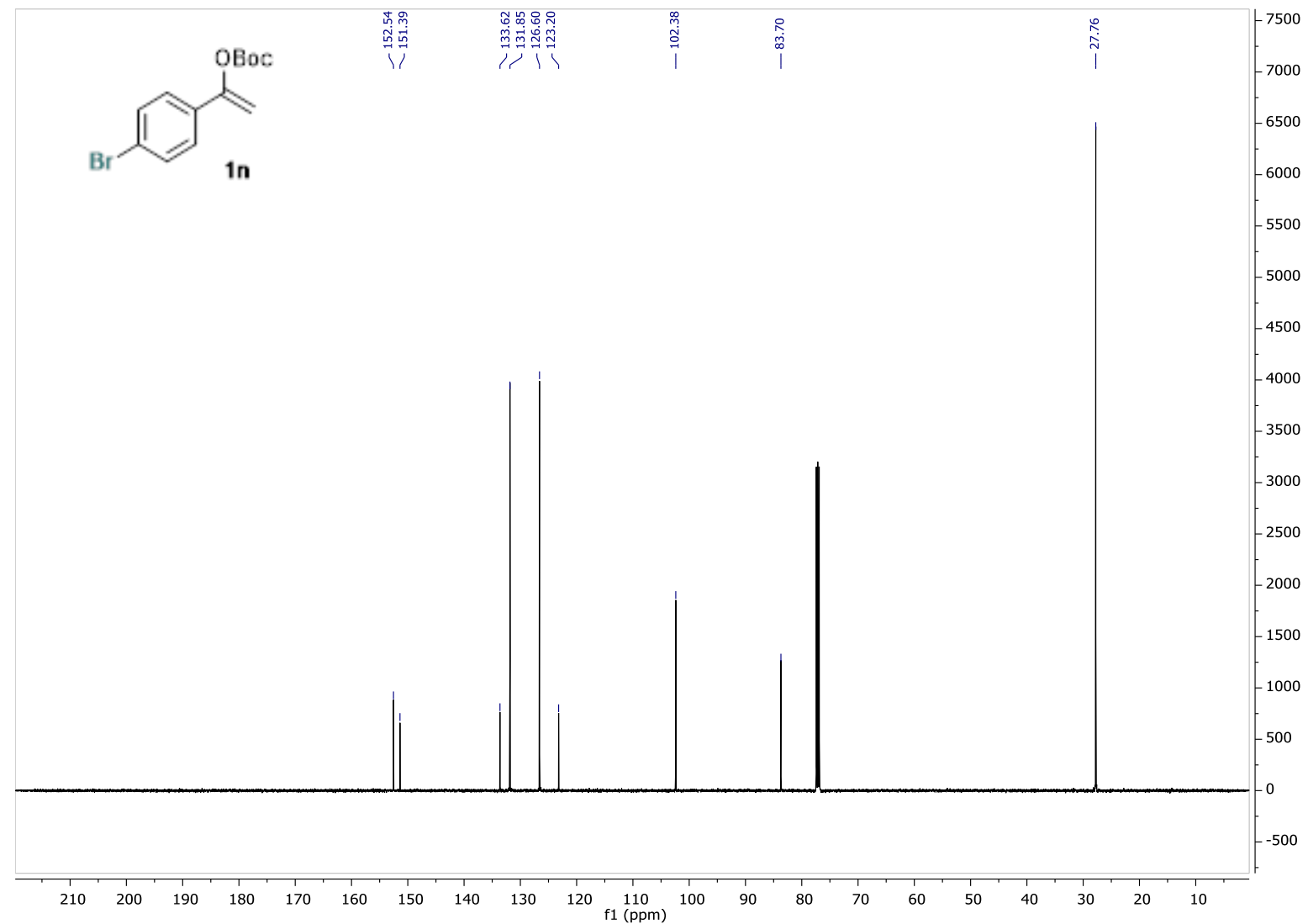

tert-butyl 1-(2,5-dichlorophenyl)vinyl carbonate 10

${ }^{1} \mathrm{H}$ NMR (400 MHz, $\mathrm{CDCl}_{3}$ )

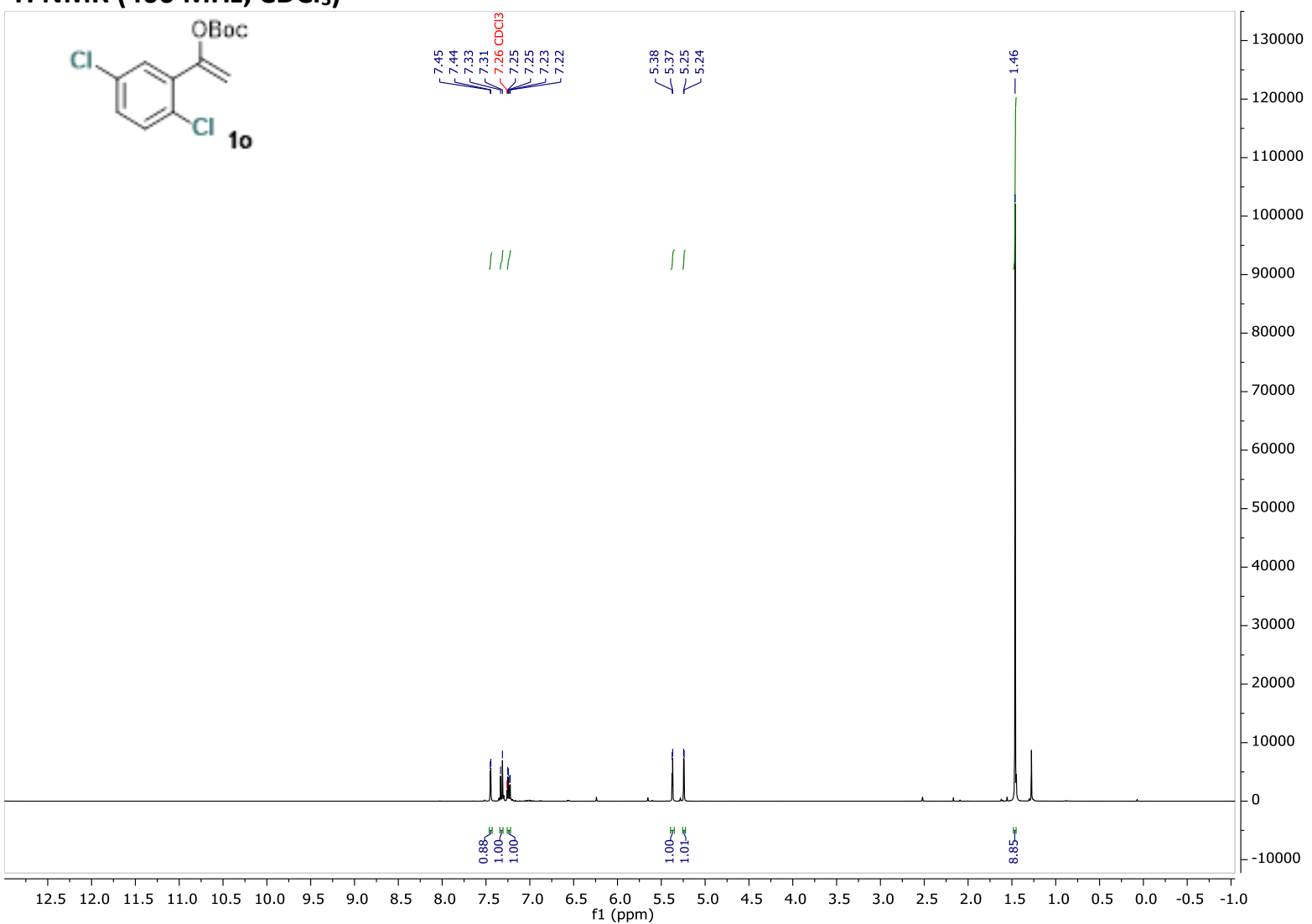


${ }^{13} \mathrm{C}$ NMR (101 MHz, $\left.\mathrm{CDCl}_{3}\right)$

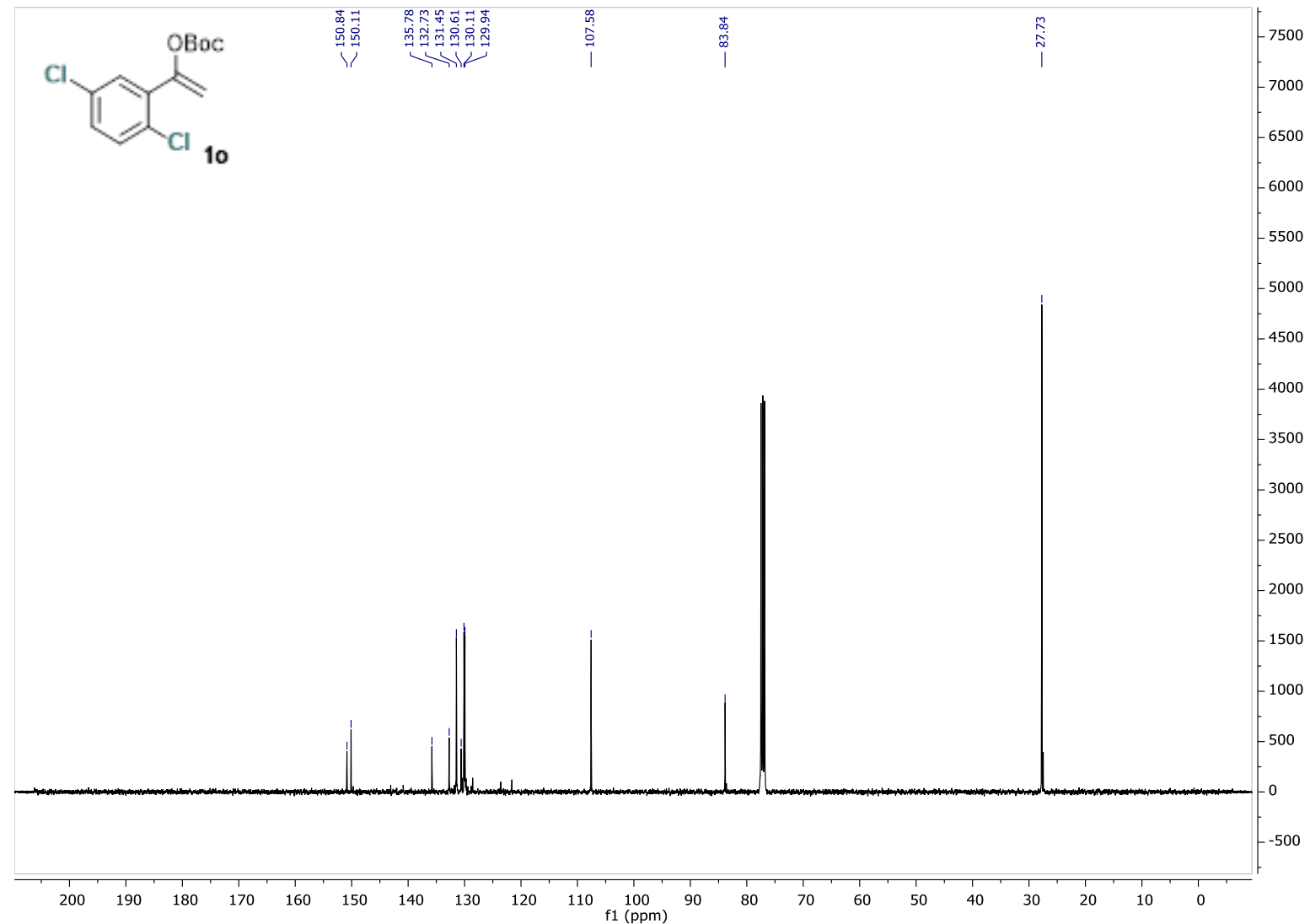

1-(4-acetylphenyl)vinyl tert-butyl carbonate 1p

${ }^{1} \mathrm{H}$ NMR (400 MHz, $\mathrm{CDCl}_{3}$ )

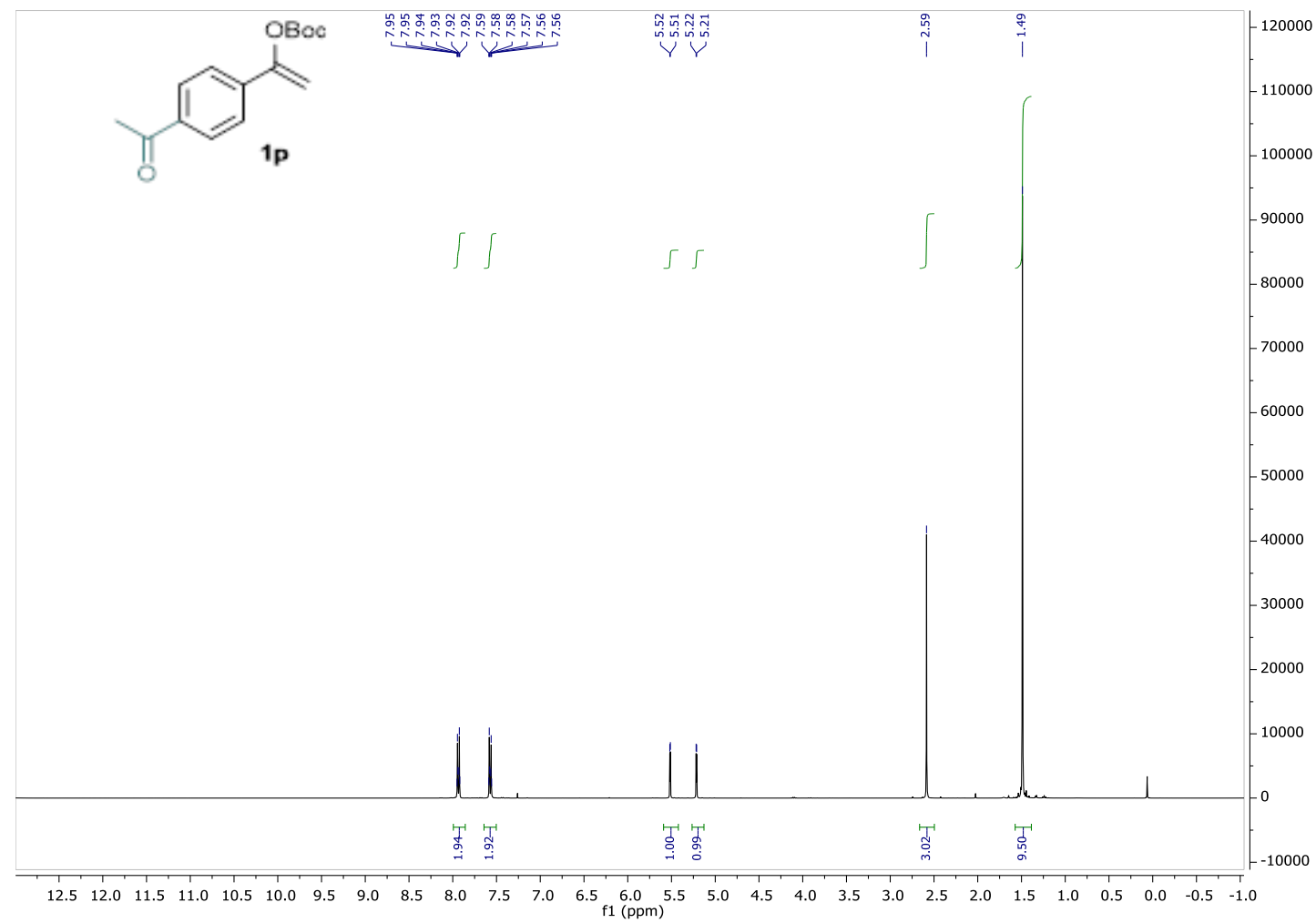


${ }^{13} \mathrm{C}$ NMR (101 MHz, $\mathrm{CDCl}_{3}$ )

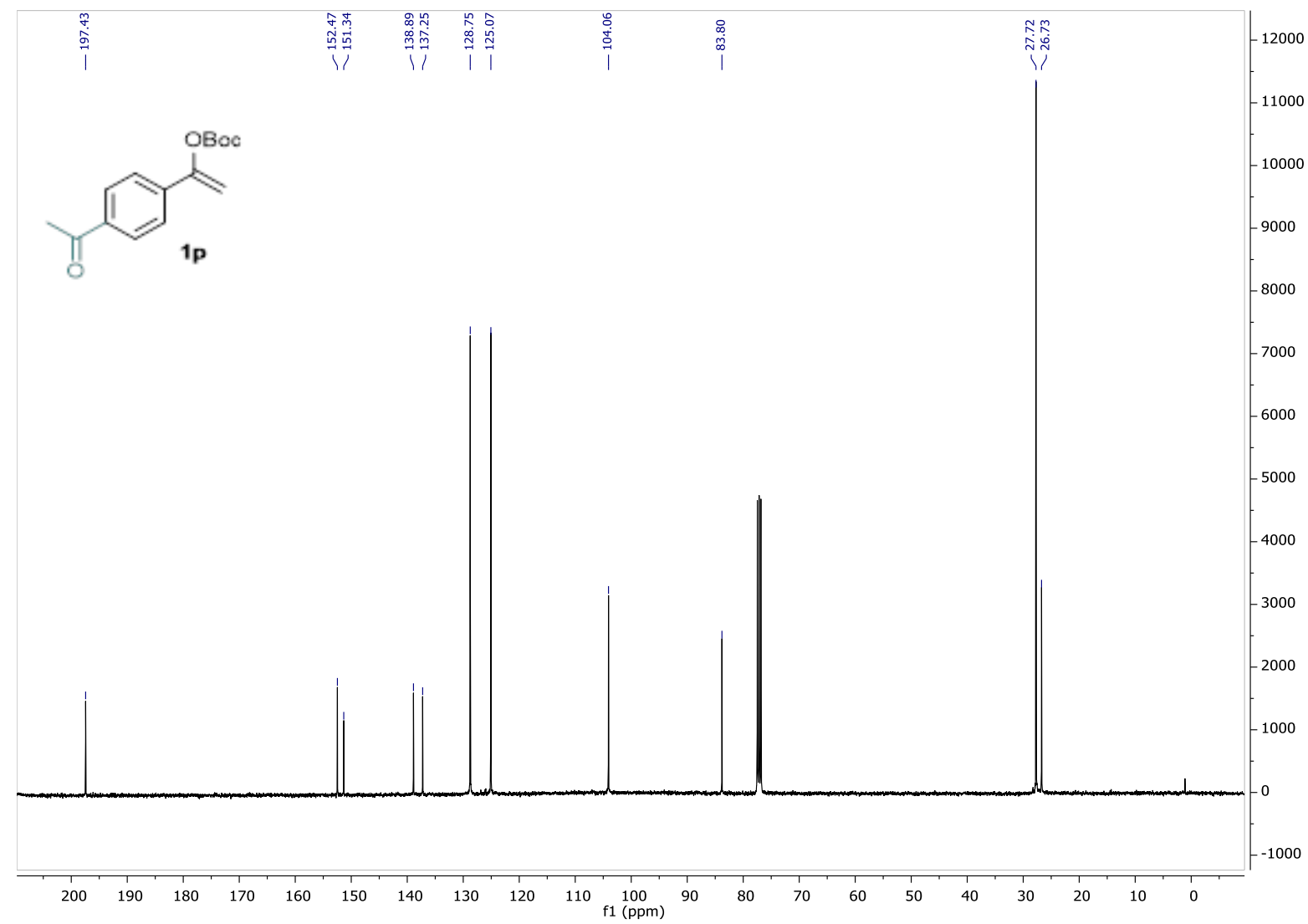

tert-butyl (1-phenylprop-1-en-1-yl) carbonate 1q

${ }^{1} \mathrm{H}$ NMR $\left(500 \mathrm{MHz}, \mathrm{CDCl}_{3}\right)$

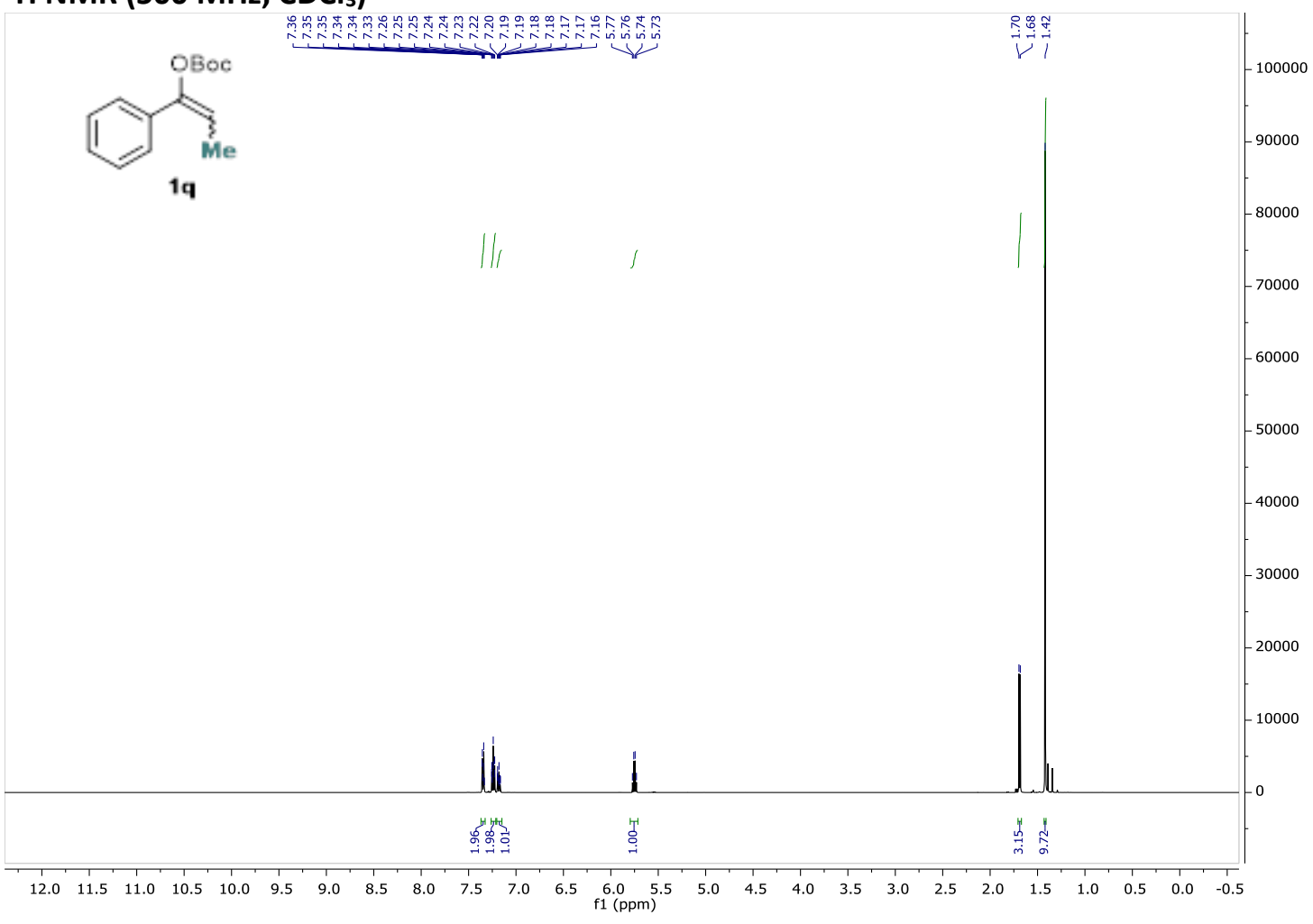


${ }^{13} \mathrm{C}$ NMR (126 MHz, $\left.\mathrm{CDCl}_{3}\right)$

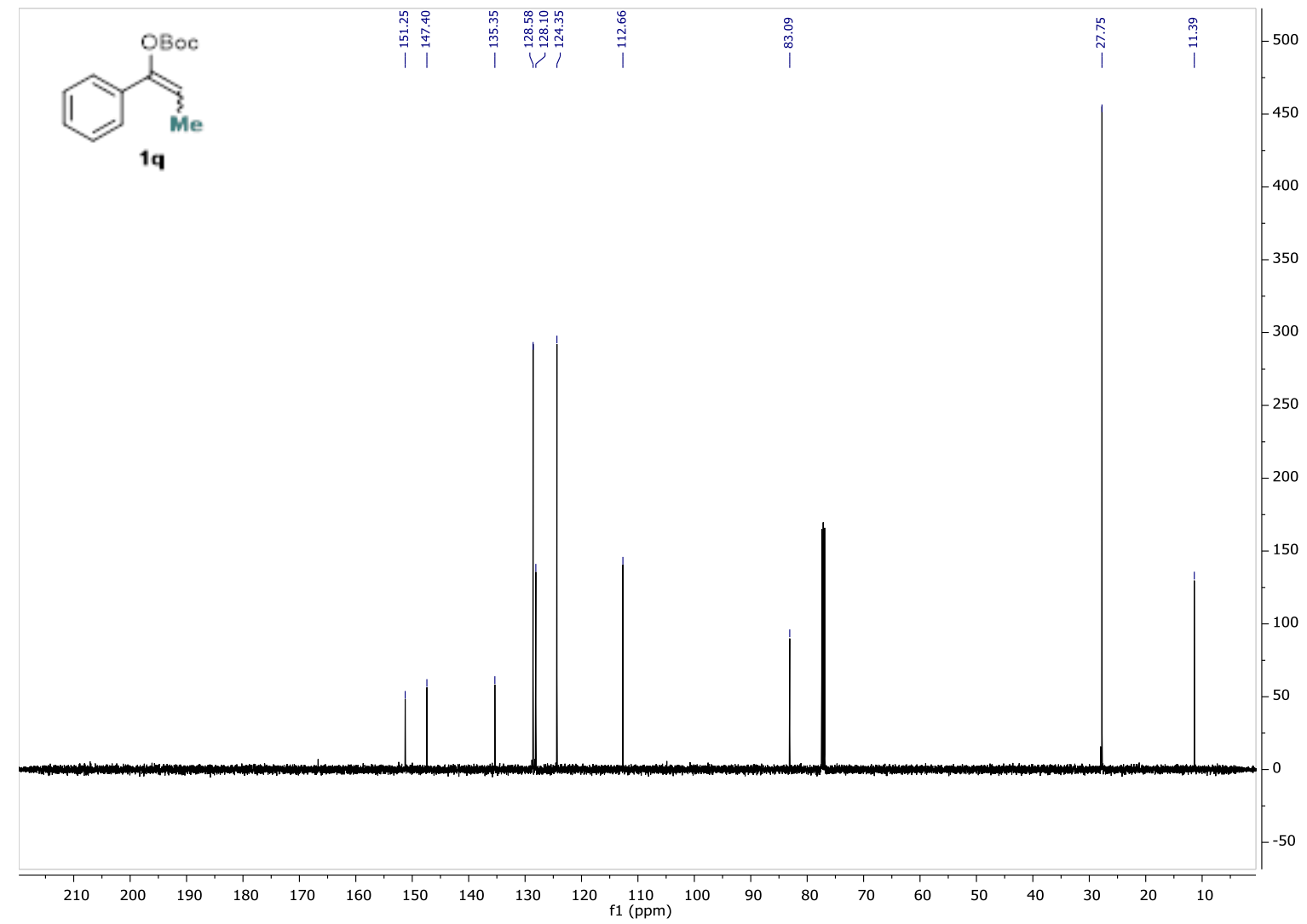

tert-butyl (1,3-diphenylprop-1-en-1-yl) carbonate 1r

${ }^{1} \mathrm{H}$ NMR (500 MHz, $\left.\mathrm{CDCl}_{3}\right)$

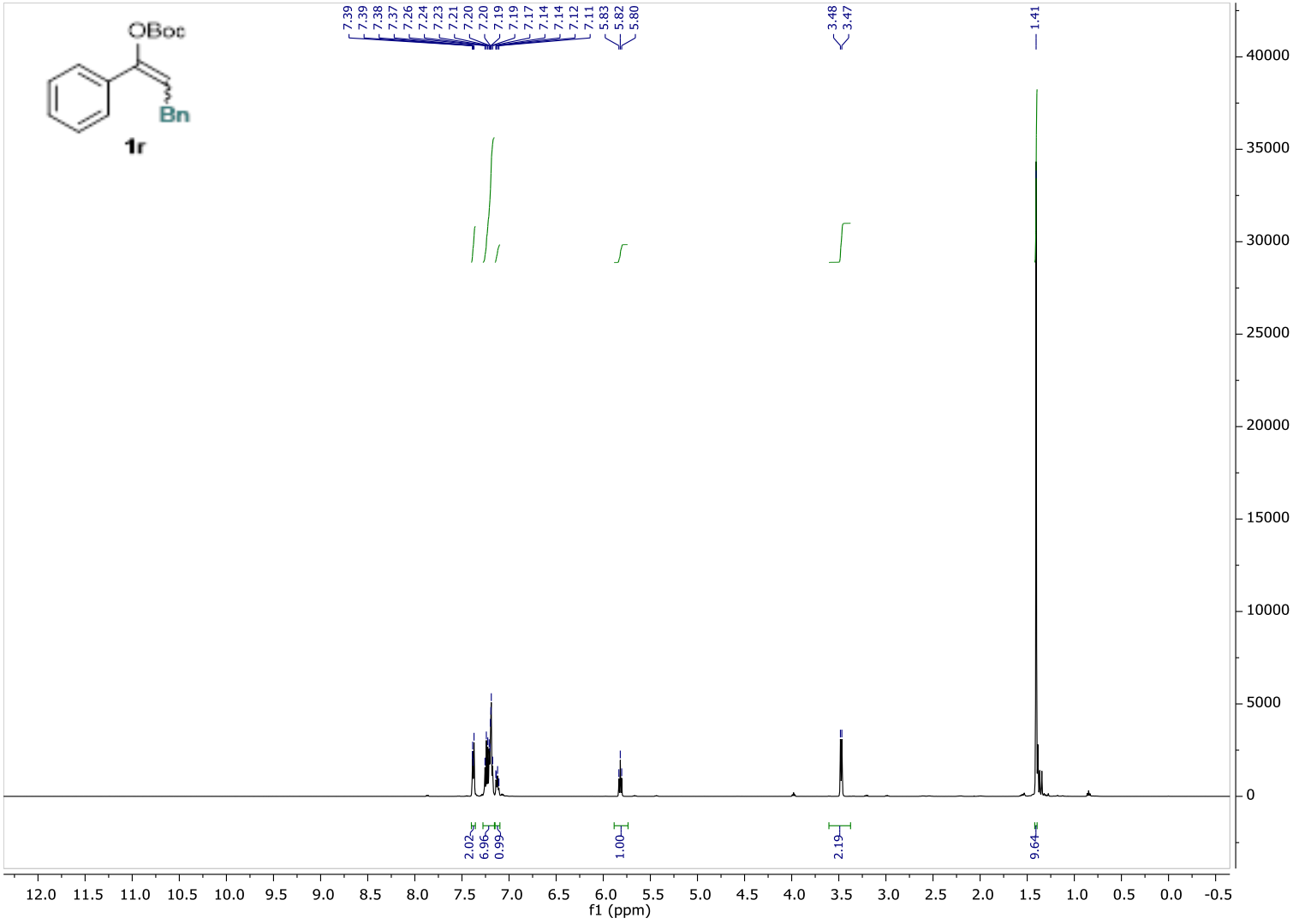


${ }^{13} \mathrm{C}$ NMR (126 MHz, $\left.\mathrm{CDCl}_{3}\right)$

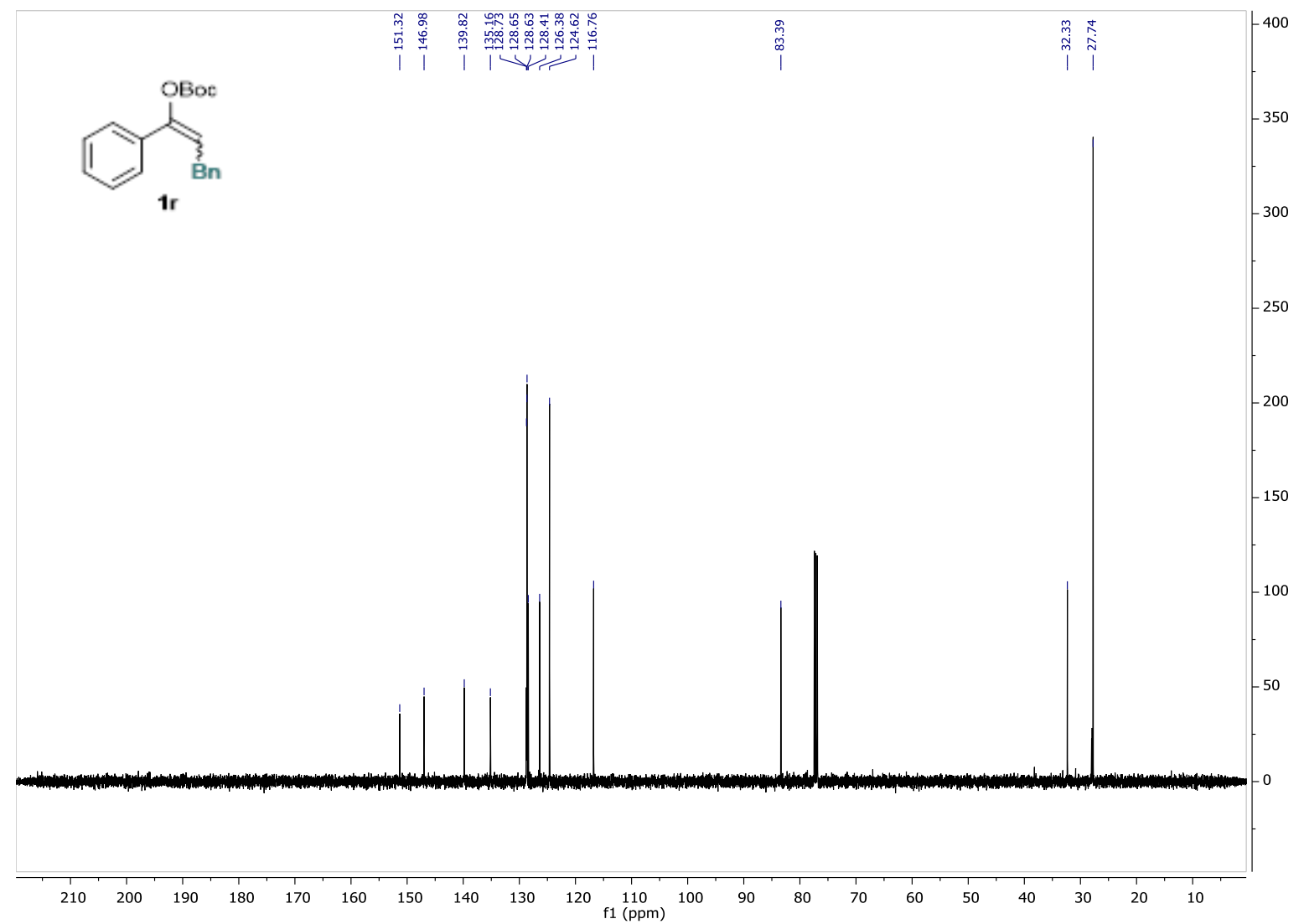

tert-butyl $1 \mathrm{H}$-inden-3-yl carbonate $1 \mathrm{~s}$

${ }^{1} \mathrm{H}$ NMR (300 MHz, $\mathrm{CDCl}_{3}$ )

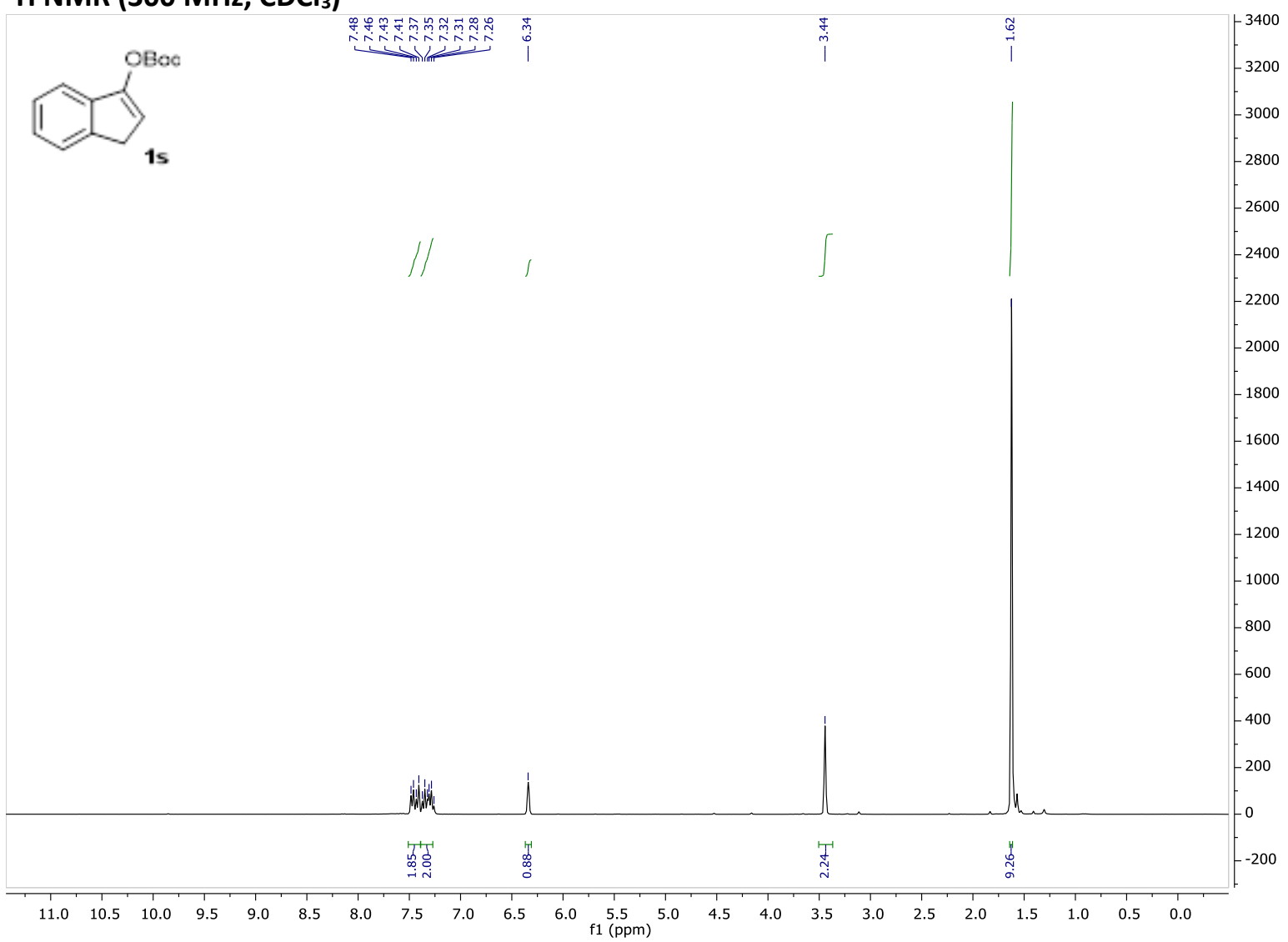


${ }^{13} \mathrm{C}$ NMR $\left(75 \mathrm{MHz}, \mathrm{CDCl}_{3}\right)$

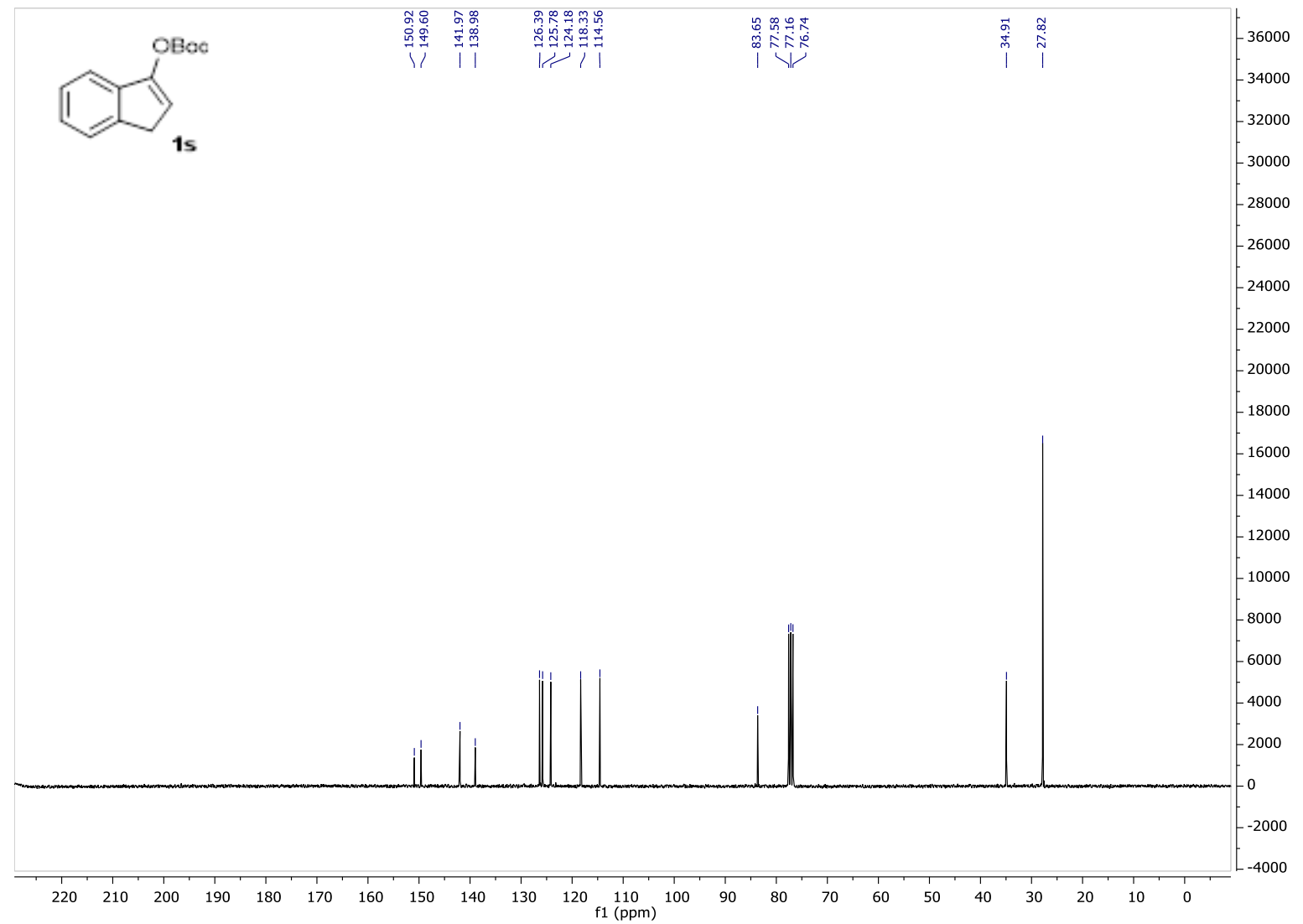

tert-butyl (5-methoxy-1H-inden-3-yl) carbonate 1t

${ }^{1} \mathrm{H}$ NMR (300 MHz, $\mathrm{CDCl}_{3}$ )

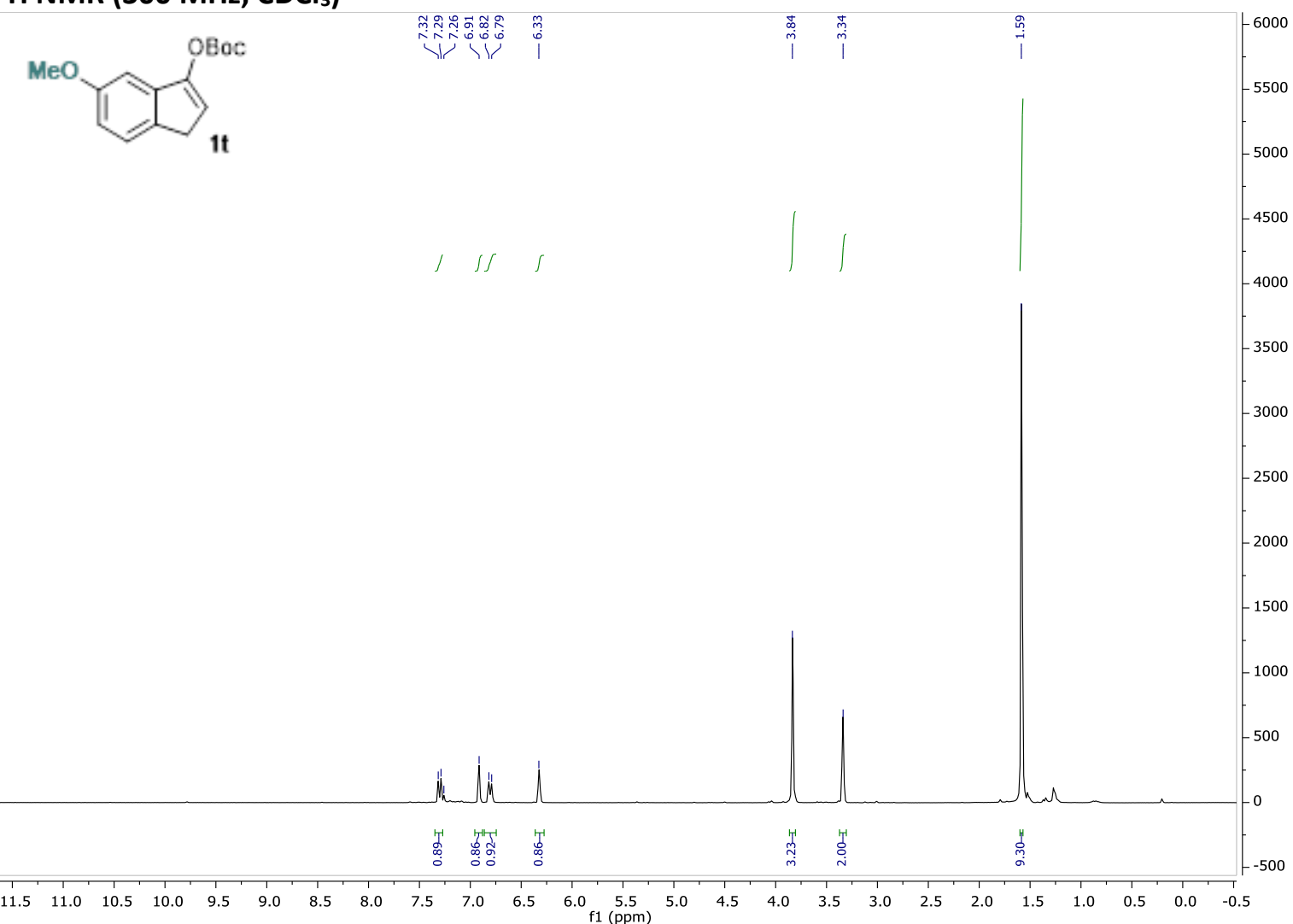


${ }^{13} \mathrm{C}$ NMR (75 MHz, $\mathrm{CDCl}_{3}$ )

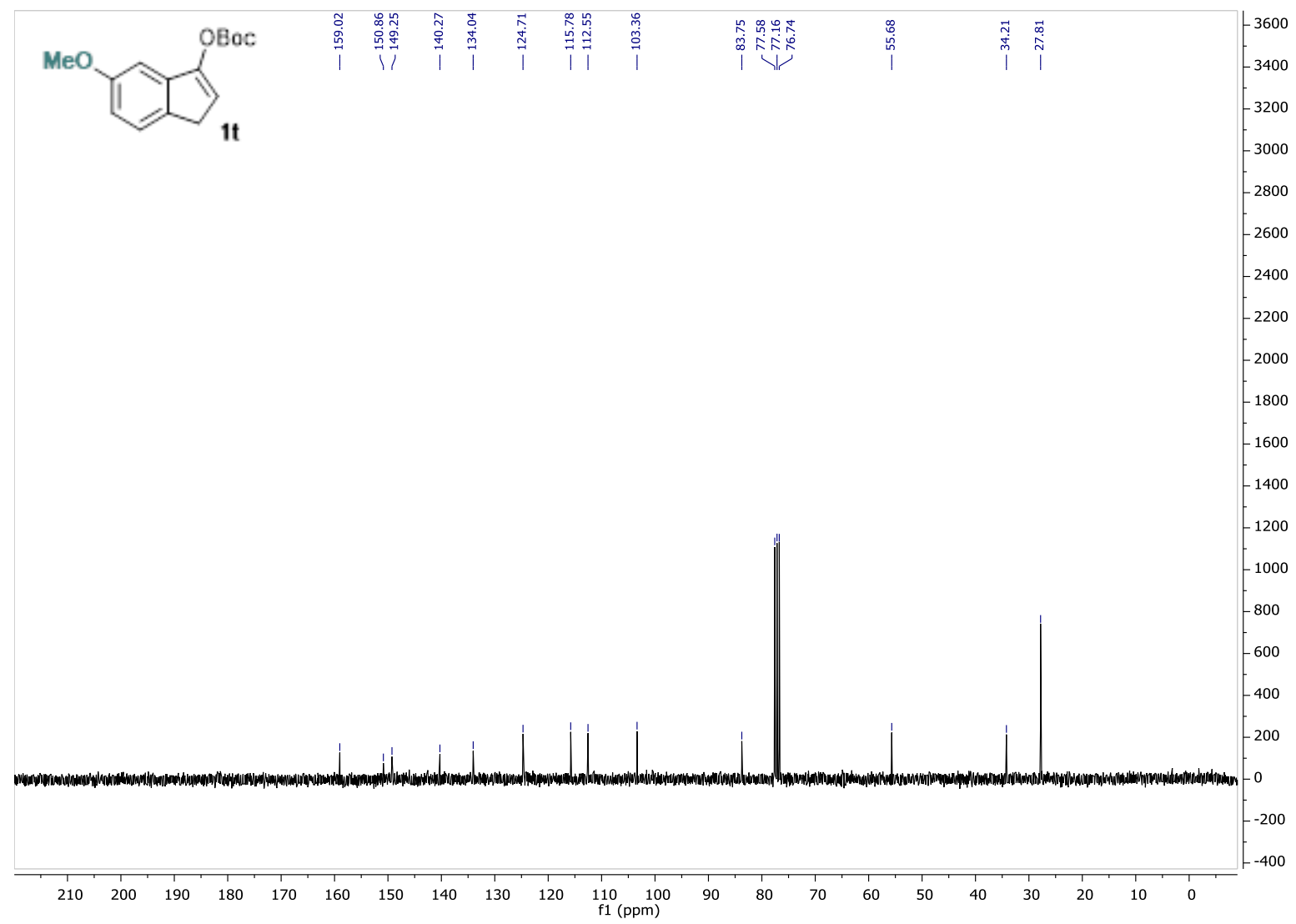

tert-butyl (6-fluoro-1H-inden-3-yl) carbonate $1 \mathrm{u}$

${ }^{1} \mathrm{H}$ NMR (300 MHz, $\mathrm{CDCl}_{3}$ )

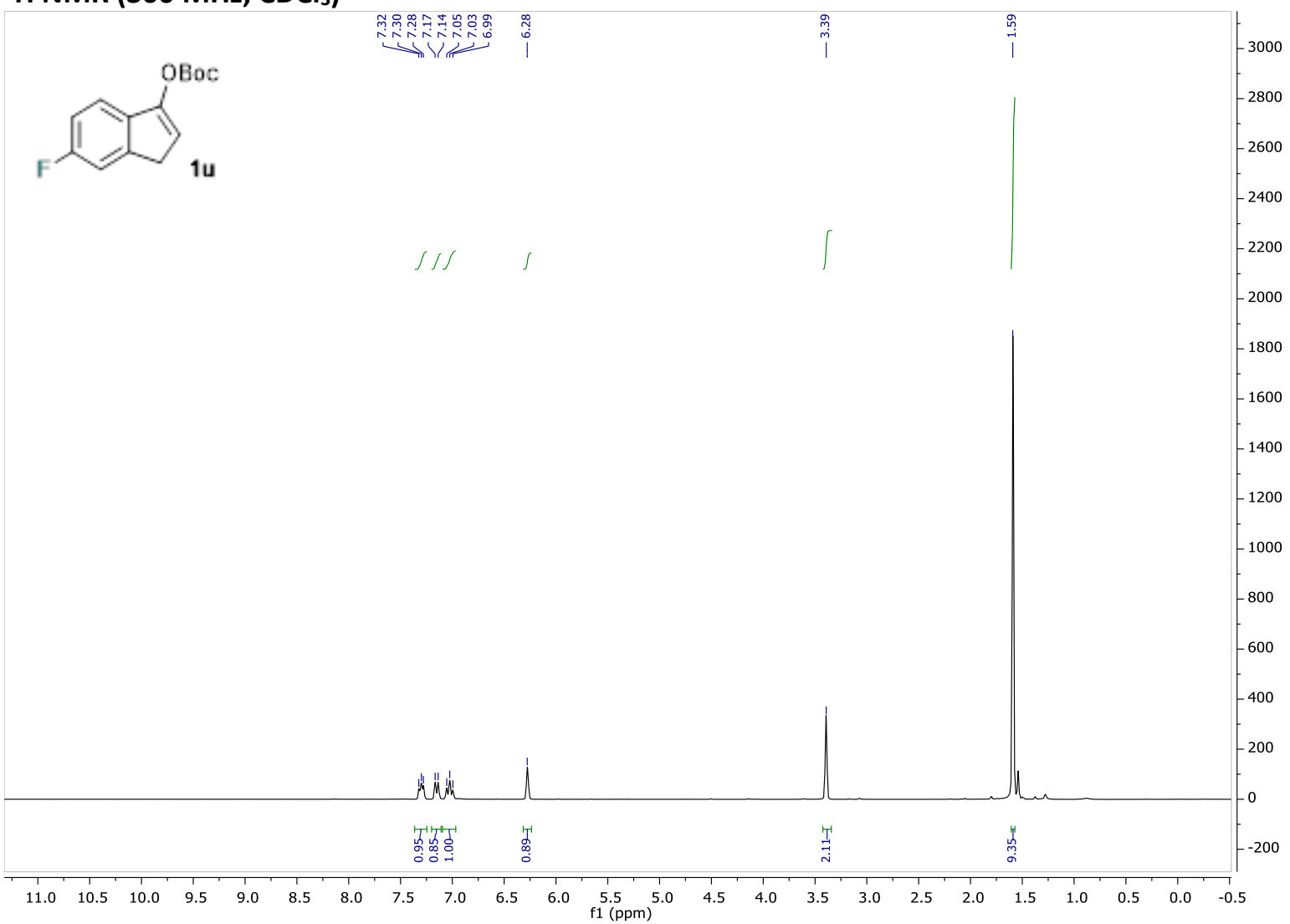




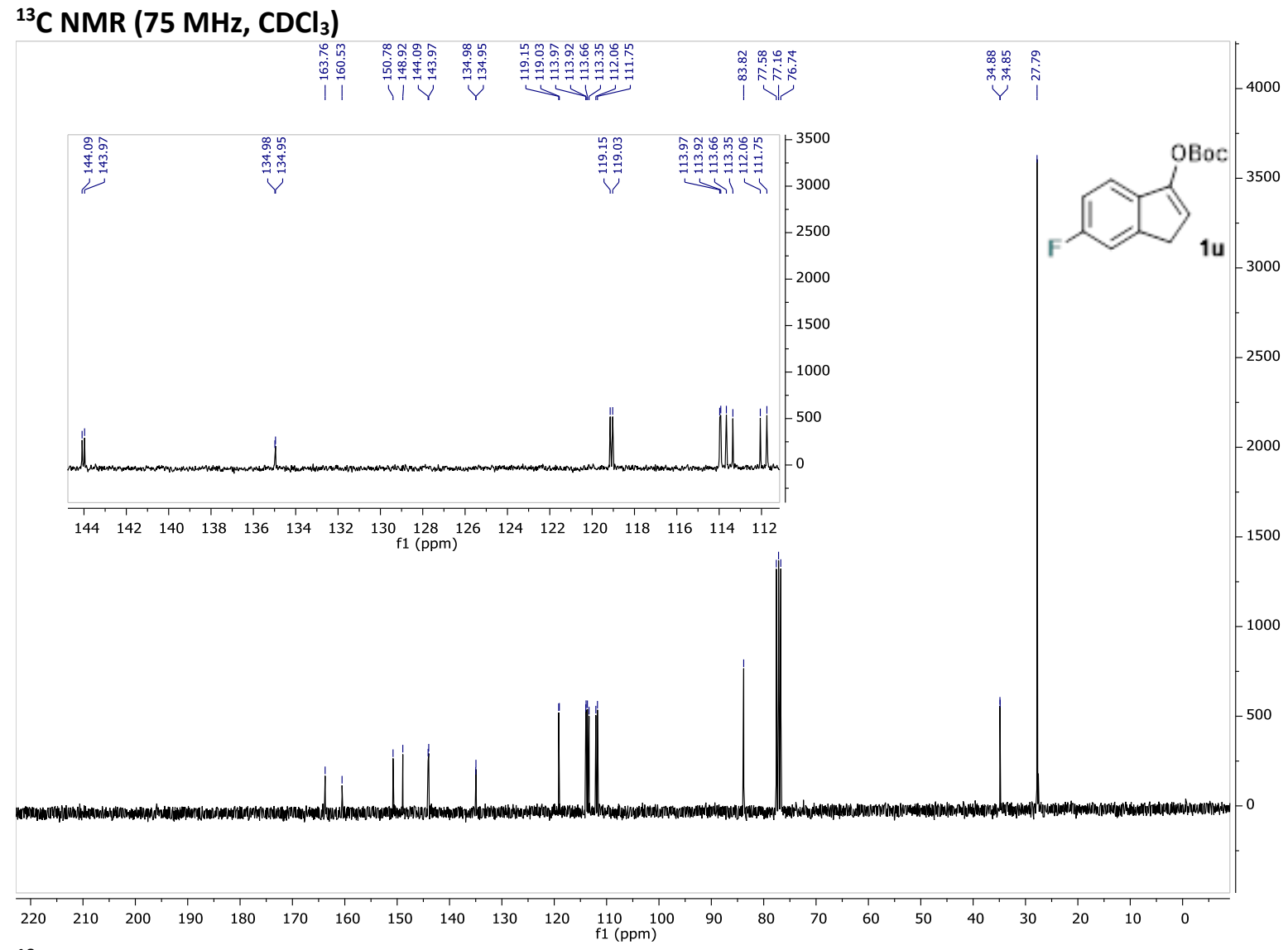

${ }^{19} \mathrm{~F}$ NMR (188 MHz, CDCl 3

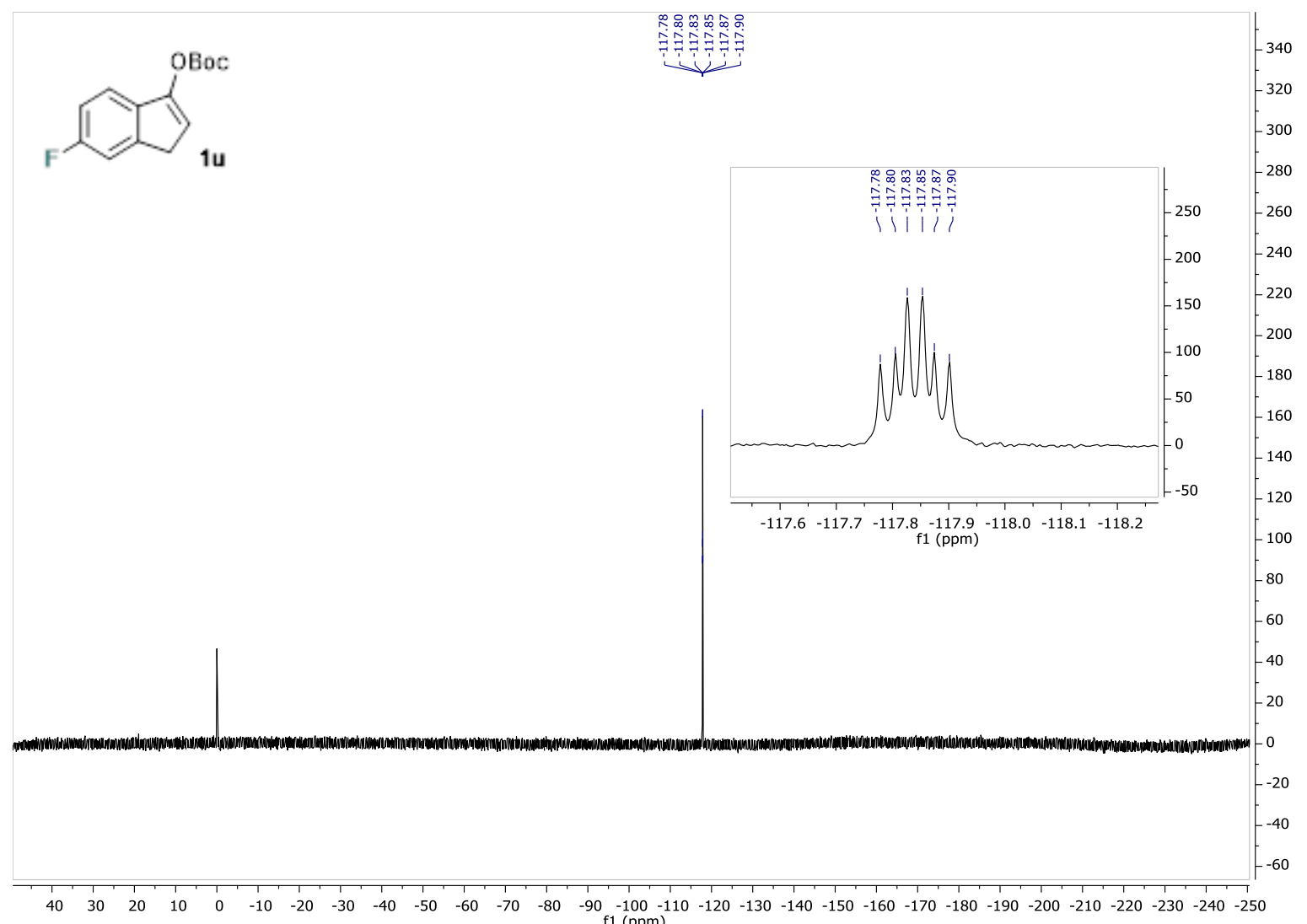


tert-butyl 3,4-dihydronaphthalen-1-yl carbonate 1v

${ }^{1} \mathrm{H}$ NMR (500 MHz, $\mathrm{CDCl}_{3}$ )

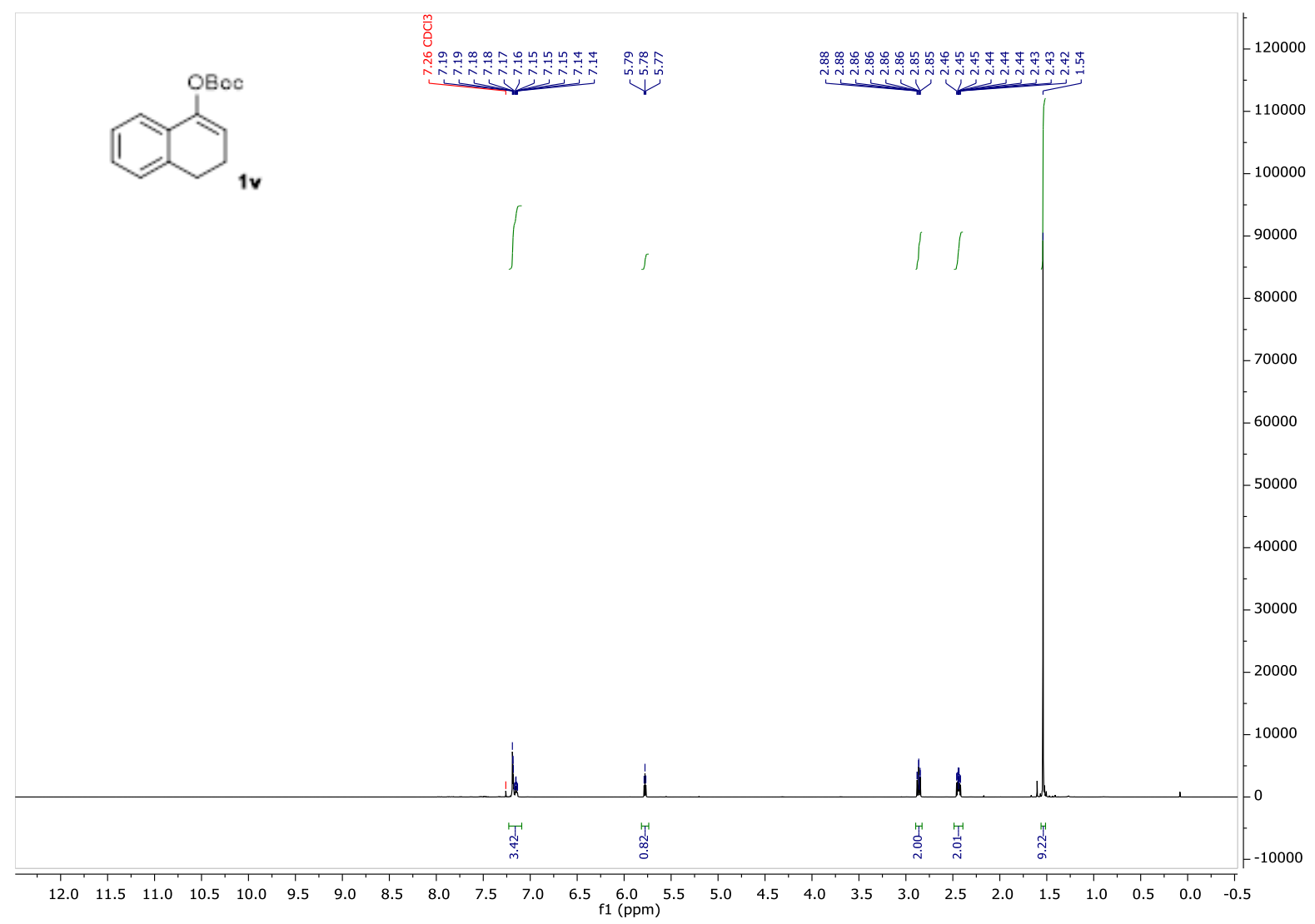

${ }^{13} \mathrm{C}$ NMR (126 MHz, $\mathrm{CDCl}_{3}$ )

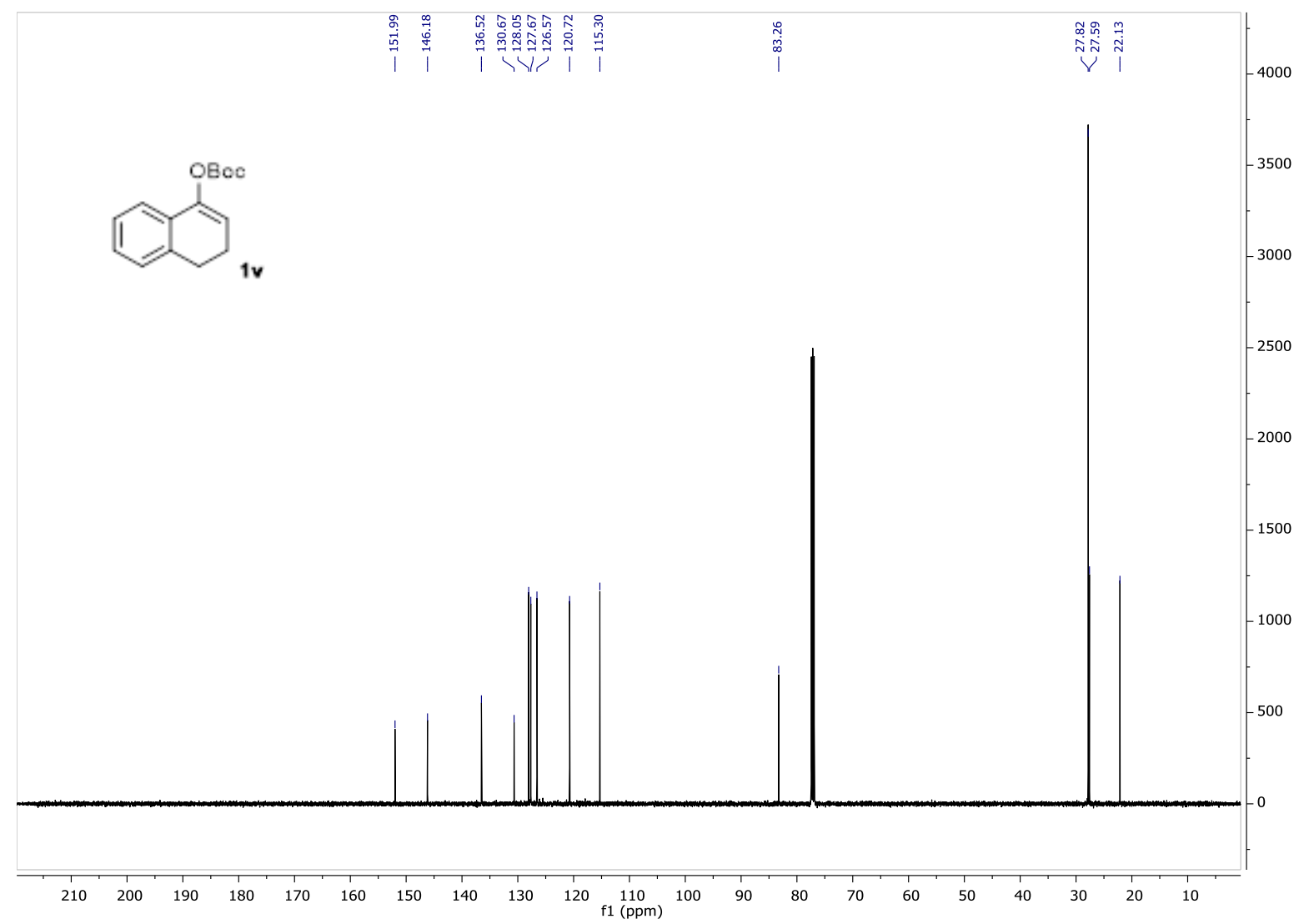


tert-butyl (6,7-dihydro-5H-benzo[7]annulen-9-yl) carbonate $1 w$

${ }^{1} \mathrm{H}$ NMR $\left(300 \mathrm{MHz}, \mathrm{CDCl}_{3}\right)$

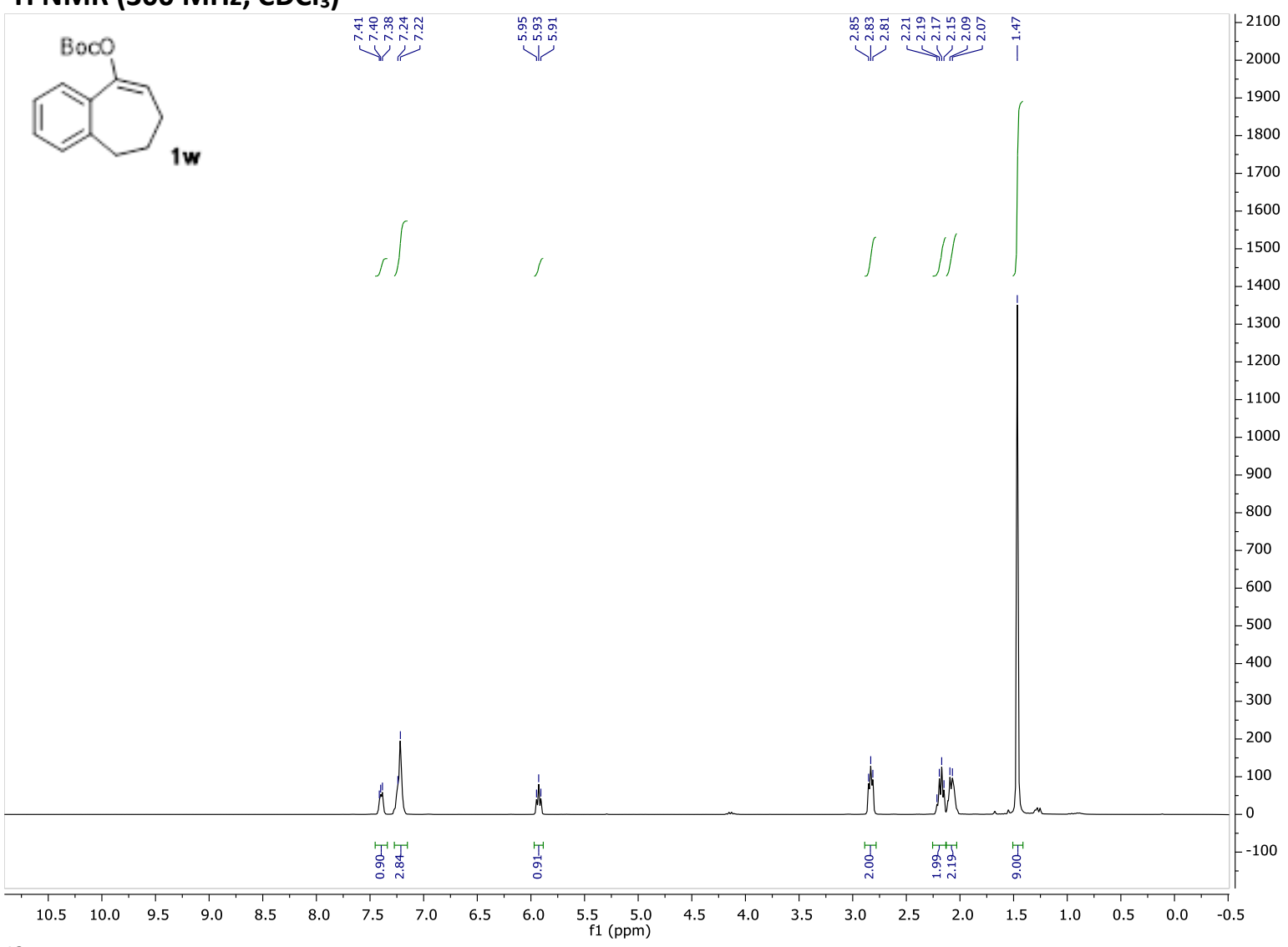

${ }^{13} \mathrm{C}$ NMR $\left(75 \mathrm{MHz}, \mathrm{CDCl}_{3}\right)$

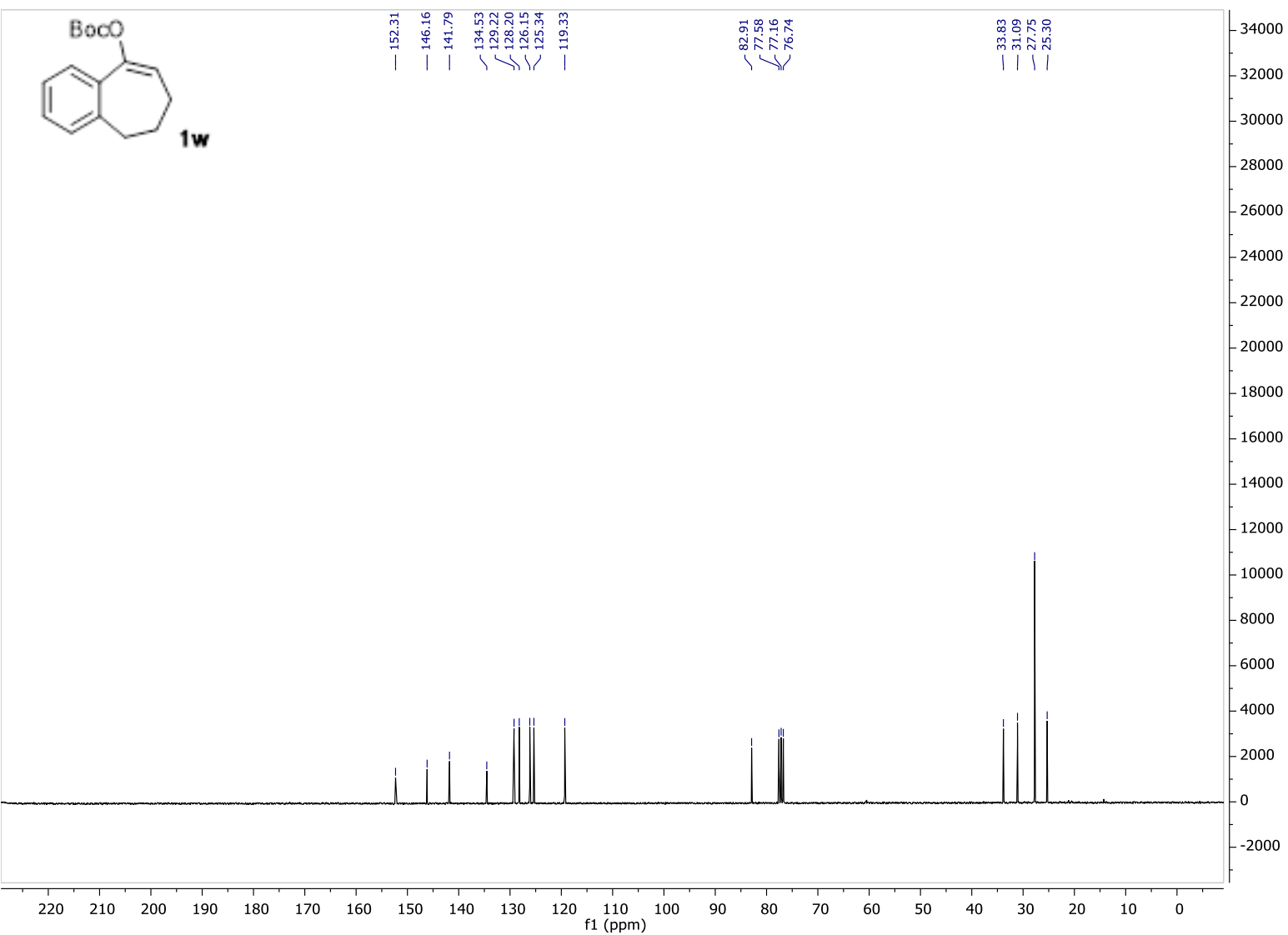


1-(benzo[b]thiophen-2-yl)vinyl tert-butyl carbonate 1x

${ }^{1} \mathrm{H}$ NMR (300 MHz, $\left.\mathrm{CDCl}_{3}\right)$
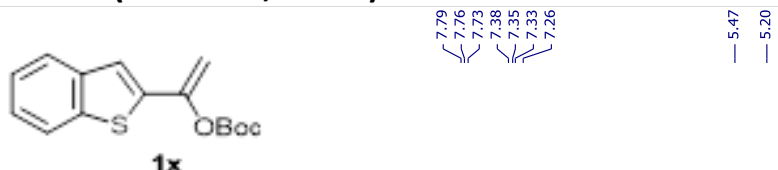

$1 \mathrm{x}$

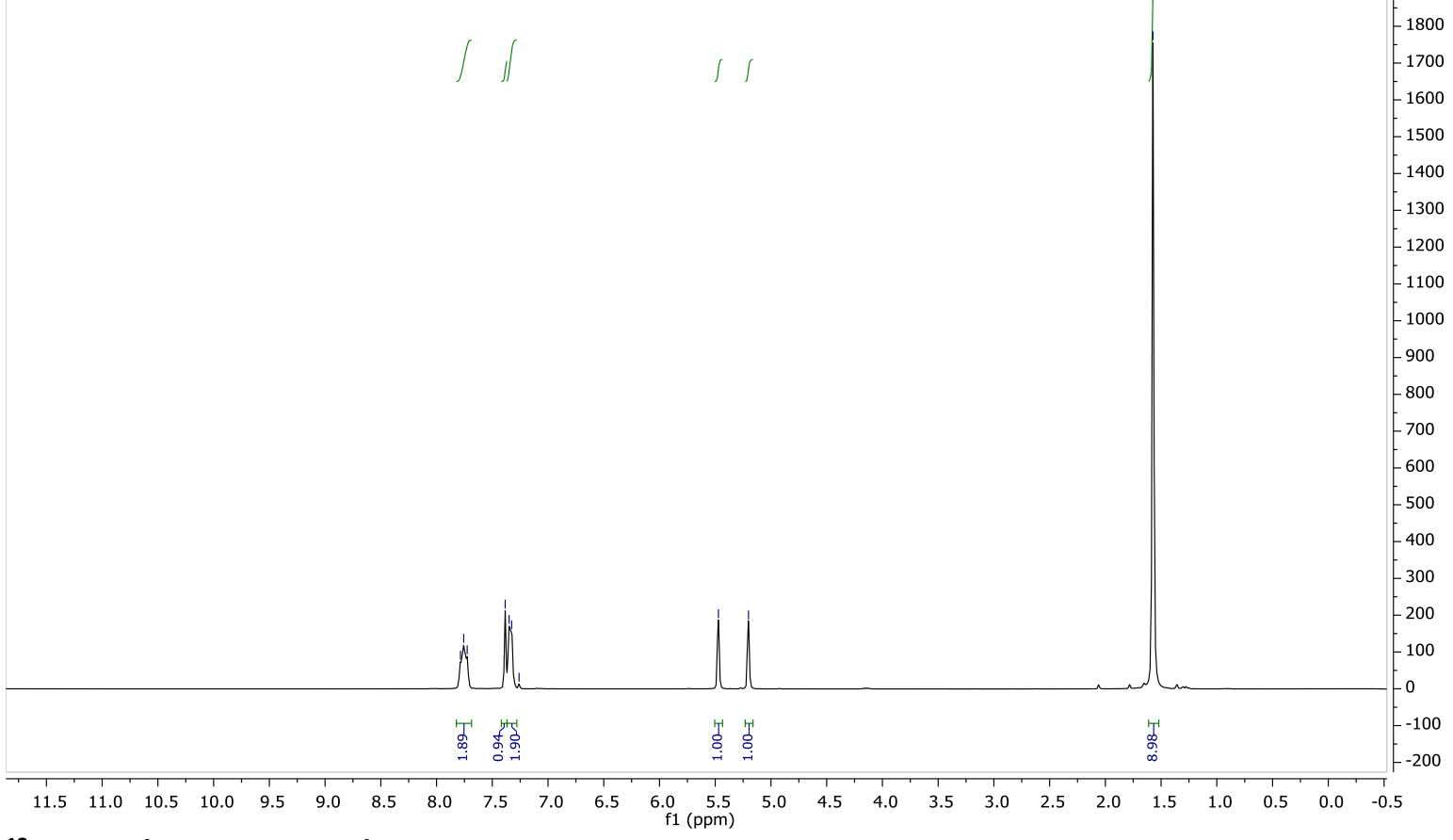

${ }^{13} \mathrm{C} \mathrm{NMR} \mathrm{(75} \mathrm{MHz}, \mathrm{CDCl}_{3}$ )
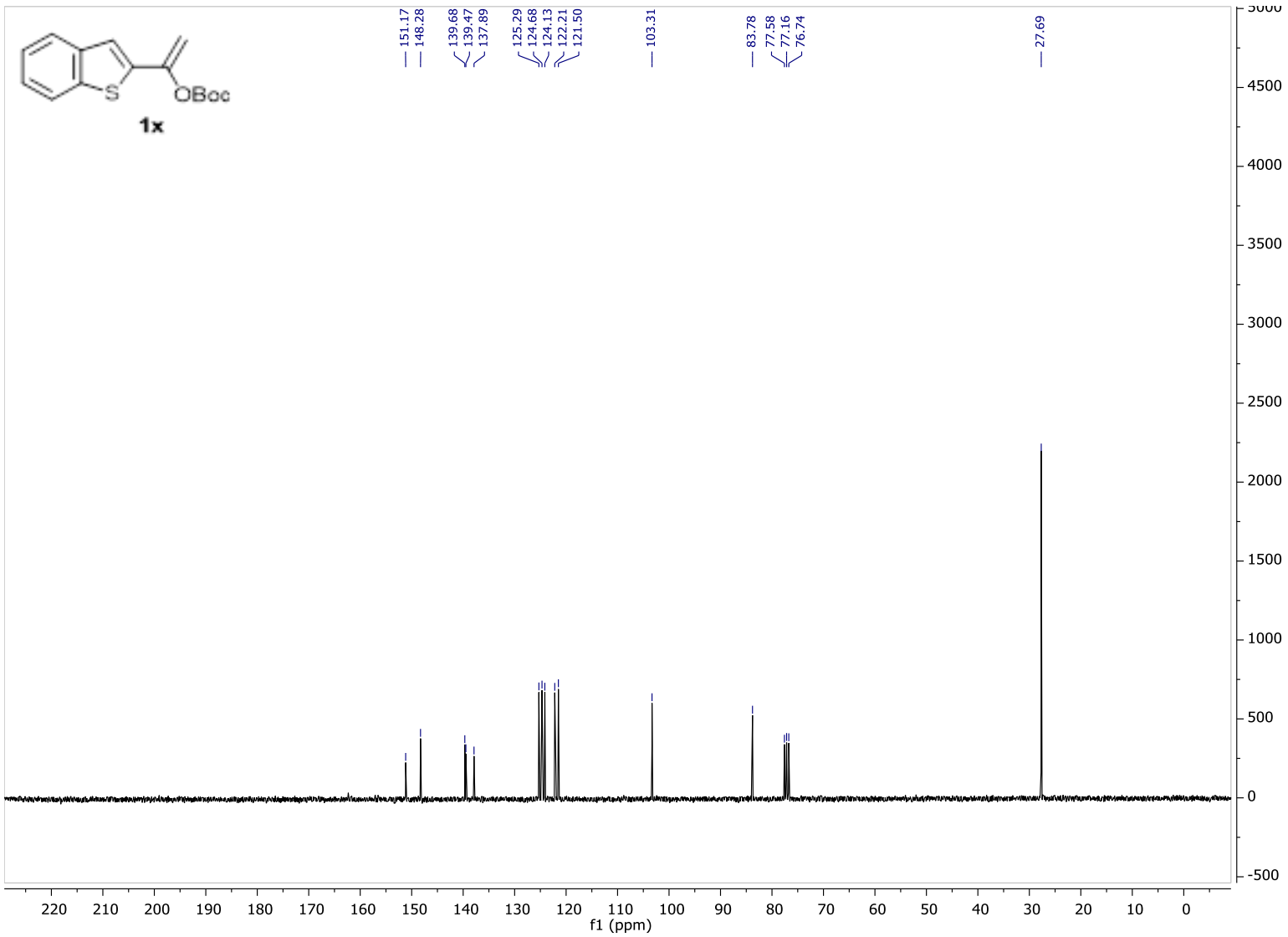
tert-butyl 1-(naphthalen-2-yl)vinyl carbonate 1y

${ }^{1} \mathrm{H}$ NMR (400 MHz, $\left.\mathrm{CDCl}_{3}\right)$

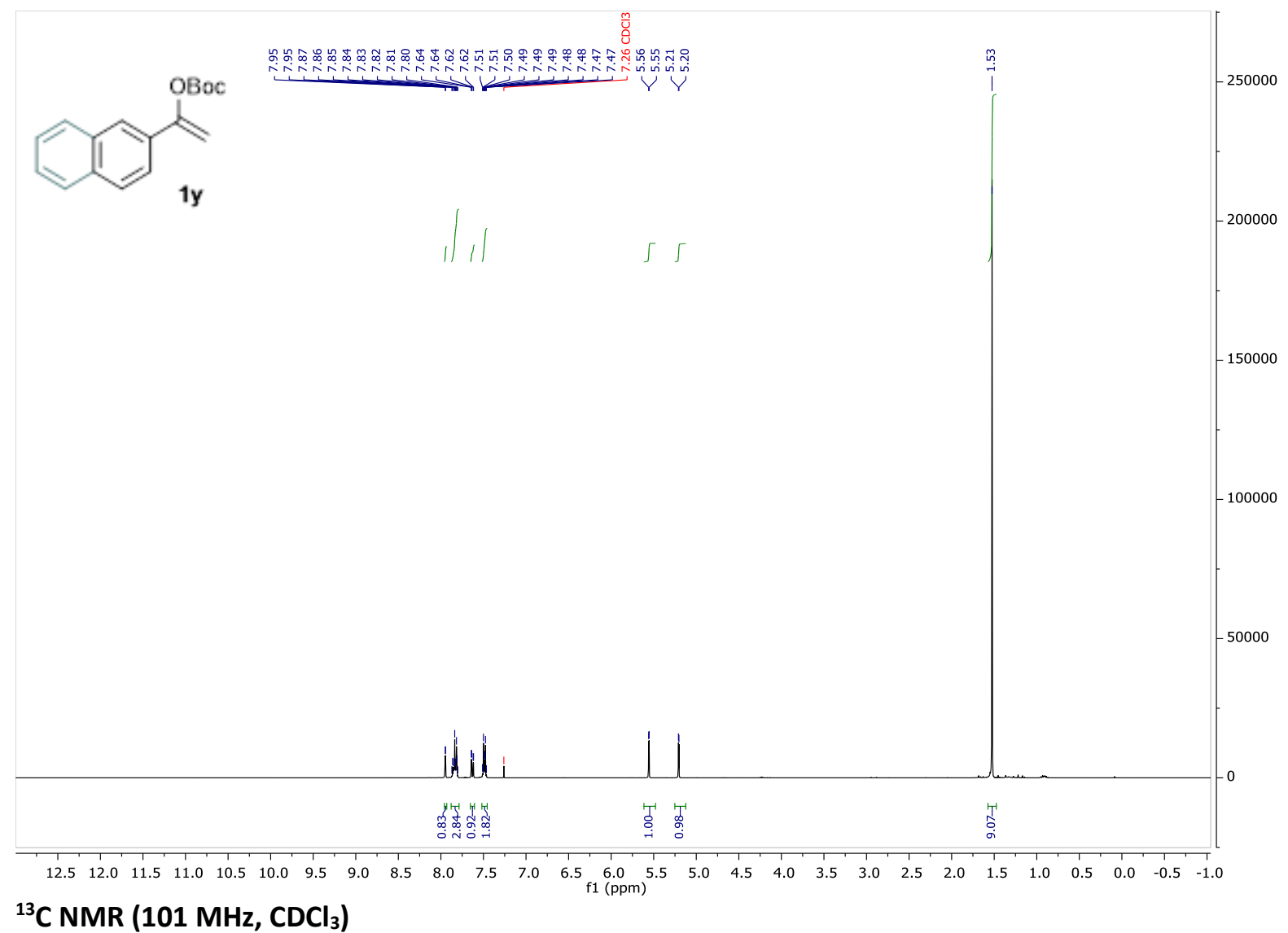

${ }^{13} \mathrm{C}$ NMR (101 MHz, $\left.\mathrm{CDCl}_{3}\right)$
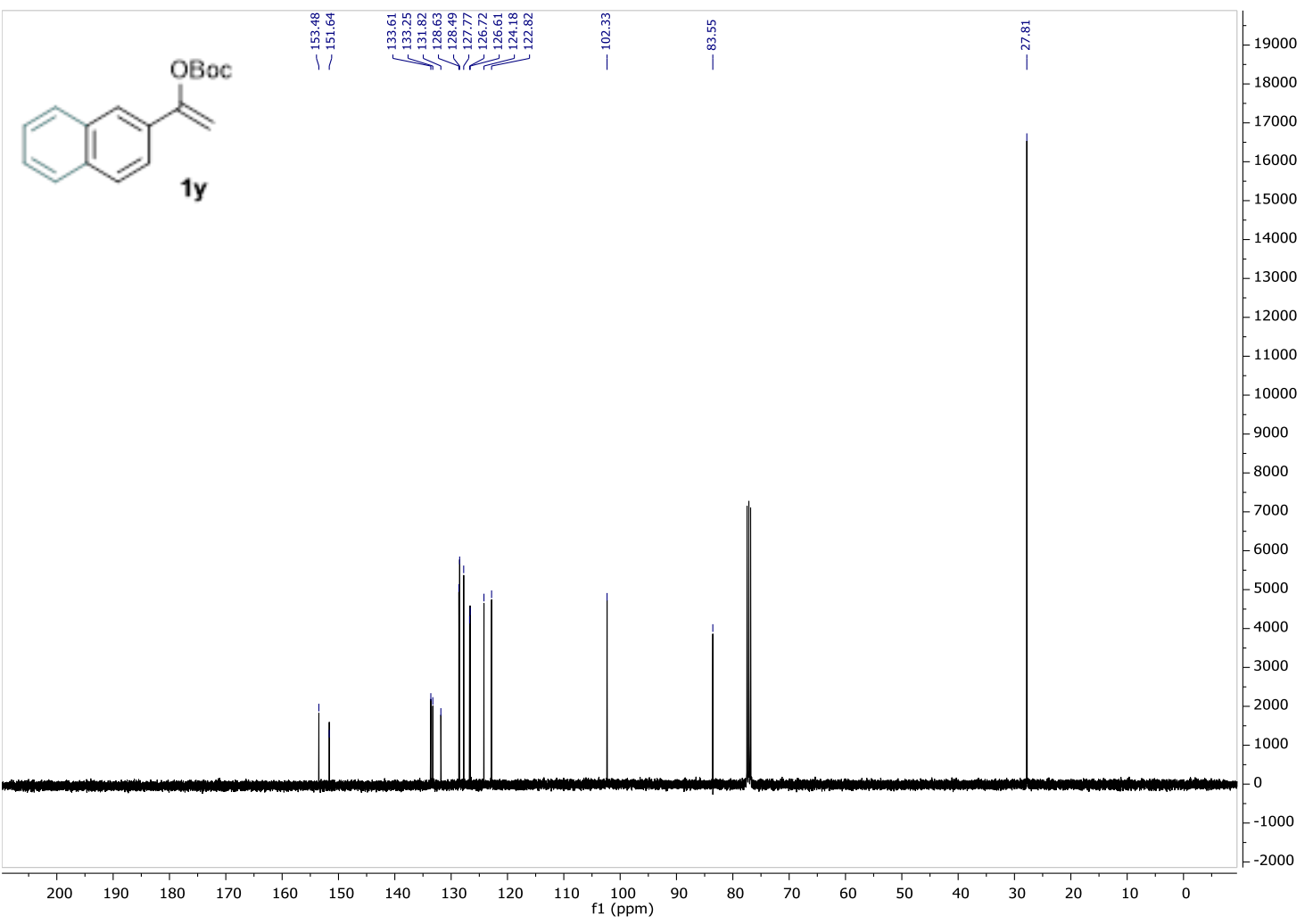
tert-butyl 2,2-difluoro-1-(naphthalen-2-yl)vinyl carbonate 1aa

${ }^{1} \mathrm{H}$ NMR $\left(500 \mathrm{MHz}, \mathrm{CDCl}_{3}\right)$

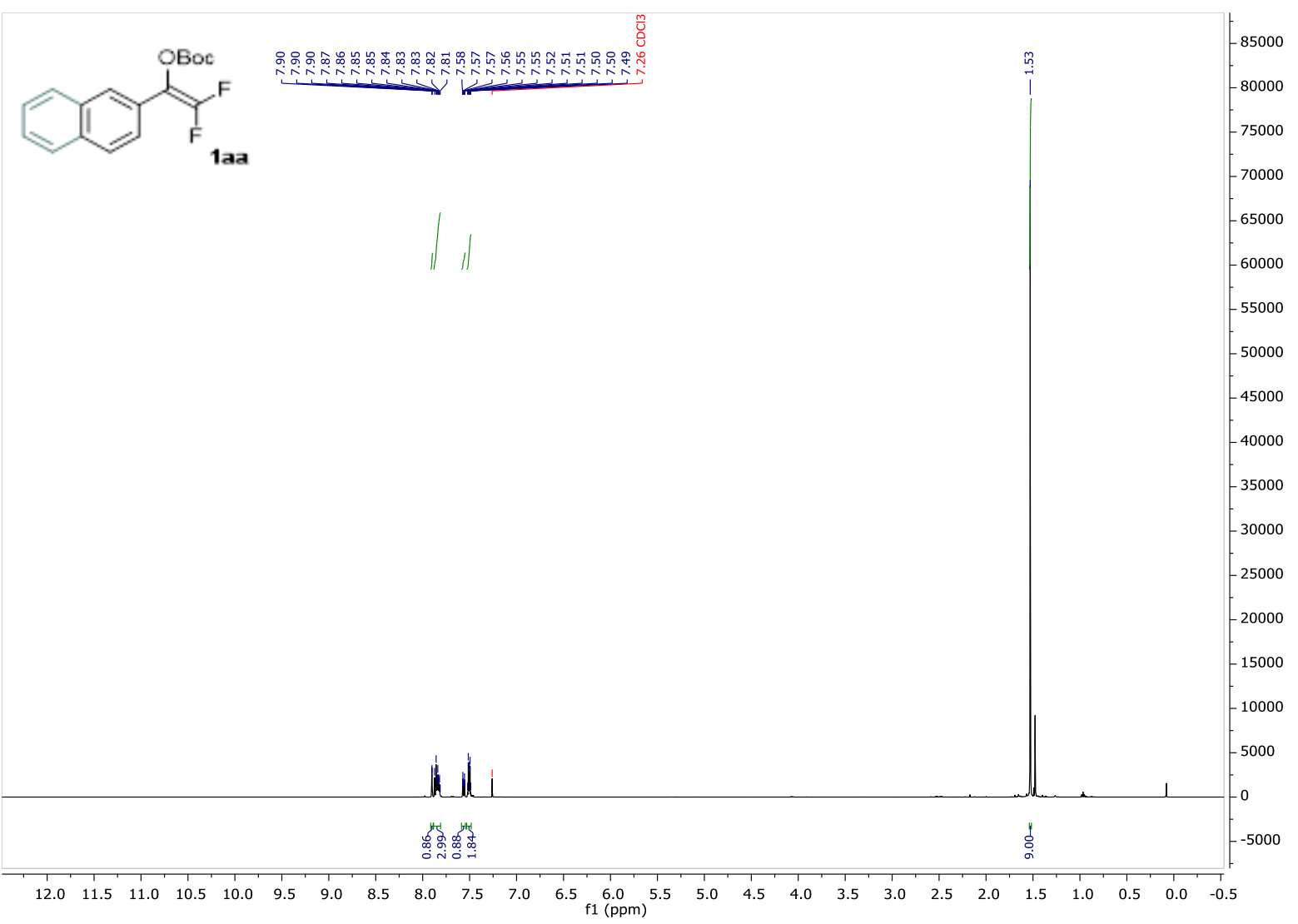

${ }^{13} \mathrm{C}$ NMR (126 MHz, $\left.\mathrm{CDCl}_{3}\right)$

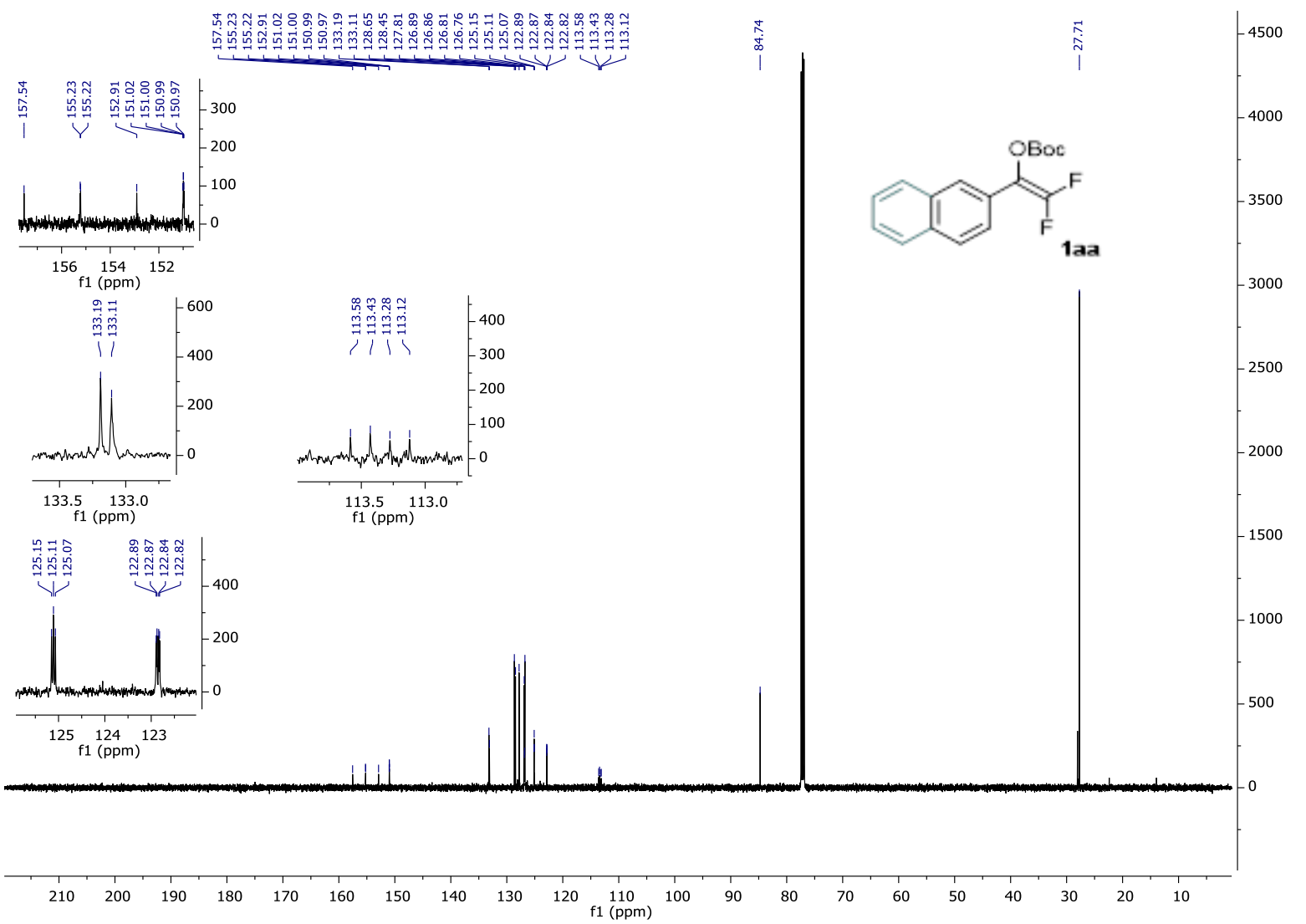


${ }^{19} \mathrm{~F}$ NMR (188 MHz, $\left.\mathrm{CDCl}_{3}\right)$

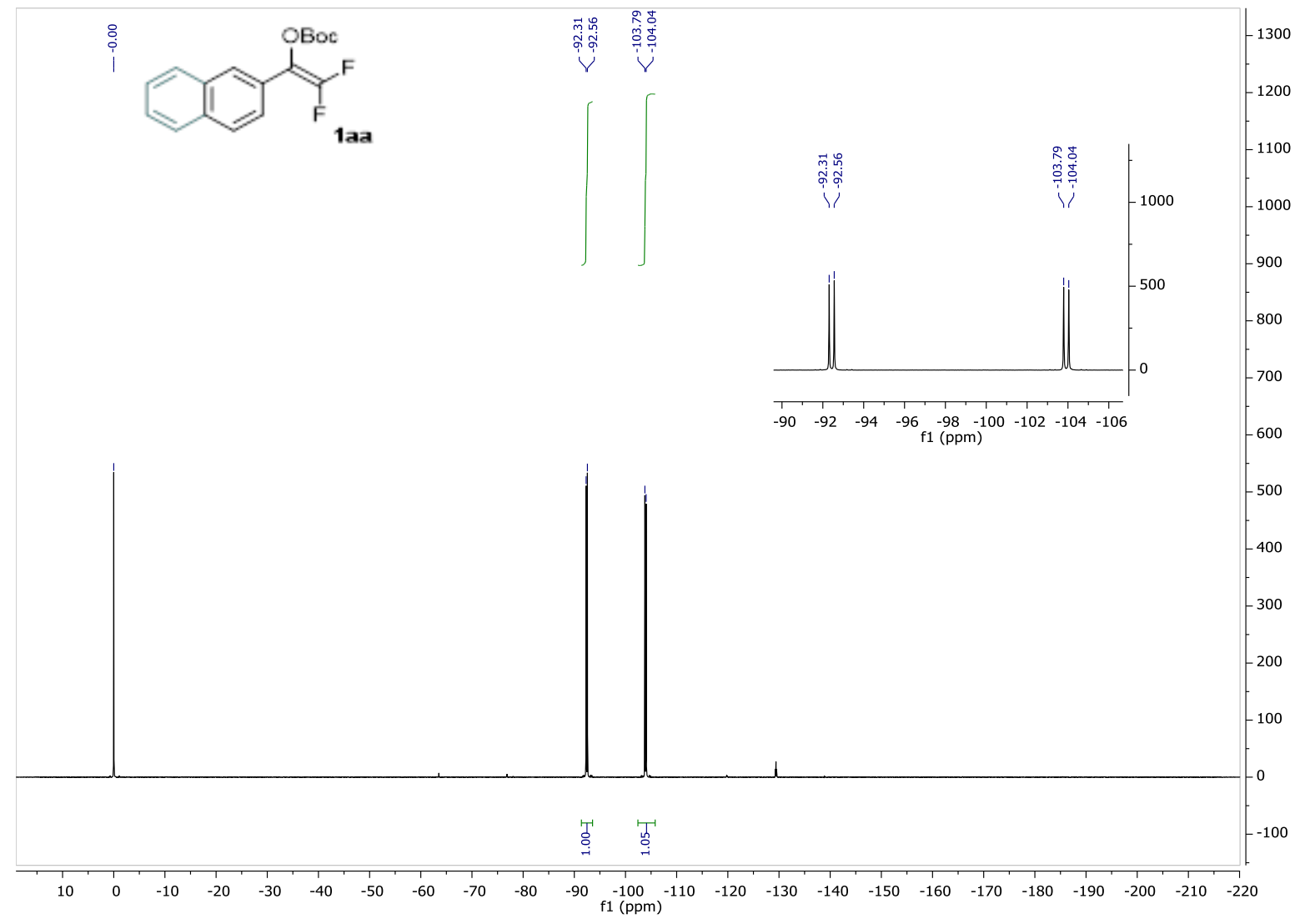

tert-butyl oct-1-en-2-yl carbonate 1ab

${ }^{1} \mathrm{H}$ NMR (400 MHz, $\mathrm{CDCl}_{3}$ )

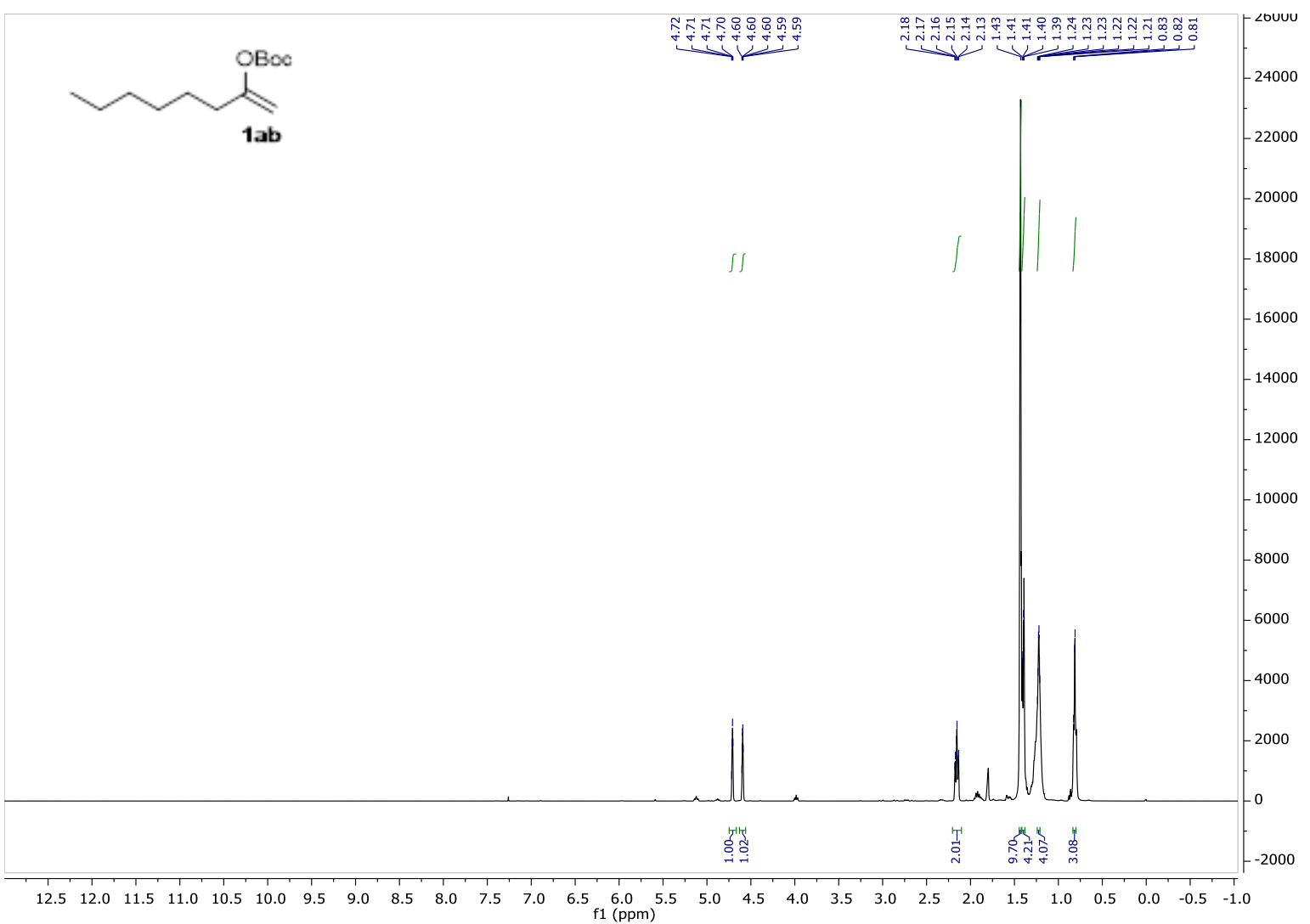


${ }^{13} \mathrm{C}$ NMR (101 MHz, $\left.\mathrm{CDCl}_{3}\right)$

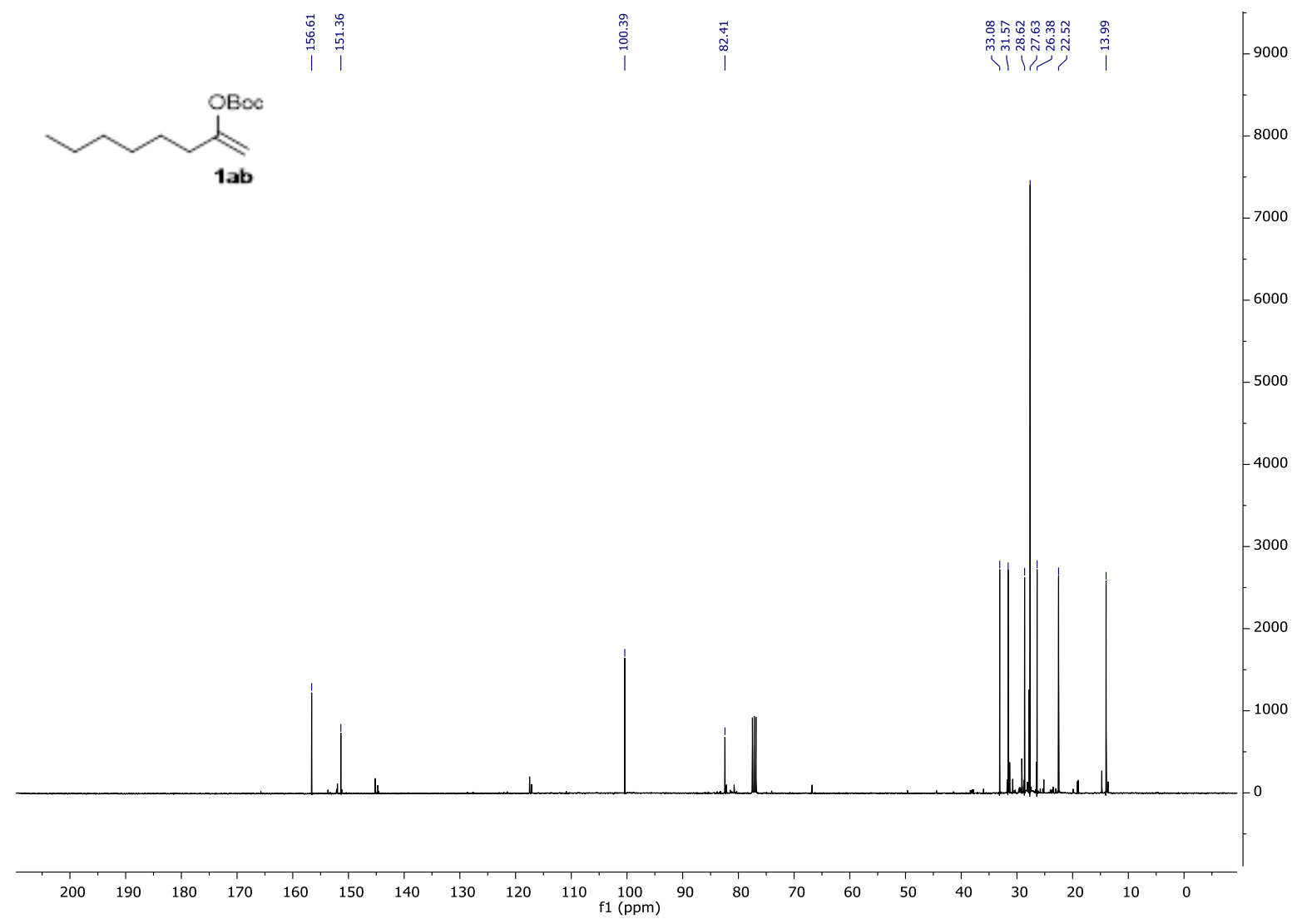

(E)-tert-butyl (4-phenylbuta-1,3-dien-2-yl) carbonate 1ac

${ }^{1} \mathrm{H}$ NMR (300 MHz, $\left.\mathrm{CDCl}_{3}\right)$

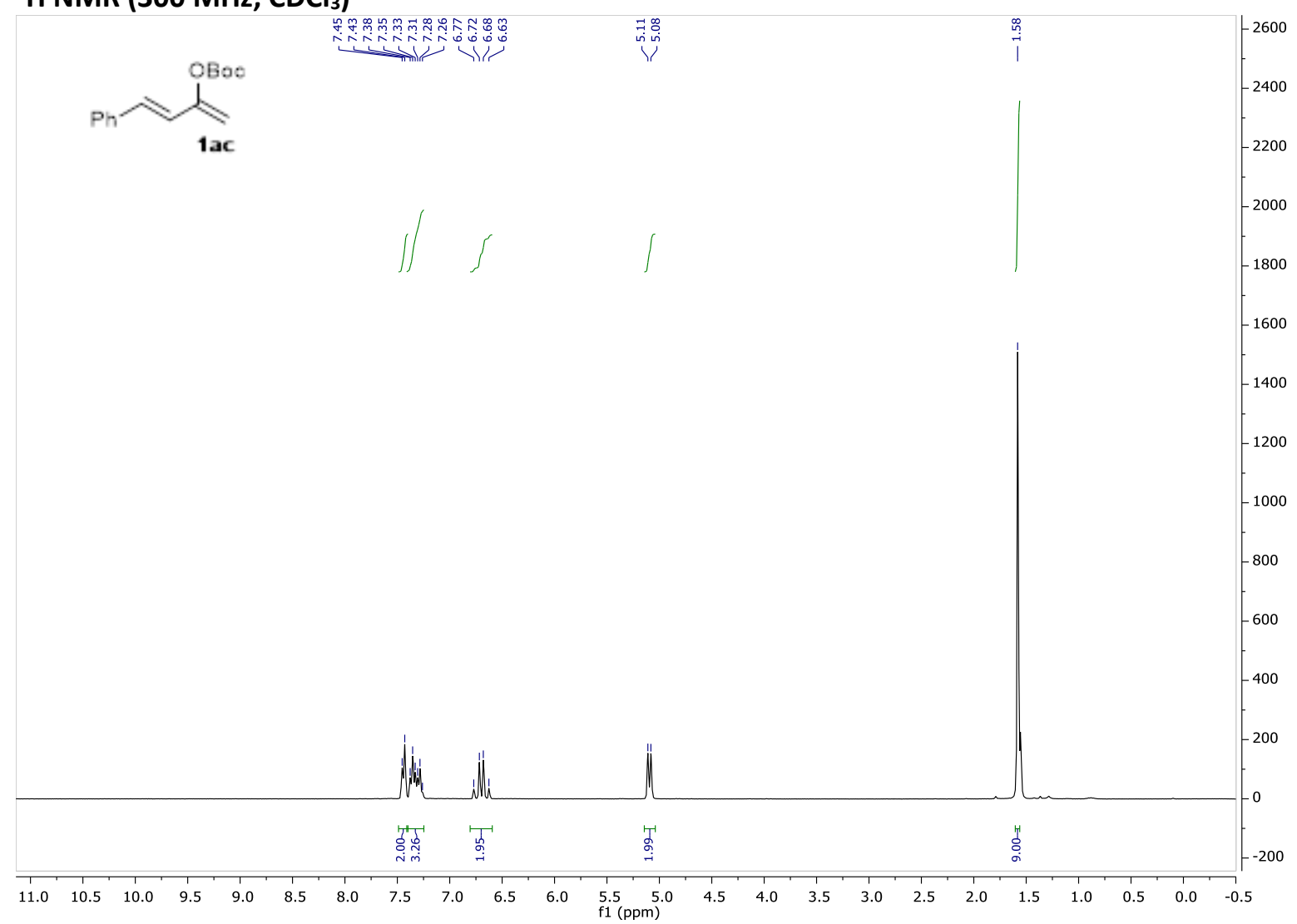


${ }^{13} \mathrm{C}$ NMR (75 MHz, $\left.\mathrm{CDCl}_{3}\right)$

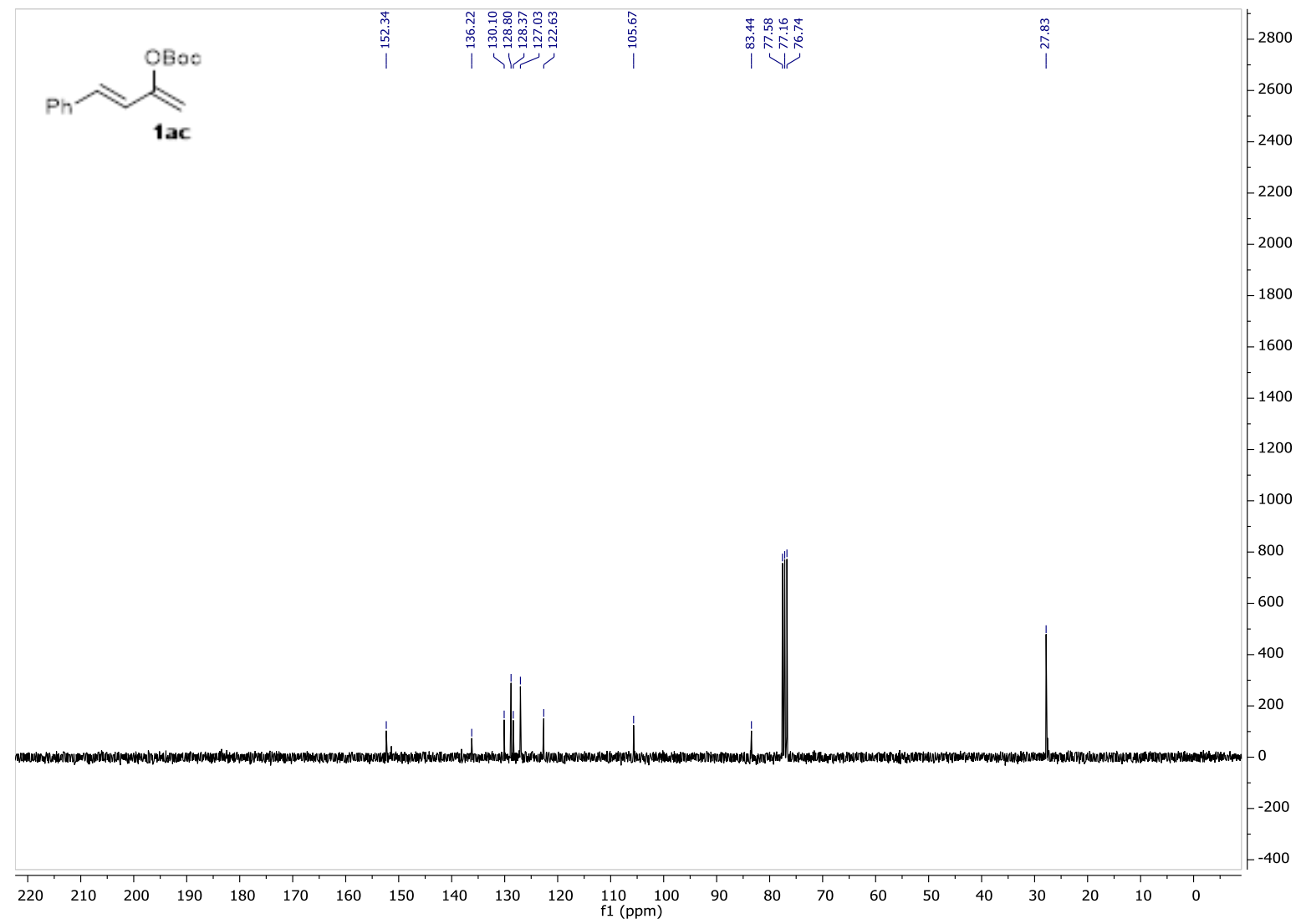

(E)-tert-butyl nona-1,3-dien-2-yl carbonate 1ad

${ }^{1} \mathrm{H}$ NMR $\left(300 \mathrm{MHz}, \mathrm{CDCl}_{3}\right)$

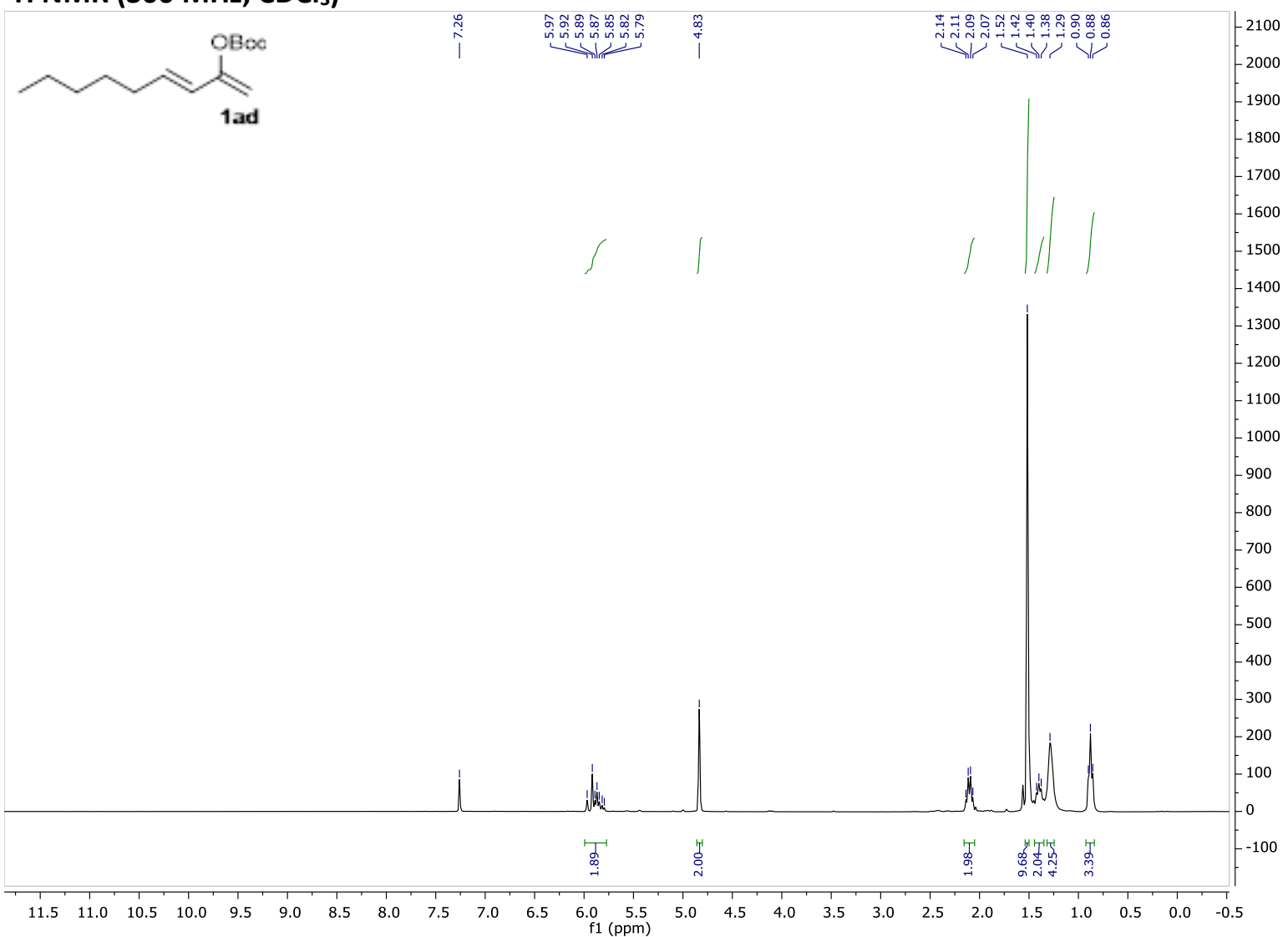




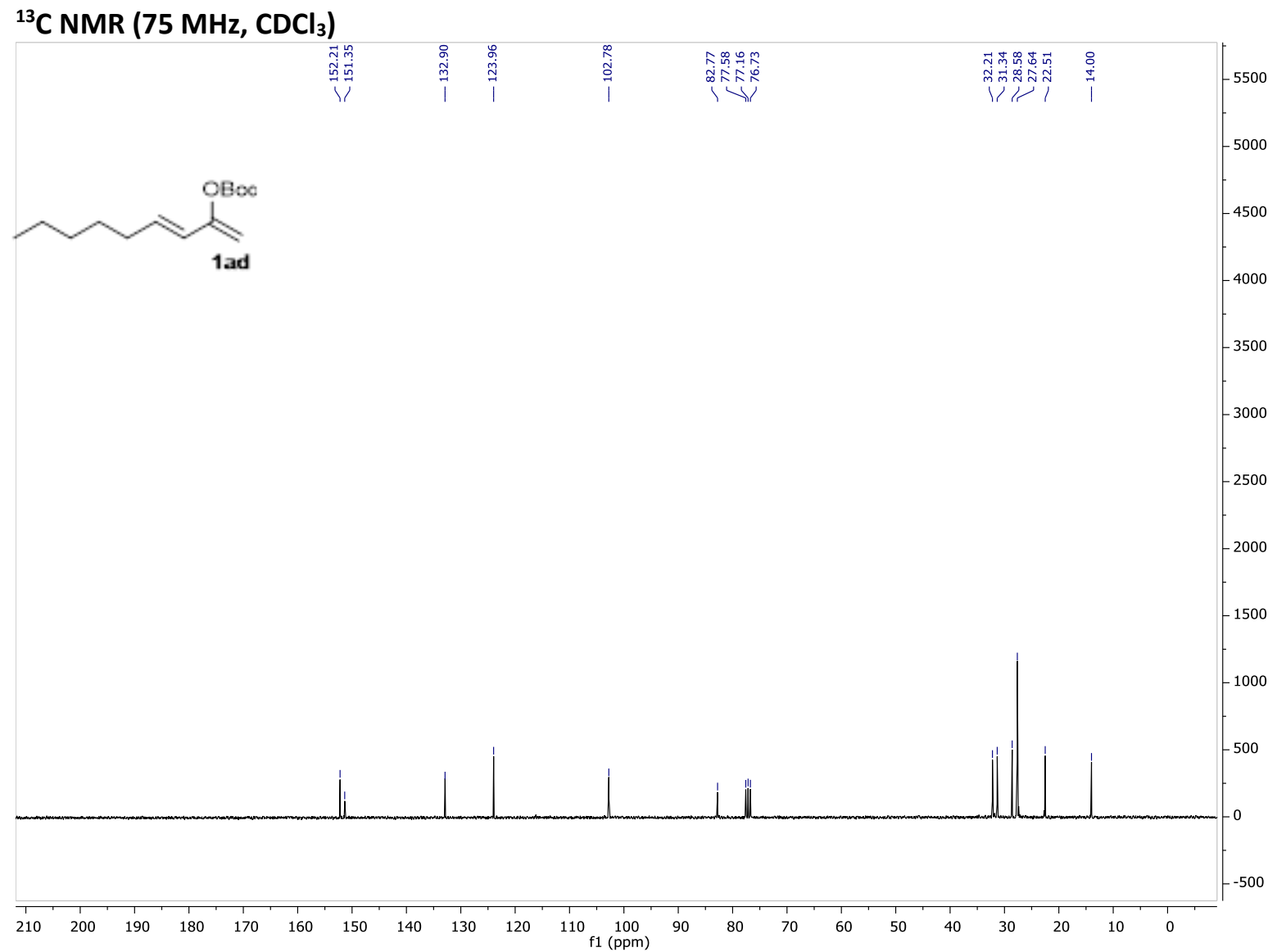

tert-butyl 1-(3,5,5,6,8,8-hexamethyl-5,6,7,8-tetrahydronaphthalen-2-yl)vinyl carbonate 1ae ${ }^{1} \mathrm{H}$ NMR $\left(500 \mathrm{MHz}, \mathrm{CDCl}_{3}\right)$

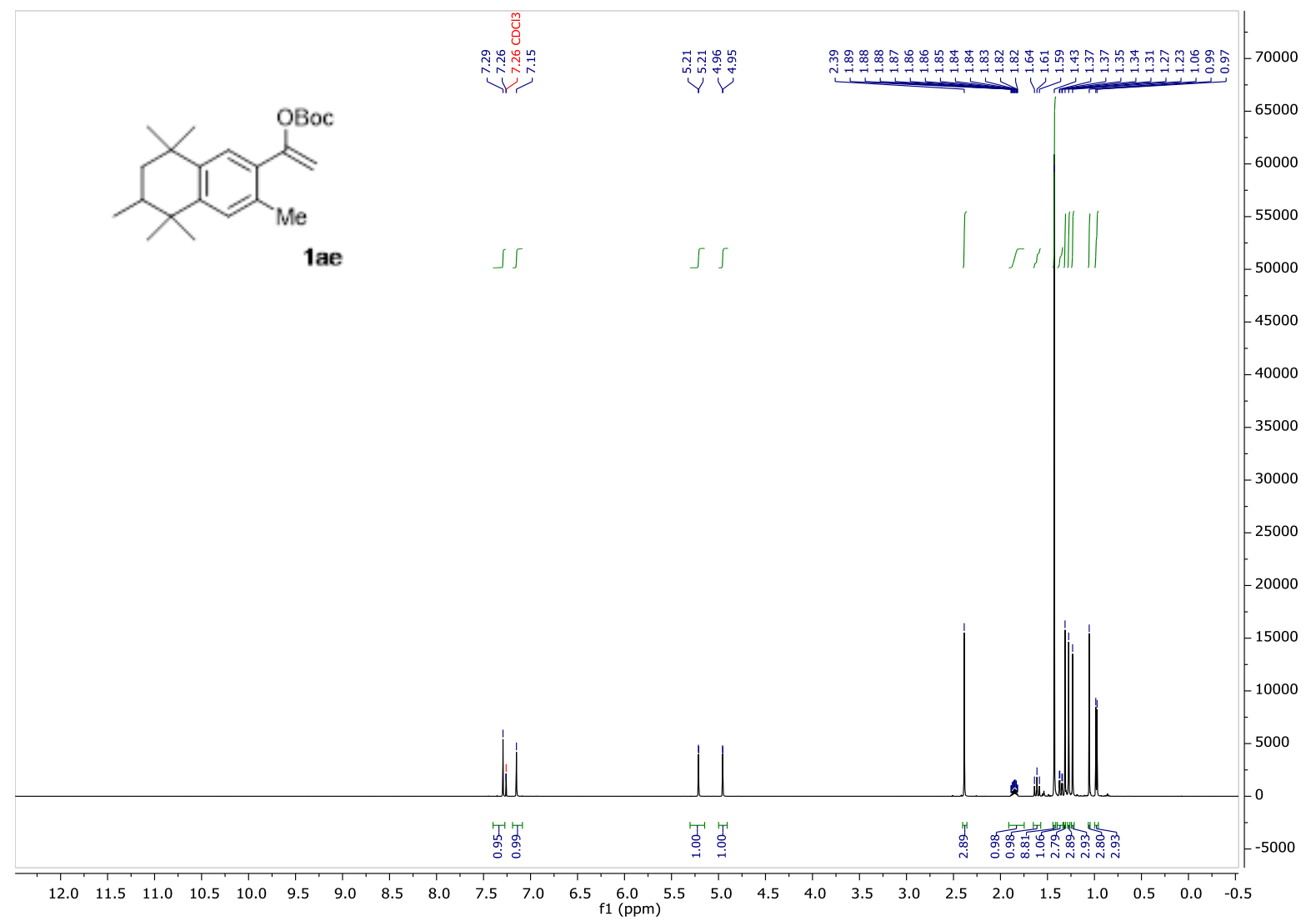


${ }^{13} \mathrm{C}$ NMR (126 MHz, $\left.\mathrm{CDCl}_{3}\right)$

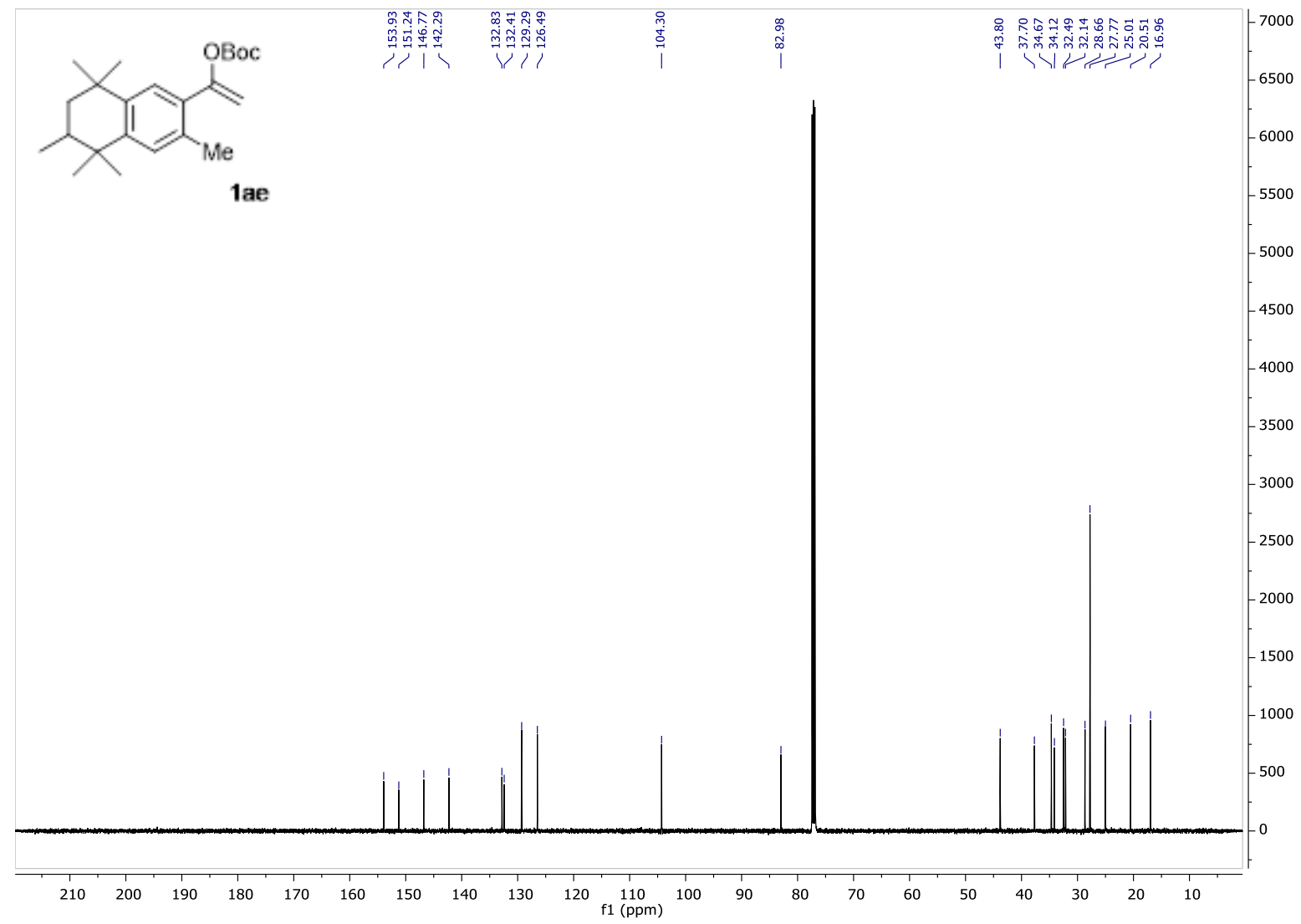

tert-butyl (1-(4-(tert-butyl)-2,6-dimethyl-3,5-dinitrophenyl)vinyl) carbonate 1af

${ }^{1} \mathrm{H}$ NMR $\left(300 \mathrm{MHz}, \mathrm{CDCl}_{3}\right)$

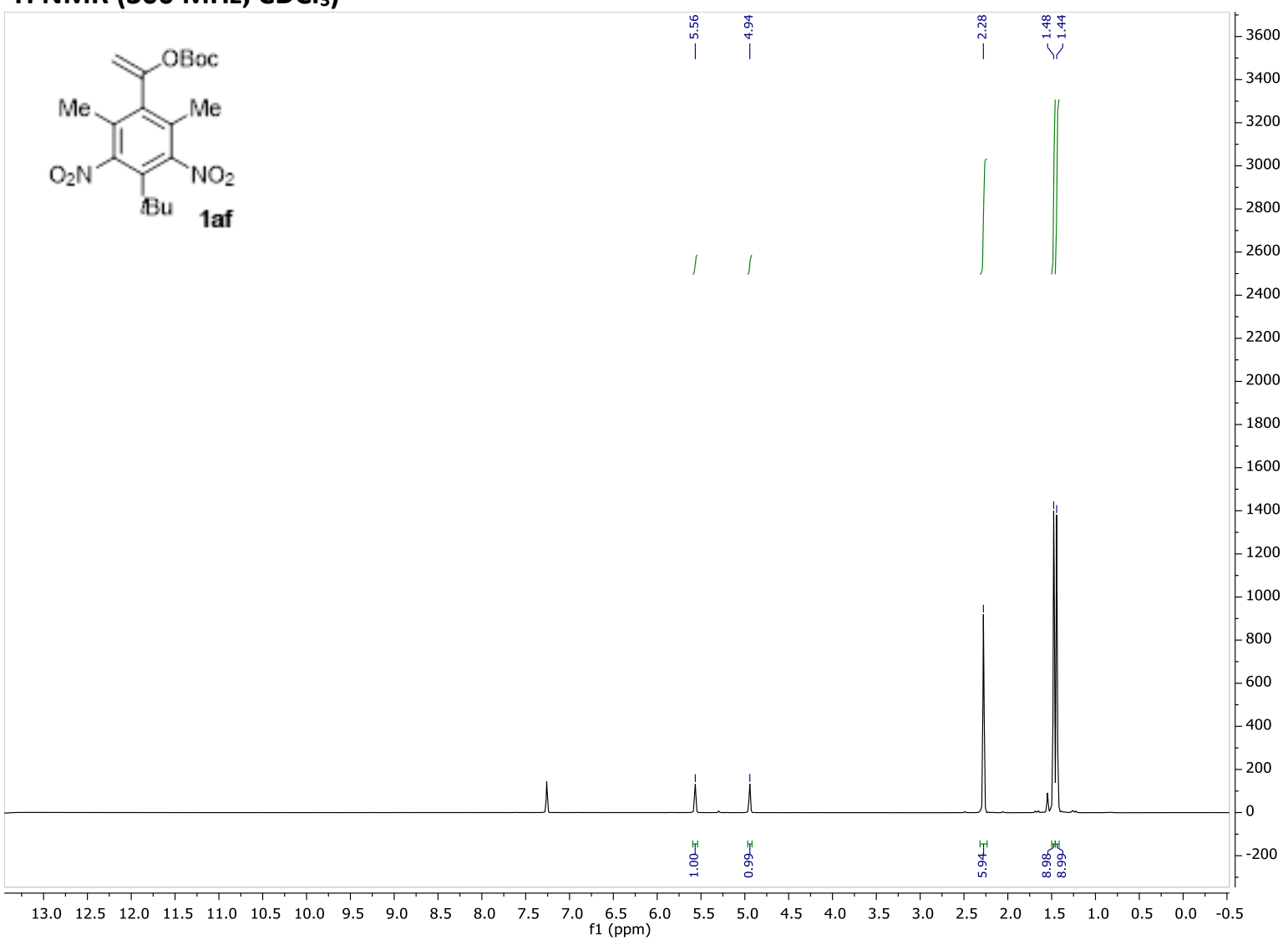


${ }^{13} \mathrm{C}$ NMR $\left(75 \mathrm{MHz}, \mathrm{CDCl}_{3}\right)$
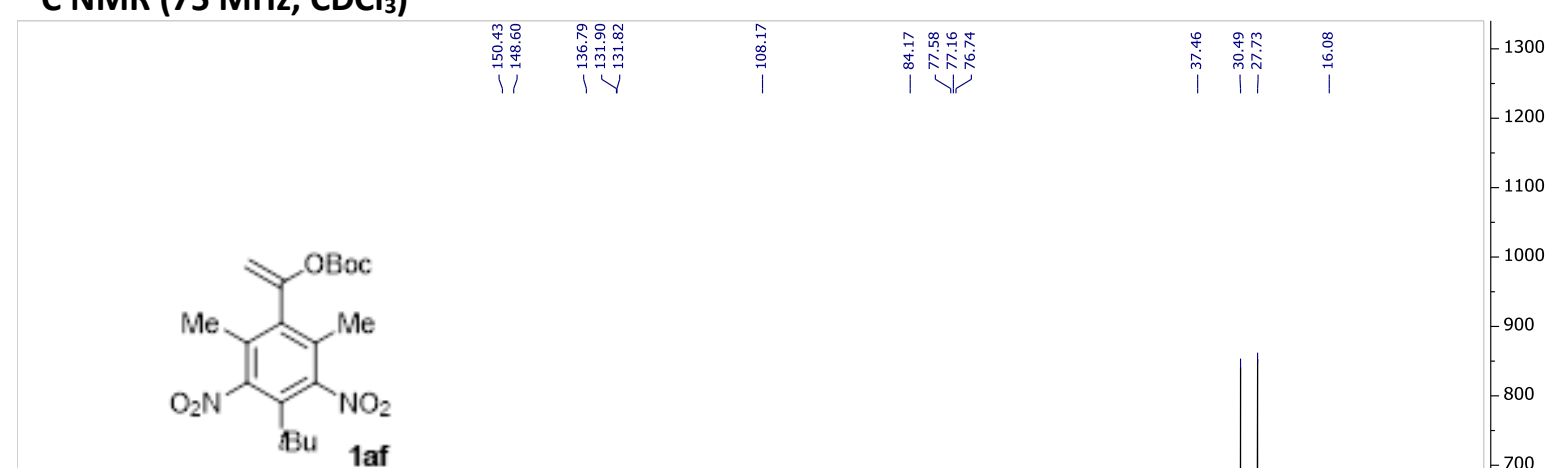

$$
\text { at }
$$

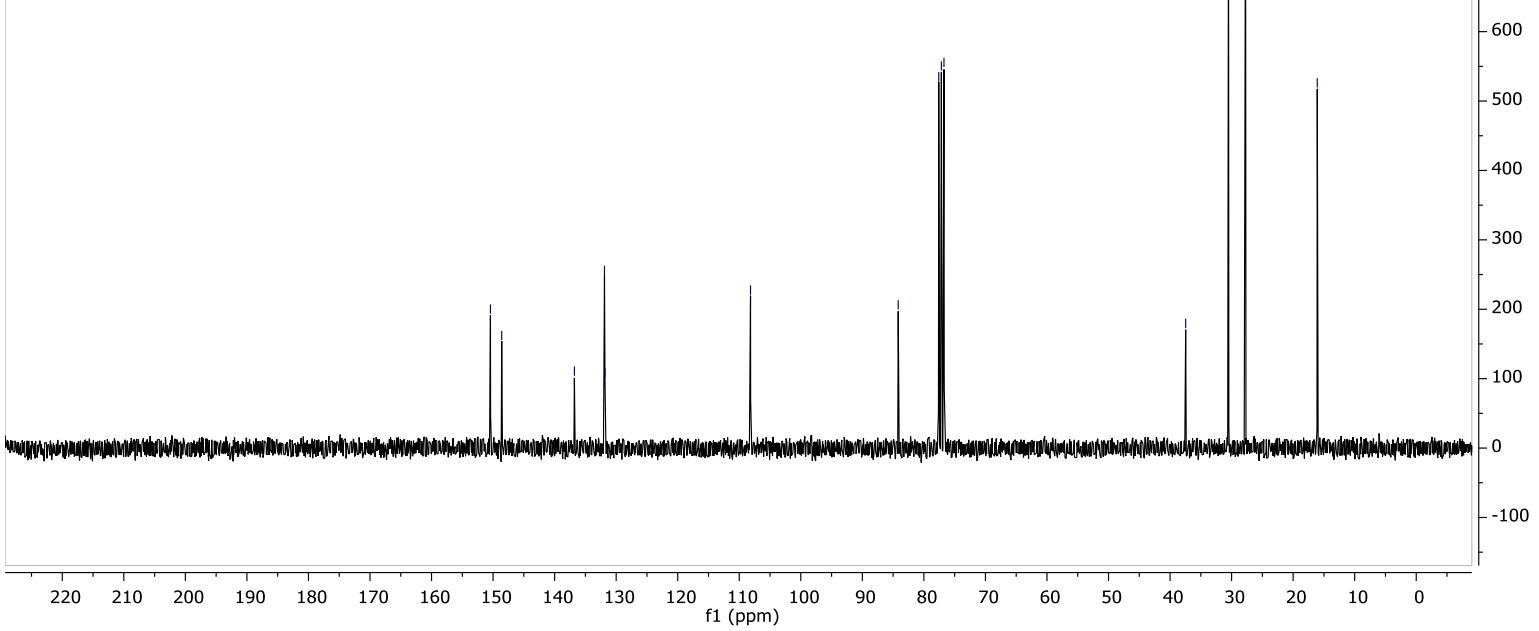

tert-butyl (1-(6-(tert-butyl)-1,1-dimethyl-2,3-dihydro-1H-inden-4-yl)vinyl) carbonate 1ag ${ }^{1} \mathrm{H}$ NMR $\left(300 \mathrm{MHz}, \mathrm{CDCl}_{3}\right)$

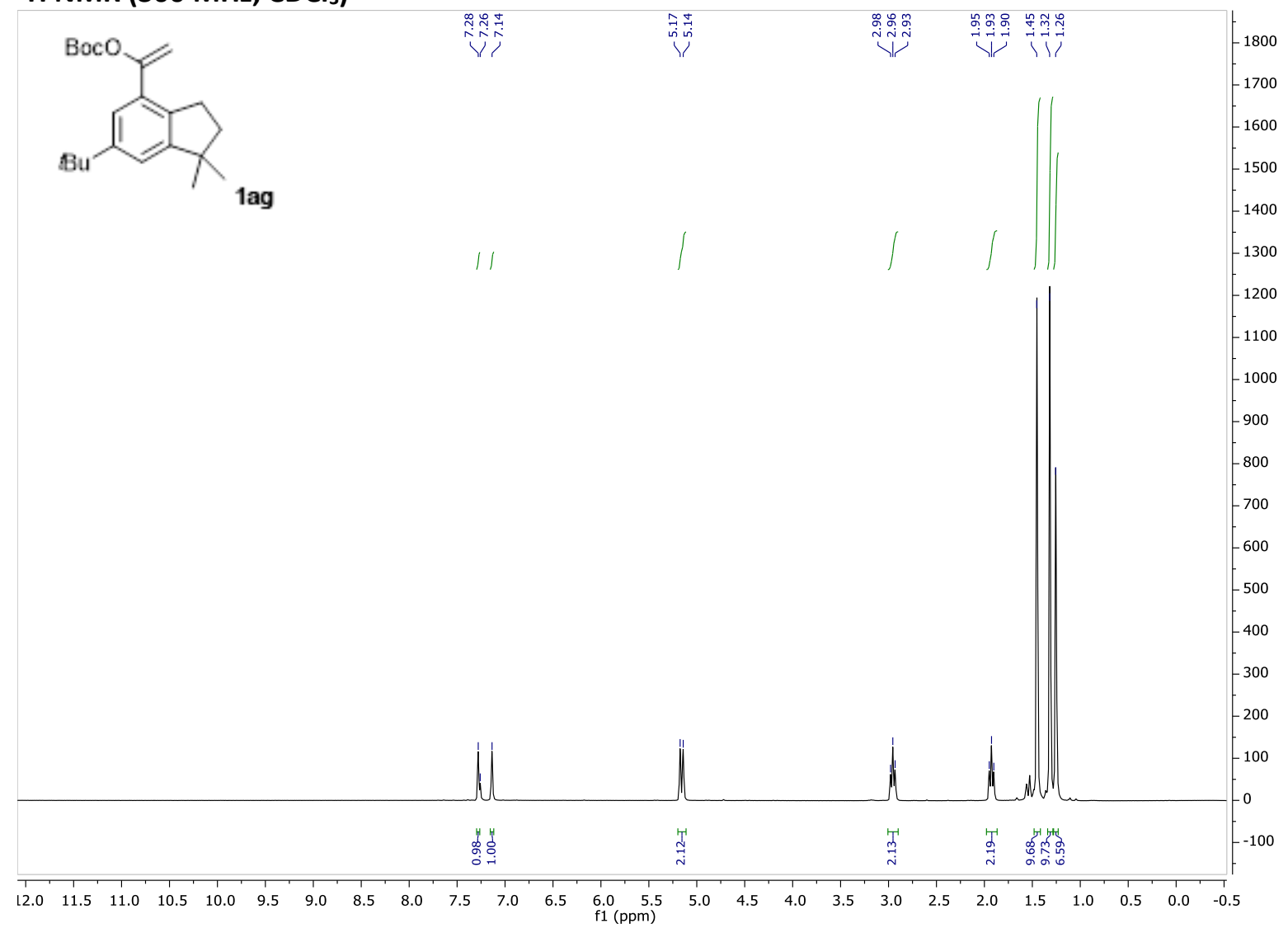




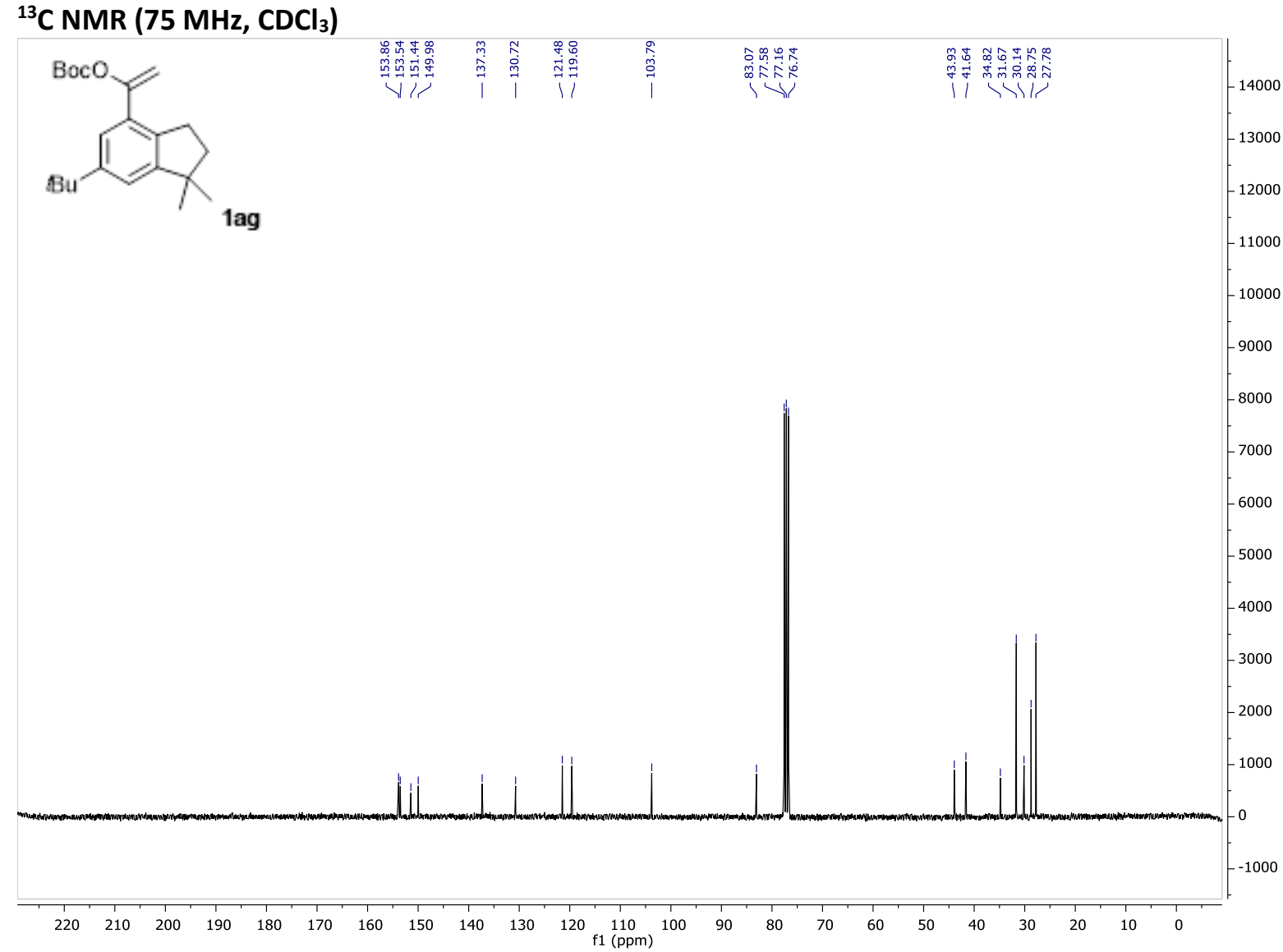

(E)-tert-butyl (4-(2,6,6-trimethylcyclohex-2-en-1-yl)buta-1,3-dien-2-yl) carbonate 1ah

${ }^{1} \mathrm{H}$ NMR (300 MHz, $\mathrm{CDCl}_{3}$ )

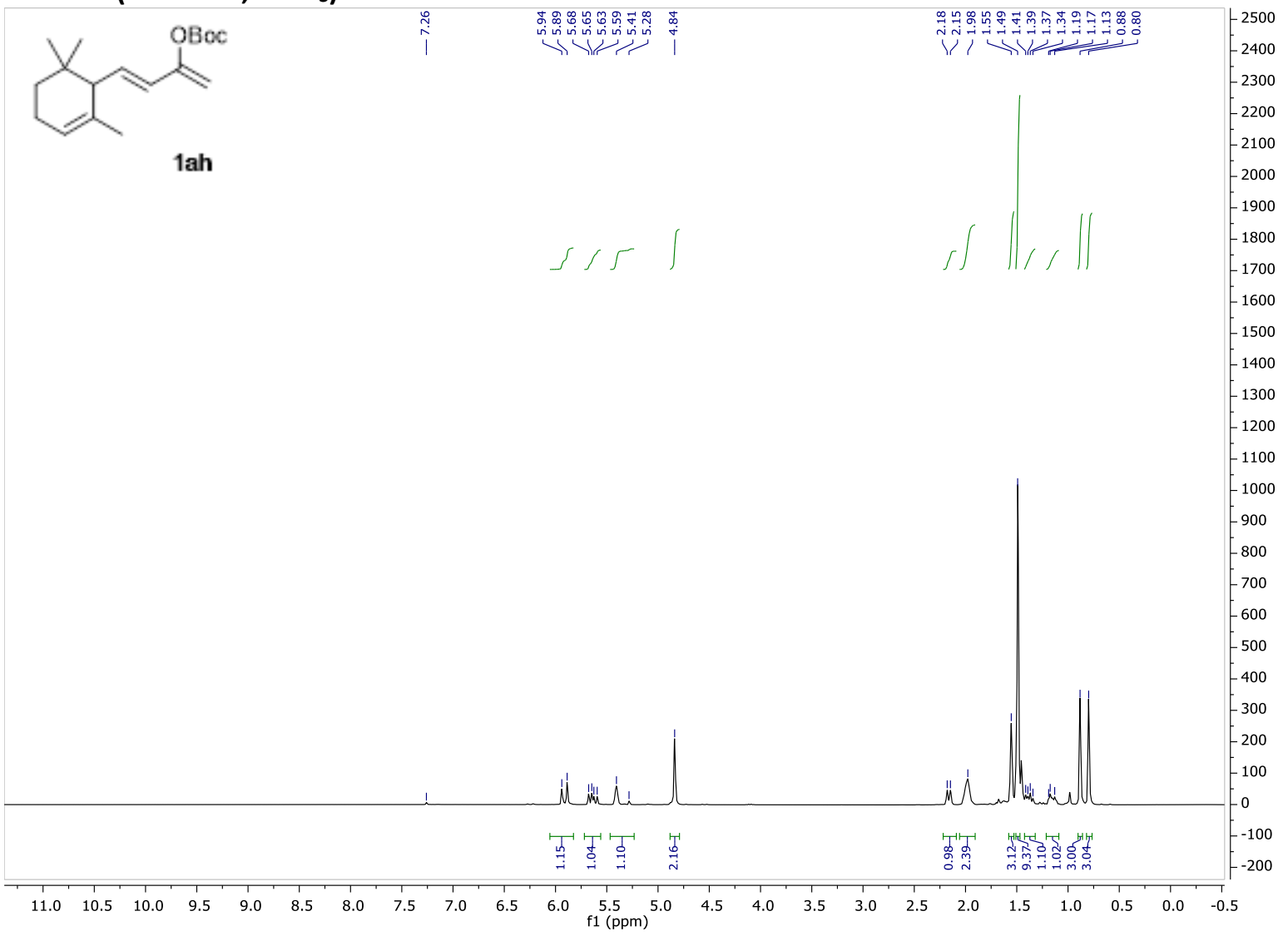




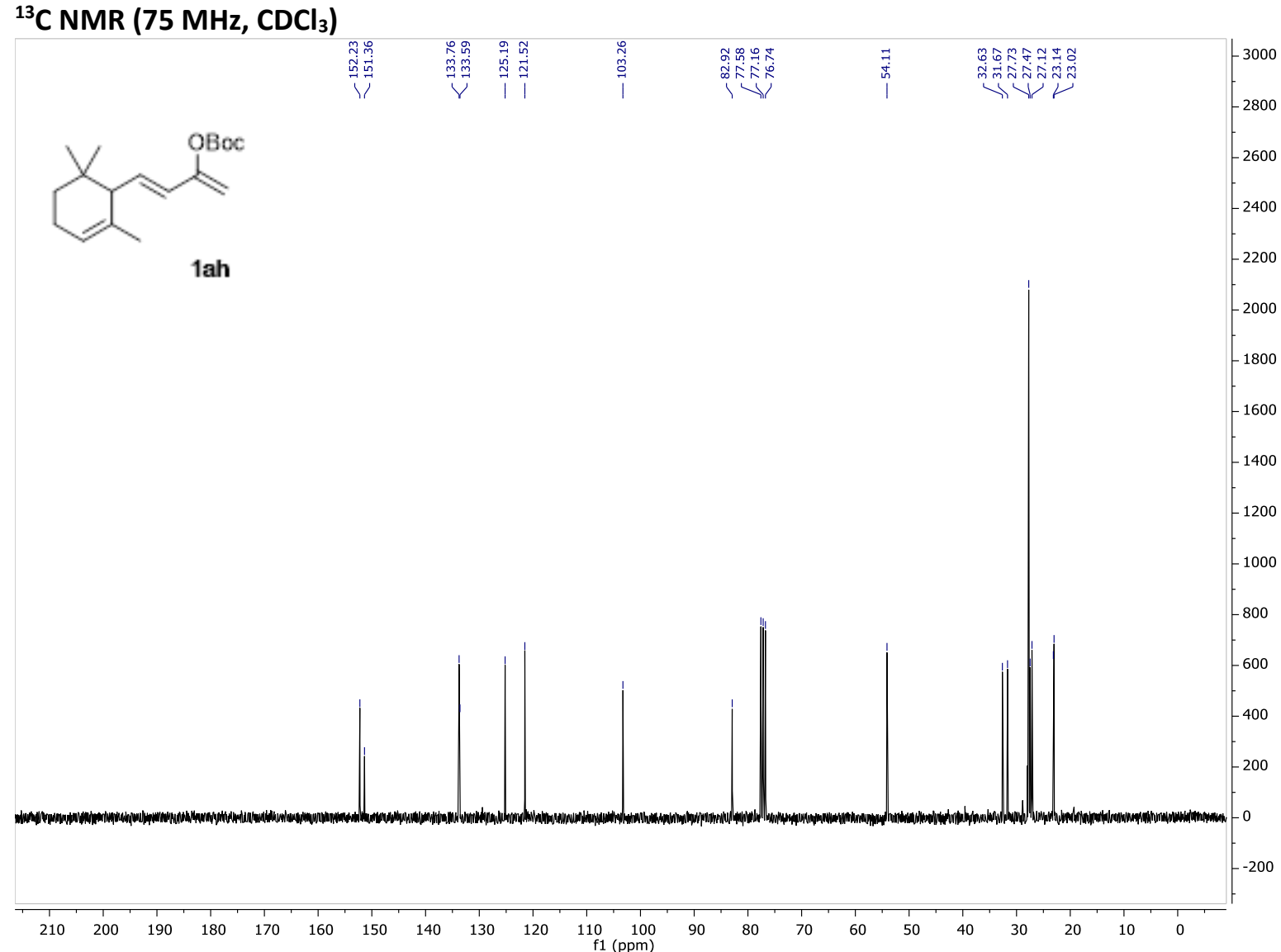

tert-butyl $\quad(1-((3 S, 10 R, 13 S, 17 S)-3-m e t h o x y-10,13-d i m e t h y l-2,3,4,7,8,9,10,11,12,13,14,15,16,17-$ tetradecahydro-1H-cyclopenta[a]phenanthren-17-yl)vinyl) carbonate 1ai

${ }^{1} \mathrm{H}$ NMR $\left(500 \mathrm{MHz}, \mathrm{CDCl}_{3}\right)$

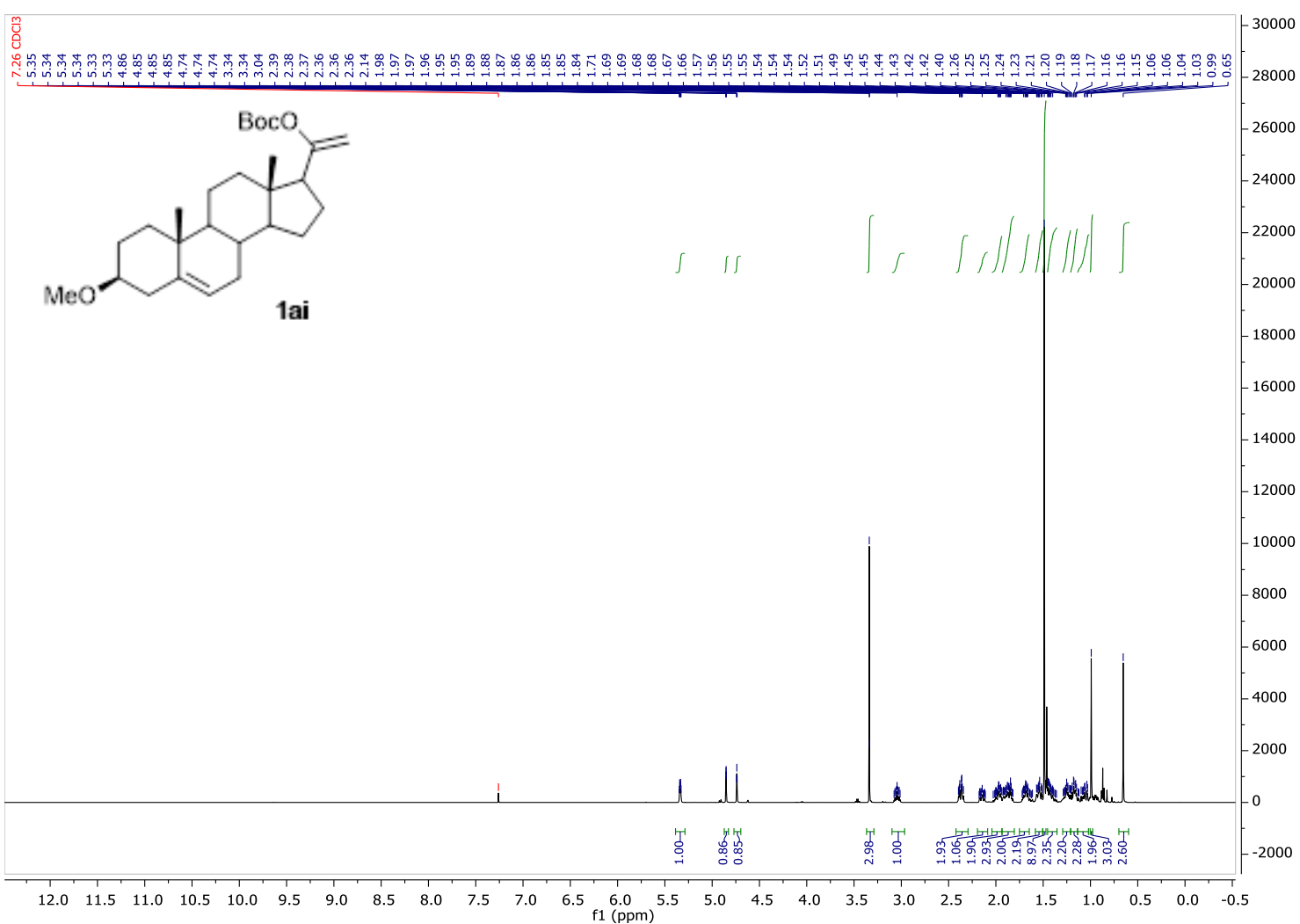


$\left.{ }^{13} \mathrm{C} \mathrm{NMR} \mathrm{(126} \mathrm{MHz,} \mathrm{CDCl}_{3}\right)$

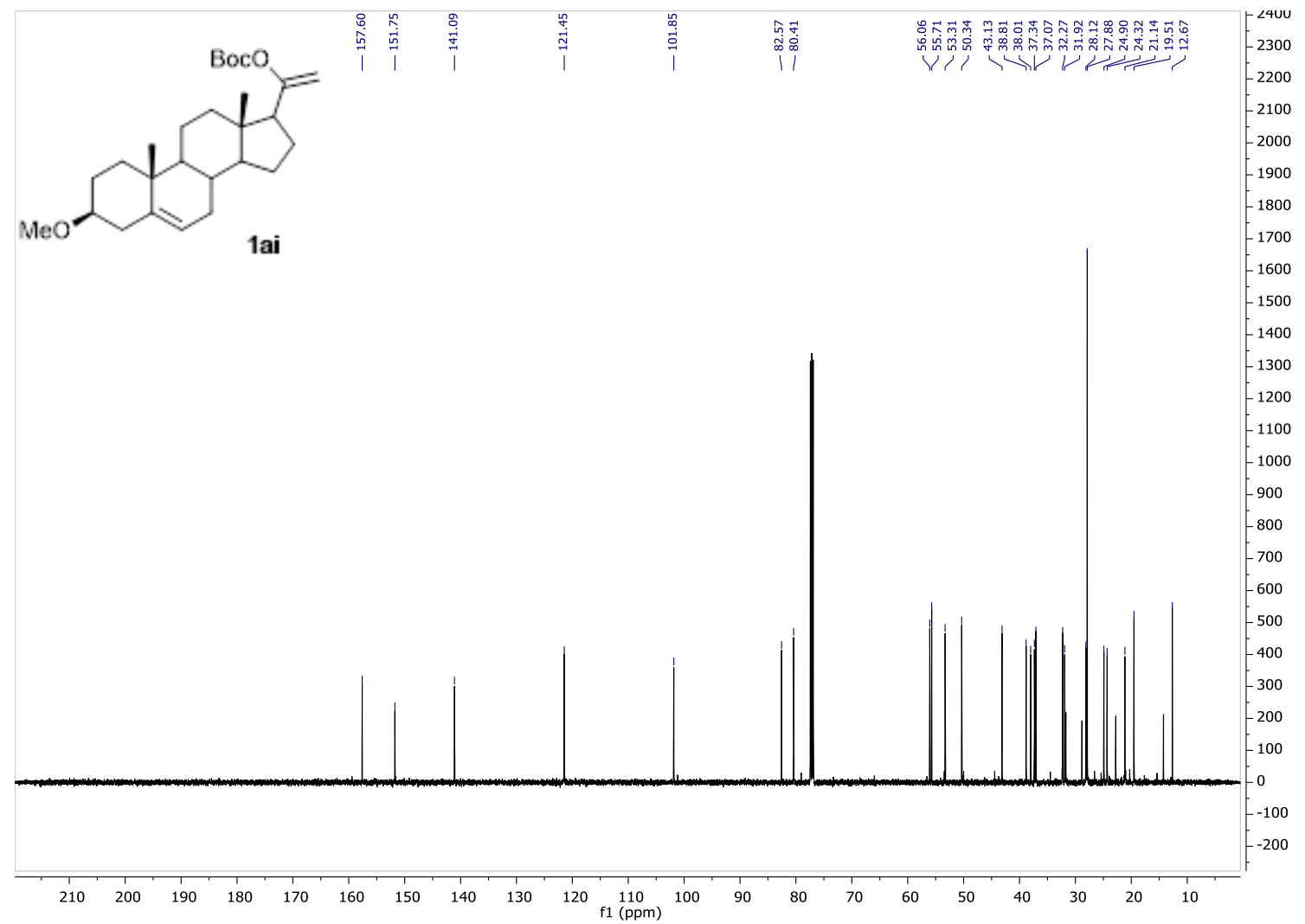

tert-butyl

((10R,13S,17S)-17-methoxy-10,13-dimethyl-2,7,8,9,10,11,12,13,14,15,16,17dodecahydro-1H-cyclopenta[a]phenanthren-3-yl) carbonate 1aj

${ }^{1} \mathrm{H}$ NMR (300 MHz, $\mathrm{CDCl}_{3}$ )

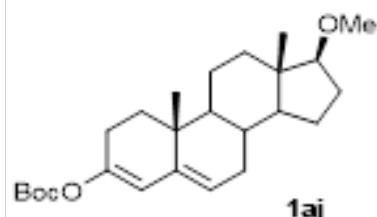

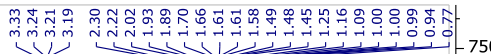

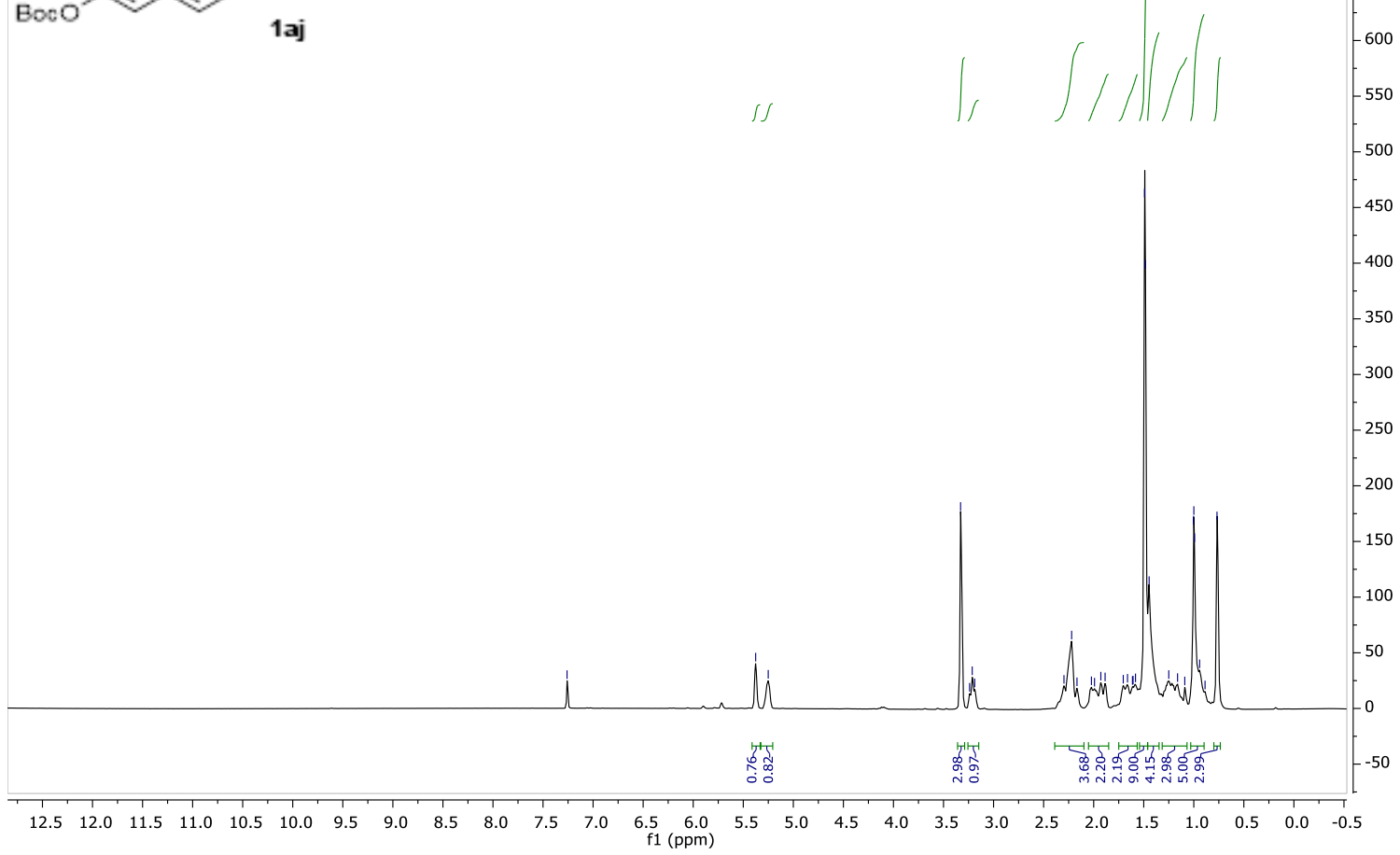




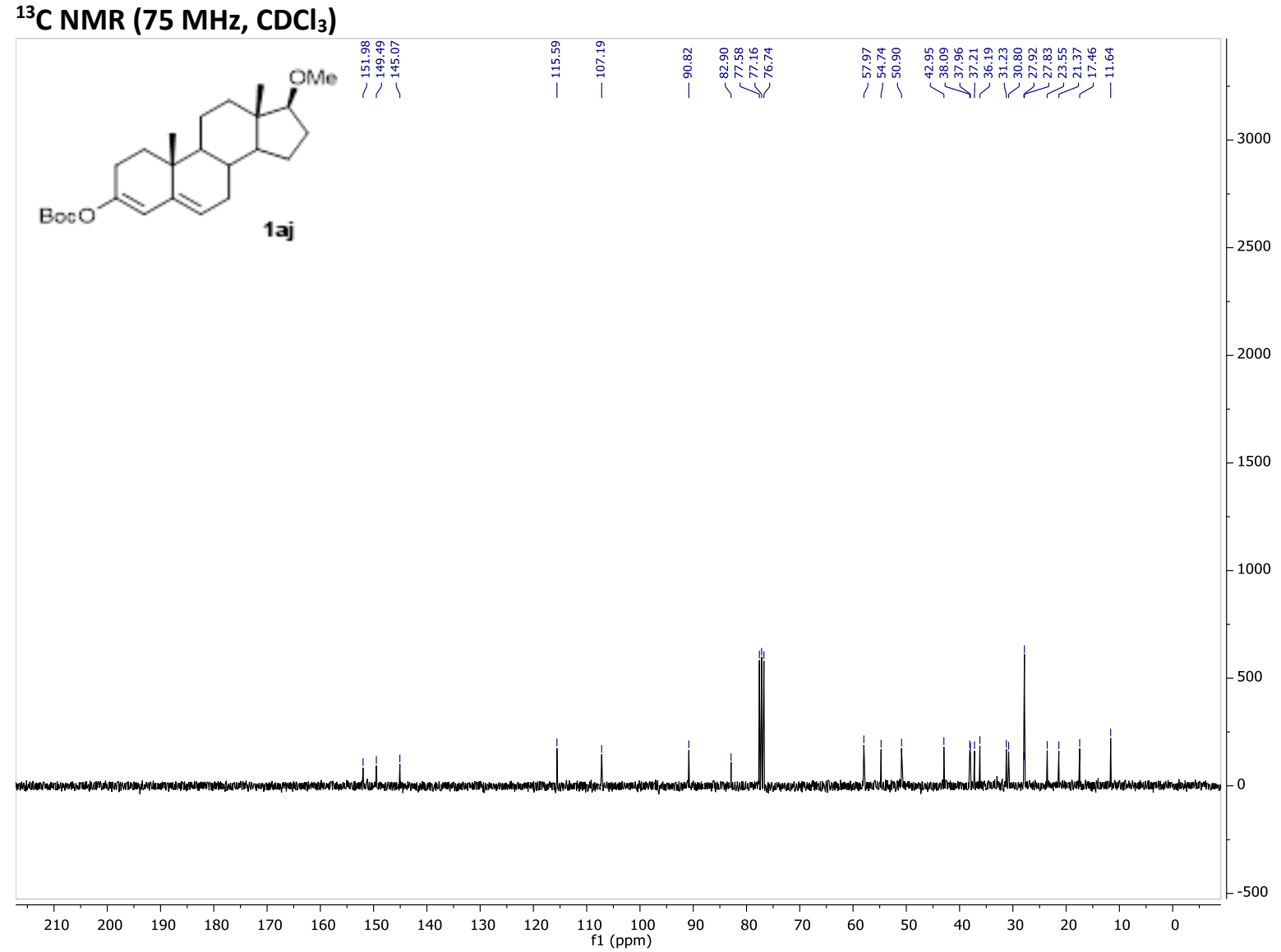

1-(4-fluorophenyl)-2-(trifluoromethoxy)ethanone 3

${ }^{1} \mathrm{H}$ NMR $\left.\left(300 \mathrm{MHz}^{\mathrm{CDCl}}\right)_{3}\right)$

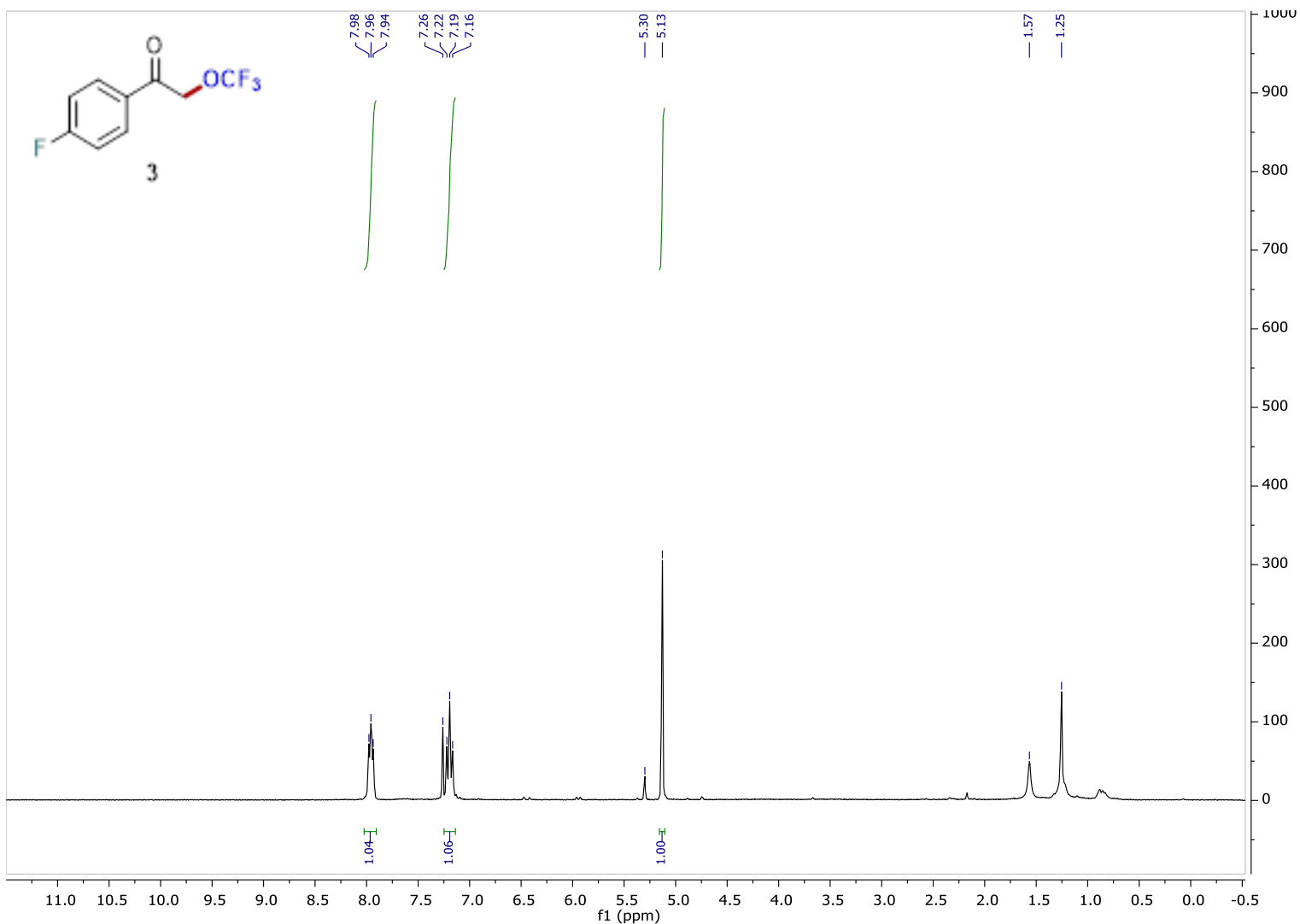


${ }^{13} \mathrm{C}$ NMR (75 MHz, $\mathrm{CDCl}_{3}$ )

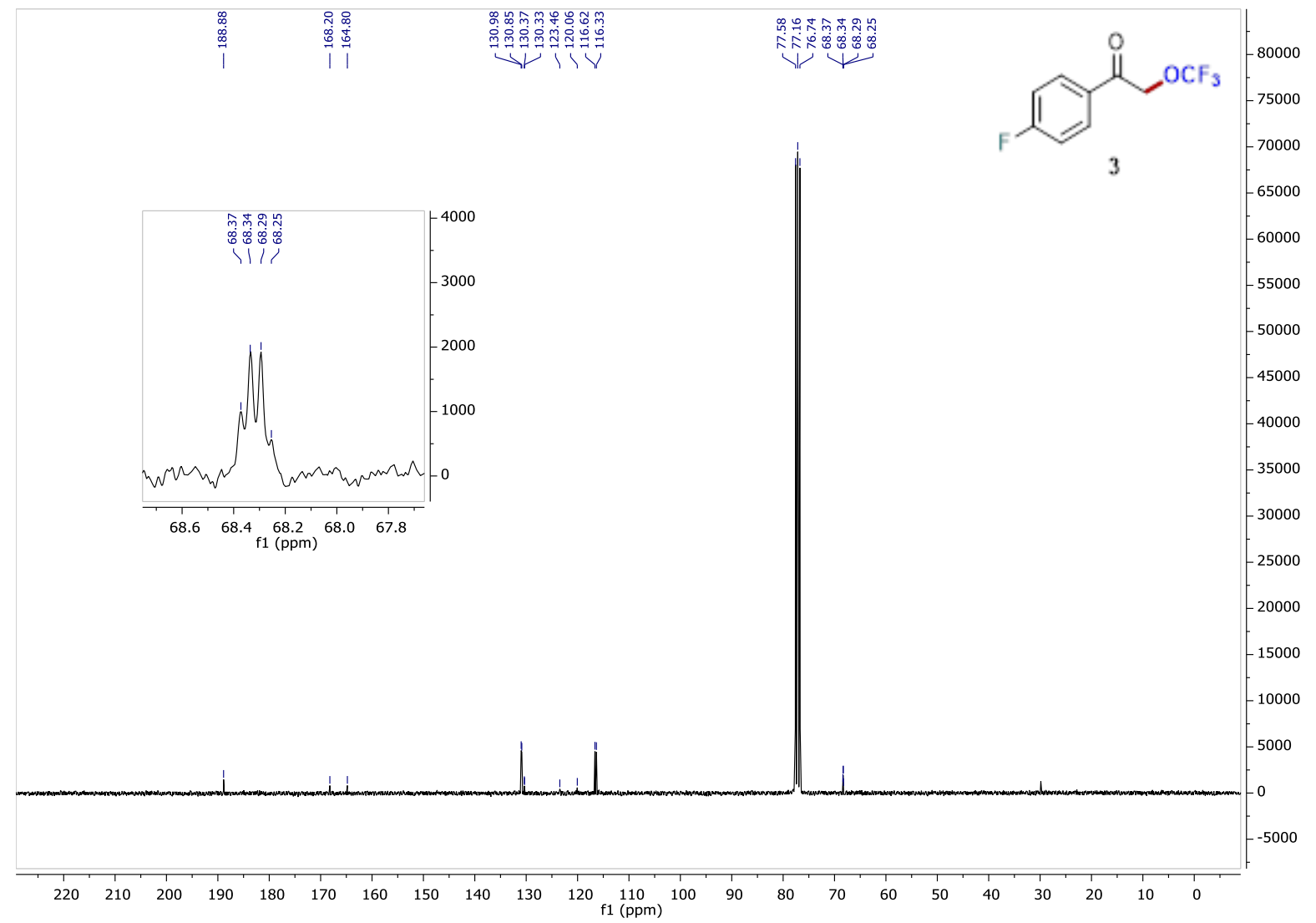

${ }^{19} \mathrm{~F}$ NMR (188 MHz, $\left.\mathrm{CDCl}_{3}\right)$

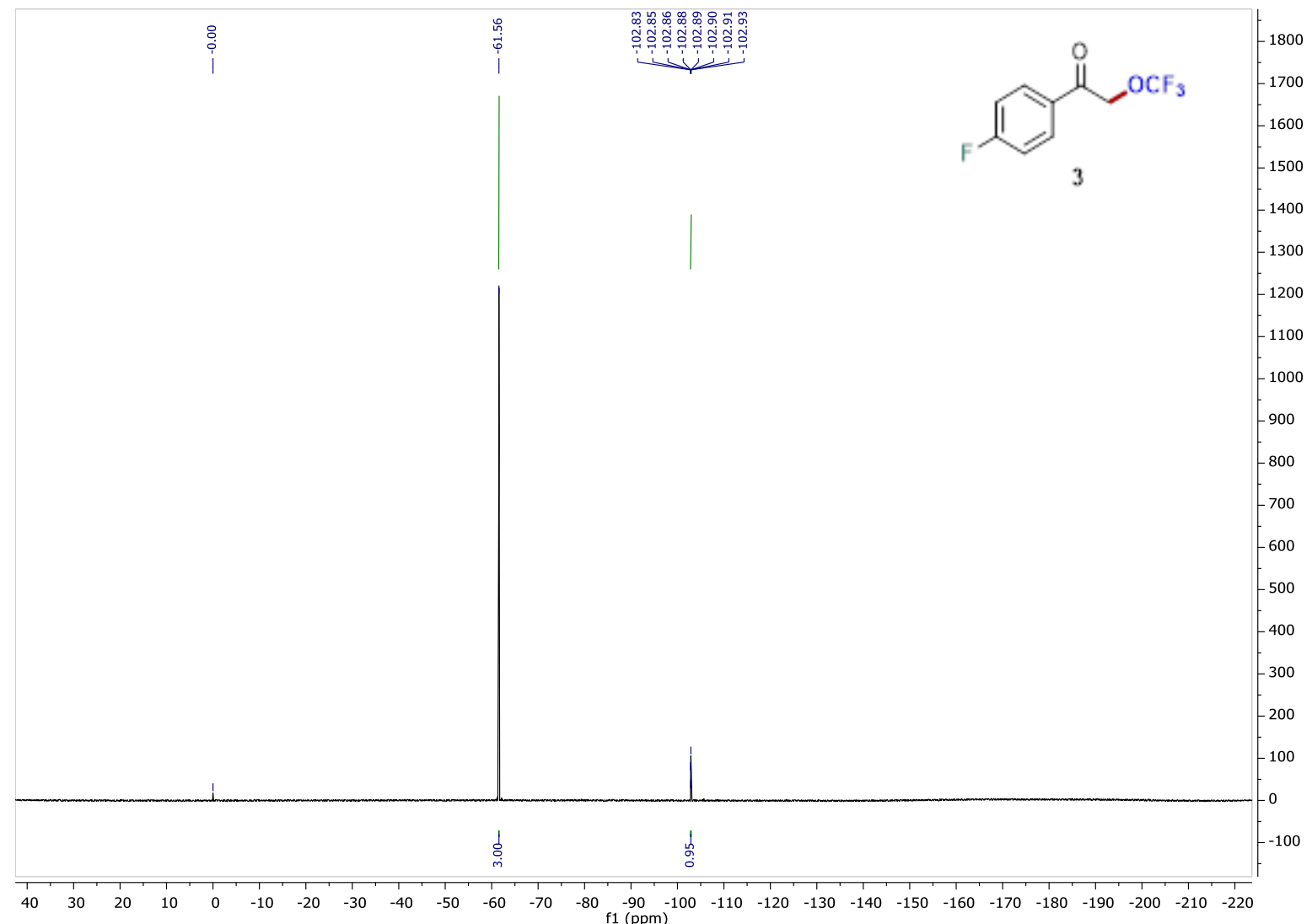


1-(o-tolyl)-2-(trifluoromethoxy)ethanone 7

${ }^{1} \mathrm{H}$ NMR (300 MHz, $\mathrm{CDCl}_{3}$ )

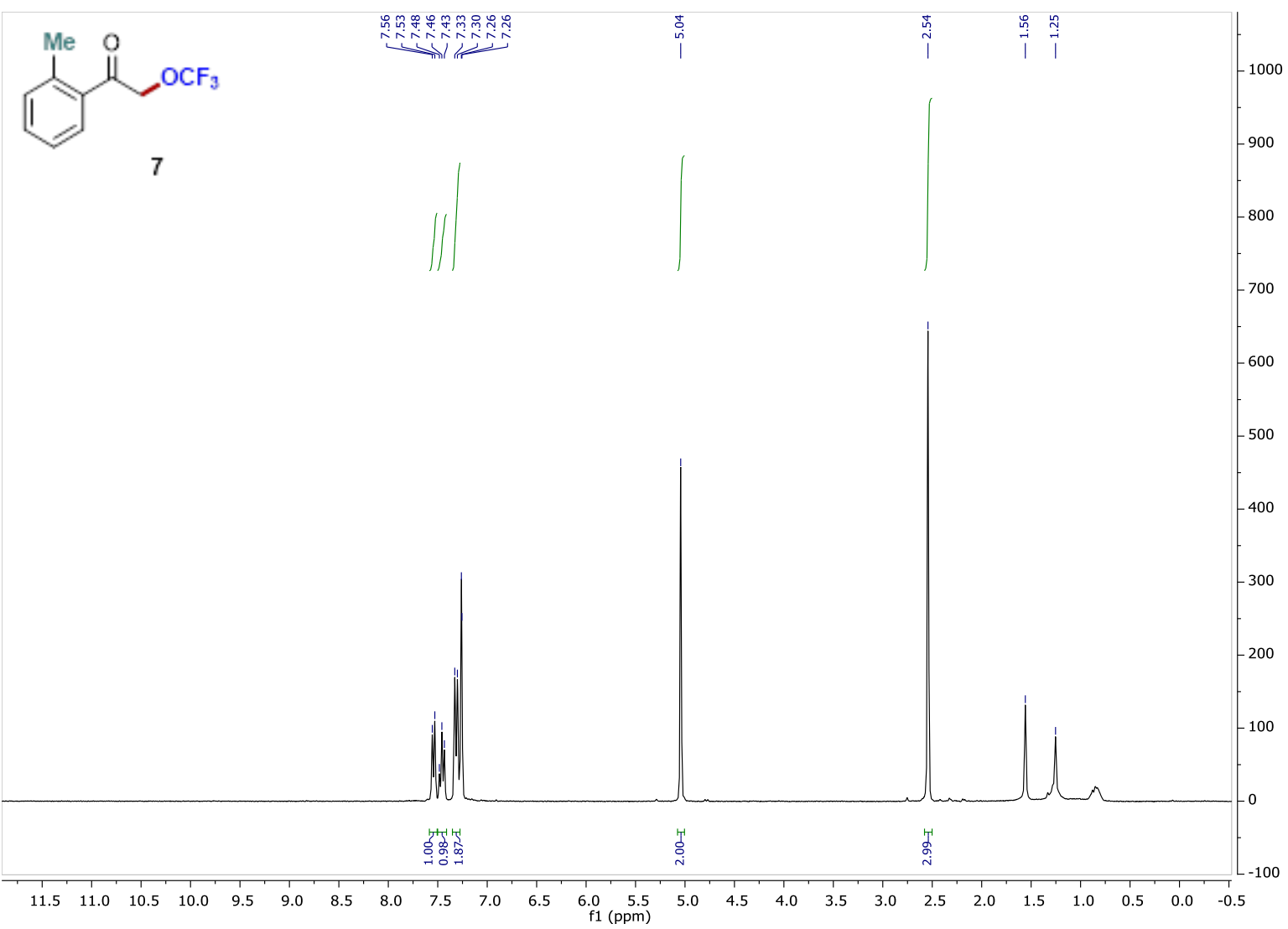

${ }^{13} \mathrm{C}$ NMR (75 MHz, $\left.\mathrm{CDCl}_{3}\right)$

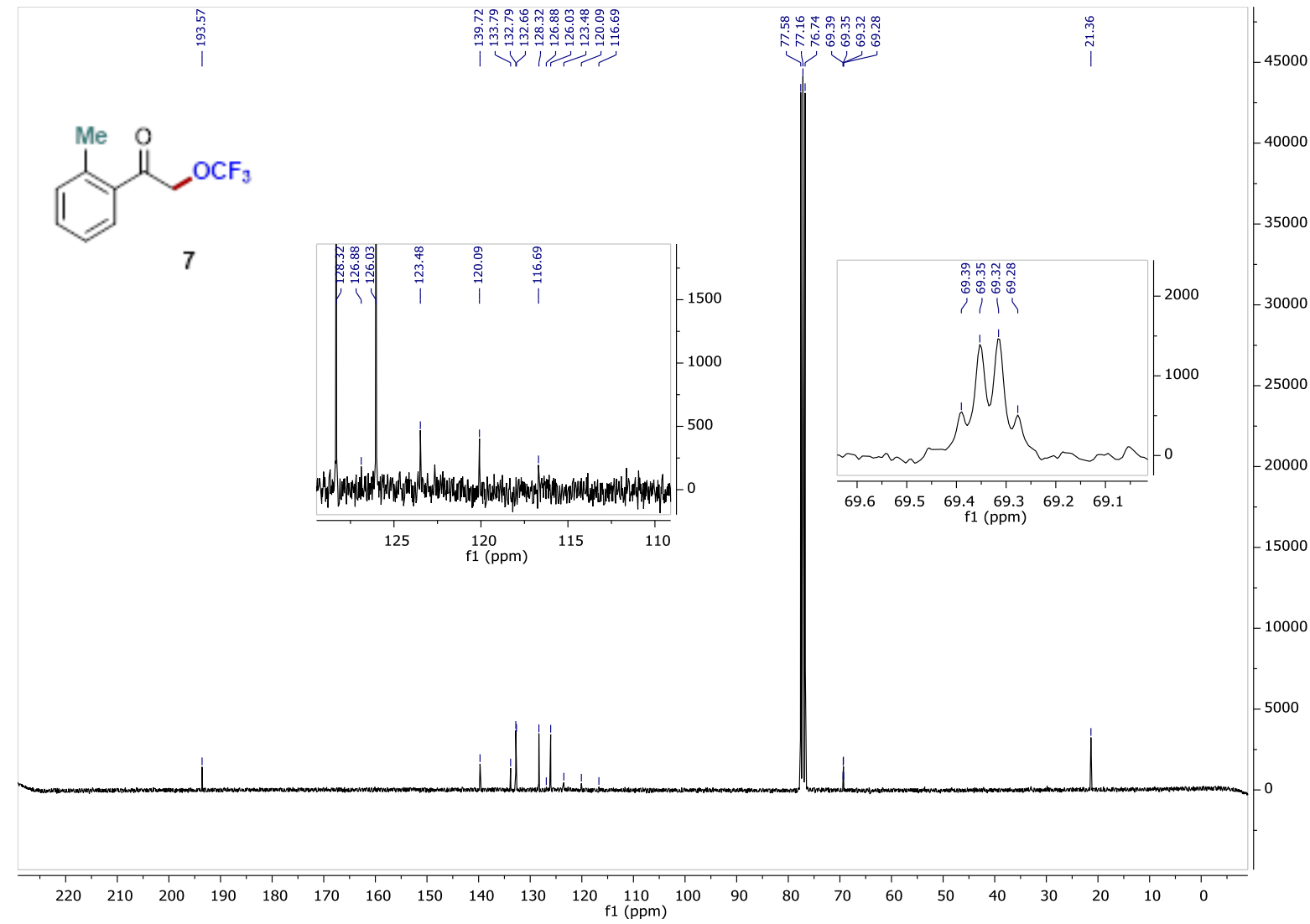


${ }^{19} \mathrm{~F}$ NMR (188 MHz, $\left.\mathrm{CDCl}_{3}\right)$

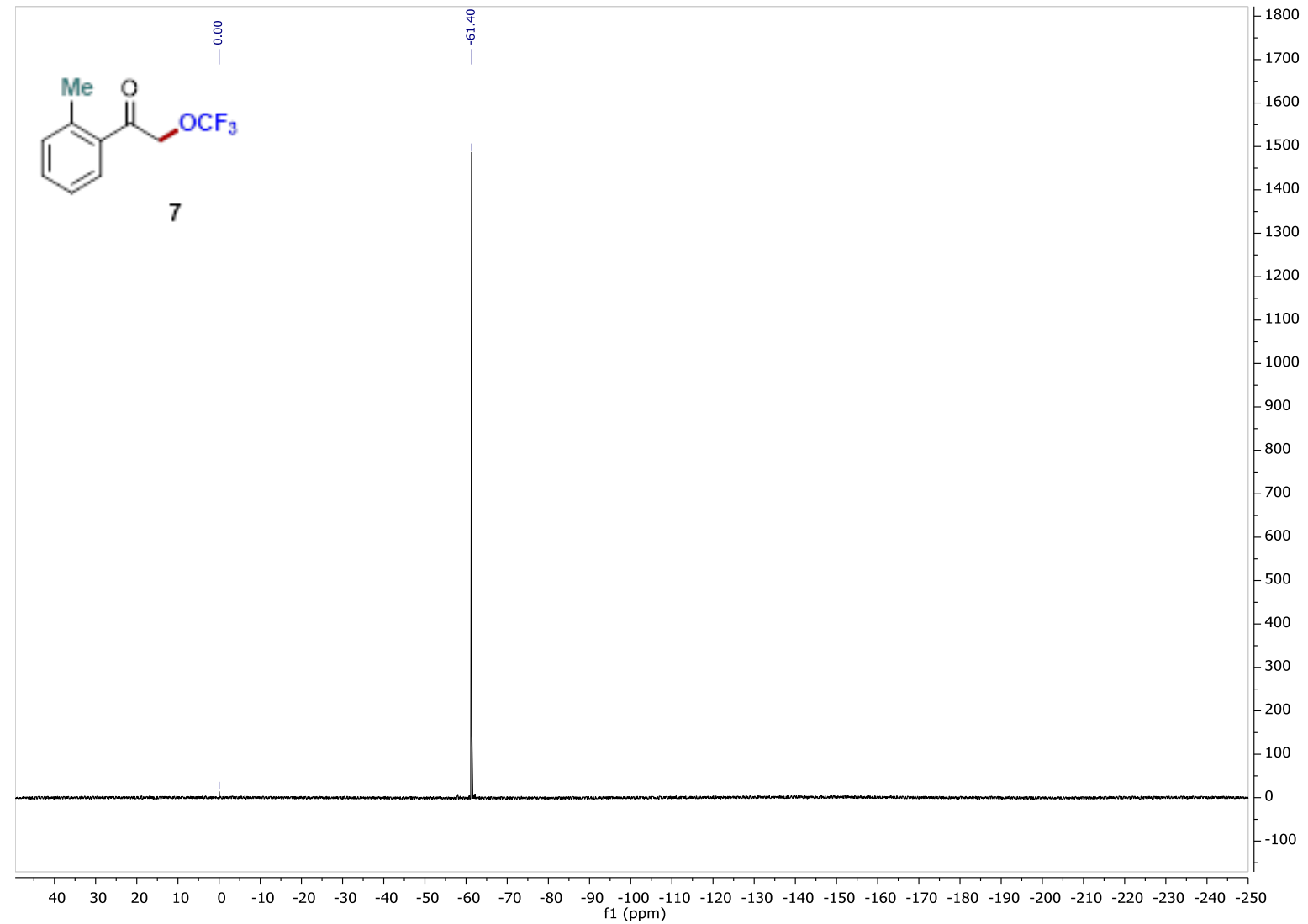

1-(m-tolyl)-2-(trifluoromethoxy)ethanone 8

${ }^{1} \mathrm{H}$ NMR $\left(300 \mathrm{MHz}, \mathrm{CDCl}_{3}\right)$

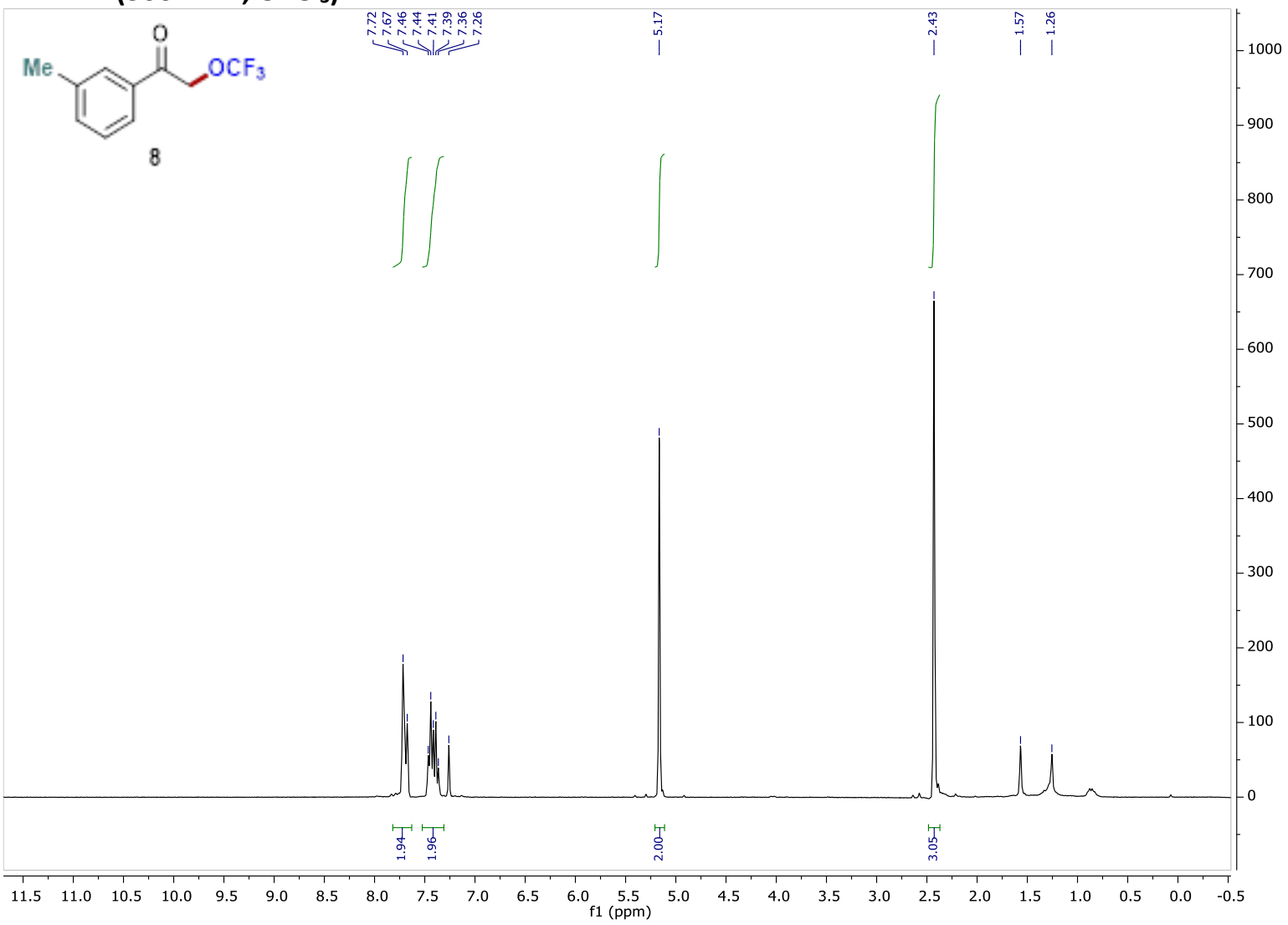


${ }^{13} \mathrm{C}$ NMR (75 MHz, $\left.\mathrm{CDCl}_{3}\right)$

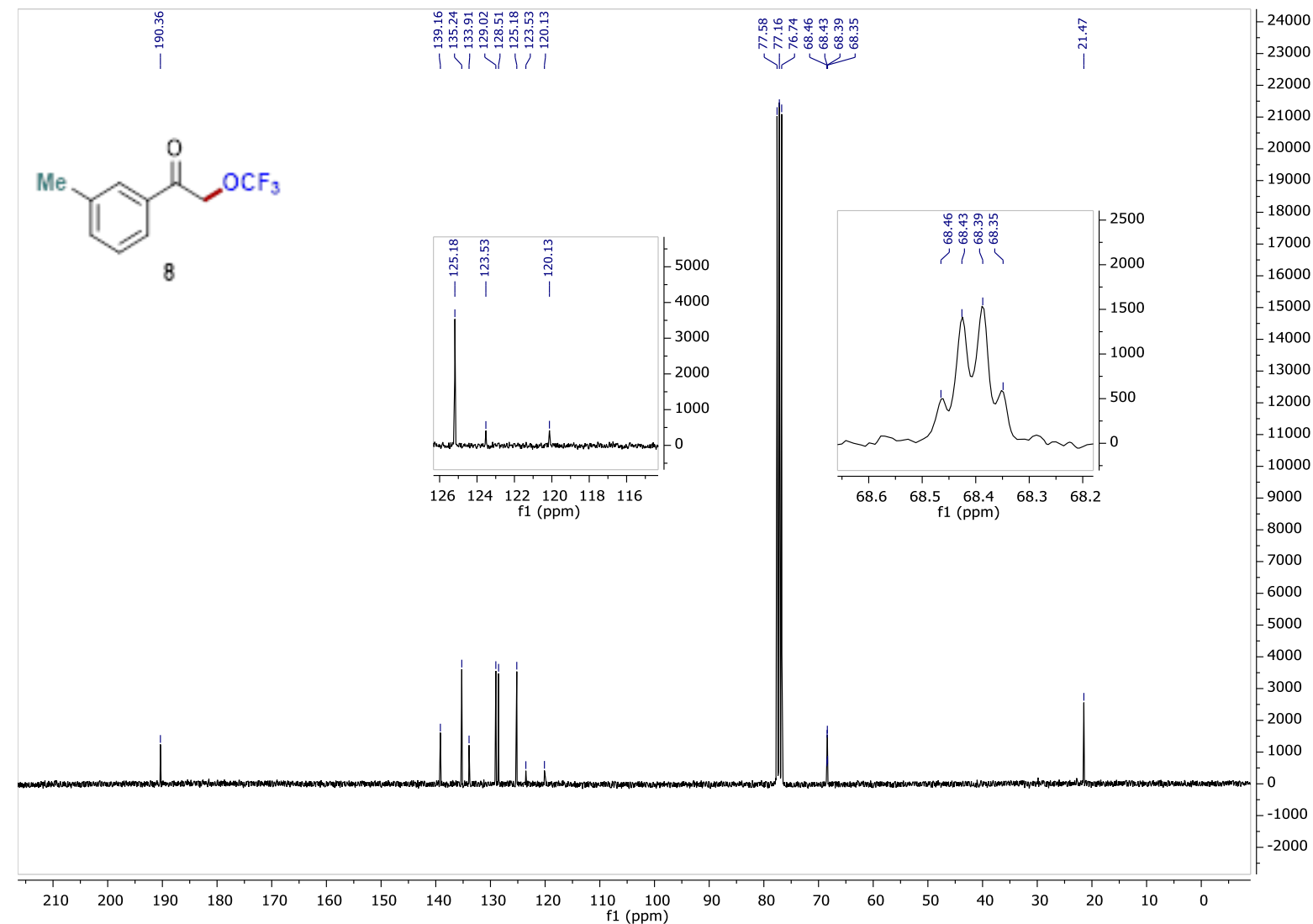

${ }^{19} \mathrm{~F}$ NMR (188 MHz, $\left.\mathrm{CDCl}_{3}\right)$

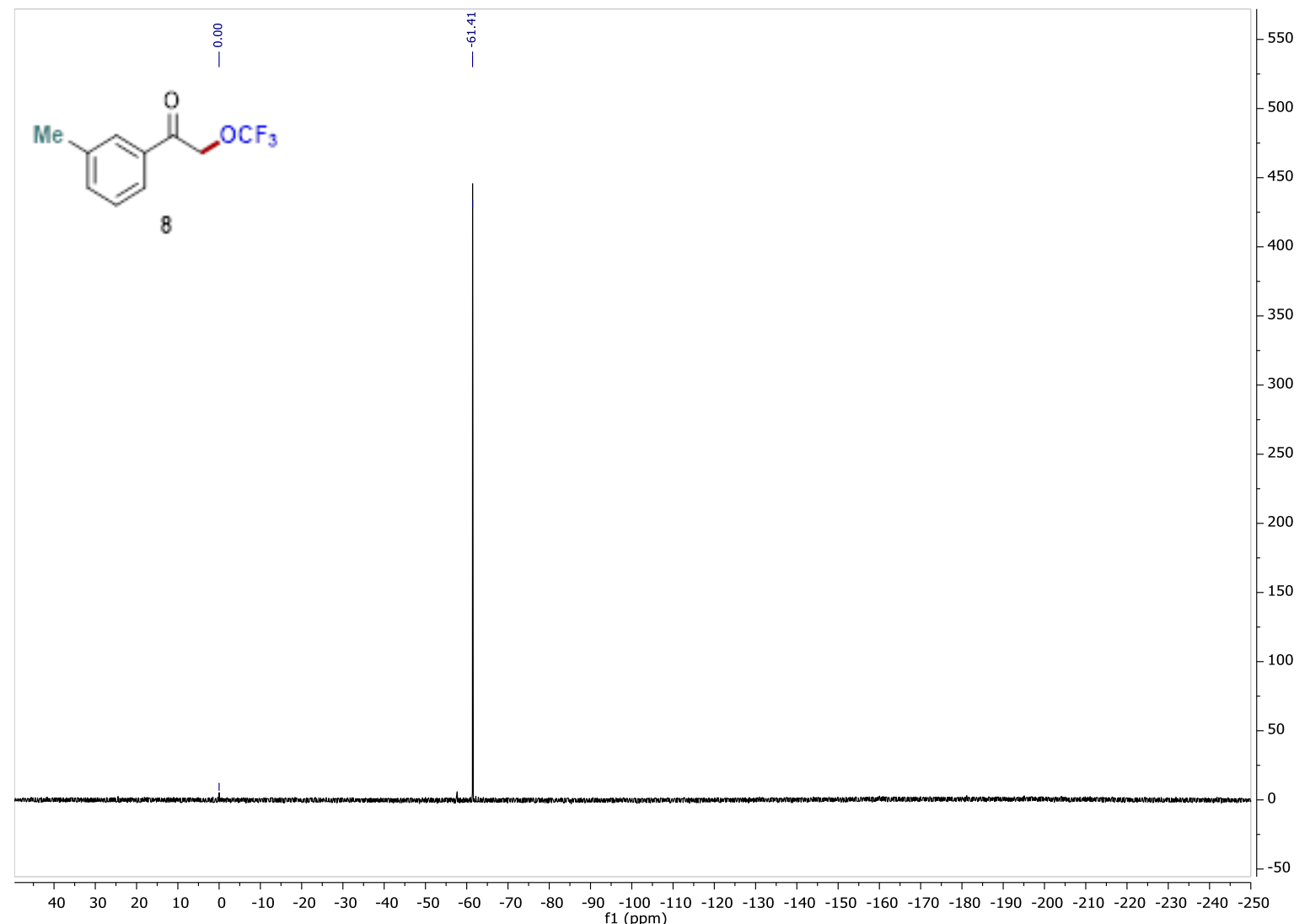


1-(p-tolyl)-2-(trifluoromethoxy)ethanone 9

${ }^{1} \mathrm{H}$ NMR $\left(300 \mathrm{MHz}, \mathrm{CDCl}_{3}\right)$

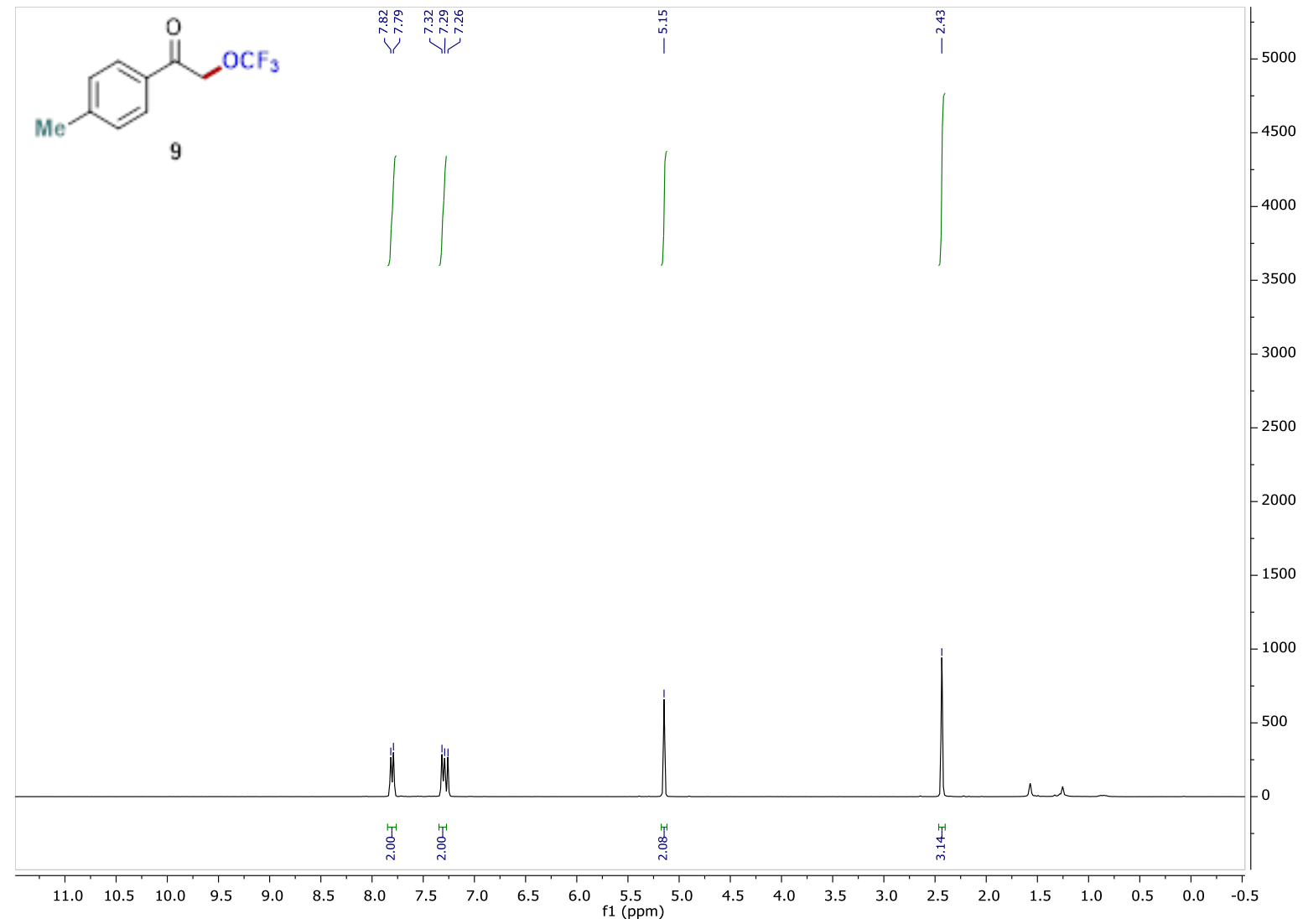

${ }^{13} \mathrm{C}$ NMR $\left(75 \mathrm{MHz}, \mathrm{CDCl}_{3}\right)$

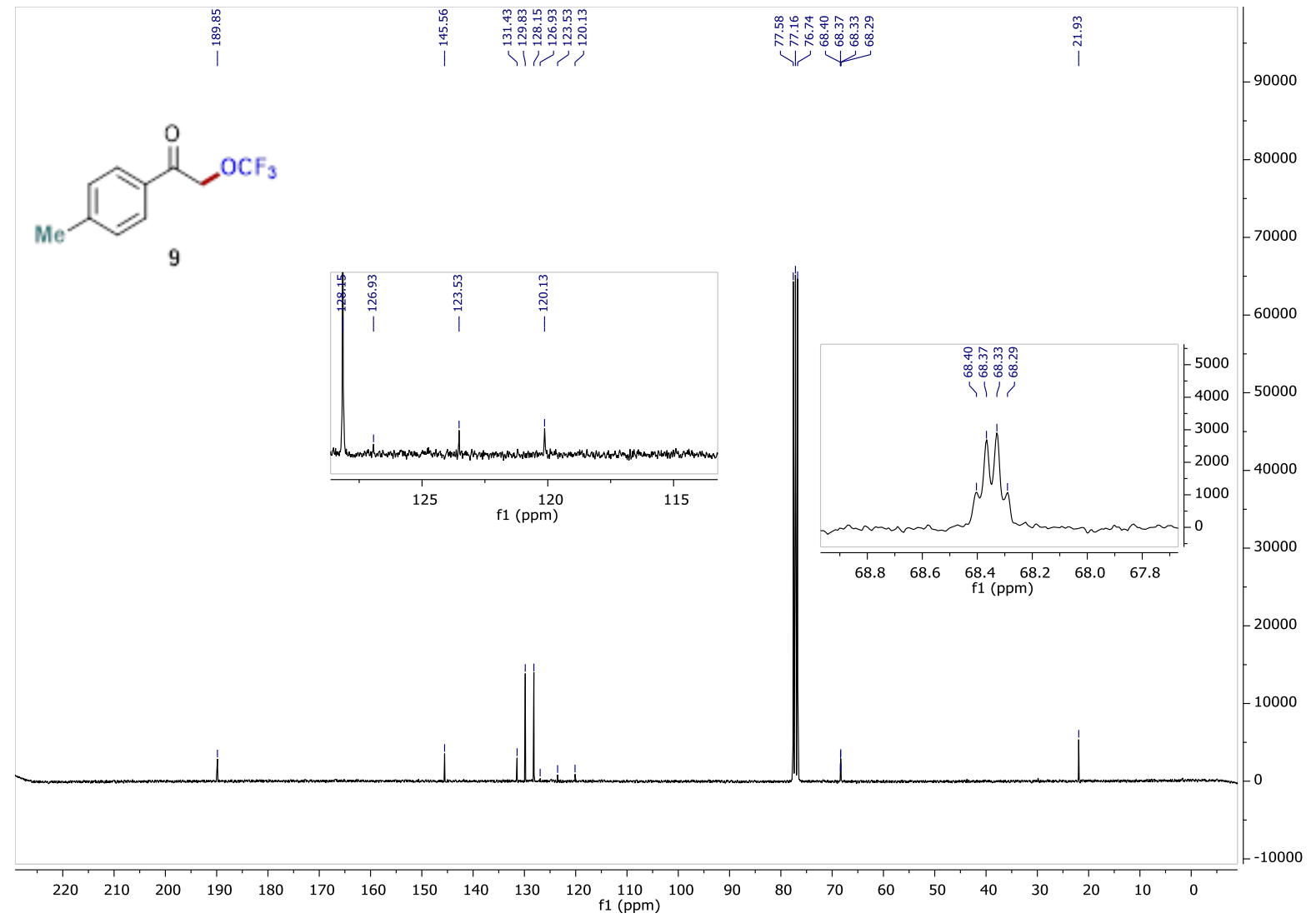


${ }^{19} \mathrm{~F}$ NMR (188 MHz, $\left.\mathrm{CDCl}_{3}\right)$

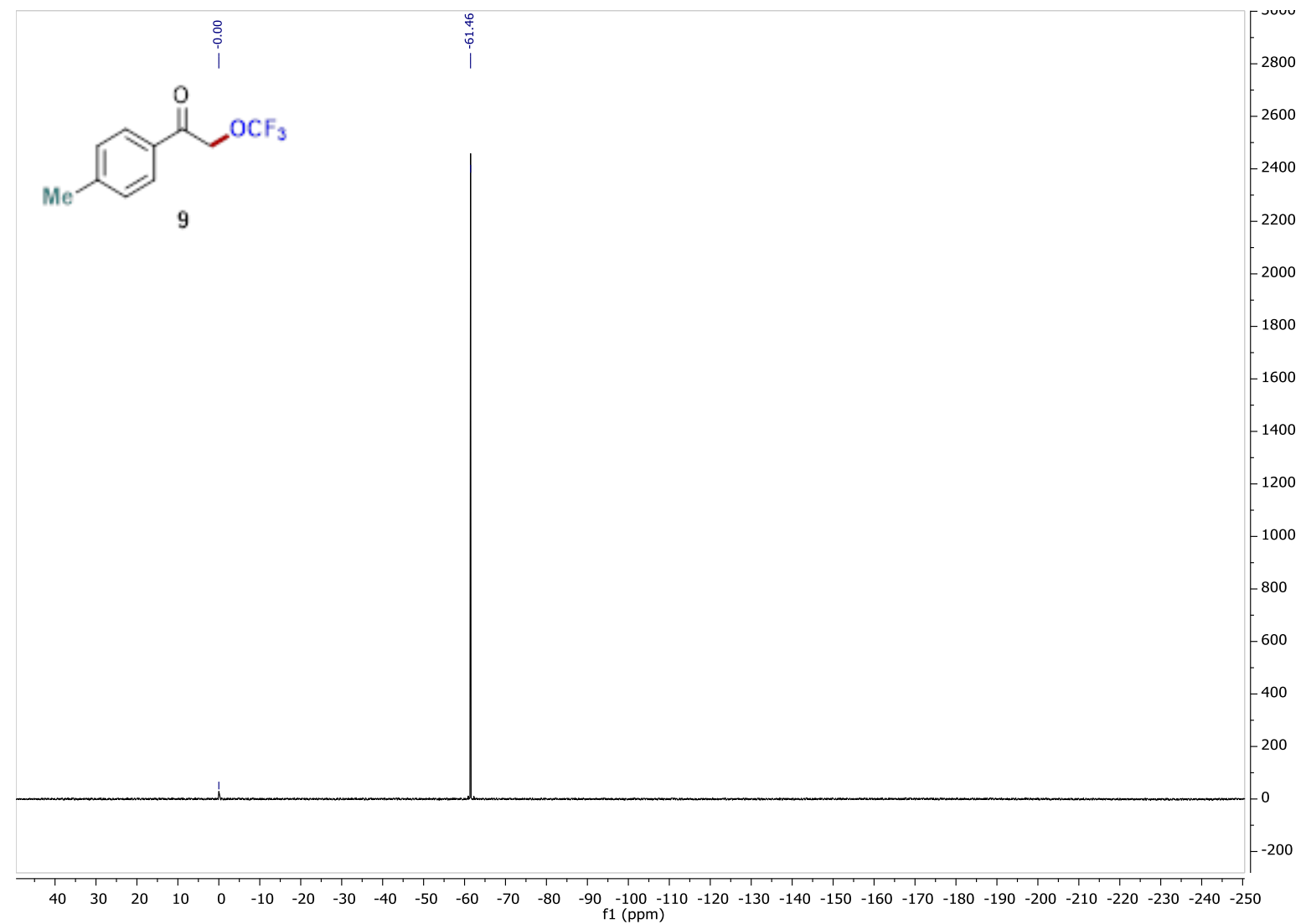

4-(2-(trifluoromethoxy)acetyl)benzonitrile 12

${ }^{1} \mathrm{H}$ NMR $\left(300 \mathrm{MHz}, \mathrm{CDCl}_{3}\right)$

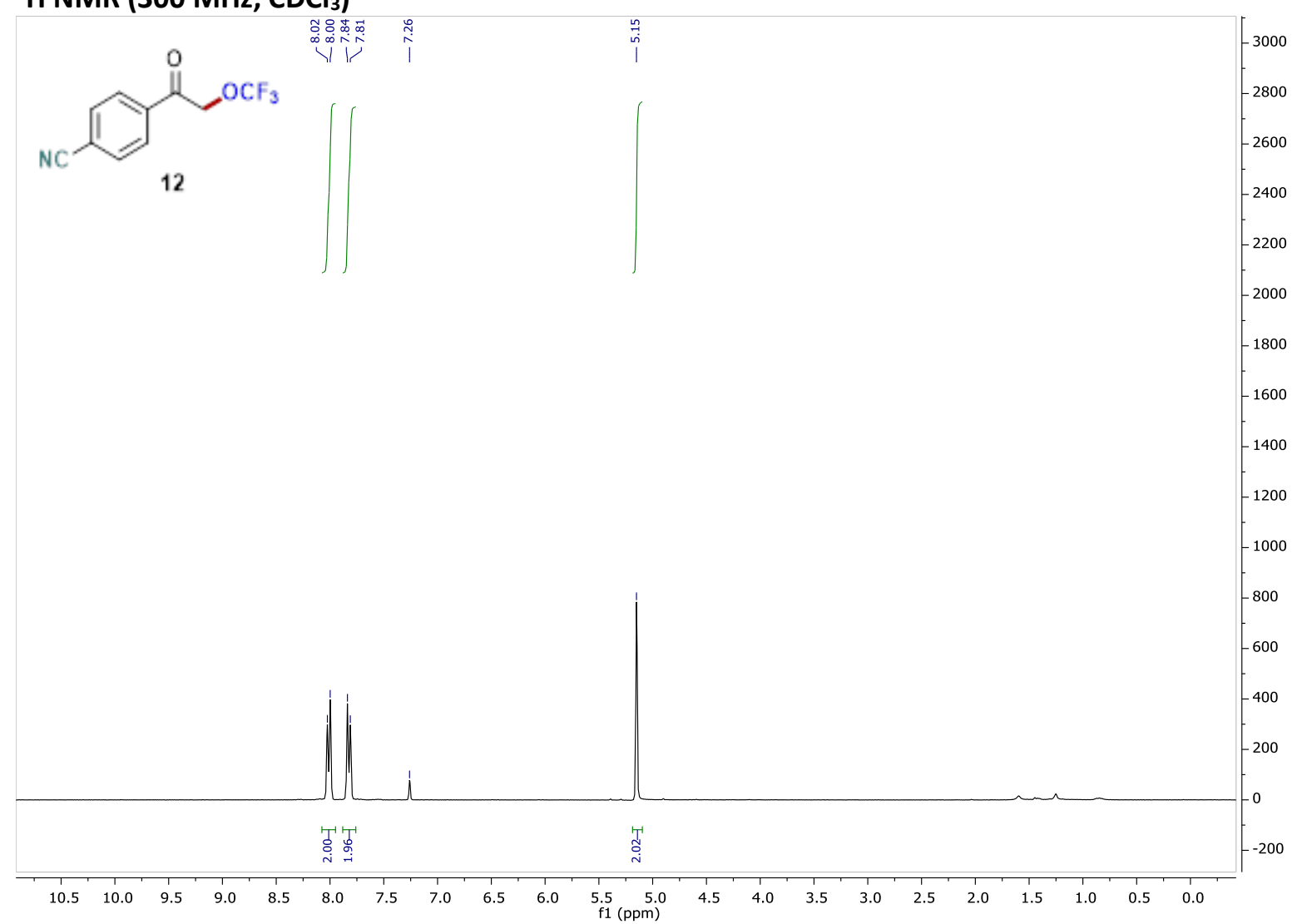


${ }^{13} \mathrm{C}$ NMR (75 MHz, $\mathrm{CDCl}_{3}$ )

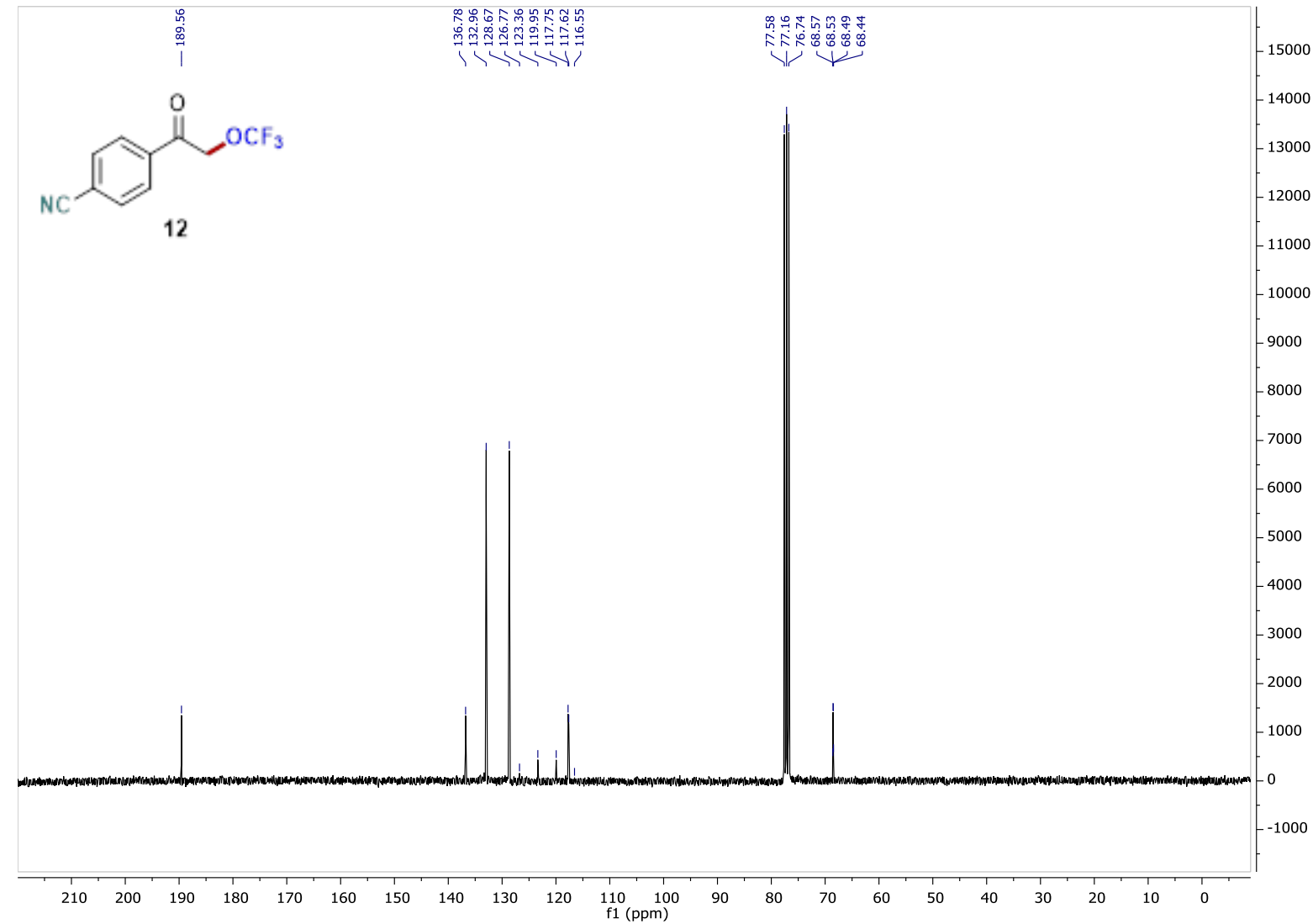

${ }^{19} \mathrm{~F} \mathrm{NMR} \mathrm{(188} \mathrm{MHz,} \mathrm{CDCl}_{3}$ )

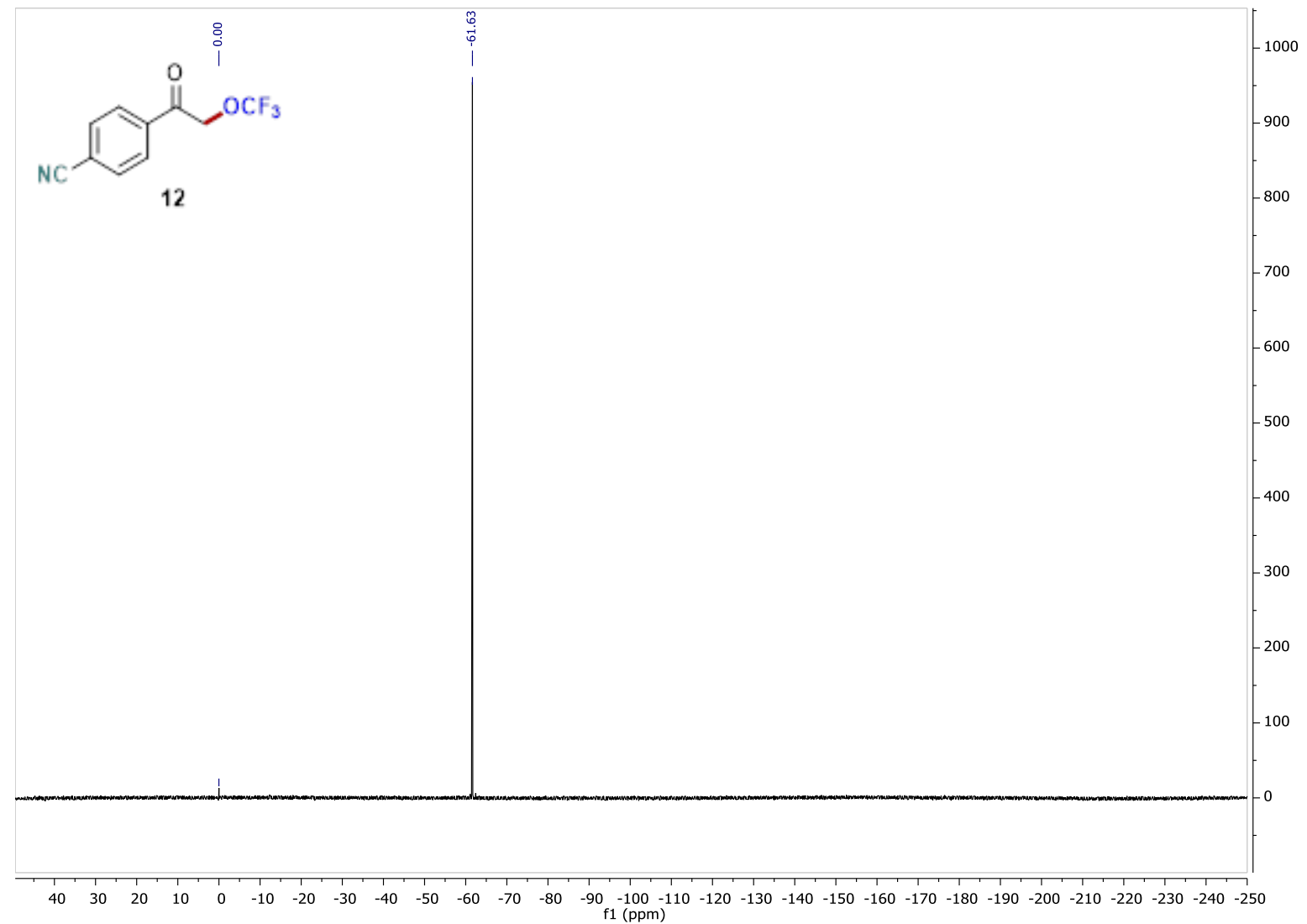


2-(trifluoromethoxy)-1-(3-(trifluoromethyl)phenyl)ethanone 13

${ }^{1} \mathrm{H}$ NMR $\left(300 \mathrm{MHz}, \mathrm{CDCl}_{3}\right)$

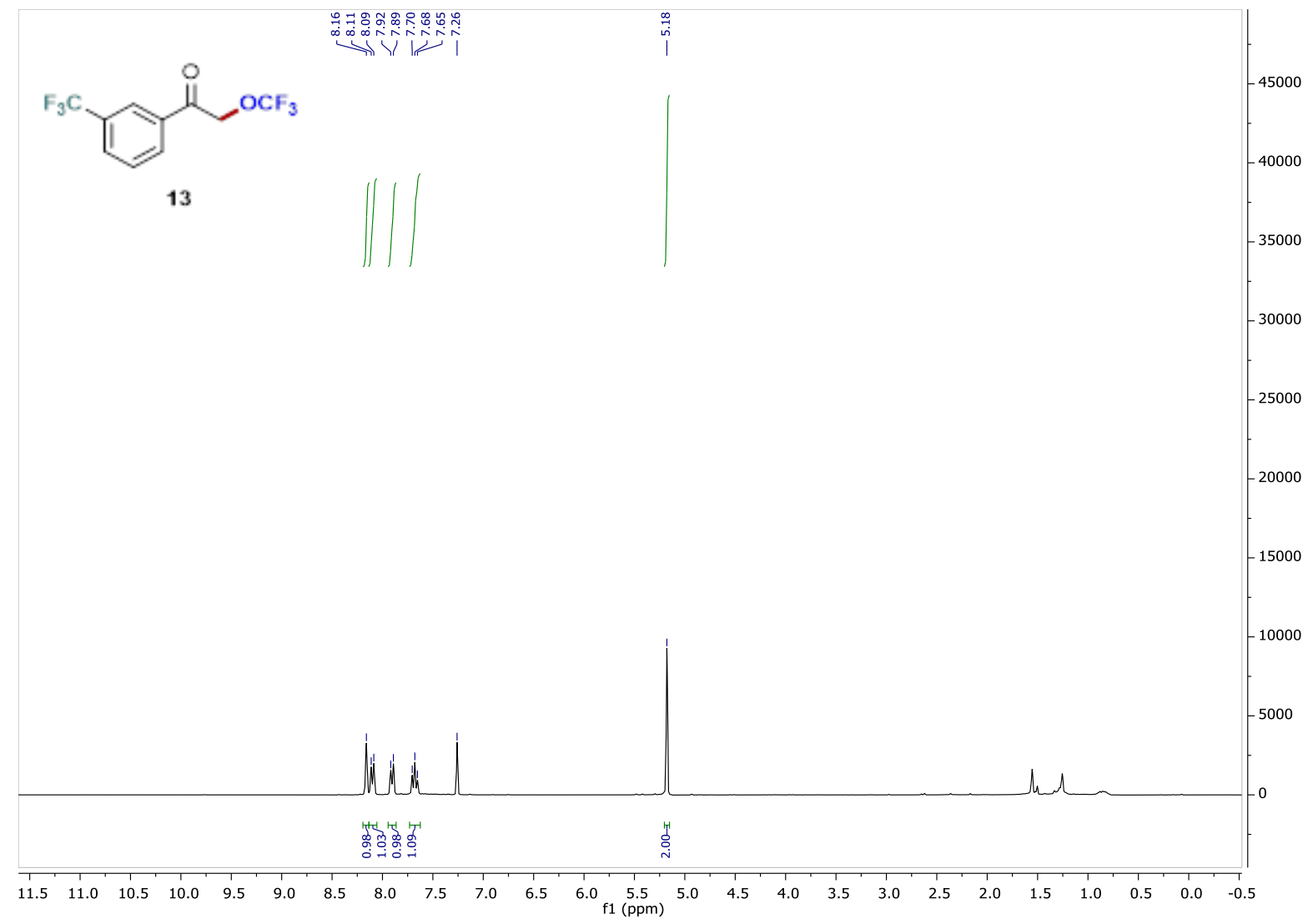

${ }^{13} \mathrm{C}$ NMR (75 MHz, $\mathrm{CDCl}_{3}$ )

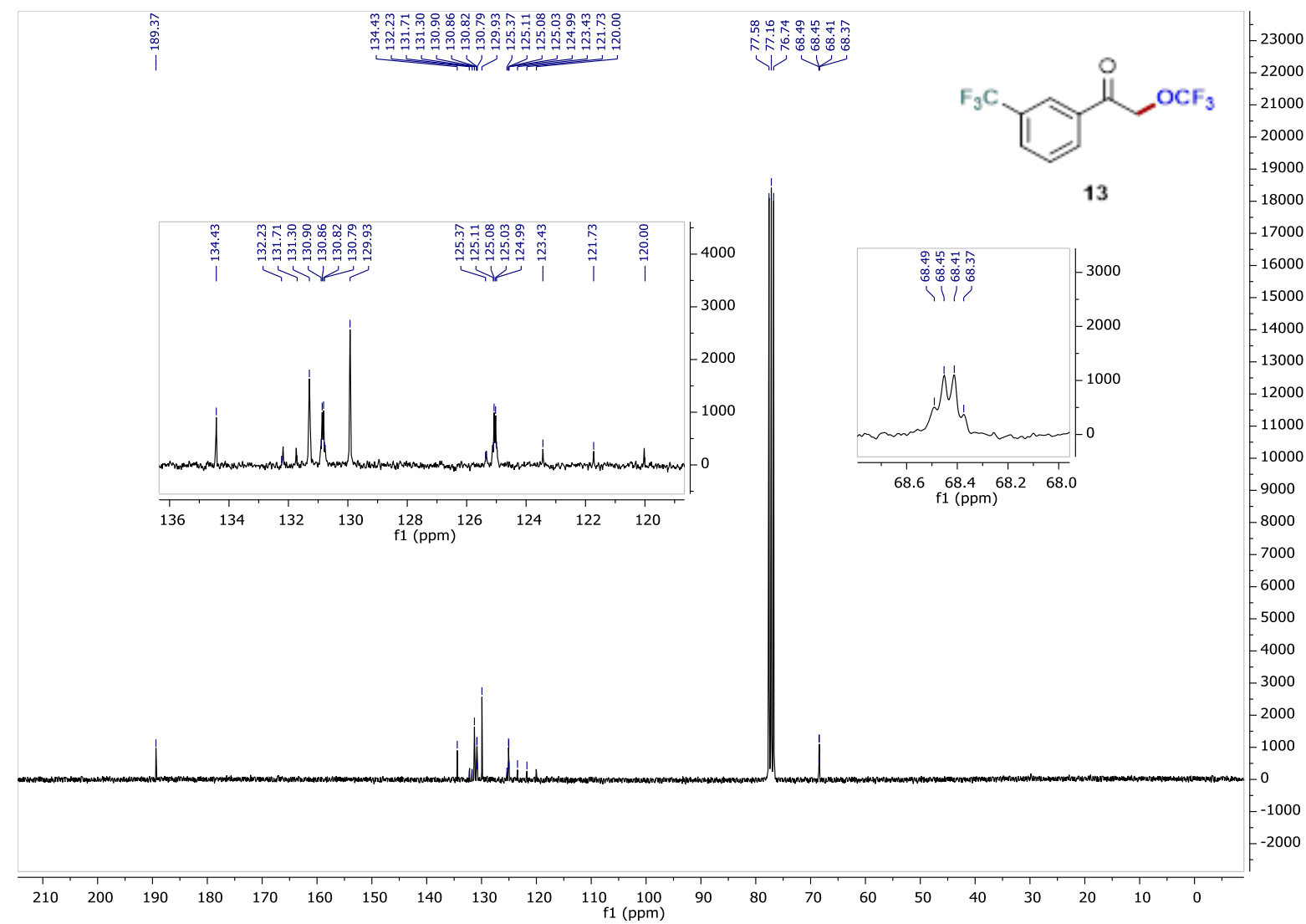


${ }^{19} \mathrm{~F}$ NMR (188 MHz, $\left.\mathrm{CDCl}_{3}\right)$

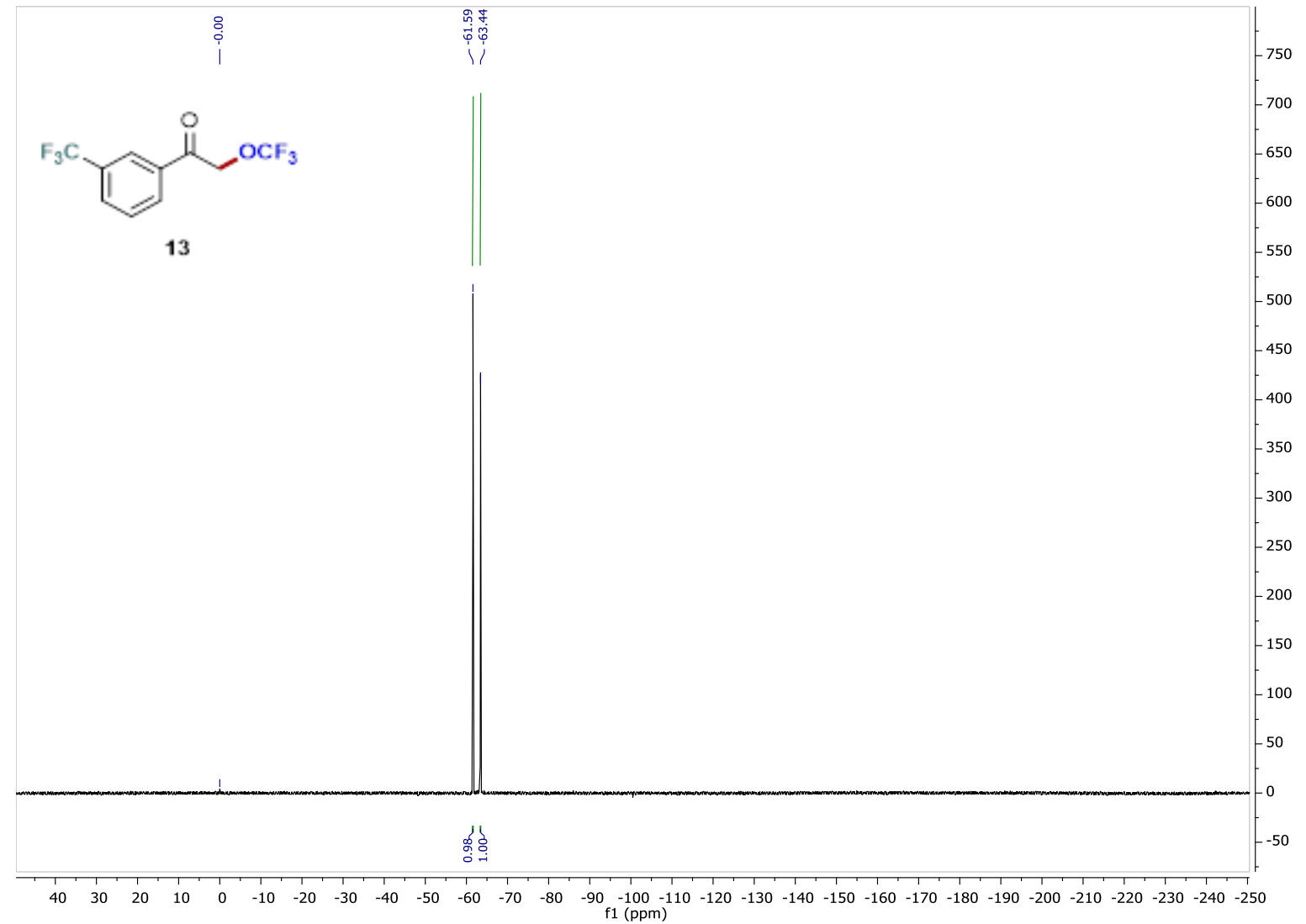

1-(2-bromophenyl)-2-(trifluoromethoxy)ethan-1-one 15

${ }^{1} \mathrm{H}$ NMR $\left(500 \mathrm{MHz}, \mathrm{CDCl}_{3}\right)$

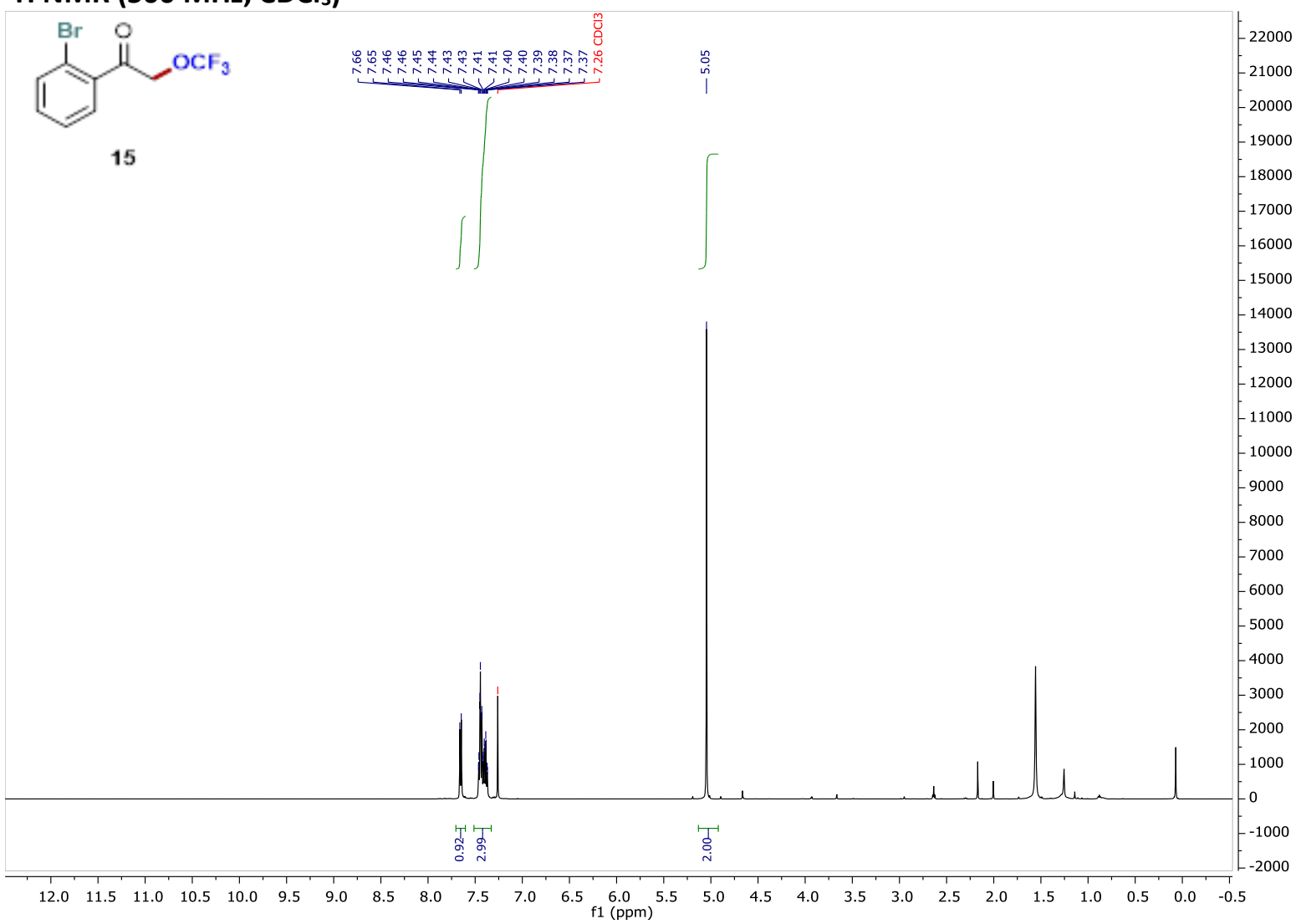


${ }^{13} \mathrm{C} \mathrm{NMR} \mathrm{(126} \mathrm{MHz,} \mathrm{CDCl}_{3}$ )

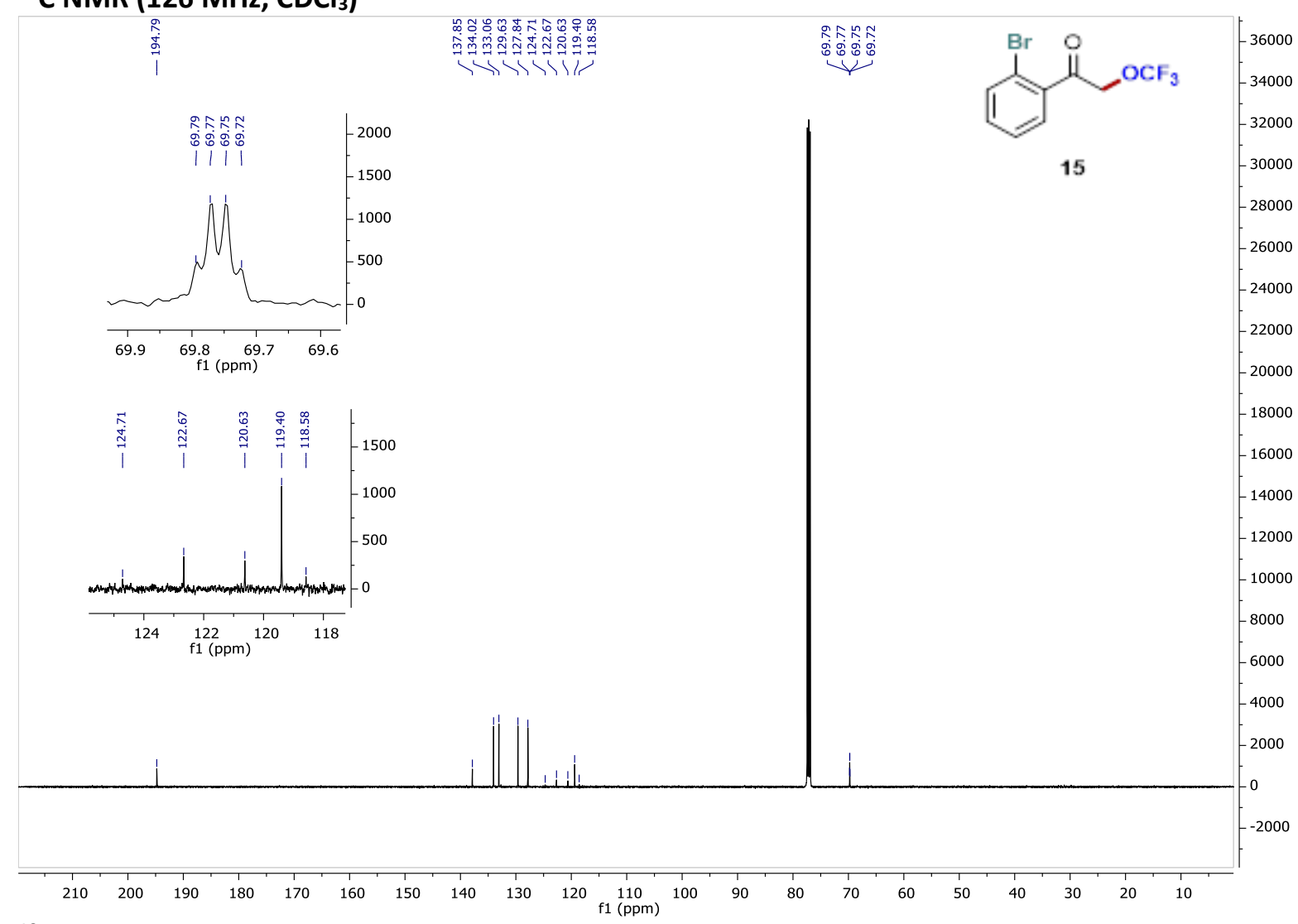

$\left.{ }^{19} \mathrm{~F} \mathrm{NMR} \mathrm{(188} \mathrm{MHz,} \mathrm{CDCl}_{3}\right)$

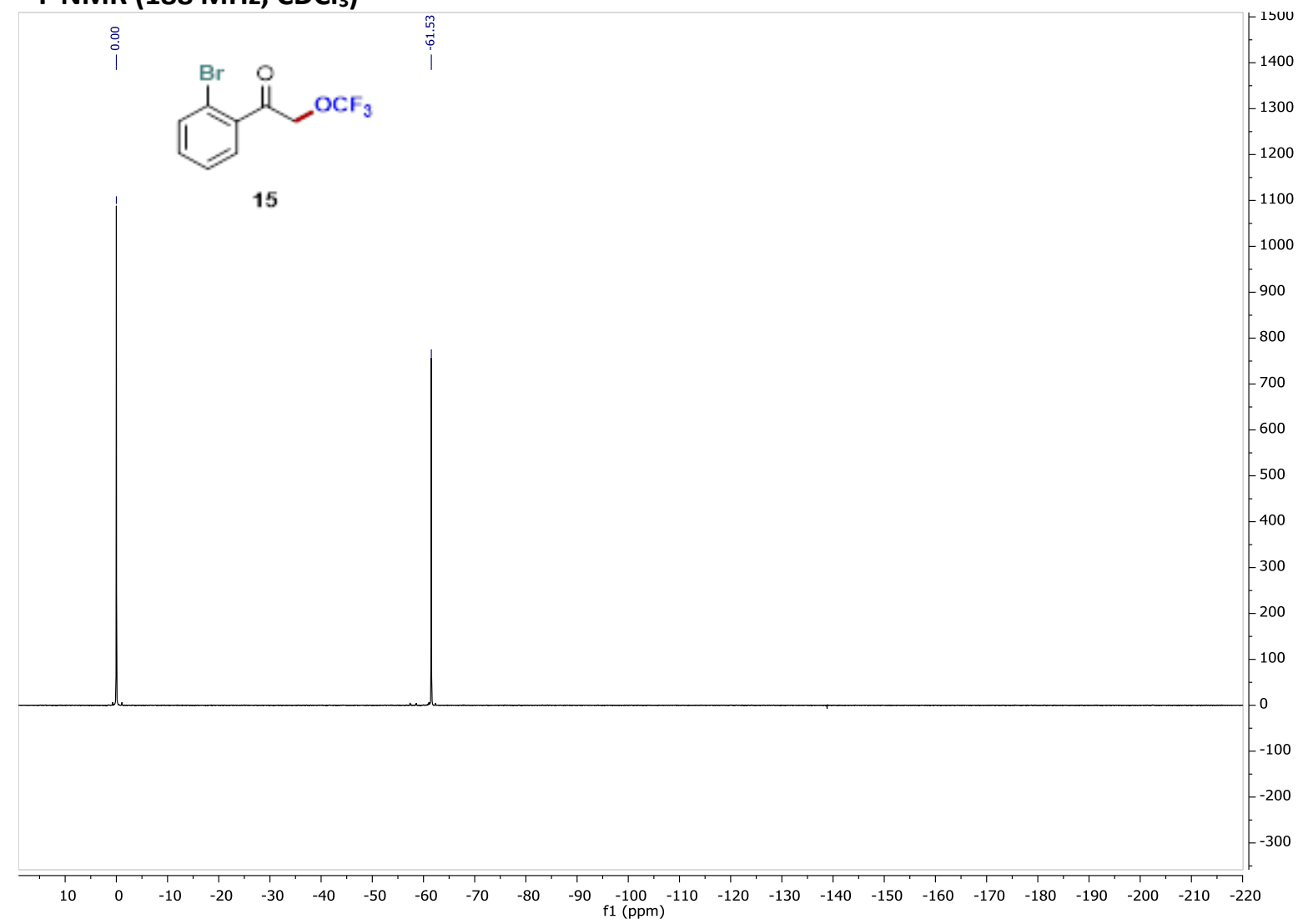




\section{1-(3-bromophenyl)-2-(trifluoromethoxy)ethan-1-one 16}

${ }^{1} \mathrm{H}$ NMR (500 MHz, $\mathrm{CDCl}_{3}$ )

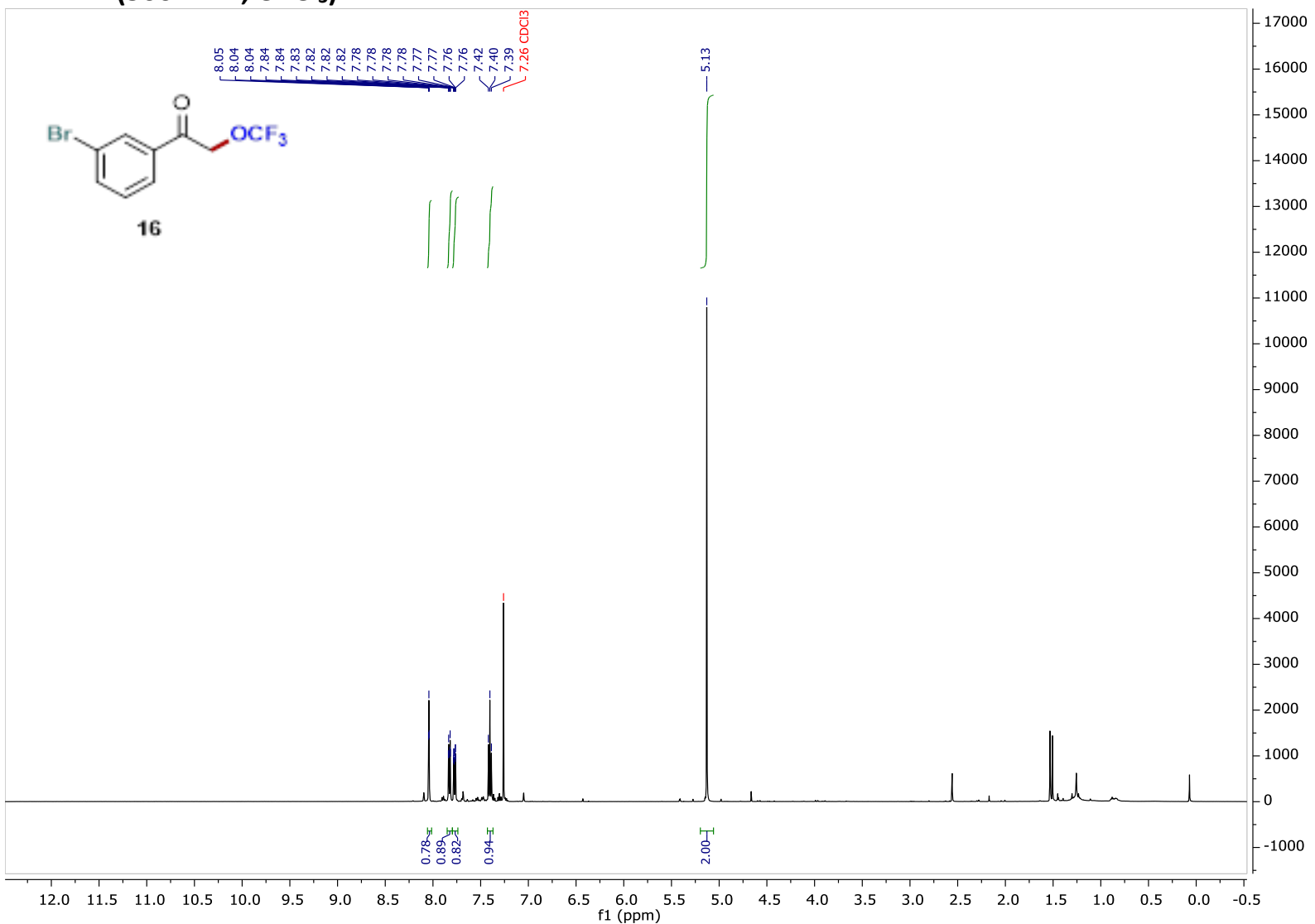

$\left.{ }^{13} \mathrm{C} \mathrm{NMR} \mathrm{(126} \mathrm{MHz,} \mathrm{CDCl}_{3}\right)$

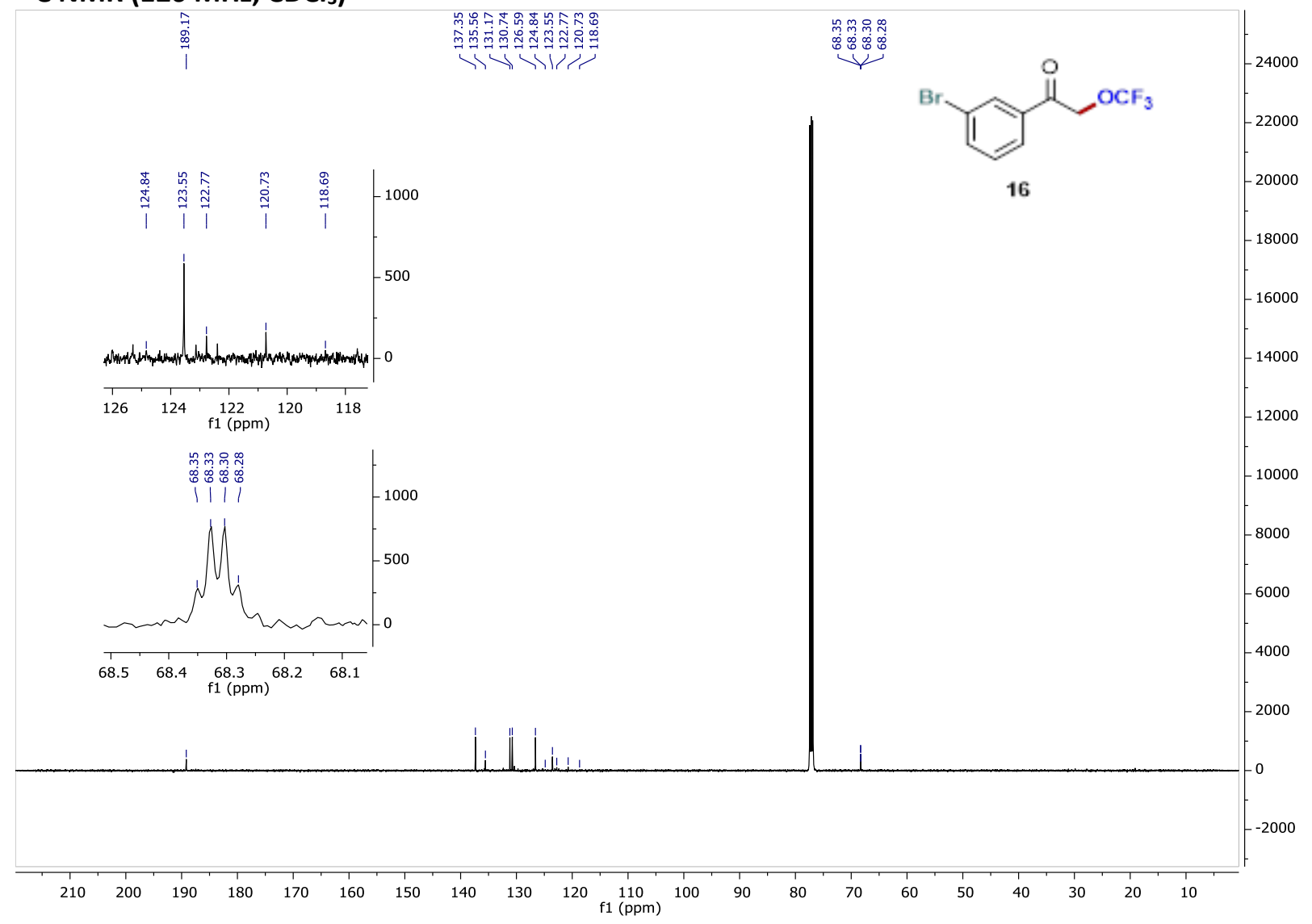


${ }^{19} \mathrm{~F}$ NMR (188 MHz, $\left.\mathrm{CDCl}_{3}\right)$

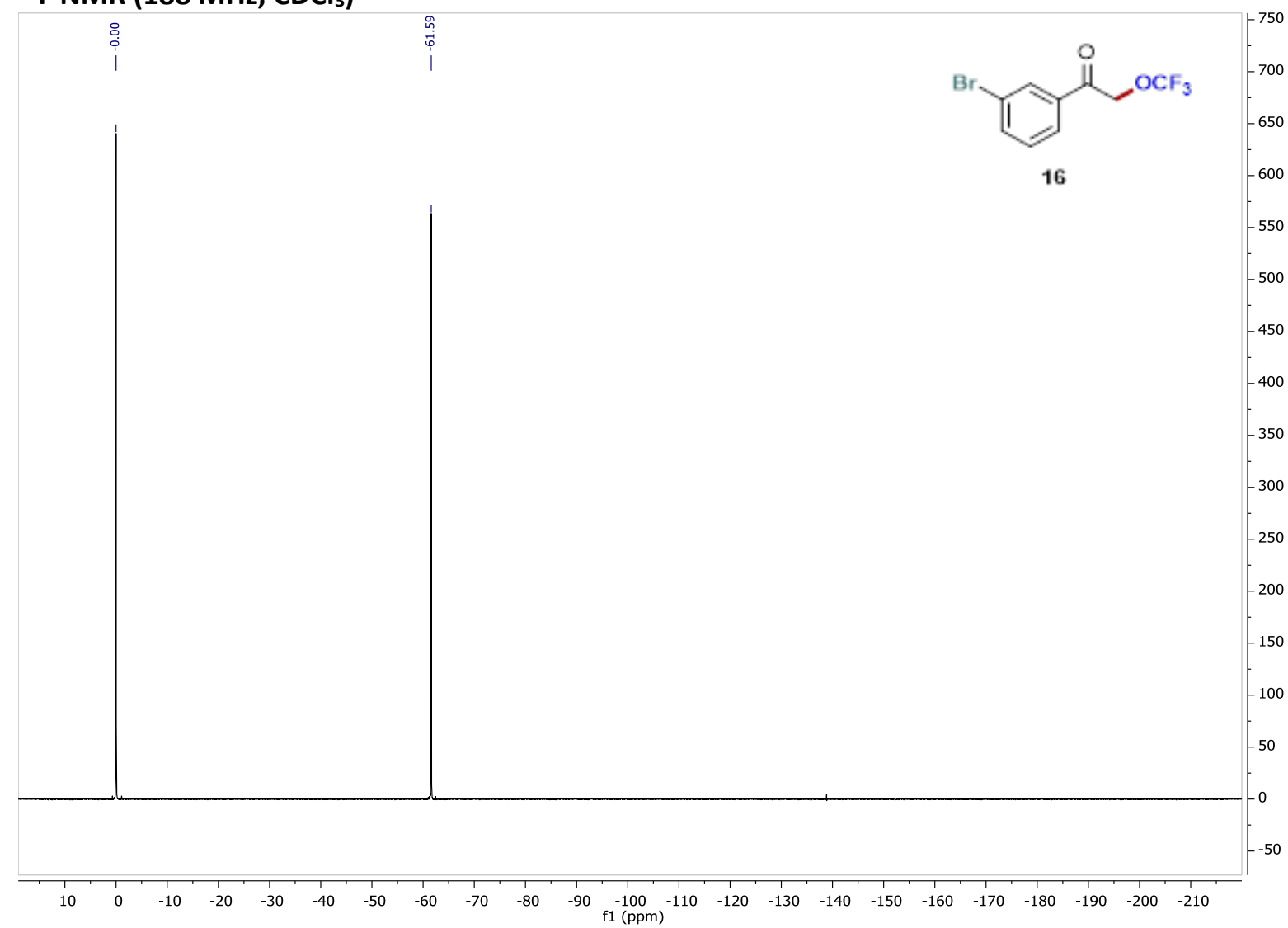

1-(4-bromophenyl)-2-(trifluoromethoxy)ethan-1-one 17

${ }^{1} \mathrm{H}$ NMR $\left(500 \mathrm{MHz}, \mathrm{CDCl}_{3}\right)$

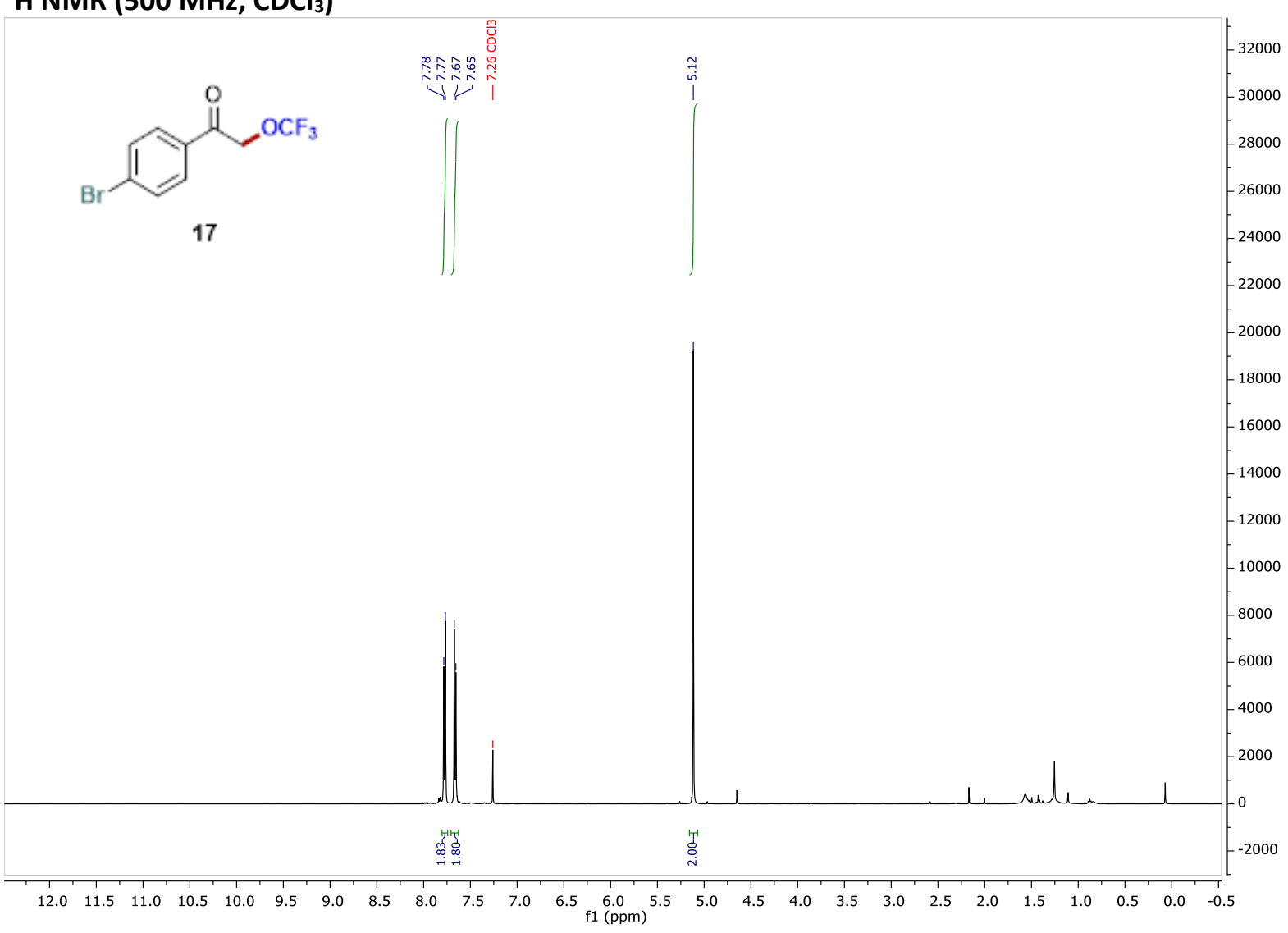


${ }^{13} \mathrm{C} \mathrm{NMR} \mathrm{(126} \mathrm{MHz,} \mathrm{CDCl}_{3}$ )

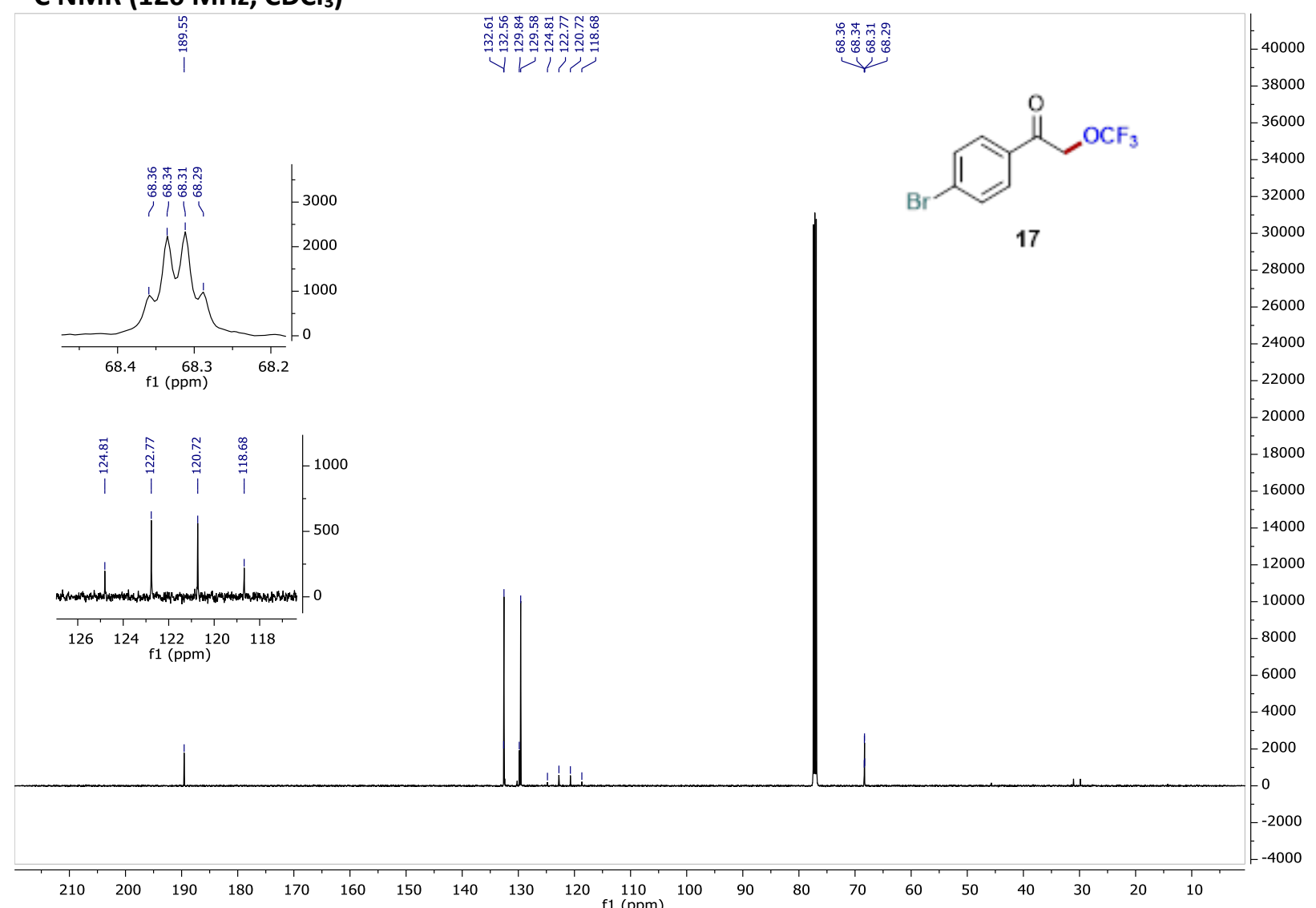

${ }^{19} \mathrm{~F}$ NMR (188 MHz, $\mathrm{CDCl}_{3}$ )

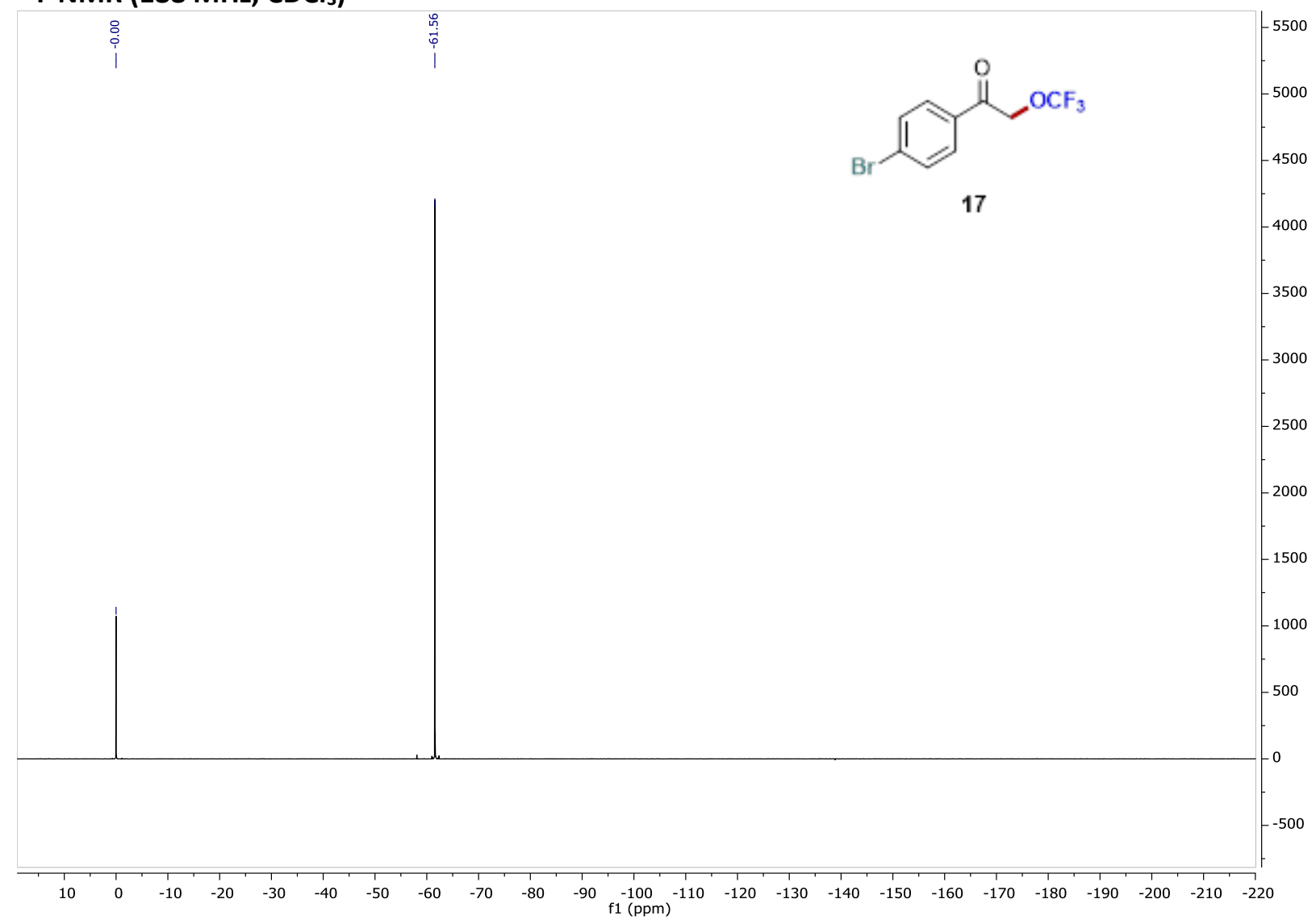


1-phenyl-2-(trifluoromethoxy)propan-1-one 20

${ }^{1} \mathrm{H}$ NMR (500 MHz, $\left.\mathrm{CDCl}_{3}\right)$

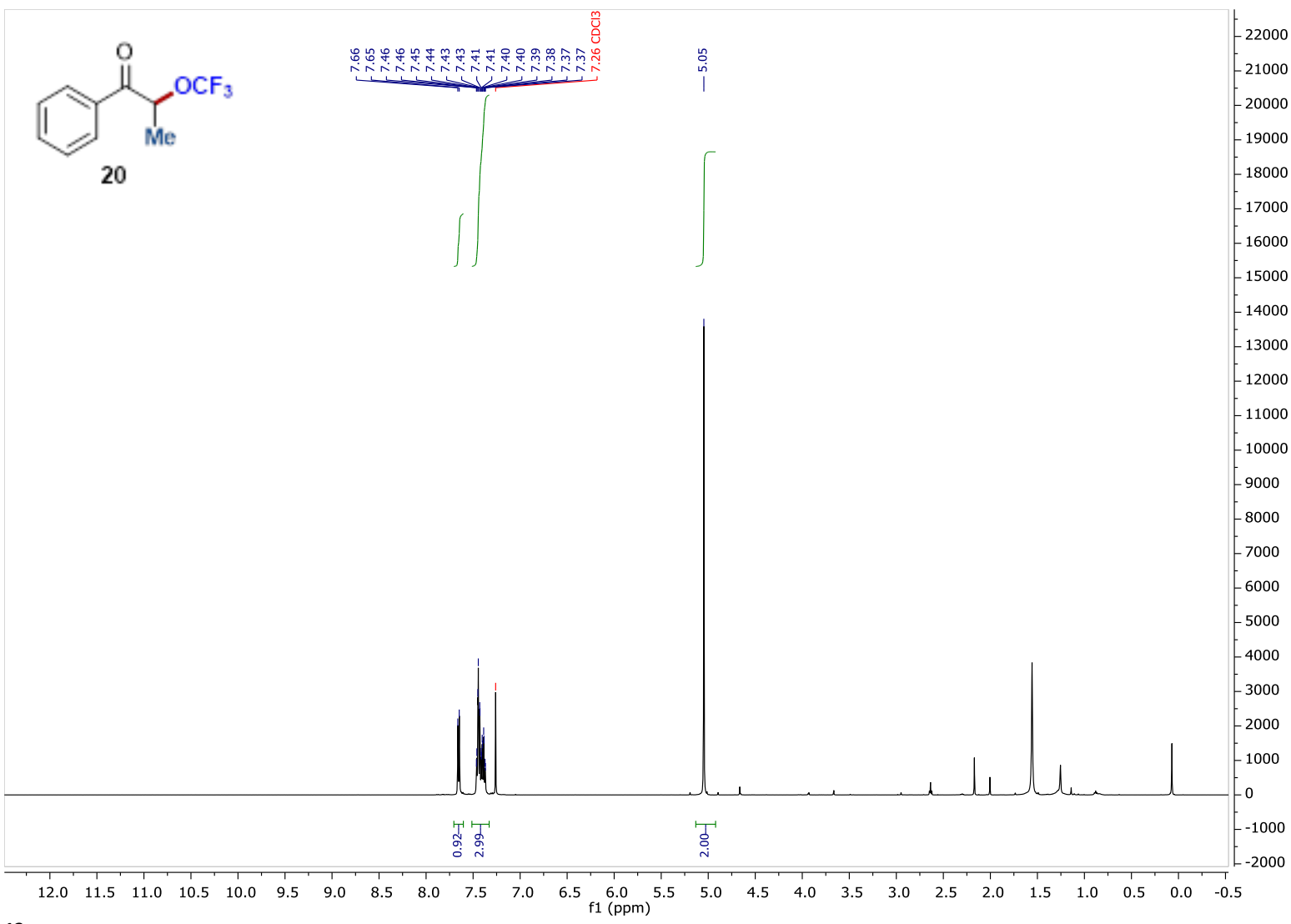

$\left.{ }^{13} \mathrm{C} \mathrm{NMR} \mathrm{(126} \mathrm{MHz,} \mathrm{CDCl}_{3}\right)$

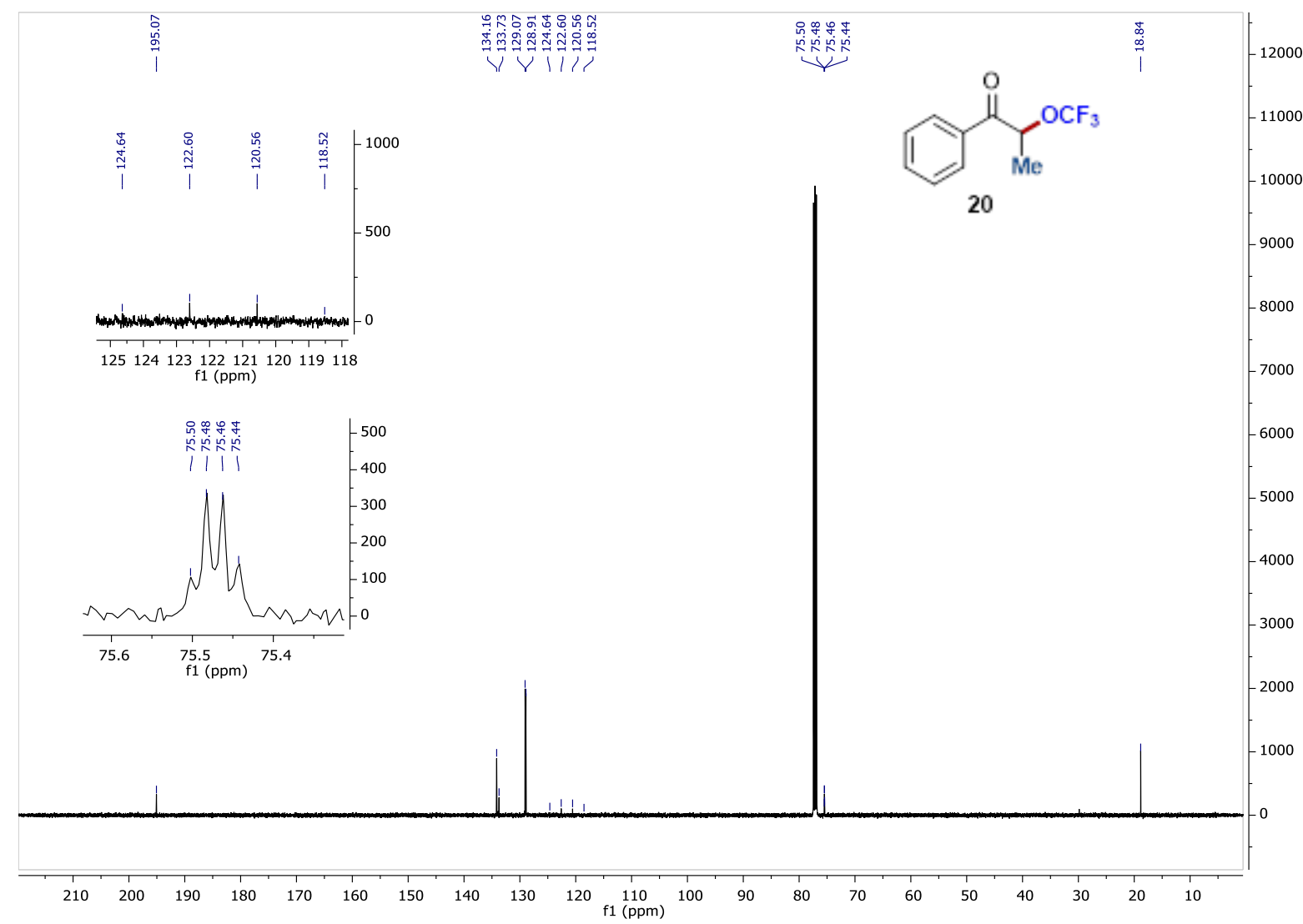


${ }^{19} \mathrm{~F}$ NMR (188 MHz, $\left.\mathrm{CDCl}_{3}\right)$

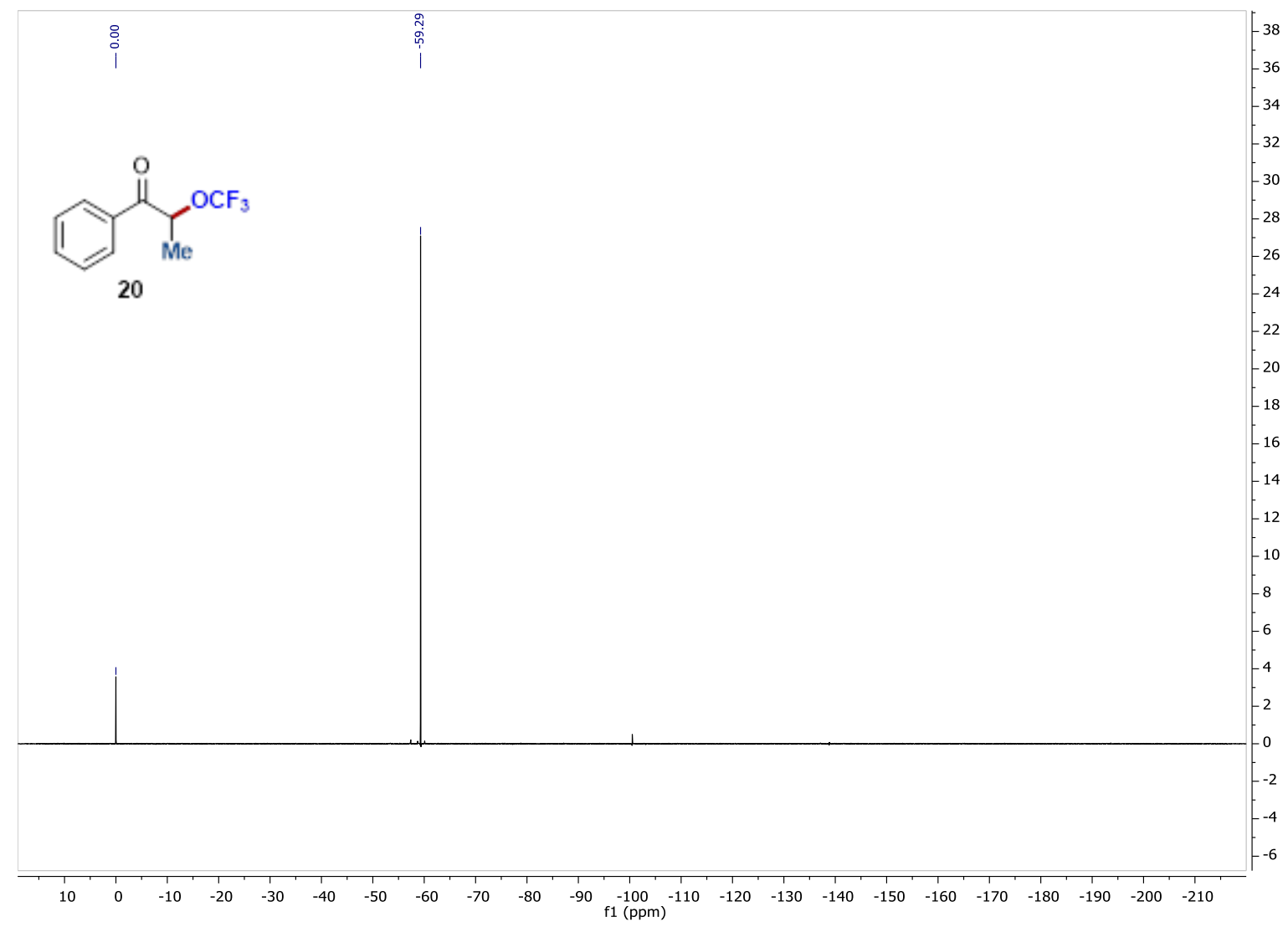

\section{1,3-diphenyl-2-(trifluoromethoxy)propan-1-one 21}

${ }^{1} \mathrm{H}$ NMR $\left(500 \mathrm{MHz}, \mathrm{CDCl}_{3}\right)$

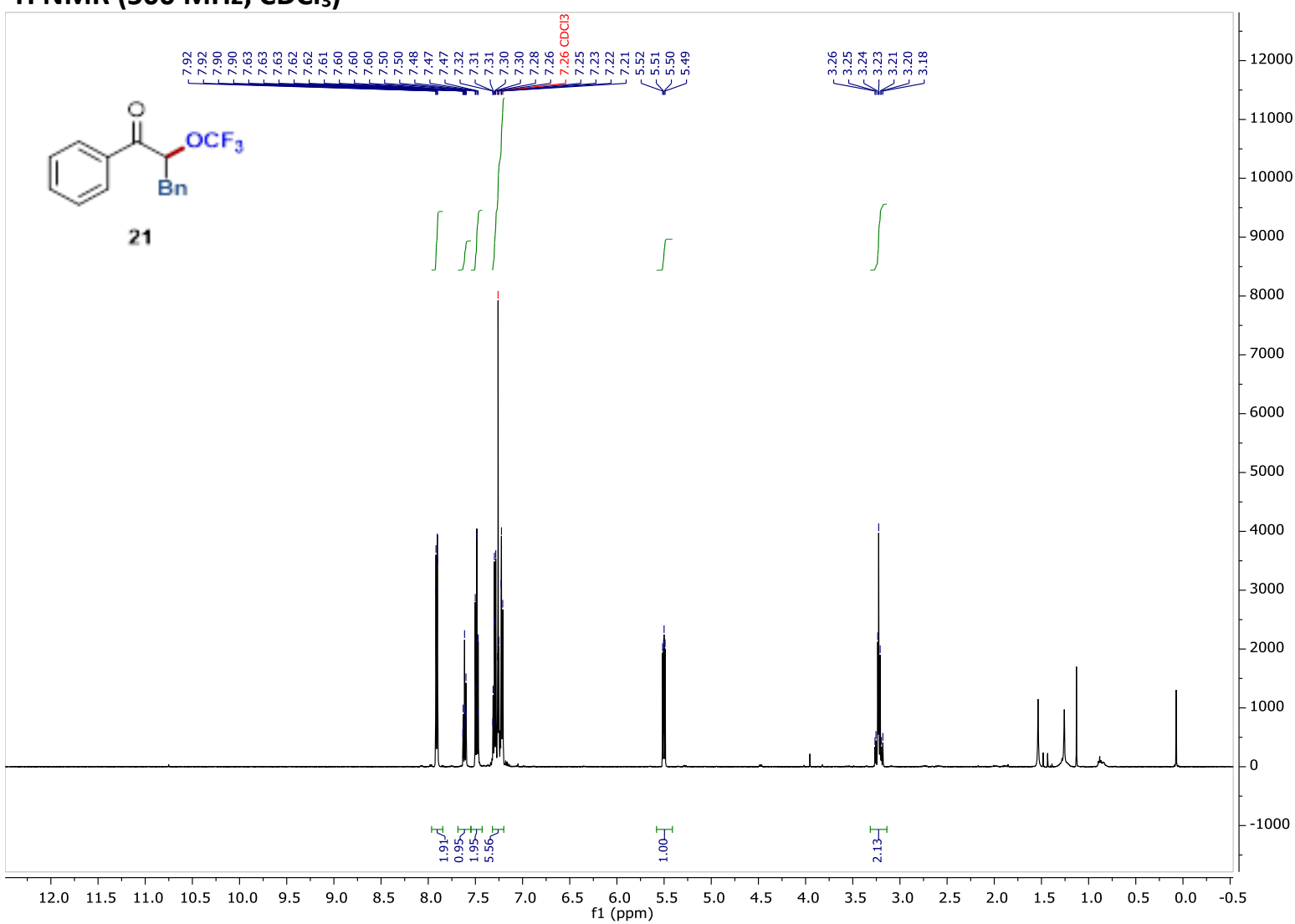


$\left.{ }^{13} \mathrm{C} \mathrm{NMR} \mathrm{(126} \mathrm{MHz,} \mathrm{CDCl}_{3}\right)$

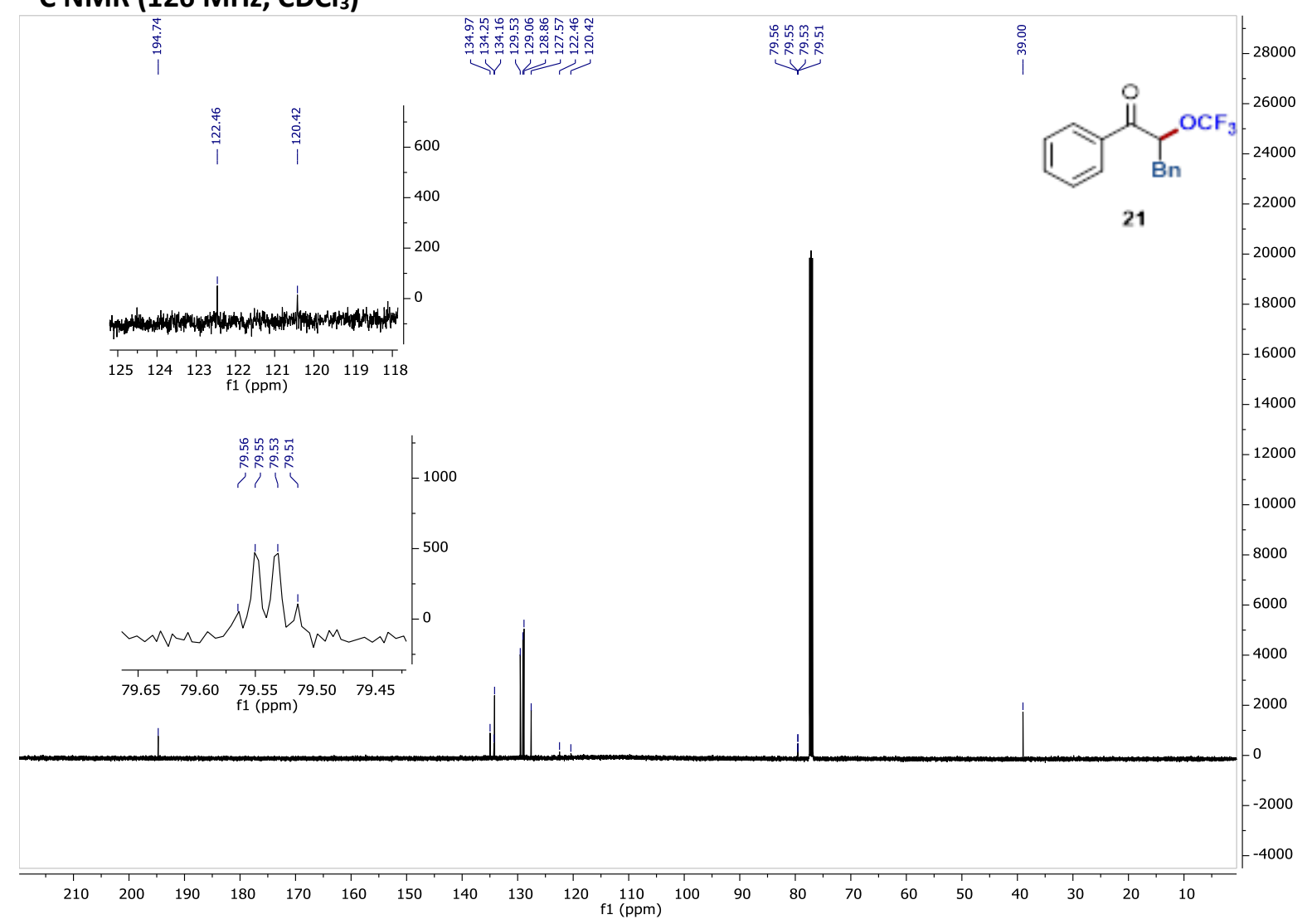

${ }^{19} \mathrm{~F} \mathrm{NMR} \mathrm{(188} \mathrm{MHz,} \mathrm{CDCl} 3$ )

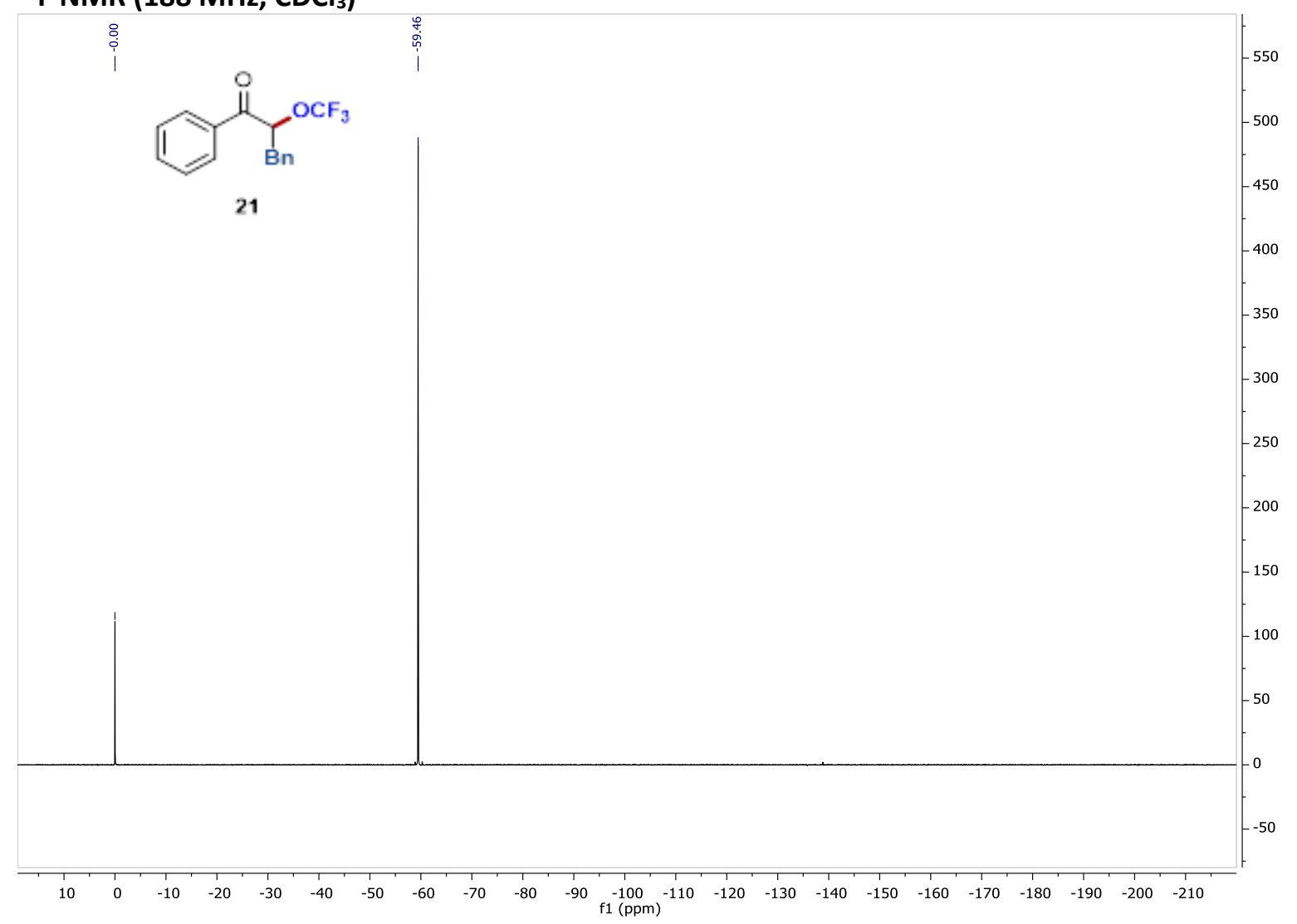


2-(trifluoromethoxy)-2,3-dihydro-1H-inden-1-one 22

${ }^{1} \mathrm{H}$ NMR (300 MHz, $\mathrm{CDCl}_{3}$ )

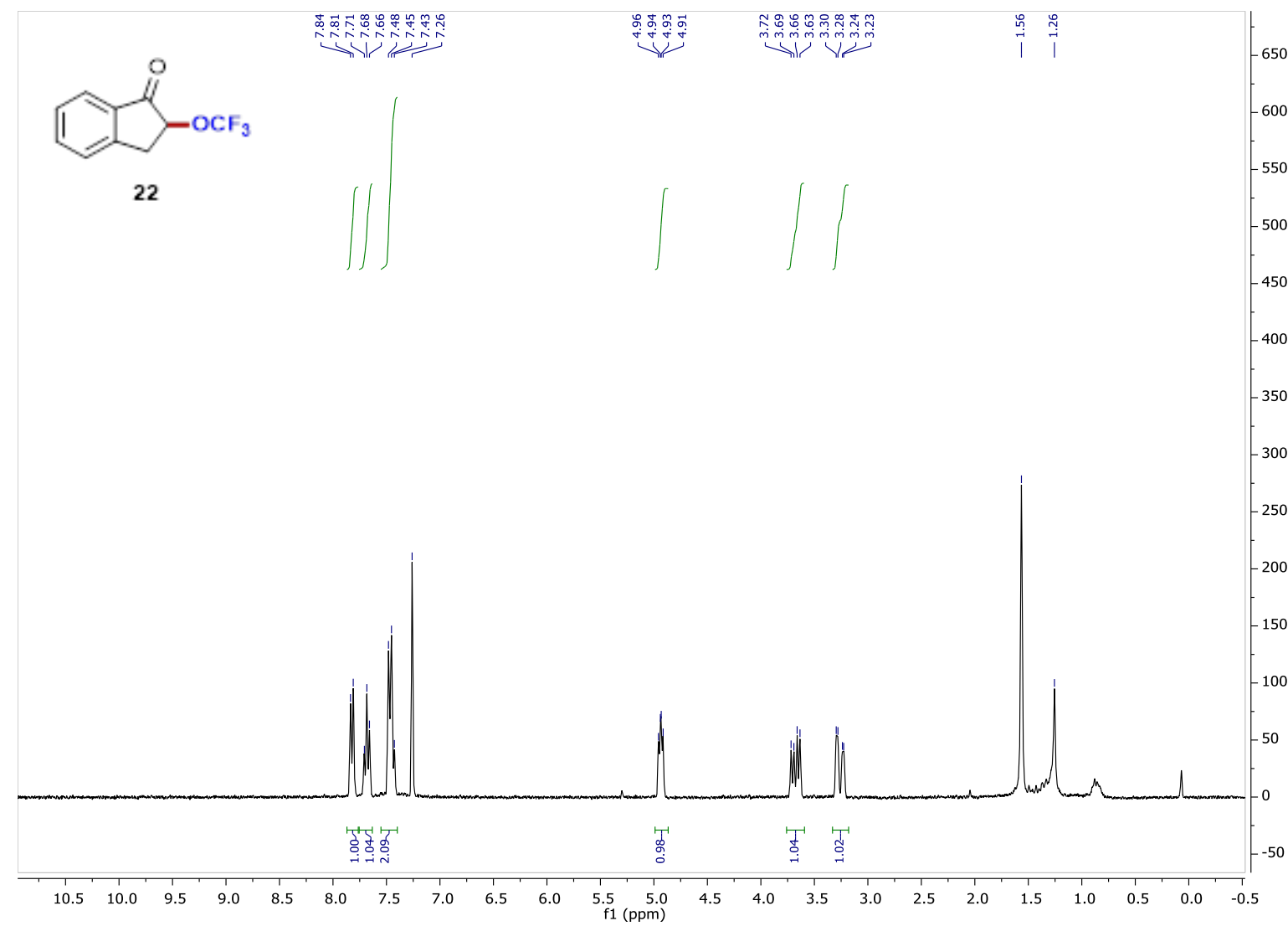

${ }^{13} \mathrm{C}$ NMR $\left(75 \mathrm{MHz}, \mathrm{CDCl}_{3}\right)$

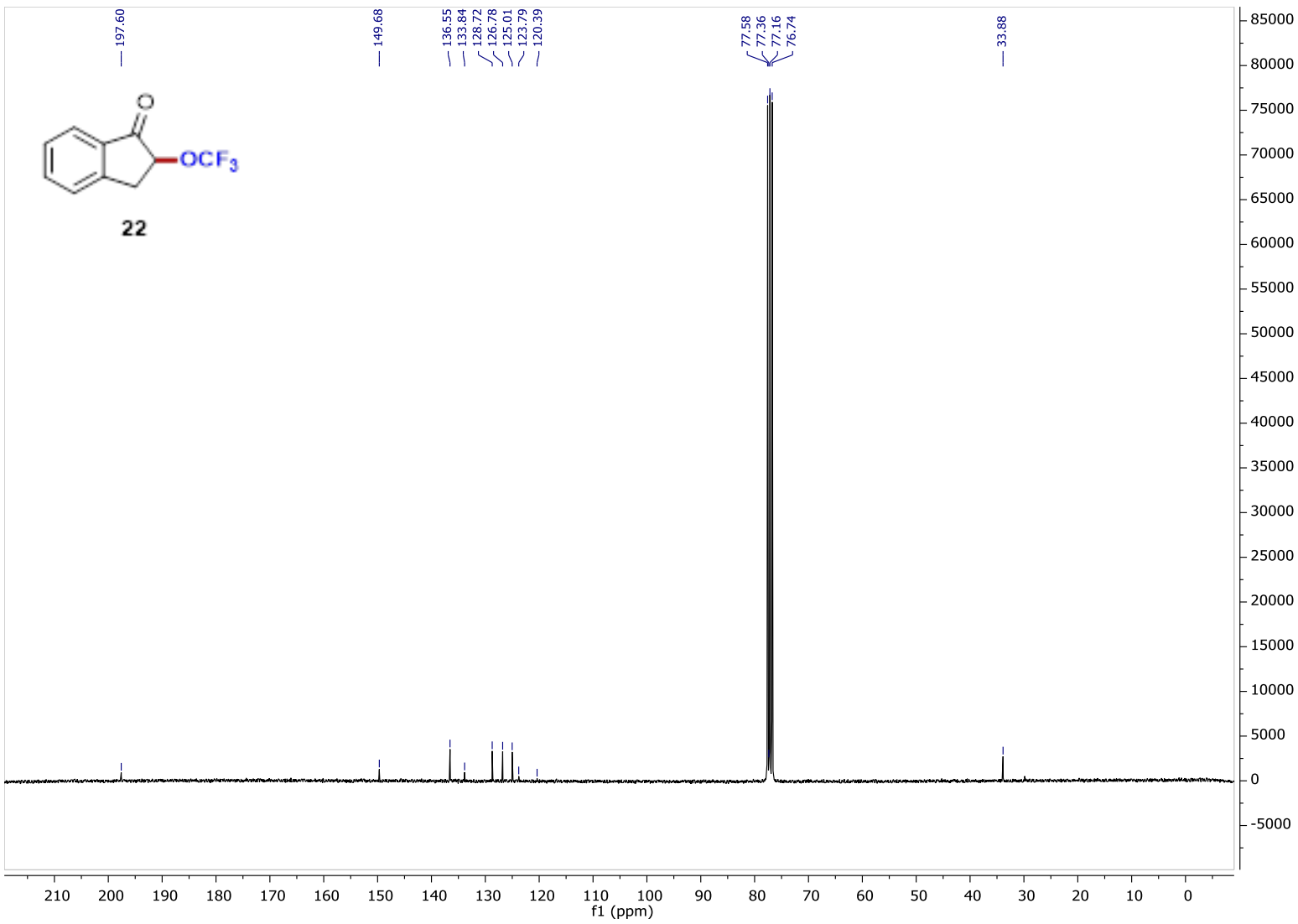


${ }^{19} \mathrm{~F}$ NMR (188 MHz, $\left.\mathrm{CDCl}_{3}\right)$

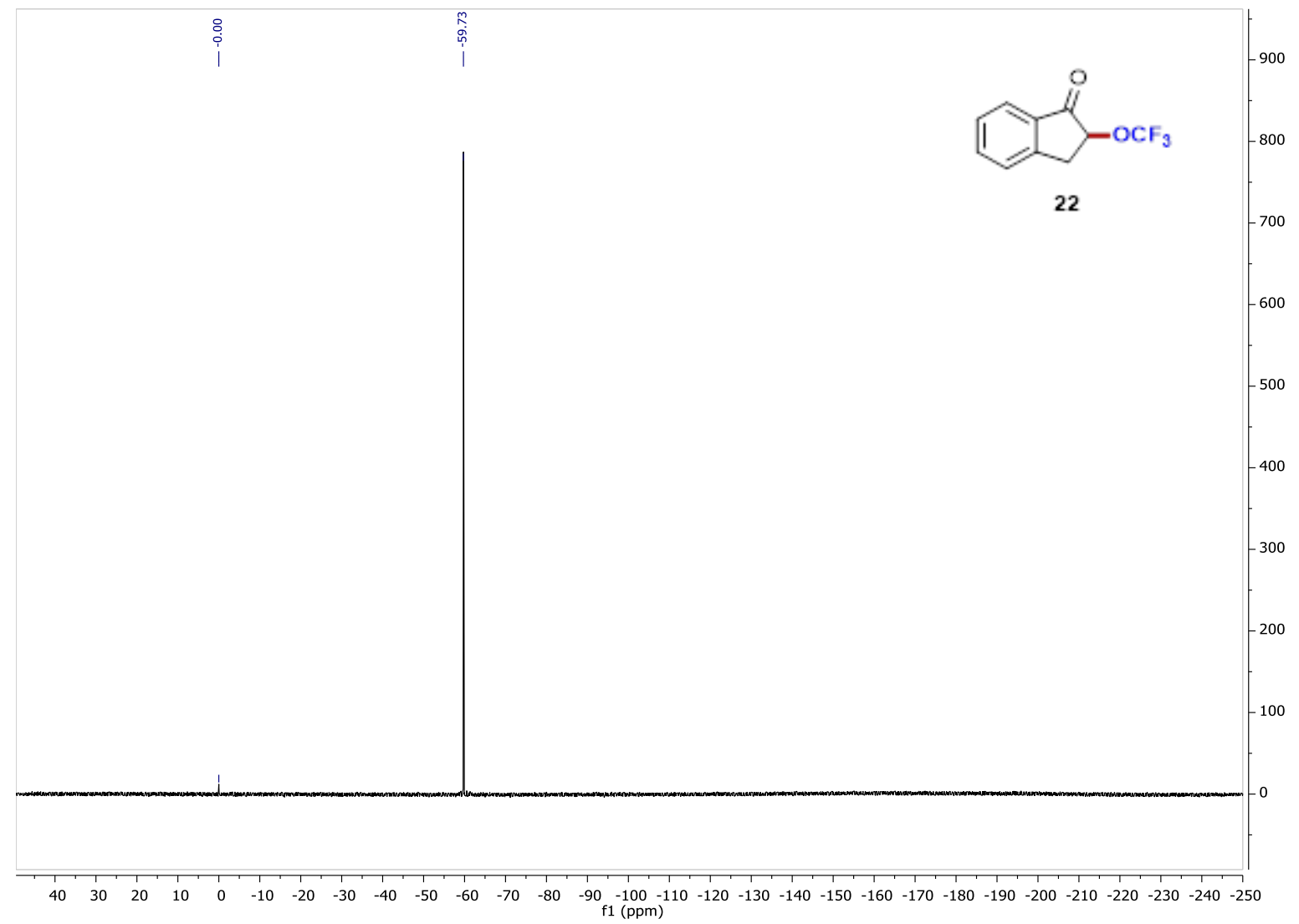

6-methoxy-2-(trifluoromethoxy)-2,3-dihydro-1H-inden-1-one 23

${ }^{1} \mathrm{H}$ NMR $\left.\left(300 \mathrm{MHz}^{\mathrm{CDCl}}\right)_{3}\right)$

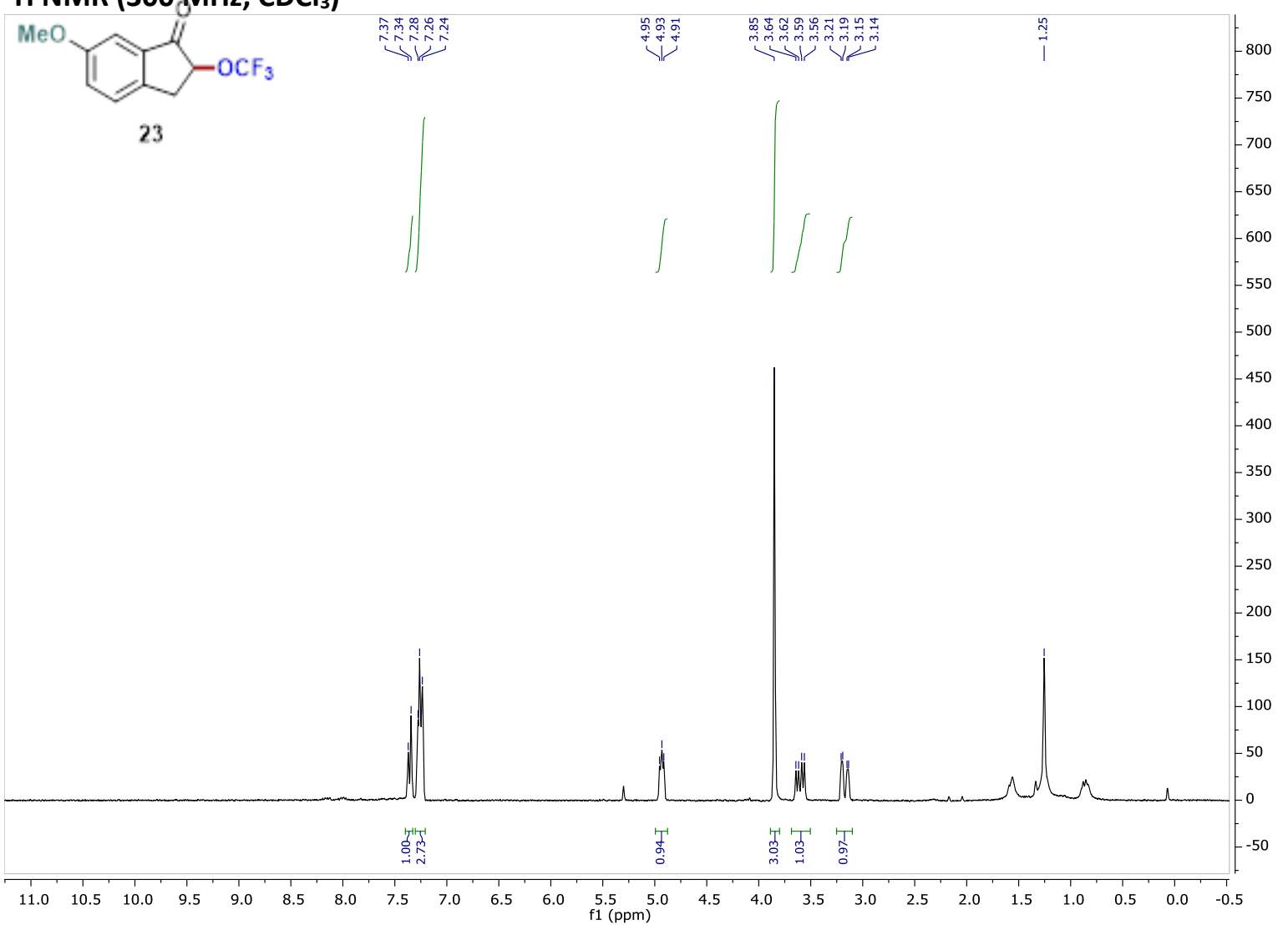




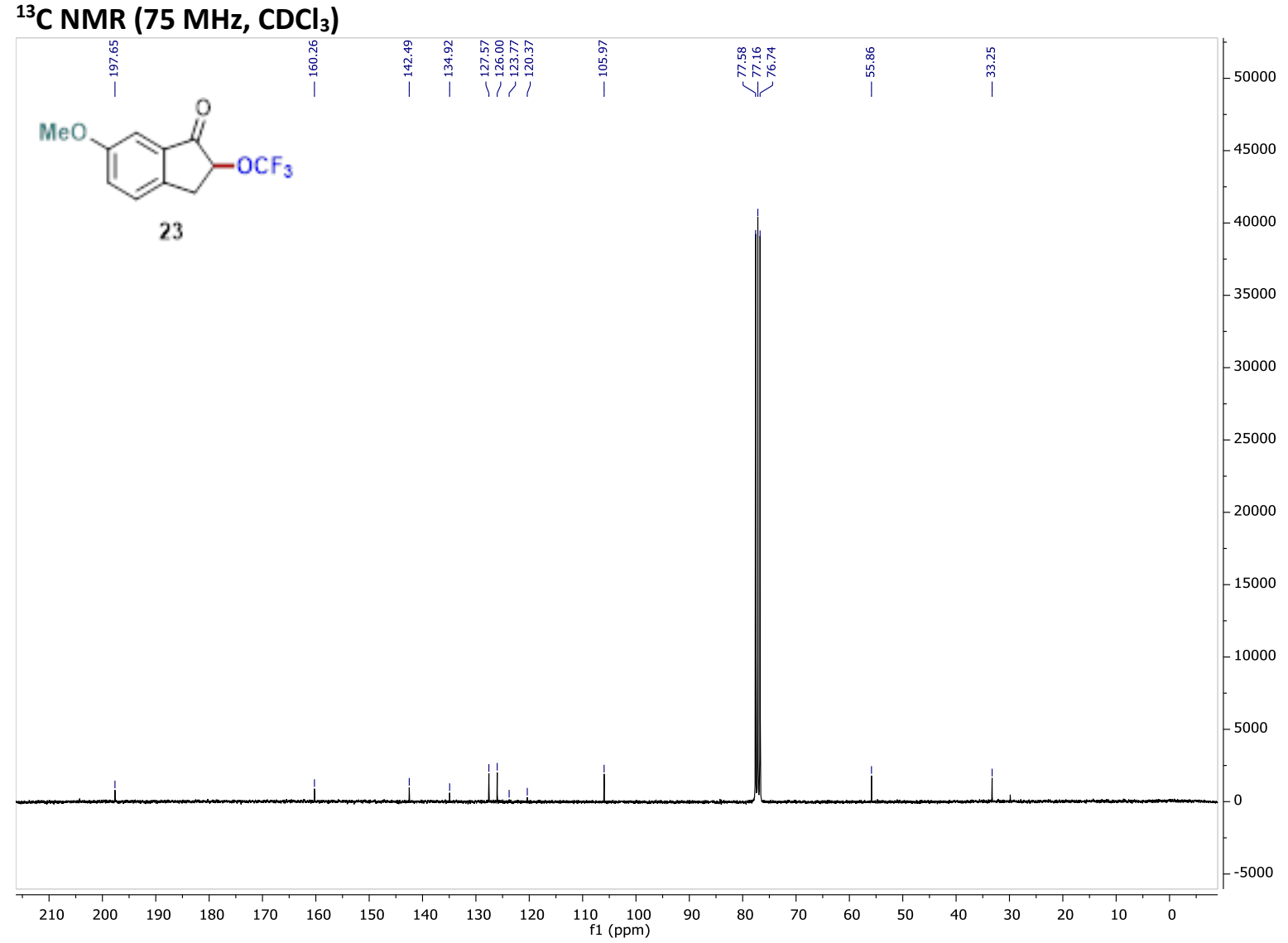

$\left.{ }^{19} \mathrm{~F} \mathrm{NMR} \mathrm{(188} \mathrm{MHz,} \mathrm{CDCl}_{3}\right)$

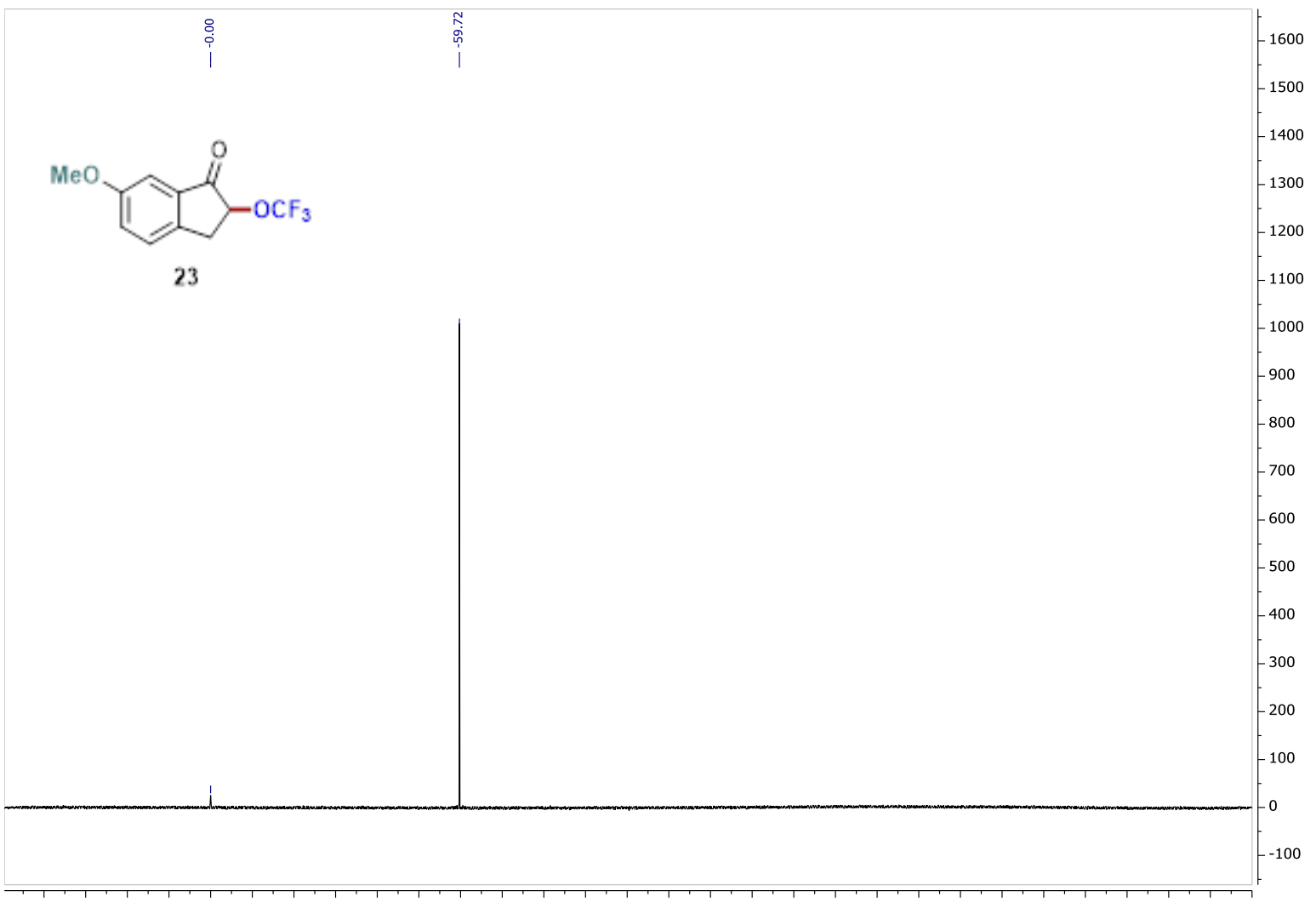

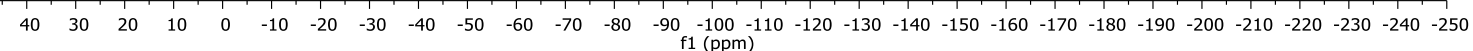


5-fluoro-2-(trifluoromethoxy)-2,3-dihydro-1H-inden-1-one 24

${ }^{1} \mathrm{H}$ NMR $\left(300 \mathrm{MHz}, \mathrm{CDCl}_{3}\right)$

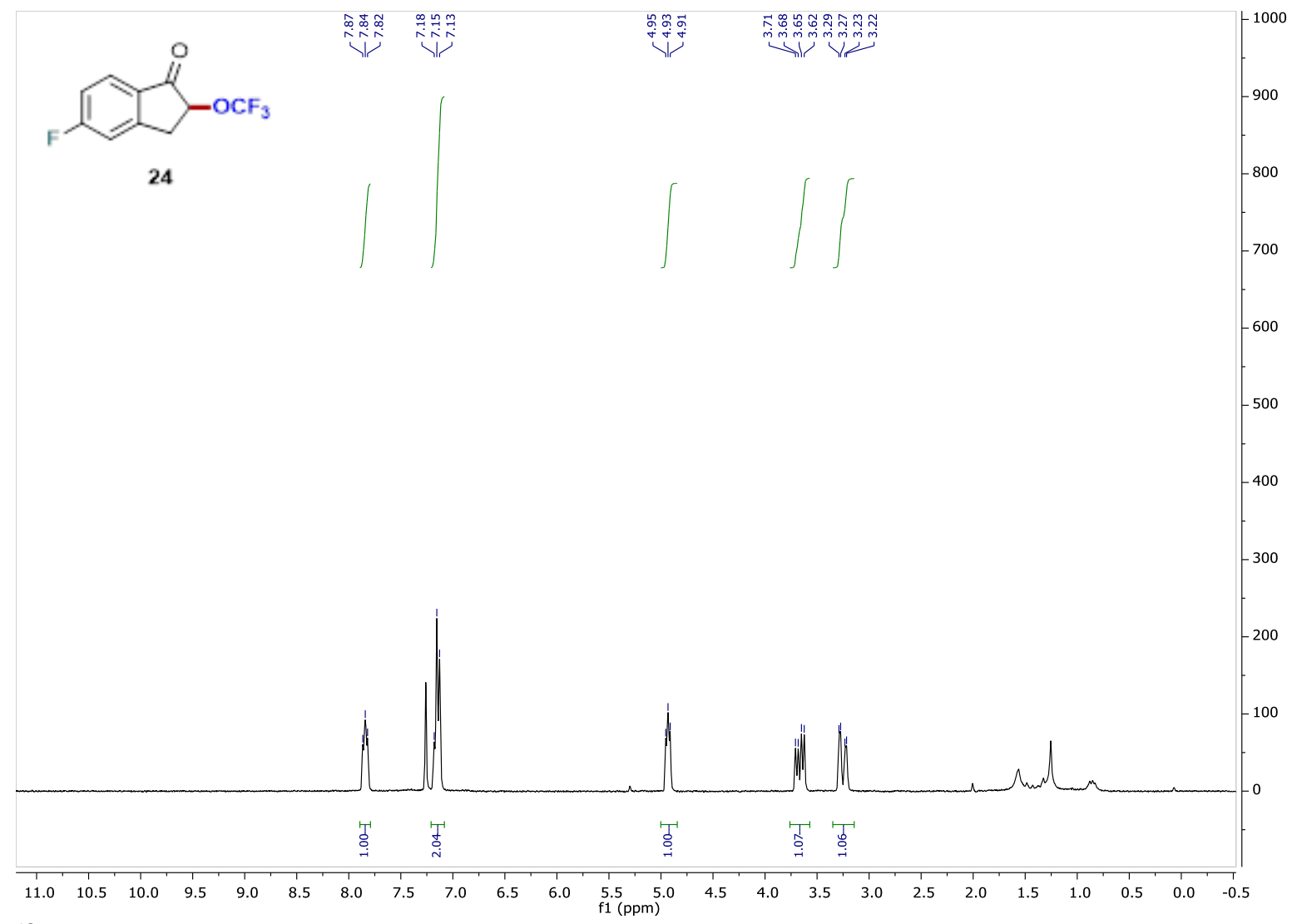

${ }^{13} \mathrm{C}$ NMR (75 MHz, $\left.\mathrm{CDCl}_{3}\right)$

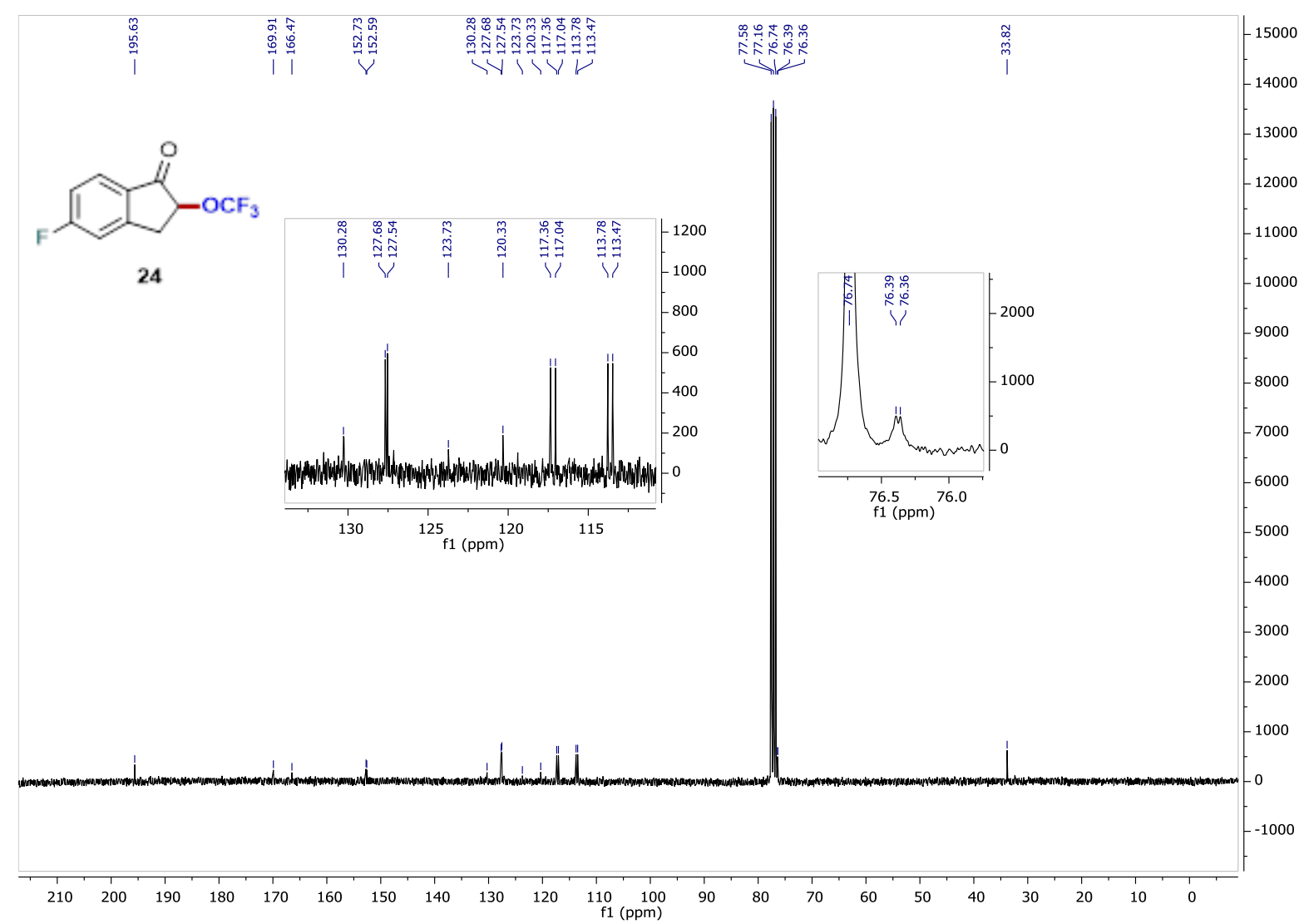


${ }^{19} \mathrm{~F}$ NMR (188 MHz, $\left.\mathrm{CDCl}_{3}\right)$

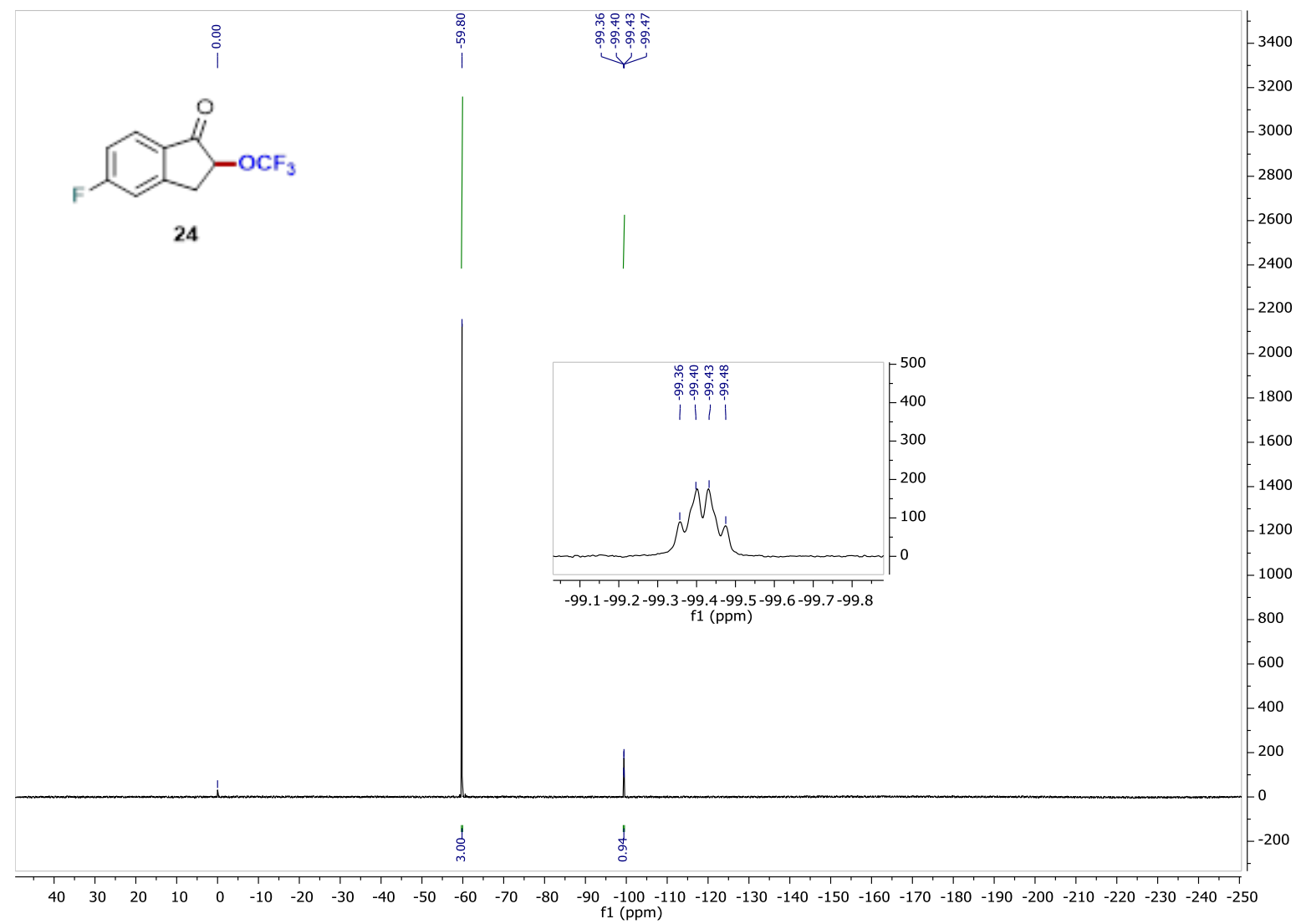

6-(trifluoromethoxy)-6,7,8,9-tetrahydro-5H-benzo[7]annulen-5-one 26

${ }^{1} \mathrm{H}$ NMR $\left(300 \mathrm{MHz}, \mathrm{CDCl}_{3}\right)$

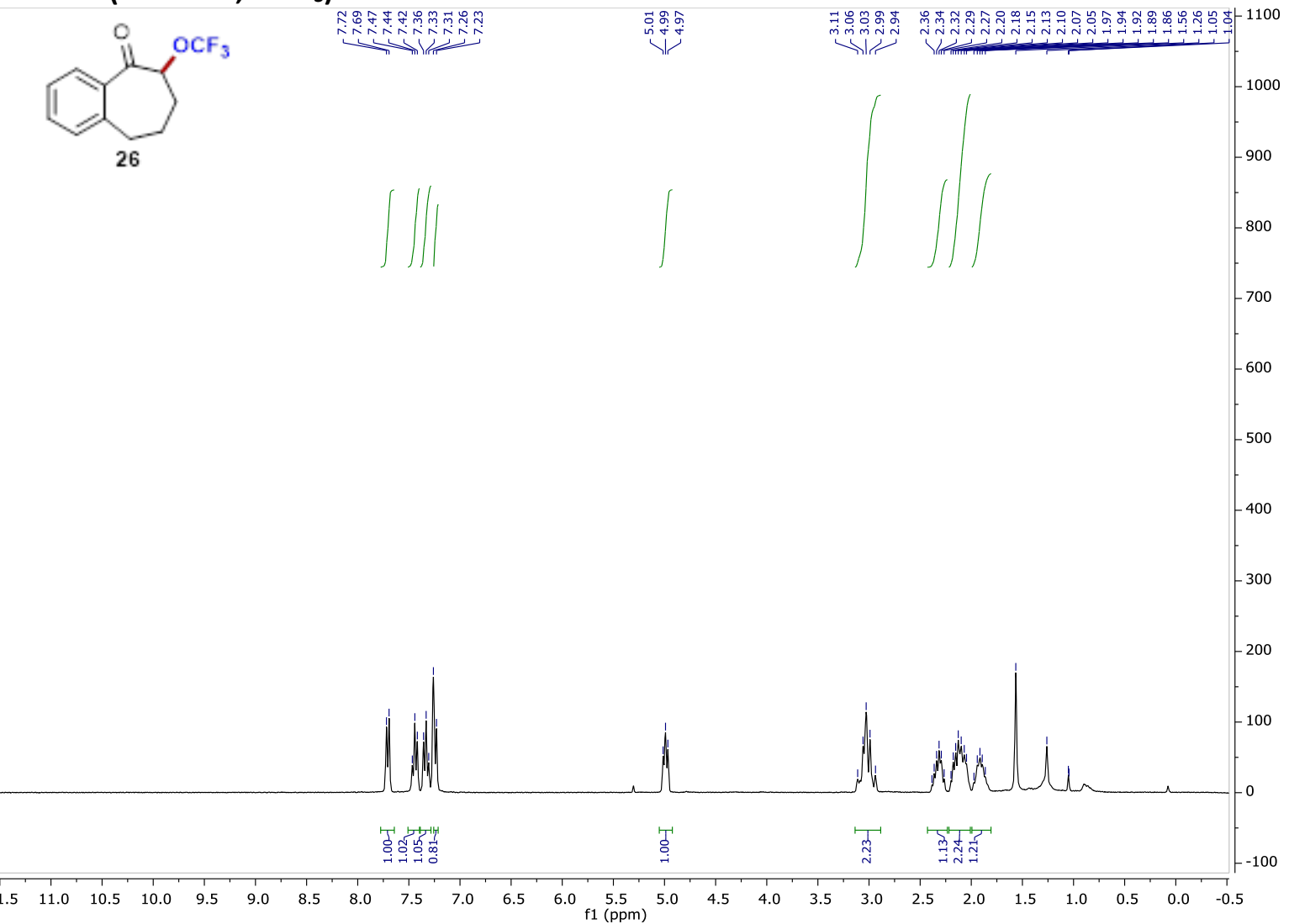


${ }^{13} \mathrm{C}$ NMR $\left(75 \mathrm{MHz}, \mathrm{CDCl}_{3}\right)$

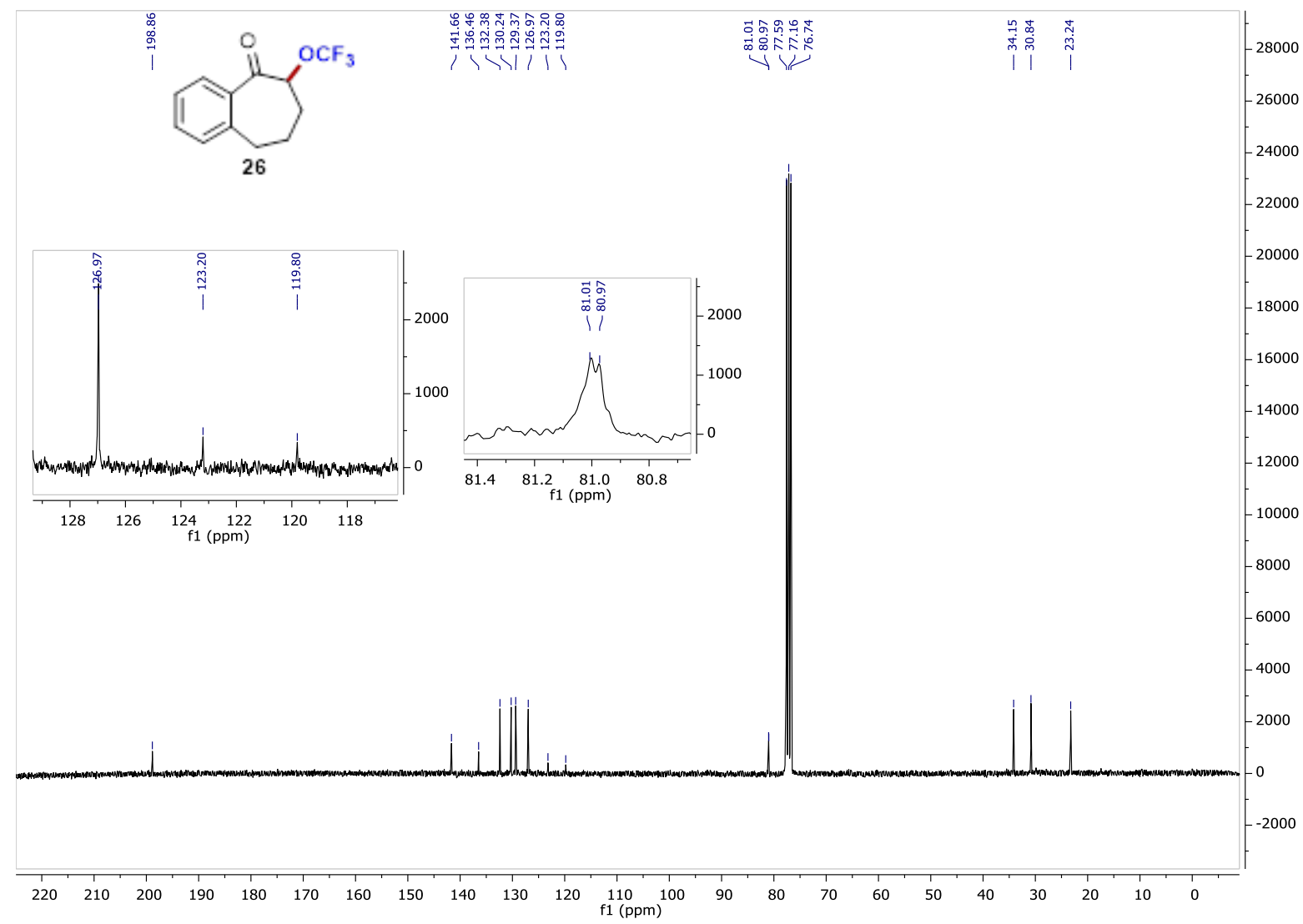

${ }^{19} \mathrm{~F}$ NMR (188 MHz, $\left.\mathrm{CDCl}_{3}\right)$

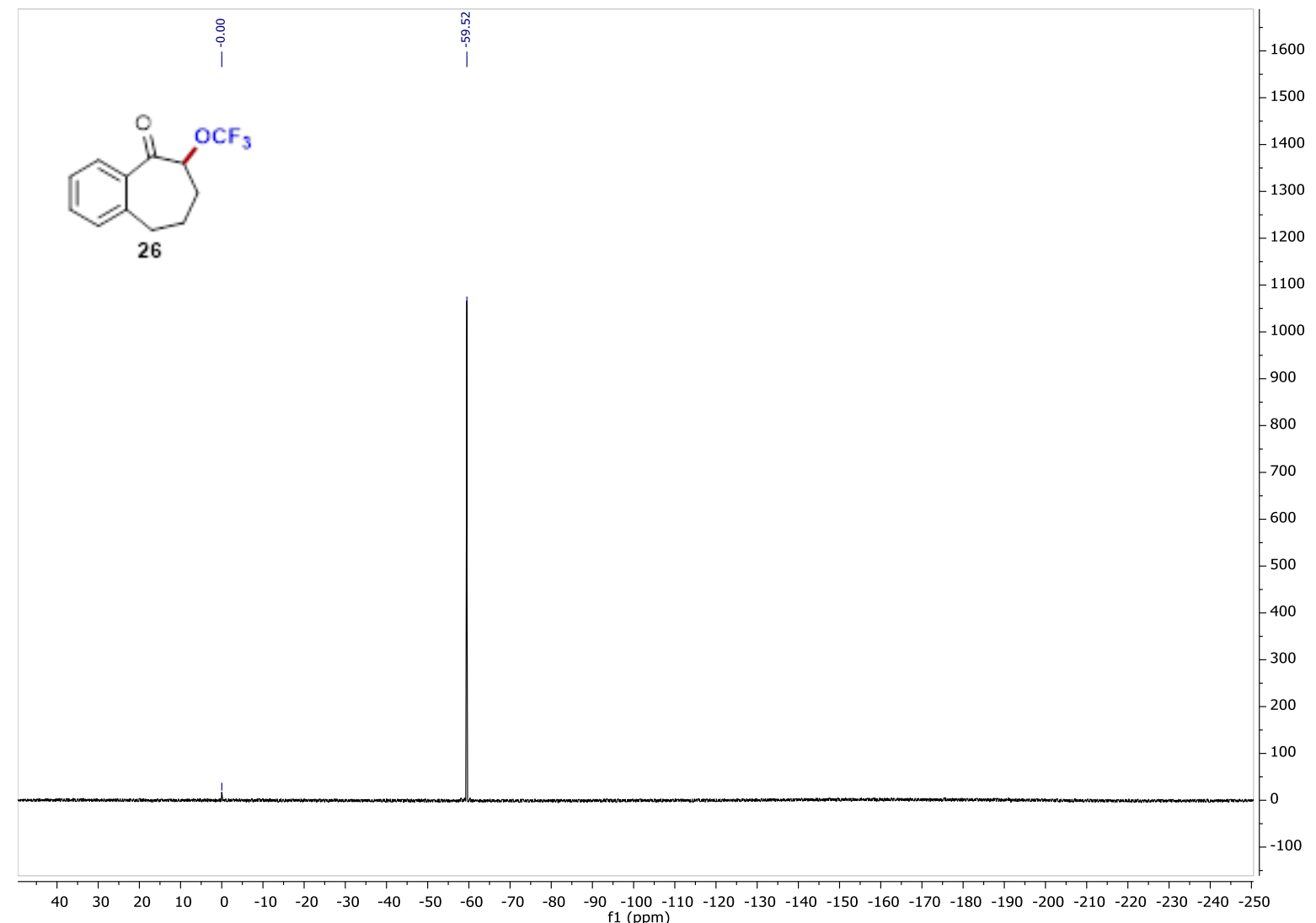


1-(benzo[b]thiophen-2-yl)-2-(trifluoromethoxy)ethanone 27

${ }^{1} \mathrm{H}$ NMR (300 MHz, $\mathrm{CDCl}_{3}$ )

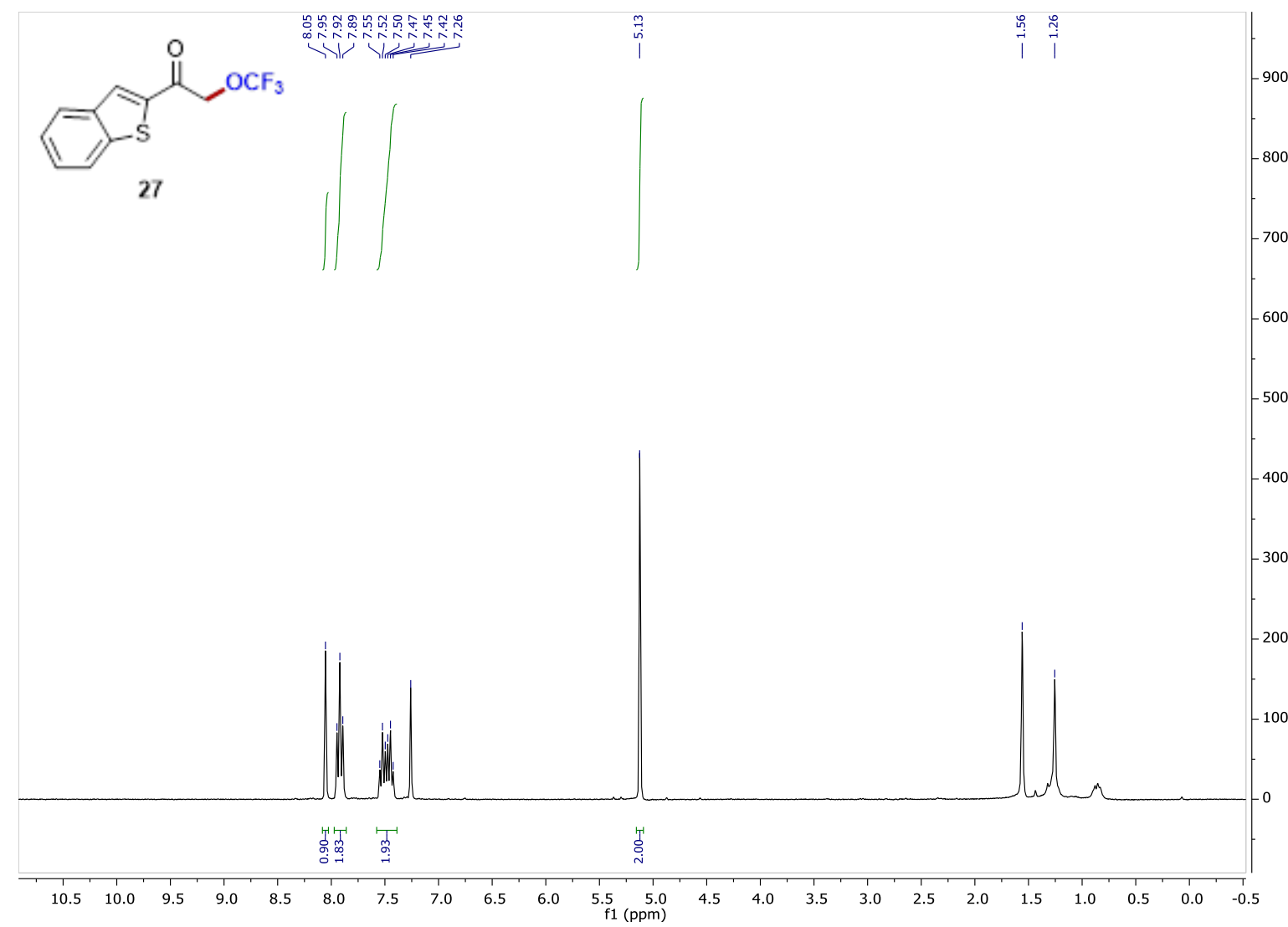

${ }^{13} \mathrm{C}$ NMR (75 MHz, $\mathrm{CDCl}_{3}$ )

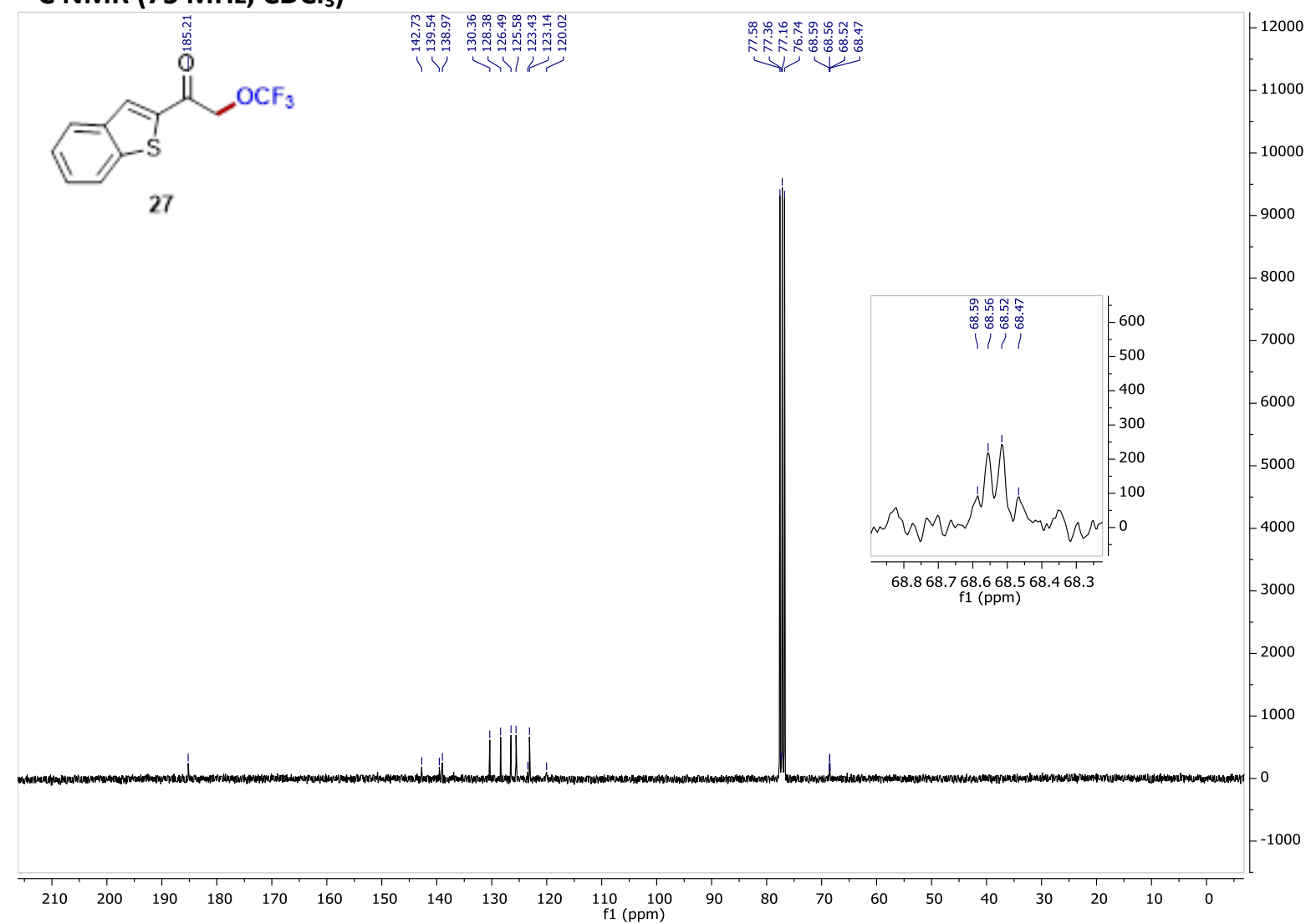


${ }^{19} \mathrm{~F}$ NMR (188 MHz, $\left.\mathrm{CDCl}_{3}\right)$

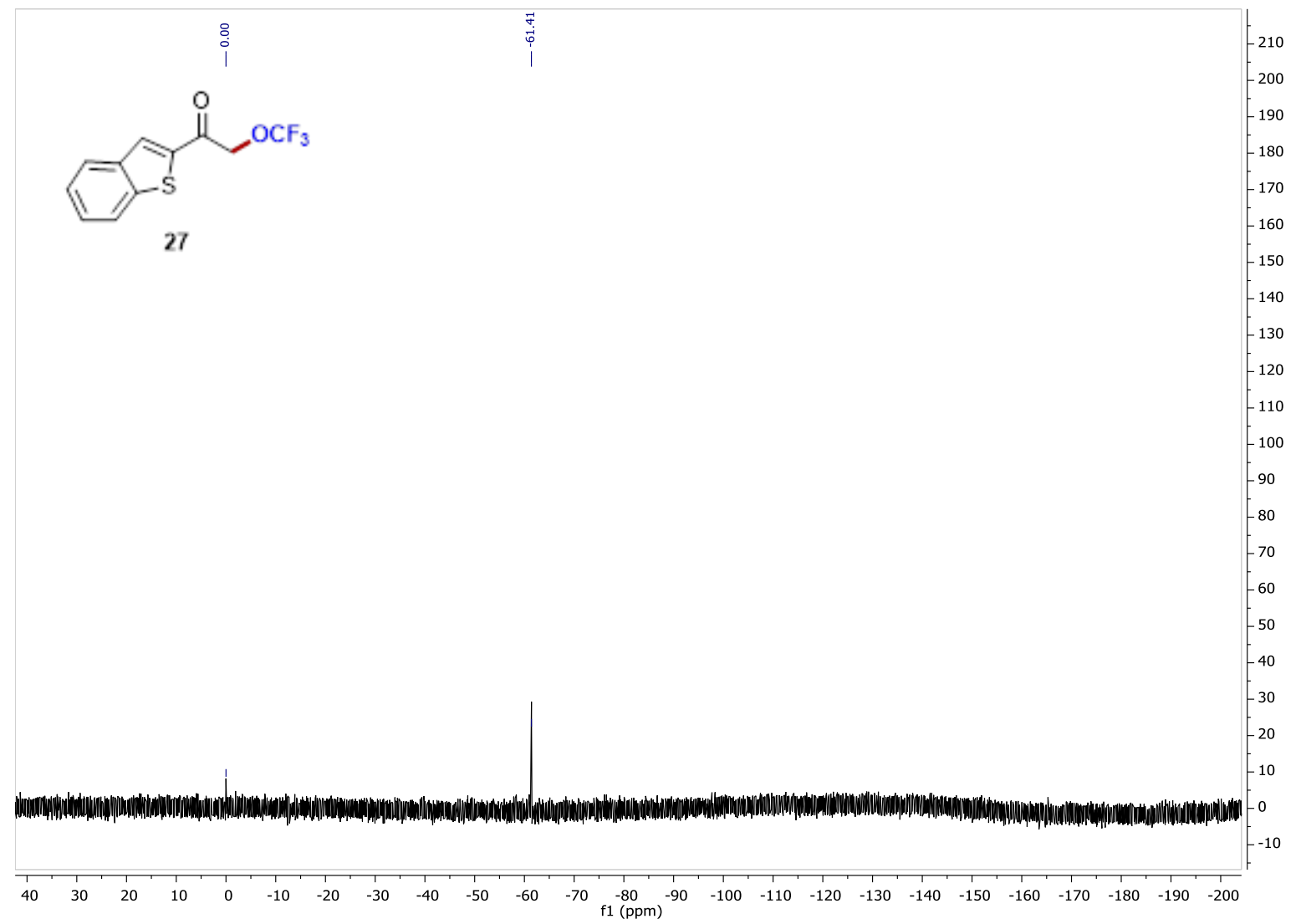

(E)-4-phenyl-1-(trifluoromethoxy)but-3-en-2-one 32

${ }^{1} \mathrm{H}$ NMR (300 MHz, $\left.\mathrm{CDCl}_{3}\right)$

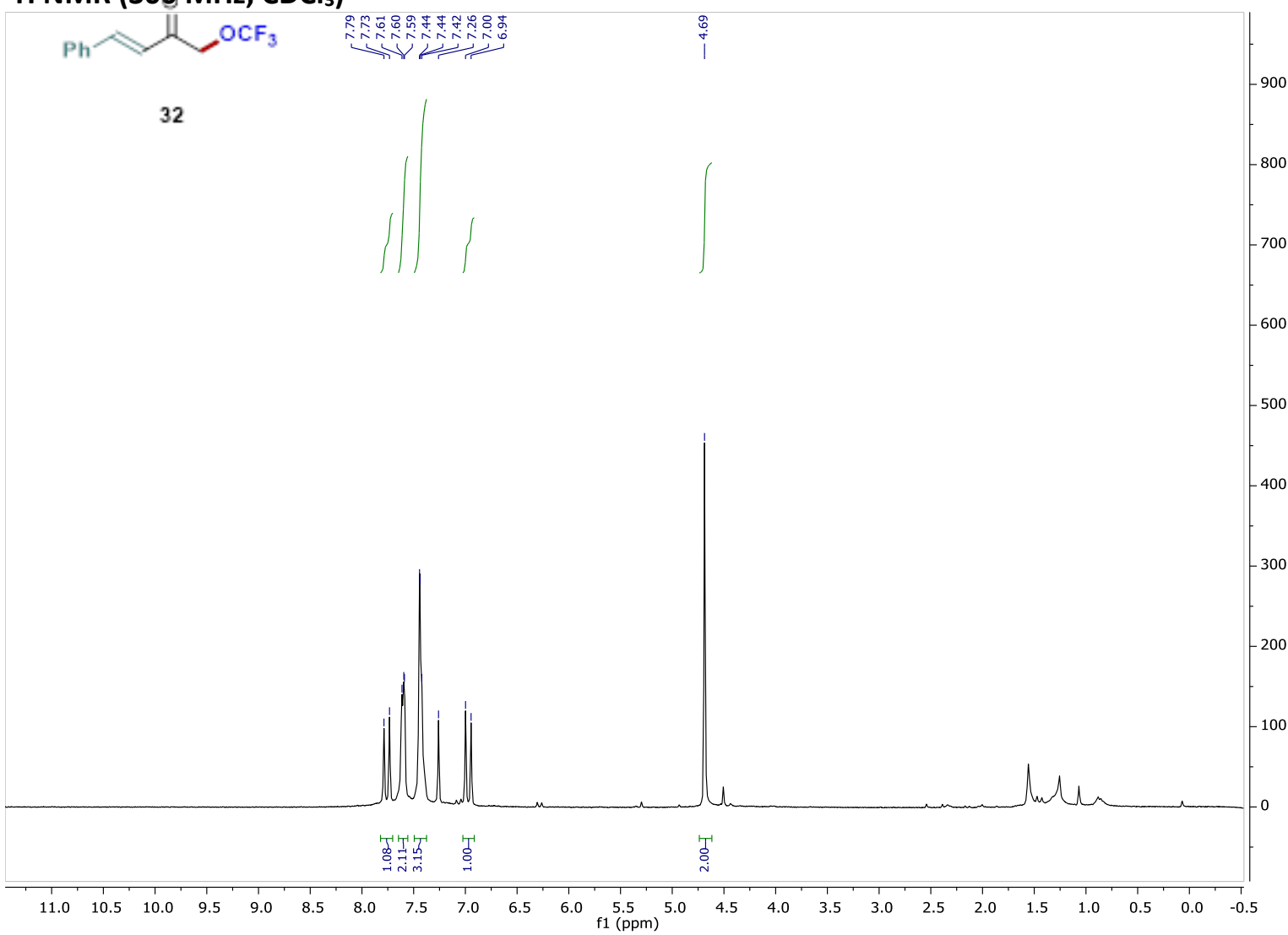


${ }^{13} \mathrm{C}$ NMR (75 MHz, $\mathrm{CDCl}_{3}$ )

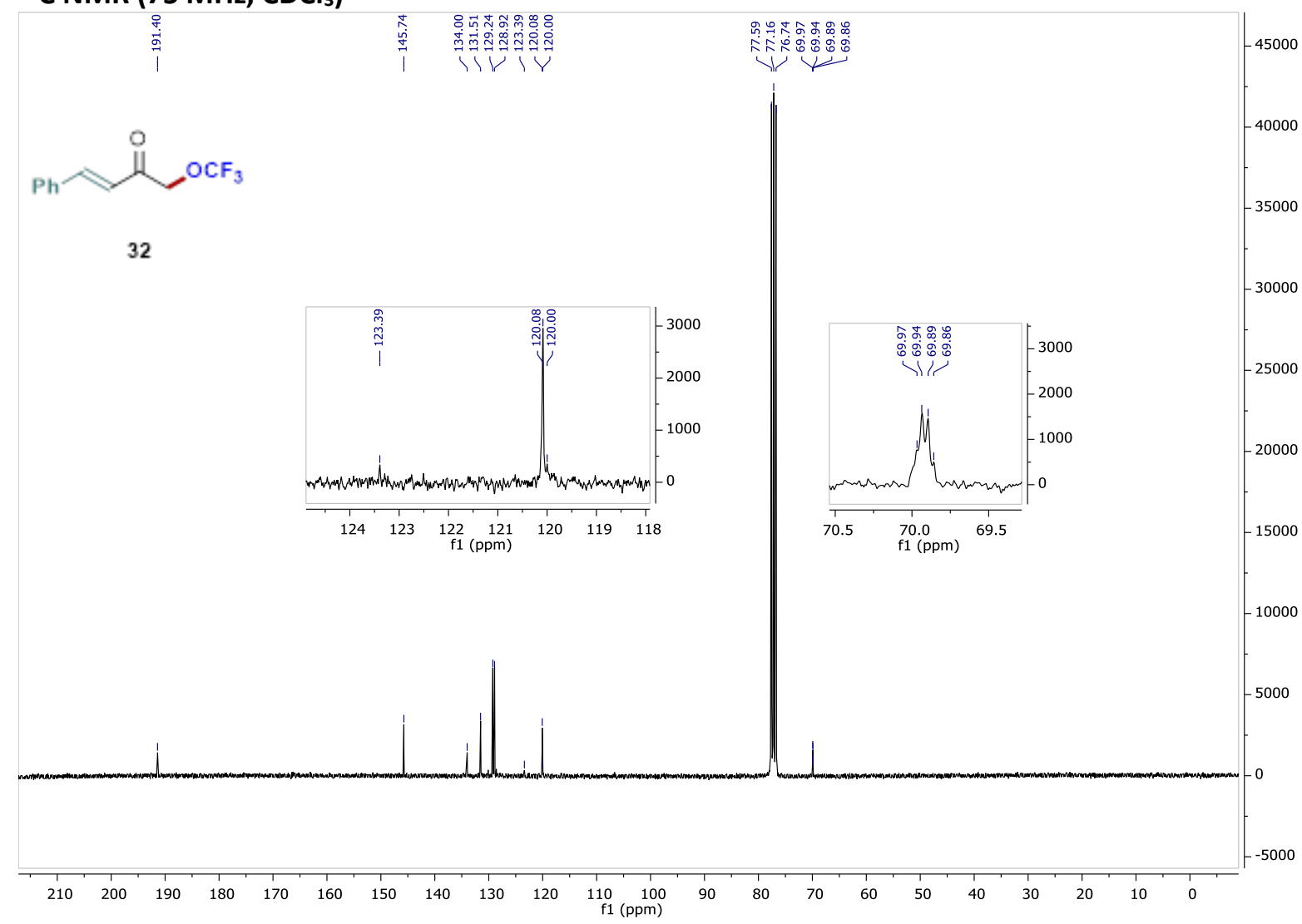

$\left.{ }^{19} \mathrm{~F} \mathrm{NMR} \mathrm{(188} \mathrm{MHz,} \mathrm{CDCl}_{3}\right)$

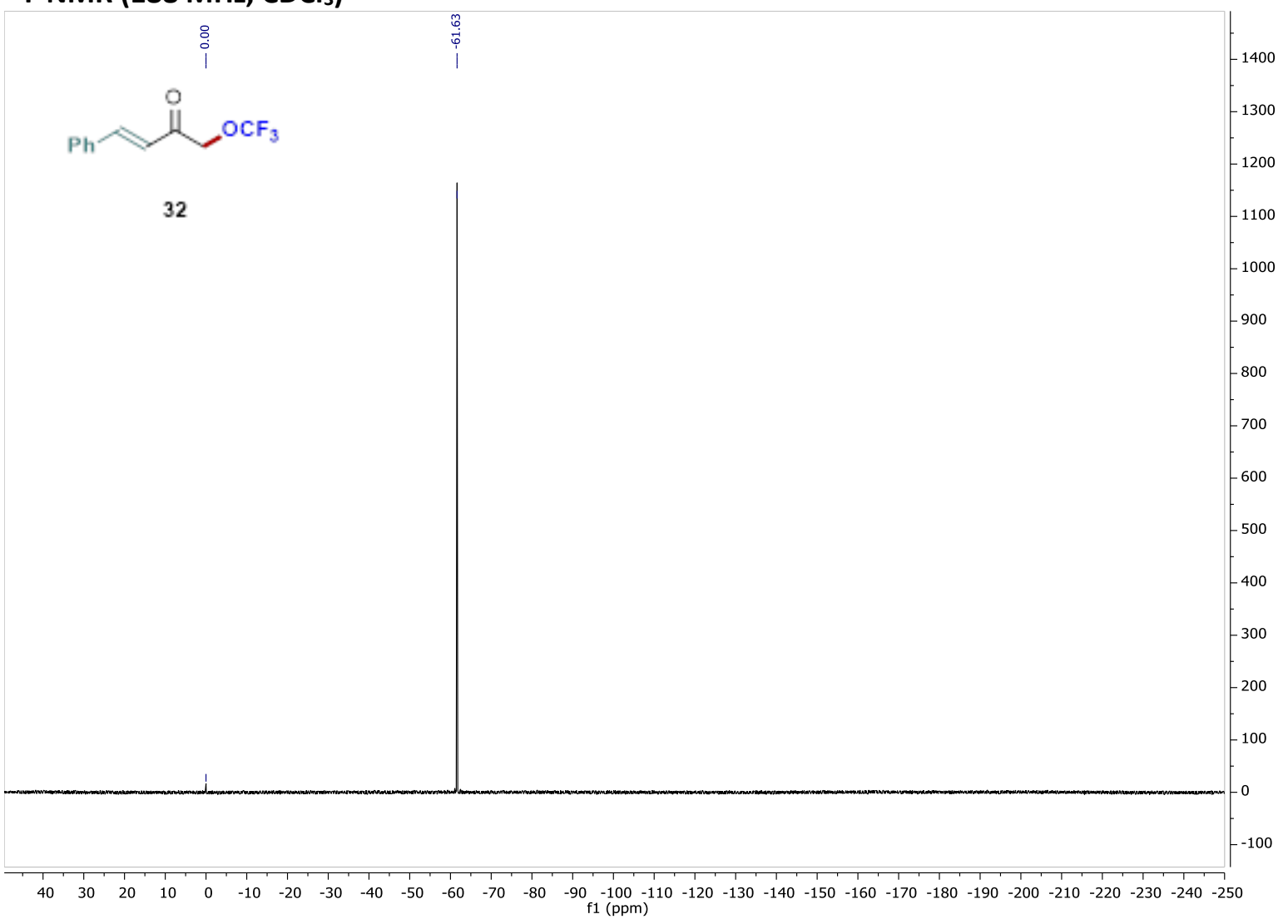


(E)-1-(trifluoromethoxy)non-3-en-2-one 33

${ }^{19}$ F NMR (188 MHz, $\left.\mathrm{CDCl}_{3}\right)$

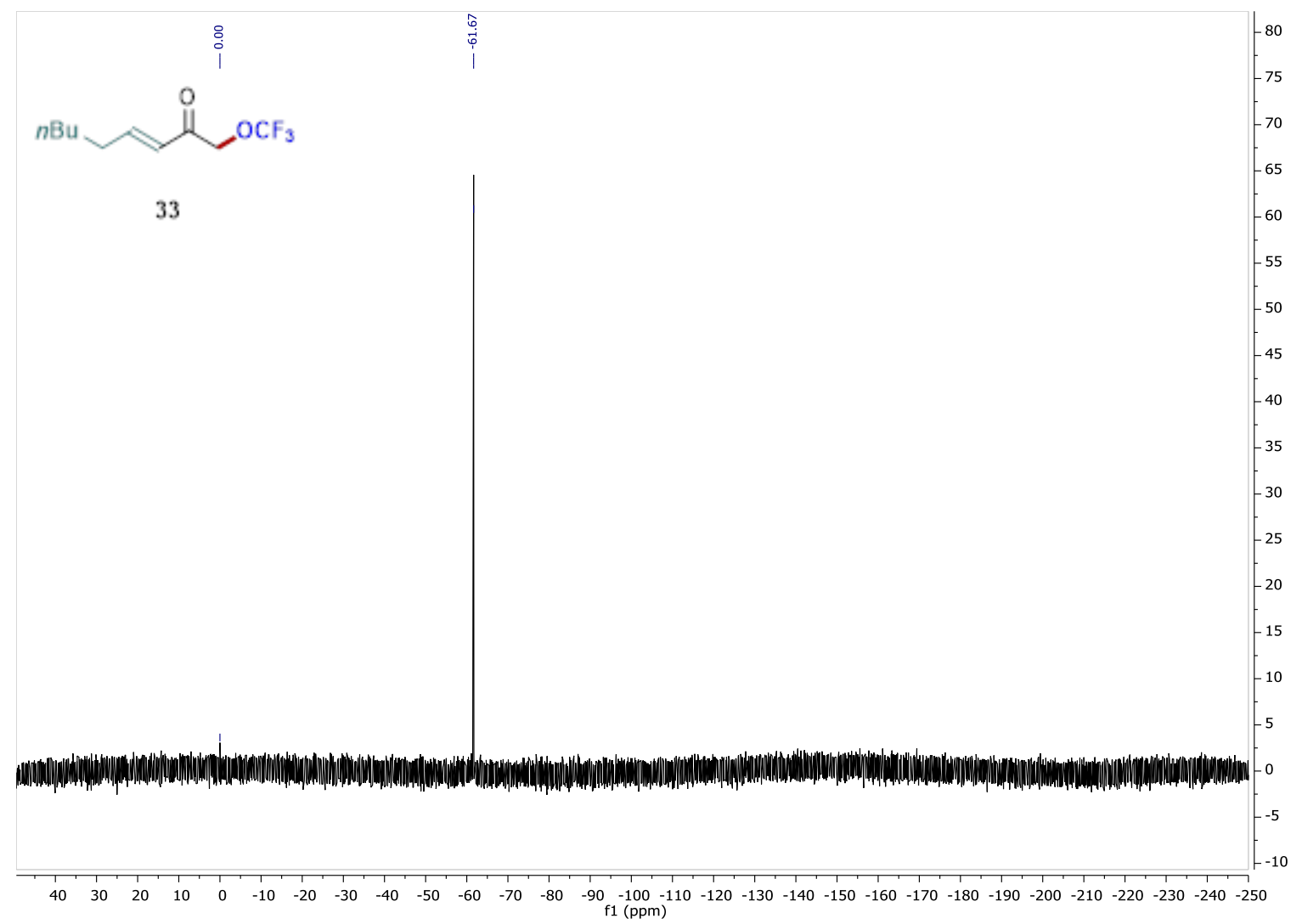

1-(3,5,5,6,8,8-hexamethyl-5,6,7,8-tetrahydronaphthalen-2-yl)-2-(trifluoromethoxy)ethan-1-one 34 ${ }^{1} \mathrm{H}$ NMR $\left(500 \mathrm{MHz}, \mathrm{CDCl}_{3}\right)$

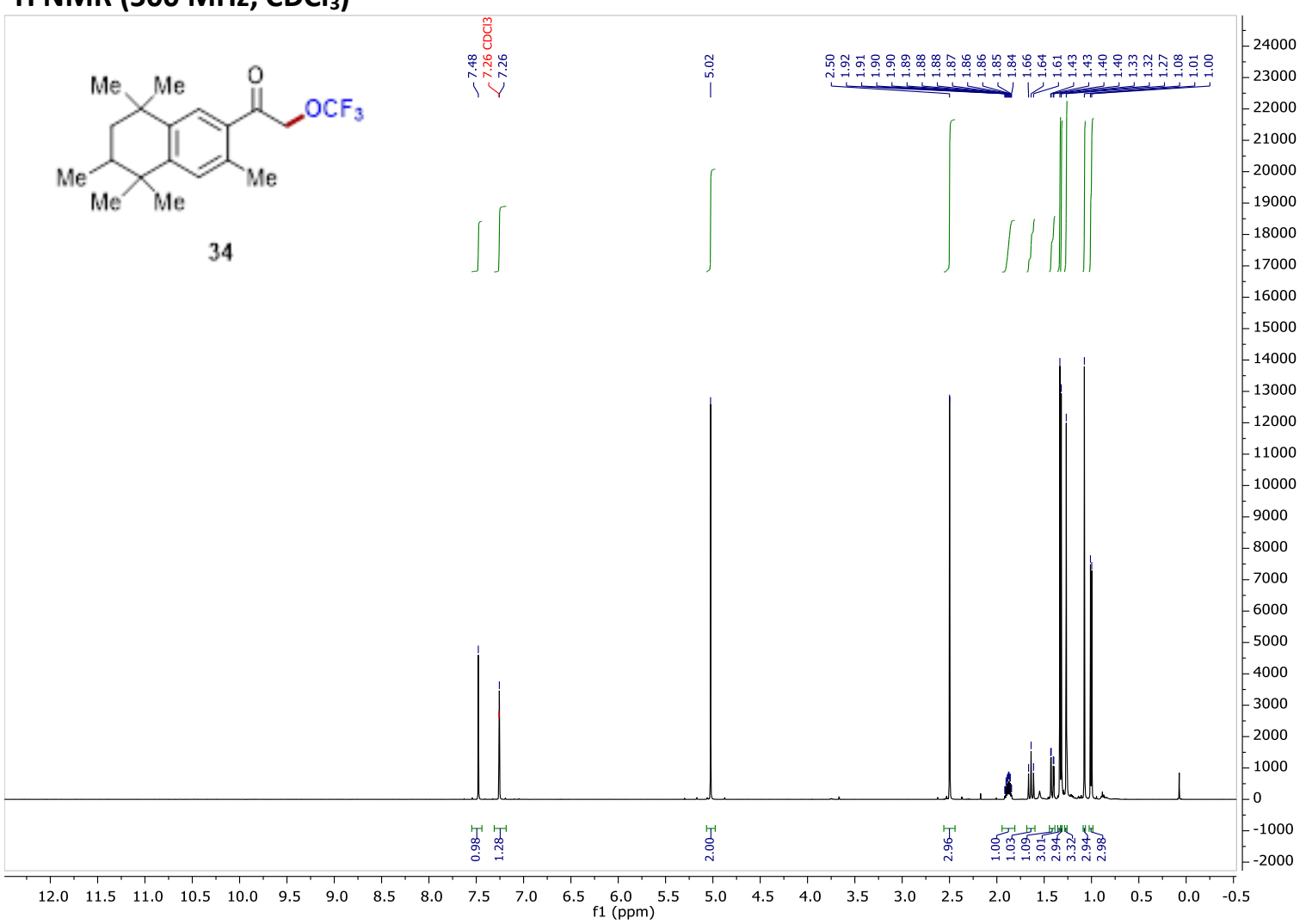


${ }^{13} \mathrm{C}$ NMR (126 MHz, $\mathrm{CDCl}_{3}$ )

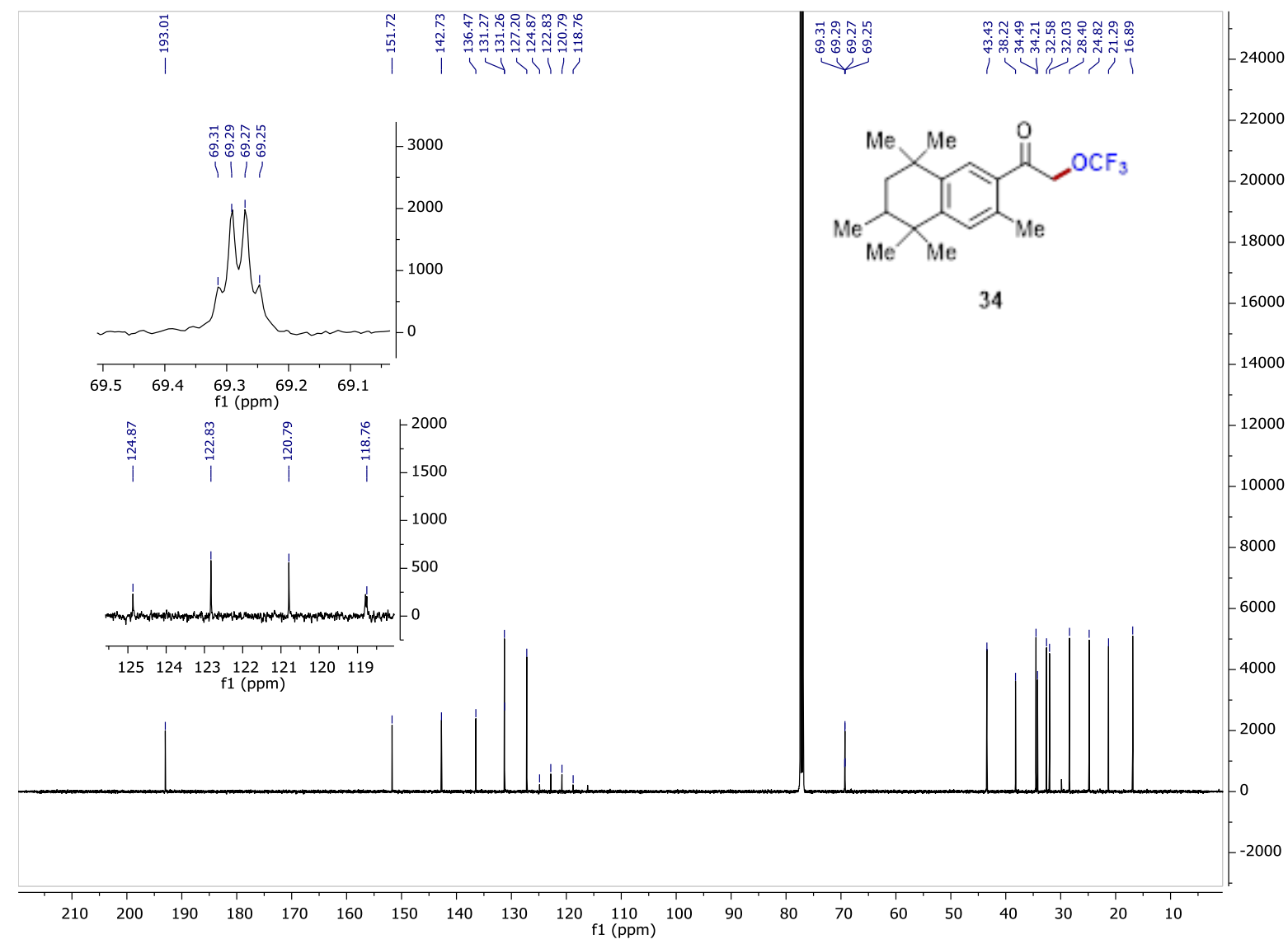

$\left.{ }^{19} \mathrm{~F} \mathrm{NMR} \mathrm{(188} \mathrm{MHz,} \mathrm{CDCl}_{3}\right)$

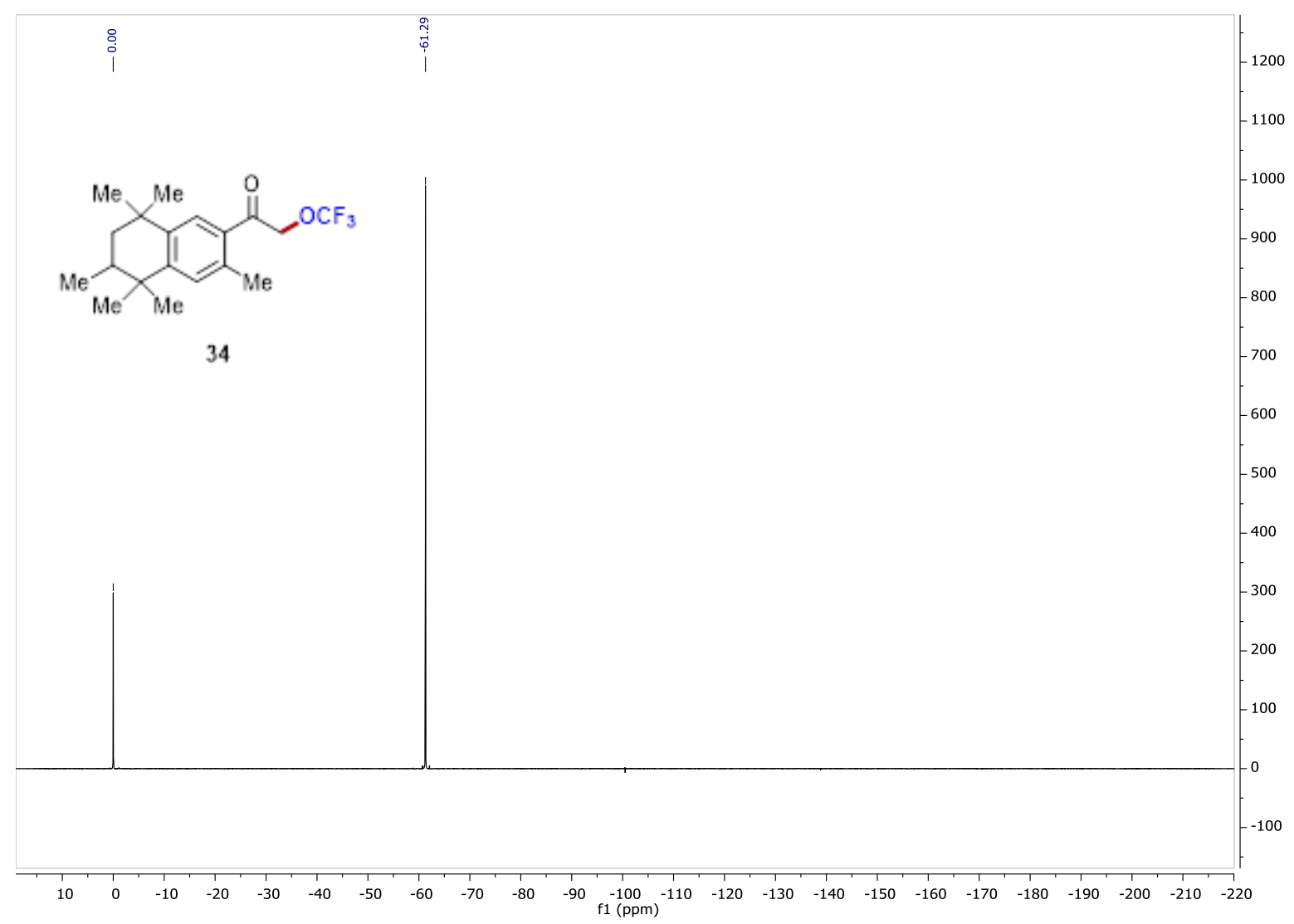


1-(4-(tert-butyl)-2,6-dimethyl-3,5-dinitrophenyl)-2-(trifluoromethoxy)ethanone 35

${ }^{1} \mathrm{H}$ NMR $\left(300 \mathrm{MHz}, \mathrm{CDCl}_{3}\right)$

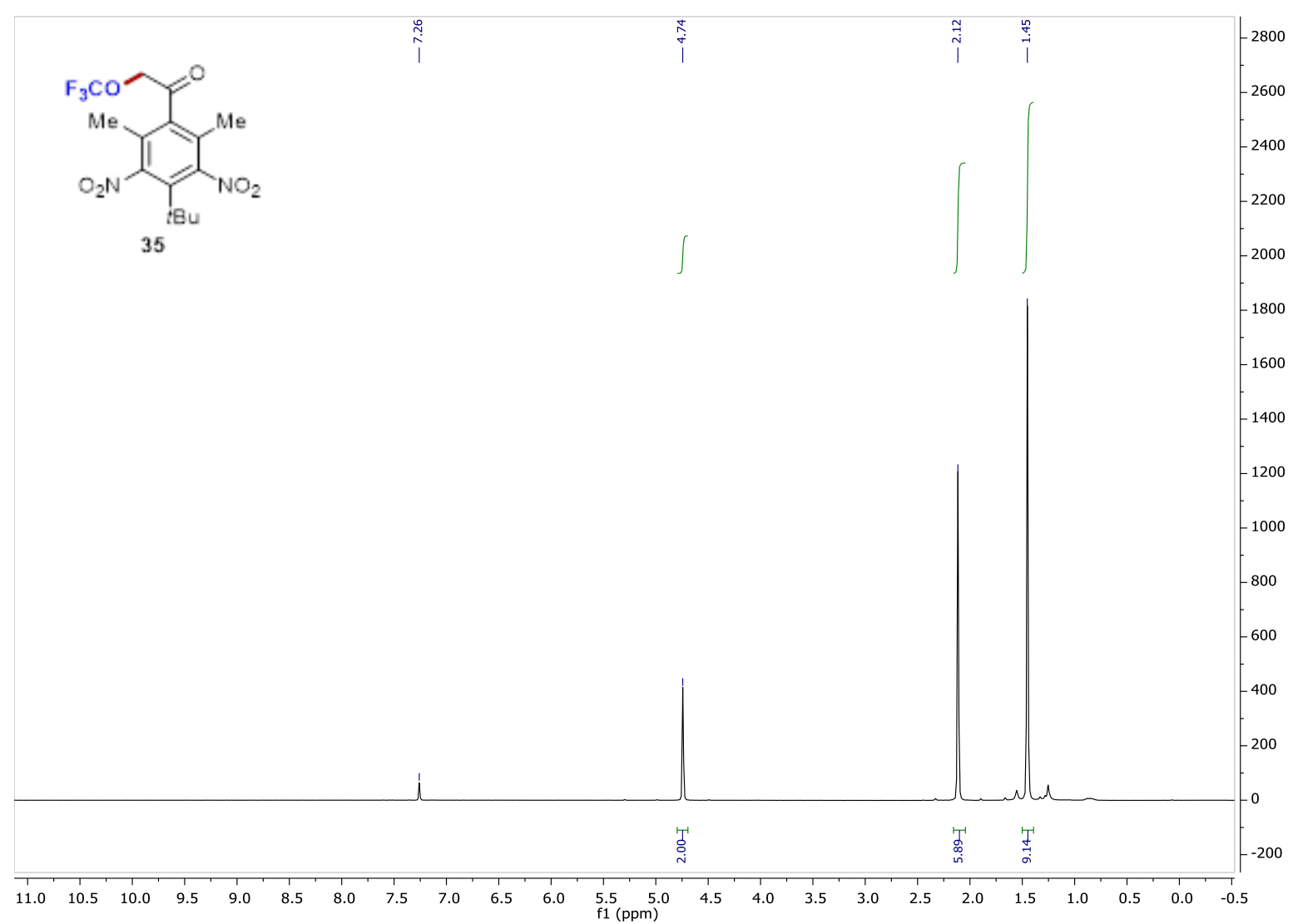

${ }^{13} \mathrm{C} \mathrm{NMR}\left(75 \mathrm{MHz}, \mathrm{CDCl}_{3}\right)$

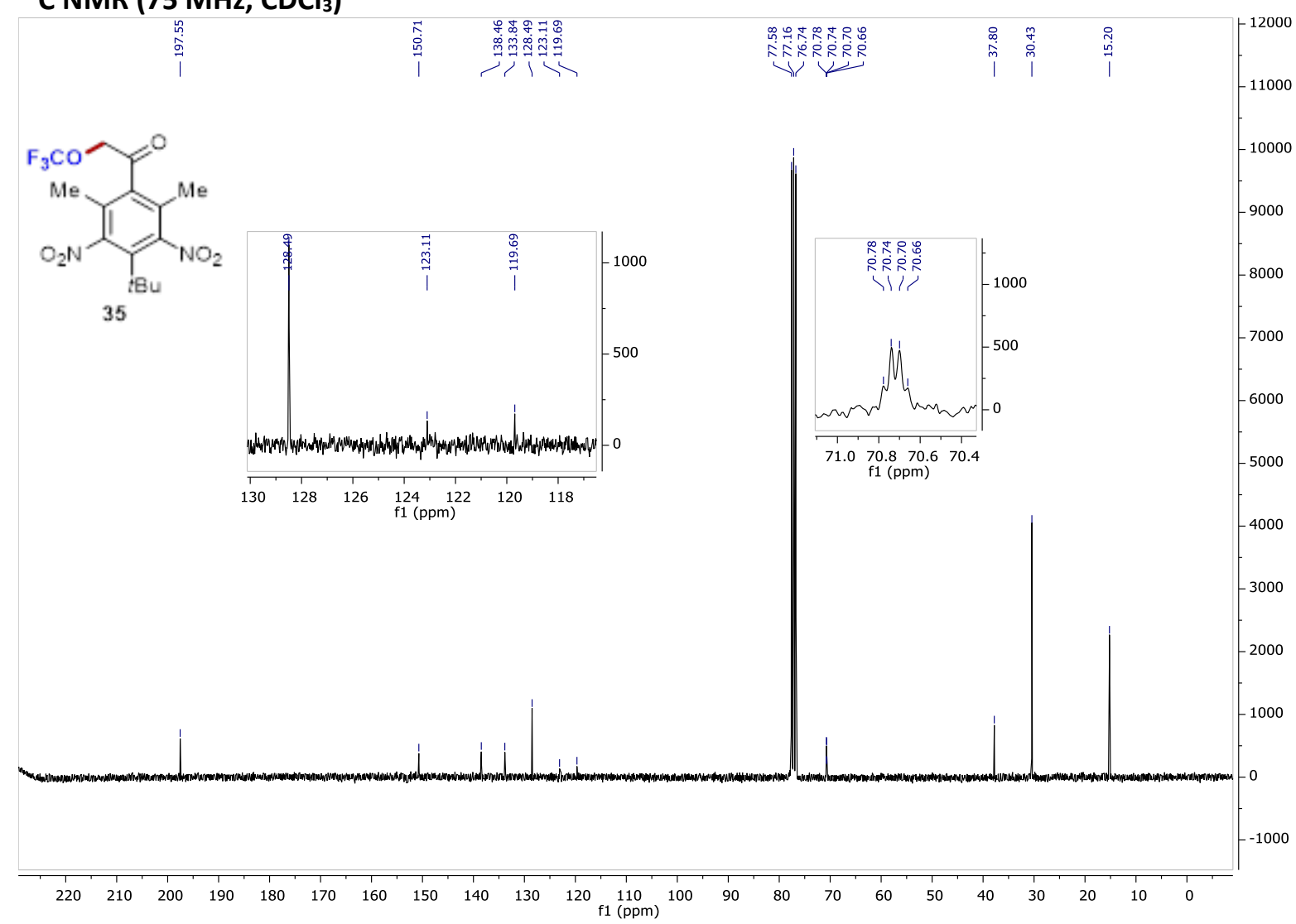


${ }^{19} \mathrm{~F}$ NMR (188 MHz, $\left.\mathrm{CDCl}_{3}\right)$

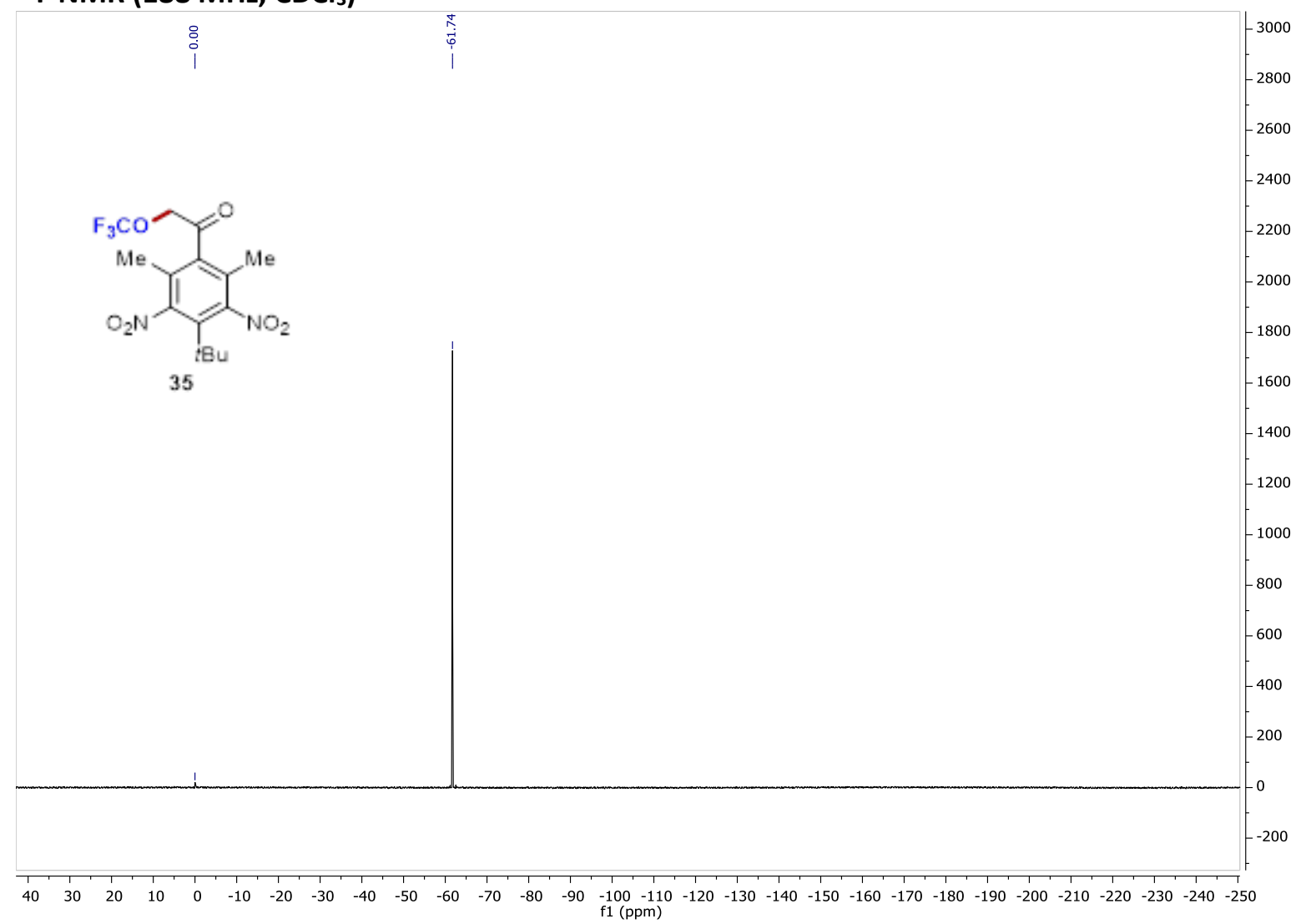

1-(6-(tert-butyl)-1,1-dimethyl-2,3-dihydro-1H-inden-4-yl)-2-(trifluoromethoxy)ethanone 36

${ }^{1} \mathrm{H}$ NMR $\left(300 \mathrm{MHz}, \mathrm{CDCl}_{3}\right)$

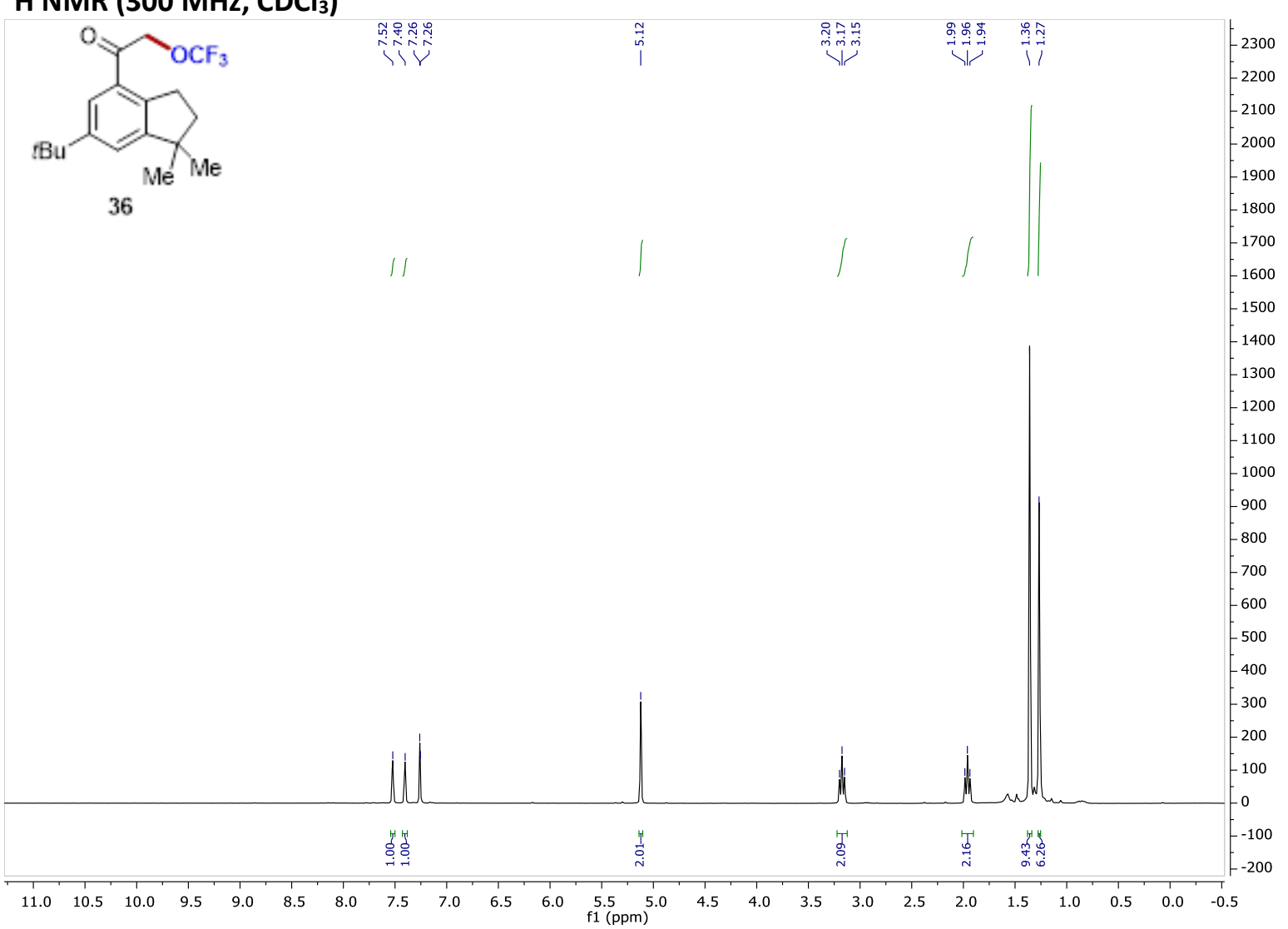


${ }^{13} \mathrm{C}$ NMR (75 MHz, $\mathrm{CDCl}_{3}$ )

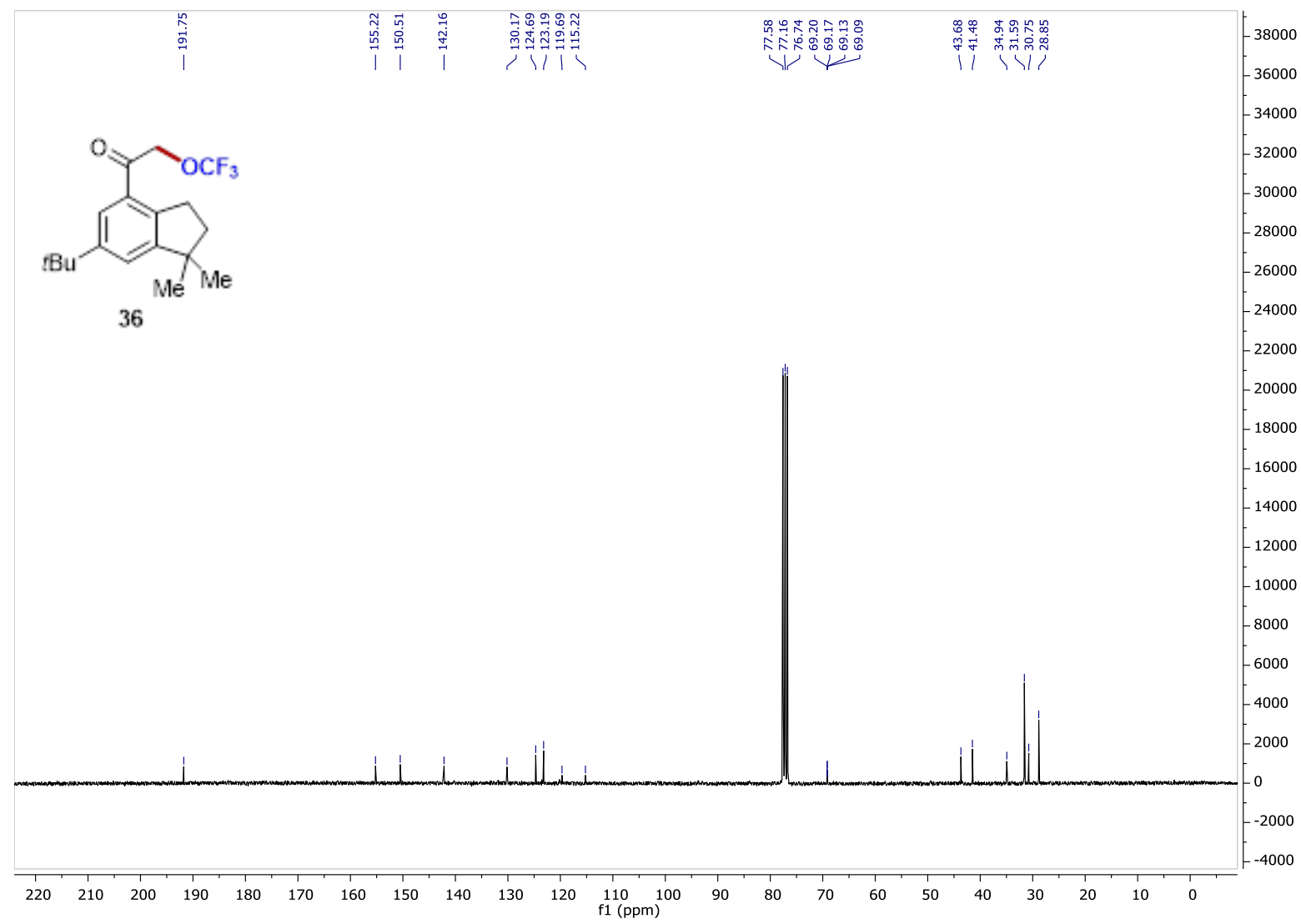

${ }^{19} \mathrm{~F}$ NMR (188 MHz, $\left.\mathrm{CDCl}_{3}\right)$

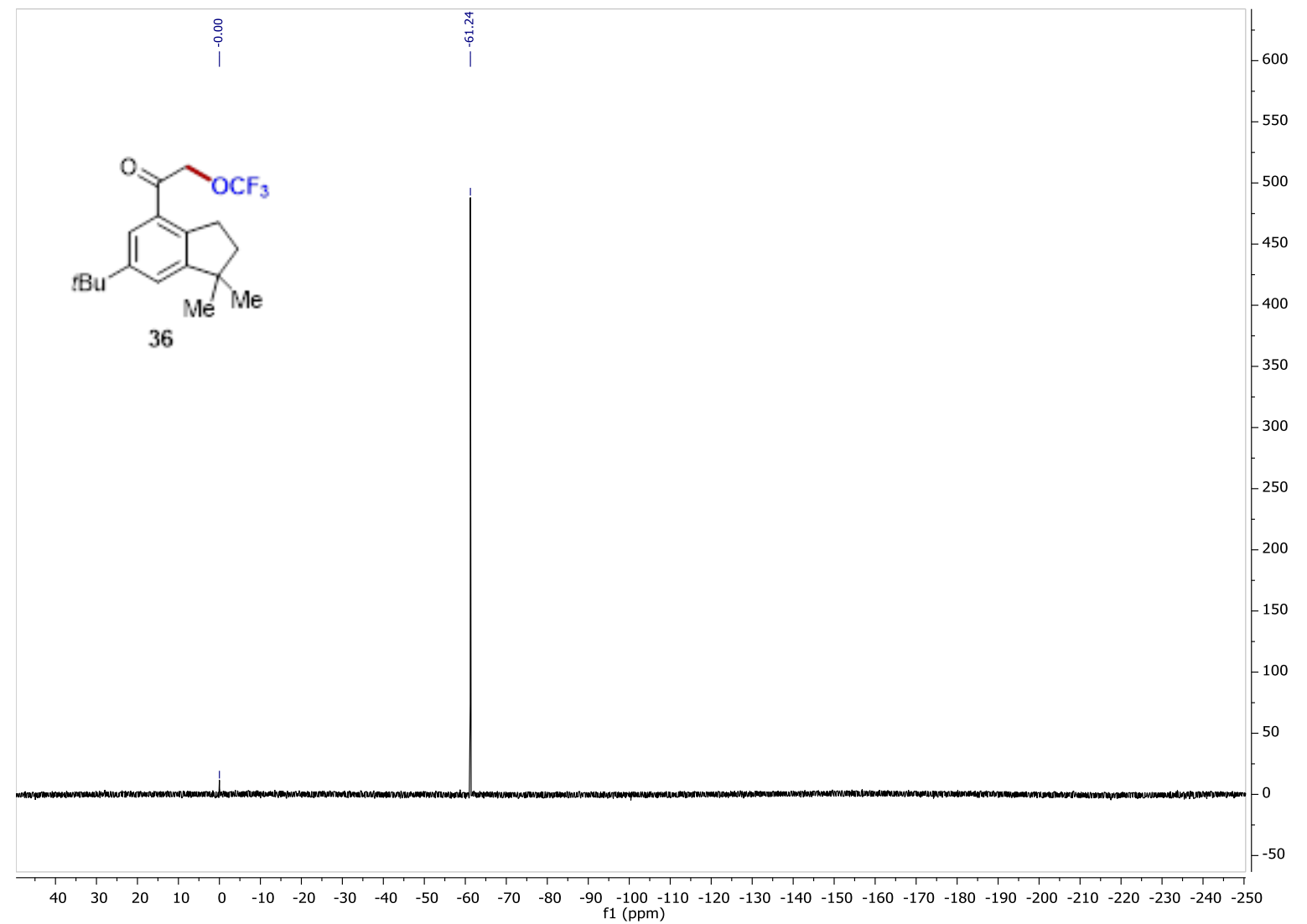


(E)-1-(trifluoromethoxy)-4-(2,6,6-trimethylcyclohex-2-en-1-yl)but-3-en-2-one 37

${ }^{1} \mathrm{H}$ NMR (200 MHz, $\mathrm{CDCl}_{3}$ )

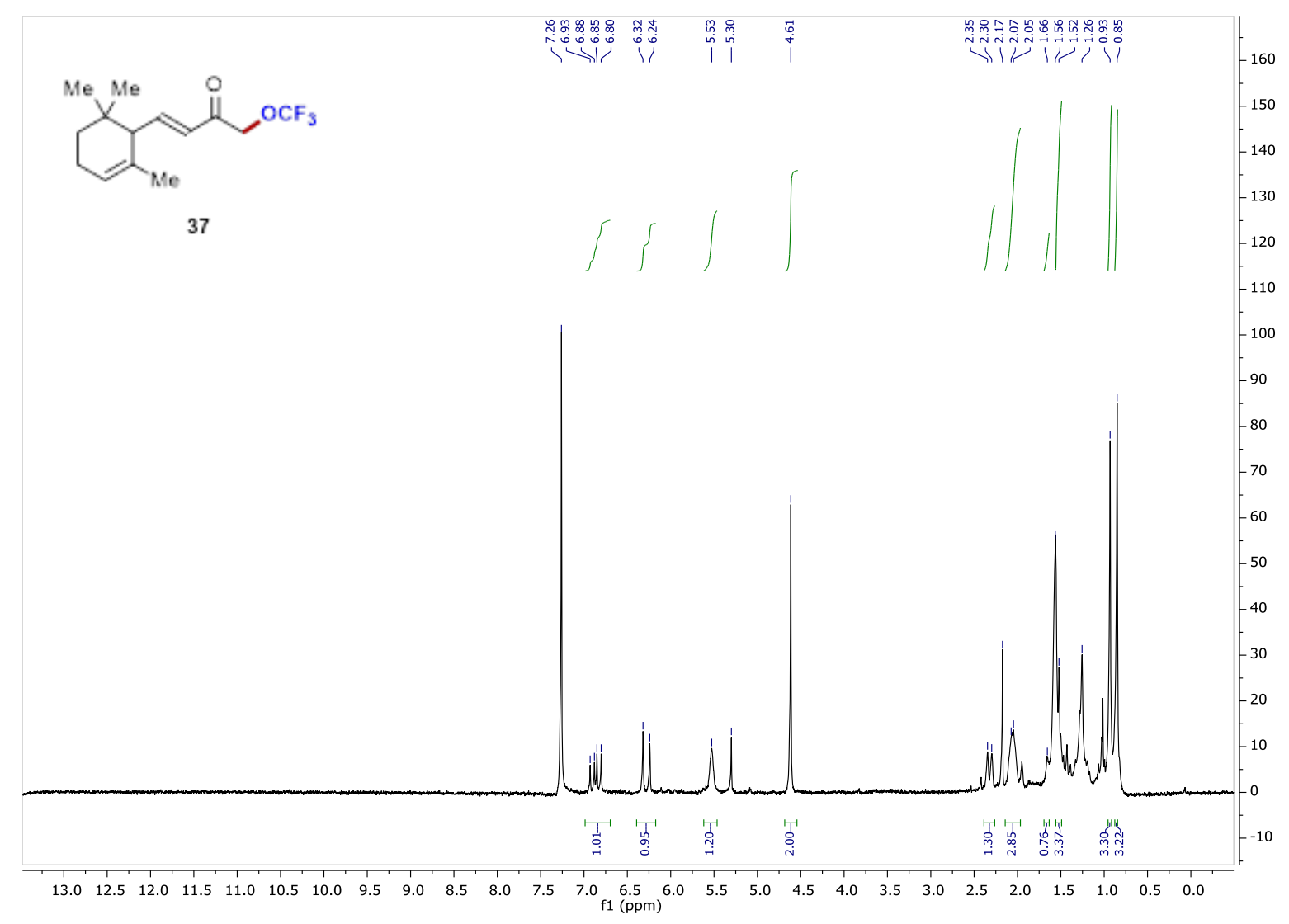

${ }^{13} \mathrm{C}$ NMR (151 MHz, $\left.\mathrm{CDCl}_{3}\right)$

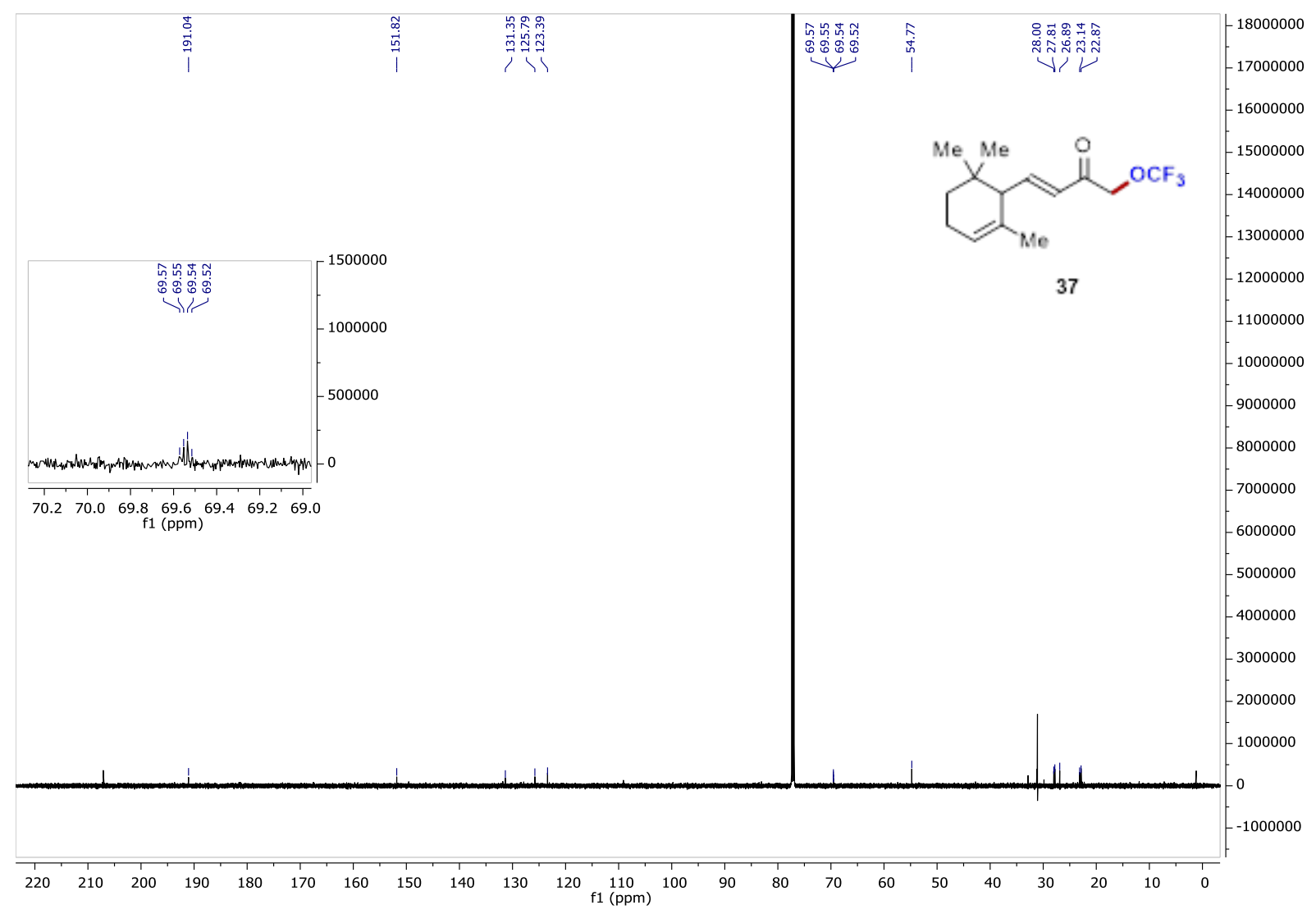


${ }^{19} \mathrm{~F}$ NMR (188 MHz, $\left.\mathrm{CDCl}_{3}\right)$

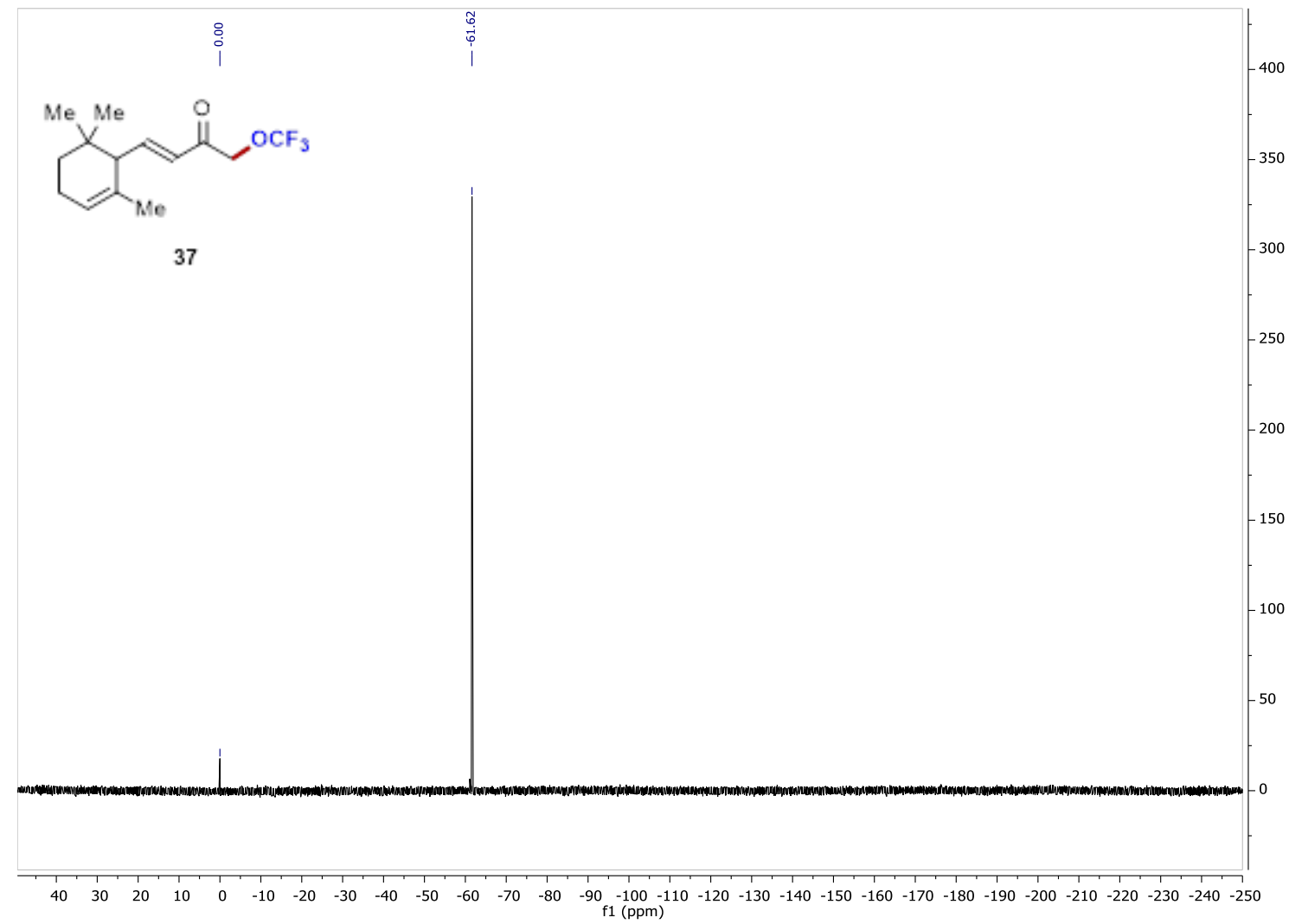

(8R,9S,10R,13S,14S,17S)-17-methoxy-10,13-dimethyl-6-(trifluoromethoxy)$6,7,8,9,10,11,12,13,14,15,16,17$-dodecahydro-1H-cyclopenta[a]phenanthren-3(2H)-one 39 ${ }^{1} \mathrm{H}$ NMR (600 MHz, $\mathrm{CDCl}_{3}$ )

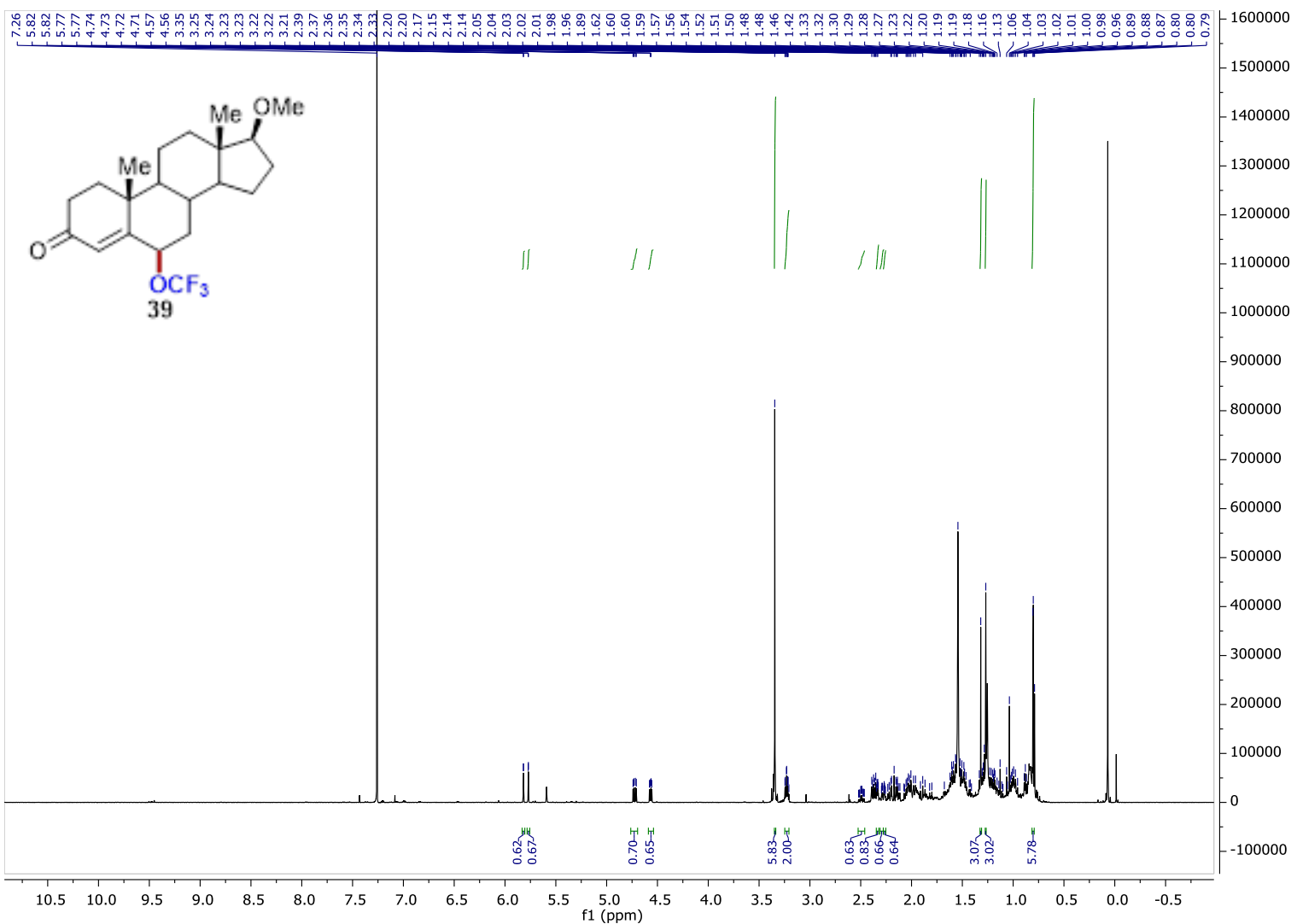




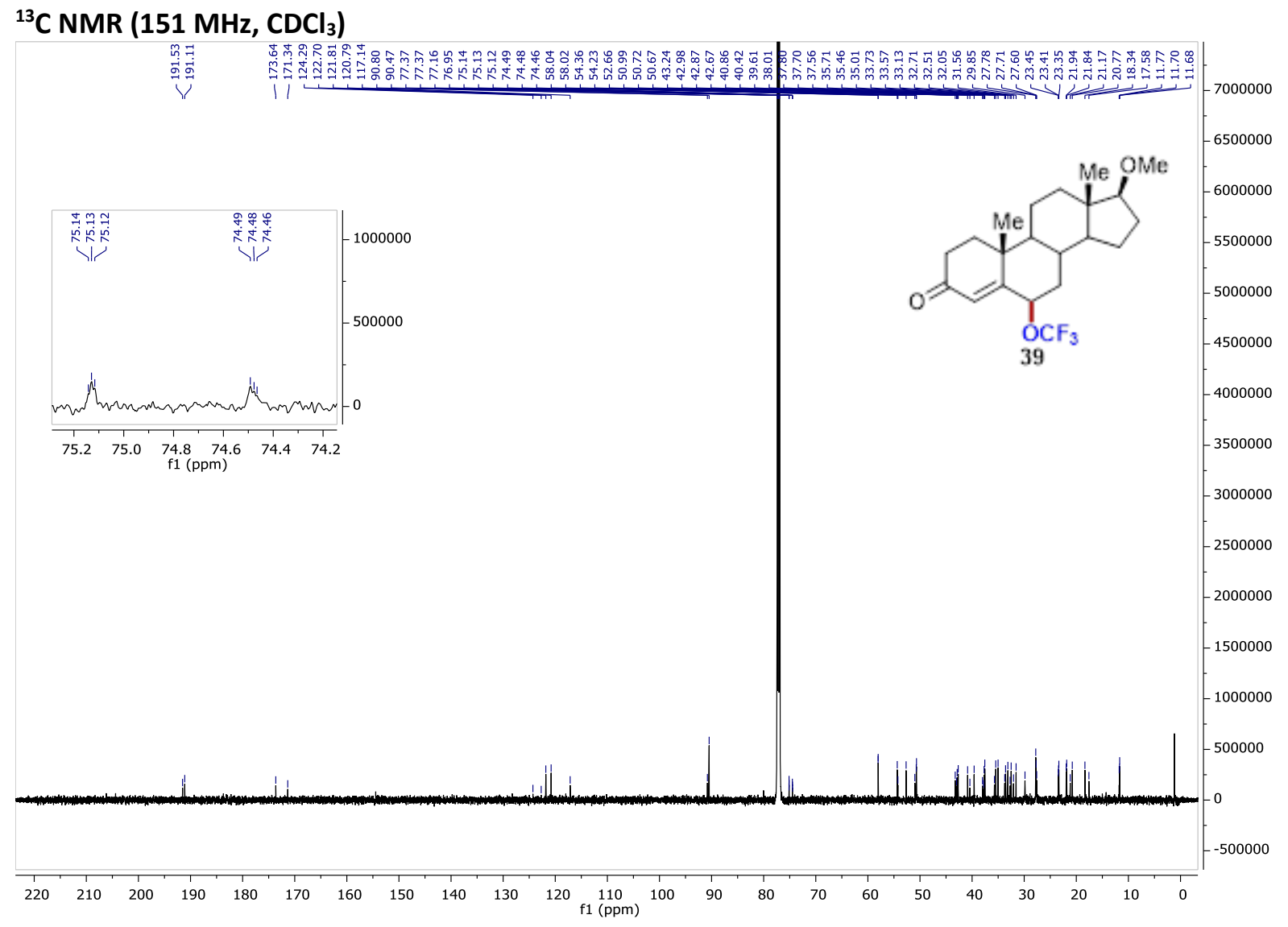

${ }^{19} \mathrm{~F}$ NMR (188 MHz, $\left.\mathrm{CDCl}_{3}\right)$

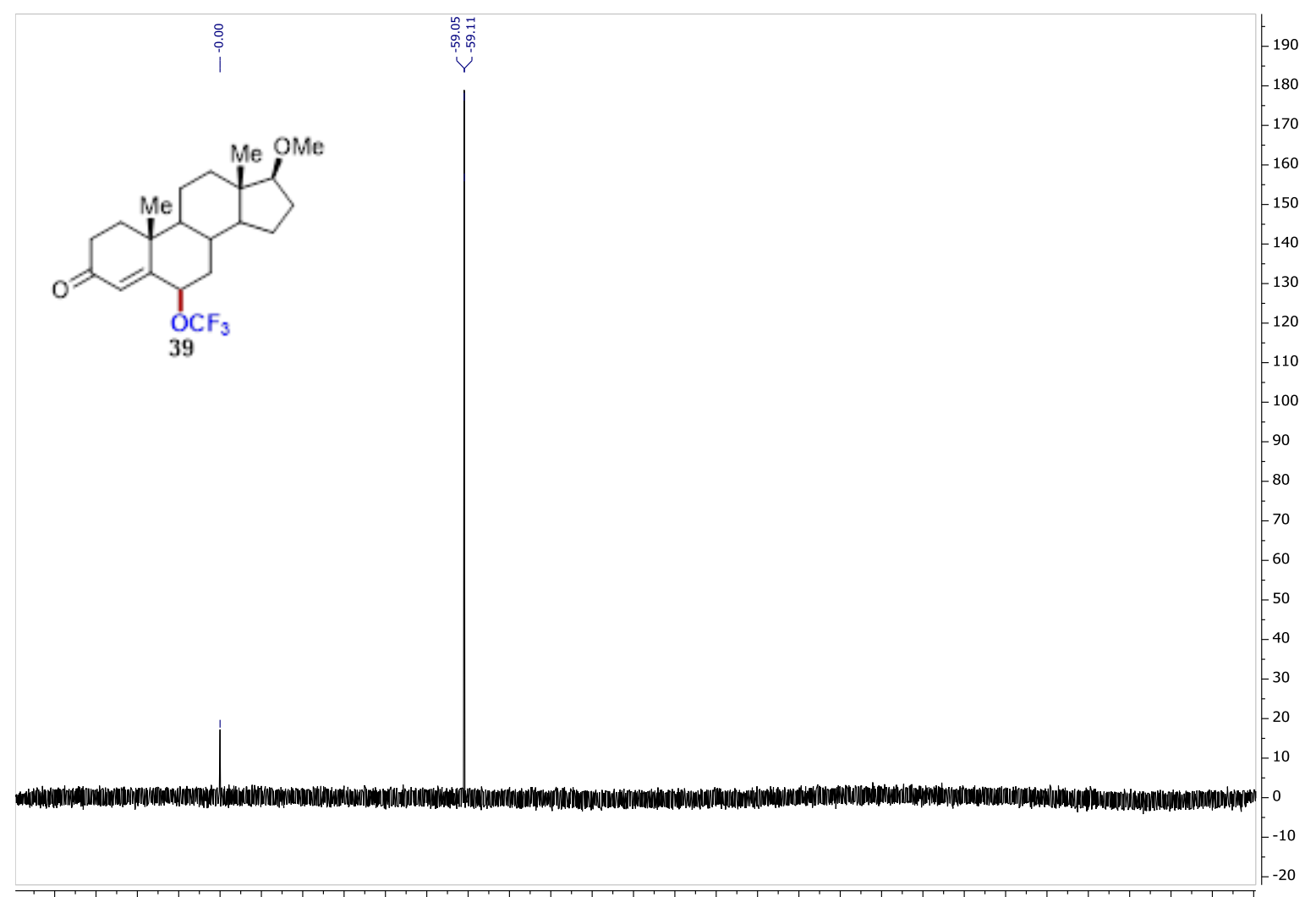

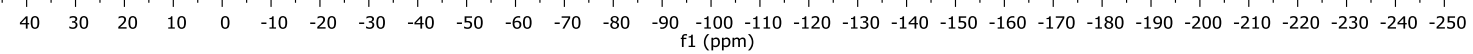




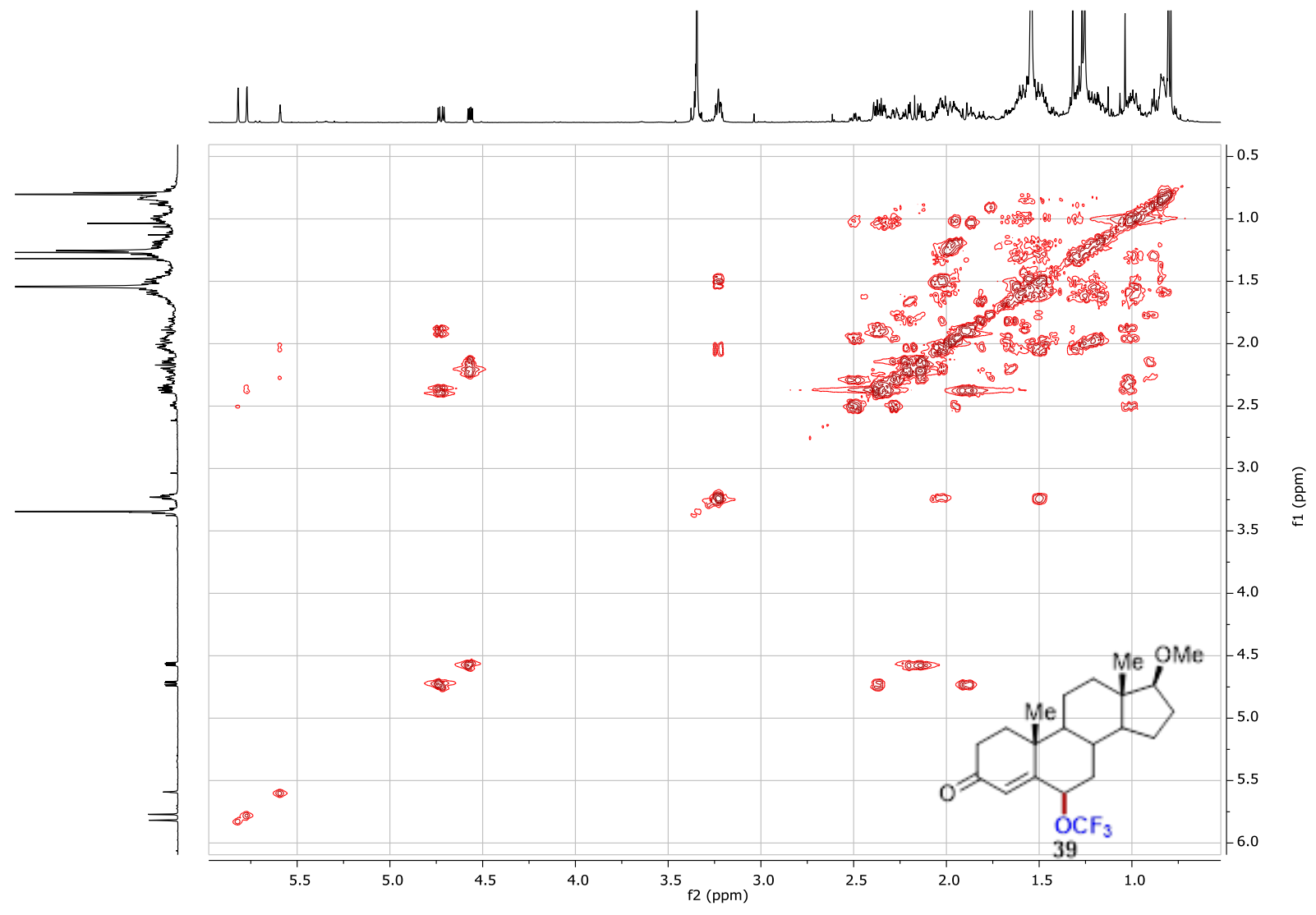

HSQC (600 MHz, $\left.\mathrm{CDCl}_{3}\right)$

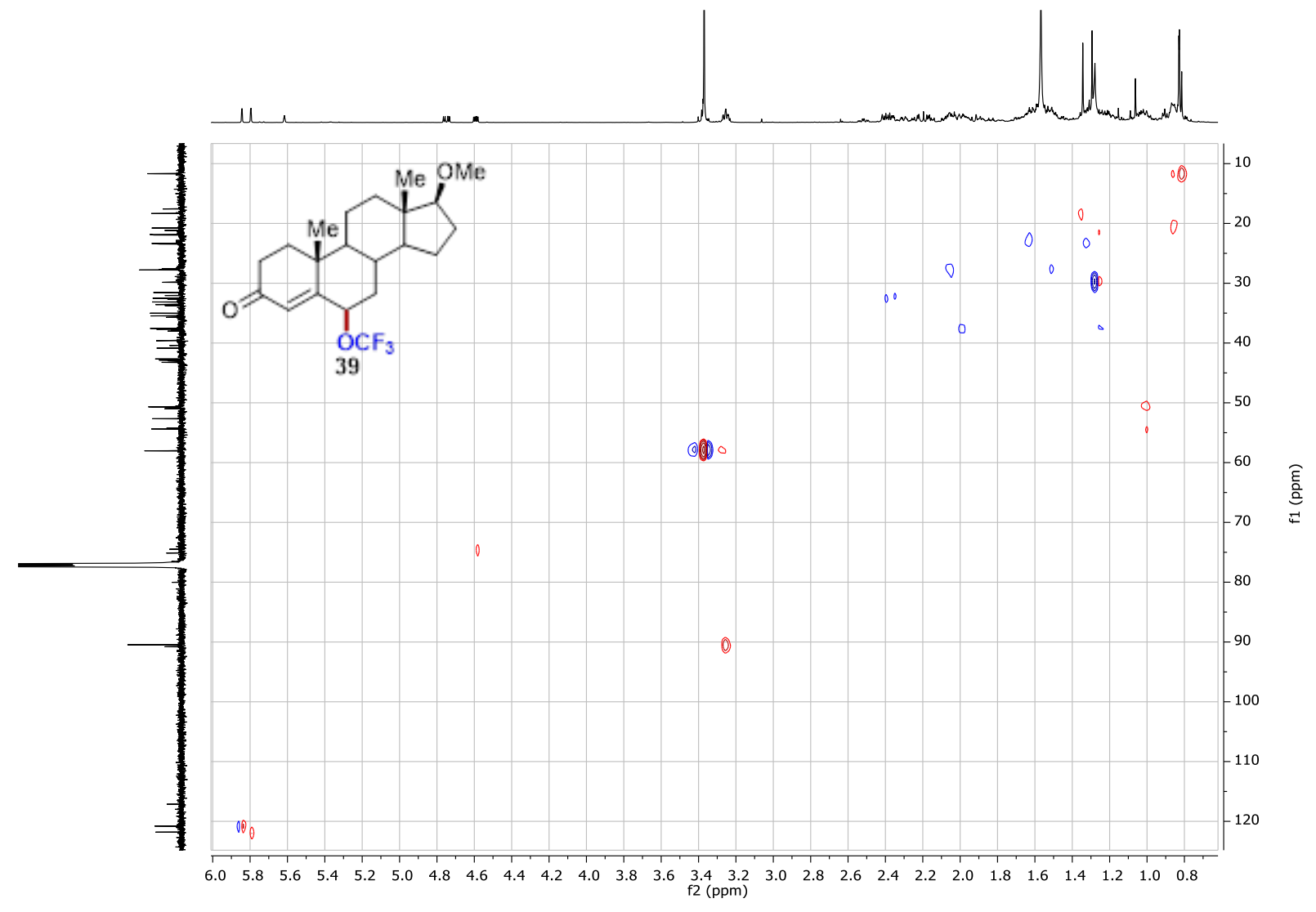


1-(4-bromophenyl)-2-(trifluoromethoxy)ethan-1-ol 40

${ }^{1} \mathrm{H}$ NMR $\left(500 \mathrm{MHz}, \mathrm{CDCl}_{3}\right)$<smiles>OC(COC(F)(F)F)c1ccc(Br)cc1</smiles>

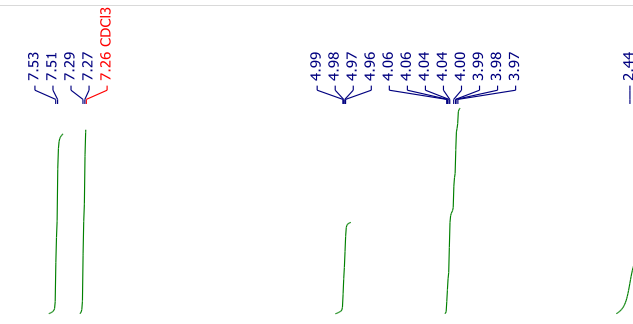

$-5000$

4500

4000

3500

3000

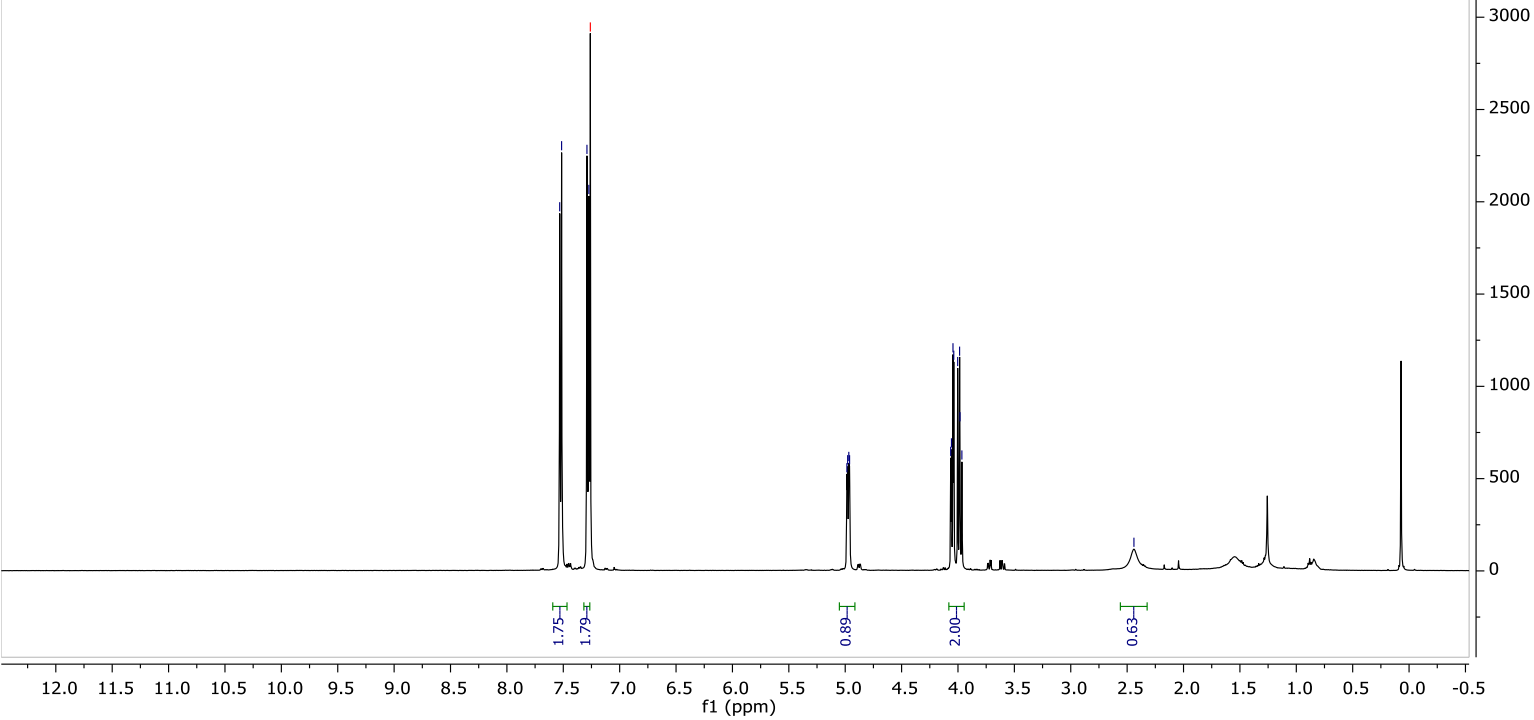

${ }^{13} \mathrm{C}$ NMR (126 MHz, $\left.\mathrm{CDCl}_{3}\right)$

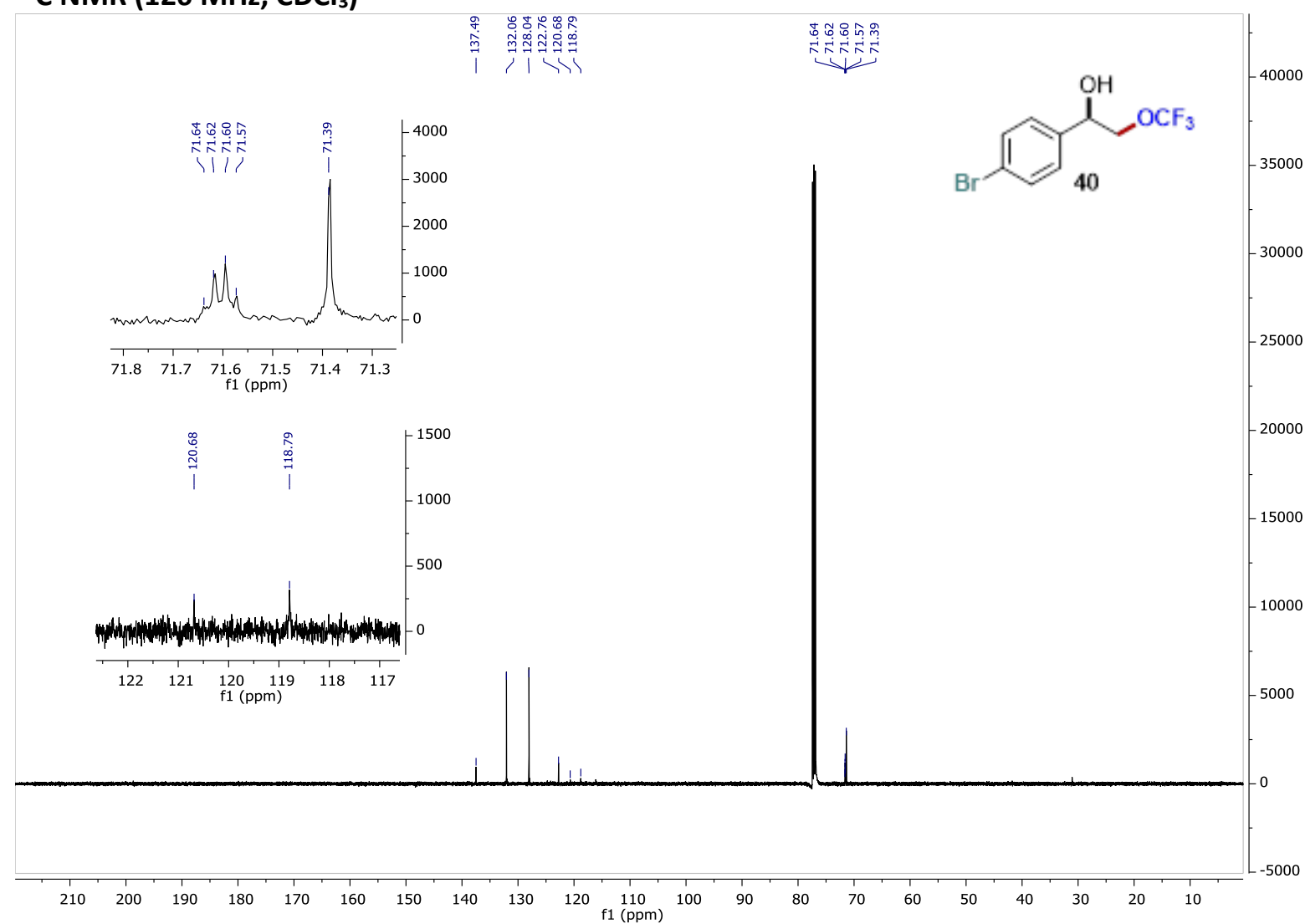




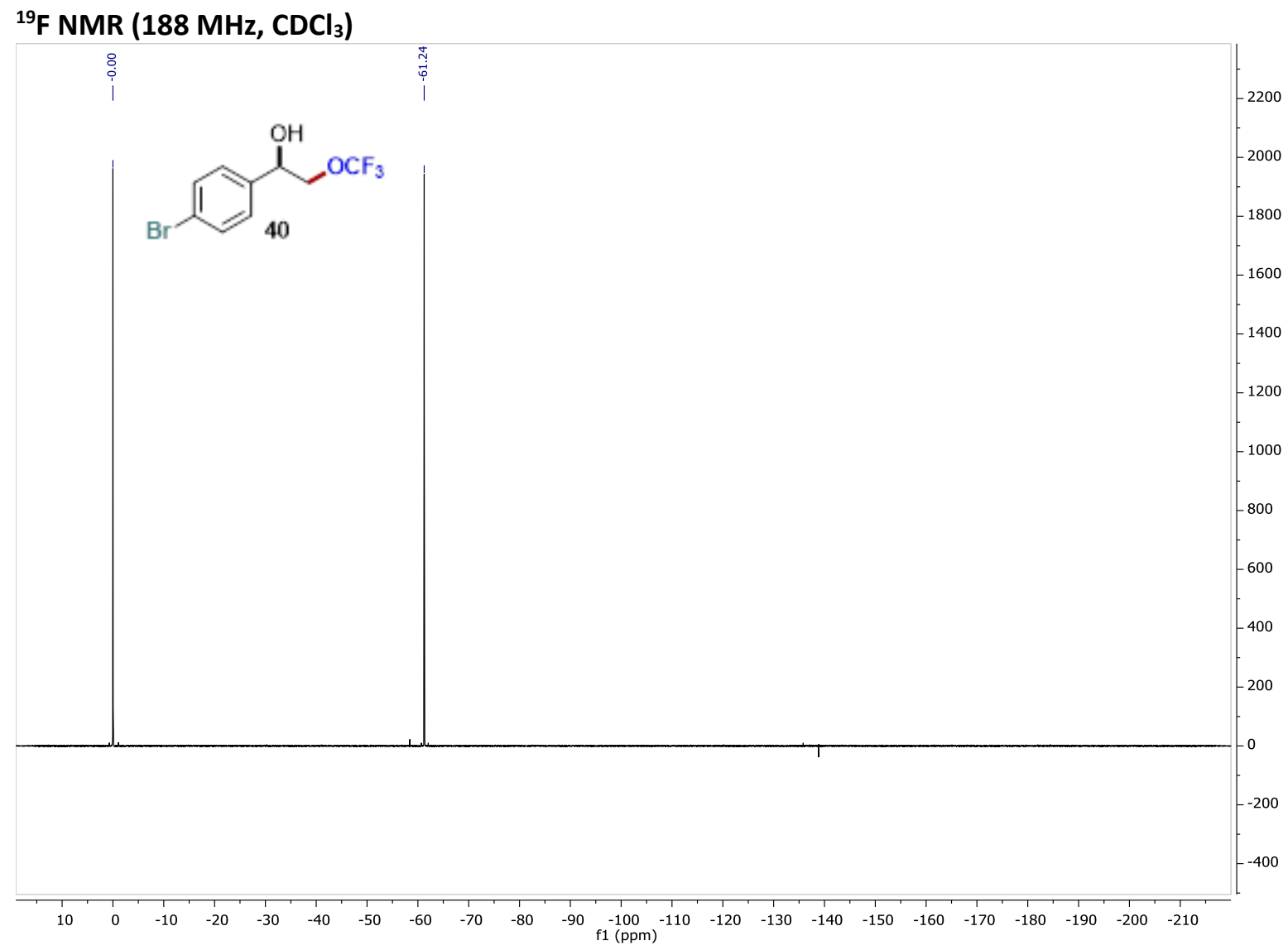

1-(4-morpholinophenyl)-2-(trifluoromethoxy)ethan-1-ol 41

${ }^{1} \mathrm{H}$ NMR (500 MHz, $\mathrm{CDCl}_{3}$ )

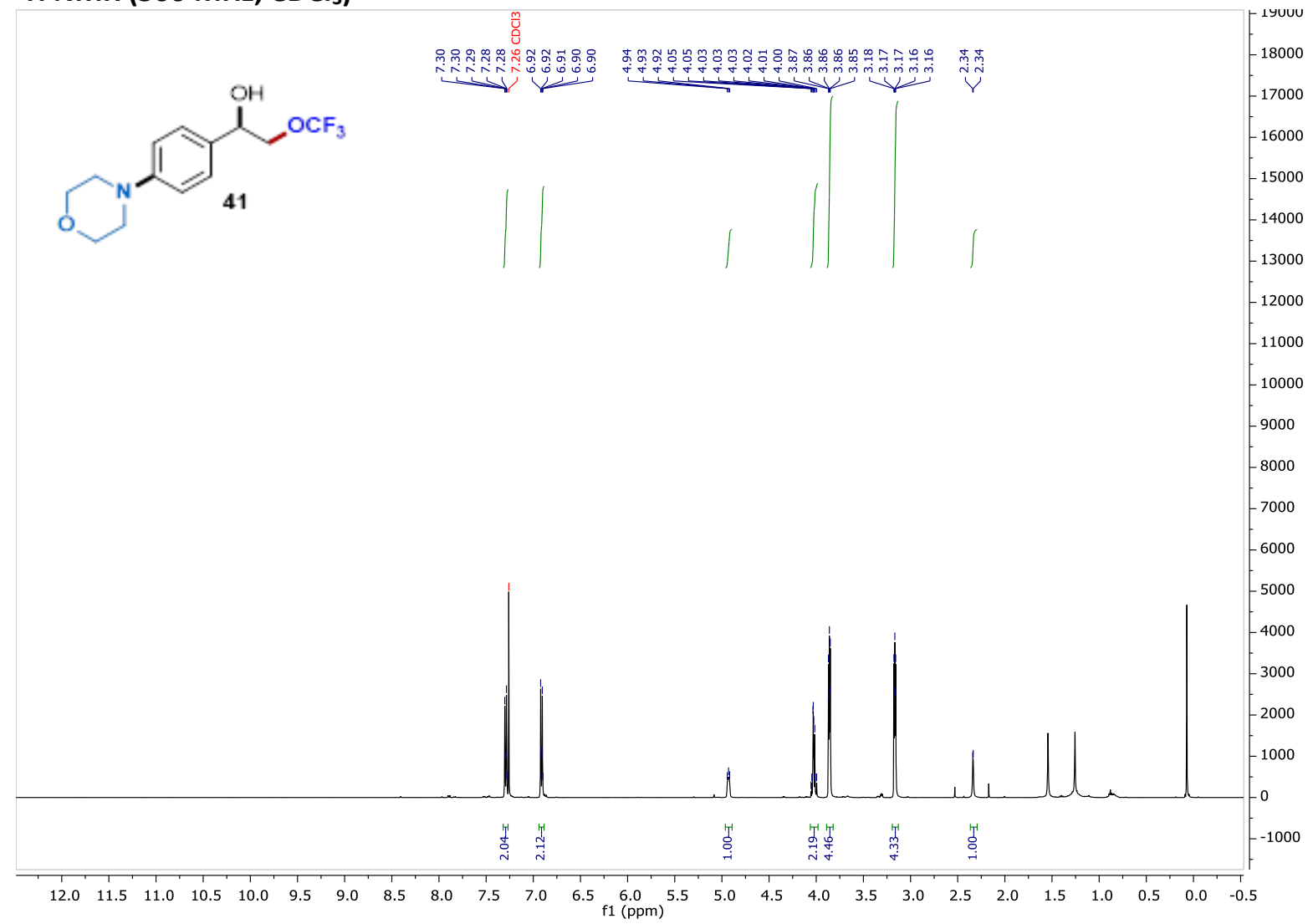




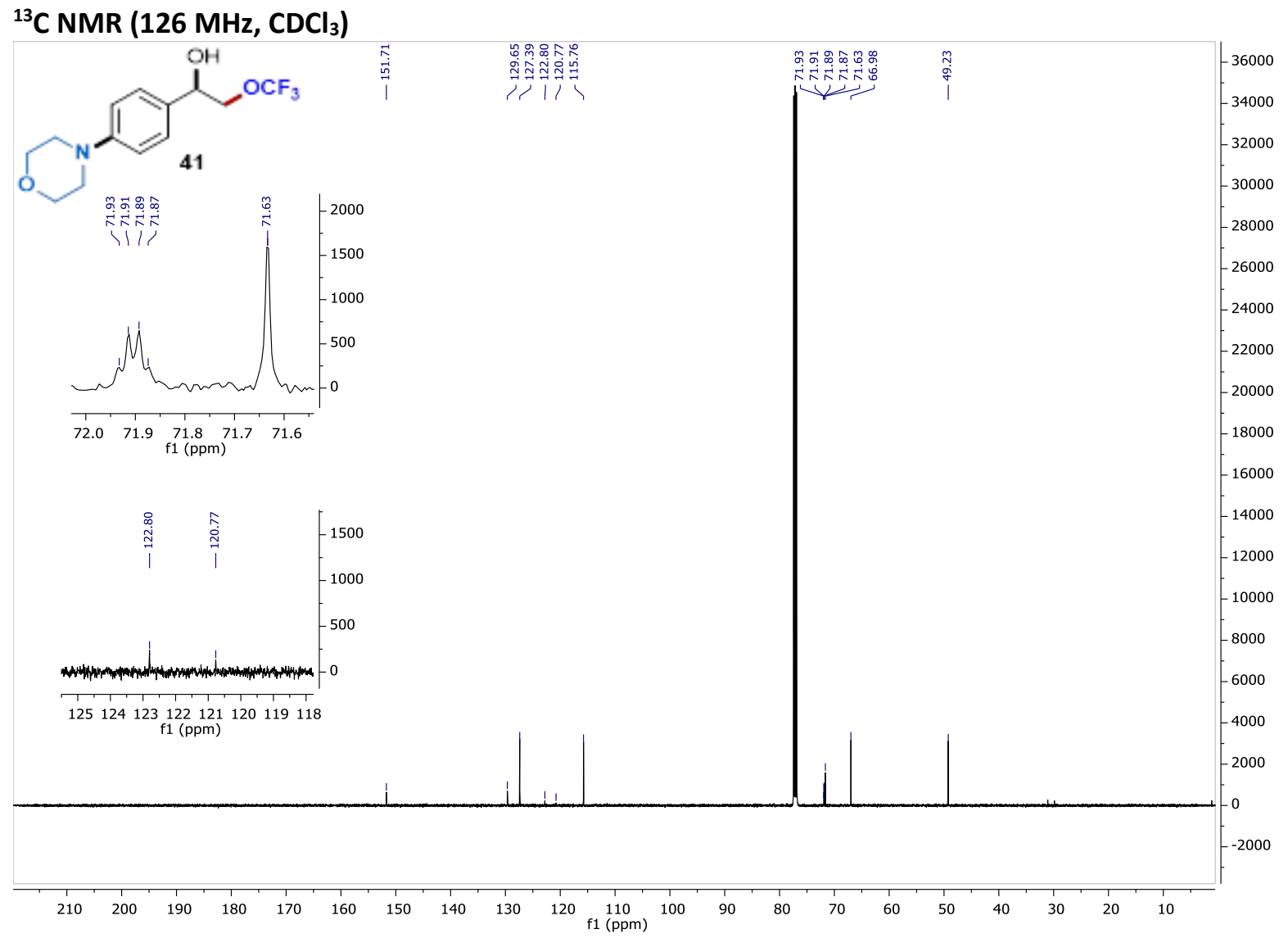

${ }^{19} \mathrm{~F}$ NMR (188 MHz, $\left.\mathrm{CDCl}_{3}\right)$

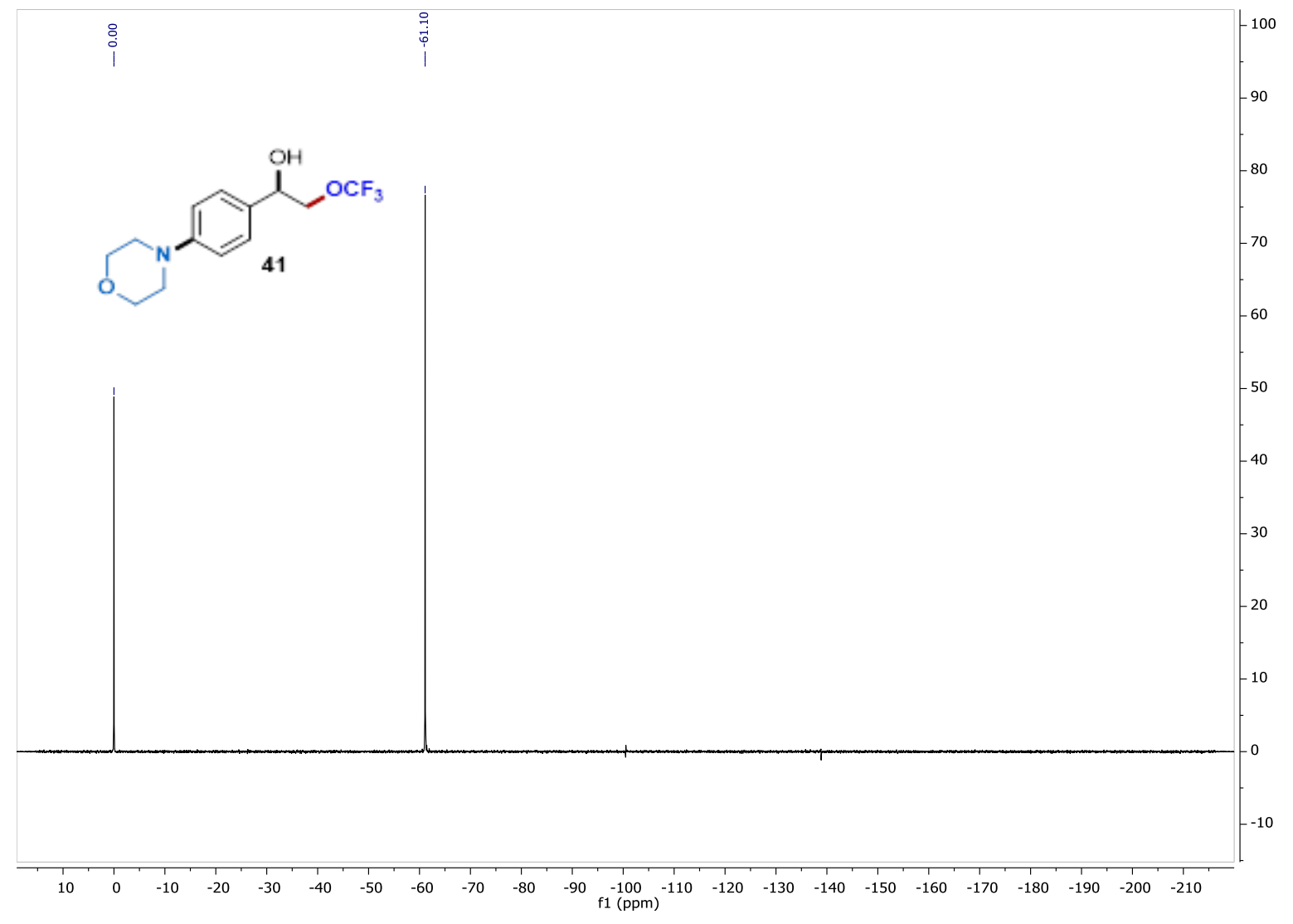


1-(4-bromophenyl)-2-(trifluoromethoxy)ethan-1-aminium acetate 42

${ }^{1} \mathrm{H}$ NMR (500 MHz, $\mathrm{CDCl}_{3}$ )

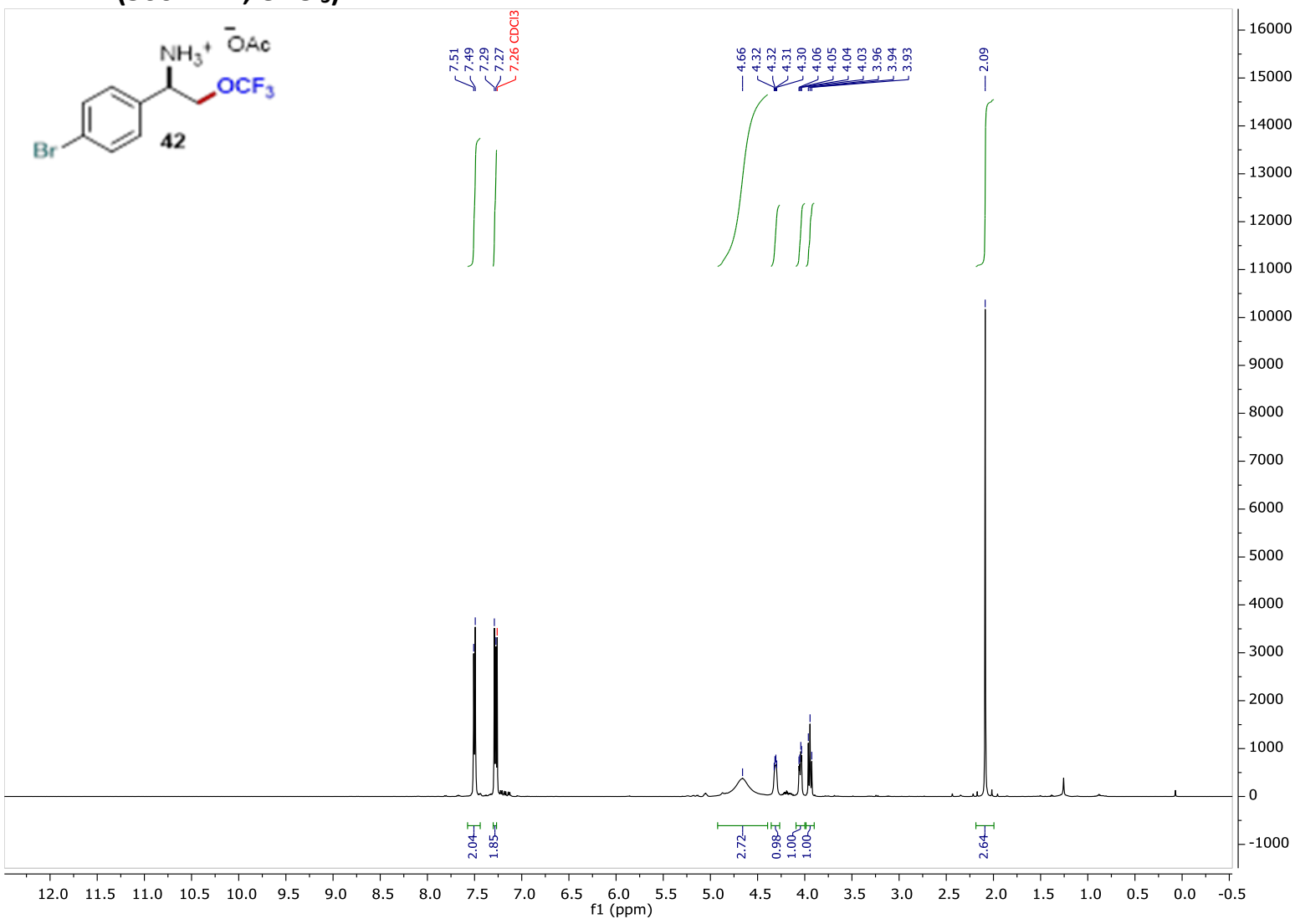

${ }^{13} \mathrm{C} \mathrm{NMR} \mathrm{(126} \mathrm{MHz,} \mathrm{CDCl}_{3}$ )

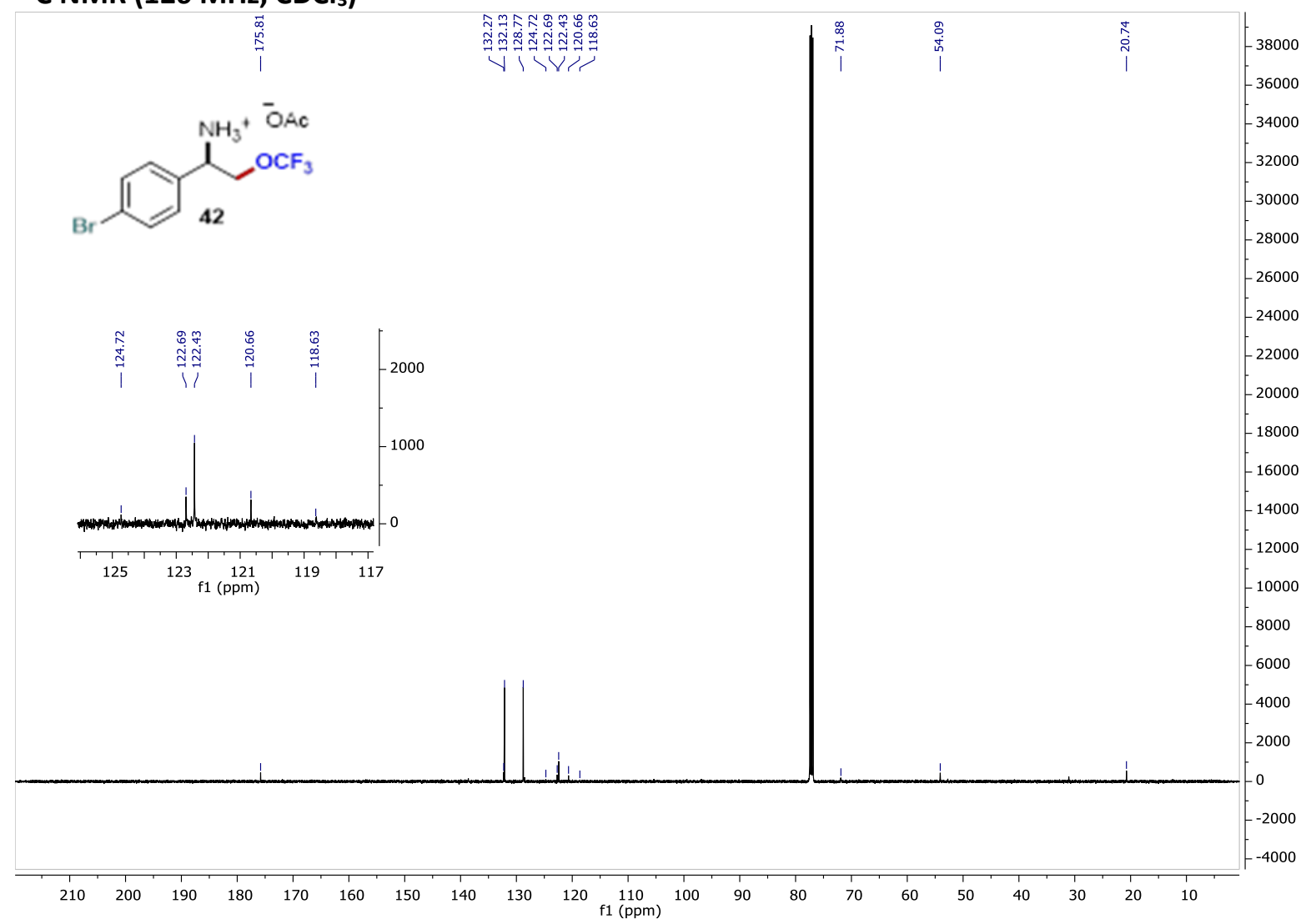


${ }^{19} \mathrm{~F}$ NMR (188 MHz, $\left.\mathrm{CDCl}_{3}\right)$

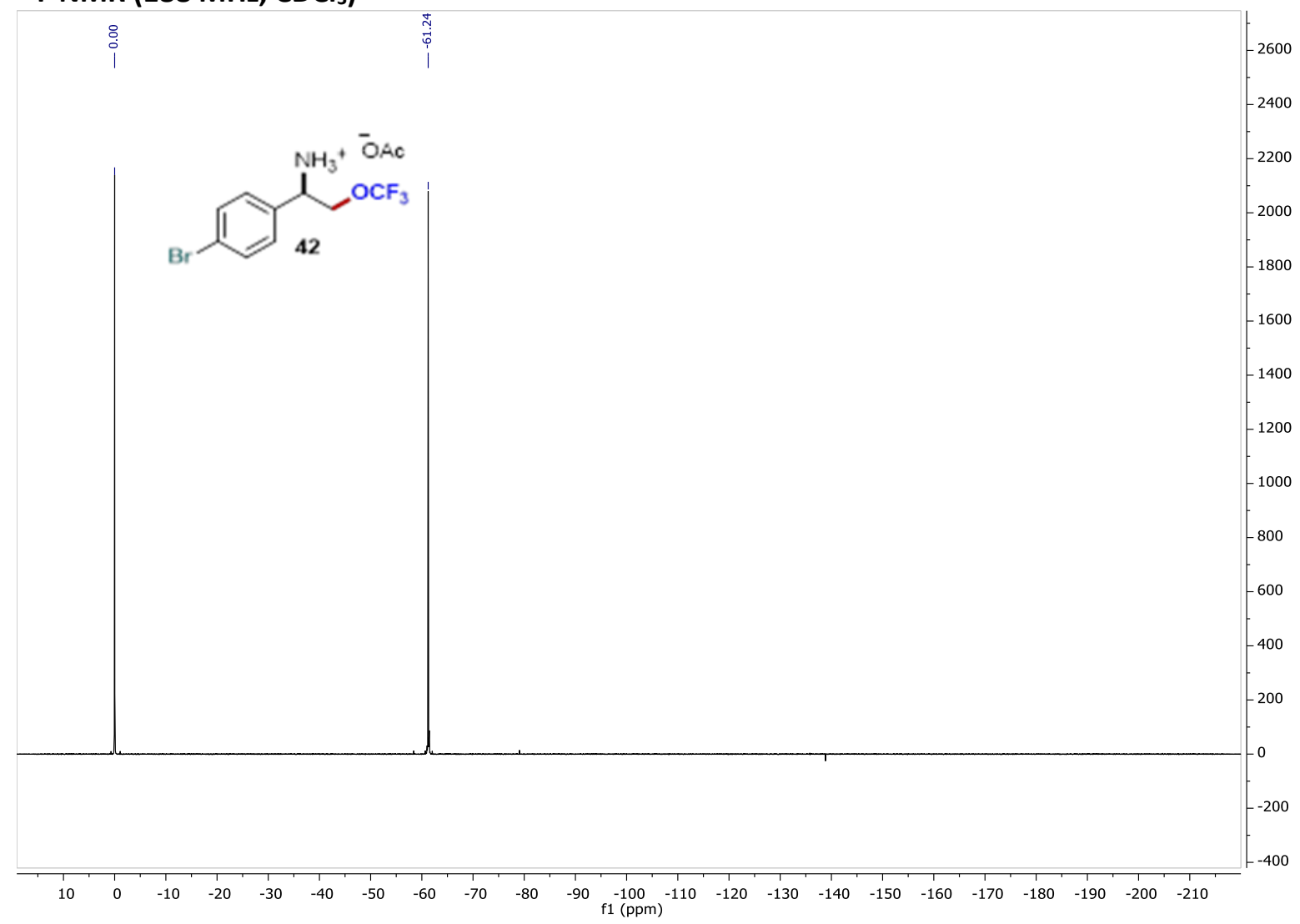

4-(3-(dimethylamino)-2-(trifluoromethoxy)acryloyl)benzonitrile 43

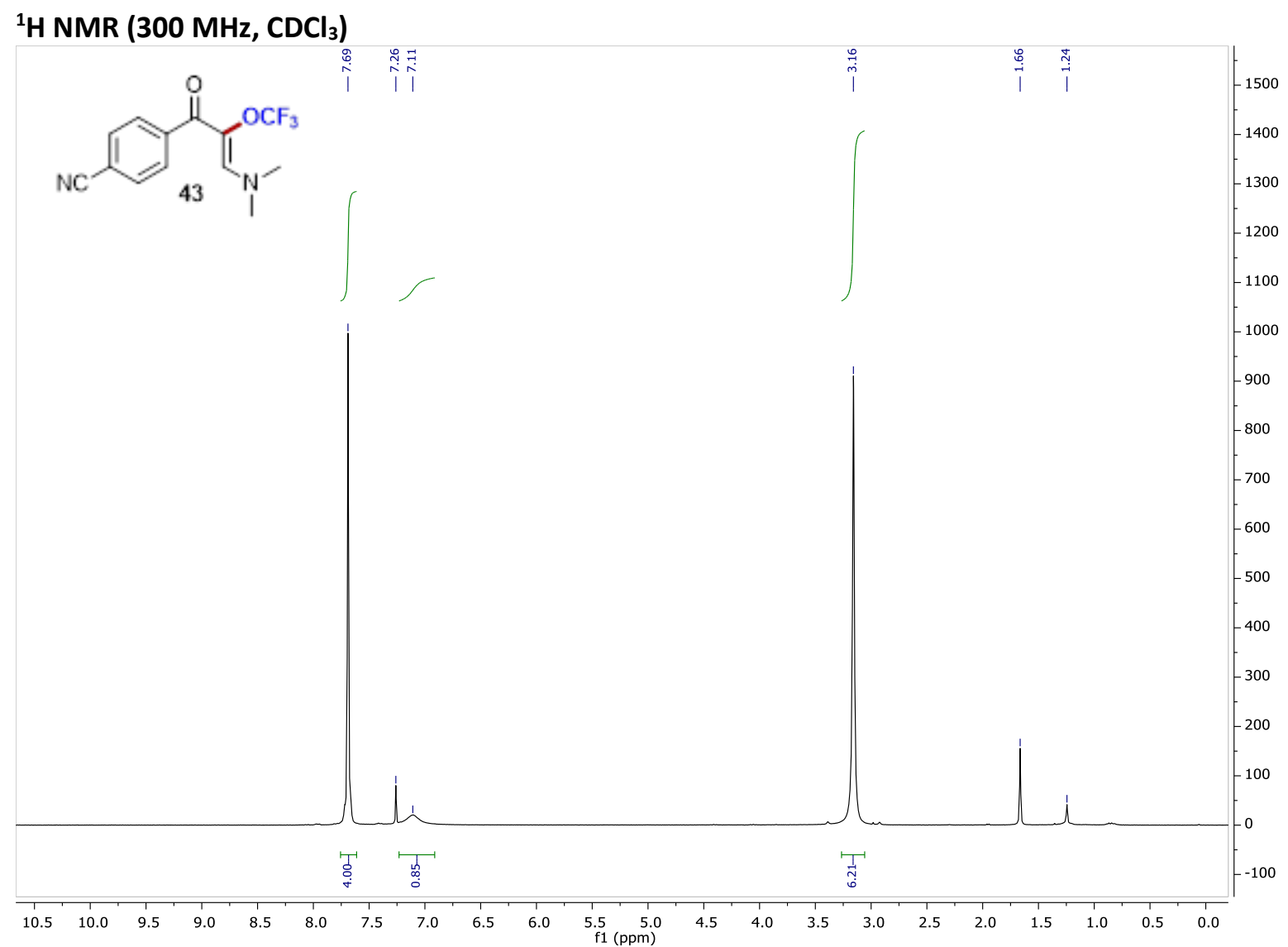


${ }^{13} \mathrm{C}$ NMR $\left(75 \mathrm{MHz}, \mathrm{CDCl}_{3}\right)$

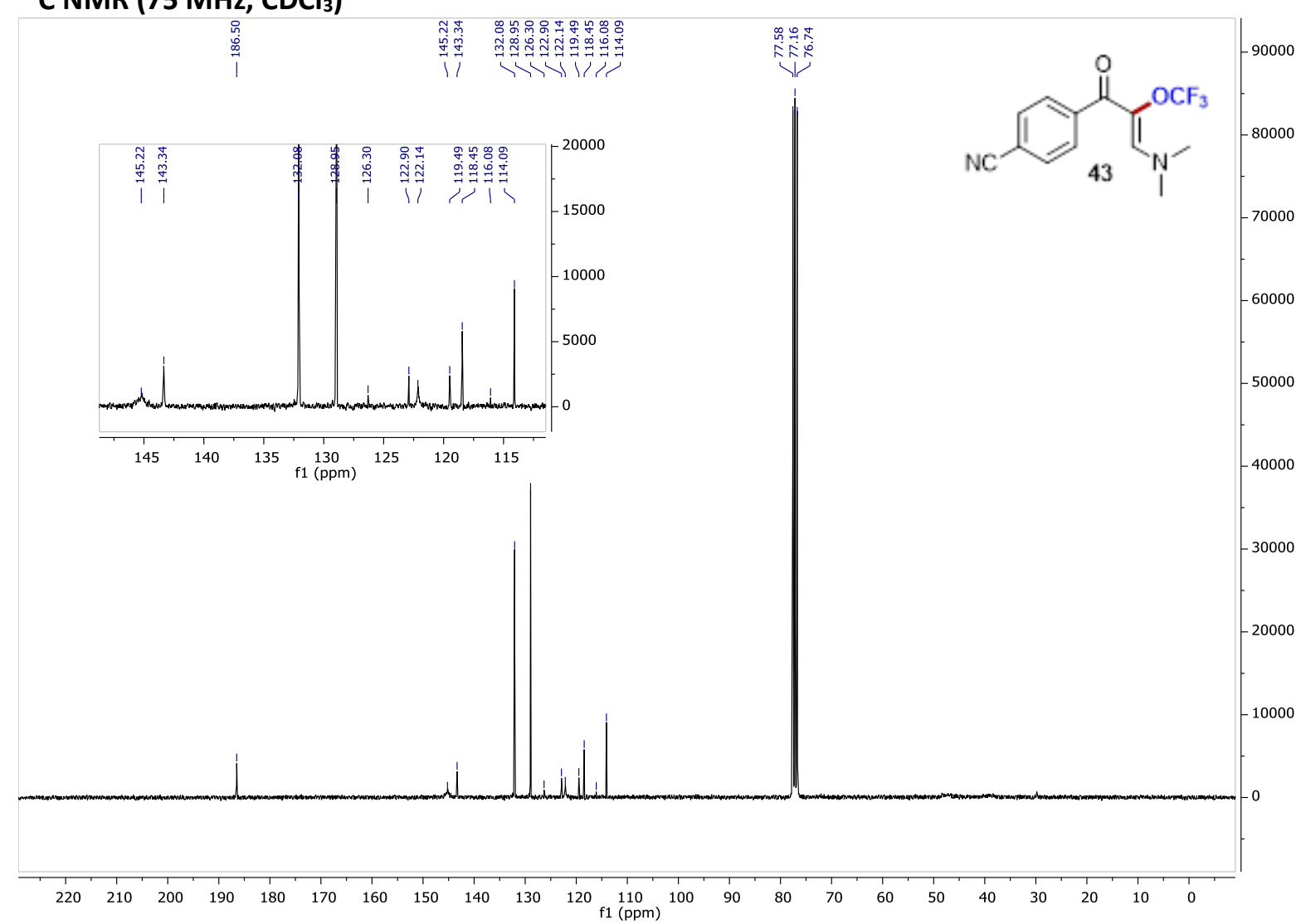

${ }^{19} \mathrm{~F} \mathrm{NMR} \mathrm{(188} \mathrm{MHz,} \mathrm{CDCl}_{3}$ )

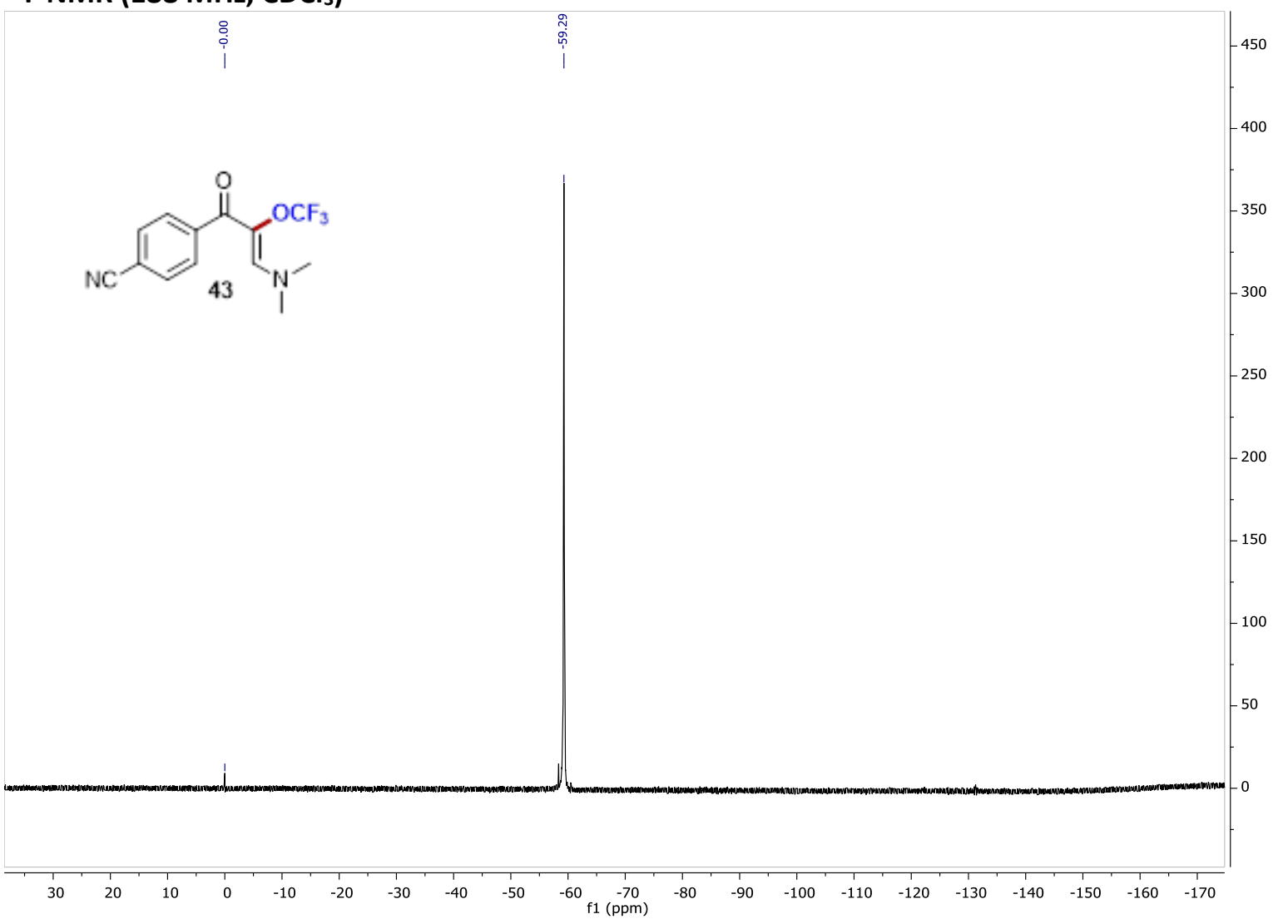

